University of Tennessee Health Science Center

UTHSC Digital Commons

\title{
Quantitative Analysis of First- versus Second-Premolar Extraction Effects in Orthodontic Treatment
}

Robert A. Turner

University of Tennessee Health Science Center

Follow this and additional works at: https://dc.uthsc.edu/dissertations

Part of the Orthodontics and Orthodontology Commons

\section{Recommended Citation}

Turner, Robert A. , "Quantitative Analysis of First- versus Second-Premolar Extraction Effects in Orthodontic Treatment" (2007). Theses and Dissertations (ETD). Paper 268. http://dx.doi.org/10.21007/ etd.cghs.2007.0326.

This Thesis is brought to you for free and open access by the College of Graduate Health Sciences at UTHSC Digital Commons. It has been accepted for inclusion in Theses and Dissertations (ETD) by an authorized administrator of UTHSC Digital Commons. For more information, please contact jwelch30@uthsc.edu. 


\title{
Quantitative Analysis of First- versus Second-Premolar Extraction Effects in Orthodontic Treatment
}

\author{
Abstract \\ Malocclusions can involve arch-size tooth-size discrepancies that have to be resolved by premolar \\ extractions. Treatment planning in such cases usually involves removing first (P1) or second premolars \\ (P2). Choosing an extraction pattern is a learned skill that incorporates many diagnostic factors. This \\ study compared the amounts of in-treatment tooth movement in two common extraction patterns (4/4 \\ and $5 / 5$ ) in order to gain insight into the criteria used for differential diagnosis and treatment planning. \\ Objective: A method of cast analysis was developed to measure the anteroposterior and transverse \\ changes in tooth positions during orthodontic treatment. Data were used (1) to assess how the extraction \\ space was used to resolve the malocclusion and (2) to identify pretreatment occlusal variables \\ associated with the amounts of orthodontic tooth movement. Methods: Dental casts of 90 orthodontic \\ cases with Class I malocclusions from American white adolescents were digitized (70 landmarks) at the \\ start and end of treatment to capture the in-treatment tooth movements. A battery of 20 pretreatment \\ occlusal variables (overjet, overbite, arch length, etc.) was used as predictor variables, and the in- \\ treatment changes in each tooth's position were the outcomes. Multivariate linear regression was used to \\ find pretreatment characteristics that predicted which premolars had been extracted. Results: Cases \\ treated with P1 extractions exhibited (1) greater incisor irregularity, (2) greater overjet, (3) more frequent \\ interdental spacing, (4) longer arch lengths (1-3 and 1-6), (5) greater overbite, and (6) broader arch widths \\ (3-3 and 6-6). Predictably, the occlusal variables in combination were more predictive than when studied \\ univariately. Only about half the extraction space was used to resolve anterior problems in the average P1 \\ case, and less was used in the average P2 case. Conclusions: Choosing an extraction pattern appropriate \\ to a malocclusion is a learned skill. This study quantifies some of those diagnostic characteristics that \\ specialists use to choose a premolar extraction pattern suited to a particular malocclusion.

\section{Document Type} \\ Thesis

\section{Degree Name} \\ Master of Dental Science (MDS)

\section{Program} \\ Orthodontics

\section{Research Advisor} \\ Edward F. Harris, PhD

\section{Keywords} \\ Orthodontics, Malocclusion, Tooth movement, Dental arch form, Extraction treatment, Premolar extraction

\section{Subject Categories} \\ Dentistry | Medicine and Health Sciences | Orthodontics and Orthodontology
}




\title{
QUANTITATIVE ANALYSIS OF FIRST- VERSUS SECOND-PREMOLAR EXTRACTION EFFECTS IN ORTHODONTIC TREATMENT
}

\author{
A Thesis \\ Presented for \\ The Graduate Studies Council \\ The University of Tennessee \\ Health Science Center
}

\author{
In Partial Fulfillment \\ Of the Requirements for the Degree \\ Master of Dental Science \\ From The University of Tennessee
}

By

Robert Andrew Turner, D.D.S

May 2007 
Copyright $@ 2007$ by Robert Andrew Turner All rights reserved 


\section{DEDICATION}

This thesis is dedicated in loving memory of my father

Robert Lee Turner

in recognition of his love, guidance and support

of my education 


\section{ACKNOWLEDGMENTS}

I would first like to thank my thesis committee, Dr. Quinton Robinson and Dr. Richard Williams for their guidance and support in making this project possible. The true hero of this thesis would have to be Dr. Ed Harris, who graciously spent many long hours helping and directing me through it. This thesis could have never been with out his assistance. I would also like to thank my wife, Diana, not only for her support, but for putting up with me as I remained frustrated, stayed up late and ignored other duties around the house to finish this to completion. And lastly I would like to give special recognition to my father, Robert Lee Turner, whose hard work, love, and many sacrifices allowed me the opportunity to attend college, dental school, and complete this program. 


\begin{abstract}
Malocclusions can involve arch-size tooth-size discrepancies that have to be resolved by premolar extractions. Treatment planning in such cases usually involves removing first (P1) or second premolars (P2). Choosing an extraction pattern is a learned skill that incorporates many diagnostic factors. This study compared the amounts of in-treatment tooth movement in two common extraction patterns $(4 / 4$ and $5 / 5)$ in order to gain insight into the criteria used for differential diagnosis and treatment planning. Objective: A method of cast analysis was developed to measure the anteroposterior and transverse changes in tooth positions during orthodontic treatment. Data were used (1) to assess how the extraction space was used to resolve the malocclusion and (2) to identify pretreatment occlusal variables associated with the amounts of orthodontic tooth movement. Methods: Dental casts of 90 orthodontic cases with Class I malocclusions from American white adolescents were digitized (70 landmarks) at the start and end of treatment to capture the in-treatment tooth movements. A battery of 20 pretreatment occlusal variables (overjet, overbite, arch length, etc.) was used as predictor variables, and the in-treatment changes in each tooth's position were the outcomes. Multivariate linear regression was used to find pretreatment characteristics that predicted which premolars had been extracted. Results:
\end{abstract}


Cases treated with P1 extractions exhibited (1) greater incisor irregularity, (2) greater overjet, (3) more frequent interdental spacing, (4) longer arch lengths (1-3 and 1-6), (5) greater overbite, and (6) broader arch widths (3-3 and 6-6). Predictably, the occlusal variables in combination were more predictive than when studied univariately. Only about half the extraction space was used to resolve anterior problems in the average P1 case, and less was used in the average P2 case. Conclusions: Choosing an extraction pattern appropriate to a malocclusion is a learned skill. This study quantifies some of those diagnostic characteristics that specialists use to choose a premolar extraction pattern suited to a particular malocclusion. 


\section{TABLE OF CONTENTS}

CHAPTER

PAGE

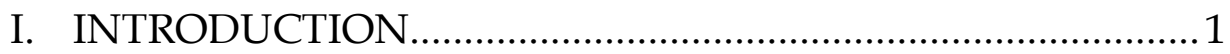

II. REVIEW OF THE LITERATURE ........................................... 4

Quantifying Extraction Decisions.................................... 4

First Premolar Extractions ...................................... 4

Second Premolar Extractions....................................6

Other Considerations .............................................. 8

Premolar Extraction Effects.......................................... 8

Incisor Angulation............................................... 10

Incisor versus Molar Changes ............................... 11

Incisor Retraction ...................................... 12

Molar Protraction...................................... 15

Temporary Implants for Anchorage....................... 17

Occlusal Plane........................................................ 17

Vertical Dimension................................................ 18

Arch Changes....................................................... 19

Arch Width .................................................. 19

Arch Depth .............................................. 21

Tooth Size-Arch Length Discrepancy ..................... 22

Tooth Size Discrepancy....................................... 23

Stability of Palatal Rugae................................................ 23

Current Use of the Microscribe GX2 ............................... 27

III. MATERIALS AND METHODS ............................................. 29

Sample Characteristics................................................. 29

Data Collection................................................................ 30

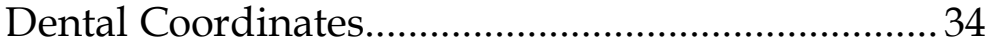

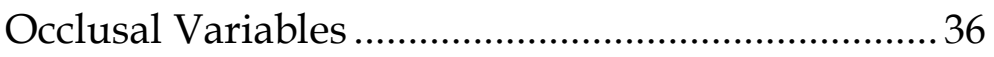

Tooth Designations ........................................................... 46

Statistical Analyses .................................................... 46

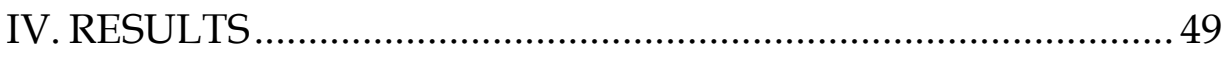

Occlusal Relationships...................................................49

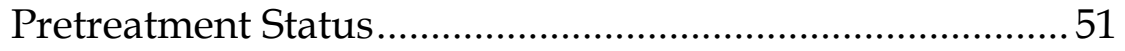

Mesiodistal Axis ................................................. 54

vii 


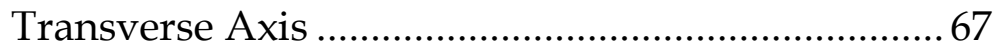

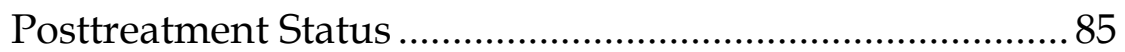

Mesiodistal Axis ……………………………….......... 86

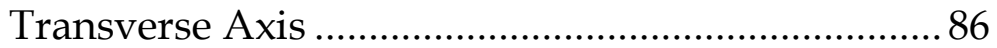

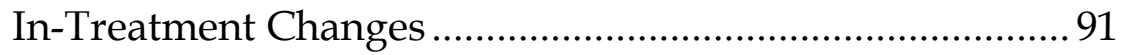

Transverse Changes ……………………………….... 91

Mesiodistal Changes ................................................... 97

Proxy Tooth Sizes............................................................... 100

Predicting Tooth Movements from the Malocclusion ....... 103

Complete Sample...................................................... 103

Incisors ....................................................... 106

Canine ......................................................... 124

First Premolar................................................. 131

Second Premolar …........................................ 135

First Molar .................................................... 142

Second Molar................................................... 151

First Premolar Extractions ........................................ 158

Central Incisor .............................................. 163

Lateral Incisor.............................................. 172

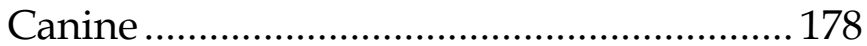

Second Premolar ........................................... 188

First Molar ...................................................... 192

Second Molar............................................... 202

Second Premolar Extraction ..................................... 211

Central Incisor ................................................. 211

Lateral Incisor............................................ 217

Canine .................................................... 224

First Premolar............................................... 231

First Molar .................................................... 236

Second Molar................................................. 249

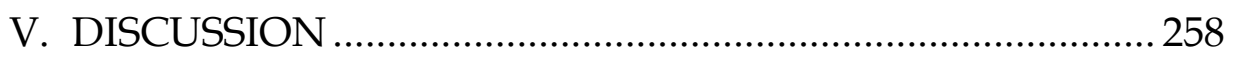

Complete Sample .............................................................. 260

Occlusal Characteristics ........................................... 260

Pretreatment Status..................................... 260

Posttreatment Status .................................... 261

Sexual Dimorphism ........................................ 262

In-Treatment Changes ................................... 263

Predicting Tooth Movements .................................... 264

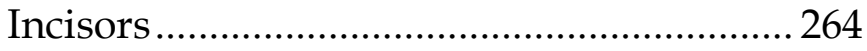


Canine .......................................................... 266

First Molar .................................................. 267

Second Molar.............................................. 269

First Premolar Sample ..................................................... 270

Predicting Tooth Movements ................................ 270

Incisors ................................................... 270

Canine ....................................................... 271

Molar........................................................... 272

Second Premolar Sample.............................................. 273

Predicting Tooth Movements .............................. 273

Incisors ....................................................... 273

Canine ..................................................... 274

Molar........................................................... 274

VI. SUMMARY AND CONCLUSIONS .................................. 276

LIST OF REFERENCES...................................................... 280

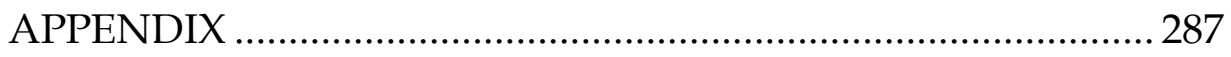

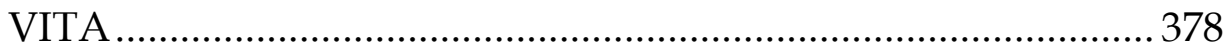




\section{LIST OF TABLES}

TABLE

PAGE

1. Occlusal conditions, by sex and group.................................................50

2. Assessments of proxy maxillary mesiodistal tooth dimensions, by extraction pattern, sex, and side.

3. Mesiodistal (X-axis) relationships at the pretreatment examination

4. Transverse (Y-axis) relationships at the pretreatment

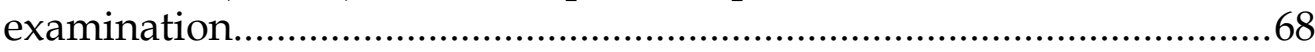

5. Mesiodistal (X-axis) relationships at the posttreatment

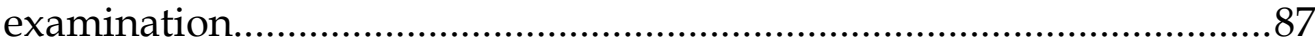

6. Transverse (Y-axis) relationships at the posttreatment examination

7. Descriptive statistics and one-sample tests for the change in the transverse (X-axis) arch dimension

8. Descriptive statistics and one-sample tests for the change in the mesiodistal (Y-axis) arch dimension

9. In-treatment changes in the mesiodistal (X-axis) relationships.

10. In-treatment changes in the transverse (Y-axis) relationships.

11. Listing of the occlusal and demographic variables available for selection into the stepwise multiple linear regression model 
12. Results of stepwise multiple regression analysis with the in-treatment change in the maxillary left central incisor (mesial) as the dependent variable

13. Results of stepwise multiple regression analysis with the in-treatment change in the maxillary right central incisor (mesial) as the dependent variable

14. Results of stepwise multiple regression analysis with the in-treatment change in the maxillary left central incisor (distal) as the dependent variable

15. Results of stepwise multiple regression analysis with the in-treatment change in the maxillary right central incisor (distal) as the dependent variable.

16. Results of stepwise multiple regression analysis with the in-treatment change in the maxillary left lateral incisor (mesial) as the dependent variable

17. Results of stepwise multiple regression analysis with the in-treatment change in the maxillary right lateral incisor (mesial) as the dependent variable

18. Results of stepwise multiple regression analysis with the in-treatment change in the maxillary left lateral incisor (distal) as the dependent variable

19. Results of stepwise multiple regression analysis with the in-treatment change in the maxillary right lateral incisor (distal) as the dependent variable.

20. Results of stepwise multiple regression analysis with the in-treatment change in the maxillary left canine (mesial) as the dependent variable

21. Results of stepwise multiple regression analysis with the in-treatment change in the maxillary right canine (mesial) as the dependent variable 126 
22. Results of stepwise multiple regression analysis with the in-treatment change in the maxillary left canine (distal) as the dependent variable

23. Results of stepwise multiple regression analysis with the in-treatment change in the maxillary right canine (distal) as the dependent variable

24. Results of stepwise multiple regression analysis with the in-treatment change in the maxillary left first premolar (mesial) as the dependent variable.

25. Results of stepwise multiple regression analysis with the in-treatment change in the maxillary right first premolar (mesial) as the dependent variable.....

26. Results of stepwise multiple regression analysis with the in-treatment change in the maxillary left first premolar (distal) as the dependent variable

27. Results of stepwise multiple regression analysis with the in-treatment change in the maxillary right first premolar (distal) as the dependent variable

28. Results of stepwise multiple regression analysis with the in-treatment change in the maxillary left second premolar (mesial) as the dependent variable.

29. Results of stepwise multiple regression analysis with the in-treatment change in the maxillary right second premolar (mesial) as the dependent variable

30. Results of stepwise multiple regression analysis with the in-treatment change in the maxillary left second premolar (distal) as the dependent variable

31. Results of stepwise multiple regression analysis with the in-treatment change in the maxillary right second premolar (distal) as the dependent variable 
32. Results of stepwise multiple regression analysis with the in-treatment change in the maxillary left first molar (mesial) as the dependent variable

33. Results of stepwise multiple regression analysis with the in-treatment change in the maxillary right first molar (mesial) as the dependent variable

34. Results of stepwise multiple regression analysis with the in-treatment change in the maxillary left first molar (distal) as the dependent variable

35. Results of stepwise multiple regression analysis with the in-treatment change in the maxillary right first molar (distal) as the dependent variable

36. Results of stepwise multiple regression analysis with the in-treatment change in the maxillary left second molar (mesial) as the dependent variable

37. Results of stepwise multiple regression analysis with the in-treatment change in the maxillary right second molar (mesial) as the dependent variable

38. Results of stepwise multiple regression analysis with the in-treatment change in the maxillary left second molar (distal) as the dependent variable

39. Results of stepwise multiple regression analysis with the in-treatment change in the maxillary right second molar (distal) as the dependent variable

40. Results of stepwise linear regression predicting the amount of mesiodistal tooth movement from the pretreatment malocclusion: results for maxillary left central incisor (mesial) in the subset in whom first premolars were extracted. 
41. Results of stepwise linear regression predicting the amount of mesiodistal tooth movement from the pretreatment malocclusion: results for maxillary right central incisor (mesial) in the subset in whom first premolars were extracted.

42. Results of stepwise linear regression predicting the amount of mesiodistal tooth movement from the pretreatment malocclusion: results for maxillary left central incisor (distal) in the subset in whom first premolars were extracted.

43. Results of stepwise linear regression predicting the amount of mesiodistal tooth movement from the pretreatment malocclusion: results for maxillary right central incisor (distal) in the subset in whom first premolars were extracted.

44. Results of stepwise linear regression predicting the amount of mesiodistal tooth movement from the pretreatment malocclusion: results for maxillary left lateral incisor (mesial) in the subset in whom first premolars were extracted....

45. Results of stepwise linear regression predicting the amount of mesiodistal tooth movement from the pretreatment malocclusion: results for maxillary right lateral incisor (mesial) in the subset in whom first premolars were extracted.

46. Results of stepwise linear regression predicting the amount of mesiodistal tooth movement from the pretreatment malocclusion: results for maxillary left lateral incisor (distal) in the subset in whom first premolars were extracted.

47. Results of stepwise linear regression predicting the amount of mesiodistal tooth movement from the pretreatment malocclusion: results for maxillary right lateral incisor (distal) in the subset in whom first premolars were extracted. 
48. Results of stepwise linear regression predicting the amount of mesiodistal tooth movement from the pretreatment malocclusion: results for maxillary left canine (mesial) in the subset in whom first premolars were extracted.

49. Results of stepwise linear regression predicting the amount of mesiodistal tooth movement from the pretreatment malocclusion: results for maxillary right canine (mesial) in the subset in whom first premolars were extracted.

50. Results of stepwise linear regression predicting the amount of mesiodistal tooth movement from the pretreatment malocclusion: results for maxillary left canine (distal) in the subset in whom first premolars were extracted.

51. Results of stepwise linear regression predicting the amount of mesiodistal tooth movement from the pretreatment malocclusion: results for maxillary right canine (distal) in the subset in whom first premolars were extracted.

52. Results of stepwise linear regression predicting the amount of mesiodistal tooth movement from the pretreatment malocclusion: results for maxillary left second premolar (mesial) in the subset in whom first premolars were extracted

53. Results of stepwise linear regression predicting the amount of mesiodistal tooth movement from the pretreatment malocclusion: results for maxillary right second premolar (mesial) in the subset in whom first premolars were extracted

54. Results of stepwise linear regression predicting the amount of mesiodistal tooth movement from the pretreatment malocclusion: results for maxillary left second premolar (distal) in the subset in whom first premolars were extracted 
55. Results of stepwise linear regression predicting the amount of mesiodistal tooth movement from the pretreatment malocclusion: results for maxillary right second premolar (distal) in the subset in whom first premolars were extracted

56. Results of stepwise linear regression predicting the amount of mesiodistal tooth movement from the pretreatment malocclusion: results for maxillary left first molar (mesial) in the subset in whom first premolars were extracted.

57. Results of stepwise linear regression predicting the amount of mesiodistal tooth movement from the pretreatment malocclusion: results for maxillary right first molar (mesial) in the subset in whom first premolars were extracted

58. Results of stepwise linear regression predicting the amount of mesiodistal tooth movement from the pretreatment malocclusion: results for maxillary left first molar (distal) in the subset in whom first premolars were extracted

59. Results of stepwise linear regression predicting the amount of mesiodistal tooth movement from the pretreatment malocclusion: results for maxillary right first molar (distal) in the subset in whom first premolars were extracted.

60. Results of stepwise linear regression predicting the amount of mesiodistal tooth movement from the pretreatment malocclusion: results for maxillary left second molar (mesial) in the subset in whom first premolars were extracted

61. Results of stepwise linear regression predicting the amount of mesiodistal tooth movement from the pretreatment malocclusion: results for maxillary right second molar (mesial) in the subset in whom first premolars were extracted 
62. Results of stepwise linear regression predicting the amount of mesiodistal tooth movement from the pretreatment malocclusion: results for maxillary left second molar (distal) in the subset in whom first premolars were extracted.

63. Results of stepwise linear regression predicting the amount of mesiodistal tooth movement from the pretreatment malocclusion: results for maxillary right second molar (distal) in the subset in whom first premolars were extracted.

64. Results of stepwise linear regression predicting the amount of mesiodistal tooth movement from the pretreatment malocclusion: results for maxillary right central incisor (mesial) in the subset in whom second premolars were extracted

65. Results of stepwise linear regression predicting the amount of mesiodistal tooth movement from the pretreatment malocclusion: results for maxillary left central incisor (mesial) in the subset in whom second premolars were extracted

66. Results of stepwise linear regression predicting the amount of mesiodistal tooth movement from the pretreatment malocclusion: results for maxillary left central incisor (distal) in the subset in whom second premolars were extracted

67. Results of stepwise linear regression predicting the amount of mesiodistal tooth movement from the pretreatment malocclusion: results for maxillary right central incisor (distal) in the subset in whom second premolars were extracted

68. Results of stepwise linear regression predicting the amount of mesiodistal tooth movement from the pretreatment malocclusion: results for maxillary left lateral incisor (mesial) in the subset in whom second premolars were extracted 
69. Results of stepwise linear regression predicting the amount of mesiodistal tooth movement from the pretreatment malocclusion: results for maxillary right lateral incisor (mesial) in the subset in whom second premolars were extracted.

70. Results of stepwise linear regression predicting the amount of mesiodistal tooth movement from the pretreatment malocclusion: results for maxillary left lateral incisor (distal) in the subset in whom second premolars were extracted.

71. Results of stepwise linear regression predicting the amount of mesiodistal tooth movement from the pretreatment malocclusion: results for maxillary right lateral incisor (distal) in the subset in whom second premolars were extracted 223

72. Results of stepwise linear regression predicting the amount of mesiodistal tooth movement from the pretreatment malocclusion: results for maxillary left canine (mesial) in the subset in whom second premolars were extracted 225

73. Results of stepwise linear regression predicting the amount of mesiodistal tooth movement from the pretreatment malocclusion: results for maxillary right canine (mesial) in the subset in whom second premolars were extracted 226

74. Results of stepwise linear regression predicting the amount of mesiodistal tooth movement from the pretreatment malocclusion: results for maxillary left canine (distal) in the subset in whom second premolars were extracted

75. Results of stepwise linear regression predicting the amount of mesiodistal tooth movement from the pretreatment malocclusion: results for maxillary right canine (distal) in the subset in whom second premolars were extracted 
76. Results of stepwise linear regression predicting the amount of mesiodistal tooth movement from the pretreatment malocclusion: results for maxillary left first premolar (mesial) in the subset in whom second premolars were extracted 233

77. Results of stepwise linear regression predicting the amount of mesiodistal tooth movement from the pretreatment malocclusion: results for maxillary right first premolar (mesial) in the subset in whom second premolars were extracted

78. Results of stepwise linear regression predicting the amount of mesiodistal tooth movement from the pretreatment malocclusion: results for maxillary left first premolar (distal) in the subset in whom second premolars were extracted

79. Results of stepwise linear regression predicting the amount of mesiodistal tooth movement from the pretreatment malocclusion: results for maxillary right first premolar (distal) in the subset in whom second premolars were extracted

80. Results of stepwise linear regression predicting the amount of mesiodistal tooth movement from the pretreatment malocclusion: results for maxillary left first molar (mesial) in the subset in whom second premolars were extracted

81. Results of stepwise linear regression predicting the amount of mesiodistal tooth movement from the pretreatment malocclusion: results for maxillary right first molar (mesial) in the subset in whom second premolars were extracted

82. Results of stepwise linear regression predicting the amount of mesiodistal tooth movement from the pretreatment malocclusion: results for maxillary left first molar (distal) in the subset in whom second premolars were extracted 
83. Results of stepwise linear regression predicting the amount of mesiodistal tooth movement from the pretreatment malocclusion: results for maxillary right first molar (distal) in the subset in whom second premolars were extracted

84. Results of stepwise linear regression predicting the amount of mesiodistal tooth movement from the pretreatment malocclusion: results for maxillary left second molar (mesial) in the subset in whom second premolars were extracted 250

85. Results of stepwise linear regression predicting the amount of mesiodistal tooth movement from the pretreatment malocclusion: results for maxillary right second molar (mesial) in the subset in whom second premolars were extracted

86. Results of stepwise linear regression predicting the amount of mesiodistal tooth movement from the pretreatment malocclusion: results for maxillary left second molar (distal) in the subset in whom second premolars were extracted.

87. Results of stepwise linear regression predicting the amount of mesiodistal tooth movement from the pretreatment malocclusion: results for maxillary right second molar (distal) in the subset in whom second premolars were extracted 


\section{LIST OF FIGURES}

FIGURE

PAGE

1. Maxillary dental arch showing locations of (1) the two anatomic contacts on each of the 14 permanent teeth excluding third molars and (2) a mesial and distal point along the midpalatal raphe used to define the midline of the maxillary arch

2. Maxillary dental arch showing the landmarks used in second-premolar extraction cases

3. Maxillary dental arch showing the landmarks used in first-premolar extraction cases.

4. Lateral view of the central incisors, showing the method of measuring overjet, measured parallel with Downs' occlusal plane.

5. Lateral view of the central incisors, showing the method of measuring overbite, measured perpendicular to Downs' occlusal plane.

6. Lateral view of the permanent first molars, showing the method of measuring the buccal segment relationship

7. Lateral view of the canines, showing the method of measuring canine discrepancy, which is the horizontal (parasagittal) deviation of the mandibular canine's cusp tip relative to the canine-first premolar embrasure

8. Diagram of the labial view of the central incisors, showing the method of measuring the deviation of the maxillary and mandibular dental midlines 
9. Diagrammatic illustration of a maxillary dental arch, showing the manner that, with sliding calipers, the incisor-to-canine (1-3) and the incisor-to-molar (1-6) arch chords were measured.

10. Incisor irregularity is the summation of the five distances between the anatomic contacts of the anterior six teeth

11. Plot of the mesiodistal (X axis) coordinates at the pretreatment examination showing the sexual dimorphism in arch length and, as well, greater arch length in the first-premolar extraction sample. 55

12. First-premolar extraction group of males at the pretreatment examination

13. Second-premolar extraction group of males at the pretreatment examination

14. First-premolar extraction group of females at the pretreatment examination

15. Second-premolar extraction group of females at the pretreatment examination

16. Comparison of the first-premolar extraction samples of males and females at the pretreatment examination

17. Comparison of the second-premolar extraction samples of males and females at the pretreatment examination

18. Comparison of the first- and second-premolar extraction samples of males at the pretreatment examination.

19. Comparison of the first- and second-premolar extraction samples of females at the pretreatment examination

20. Mean in-treatment mesiodistal $(\mathrm{Y})$ change, by group and side, for the distal landmark on the second molar 
21. Mean in-treatment mesiodistal ( $\mathrm{Y}$ ) change, by group and side, for the mesial landmark on the second molar.

22. Mean in-treatment mesiodistal $(\mathrm{Y})$ change, by group, and side, for the distal landmark on the first molar

23. Mean in-treatment mesiodistal $(\mathrm{Y})$ change, by group, and side, for the mesial landmark on the first molar.

24. Mean in-treatment mesiodistal $(\mathrm{Y})$ change, by group, and side, for the distal landmark on the second premolar.

25. Mean in-treatment mesiodistal $(\mathrm{Y})$ change, by group, and side, for the mesial landmark on the second premolar.

26. Mean in-treatment mesiodistal $(\mathrm{Y})$ change, by group, and side, for the distal landmark on the first premolar.

27. Mean in-treatment mesiodistal $(\mathrm{Y})$ change, by group, and side, for the mesial landmark on the first premolar. 78

28. Mean in-treatment mesiodistal $(\mathrm{Y})$ change, by group, and side, for the distal landmark on the canine

29. Mean in-treatment mesiodistal ( $Y$ ) change, by group, and side, for the mesial landmark on the canine

30. Mean in-treatment mesiodistal $(Y)$ change, by group, and side, for the distal landmark on the lateral incisor

31. Mean in-treatment mesiodistal $(\mathrm{Y})$ change, by group, and side, for the mesial landmark on the lateral incisor

32. Mean in-treatment mesiodistal $(\mathrm{Y})$ change, by group, and side, for the distal landmark on the central incisor

33. Mean in-treatment mesiodistal $(\mathrm{Y})$ change, by group, and side, for the mesial landmark on the central incisor. 84 
34. Scatterplot showing the positive association between pretreatment arch chord (1-3) and the amount of retraction of the maxillary left central incisor (mesial).

35. Scatterplot showing the positive association between pretreatment canine relationship and the amount of retraction of the maxillary left central incisor (mesial)

36. Scatterplot showing the positive association between pretreatment interdental spacing and the amount of retraction of the maxillary left central incisor (mesial)

37. Scatterplot showing the positive association between pretreatment maxillary arch chord (1-6) and the amount of retraction of the maxillary left central incisor (distal)

38. Scatterplot showing the negative association between pretreatment incisor irregularity and the amount of retraction of the maxillary left lateral incisor (mesial)

39. Scatterplot showing the positive association between pretreatment overbite and mesiodistal change of the maxillary left lateral incisor (mesial)

40. Scatterplot showing the positive association between pretreatment overbite and the amount of retraction of the maxillary left lateral incisor (distal).

41. Bar chart showing the greater retraction of the canine (left, distal contact) in the first-premolar sample (P4s) versus the sample treated with second-premolar extractions (P5s)

42. Scatterplot showing the positive association between pretreatment incisor irregularity and the amount of retraction of the maxillary right central canine (distal)

43. Scatterplot showing the negative association between pretreatment overjet and the amount of retraction of the maxillary right first premolar (mesial) 134 
44. Scatterplot showing the positive association between pretreatment canine relationship and the amount of retraction of the maxillary right first premolar (distal)....

45. Scatterplot showing the positive association between incisor irregularity and the amount of retraction of the maxillary left second premolar (mesial)

46. Scatterplot showing the negative association between pretreatment arch chord (1-6) and the amount of mesial movement of the maxillary right second premolar (mesial)

47. Scatterplot showing the positive association between pretreatment incisor irregularity and the amount of mesial movement of the maxillary right first molar (mesial)

48. Scatterplot showing the positive association between pretreatment canine relationship and the amount of mesial movement of the maxillary left first molar (mesial)

49. Scatterplot showing the positive association between pretreatment incisor irregularity and the amount of mesial movement of the maxillary left first molar (distal).

50. Scatterplot showing the positive association between pretreatment overbite and the amount of mesial movement of the maxillary left first molar (distal)

51. Scatterplot showing the positive association between pretreatment arch chord (1-6) and the amount of mesiodistal movement of the maxillary left second molar (mesial)

52. Scatterplot showing the positive association between pretreatment incisor overbite and the amount of mesial movement of the maxillary right second molar (distal).... 
53. Scatterplot showing the association between pretreatment spacing and the amount of mesiodistal change of the maxillary right central incisor (mesial) analyzed in just the first-premolar extraction sample

54. Scatterplot showing the association between pretreatment overjet and the amount of mesiodistal change of the maxillary right central incisor (mesial) analyzed in just the first-premolar extraction sample.

55. Scatterplot showing the association between pretreatment intercanine width and the amount of mesiodistal change of the maxillary right central incisor (mesial) analyzed in just the first-premolar extraction sample

56. Scatterplot showing the association between pretreatment overbite and the amount of mesiodistal change of the maxillary right central incisor (distal) analyzed in just the first-premolar extraction sample.

57. Scatterplot showing the association between pretreatment overbite and the amount of mesiodistal change of the maxillary left lateral incisor (distal) analyzed in just the first-premolar extraction sample 179

58. Scatterplot showing the association between pretreatment spacing and the amount of mesiodistal change of the maxillary left canine (mesial) analyzed in just the first-premolar extraction sample....

59. Scatterplot showing the association between pretreatment spacing and the amount of mesiodistal change of the maxillary right canine (mesial) analyzed in just the first-premolar extraction sample.

60. Scatterplot showing the association between pretreatment spacing and the amount of mesiodistal change of the maxillary left canine (distal) analyzed in just the first-premolar extraction sample. 186 
61. Scatterplot showing the association between pretreatment spacing and the amount of mesiodistal change of the maxillary right canine (distal) analyzed in just the first-premolar extraction sample.

62. Scatterplot showing the association between pretreatment maxillary 1-6 chord distance and the amount of mesiodistal change of the maxillary right second premolar (mesial) analyzed in just the first-premolar extraction sample.

63. Scatterplot showing the association between pretreatment incisor irregularity and the amount of mesiodistal change of the maxillary left first molar (mesial) analyzed in just the first-premolar extraction sample....

64. Scatterplot showing the association between maxillary 1-6 arch chord and the amount of mesiodistal change of the maxillary right first molar (mesial) analyzed in just the first-premolar extraction sample.

65. Scatterplot showing the association between maxillary 1-3 chord distance and the amount of mesiodistal change of the maxillary left second molar (mesial) analyzed in just the first-premolar extraction sample.

66. Scatterplot showing the association between maxillary 1-6 chord and the amount of mesiodistal change of the maxillary right second molar (mesial) analyzed in just the first-premolar extraction sample. 206

67. Scatterplot showing the association between incisor irregularity and the amount of mesiodistal change of the maxillary left second molar (distal) analyzed in just the first-premolar extraction sample.

68. Scatterplot showing the association between pretreatment maxillary 1-3 chord distance and the amount of mesiodistal change of the maxillary right central incisor (mesial) analyzed in just the second-premolar extraction sample 
69. Scatterplot showing the association between pretreatment incisor irregularity and the amount of mesiodistal change of the maxillary right central incisor (distal) analyzed in just the second-premolar extraction sample

70. Scatterplot showing the association between pretreatment incisor overbite and the amount of mesiodistal change of the maxillary right lateral incisor (mesial) analyzed in just the second-premolar extraction sample

71. Scatterplot showing the association between pretreatment overjet and the amount of mesiodistal change of the maxillary right canine (mesial) analyzed in just the second-premolar extraction sample

72. Scatterplot showing the association between pretreatment maxillary incisor irregularity and the amount of mesiodistal change of the maxillary right canine (distal) analyzed in just the second-premolar extraction sample

73. Scatterplot showing the association between pretreatment overjet and the amount of mesiodistal change of the maxillary right first premolar (mesial) analyzed in just the second-premolar extraction sample

74. Scatterplot showing the association between pretreatment canine discrepancy and the amount of mesiodistal change of the maxillary left first premolar (distal) analyzed in just the second-premolar extraction sample

75. Scatterplot showing the association between pretreatment canine discrepancy and the amount of mesiodistal change of the maxillary left first molar (mesial) analyzed in just the second-premolar extraction sample

76. Scatterplot showing the association between pretreatment maxillary incisor irregularity and the amount of mesiodistal change of the maxillary right first molar (mesial) analyzed in just the second-premolar extraction sample 
77. Scatterplot showing the association between pretreatment canine discrepancy and the amount of mesiodistal change of the maxillary left first molar (distal) analyzed in just the second-premolar extraction sample

78. Scatterplot showing the association between pretreatment incisor irregularity and the amount of mesiodistal change of the maxillary right first molar (distal) analyzed in just the second-premolar extraction sample

79. Scatterplot showing the association between pretreatment maxillary 6-6 width and the amount of mesiodistal change of the maxillary left second molar (mesial) analyzed in just the second-premolar extraction sample

80. Scatterplot showing the association between pretreatment overjet and the amount of mesiodistal change of the maxillary right second molar (mesial) analyzed in just the second-premolar extraction sample

81. Scatterplot showing the association between pretreatment canine discrepancy distance and the amount of mesiodistal change of the maxillary right second molar (distal) analyzed in just the second-premolar extraction sample 


\section{CHAPTER I}

\section{INTRODUCTION}

The use of premolar extractions for orthodontic treatment is historically controversial (Proffit 2000). This controversy continues today, and the decision to include premolar extraction in a patient's treatment is one of the most important decisions an orthodontist faces. This is further complicated by the fact that the relative efficacy of extraction or nonextraction strategies, in either the short or long term, has yet to be fully established (Weintraub et al. 1996).

Previous work has demonstrated that premolars are the most common teeth removed for orthodontic treatment (Proffit 1994). Conveniently located between the anterior and posterior segments, premolars would appear to be the obvious choice for correcting crowding and anterior-posterior discrepancies.

The predominant reason to seek orthodontic treatment is dental crowding and malalignment. These malocclusions commonly are due to tooth crown dimensions exceeding the available space of the supporting alveolar arches. One strategy for correcting these discrepancies is to move the teeth labially and buccally and flare them (Weinberg 1996). However, this can expand the teeth beyond the bony limits, which can adversely affect esthetics, function, and stability. To avoid expansion an alternative is to remove teeth to 
create space that resolves the tooth size-arch length discrepancies (TSALD).

Removing premolars can also be used to treat orthodontic conditions other than TSALD. It is unlikely that all the space provided by premolar extraction (ca. $15 \mathrm{~mm}$ per arch) is needed to correct a patient's TSALD. Different premolar extraction patterns provide space in different locations of the arches. For example, this extra space can be used to reduce protrusion or to camouflage skeletal Class II or Class III problems. An extraction pattern can also be selected that removes abnormally small or large premolars that contribute to a Bolton discrepancy (Proffit 2000).

During orthodontic treatment involving the extraction of teeth, arch dimensional changes occur and these dimensions can continue to change following active treatment (Ong and Woods 2001). This is further complicated by, to date; the literature fails to provide a predicable guide to predicting the use of maxillary extraction spaces (Schoppe 1964; Williams and Hosila 1976). Williams and Hosila (1976) showed varying amounts of molar and incisor movement during extraction space closure for first-premolar extraction cases.

Once the extraction decision has been made there are several factors that influence how the teeth are aligned in the arches. Differing treatment mechanics employ a range of force systems and appliances to correct the malocclusion. Aside from techniques used by the orthodontist, are there any variables that influence how the teeth move during treatment? Are there any 
unique occlusal characteristics of the malocclusion that may influence how the teeth move? There is a great range of variation present in each classification of malocclusion. The literature is inundated with information concerning posttreatment occlusal relationships and how this influences stability (e.g., Sadowsky et al. 1994; Kahl-Nieke et al. 1996; Housley et al. 2003). However, little is known concerning how these pretreatment relationships affect movement during treatment. 


\section{CHAPTER II \\ REVIEW OF THE LITERATURE}

\section{Quantifying Extraction Decisions}

When comparing the different treatment philosophies among orthodontists, one can find a great range of techniques, appliances, and axillaries. A common variant among orthodontists is the frequency of which the orthodontist determines that cases require premolar extractions for treatment. It is estimated that one-third of all orthodontic patients have such a severe malocclusion that some pattern of premolar extraction is deemed necessary to resolve the problems and align the teeth (Proffit 2000). TSALD is the most important factor necessitating the decision to extract premolars (Baumrind et al. 1996). Differential diagnosis involves determining whether first- or second-premolars should be extracted in the maxilla and/or in the mandible. Basic guidelines towards choosing premolar extraction patterns are available in textbooks (e.g., Moyers 1973; Proffit 2000), but more detailed factors for consideration are available in the primary orthodontic literature.

\section{First Premolar Extractions}

General guidelines suggest extracting first premolars when the TSALD source area is primarily in the anterior portion of the arch. Removing the first 
premolars is a straightforward way to correct anterior crowding, excessive overjet and protrusion. This correction works by making space for the alignment of teeth or the retraction of canines and incisors. Extracting premolars close to the area of crowding is beneficial because at the point when crowding or protrusion is corrected, little extraction space remains to be closed (Schoppe 1964; Graber 1972; Dewel 1973; Moyers 1973; Proffit 2000).

Other indications for a first premolar extraction pattern instead of a second premolar extraction pattern include overbite, malocclusion type, and serial extraction therapy. Brandt and Safirstein (1975) stated that placing the extraction site closer to the anterior gives a mechanical advantage in leveling the arch as space is closed. This advantage is helpful when treating patients with a deep bite. Creekmore (1997) said that, as a rule of thumb, he preferred extracting maxillary first premolars for non-surgical treatments of nongrowing Class II, division 2 malocclusions. He specified that in these cases he treated the mandible without premolar extraction. Dewel (1973) specified one more reason for first premolar extractions over second premolar extractions. He stated that when treating a young patient with serial extraction, who will require the subsequent removal of permanent teeth, the first premolars should be removed so that erupting canines can more easily drift distally into the extraction space. 


\section{Second Premolar Extractions}

The basic indication for second premolar extraction is when there is moderate anterior crowding with no protrusion and the patient has good facial balance (Dewel 1955; Graber 1972; Dewel 1973; Moyers 1973; Brandt and Safirstein 1975; Creekmore 1997; Proffit 2000). Some subjectivity of these guidelines is shown because de Castro (1974) describes this instance of "moderate" crowding as being when there is a TSALD of $5 \mathrm{~mm}$ or more, while Schoppe (1964) describes it as being a TSALD of $7.5 \mathrm{~mm}$ or less. Either way, removing the second premolars will give enough space to resolve minor crowding while not changing the profile. It also leaves the incisors in their original positions over basal bone without inclining them labially, which is undesirable (Dewel 1955; Schoppe 1964).

Other considerations for removing second premolars instead of first premolars include posterior crowding, anterior openbite, Class III correction, and facilitation of intentional anchorage slippage. When second or third molars are crowded, ectopic, or impacted, they can be helped by increasing space in the posterior segments. This space is created by extracting second premolars so that the first molar can move mesially (Logan 1973; de Castro 1974). This extraction pattern also is advantageous for correcting anterior openbites. This effect is seen because it is easier to accentuate the curve of Spee and lessen relapse of the open bite after treatment (Brandt and Safirstein 
1975). Also, by reducing the posterior vertical dimension, an immediate increase in anterior overbite is accomplished, facilitating closure of an open bite (Logan 1973). Second-premolar extraction in the maxilla can also be helpful in camouflaging Class III malocclusions when combined with firstpremolar extraction in the mandible. This pattern allows more retraction of the lower incisors while allowing more mesial maxillary molar movement to correct the malocclusion (Schoppe 1964).

Intentional anchorage slippage can be greatly facilitated by the extraction of second premolars. This is desirable when there is excess extraction space remaining after TSALD resolution and the patient has good facial harmony. De Castro (1974) specifies that when needing to move the molars forward more than $2.5 \mathrm{~mm}$ on each side, a second premolar extraction pattern is indicated. By removing second premolars instead of first premolars, first molars are easily slipped forward instead of necessitating unwanted retraction of the anterior teeth to close the remaining space (Dewel 1955; Schoppe 1964; Dewel 1973; Logan 1973; de Castro 1974). This occurs not only because the teeth mesial to the first molars are removed, giving a clear path of movement, but because the weaker anterior anchorage is increased from six to eight teeth, thereby producing more resistance (Dewel 1973). 


\section{Other Considerations}

Individual tooth conditions should also be considered before deciding which premolars to extract. When teeth are carious, ankylosed, or impacted, special consideration should be given to extracting these teeth instead of other healthy teeth. Also, when teeth in the arch are congenitally missing, this can affect extraction pattern, because an equivalent tooth should be extracted to maintain left-right arch symmetry (Brandt and Safirstein 1975).

\section{$\underline{\text { Premolar Extraction Effects }}$}

Much has been written over the years about changes that occur with premolar extractions. Premolar extraction treatment is well described because of its widespread usage. Keim et al. (2002) reported from a survey of 789 orthodontists that over $95 \%$ had extracted teeth in the last year. Virtually every orthodontist extracts premolars in some situations as it greatly helps with crowding, protrusion, retraction, vertical problems, and other factors (Proffit 2000). When reviewing pretreatment records for 148 subjects, a group of orthodontist showed a $65 \%$ agreement when asked if premolars needed to be extracted or not (Baumrind et al. 1996). The debate concerning premolar extraction today does not center on whether it should be done, but, rather, on which malocclusions require it and which teeth should be extracted in a given situation. 
However, history shows that this was not always the trend. At the end of the nineteenth century, Edward Angle staunchly opposed all extraction therapy because he thought every person had the potential for the ideal relationship of all his teeth. This theory led to his use of expansion appliances and rubber bands to achieve overbite and overjet that accommodated all of the teeth. An article of faith for him was that a correct occlusion would result in maximum esthetics and stability. He wrote that if the final placement of the teeth was not stable, it simply pointed to the orthodontist's failure to position the teeth in an ideal occlusion and not to any failure of his theory (Proffit 2000:250). In the 1920s, Calvin Case debated Angle's student Martin Dewey over this issue and proposed the need for extraction treatment, but nonextraction sentiment continued to prevail into the late 1930s and 40s. By the 1940s, the propensity for relapse in nonextraction treatment caused many clinicians to reconsider the extraction question. Charles Tweed (1944:406) stated that:

I also maintain that when a discrepancy between tooth pattern and basal bone does exist, it is far better to remove dental units to bring about a balance between tooth anatomy and basal bone; and that if this correction is made, our patients will be benefited by a nearer approach to the normal than is possible if we retain all the dental units and in so doing are compelled to displace all the teeth off the dental ridge and into protrusion. 


\section{Incisor Angulation}

With the increased space available in the arch following the extraction of a pair of premolars, the orthodontist may use some or all of that space to correct anterior tooth inclinations and relationships. Bishara, Cummins and Zaher (1997) contrasted groups of Class II, division 1 patients treated by extraction or nonextraction. They concluded that treatment plans including first premolar extraction result in a significantly more obtuse interincisal angle. However, no description was given of the amount of crowding, overjet, or overbite with which the patients initially presented.

Carter (1988) showed that the interincisal angle increased 22 degrees in a group of first premolar extraction patients treated with edgewise appliances. There was also no account given of starting conditions for these patients. Bishara et al. (1995) found in a study of Class II patients treated with first-premolar extraction, that the increase in this angle was 4.3 degrees in males and 12.3 degrees in females. Conversely, there was a 4.6 degree decrease in males and a 1.1 degree decrease in females treated without extractions. The improvement seen in extraction groups is the result of changes that occur both to the upper and lower incisors as they are retracted several millimeters and retroclined (Darendeliler and Taner-Sarisoy 2001).

Ong and Woods (2001) conducted a cephalometric and cast study comparing first and second premolar extraction patterns in the maxilla. 
Relating the maxillary incisors to an A-Pogonion reference line, they showed that a significant reduction in incisor protrusion and proclination was noted regardless of which maxillary premolar was extracted. However, the extraction pattern was relevant as there was a maxillary incisor angulation reduction of 8.2 degrees in the first premolar extraction group compared to an angulation reduction of 3.3 degrees in the second premolar extraction group. Because the malocclusions that were selected for the first or second premolar extraction pattern were of different types and severity these data are not conclusive.

Over the years, much has been written about protrusion and the final axial inclination of the mandibular incisors and the influence this has on relapse. Many have written on the disadvantages and likely hood of relapse if the mandibular incisors are protruded from their original position (Nance 1947; Brodie 1938; Cole 1948; Weinberg and Sadowsky 1996). However, Freitas et al. 2004, found that mandibular incisor inclination and linear protrusion had no influence on crowding relapse.

\section{Incisor versus Molar Changes}

Using a compressive force between the anterior and posterior segments to close space from a premolar extraction will affect teeth in both regions to different degrees. This difference occurs because large multi-rooted teeth 
resist forces more than single-rooted anterior teeth with ovoid cross-sections (Jepsen 1963). The result is that molars have more of a bodily movement, while the incisor crowns tend to move more than their roots as a combination of bodily movement and also tipping. This combination results in more anterior retraction than would occur with bodily movement alone (Williams and Hosila 1976).

Incisor Retraction

If the extraction area is closer to the anterior region, a greater amount of incisor retraction can occur. This statement has been borne out by several researchers to varying degrees (Williams and Hosila 1976; Steyn et al. 1997; Ong and Woods 2001). First-premolar extractions have been measured to give an average $4.7 \mathrm{~mm}$ of incisor retraction in the maxilla in relation to the facial plane (Nasion-Pogonion), while second-premolar extractions yielded a similar $4.2 \mathrm{~mm}$ mean incisor retraction (Steyn et al. 1997). Ong and Woods (2001) studied treatment groups containing a mixture of Class I and II patients. They found more significant differences with a maxillary incisor retraction of 4.2 $\mathrm{mm}$ related to the A-Pogonion line in the first-premolar extraction group and only a $2.3 \mathrm{~mm}$ incisor retraction in the second-premolar extraction group. Using the palatal anatomy as a reference point and the Begg technique for treatment, Williams and Hosila (1976) found combined upper and lower 
retraction to be $10.3 \mathrm{~mm}$ in a four-first-premolar extraction group. They concluded that when taking out first premolars, “... approximately 66.5 per cent of the available extraction space was occupied by retracted anterior segments." This agrees with Creekmore's (1997) rule of thumb that you use two-thirds of first premolar extraction space for incisor retraction and for the correction of crowding, and the other one-third is used by the posterior teeth. Extraction patterns involving a combination of maxillary first premolars and mandibular second premolars are too variable in incisor retraction amounts to make significant conclusions. A mean of $9.3 \mathrm{~mm}$ of combined retraction was found in an upper first and lower second premolar extraction group by Williams and Hosila (1976). Ong and Woods (2001) recorded $3.7 \mathrm{~mm}$ of maxillary incisor retraction in this instance, while Steyn, du Preez and Harris (1997) recorded $6.6 \mathrm{~mm}$ of maxillary incisor retraction.

Incisor retraction can be greatly affected by the use of headgear, because it reduces the amount of anchorage that is lost during retraction of the incisors and preserves more of the extraction space for retraction. Schwab (1963) states in his study, that the use of headgear can increase the attainable amount of incisor retraction in second premolar cases up to the level of first premolar extraction cases without headgear. In the previously mentioned research on incisor retraction, none of the treatment groups used headgear, but there are several more studies reporting incisor retraction in which 
headgear use is a factor. Cusimano, McLaughlin, and Zernik (1993) studied a group of Class I or II high-angle patients with four first premolars extracted. Using the contour of the palate as a reference, they found there was an average $1.9 \mathrm{~mm}$ of maxillary incisor retraction. Carter (1988), using the Nasion-Pogonion reference line to the maxillary incisal edge, recorded $8.2 \mathrm{~mm}$ of incisor retraction in Class II first premolar extraction cases. Measuring to the incisor apex, he recorded only $0.6 \mathrm{~mm}$ of retraction. Bishara et al. (1995) showed a mean retraction of $4.6 \mathrm{~mm}$ in the maxillary anterior in Class II first premolar extraction cases using A-Pogonion as a reference. The Nasion-A line was used as a reference by Luppanapornlarp and Johnston (1993). They found a mean of $2.8 \mathrm{~mm}$ of maxillary incisor retraction in a group of Class II patients treated with first premolar extractions.

The limitation of using these studies to evaluate the use of extraction space is based on the fact that all these samples were not comparable at the start of treatment. The extraction patterns used were not randomized, but based upon what changes the clinicians were intending to develop. Thus, the differential treatments should lead to differences in incisor retraction amounts if the orthodontists are competent. However, taking these limitations into account, the careful observer can still get some glimpse into how much TSALD can be resolved by different treatments. These limitations are also 
true of the studies described in the following section on molar protraction as it is directly related to incisor retraction.

\section{Molar Protraction}

In contrast to retraction that occurs in the anterior segment is a mesial movement that occurs in the posterior segment with the molars. Less study has been done on the movement of these teeth than the incisors, but some data have been collected. In premolar extraction groups without the use of headgear, Ong and Woods (2001) compared amounts of molar protraction. The four first premolar extraction group was made up of mostly Class II subjects, while the four second premolar extraction group was almost entirely Class I in molar relationship. They found that the mean forward movement of the maxillary first molar ranged from $3.7 \mathrm{~mm}$ in four first premolar extraction groups to $4.5 \mathrm{~mm}$ in four second premolar extraction groups. Comparing this movement to the retraction of incisors in those cases revealed that $80 \%$ of the time, the molars experienced greater movement than the incisors, and thus, the majority of space created by extraction was filled by mesial movement of the molars. They found that this occurred most frequently in the patients who had decreased crowding and natural spacing between teeth. Williams and Hosila (1976) recorded that without headgear use, the combined upper and lower posterior protraction was $5.2 \mathrm{~mm}$ in their four first premolar extraction 
group and $7.2 \mathrm{~mm}$ in their group with upper first and lower second premolar extractions. These results showed more anchorage loss occurred when the extraction sites were more posterior.

Like incisor retraction, headgear may have an effect on the amount of molar protraction recorded as shown by Ashmore et al. (2002). Using palatal rugae as fiducial landmarks, they recorded that the maxillary first-molar moved an average of $2.2 \mathrm{~mm}$ distally in a Class II treatment group using headgear. They showed that molars move mesially almost a full millimeter over a 24 month period in the absence of treatment. Combining these two findings, an adjusted mean molar distalization of $3.0 \mathrm{~mm}$ is found when headgear is used. This result shows why headgear use must be considered in these comparisons, as it can drastically decrease molar protraction.

Other studies using various treatments with headgear include Carter, (1988) who, using a perpendicular to Sella-Nasion reference line found a 3.8 $\mathrm{mm}$ maxillary molar mesialization in a group treated with first premolar extractions and headgear. Counter-intuitively, he found in another premolar extraction group treated with the Begg appliance and no headgear that the maxillary molar mesialization was quite similar at $3.4 \mathrm{~mm}$. Luppanapornlarp and Johnston (1993) used a vertical reference line to Pterygoid Vertical and found a $2.4 \mathrm{~mm}$ molar protraction in patients treated with first premolar extraction. Cusimano, McLaughlin and Zernik (1993) recorded a $5.0 \mathrm{~mm}$ 
maxillary molar mesialization in a high angle Class I or II first premolar extraction group using the palate contour as a reference.

\section{Temporary Implants for Anchorage}

More recently, the use of implants as anchorage devices has allowed greater control of the maxillary molars. Thiruvenkatachari et al. (2006) used a split mouth design to demonstrate no mesial movement of the maxillary firstmolar on the side with the microimplant during canine retraction. On the control side the maxillary first-molar moved in a mesial direction $1.6 \mathrm{~mm}$. They determined the anchorage loss using superimposed lateral cephalograms measured at Pterygoid Vertical.

\section{Occlusal Plane}

It is controversial whether a change to the inclination of the occlusal plane occurs secondary to premolar extraction. No difference has been observed clinically although it has been theorized. Sheppe (1969), using plastic teeth arranged in wax arch forms to represent ideal dentitions, predicted occlusal changes from premolar extraction by carrying out the extractions and treatment on the teeth in the wax. He stated there should be a change in the occlusal plane due to retraction of the mandibular anterior region. His prediction showed that, as the incisors are retracted, the crowns 
move more lingually than the roots, so the teeth become more upright. This uprighting causes Downs' occlusal plane to tip superiorly in the anterior. Cephalometric studies show that this change never takes place, at least to any clinically significant level (Darendelier and Taner-Sarisoy 2001). This is in agreement with Luppanapornlarp and Johnston (1993) who found that there was no significant change in occlusal plane as related to the Sella-Nasion line in extraction and nonextraction groups.

\section{Vertical Dimension}

Research on the vertical dimension in premolar extraction situations is currently limited to cephalometric studies. Staggers (1994) and Kocadereli (1999) investigated what differences occur in the vertical dimension between nonextraction groups and extraction groups. Vertical changes occurring after first premolar extractions were no different from those observed in nonextraction cases. In both groups, an increase in the vertical dimension was found. As Staggers noted, "Most of orthodontic mechanics are extrusive in nature, and this extrusion appears to maintain or even increase the vertical dimension." The fact that extrusion occurred equivalently in both groups seems to place doubt as to whether premolar extraction can be linked to TMJ disorders (Staggers 1994). 


\section{Arch Changes}

Various changes take place in arch form during orthodontic treatment. Depending on the sort of treatment, there can be increase or decrease in arch width, and arch length.

\section{$\underline{\text { Arch Width }}$}

Using the standard edgewise appliance, the clinician can expect some expansion of the arch width, because the wire is engaged into bracket slots that are on the labial or buccal sides of the teeth. Orthodontists have suggested that, with premolar extraction treatment, the removal of teeth negates this expansion and leads to a "collapse" of the arch, yielding narrower intercanine, interincisal, and intermolar widths. Research directed on arch width has not agreed with the collapse premise. In several studies, not only has it been shown that there is an increase in arch width in extraction cases, but that there is more arch width expansion when the patient is treated with premolar extractions than when they are not (Bishara et al. 1994; O'Higgins and Lee 2000; Gianelly 2003). How premolar extraction could lead to arch width expansion is hard to explain, but O'Higgins and Lee (2000) and Gianelly (2003) consider that it is probably related to moving the canines distally into a larger arch diameter where the premolars were while maintaining intermolar width. Along these lines, Gianelly (2003) and Bishara 
et al. (1994) showed that there is a significant increase in intercanine width in extraction cases compared to nonextraction cases, but they differed in the amount of change they reported in intermolar width. However, the relevancy of these findings is diminished since the groups are not comparable in the amount of change needed. Gianelly (2003) used orthodontic dental casts to compare Class I and II patients treated with the extraction of four first premolars to Class I, II, and III patients treated with no extraction. He found that there was an overall increase in intercanine width in both groups with mandibular intercanine width in the extraction group being significantly more than the nonextraction group. He found no significant change in the intermolar width between the extraction and nonextraction groups.

Bishara et al. (1994) measured casts on Class II patients treated with four first premolar extractions and found a significantly greater increase in maxillary and mandibular intercanine width in that group compared to a Class II group treated without extractions. Net intercanine expansion was 3.2 $\mathrm{mm}$ in males and $3.1 \mathrm{~mm}$ in females, compared to only a $0.5 \mathrm{~mm}$ and $2.0 \mathrm{~mm}$ expansion, respectively, in the nonextraction group. They found a net decrease in intermolar width in the extraction group while the nonextraction group had an increase in this dimension. Whether or not arch width expansion remains after treatment is a different matter, and this aspect has been explored by Vaden et al. (1997). They found by cast measurements that 
although the arches become narrower with age, there still was an overall increase in arch width in premolar extraction cases up to 15 years posttreatment. Erdinc et al. (2006) compared arch width changes for firstpremolar extraction cases and non-extraction cases. They found that intercanine width increased significantly for both groups at the end of treatment and both had a significant decrease in intercanine width from the posttreatment value at a mean time of 2 years postretention. Interestingly, they found a greater increase in intercanine width during treatment for the non-extraction group.

\section{$\underline{\text { Arch Depth }}$}

An extraction pattern with premolar extractions will result in a mesiodistal tooth structure loss of around $15 \mathrm{~mm}$ per arch. This will result in a decrease of the anteroposterior dimension of the arch depth, but to what extent? All of the studies reviewed concluded that there was a significant reduction in arch depth following premolar extractions (Bishara et al. 1994, 1997; Vaden et al. 1997; O'Higgins and Lee 2000). O'Higgins and Lee (2000) found that arch depth reduces to a greater extent than even the mesiodistal width of the premolars based on orthodontic cast studies and Vaden and coworkers (1997) stated that the arches continually lose arch length with age. These results indicate that overjet and anteroposterior changes are not directly 
coordinated with mesiodistal tooth structure loss, because other factors such as arch shape, width, and age must be considered.

\section{Tooth Size-Arch Length Discrepancy}

The difference between tooth structure and arch length available to support it is a relevant concern for the orthodontist. It would be beneficial to understand how premolar extractions affect this discrepancy during and after treatment, because treatment decisions are affected by this factor. Bishara et al. (1994) confirmed what was already supposed when they concluded that extracting premolars significantly improves the discrepancy between tooth size and arch length. Using cast measurements, it was found in another study that there was a gradual increase in TSALD in both extraction and nonextraction treatment groups following the end of treatment as both groups have stability problems (Bishara et al. 1997). O'Higgins and Lee (2000) theorized from conducting a study using plastic teeth in wax arch forms that the arch length space available after premolar extractions should be greater than even the size of the premolars removed due to the phenomenon of retracting the incisors into a larger arch diameter as was discussed above. 


\section{Tooth Size Discrepancy}

Tooth-size discrepancy is a disharmony between mesiodistal widths of the maxillary and mandibular arch as described by Bolton (1958). A study by Saatci and Yukay (1997) on 100 patients with no pretreatment tooth-size discrepancy used Bolton's analysis to determine if the removal of first or second premolars would produce any discrepancy. They showed that an average $1.25 \mathrm{~mm}$ tooth-size discrepancy resulted from first premolar extraction treatments in 31 of 50 patients. In second premolar extraction treatments, only a mean $0.84 \mathrm{~mm}$ tooth-size discrepancy resulted in 17 of 50 patients. It should be noted that this study was not done by comparing pretreatment and posttreatment casts, but rather by simulating extractions on the pretreatment casts by using a computer program to compute the resulting discrepancies.

\section{$\underline{\text { Stability of Palatal Rugae }}$}

An area in which it is important to describe change during premolar extractions is the palate. Its importance comes not in how it affects making treatment decisions, but in the way it may be used as a landmark for dental research. Lysell (1955) developed a classification system for the palatal rugae pairs, and, using a symmetrograph, described their general stability. His study did not include changes that occur during orthodontic treatment, but it 
did describe the effects that extractions without treatment have on the rugae. He concluded that "extractions have a local but no general effect on the direction of the rugae." The uniqueness and overall stability of the rugae suggested their use for forensics and even general anthropological studies of paternity determination. This appreciation for the rugae as unique and stable landmarks is further substantiated as English et al. (1988) concluded that the palatal rugae pattern is sufficiently characteristic to discriminate between individuals; but they found it was legitimate to base identification on their comparison.

Many studies have been completed showing that rugal landmarks are applicable for dental research specifically. Van der Linden (1978) concluded that little change takes place in rugae length and interruga distance through growth using the occlusal plane as a reference. He stated that the rugae points demonstrate "... remarkable stability in their anteroposterior relationships to each other and lend themselves quite well to the analysis of the changes in mesiodistal locations in buccal teeth in normal developing dental arches." This result was based on cast and cephalometric measurements in children from 6 to 16 years old without orthodontic treatment. In another smaller group of treated subjects, he did the same analysis and found that orthodontic treatment can influence rugae position, particularly anteroposterior distances between medial and lateral points of the same rugae. Most of the changes 
were due to movement in the lateral and anterior rugae points only, so the posterior medial rugae points were advocated as stable landmarks for research involving orthodontic treatment. This finding was similar to Hausser's study (1950) that described rugae movement after extraction of teeth. He observed the lateral parts moved half the distance that the teeth moved, while the medial parts did not seem to move at all (cited in Lysell 1955). The stability of the medial points over the lateral points was also observed by Peavy and Kendrick (1967) who said that, the "... closer the rugae are to the teeth, the more prone they are to 'stretch' in the direction that their associated teeth move." Almeida et al. (1995) demonstrated in a cast study that these medial rugae points were stable in both the transverse and sagittal planes and useful for longitudinal cast analysis even when the patients were treated with headgear or functional appliances.

In addition to these findings of the importance of using medial points, has been the finding that the more posterior rugae are less susceptible to changes with tooth movement. In a study of patients treated with maxillary first premolar extractions it was concluded that rugae in the canine area were unstable moving $1.94 \mathrm{~mm}$ on average, but the most posterior rugae averaged only $0.19 \mathrm{~mm}$ of movement and were stable (Peavy and Kendrick 1967). In another study, orthodontic treatment was shown to change rugae position in the sagittal dimension more in the anterior region (Almeida et al. 1995). This 
difference was demonstrated because there was an increased anteroposterior change in medial points between the first and second pair of rugae, going from anterior to posterior, compared to between the second and third pair. The shortcoming of this data is that it cannot be determined which rugae points were moving to cause this change.

Although posterior rugae are more stable in general, it is the third palatal rugae pair in particular that has been shown to be the most stable reference for evaluating transverse and anteroposterior change. Bailey, Esmailnejad and Almeida (1996) studied palatal rugae changes in adults undergoing both extraction and nonextraction treatment and concluded that the third palatal rugae points are best for evaluating tooth movement in a linear, transverse, and anteroposterior direction, regardless of treatment method. Hoggan and Sadowsky (2001) used cephalometry to evaluate the anteroposterior measurements that were derived by using palatal rugae. Their findings also suggested the accuracy of using the mesial point of the third pair of rugae. They concluded that measuring sagittal distances by relating the teeth to this landmark was as accurate as measurements from a cephalogram and suggested that to determine sagittal anchorage loss, “... progress dental casts can be used instead of a lateral cephalometric radiograph." 
When using palatal rugae points as landmarks, their association with the occlusal plane is important. The occlusal plane is often used as a reference plane to relate the teeth to the rugae, but it often changes during growth and orthodontic treatment. Van der Linden (1978) has shown that a 3 degree decrease in the occlusal plane results in a distal displacement of $1 \mathrm{~mm}$ for a molar point being related to a medial ruga point on the occlusal plane. This change occurs because the perpendicular lines dropped to the occlusal plane are separated as the plane rotates counter-clockwise causing increased distance between the molar and the ruga point. The increased distance makes it appear that the molars have distalized. This distalization would mask some of the mesial movement of molars if a correction were not made for the change in occlusal plane.

The summation of this research on rugae suggests the use of medial points of the third (distal) palatal rugae as stable landmarks for determining tooth movement.

\section{Current Use of the Microscribe GX2}

Recently, investigators have sought an accurate means of collecting data from dental casts in both two and three dimensions and a direct way of imputing the data into computer for analyzing. In literature, the Microscribe has been used to fulfill both the needs. Camporesi et al. (2006) used the 
Microscribe to analyze arch forms in a Southern European population. Dental casts were made and the coordinates of the facial height of contour of each tooth were taken with the Microscribe and imputed into Rhinoceros. This allowed them to compare arch forms of natural ideal occlusions to commercially available preformed arch wires. Yoa et al. (2005) also incorporated the Microscribe digitizer and the program Rhinoceros into their study of maxillary molar intrusion with mini-implant anchorage. In this study, the amount of intrusion was measured for overerupted maxillary molars by entering the coordinates of the pre- and posttreatment maxillary cusp tips and superimposing on the marked palatal rugae 


\section{CHAPTER III \\ MATERIALS AND METHODS}

\section{$\underline{\text { Sample Characteristics }}$}

A sample of 90 completed orthodontic cases was collected from the archived records of the graduate orthodontic program at the University of Tennessee, Memphis. Fifty-four of the subjects are female and the remaining 36 are male. Cases were selected at random that met the following three criteria, (1) all permanent teeth were present and fully-erupted, disregarding third molars (and second molars at the pretreatment examination), (2) each case was maintained in a Class I buccal segment relationship, both pre- and posttreatment, and (3) each case was treated with the extraction of premolars, one per each quadrant. Two groups were compared based on the premolar extraction pattern. In 50 cases, the first premolars had been extracted in each quadrant; in the other 40 cases, the second premolars had been extracted. These two extraction patterns constituted the major comparison in this study, namely, what occlusal characteristics differed between the first- and secondpremolar extraction groups at pretreatment that colored the orthodontist's decision to remove one set over the other? 


\section{$\underline{\text { Data Collection }}$}

Tooth movements from pre- to posttreatment were measured from the maxillary dental casts as Cartesian coordinates using a MicroScribe G2X 3D digitizing instrument (Immersion Corporation, San Jose, CA).

There are several steps here. First, the idealized mesial and distal contacts of each of the 14 permanent teeth (ignoring third molars) is marked as seen in occlusal view. These landmarks are illustrated for the pretreatment arrangement (Fig. 1), the posttreatment situation in which first premolars had been extracted (Fig. 2), and the posttreatment situation in which second premolars had been extracted (Fig. 3). Then the cast is held rigid in the dental surveyor mount, which is immobile relative to the MicroScribe, and the stylus is touched to each landmark in turn. This digitizer is comparable to a CAD/CAM instrument and accurate to $0.23 \mathrm{~mm}$. Depressing the instrument's foot pedal sends the $X, Y$, and $Z$ coordinates of that point to the computer. As described below, coordinates of 64 landmarks were collected on each cast. Coordinates of homologous landmarks were collected on each subject's preand posttreatment dental cast. Data collection was driven by a computer program titled Rhinoceros 2.0 (Robert McNeel \& Associates, Seattle, WA). Intreatment changes in the $\mathrm{X}$ (mesiodistal), $\mathrm{Y}$ (mesiolateral), and $\mathrm{Z}$ 


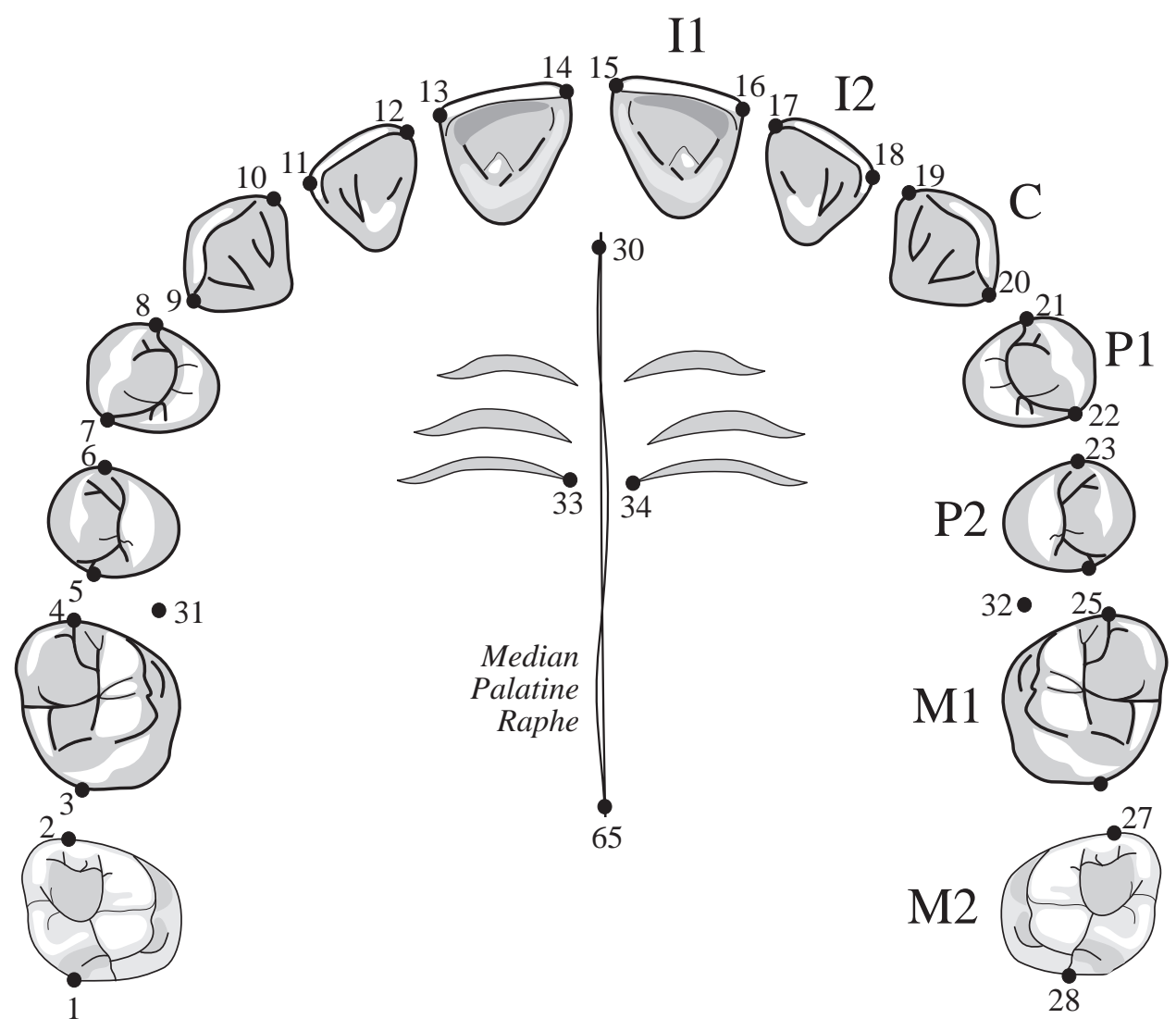

Fig. 1. Maxillary dental arch showing locations of (1) the two anatomic contacts on each of the 14 permanent teeth excluding third molars and (2) a mesial and distal point along the midpalatal raphe used to define the midline of the maxillary arch. Landmarks 31 and 32 are positioned on the gingival in the embrasure between P2 and M1 and are used to orient the dental cast. Teeth are displaced to more clearly show the landmarks digitized on each dental cast. 


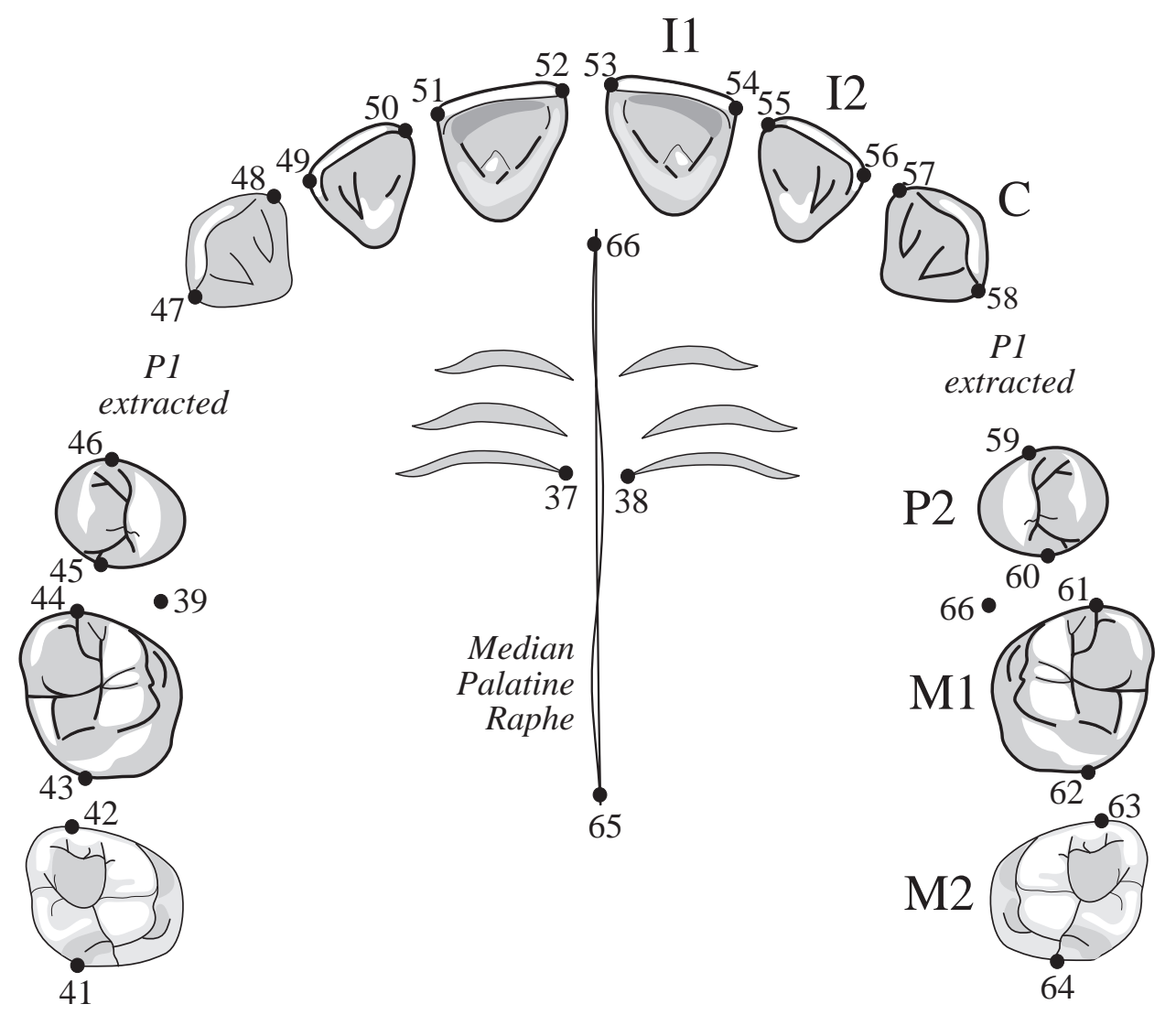

Fig. 2. Maxillary dental arch showing the landmarks used in secondpremolar extraction cases. There are two anatomic contacts on each of the 12 permanent teeth and a mesial and distal point along the midpalatal raphe used to define the midline of the maxillary arch. Teeth are displaced to more clearly show the landmarks digitized on each dental cast. 


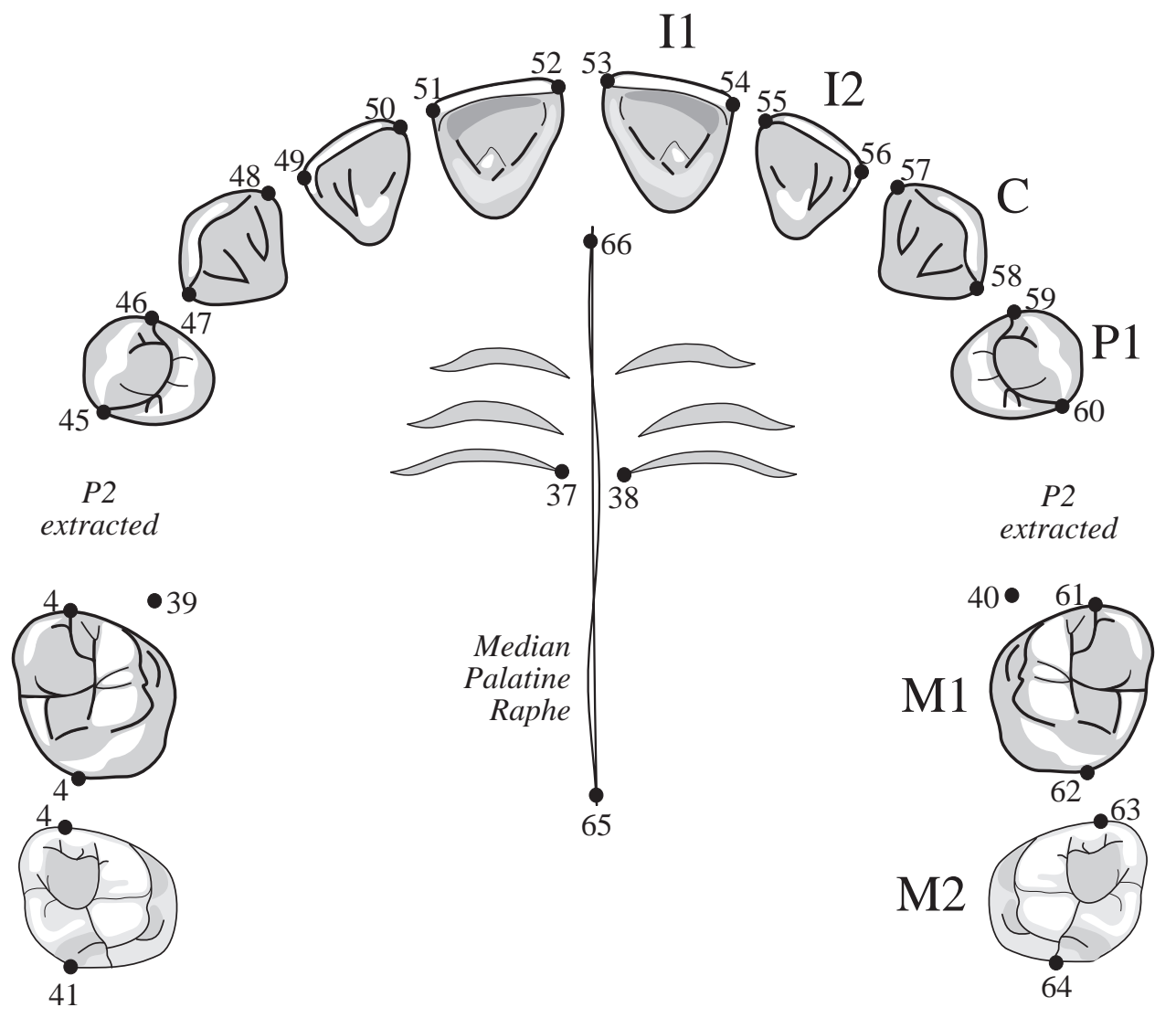

Fig. 3. Maxillary dental arch showing the landmarks used in firstpremolar extraction cases. There are two anatomic contacts on each of the 12 permanent teeth and a mesial and distal point along the midpalatal raphe used to define the midline of the maxillary arch. Teeth are displaced to more clearly show the landmarks digitized on each dental cast. 
(craniocaudal) axes were computed trigonometrically and stored in Excel spreadsheets.

\section{Dental Coordinates}

Analysis was limited to changes in the maxilla where the medial endpoints of the third pair of palatal rugae were used as fiducial landmarks against which tooth movements were quantified (van der Linden 1978; Almeida et al. 1995; Bailey et al. 1996; Hoggan and Sadowsky 2001). Accuracy of landmark identification was improved by wearing $3 X$ dental loupes and by using a mechanical pencil with $0.5 \mathrm{~mm}$ lead to mark the landmarks on the casts. The following sets of landmarks were marked on the pre- and posttreatment casts. These landmarks are illustrated in Figs. 1, 2 and 3.

1. The mesial and distal limits of the midpalatal raphe, which are used to define the midline of the dental arch (Points 29-30; 65-66).

2. The idealized distal and mesial contact was marked from second molar to second molar. These points were identified first on the posttreatment casts and were often coincident with the actually posttreament contact points. These same points were then marked on the pretreatment casts. Points are located independently in the left and right quadrants (Points 1-28 and 41$64)$.

3. The medial limits of the distal pair of palatal rugae were marked. Based on prior analysis (e.g., Almeida et al. 1995; Bailey et al. 1996), the distal rugae 
are most stable during orthodontic treatment, so the medial limits of the distal left-right pair of rugae are used as the fiducial points against which tooth movements are measured (Points 33-34 and 37-38).

4. Points were also marked in the palatal embrasure area between the first molar and second premolar for use as additional reference positions as needed (Points 31-32 and 39-40).

After the casts were placed in the rigid dental surveyor mount, the origin was established, where the value of zero was assigned to the $X, Y$, and $\mathrm{Z}$ axes. The origin was assigned, using the MicroScribe GX2 3D, by marking the mesial contact point of the central incisors. Rhinoceros 2.0 requires that the $\mathrm{X}$ and $\mathrm{Y}$ axes are defined in their respective planes. The $\mathrm{Y}$ axis was established at the mesiobuccal cusp of the maxillary right first-molar. The $\mathrm{X}$ axis was established at midpoint between the mesiobuccal cusps of the maxillary first-molars. To determine the midpoint, a rigid ruler was used to measure the distance between the cusps and then the midpoint was assigned at the height of the cusps.

After the pre- and posttreatment points were marked and then were entered into Rhinoceros 2.0 using the MicroScribe, the points were superimposed using the medial limit of the distal rugae, assigned points numbered $34-38$ on the pre- and posttreatment casts. Rhinoceros 2.0 allows the points from either cast to be moved in unison, without altering the point- 
to-point relationship. To superimpose the pre and posttreatment casts, the marked points from the posttreatment casts were moved in the $X, Y$, and $Z$ axes in order to superimpose the medial limit of the distal palatal rugae from both casts. Every attempt was made to find the "best fit" of the palatal rugae, using the mesial and distal limits of the midpalatal raphe to aid in alignment when needed. By moving only the posttreatment points, the pretreatment zero values for $\mathrm{X}, \mathrm{Y}$, and $\mathrm{Z}$ were maintained.

All of the in-treatment changes in the $X, Y$, and $Z$ axes were calculated as posttreatment value minus pretreatment value. Consequently, positive values denote increases, and negative values denote decreases (diminished arch dimensions).

\section{Occlusal Variables}

Data were collected concerning the nature and extent of the malocclusion. The maxillary and mandibular casts were occluded in maximum intercuspation, and six variables were measured.

1. incisor overjet

2. incisor overbite

3. incisor midline discrepancy

4. buccal segment relationship of the first molars

5. canine relationship 
6. incisor irregularity of the maxillary incisors

Definitions of these variables are listed below. Measurements were made in millimeters using digital-readout sliding calipers (Chicago Brand Industrial, Inc., Fremont, CA).

Incisor overjet (Baume et al. 1973; Smith and Bailit 1977) is the horizontal distance (parallel with the occlusal plane) between the facial surface of the most protrusive maxillary central incisor and the facial of the corresponding mandibular incisor (Fig 4). In practice, the depth gauge on the calipers was used to obtain the distance.

Incisor overbite (Baume et al. 1973) was the distance perpendicular to the occlusal plane that the incisal edge of the maxillary central incisor overlapped the mandibular central incisor (Fig 5). In practice, a light pencil mark was placed on the facial surface of the mandibular incisor where the incisal limit of the upper incisor was located, then the maxillary cast was removed and the distance from the pencil mark occlusally to the incisal edge of the tooth was measured. If there was left-right asymmetry, the side with greater overbite was measured.

Buccal segment relationship, abbreviated as BSR (Baume et al. 1973) is horizontal distance (parallel with the occlusal plane) between the mesiobuccal cusp of the maxillary first molar and the buccal groove of the mandibular first 


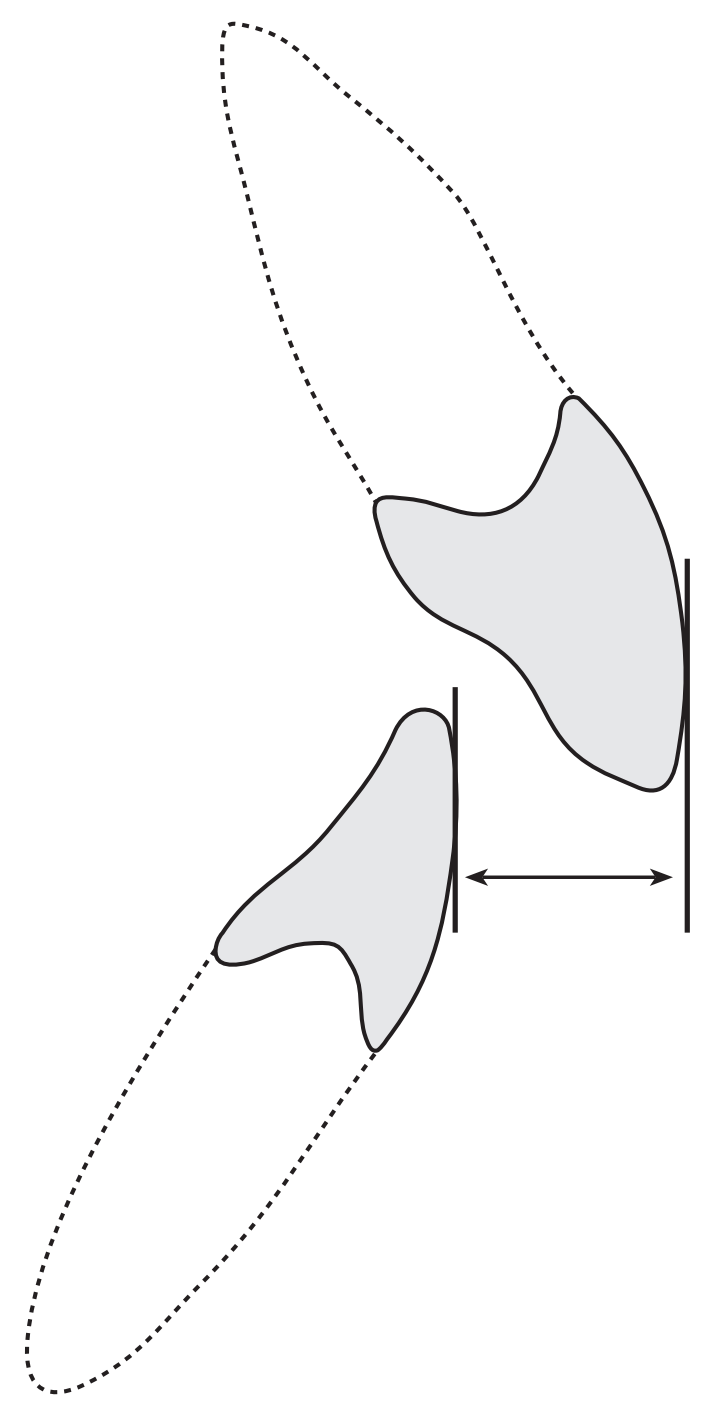

Fig. 4. Lateral view of the central incisors, showing the method of measuring overjet, measured parallel with Downs' occlusal plane. The horizontal distance was measured with sliding calipers. If the incisors are in crossbite, the measured value w2ould be negative. (Figure provided by E. F. Harris.) 


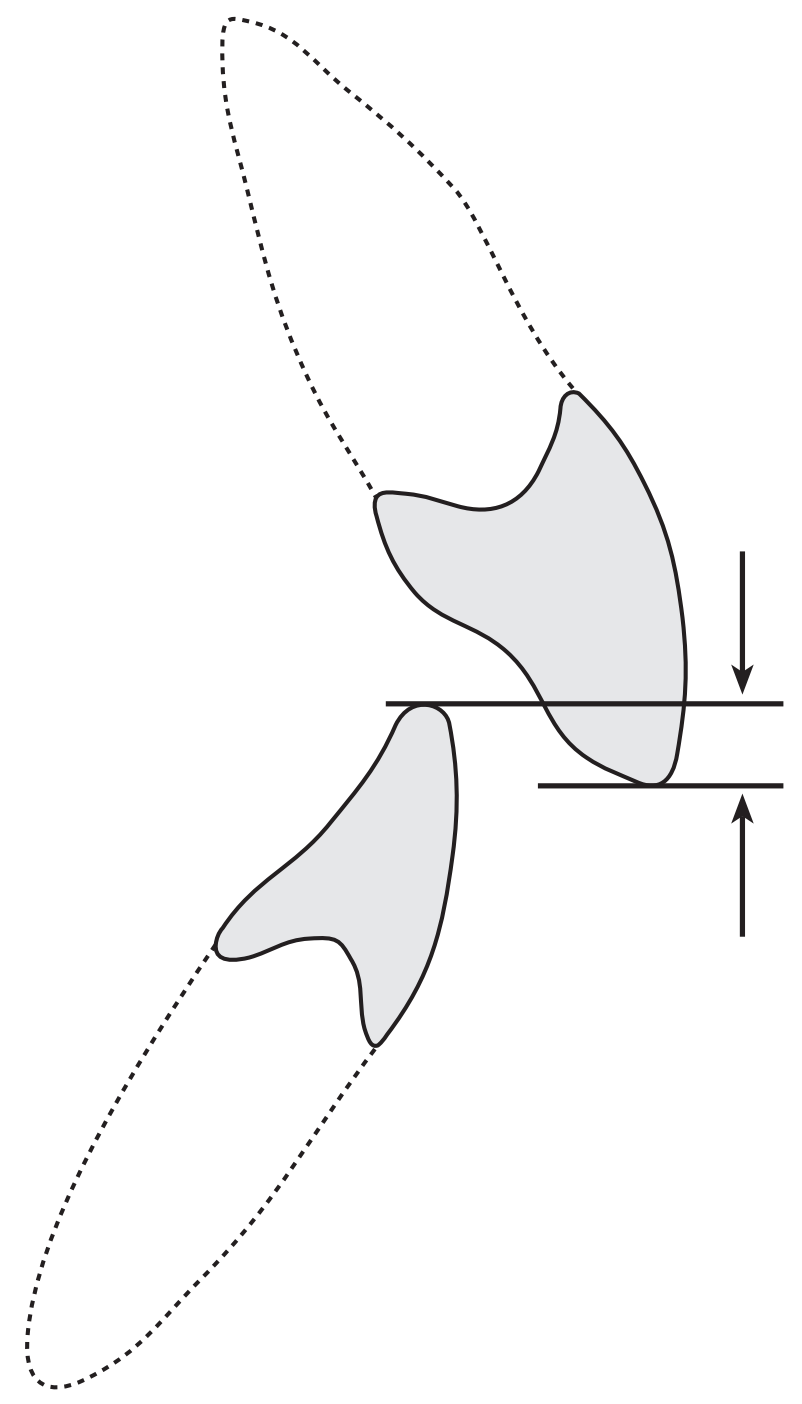

Fig. 5. Lateral view of the central incisors, showing the method of measuring overbite, measured perpendicular to Downs' occlusal plane. The vertical distance was measured with sliding calipers as the vertical distance between the maxillary and mandibular incisor's incisal edge. If there was an openbite, the measured value would be negative. (Figure provided by E. F. Harris.) 
molar (Fig. 6). If there is a Class II relationship, the distance is negative; if there is a Class III relationship, the distance is positive. A "socked-in" Class I molar relationship has a BSR of zero.

Canine relationship was measured in a fashion similar to BSR (Fig. 7). It is the horizontal distance from the cusp tip of the maxillary canine to the embrasure between the mandibular canine and first premolar. Again, if there is a Class II (canine) relationship, the distance is negative; if Class III, positive.

Dental midline discrepancy (Fig. 8) was measured metrically as the horizontal deviation of the maxillary and mandibular midline embrasures. If the dental midlines were coincident, the deviation was zero. Otherwise, a shift of the mandibular to the person's right was labeled positive.

Two arch chords were measured on each of the person's four quadrants (Fig. 9). These are labeled as the 1-3 and the 1-6 chord. The 1-3 chord was the straight-line distance, measured with sliding calipers, from the central incisor embrasure to the distal aspect of the canine. The 1-6 chord was the distance from the central incisor embrasure and the distal-buccal aspect of the permanent first molar's crown.

Incisor irregularity was measured on maxillary cast (Fig. 10) following Little's method (1975). One locates each anterior tooth's idealized mesial and distal anatomic contact, and then the actual distances are measured in the occlusal plane. Irregularity is the sum of the five distances (labeled A through 


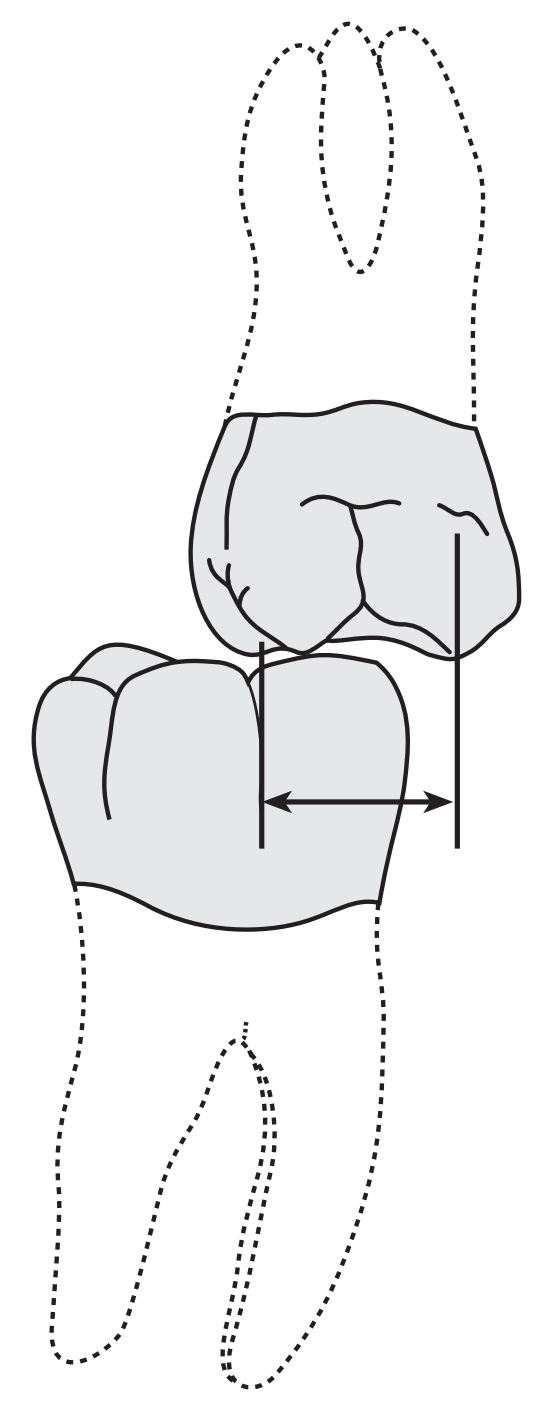

Fig. 6. Lateral view of the permanent first molars, showing the method of measuring the buccal segment relationship. This is the horizontal (parasagittal) distance of the maxillary molar's mesiobuccal cusp from the buccal groove of the mandibular molar. The horizontal discrepancy was measured with sliding calipers. If, as diagrammed here, the molars have a Class II relationship. the distance is negative. (Figure provided by E. F. Harris.) 


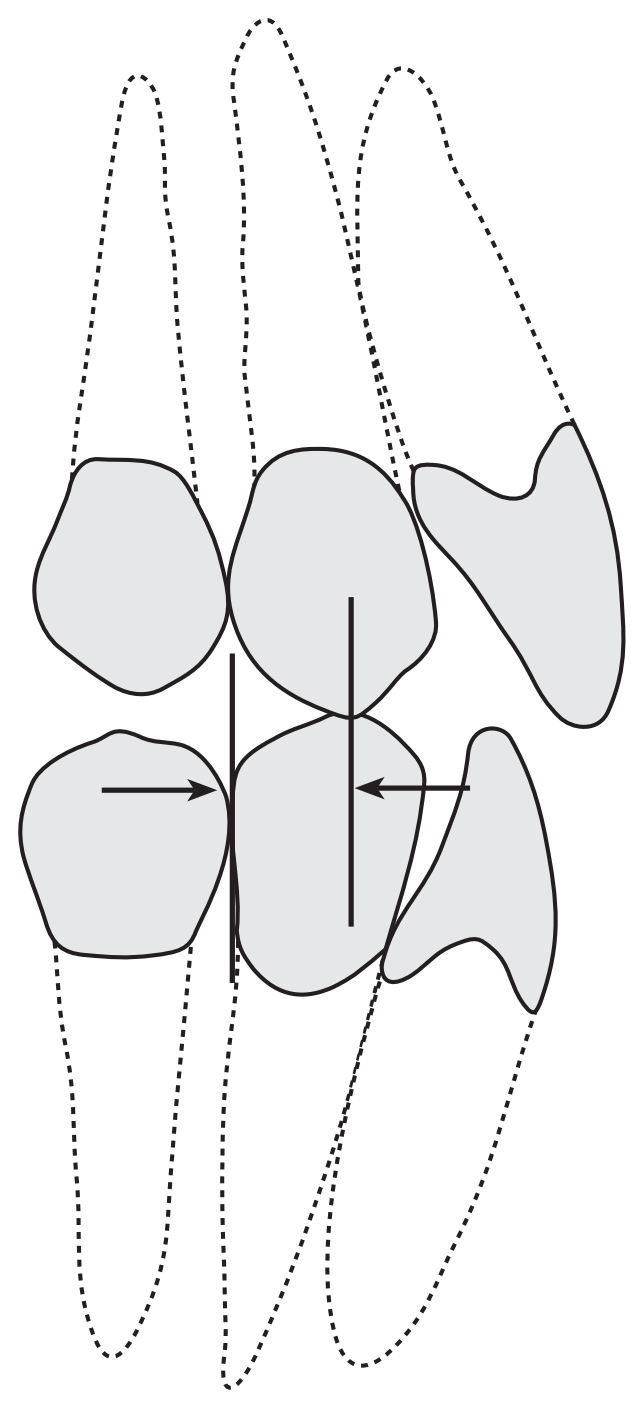

Fig. 7. Lateral view of the canines, showing the method of measuring canine discrepancy, which is the horizontal (parasagittal) deviation of the mandibular canine's cusp tip relative to the canine-first premolar embrasure. The horizontal discrepancy was measured with sliding calipers. If, as diagrammed here, the mandibular canine is mesial (Class III) of its ideal position, the value is defined as positive. (Figure provided by E. F. Harris.) 


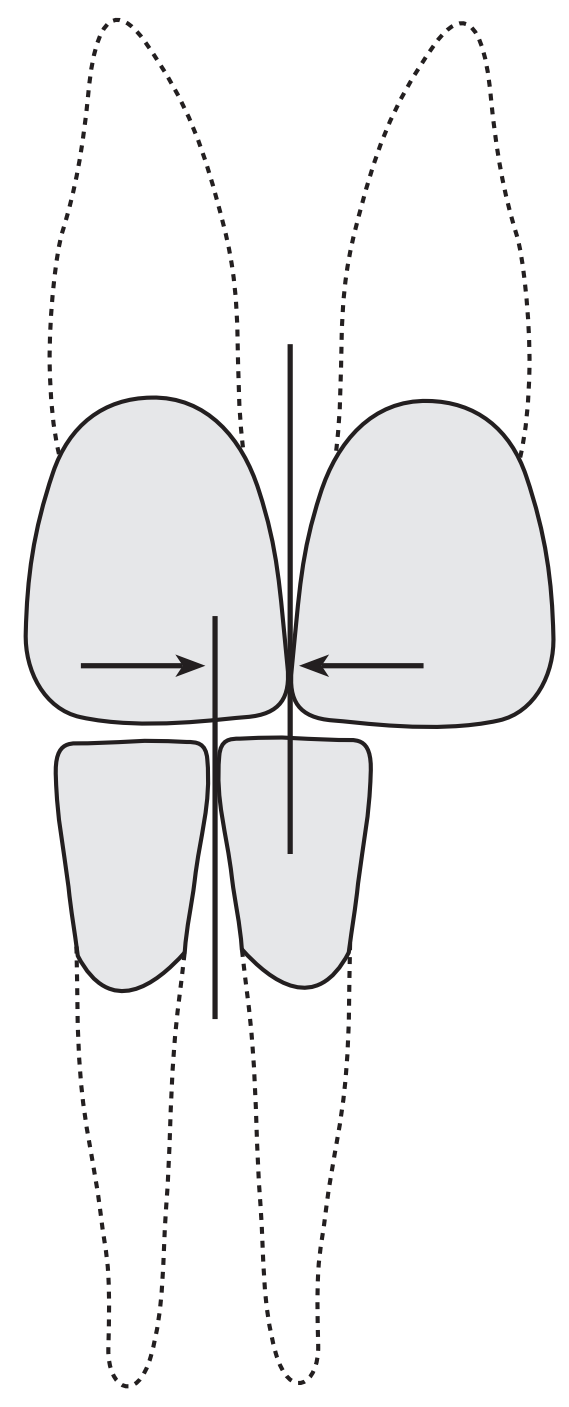

Fig. 8. Diagram of the labial view of the central incisors, showing the method of measuring the deviation of the maxillary and mandibular dental midlines. If the midlines are coincident, the discrepancy is zero. The horizontal discrepancy was measured with sliding calipers. Mandibular shifts to the right were labeled positive. (Figure provided by E. F. Harris.) 


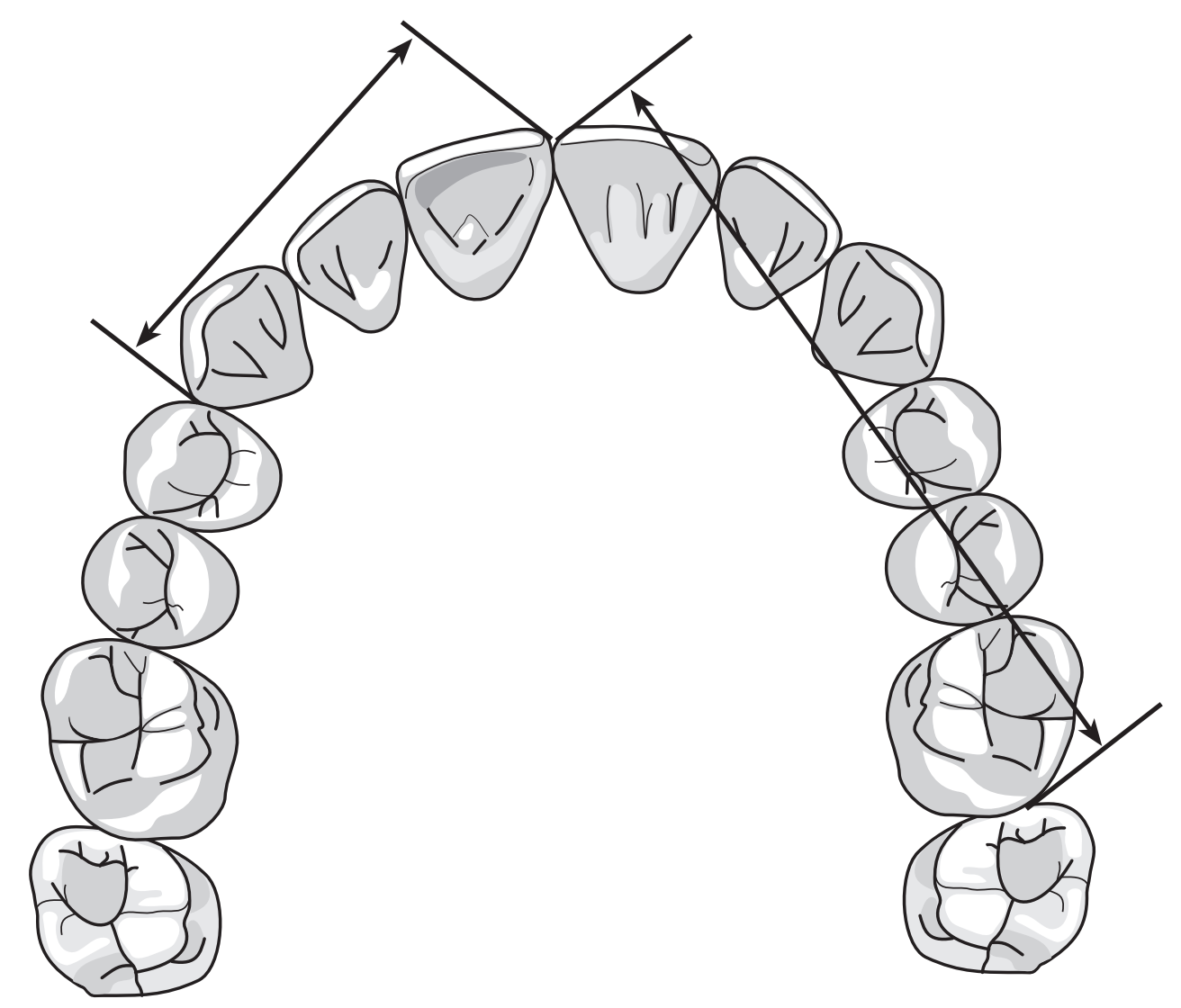

Fig. 9. Diagrammatic illustration of a maxillary dental arch, showing the manner that, with sliding calipers, the incisor-to-canine (1-3) and the incisorto-molar (1-6) arch chords were measured. In practice, both of these chords were measured on the left and right sides of both the maxillary and mandibular arches. (Figure provided by E. F. Harris.) 


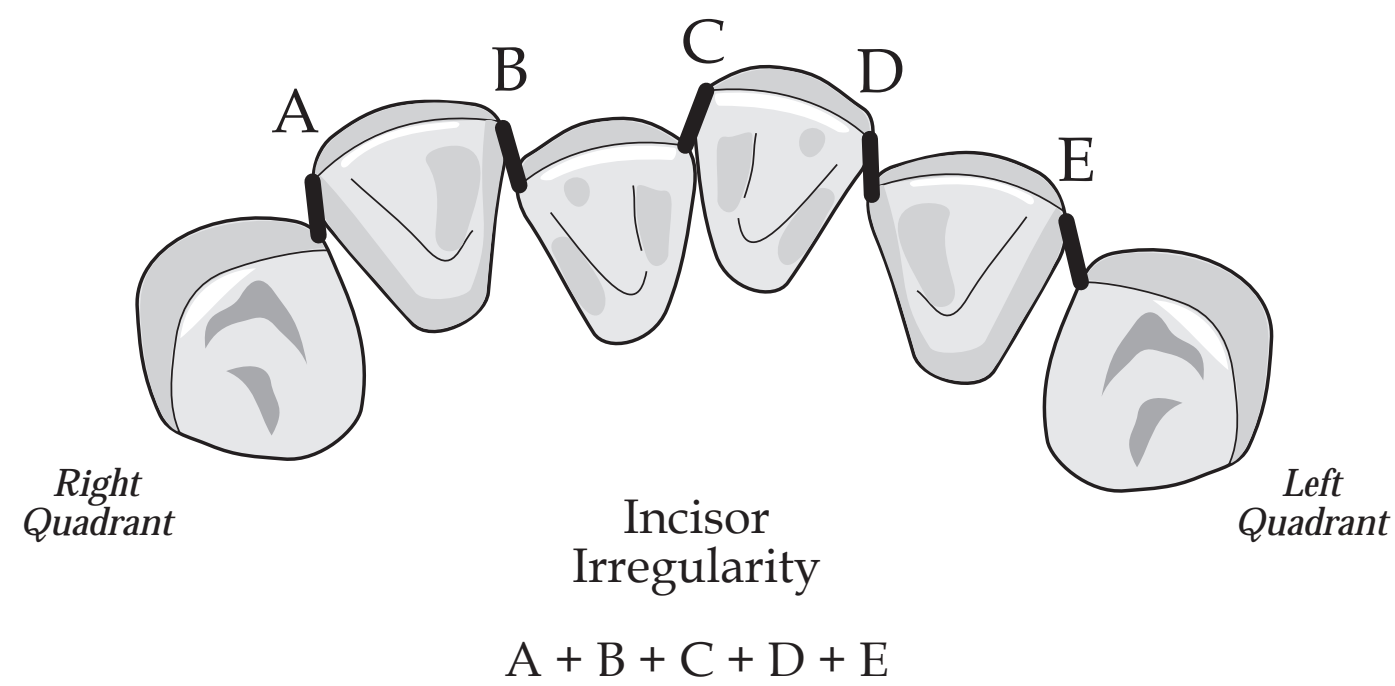

Fig. 10. Incisor irregularity is the summation of the five distances between the anatomic contacts of the anterior six teeth. If the anatomic contacts of two adjacent teeth are approximated, the distance is zero. Incisor irregularity is, then, the millimetric sum of the five contacts labeled A through E. 
E in the figure). If the two idealized contacts are abutted, regardless of each tooth's axioversion, the distance is zero. As such, irregularity is not sensitive to axioversion so long as the contacts are tight.

\section{$\underline{\text { Tooth Designations }}$}

Just the maxillary teeth are dealt with in these analyses. Two naming systems are used interchangeably. The actual names commonly are written out, but we also use the anatomical coding system (Peck and Peck 1993; Harris 2005), where one letter is used to code for the tooth type and the number designates the tooth's position in the morphogenetic field, either mesial (1) or distal (2). The codes are:

$\begin{aligned} \text { I1 } & \text { central incisor } \\ \text { I2 } & \text { lateral incisor } \\ \text { C } & \text { canine (cuspid) } \\ \text { P1 } & \text { first premolar (bicuspid) } \\ \text { P2 } & \text { second premolar (bicuspid) } \\ \text { M1 } & \text { first molar } \\ \text { M2 } & \text { second molar }\end{aligned}$

\section{$\underline{\text { Statistical Analyses }}$}

Data were collated into an Excel spreadsheet (Microsoft ${ }^{\circledR}$ 2002), and then transferred to the statistical package termed JMP 5.0.2 (SAS Institute Inc., Cary, NC). Exploratory data analysis (Tukey 1977) was performed, searching for outliers; those due to technical errors were corrected. 
Conventional descriptive statistics (e.g., Sokal and Rohlf 1995) were calculated; these (and abbreviations) are sample size (n, taken as counts of individuals, not sides), the arithmetic mean $(\bar{x})$, the standard deviation (sd), and the standard error of the mean (sem, calculated as $\mathrm{sd} / \sqrt{ } \mathrm{n})$.

One-sample t-tests were used to assess whether the in-treatment changes were systematically difference from zero (two tail tests).

Most inferential tests involved some sort of analysis of variance (e.g. Winer et al. 1991), primarily a mixed-model ANOVA that incorporated (1) extraction pattern (P1 or P2 extractions), (2) sex of the patient, and (3) side of the arch (since data were collected on the left and right quadrants). Of these, extraction pattern and sex are fixed effects, but "side" is a repeated measures variable in that there are no expected differences between tooth movements in the two maxillary quadrants; tooth movements in the left and right sides should be the same.

This mixed-model design is used several places in the analysis, so it merits providing the whole model in some detail. The form is:
Between subject
Extraction Pattern
Sex
Extraction-by-Sex
Within subject
Side
Side-by-Extraction
Side-by-Sex
Side-by-Extraction-by-Sex 
There is, then, a different error term used to evaluate the between- and within-subject effects (Winer et al., 1991). In practice, the interaction terms within-subject were rarely significant in this study, and so they often are omitted to conserve space.

Linear regression analysis (e.g., Freund and Little 1991) was used test for statistical dependencies between the amounts of tooth movement used to resolve the malocclusions as quantified by the pretreatment occlusal variables.

The conventional alpha level of 0.05 was used throughout, and all of the tests were two-tail. No correction was made for multiple comparisons. Salient results of the analysis were graphed using Delta Graph 4.0.5 (Delta Point, Inc., Monterey, CA) to show the tooth movements. 


\section{CHAPTER IV}

\section{RESULTS}

\section{Occlusal Relationships}

Caliper measurements of the dental casts (in maximum intercuspation) are listed in Table 1 . There are 24 variables assessed from the pretreatment dental casts, and we were able to study the occlusal relationships at this level of intensity because of the assistance of Dr. Kate Bodford who used the same cases as part of her study (Bodford and Harris 2007; Harris and Bodford 2007). These same variables were included in this study to further broaden the scope of the study and increase the available predictive variables. The ANOVA tests show that incisor irregularity in the maxillary arch is marginally greater in the second-premolar extraction sample, but not significantly so $(\mathrm{P}=$ 0.066). Overjet is significantly greater in the first-premolar extraction sample. Inspection of Table 1 shows that there is a large block of highly significant variables relating to the arch chords. Arch chords are straight-line distances that span the curvature of the dental arch in the anterior segments, so they capture a mix of both arch length and arch width components. The two arch chords measured in each quadrant were (1) the distance from the mesial of the central incisor to the distobuccal heel of the canine and (2) the distance from the central incisor to the distobuccal heel of the permanent first molar. These 


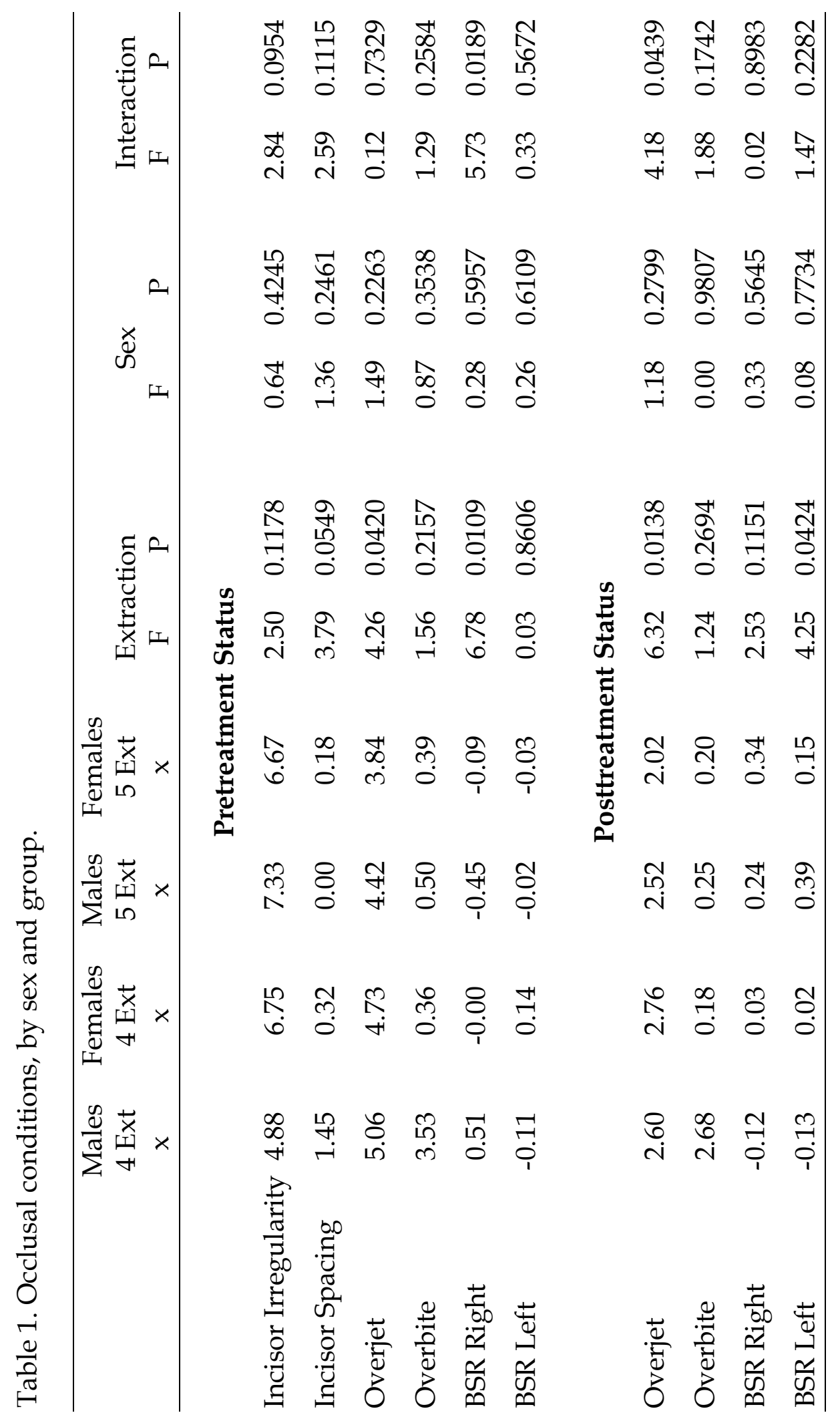


distances are far from parallel on to another, but the 1-6 chord does overlap the 1-3 chord.

For each of these eight variables, chord length is significantly longer in the first-premolar extraction sample. Presumably these differences are contributed to by the more tapered anterior arch form of the first-premolar sample, with its greater incisor overjet. On inspection, though, intercanine widths are insignificantly larger in the first-premolar sample, so the greater tapering, if it exists, would appear to be limited to the incisor. Again, cases in the first-premolar extraction sample do exhibit greater incisor overjet.

Assessment of the occlusions at the end of treatment (Table 2) shows that overjet is still somewhat larger in the first premolar group, especially in females. BSR was reduced closer to zero in the first premolar group.

\section{$\underline{\text { Pretreatment Status }}$}

Initial analysis consisted of computing ANOVA models for the mesiodistal ( $\mathrm{X}$ axis), transverse ( $\mathrm{Y}$ axis), and craniocaudal ( $\mathrm{Z}$ axis) coordinates of the pretreatment dental casts. A mixed-model ANOVA was used to test simultaneously for (1) differences between the two premolar extraction patterns, (2) differences between the sexes (due to sexual dimorphism), (3) differences in the extraction-by-sex interactions, and (4) left-right side differences. Concerning this last test, both the left and right sides of the 


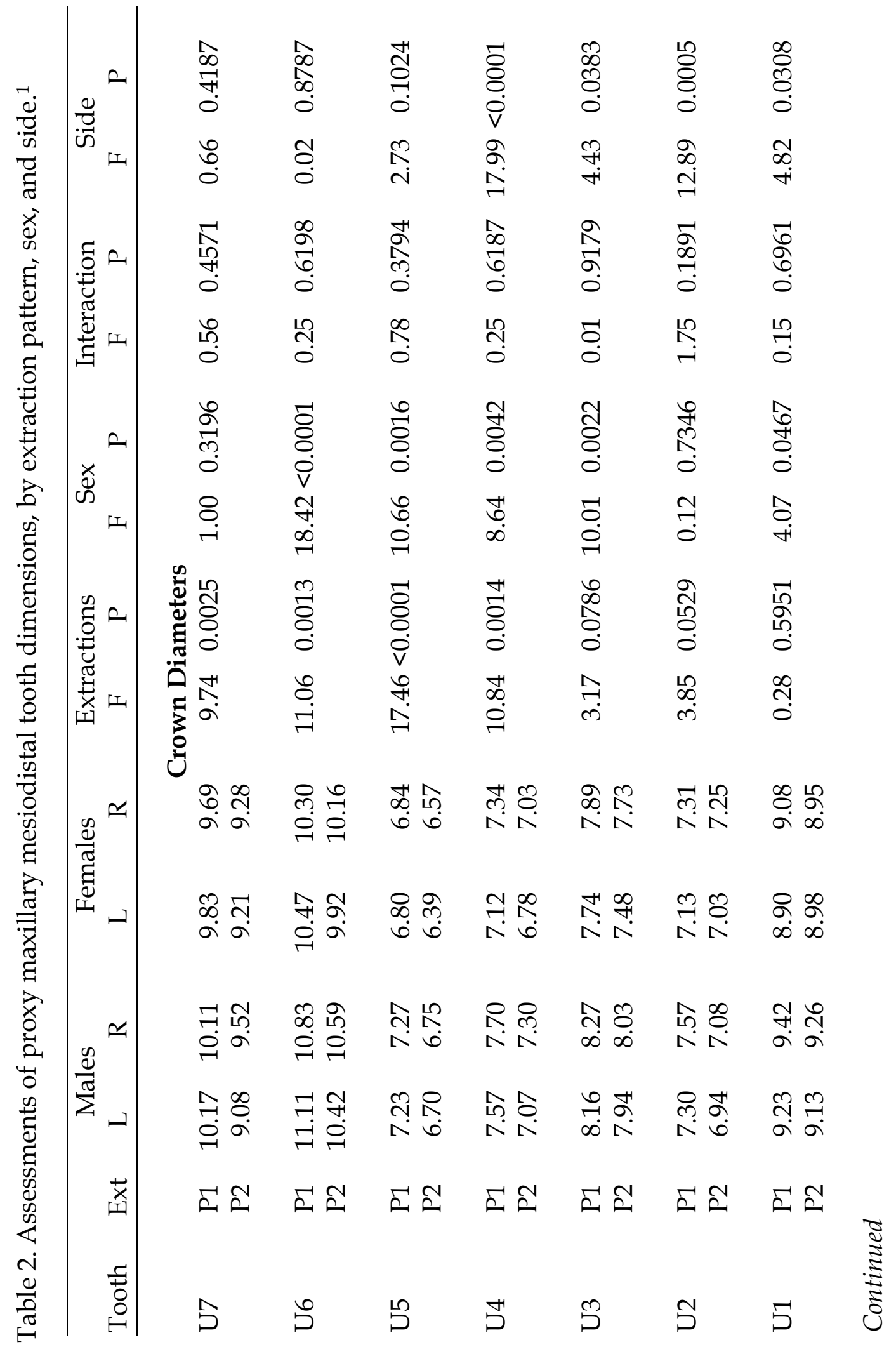




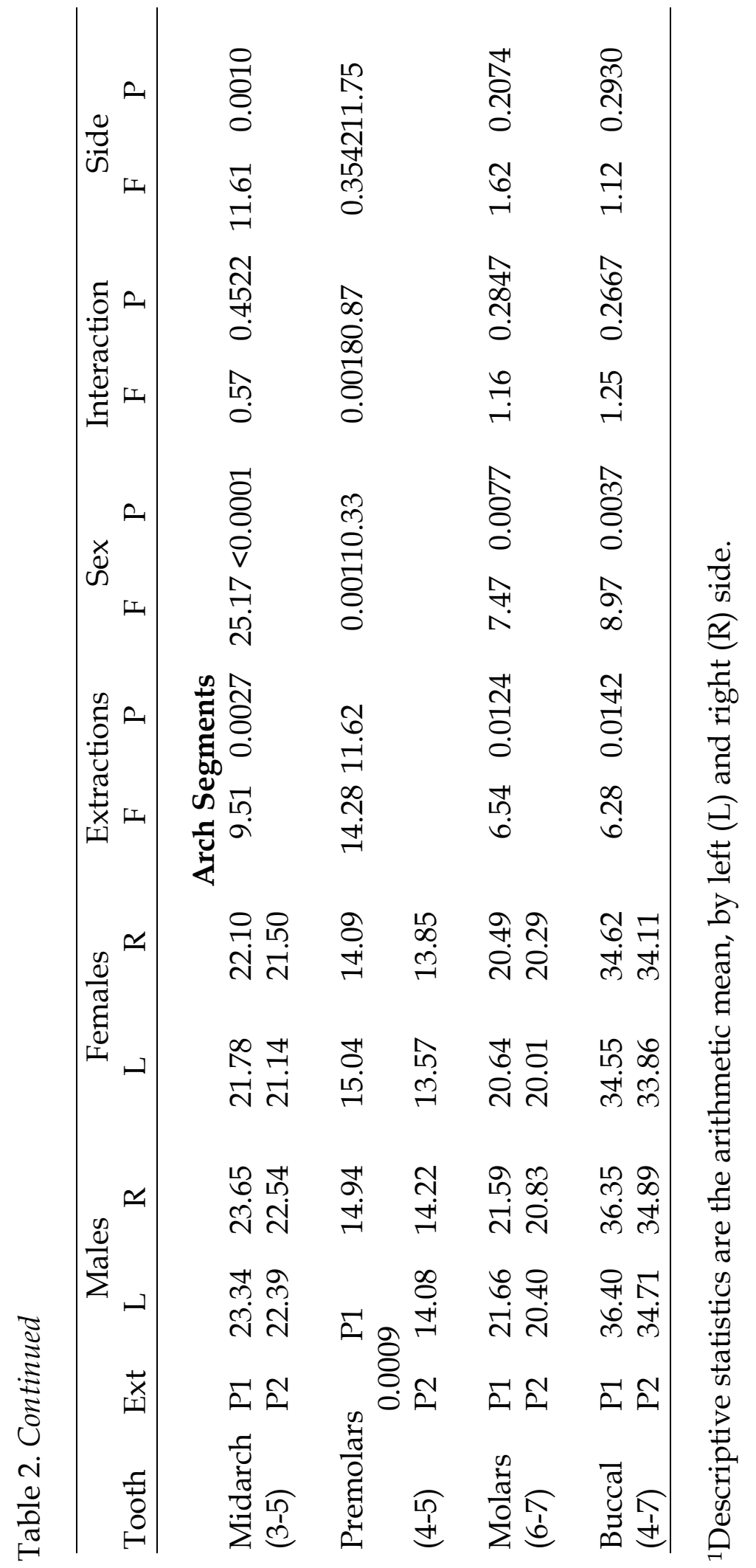


maxilla were measured throughout the study. Arch form is fundamentally symmetrical (Moorrees 1959; Sillman 1964), but minor asymmetries are not uncommon (Cassidy et al. 1998). Including "side" in the ANOVA model provides a sensitive test for detecting systematic side differences, but it also extracts this variation from the other tests it the model (Winer et al. 1991; Sokal and Rohlf 1995), so effects due to extraction pattern and/or sex can be evaluated while holding the variation due to "side" constant.

\section{Mesiodistal Axis}

Mesiodistal arch depths were measured parallel with the midpalatal raphe, with zero set at the mesial limit of the central incisors. Mean values of the $\mathrm{X}$ coordinates, by extraction pattern and sex, are graphed in Fig. 11. Figs. 12 through 15 are scaled plots of the arch forms in the four groupings (firstand second-premolar extraction patterns in males and females, respectively). Some features visually distinguish between these four graphs. Plots of the males appear larger, especially mediolaterally. This is expected given the well-documented sex differences in palatal dimensions (e.g., Moorrees 1959; Sillman 1964). The central incisors appear farther forward and more "square" (greater mesial rotation of the distal aspects) than in the other samples. The canines appear to be "blocked-out" more to the buccal in the first-premolar extraction female and second-premolar extraction male samples. Arch form 


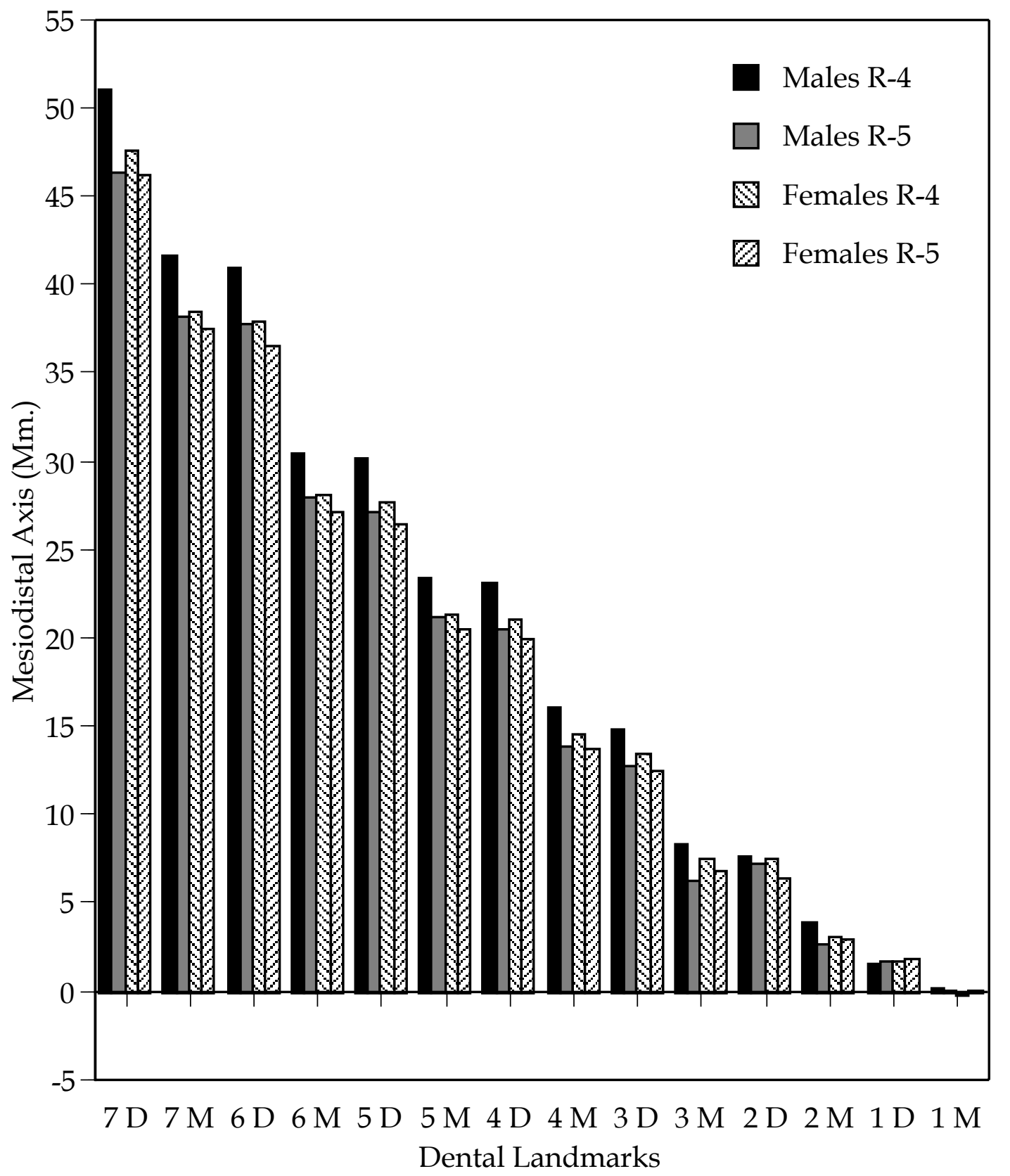

Fig. 11. Plot of the mesiodistal (X axis) coordinates at the pretreatment examination showing the sexual dimorphism in arch length and, as well, greater arch length in the first-premolar extraction sample. Just teeth in the right quadrant are shown for simplicity. Notice how the mesial landmark of one tooth is very close to the distal landmark of the tooth in front of it; this substantiates the coordinate-acquisition technique and it shows the small interproximal distances between the occlusally-observable margins of the teeth. 


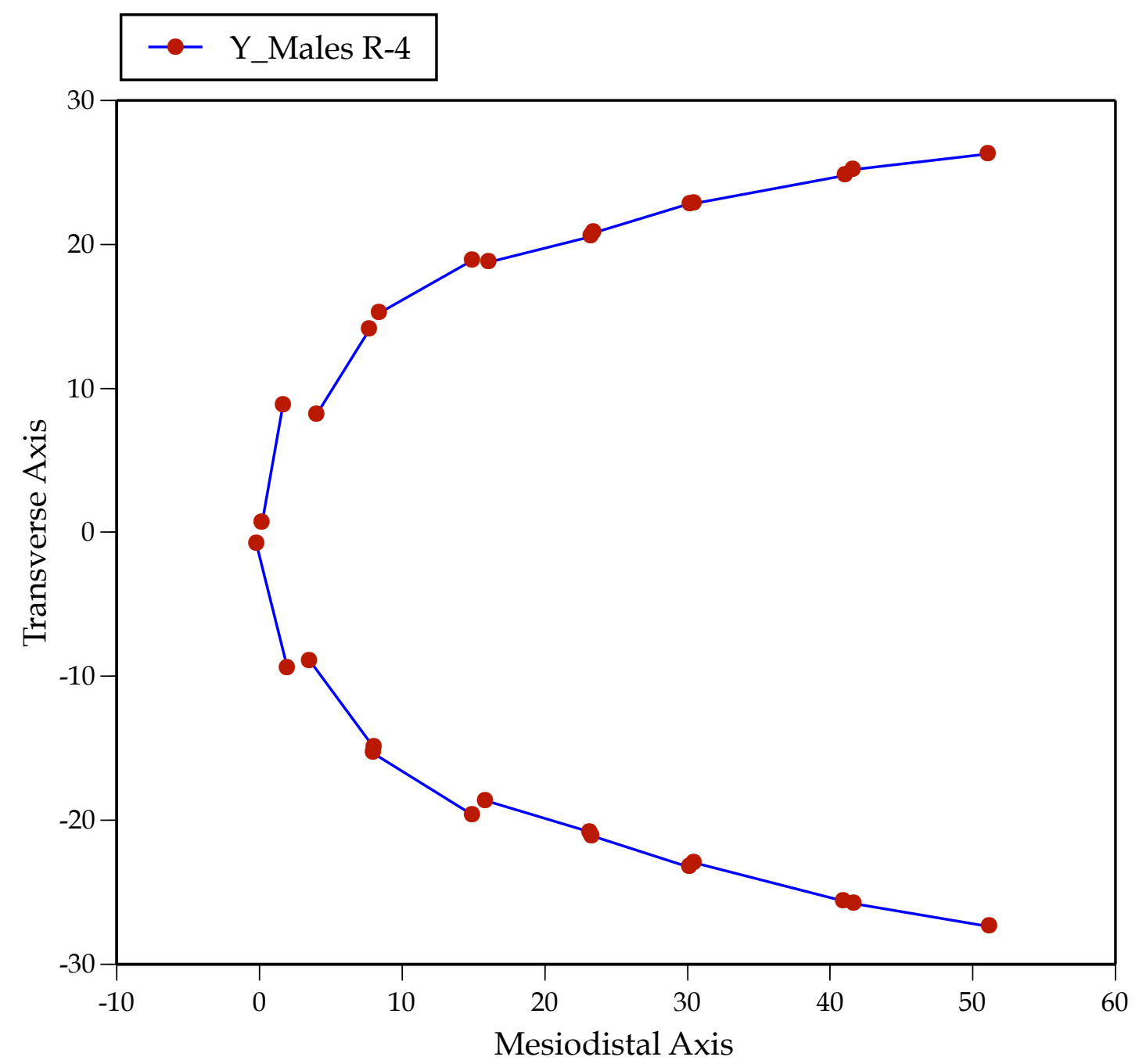

Fig. 12. First-premolar extraction group of males at the pretreatment examination. Negative transverse values are the right quadrant, so it is the occlusal view of the maxillary arch. Scales are in millimeters. 


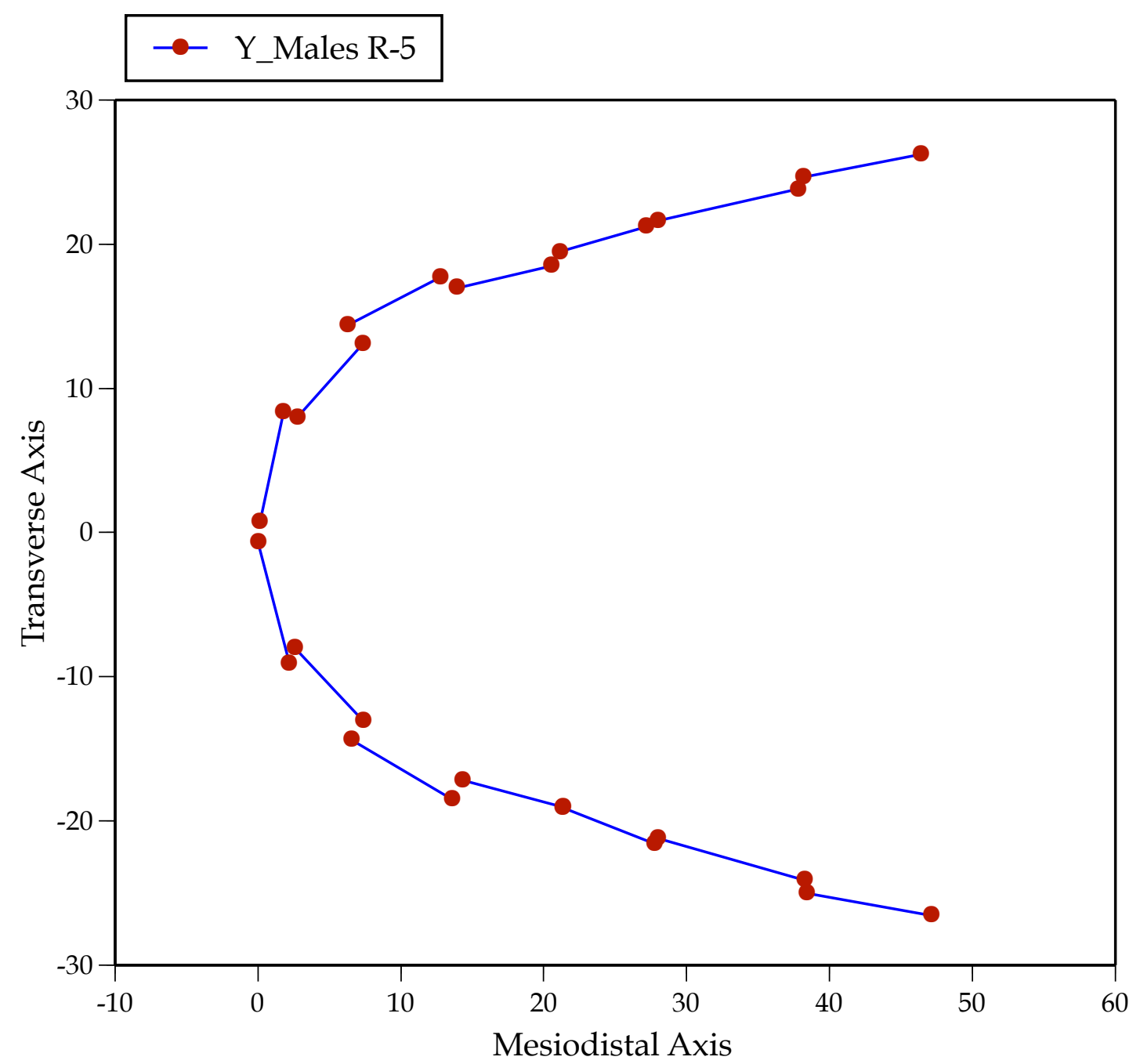

Fig. 13. Second-premolar extraction group of males at the pretreatment examination. Negative transverse values are the right quadrant, so it is the occlusal view of the maxillary arch. Scales are in millimeters. 


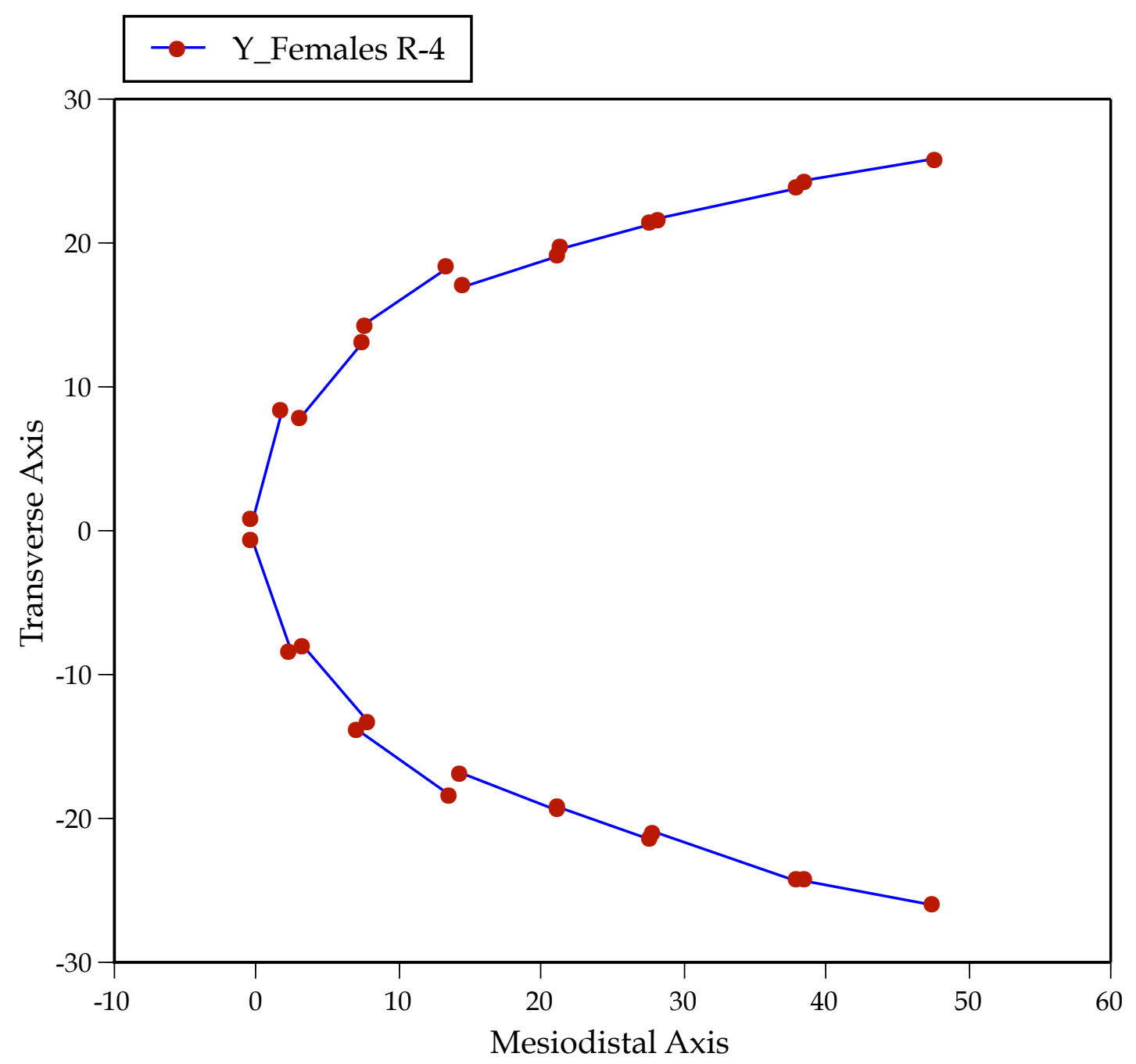

Fig. 14. First-premolar extraction group of females at the pretreatment examination. Negative transverse values are the right quadrant, so it is the occlusal view of the maxillary arch. Scales are in millimeters. 


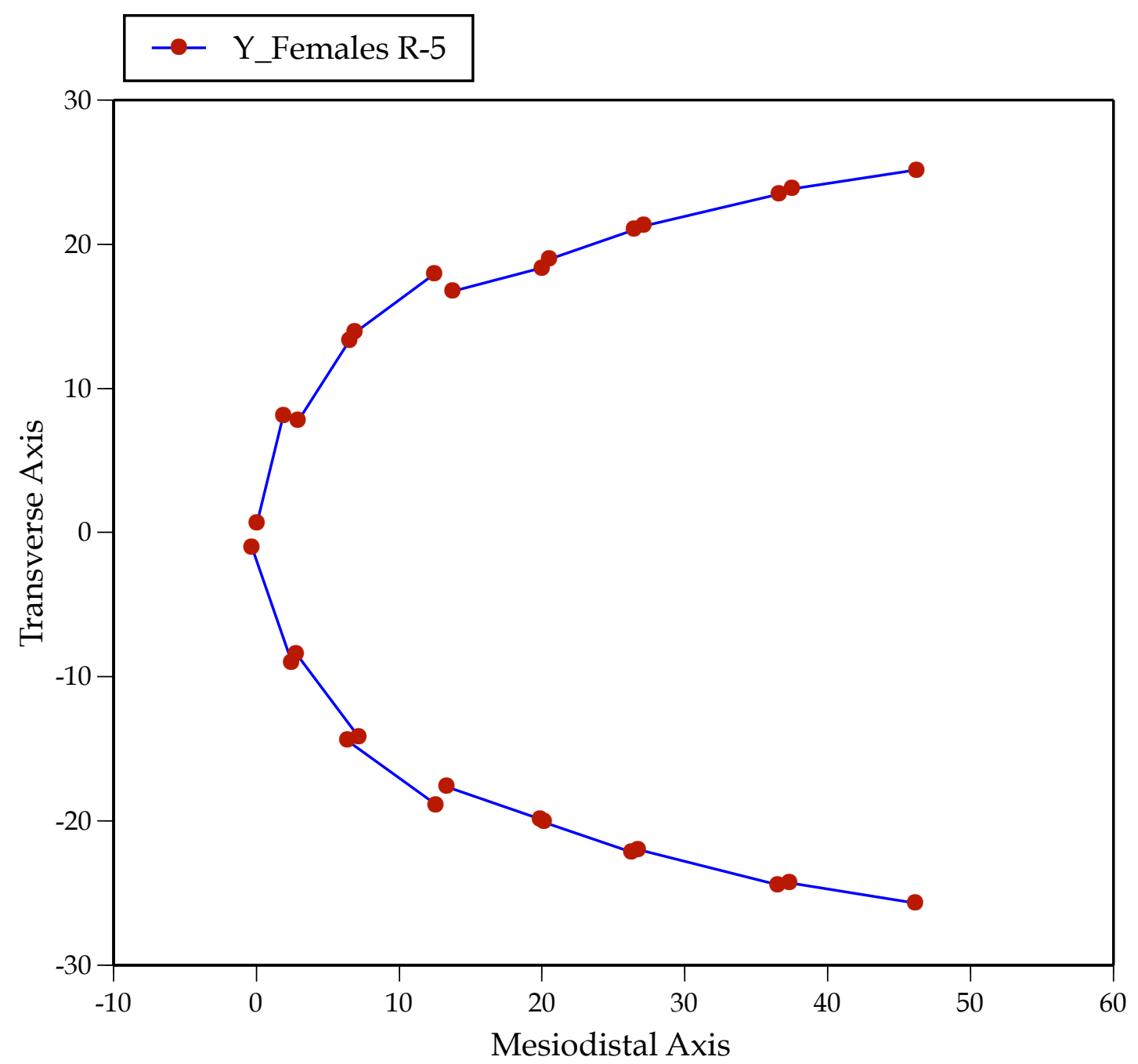

Fig. 15. Second-premolar extraction group of females at the pretreatment examination. Negative transverse values are the right quadrant, so it is the occlusal view of the maxillary arch. Scales are in millimeters. 
seems to be broader and more rounded in the first-premolar extraction male and second-premolar extraction female samples.

Fig. 16 compares the male and female samples in the first-premolar extraction samples, with registration on the mesial contacts of the central incisors. Males have discernibly broader arches throughout the tooth types. The greater arch length in males is obvious given the greater arch length of about $3 \mathrm{~mm}$ seen in the molars.

Sexual dimorphism is not as obvious in the second-premolar extraction sample (Fig. 17), where the male and female coordinates are commonly superimposed.

Fig. 18 compares the first-premolar and second-premolar extraction samples in males, and Fig. 19 is the comparable plot for females. Both of these plots exhibit considerable similarity in arch form. The lateral incisors are characteristically "ducked in" behind the earlier-emerging central incisors. The late-emerging canines are characteristically "blocked out" due to inadequate arch space.

Results of the ANOVA tests are listed in Table 3. Because of the coordinate system used, almost all of the tests between the two premolarextraction groups are statistically significant because the first-premolar sample exhibits greater overjet - so all mesiodistal coordinates measured to the incisal edge are longer in the first-premolar sample. This is true for all the tooth 


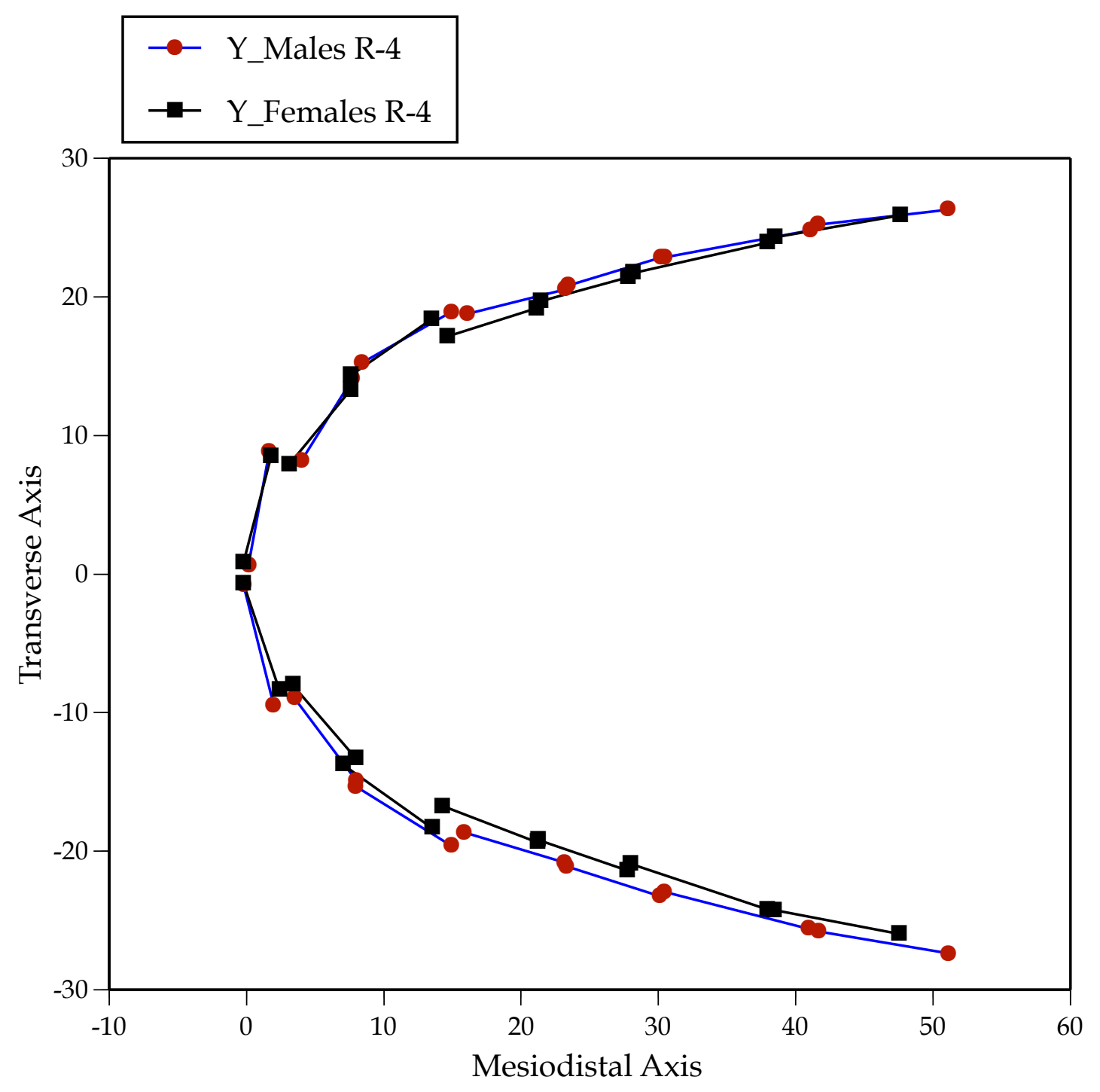

Fig. 16. Comparison of the first-premolar extraction samples of males and females at the pretreatment examination. Negative transverse values are the right quadrant, so it is the occlusal view of the maxillary arch. Scales are in millimeters. 


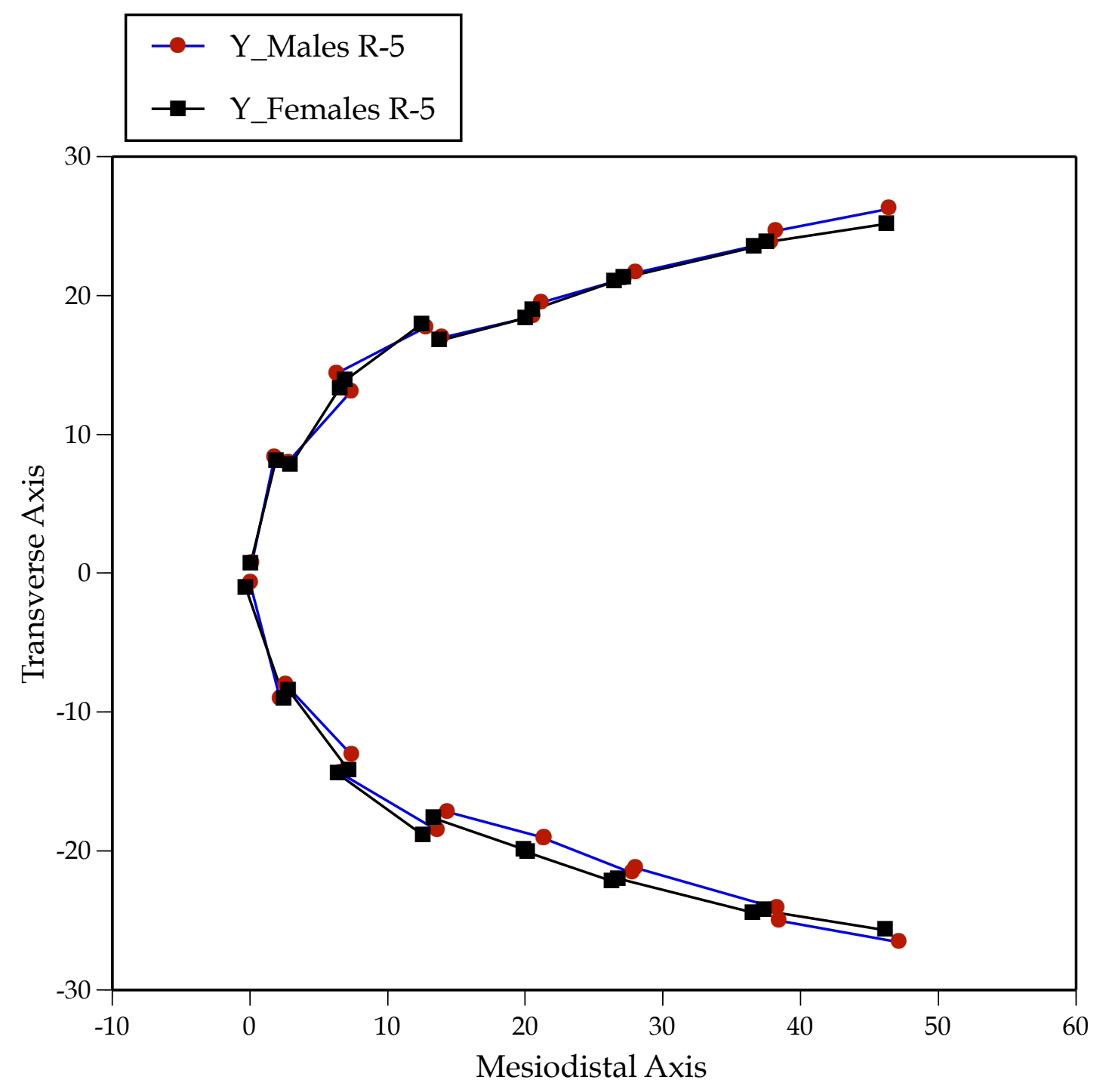

Fig. 17. Comparison of the second-premolar extraction samples of males and females at the pretreatment examination. Negative transverse values are the right quadrant, so it is the occlusal view of the maxillary arch. Scales are in millimeters. 


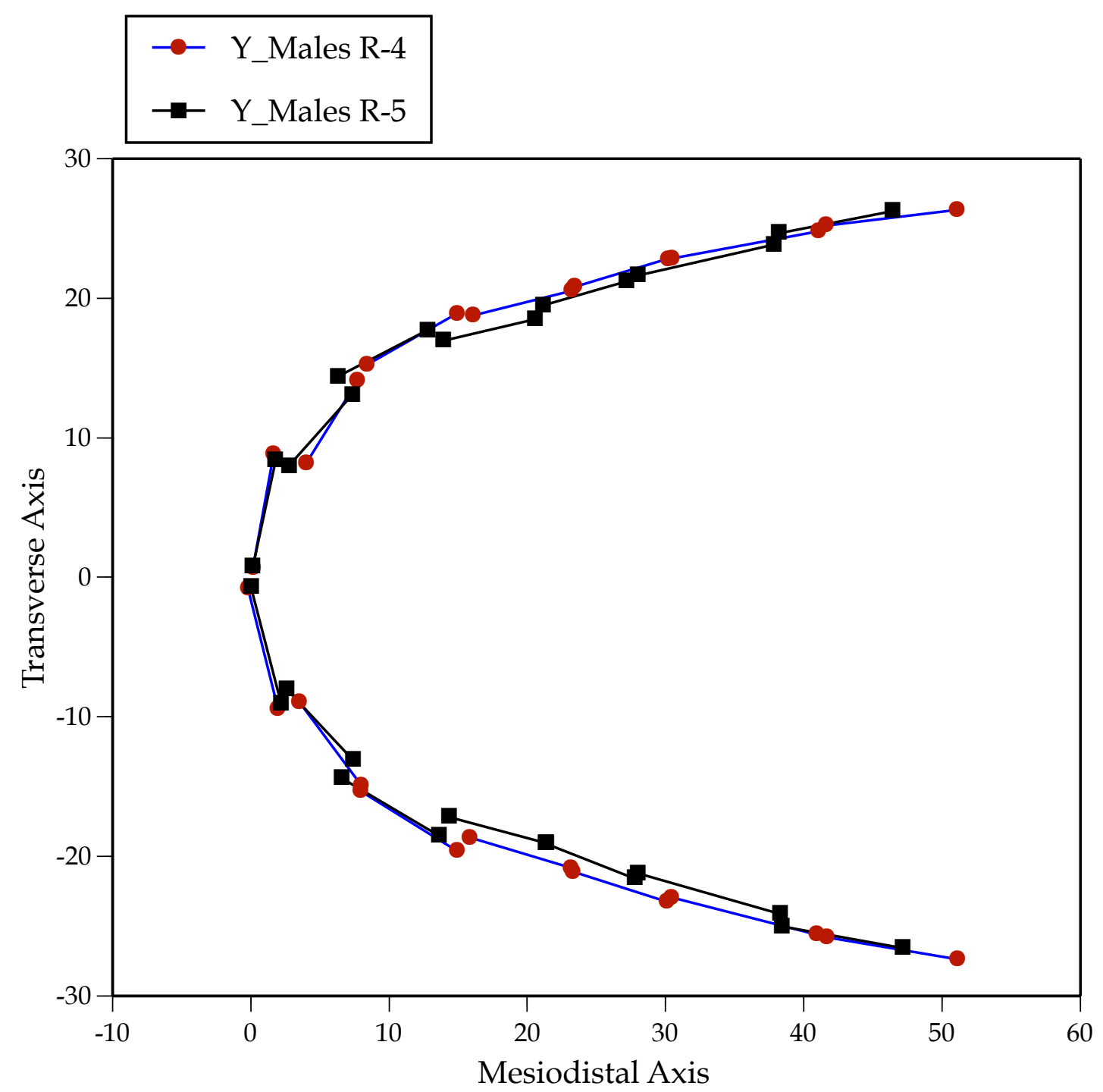

Fig. 18. Comparison of the first- and second-premolar extraction samples of males at the pretreatment examination. Negative transverse values are the right quadrant, so it is the occlusal view of the maxillary arch. Scales are in millimeters. 


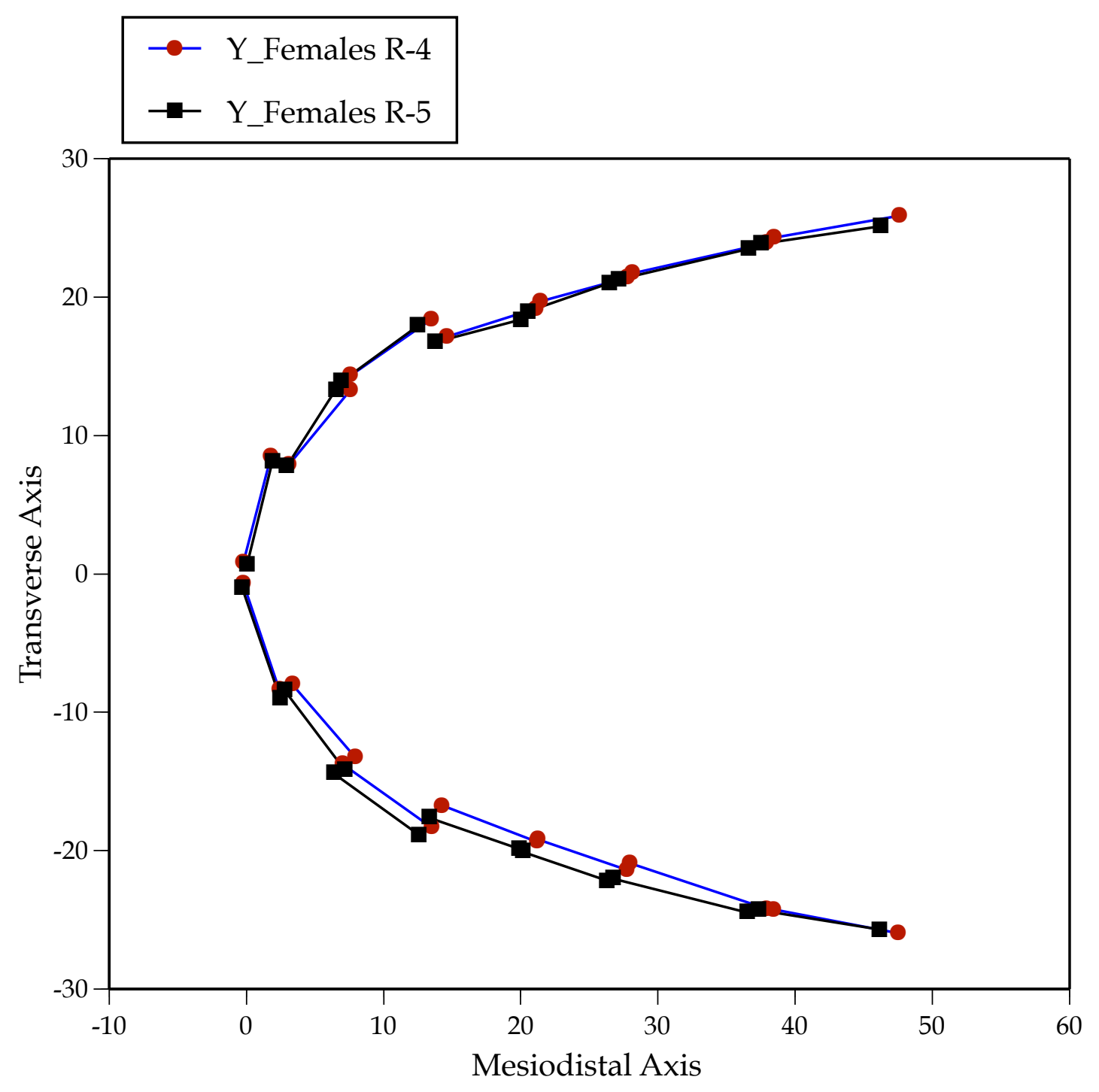

Fig. 19. Comparison of the first- and second-premolar extraction samples of females at the pretreatment examination. Negative transverse values are the right quadrant, so it is the occlusal view of the maxillary arch. Scales are in millimeters. 


\begin{tabular}{|c|c|c|c|c|c|c|c|}
\hline $0_{0}^{f}$ & $\begin{array}{l}\stackrel{N}{N} \\
\stackrel{\leftrightarrow}{\leftrightarrow} \\
\stackrel{0}{0}\end{array}$ & $\begin{array}{l}\text { రి } \\
\text { రి } \\
0\end{array}$ & \begin{tabular}{l}
0 \\
$\infty$ \\
\multirow{+}{+}{}
\end{tabular} & $\begin{array}{l}\vec{\infty} \\
\stackrel{+}{+} \\
0\end{array}$ & $\begin{array}{l}0 \\
0 \\
\infty \\
0 \\
0 \\
0\end{array}$ & $\begin{array}{l}\text { Ñ } \\
\infty \\
\infty \\
0 \\
0\end{array}$ & 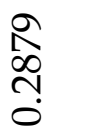 \\
\hline क्य & $\begin{array}{l}\ddot{6} \\
0\end{array}$ & $\stackrel{8}{0}$ & $\begin{array}{l}0 \\
\stackrel{0}{0}\end{array}$ & के & @ִ & $\frac{0}{0}$ & $\stackrel{\rightleftarrows}{\stackrel{\rightleftarrows}{\sim}}$ \\
\hline 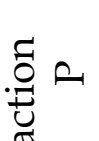 & $\begin{array}{l}1 \\
\infty \\
0 \\
0 \\
0 \\
0\end{array}$ & \begin{tabular}{l}
0 \\
\multirow{1}{0}{} \\
$\stackrel{0}{0}$
\end{tabular} & $\begin{array}{l}\text { হి } \\
\text { ஸे } \\
0\end{array}$ & $\begin{array}{l}\text { నે } \\
\text { స్ } \\
0\end{array}$ & $\begin{array}{l}\text { Nิ } \\
\text { N̦} \\
0\end{array}$ & 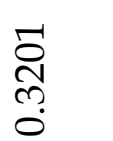 & $\begin{array}{l}\infty \\
\infty \\
m \\
n \\
0\end{array}$ \\
\hline 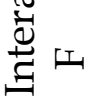 & 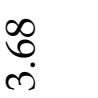 & $\begin{array}{l}\hat{\sigma} \\
\text { ì }\end{array}$ & 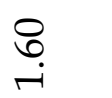 & 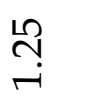 & సิ & تُ & ڤ̊ \\
\hline$x^{f}$ & $\begin{array}{l}8 \\
8 \\
8 \\
ᄋ \\
\circ\end{array}$ & $\begin{array}{l}\infty \\
\stackrel{\infty}{8} \\
\stackrel{0}{0}\end{array}$ & $\begin{array}{l}\qquad 0 \\
\stackrel{8}{8} \\
\varnothing \\
0\end{array}$ & $\begin{array}{l}\text { F्子 } \\
8 \\
\\
0\end{array}$ & 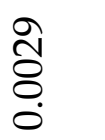 & $\begin{array}{l}8 \\
\& \\
\varnothing \\
0 \\
0\end{array}$ & $\begin{array}{l}\stackrel{ }{\&} \\
\stackrel{8}{8} \\
\stackrel{0}{0}\end{array}$ \\
\hline ळ & $\stackrel{\sim}{\sim}$ & $\underset{\infty}{\mathscr{q}}$ & $\begin{array}{l}\qquad 0 \\
\stackrel{0}{0}\end{array}$ & $\begin{array}{c}\widetilde{1} \\
\infty\end{array}$ & ఫ̆ & ले & $\widehat{\infty}$ \\
\hline . ્ْટ & $\begin{array}{l}\dot{H} \\
8 \\
\varnothing \\
0\end{array}$ & $\begin{array}{l}\stackrel{0}{\mathcal{O}} \\
\stackrel{8}{\delta} \\
\stackrel{0}{0}\end{array}$ & 훙 & $\begin{array}{l}\text { के } \\
\text { రे } \\
0 \\
0\end{array}$ & $\begin{array}{l}0 \\
8 \\
8 \\
\circ\end{array}$ & $\begin{array}{l}\hat{1} \\
\stackrel{0}{\circ} \\
0 \\
0\end{array}$ & $\begin{array}{l}\frac{10}{8} \\
\frac{8}{0} \\
0\end{array}$ \\
\hline 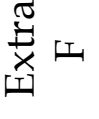 & $\begin{array}{l}\infty \\
\ddot{\theta} \\
\stackrel{\oplus}{\longrightarrow}\end{array}$ & $\begin{array}{l}\hat{\sigma} \\
\sigma\end{array}$ & 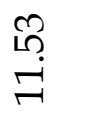 & $\begin{array}{l}\widehat{O} \\
0\end{array}$ & $\begin{array}{l}\text { ְర } \\
\stackrel{\sim}{7}\end{array}$ & $\underset{\infty}{\Delta}$ & $\begin{array}{l}\hat{N} \\
0 \\
0\end{array}$ \\
\hline 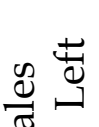 & 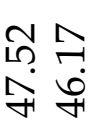 & 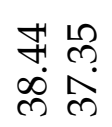 & 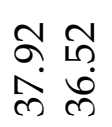 & 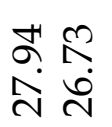 & 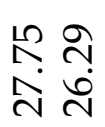 & 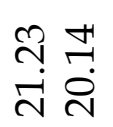 & 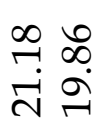 \\
\hline 苾 & 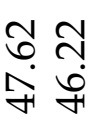 & $\begin{array}{l}\text { शे ñ } \\
\infty \\
\infty \\
\text { ñ }\end{array}$ & 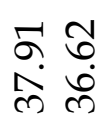 & 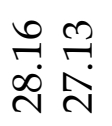 & 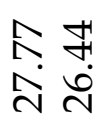 & 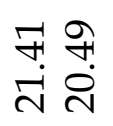 & 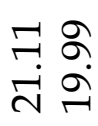 \\
\hline 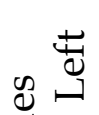 & 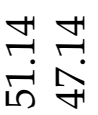 & 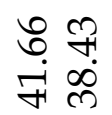 & 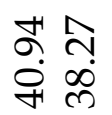 & 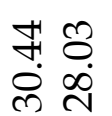 & $\begin{array}{l}m \\
\stackrel{n}{ }\end{array}$ & $\begin{array}{l}\stackrel{\infty}{N} \underset{n}{\sim} \\
\stackrel{n}{\sim}\end{array}$ & $\begin{array}{l}\text { م) } \\
\stackrel{2}{\sim} \\
\stackrel{\sim}{N}\end{array}$ \\
\hline 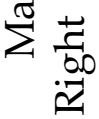 & 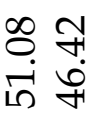 & 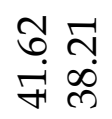 & 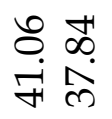 & 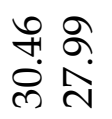 & 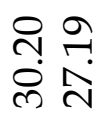 & \begin{tabular}{l}
\multirow{+}{*}{$\underset{\sim}{\sim}$} \\
$\stackrel{\sim}{N}$
\end{tabular} & 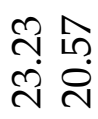 \\
\hline$\underset{x}{\vec{x}}$ & $\Xi \cong$ & $\sqsubseteq \cong$ & $\check{L}_{\mathcal{L}}$ & $\overbrace{1} \cong$ & $\Xi \cong$ & $\sqsubseteq \cong$ & $\Sigma \cong$ \\
\hline $\begin{array}{l}\frac{0}{0} \\
\frac{\pi}{\pi} \\
\frac{\pi}{\pi} \\
>\end{array}$ & $\stackrel{\ominus}{\widehat{D}}$ & $\sum_{S}$ & $\begin{array}{l}\oplus \\
\qquad \\
ص\end{array}$ & $\begin{array}{l}\sum_{0} \\
b \\
b\end{array}$ & ص & $\sum_{0}$ & $\stackrel{9}{\$}$ \\
\hline
\end{tabular}




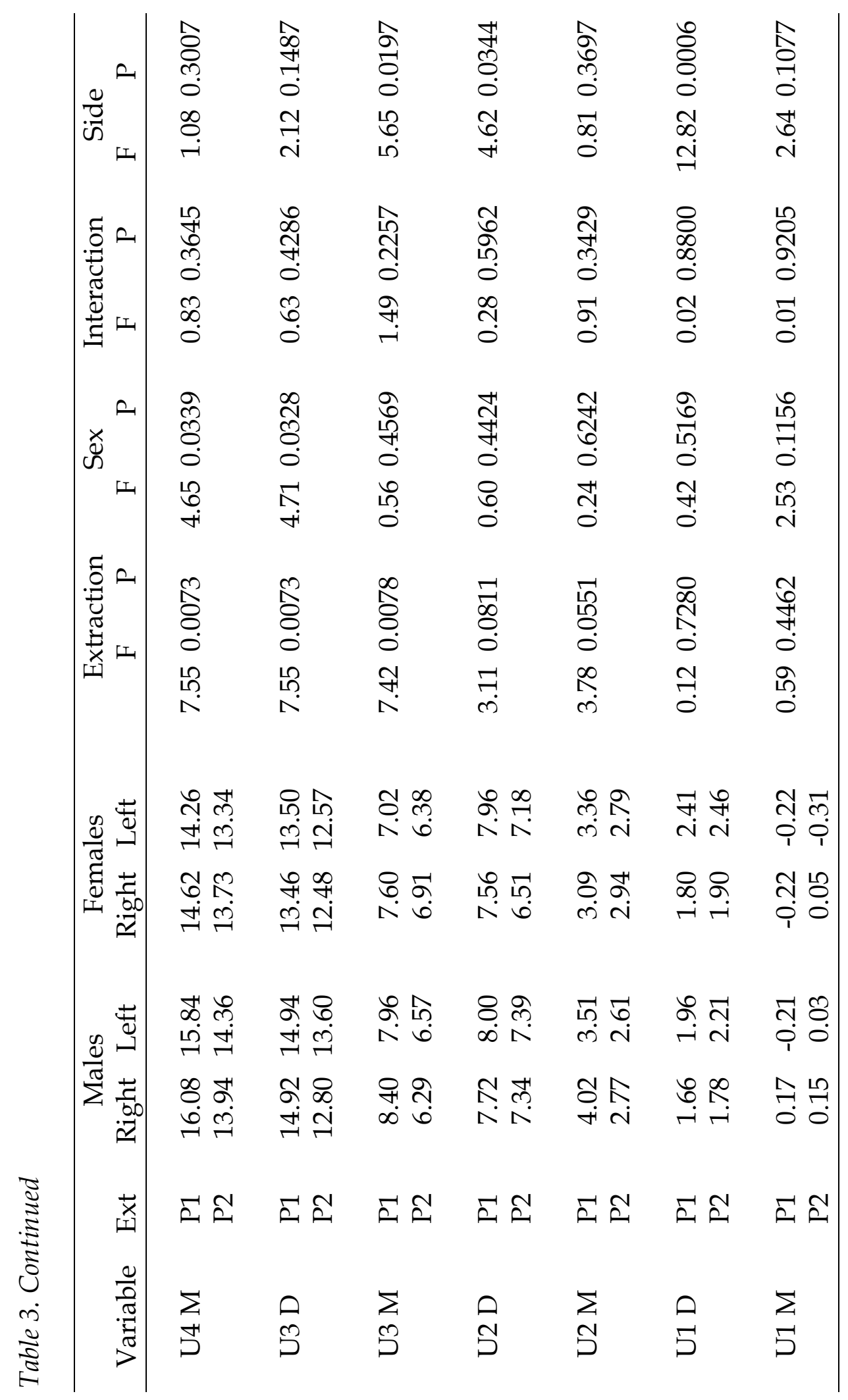


types except the incisors where the variation among these teeth obscures the differences.

Most of the tests for sex differences also are significant, especially for the teeth in the buccal segments. These differences are predictable; it is well known that palatal dimensions are appreciably larger in males than females (e.g., Moorrees 1959; Knott 1961; Burris and Harris 1998). Indeed, "sex" is included in the ANOVA design so sample sizes (and, thus, degrees of freedom) can be increased in each test and the number of test is reduced by half compared to evaluating the differences separately for the left and right sides. Analogously, "side" is included in the design as a statistically efficient means of including all the data in fewer comprehensive tests.

\section{Transverse Axis}

The $\mathrm{Y}$ axis in our coordinate system is the transverse (mediolateral) dimension. Figs. 12 through 17 are plots of the arch forms at the start of treatment. Results of the mixed-model ANOVA tests are listed in Table 4. There are significant differences between the two premolar extraction samples, but just in the premolar segment. Interestingly, no significant differences were noted in the anterior segment. At the level of both the first and second premolars, arch widths are broader prior to treatment in the first premolar sample. That is, arch form is longer overall and significantly 


\begin{tabular}{|c|c|c|c|c|c|c|c|c|}
\hline & $0_{1}$ & $\begin{array}{l}\frac{1}{8} \\
8 \\
0 \\
0\end{array}$ & 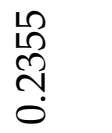 & $\begin{array}{l}\sqrt{n} \\
\stackrel{0}{0} \\
0\end{array}$ & \begin{tabular}{l}
0 \\
\multirow{2}{*}{} \\
ర్ \\
0
\end{tabular} & 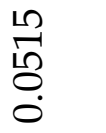 & $\begin{array}{l}10 \\
6 \\
\qquad \\
0 \\
0\end{array}$ & $\begin{array}{l}8 \\
8 \\
8 \\
0\end{array}$ \\
\hline & 苛 & $\begin{array}{l}\vec{\sigma} \\
\dot{\theta}\end{array}$ & & $\stackrel{10}{\overparen{0}}$ & 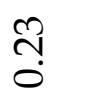 & $\begin{array}{l}\text { ஜे } \\
\text { ले }\end{array}$ & ָ̊ & 齐 \\
\hline & . & $\begin{array}{l}\hat{\tilde{N}} \\
\hat{\Omega} \\
\hat{0}\end{array}$ & $\begin{array}{l}\stackrel{ }{N} \\
\text { in } \\
0\end{array}$ & 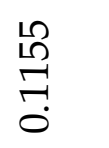 & 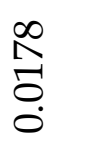 & $\begin{array}{l}\infty \\
\infty \\
\\
0\end{array}$ & $\underset{0}{N}$ & 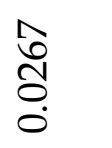 \\
\hline : & 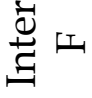 & $\stackrel{8}{8}$ & तै & 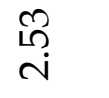 & $\begin{array}{l}\$ \\
\infty \\
10\end{array}$ & $\begin{array}{l}2 \\
\frac{2}{10}\end{array}$ & $\begin{array}{l}8 \\
10\end{array}$ & $\begin{array}{l}\infty \\
0 \\
10\end{array}$ \\
\hline $\begin{array}{l}\text { त) } \\
\stackrel{2}{2} \\
0 \\
0 \\
0\end{array}$ & $\underset{\mathscr{W}}{\times}$ & $\begin{array}{l}1 \\
\text { Oे } \\
0 \\
0\end{array}$ & $\begin{array}{l}\infty \\
0 \\
0 \\
0 \\
0\end{array}$ & 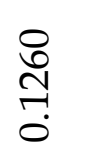 & $\begin{array}{l}\stackrel{0}{\mathcal{1}} \\
\stackrel{5}{0} \\
\stackrel{0}{0}\end{array}$ & \begin{tabular}{l}
\multicolumn{8}{c}{} \\
0 \\
0 \\
0
\end{tabular} & $\begin{array}{l}\infty \\
\stackrel{\infty}{1} \\
\stackrel{0}{0} \\
0\end{array}$ & \begin{tabular}{l}
0 \\
0 \\
0 \\
\hdashline \\
0
\end{tabular} \\
\hline 祘 & I & $\begin{array}{l}\infty \\
\stackrel{+}{+}\end{array}$ & 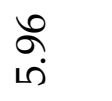 & $\begin{array}{l}\text { वे } \\
\text { i }\end{array}$ & ले & $\begin{array}{l}10 \\
10 \\
0\end{array}$ & $\begin{array}{l}\infty \\
\stackrel{n}{m}\end{array}$ & 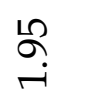 \\
\hline & $A_{1}$ & 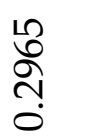 & 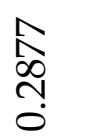 & $\begin{array}{l}\stackrel{n}{1} \\
\stackrel{0}{0} \\
0\end{array}$ & $\begin{array}{l}\stackrel{\infty}{N} \\
\stackrel{\sim}{0}\end{array}$ & $\begin{array}{l}\stackrel{\leftrightarrow}{\mathbb{V}} \\
\stackrel{0}{0}\end{array}$ & $\begin{array}{l}\text { ㅇ } \\
\stackrel{N}{O} \\
\stackrel{0}{0}\end{array}$ & $\begin{array}{l}0 \\
0 \\
0 \\
0\end{array}$ \\
\hline & 工 & $\stackrel{\circ}{\stackrel{ }{ }}$ & $\stackrel{n}{\sim}$ & $\underset{\sim}{\mathbb{N}}$ & 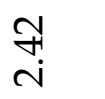 & $\begin{array}{l}\hat{0} \\
\text { ले }\end{array}$ & જे & 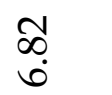 \\
\hline & 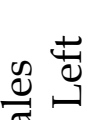 & 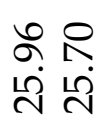 & $\begin{array}{l}\stackrel{\infty}{\sim} \underset{N}{\sim} \\
\stackrel{+}{\sim}\end{array}$ & 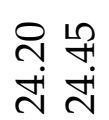 & 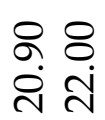 & 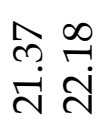 & 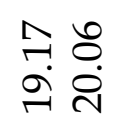 & 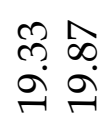 \\
\hline & 苞 & $\begin{array}{l}\infty \\
\infty \\
\stackrel{m}{\sim} \stackrel{m}{\sim} \\
\stackrel{n}{N}\end{array}$ & $\begin{array}{l}0 \\
\infty \\
\stackrel{\infty}{\infty} \\
\stackrel{\mathbb{N}}{N}\end{array}$ & $\begin{array}{l}\infty \\
\infty \\
\stackrel{\infty}{+} \\
\stackrel{\sim}{N}\end{array}$ & 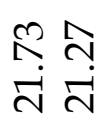 & 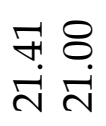 & $\begin{array}{l}\text { बे } \\
\stackrel{\sigma}{ } \\
\stackrel{\infty}{\sigma}\end{array}$ & 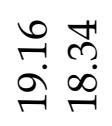 \\
\hline & 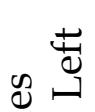 & 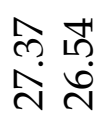 & $\begin{array}{l}\wedge 8 \\
8 \\
\stackrel{2}{N}\end{array}$ & 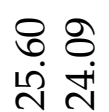 & 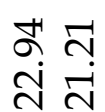 & 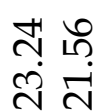 & 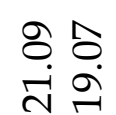 & 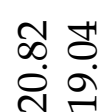 \\
\hline & $\sum_{i}^{\pi} \frac{\pi}{\sigma}$ & 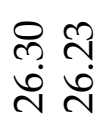 & 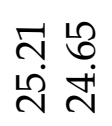 & $\begin{array}{l}\infty \\
\infty \\
\stackrel{+}{\sim} \dot{~}\end{array}$ & 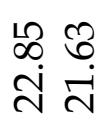 & $\begin{array}{l}\infty ָ \\
\infty \\
\\
\end{array}$ & $\begin{array}{l}\text { No } \\
\infty \\
\stackrel{\sim}{+}\end{array}$ & 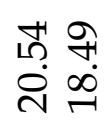 \\
\hline & $\underset{\substack{x\\
}}{\vec{x}}$ & $\curvearrowleft \cong$ & $\tilde{\sim} \cong$ & $\Xi \cong$ & $\Xi \cong$ & $\overbrace{} \cong$ & $\Sigma \cong$ & $\Xi \cong$ \\
\hline & & $\stackrel{\ominus}{\triangle}$ & $\sum_{5}$ & $\begin{array}{l}0 \\
b \\
ص\end{array}$ & $\begin{array}{l}\sum_{0} \\
b \\
b\end{array}$ & $\begin{array}{l}\oplus \\
\stackrel{0}{p}\end{array}$ & $\sum_{10}$ & $\stackrel{D}{\square}$ \\
\hline
\end{tabular}




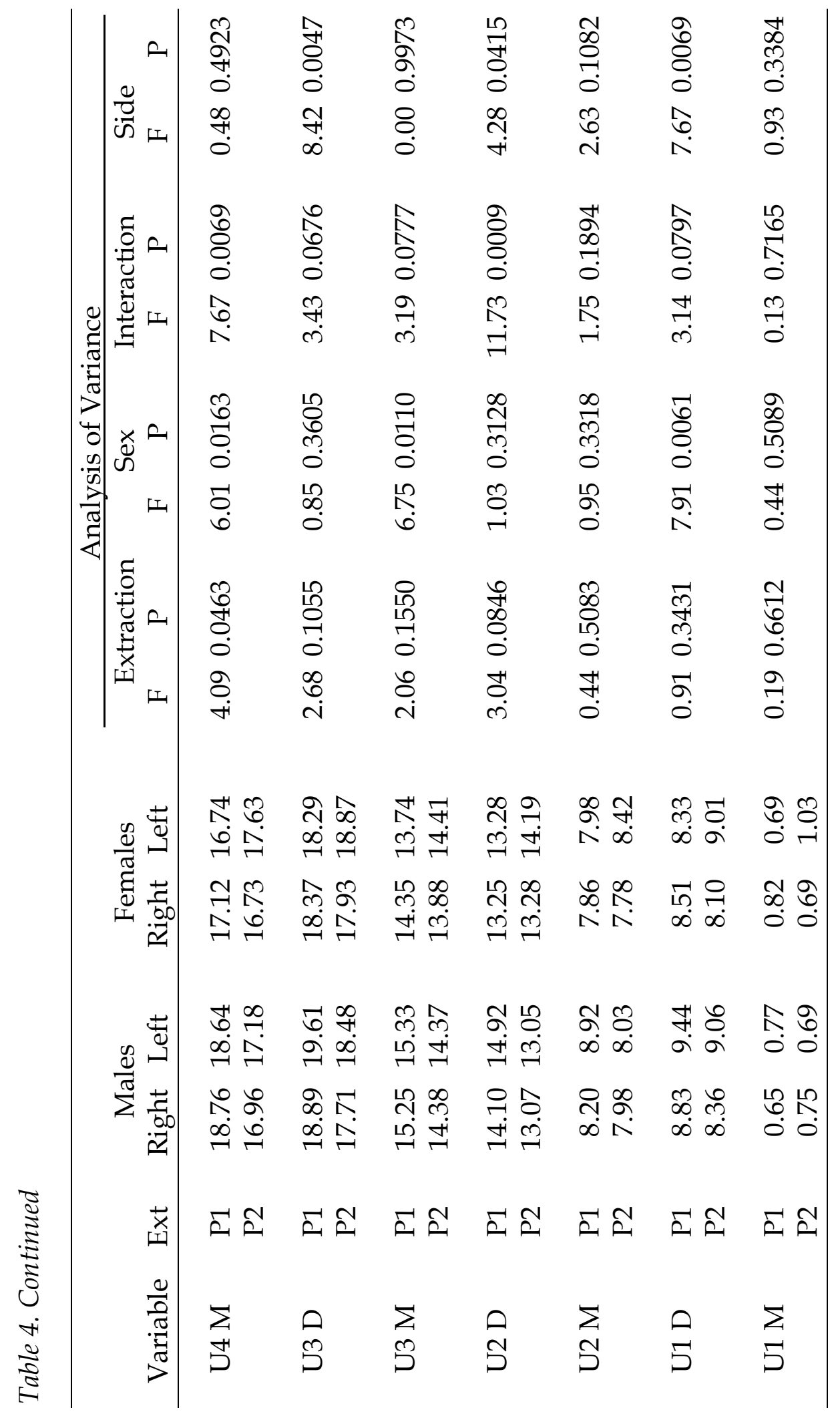


broader in the premolar region in cases where the first premolars subsequently were extracted.

In several cases scattered throughout the arch (5 of 14 tests), palatal width is significantly broader in boys than girls. Of note, however, several of the extraction-by-sex interaction tests are significant, which means that the main effects are biased. That is, one of the underlying assumptions of analysis of variance is "additivity," which means that the effects are proportional across the samples. In the present case, expectation is that the arch sizes in the first-premolar and second-premolar extraction groups will be proportional ("additive") in males and females. Lack of additivity (i.e., a significant interaction effect) is tested for in each of the ANOVA models (Table 4), and the source of significant interaction generally can be discerned from inspection of plots of the sample means. Six of these 14 tests disclose significant extraction-by-sex nonadditivity. For completeness, we have plotted the means for all 14 sets of variables. (Figs. 20 though 33). Fig. 23 (mesial contact of maxillary first molar) is the first variable in the table showing a significant interaction effect, and this is due to the very different rankings found in the first-premolar extraction group compared to the second-premolar extraction group. Males have broader inter-molar widths in the first-premolar extraction sample, and there is an appreciable side difference (right $>$ left) in the female first-premolar samples. Neither of these relationships occurs in the second- 


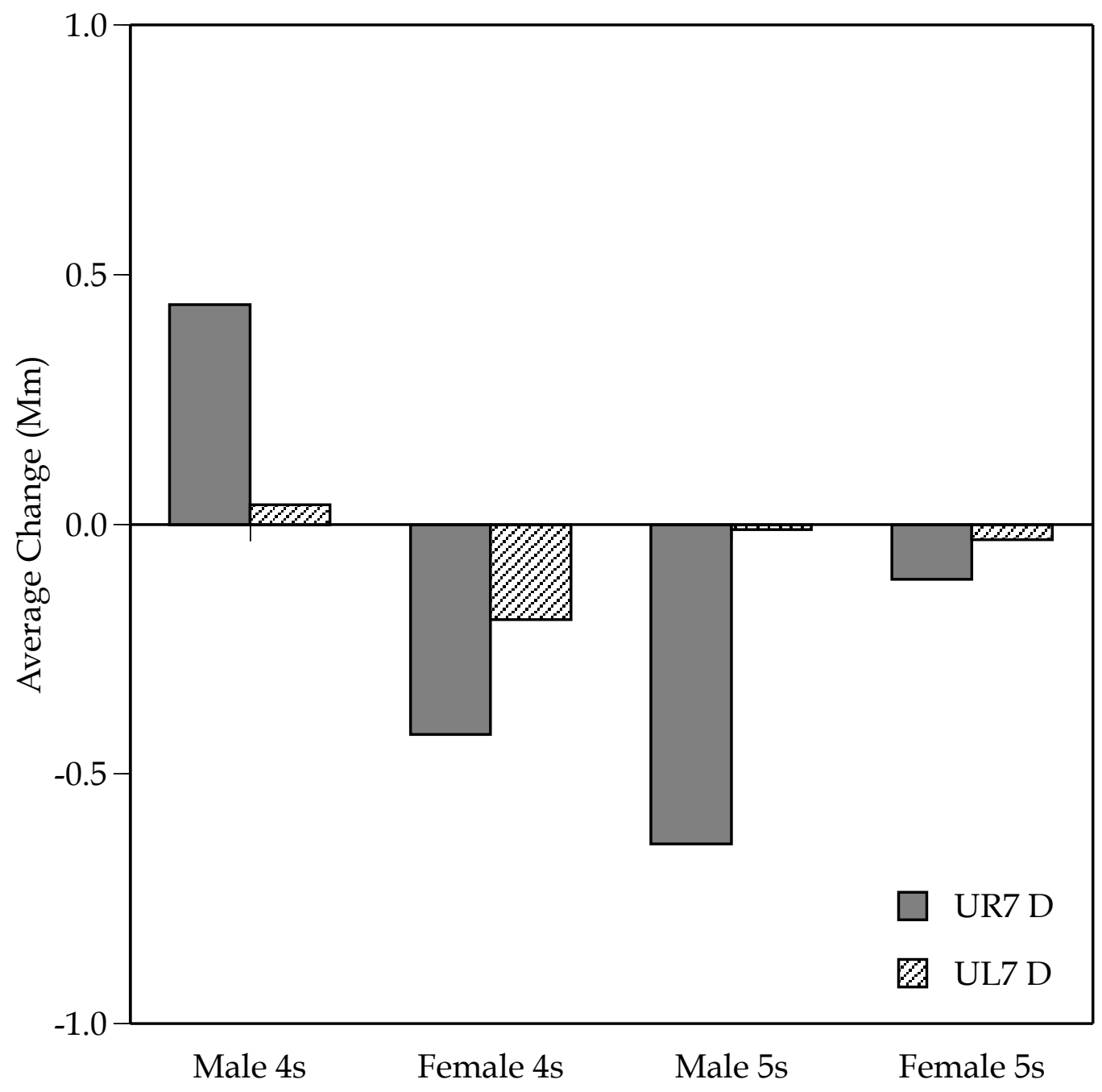

Fig. 20. Mean in-treatment mesiodistal $(Y)$ change, by group and side, for the distal landmark on the second molar. 


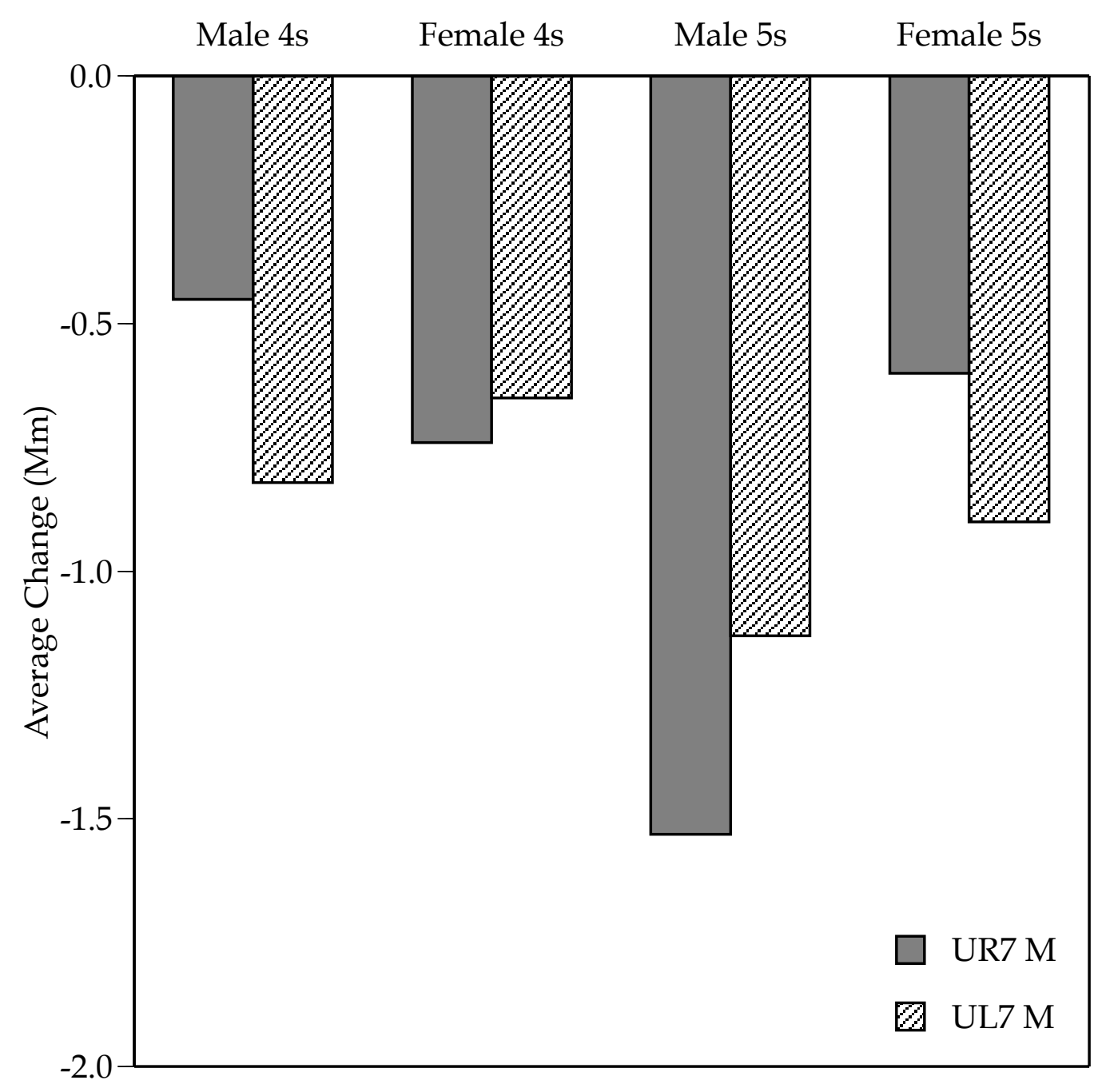

Fig. 21. Mean in-treatment mesiodistal $(\mathrm{Y})$ change, by group and side, for the mesial landmark on the second molar. 


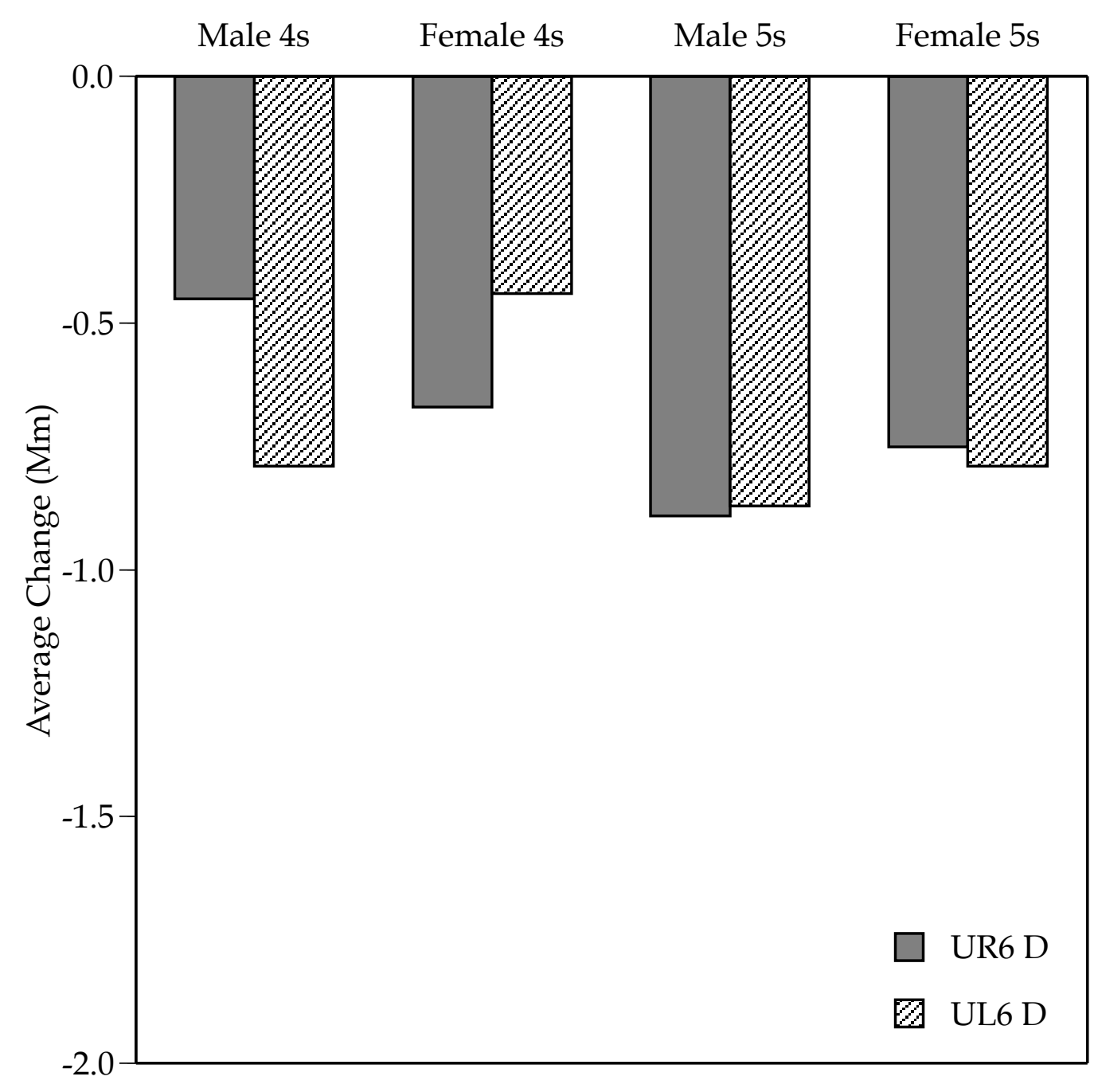

Fig. 22. Mean in-treatment mesiodistal $(\mathrm{Y})$ change, by group and side, for the distal landmark on the first molar. 


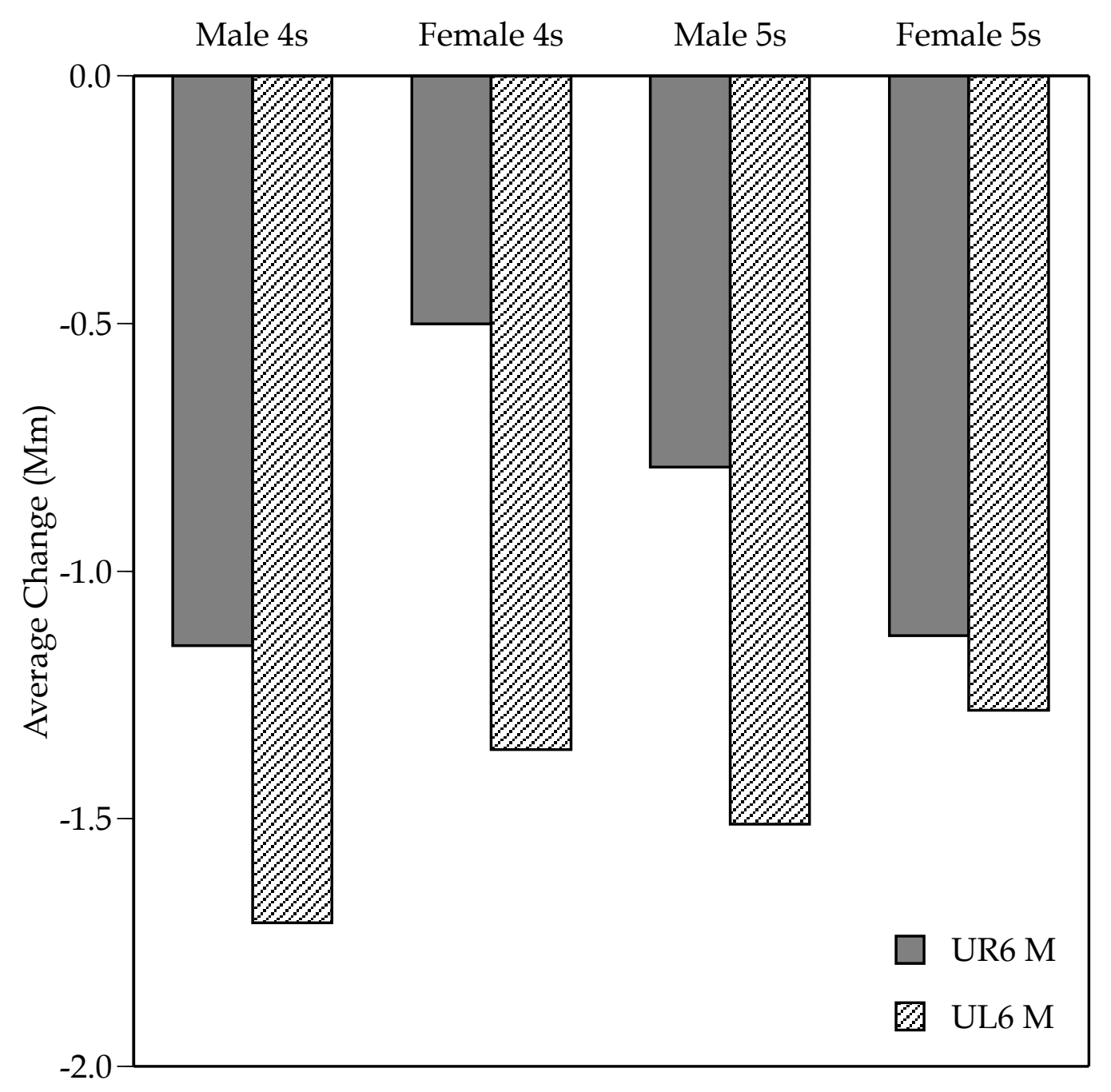

Fig. 23. Mean in-treatment mesiodistal $(\mathrm{Y})$ change, by group and side, for the mesial landmark on the first molar. 


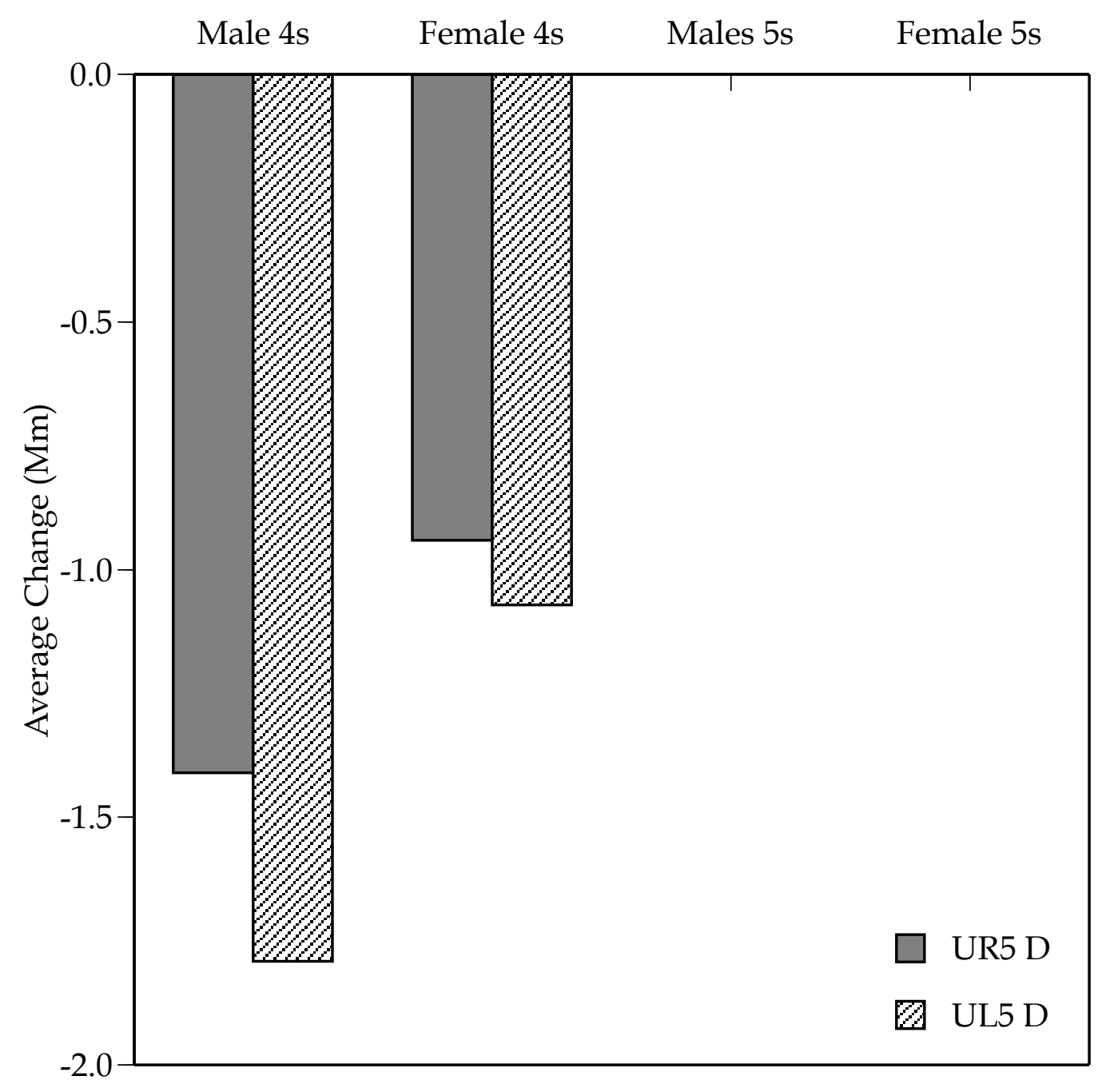

Fig. 24. Mean in-treatment mesiodistal ( $\mathrm{Y}$ ) change, by group and side, for the distal landmark on the second premolar. 


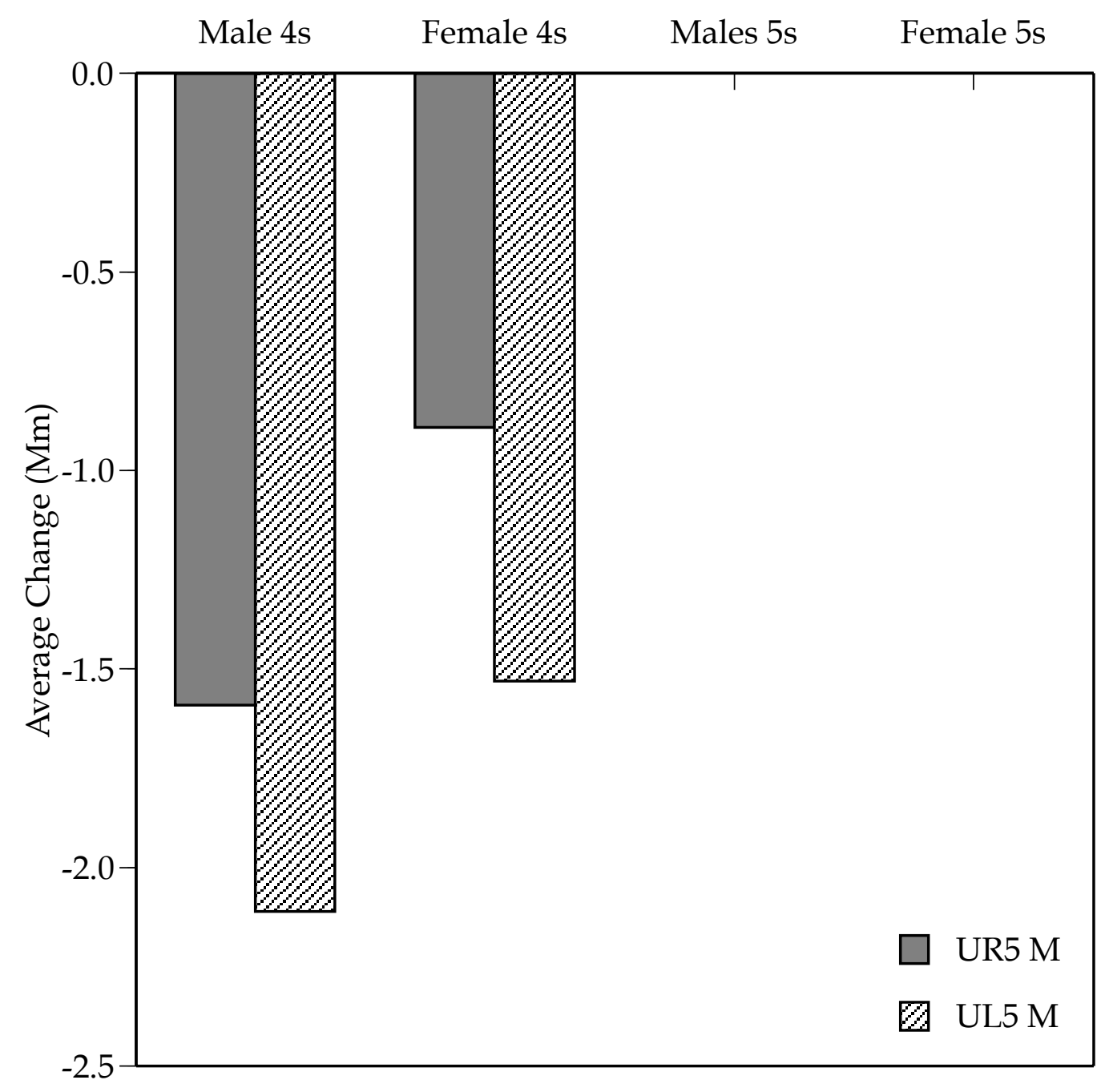

Fig. 25. Mean in-treatment mesiodistal $(\mathrm{Y})$ change, by group and side, for the mesial landmark on the second premolar. 


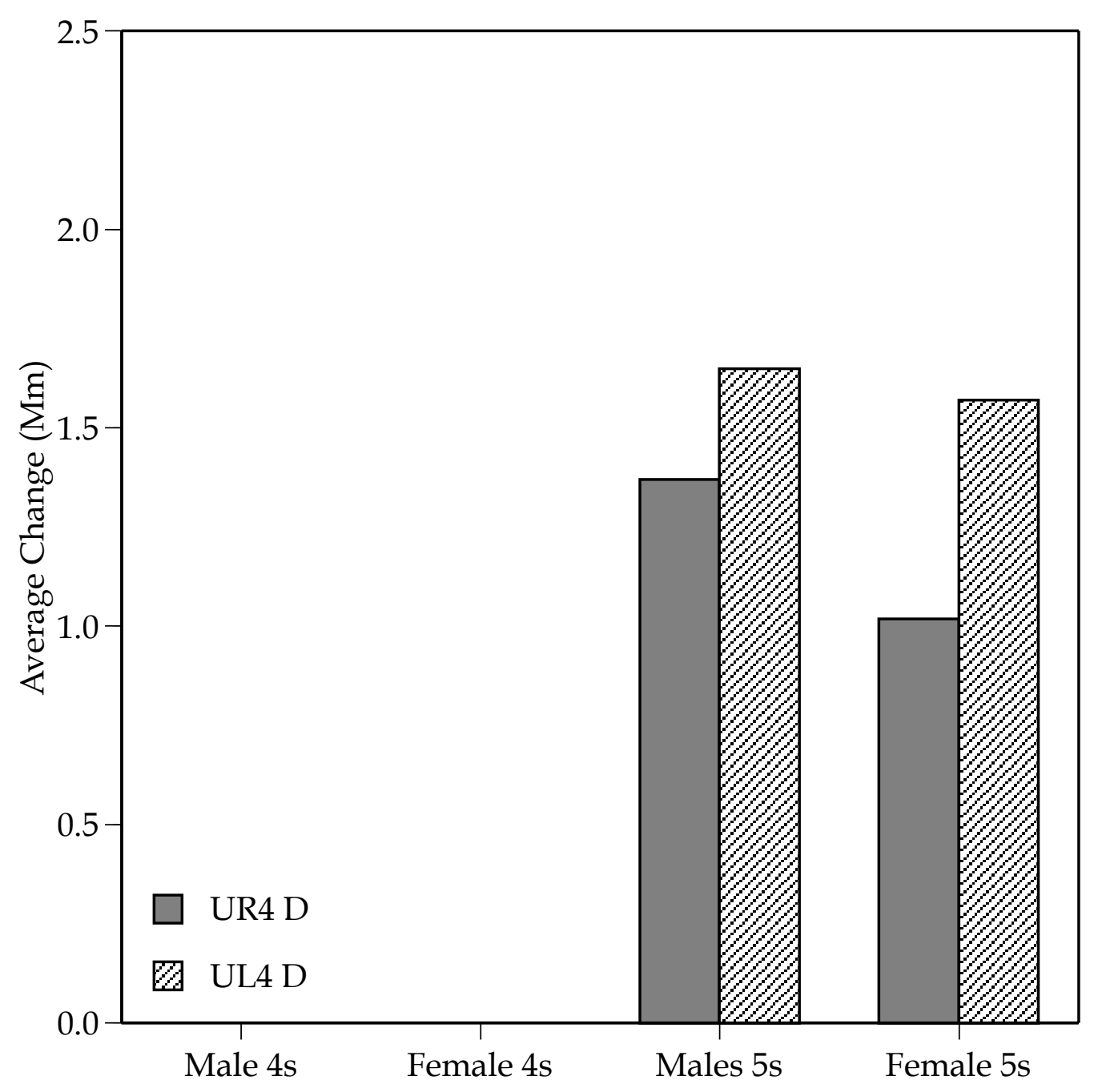

Fig. 26. Mean in-treatment mesiodistal $(\mathrm{Y})$ change, by group and side, for the distal landmark on the first premolar. 


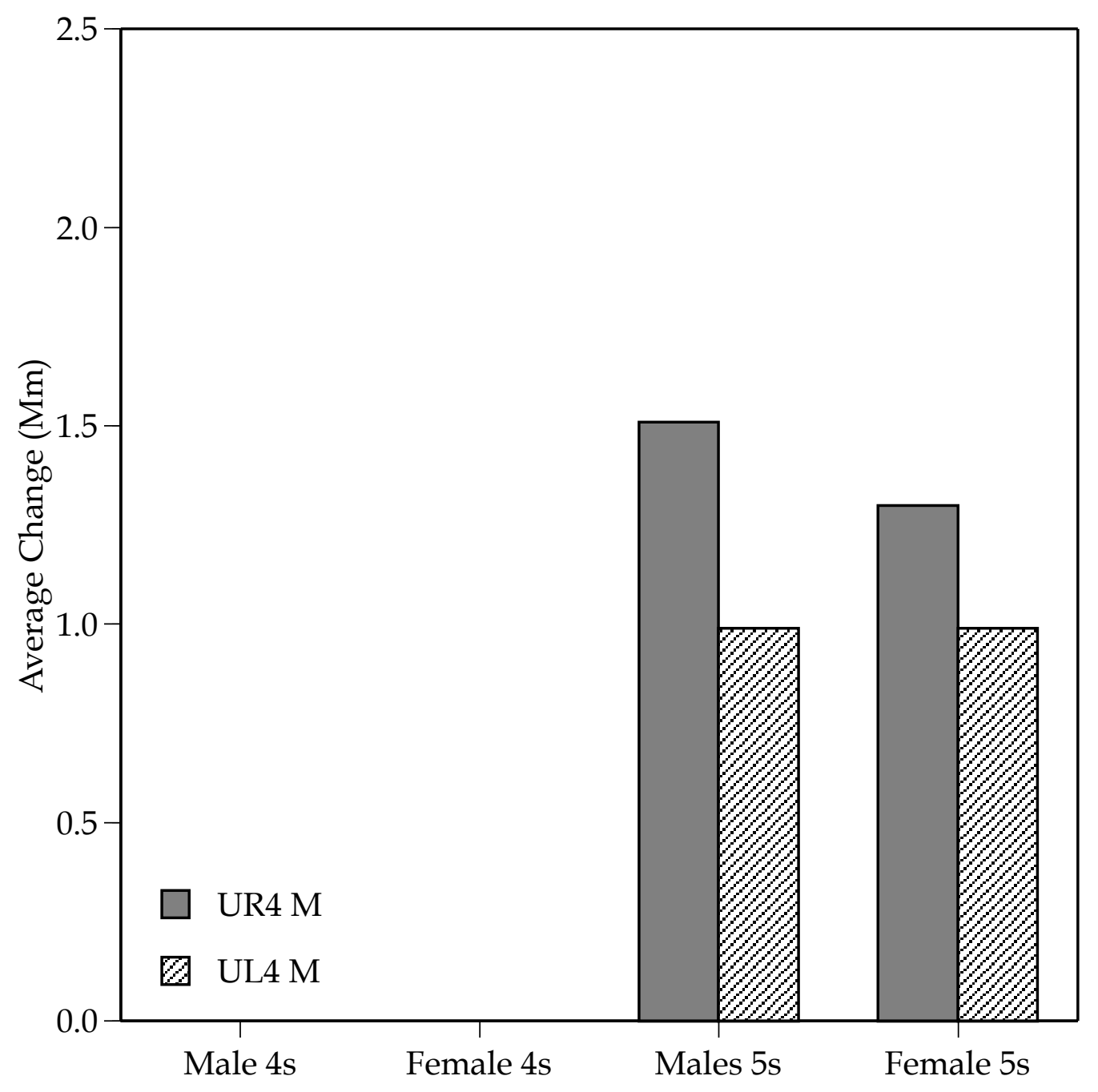

Fig. 27. Mean in-treatment mesiodistal ( $\mathrm{Y}$ ) change, by group and side, for the mesial landmark on the first premolar. 


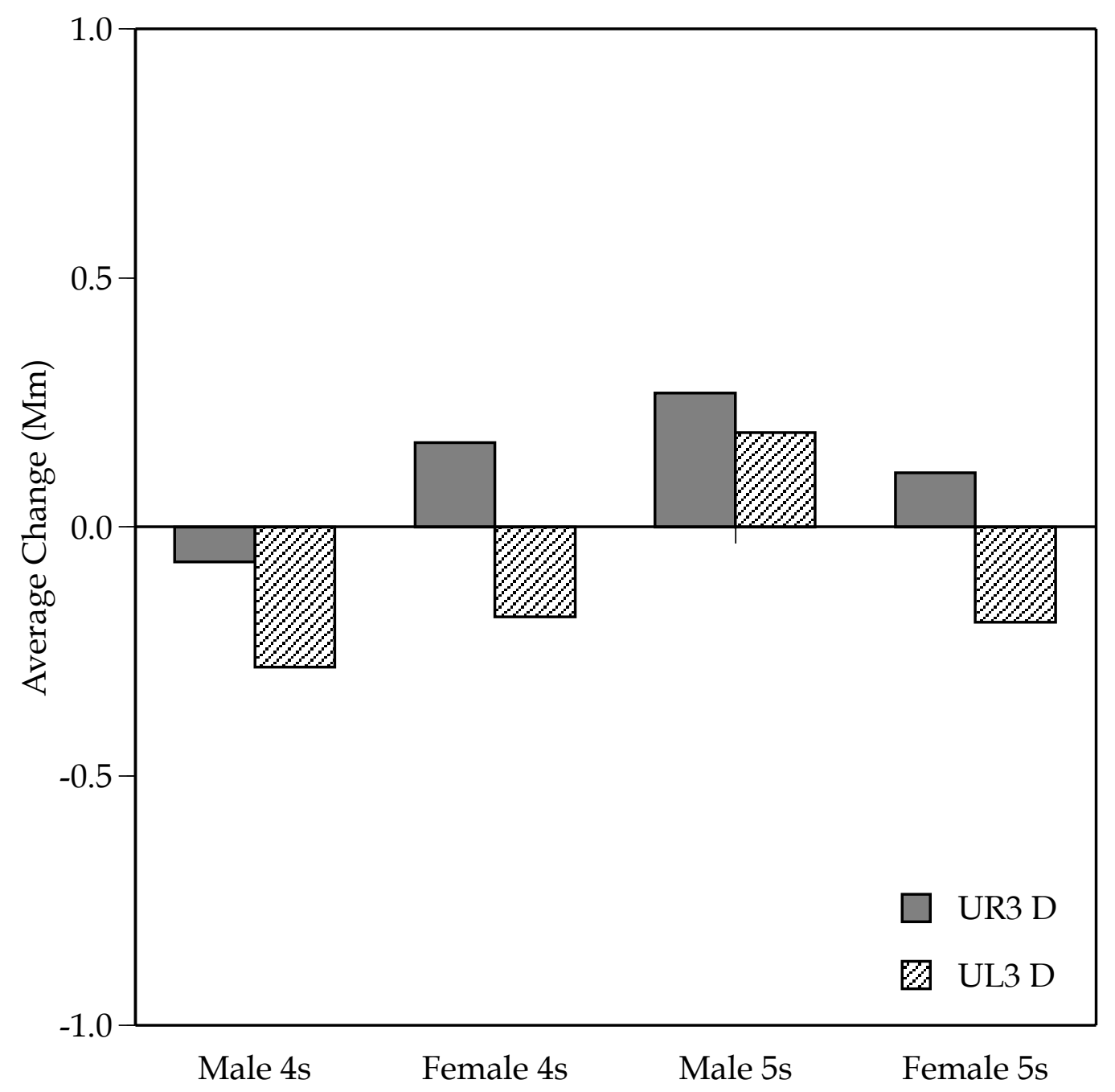

Fig. 28. Mean in-treatment mesiodistal $(\mathrm{Y})$ change, by group and side, for the distal landmark on the canine. 


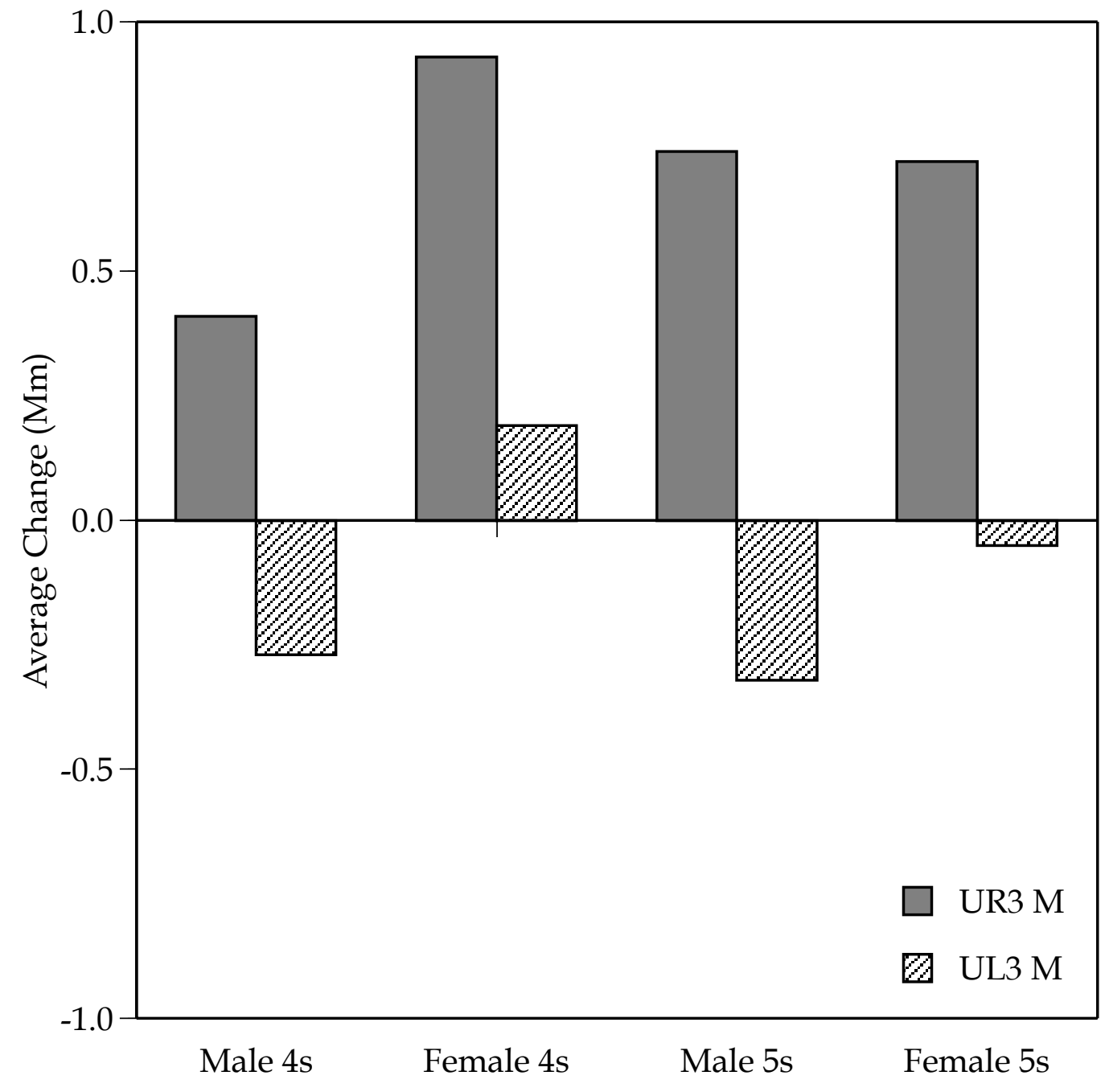

Fig. 29. Mean in-treatment mesiodistal $(Y)$ change, by group and side, for the mesial landmark on the canine. 


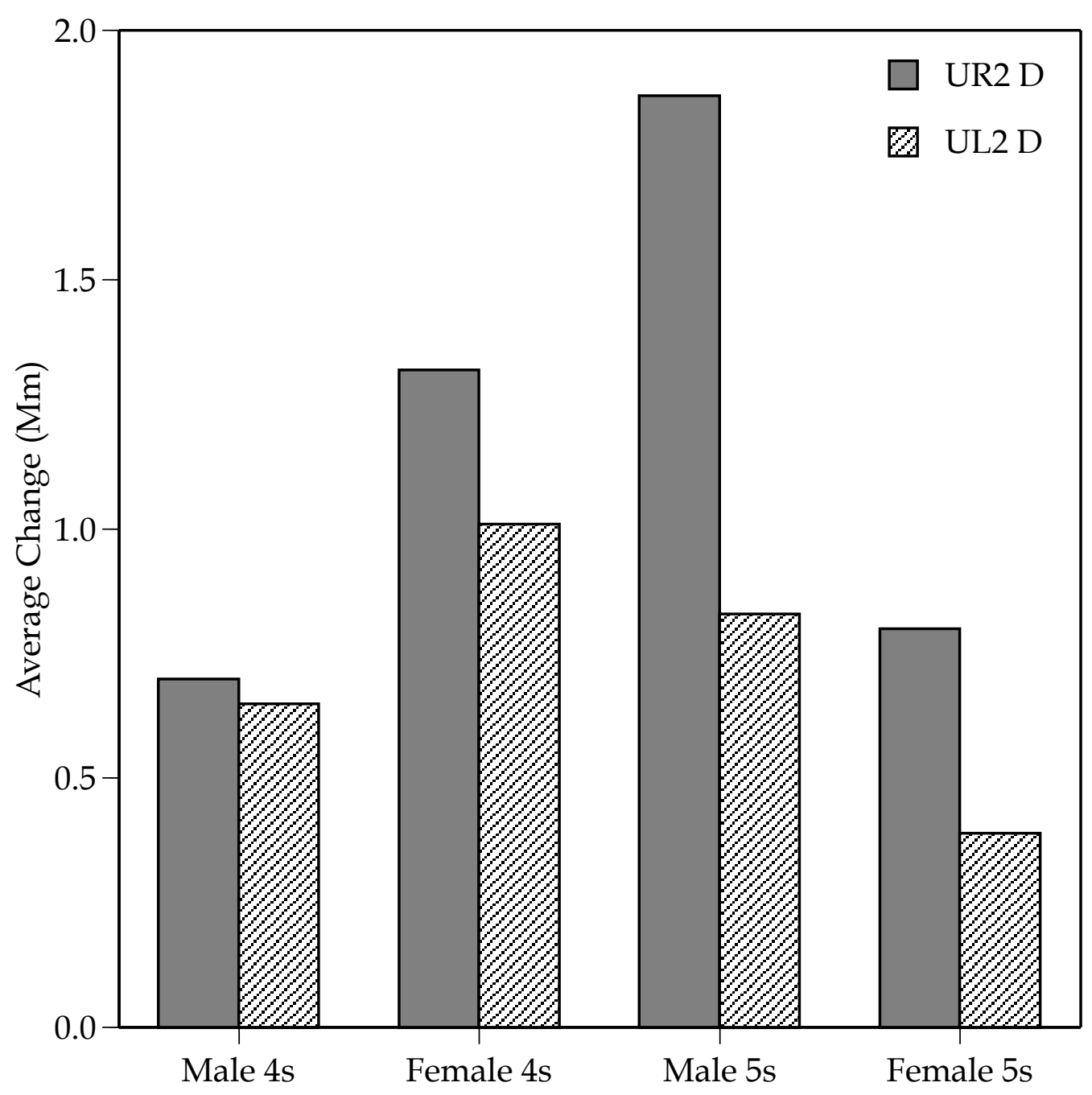

Fig. 30. Mean in-treatment mesiodistal $(\mathrm{Y})$ change, by group and side, for the distal landmark on the lateral incisor. 


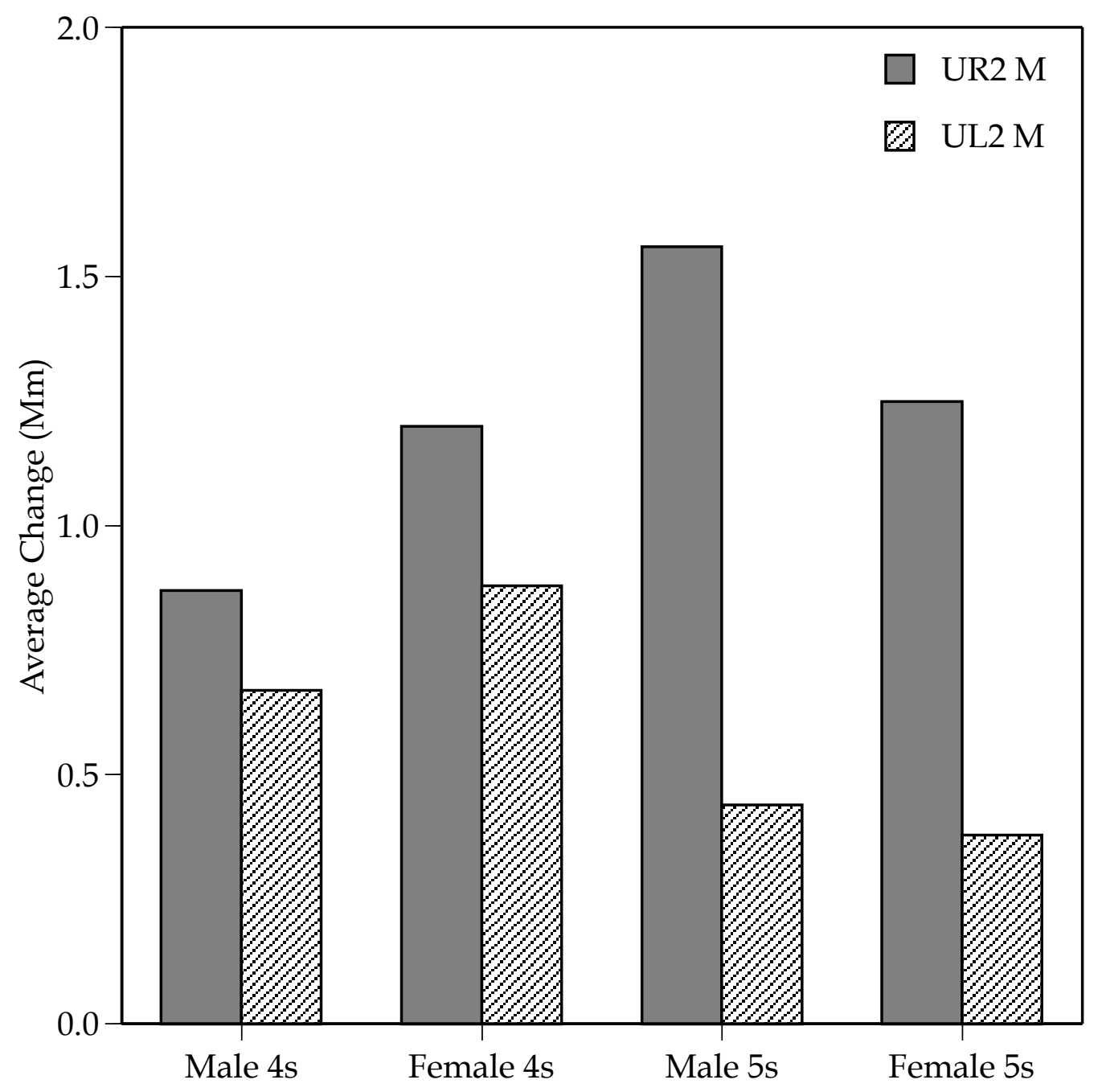

Fig. 31. Mean in-treatment mesiodistal $(Y)$ change, by group and side, for the mesial landmark on the lateral incisor. 


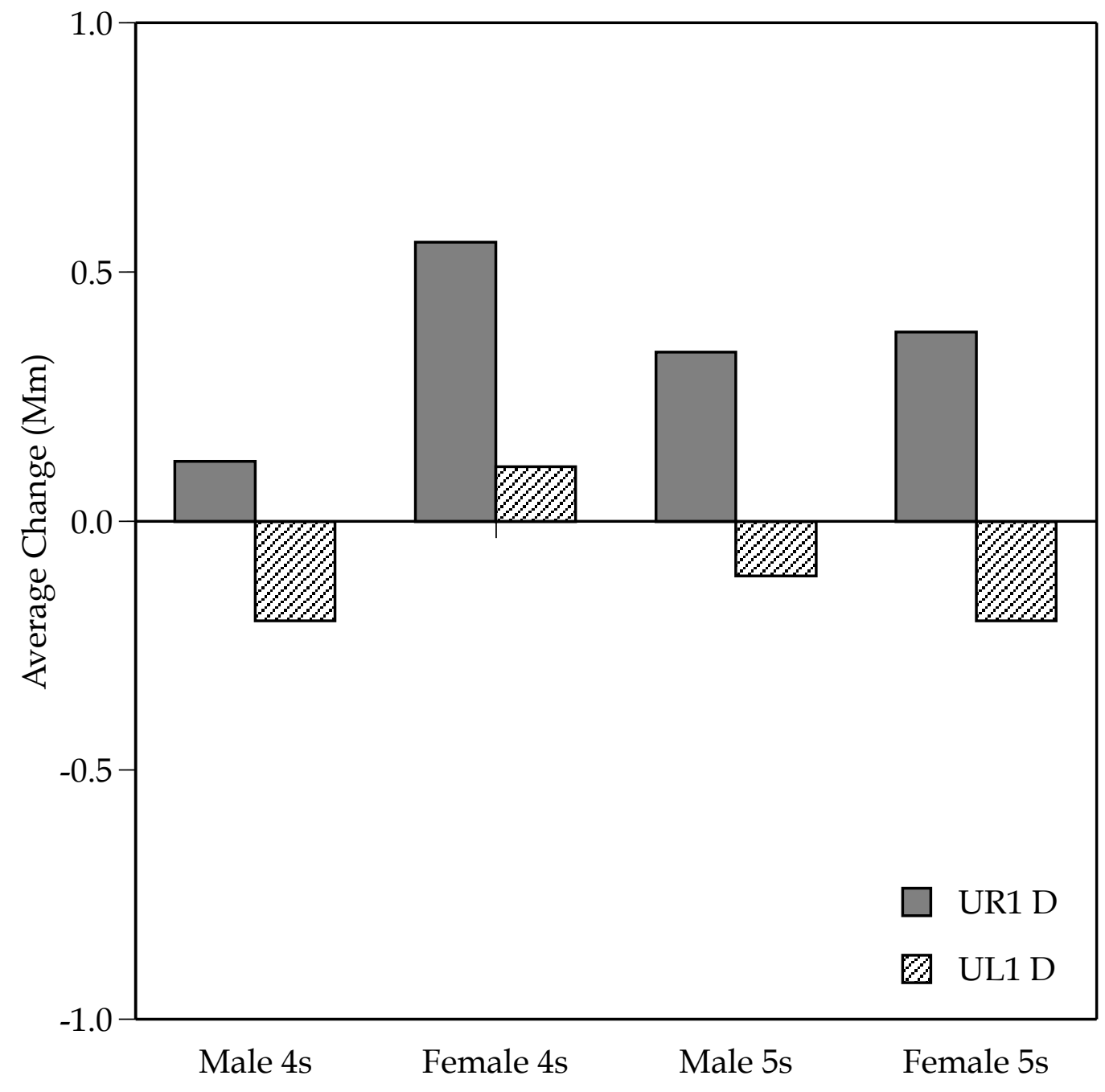

Fig. 32. Mean in-treatment mesiodistal $(\mathrm{Y})$ change, by group and side, for the distal landmark on the central incisor. 


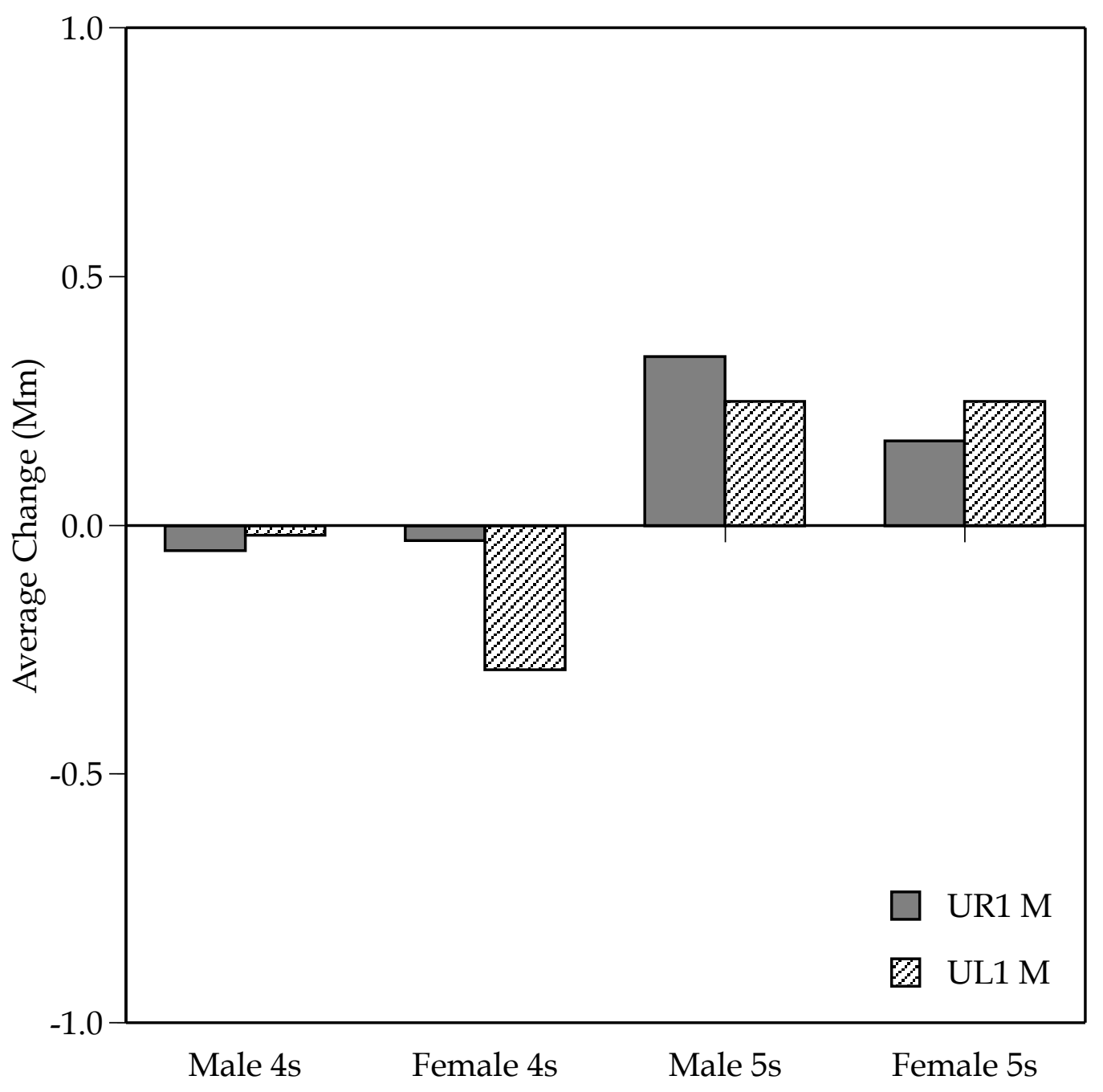

Fig. 33. Mean in-treatment mesiodistal $(\mathrm{Y})$ change, by group and side, for the mesial landmark on the central incisor. 
premolar extraction group, where there is no apparent sexual dimorphism, and the left-right side differences are smaller. Transverse breadth is greatest in the second-premolar extraction group for females on the left side.

Of note, the same differences in size relationships - the same source of a significant interaction effect-is evident for the other coordinates, specifically the mesial contact of the first molar (Fig. 23) through the distal contact of the lateral incisor (Fig. 30). Consistently, the first-premolar extraction sample of males has the largest arch breadths, and there is persistent side asymmetry in the second-premolar extraction samples, but males are not appreciable larger than females in the second-premolar groups. This latter feature (lack of obvious sexual dimorphism in the second-premolar samples) is the major cause of the significance of the interaction effects.

\section{$\underline{\text { Posttreatment Status }}$}

Since either the first or second premolar was extracted from both maxillary quadrants, there could be no comparison of the four premolar landmarks of those teeth at the posttreatment examination. There were, then, 10 instead of 14 results for the mixed-model ANOVA. On the other hand, data were analyzed for the four premolar landmarks with regards sex and side. 


\section{Mesiodistal Axis}

ANOVA results are listed in Table 5. All 10 tests show that the arch lengths are significantly shorter in the second-premolar extraction sample, which is the same relationship seen at the pretreatment examination. Therefore, the longer arch lengths in the first-premolar group at the start of treatment, which we attribute primarily to protrusion of the maxillary incisors and thus, greater overjet, was not completely resolved during treatment. This supposition is substantiated by the significantly greater overjet in the firstpremolar sample at the end of treatment (Table 1).

Table 5 shows that a scattering (4/14) of the variables still exhibit significant sexual dimorphism, particularly towards the back of the arch, but development of a consistent arch form during treatment clearly diminishes the extent of sexual dimorphism compared to the pretreatment condition.

\section{Transverse Axis}

Just as the first-premolar extraction sample has a longer arch mesiodistally, it also is broader mediolaterally (Table 6). Differences in the mean sizes are small - on the order of 1 to $2 \mathrm{~mm}$ - but the differences are highly significant statistically. These differences between the two extraction groups are in addition to several instances throughout the dental arch (6/14 tests) where arch breadth is significantly larger in males than females. 


\begin{tabular}{|c|c|c|c|c|c|c|c|}
\hline$\frac{0}{0}^{n}$ & \begin{tabular}{l}
0 \\
$\stackrel{0}{\infty}$ \\
\multirow{+}{+}{} \\
0
\end{tabular} & $\begin{array}{l}\stackrel{8}{\circ} \\
0 \\
0\end{array}$ & 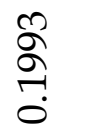 & $\begin{array}{l}\hat{L} \\
\stackrel{8}{0} \\
0\end{array}$ & $\begin{array}{l}\text { స్ } \\
\text { กุ. } \\
0\end{array}$ & 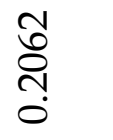 & $\begin{array}{l}\stackrel{10}{\not} \\
\text { ᄋ̆ } \\
0\end{array}$ \\
\hline के & ఫ্ & ָே & مِ & $\begin{array}{l}\infty \\
0 \\
\infty\end{array}$ & $\stackrel{\circ}{\stackrel{0}{0}}$ & $\underset{ت}{ت}$ & बे \\
\hline$\underbrace{0}_{\tilde{d}} \sim$ & 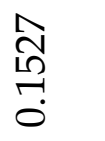 & 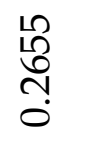 & $\begin{array}{c}\infty \\
\stackrel{\infty}{+} \\
\stackrel{0}{0}\end{array}$ & & $\cdot$ & $\cdot$ & $\cdot$ \\
\hline 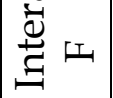 & $\begin{array}{l}\infty \\
0 \\
\text { ஸे }\end{array}$ & $\stackrel{\text { ำ }}{\sim}$ & $\begin{array}{l}\infty \\
\text { m } \\
\end{array}$ & के & $\cdot$ & $\cdot$ & $\cdot$ \\
\hline$x^{f}$ & $\begin{array}{l}\infty \\
\stackrel{\infty}{0} \\
0 \\
0\end{array}$ & $\begin{array}{l}0 \\
0 \\
0 \\
0 \\
0\end{array}$ & $\begin{array}{l}\text { L } \\
\stackrel{0}{0} \\
0 \\
0\end{array}$ & $\begin{array}{l}\stackrel{2}{1} \\
\text { ถે } \\
0 \\
0\end{array}$ & 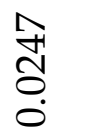 & $\begin{array}{l}8 \\
8 \\
8 \\
0\end{array}$ & $\begin{array}{l}\qquad 0 \\
\stackrel{1}{4} \\
0 \\
0\end{array}$ \\
\hline$\infty_{\text {L. }}$ & $\begin{array}{l}\mathscr{B} \\
\dot{10}\end{array}$ & 로 & $\begin{array}{l}\hat{1} \\
\text { மீ) }\end{array}$ & $\begin{array}{l}\infty \\
\infty \\
\dot{\infty}\end{array}$ & $\begin{array}{l}\infty \\
\infty \\
\infty \\
1\end{array}$ & $\begin{array}{l}R \\
\stackrel{R}{n}\end{array}$ & ָ̃ \\
\hline . & $\begin{array}{l}5 \\
8 \\
8\end{array}$ & $\begin{array}{l}5 \\
8 \\
8\end{array}$ & 용 & $\begin{array}{l}\text { 옹 } \\
8\end{array}$ & $\cdot$ & $\cdot$ & 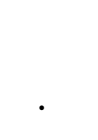 \\
\hline 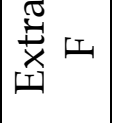 & 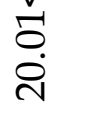 & ت্் & 文 & ฉి & . & . & . \\
\hline 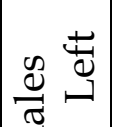 & 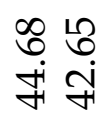 & 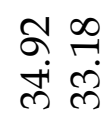 & 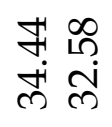 & 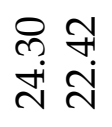 & 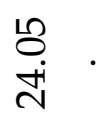 & సิ & $\stackrel{\widetilde{N}}{\check{z}}$ \\
\hline 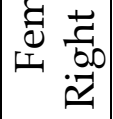 & 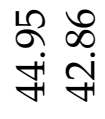 & 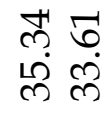 & 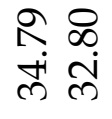 & 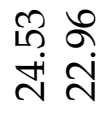 & 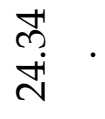 & ก̃ & $\begin{array}{l}\text { テे } \\
\stackrel{\text { N }}{ }\end{array}$ \\
\hline 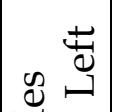 & 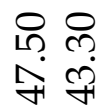 & $\begin{array}{l}\infty \\
0 \\
m \\
m\end{array}$ & 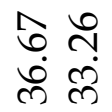 & 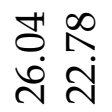 & $\begin{array}{l}\infty \\
\infty \\
\stackrel{\infty}{N}\end{array}$ & $\begin{array}{l}8 \\
\infty \\
\infty\end{array}$ & \begin{tabular}{l}
\multirow{1}{1}{} \\
Ni
\end{tabular} \\
\hline 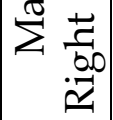 & 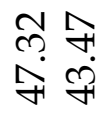 & 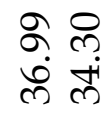 & 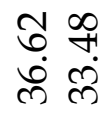 & 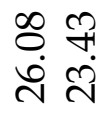 & $\begin{array}{l}\stackrel{0}{\mathrm{~N}} \\
\stackrel{\mathrm{L}}{\mathrm{N}}\end{array}$ & $\begin{array}{l}0 \\
0 \\
\infty \\
\infty\end{array}$ & $\begin{array}{l}\infty \\
\stackrel{\infty}{ } \\
\text { N }\end{array}$ \\
\hline $\begin{array}{c}\vec{x} \\
\dot{x}\end{array}$ & $\sqsubseteq \cong$ & $\Sigma \cong$ & $\Sigma \cong$ & $\tilde{\epsilon} \cong$ & $\tilde{E} \cong$ & $\tilde{F} \cong$ & $\Sigma ઈ$ \\
\hline 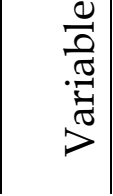 & $\stackrel{\ominus}{5}$ & $\begin{array}{l}\sum_{\Lambda} \\
S\end{array}$ & $\stackrel{\oplus}{\emptyset}$ & $\begin{array}{l}\sum_{0} \\
b\end{array}$ & $\stackrel{\oplus}{\rho}$ & $\begin{array}{l}\sum_{0} \\
\qquad\end{array}$ & 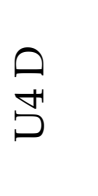 \\
\hline
\end{tabular}




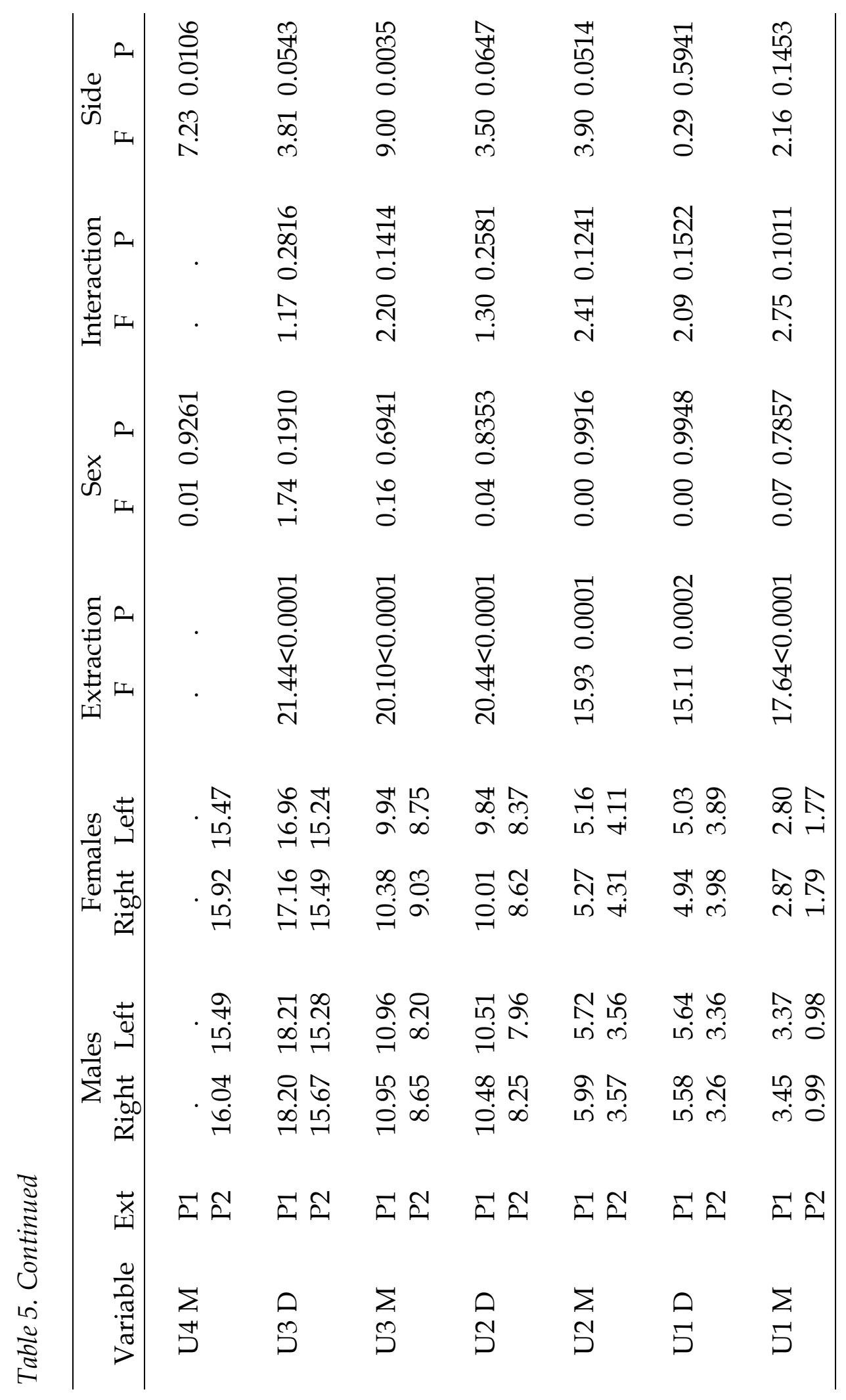




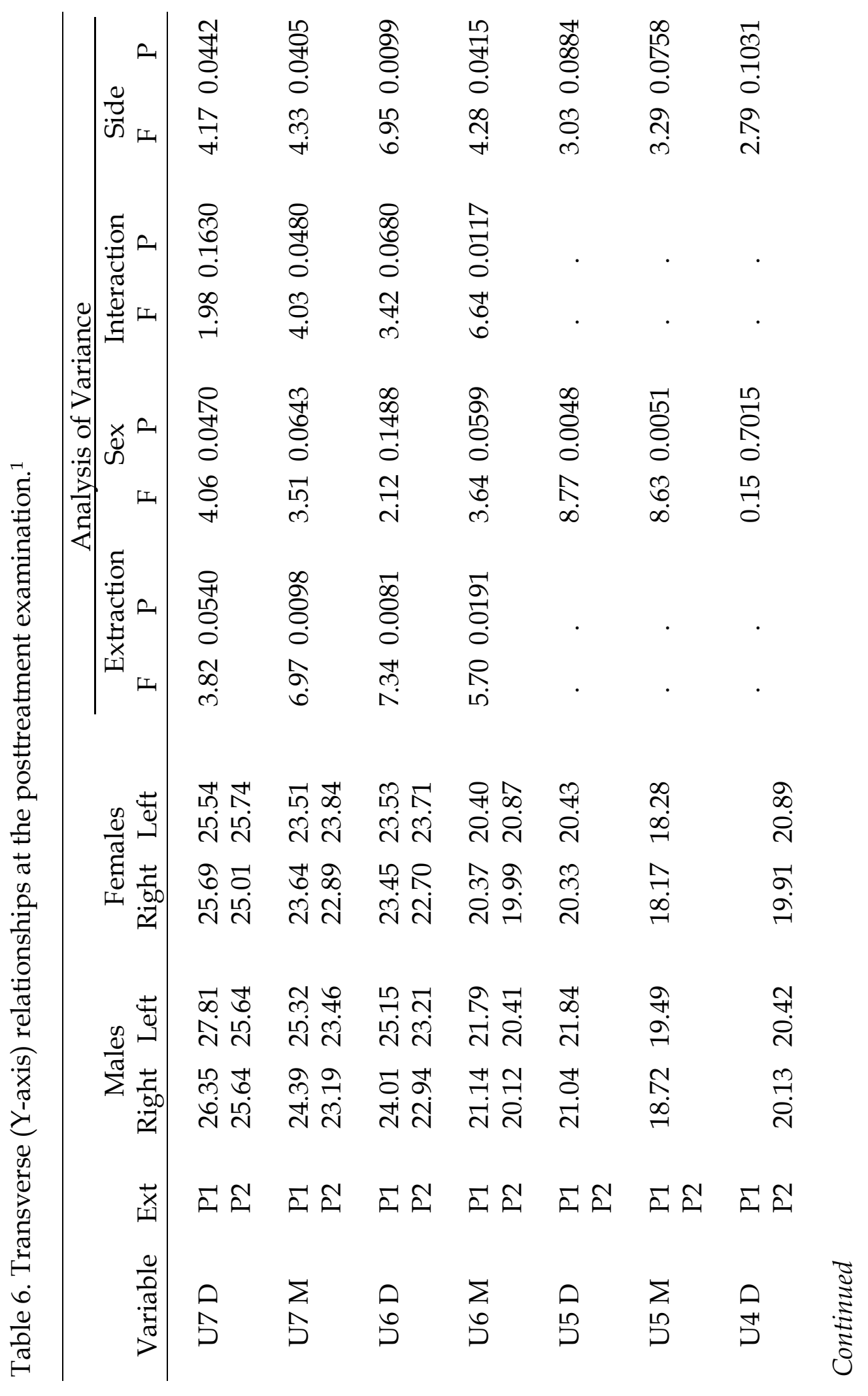




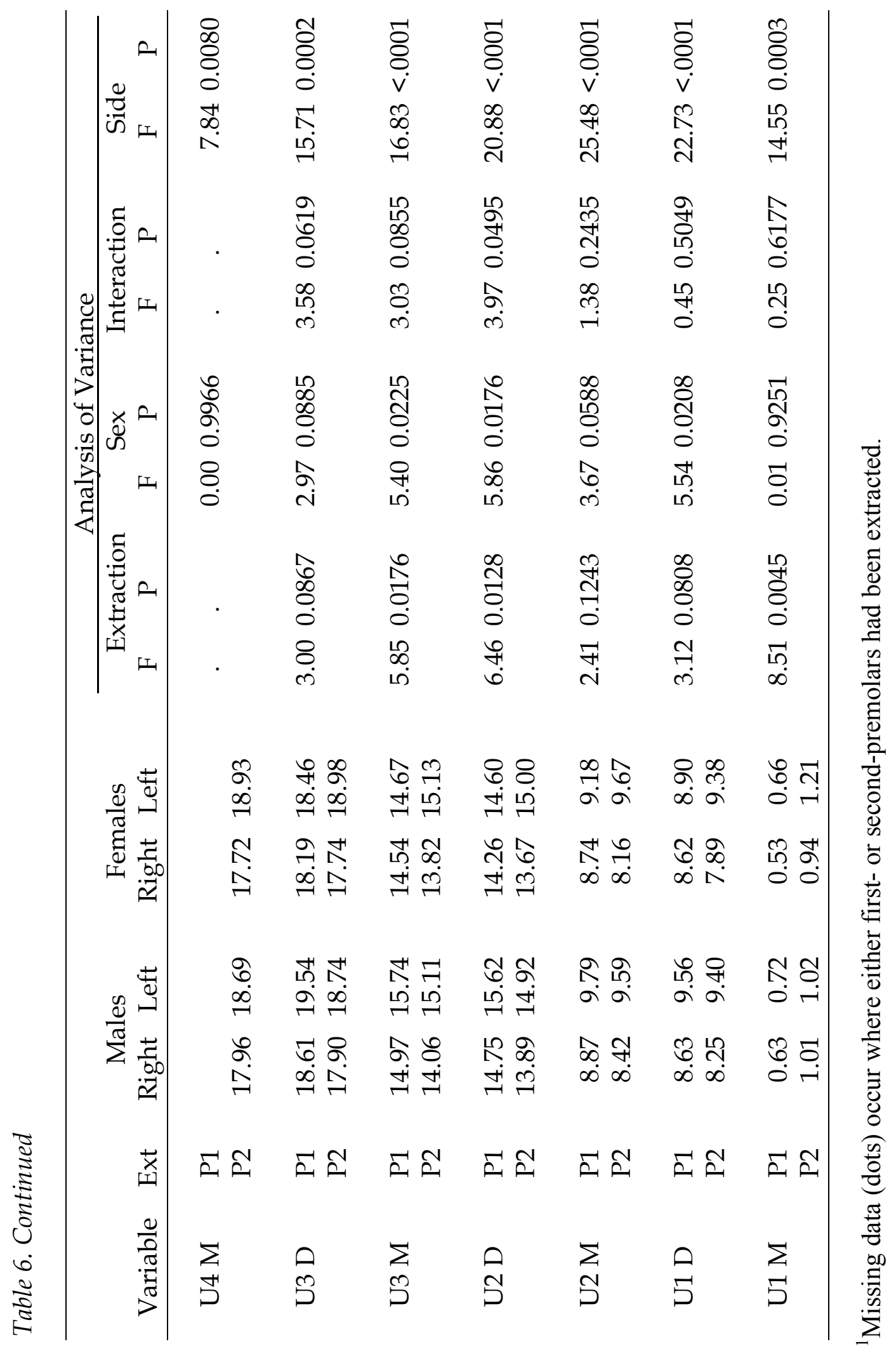


Table 6 also discloses numerous instances where the left side of the arch systematically exceeds the corresponding size on the right--so called directional asymmetry (e.g., Harris 1992; Harris and Bodford 2007). This also was commented on for the pretreatment data, but inspection shows that the sidedness is more prevalent and absolutely larger at the end of treatment than before. The causes of the differences are not obvious because several things are happening simultaneously. These adolescents experienced at least two years of fairly rapid growth during treatment. This increase in palatal size could have increased the asymmetry, but the teeth also were bonded to archwires throughout treatment, so the influence of directed tooth movement cannot be discounted. Indeed, the slight asymmetries disclosed here could have been accentuated by side differences in treatment mechanics and/or tissue responses.

\section{$\underline{\text { In-Treatment Changes }}$}

\section{Transverse Changes}

There are, grandly, two issues of particular interest when reviewing the in-treatment changes in tooth position, (1) whether a dental landmark moved systematically during treatment and (2) whether the average changes differed significantly among the samples, especially between the two extraction 
patterns. This first question was evaluated with one-sample t-tests (two-tail). That is, was there a significant trend in the directions of dental landmark changes? Table 7, for example, lists the statistical results of one-sample t-tests for the transverse changes of the whole sample. In these results (28 univariate tests), every change is seen to be highly significant statistically. Given the nature of the changes (posttreatment status minus pretreatment status), the three posterior teeth (P2, M1, M2) exhibited arch constriction whereas the four mesial tooth types characteristically experienced arch expansion (positive means). The results for the mesiodistal changes (total sample) are listed in Table 8 .

ANOVA results are listed in Table 9 testing for differences in the amount of change by extraction pattern or sex, and none of the four molar landmarks changed differently in the extraction patterns. In contrast, the six canine and incisor landmarks each was expanded significantly more in the first-premolar extraction sample. The magnitudes of the mean transverse changes tend to increase more as the front of the arch is reached, notably so in the first-premolar extraction group. This suggests that one determinant of which extraction pattern to use in a case depends on the extent of incisor crowding. The greater transverse correction in the first-premolar group suggests that they have narrower, more tapered anterior segments that merit greater transverse expansion of the teeth to achieve alignment. 
Table 7. Descriptive statistics and one-sample tests for the change in the transverse (X-axis) arch dimension.

\begin{tabular}{|c|c|c|c|c|c|c|}
\hline Variable & Mean & $\mathrm{sd}$ & sem & $\mathrm{n}$ & $t$ test & $\mathrm{P}$ \\
\hline UR7 D & -3.24 & 1.72 & 0.19 & 84 & -17.24 & $<0.0001$ \\
\hline UR7 M & -3.87 & 1.73 & 0.19 & 84 & -20.46 & $<0.0001$ \\
\hline UR6 D & -3.83 & 1.60 & 0.17 & 90 & -22.71 & $<0.0001$ \\
\hline UR6 M & -4.11 & 1.54 & 0.16 & 90 & -25.35 & $<0.0001$ \\
\hline UR5 D & -3.86 & 1.61 & 0.23 & 50 & -16.97 & $<0.0001$ \\
\hline UR5 M & -4.26 & 1.56 & 0.22 & 50 & -19.28 & $<0.0001$ \\
\hline UR4 D & 2.42 & 1.42 & 0.22 & 40 & 10.76 & $<0.0001$ \\
\hline UR4 M & 2.16 & 1.26 & 0.20 & 40 & 10.86 & $<0.0001$ \\
\hline UR3 D & 3.27 & 1.37 & 0.14 & 90 & 22.71 & $<0.0001$ \\
\hline UR3 M & 2.47 & 1.51 & 0.16 & 90 & 15.49 & $<0.0001$ \\
\hline UR2 D & 2.17 & 1.89 & 0.20 & 90 & 10.87 & $<0.0001$ \\
\hline UR2 M & 1.68 & 1.80 & 0.19 & 90 & 8.84 & $<0.0001$ \\
\hline UR1 D & 2.75 & 2.06 & 0.22 & 90 & 12.66 & $<0.0001$ \\
\hline UR1 M & 2.39 & 2.08 & 0.22 & 90 & 10.90 & $<0.0001$ \\
\hline UL1 M & 2.55 & 2.25 & 0.24 & 90 & 10.72 & $<0.0001$ \\
\hline UL1 D & 2.29 & 2.20 & 0.23 & 90 & 9.88 & $<0.0001$ \\
\hline UL2 M & 1.62 & 1.98 & 0.21 & 90 & 7.77 & $<0.0001$ \\
\hline UL2 D & 1.62 & 1.88 & 0.20 & 90 & 8.19 & $<0.0001$ \\
\hline UL3 M & 2.57 & 1.51 & 0.16 & 90 & 16.17 & $<0.0001$ \\
\hline UL3 D & 2.90 & 1.45 & 0.15 & 90 & 18.93 & $<0.0001$ \\
\hline UL4 M & 1.76 & 1.49 & 0.23 & 40 & 7.48 & $<0.0001$ \\
\hline UL4 D & 1.86 & 1.51 & 0.24 & 40 & 7.79 & $<0.0001$ \\
\hline UL5 M & -4.26 & 1.30 & 0.18 & 50 & -23.187 & $<0.0001$ \\
\hline UL5 D & -3.95 & 1.44 & 0.20 & 50 & -19.44 & $<0.0001$ \\
\hline UL6 M & -4.27 & 1.60 & 0.17 & 90 & -25.336 & $<0.0001$ \\
\hline UL6 D & -4.05 & 1.59 & 0.17 & 90 & -24.19 & $<0.0001$ \\
\hline UL7 M & -4.20 & 1.71 & 0.19 & 84 & -22.515 & $<0.0001$ \\
\hline UL7 D & -3.43 & 1.78 & 0.19 & 84 & -17.616 & $<0.0001$ \\
\hline
\end{tabular}


Table 8. Descriptive statistics and one-sample tests for the change in the mesiodistal (Y-axis) arch dimension.

\begin{tabular}{lrrrrrr}
\hline Variable & Mean & sd & sem & $\mathrm{n}$ & t test & \multicolumn{1}{c}{$\mathrm{P}$} \\
\hline UR7 D & -0.15 & 1.48 & 0.16 & 84 & -0.92 & 0.3617 \\
UR7 M & -0.73 & 1.26 & 0.14 & 84 & -5.33 & $<0.0001$ \\
UR6 D & -0.68 & 1.27 & 0.13 & 90 & -5.06 & $<0.0001$ \\
UR6 M & -0.88 & 1.43 & 0.15 & 90 & -5.81 & $<0.0001$ \\
UR5 D & -1.13 & 1.53 & 0.22 & 50 & -5.24 & $<0.0001$ \\
UR5 M & -1.18 & 1.61 & 0.23 & 50 & -5.19 & $<0.0001$ \\
UR4 D & 1.15 & 1.16 & 0.18 & 40 & 6.27 & $<0.0001$ \\
UR4 M & 1.38 & 1.14 & 0.18 & 40 & 7.68 & $<0.0001$ \\
UR3 D & 0.11 & 1.36 & 0.14 & 90 & 0.77 & 0.4413 \\
UR3 M & 0.72 & 1.13 & 0.12 & 90 & 6.05 & $<0.0001$ \\
UR2 D & 1.12 & 1.25 & 0.13 & 90 & 8.53 & $<0.0001$ \\
UR2 M & 1.20 & 1.30 & 0.14 & 90 & 8.72 & $<0.0001$ \\
UR1 D & 0.37 & 0.94 & 0.10 & 90 & 3.75 & 0.0003 \\
UR1 M & 0.08 & 0.75 & 0.08 & 90 & 1.05 & 0.2960 \\
UL1 M & 0.01 & 0.67 & 0.07 & 90 & 0.20 & 0.8387 \\
UL1 D & -0.09 & 0.94 & 0.10 & 90 & -0.88 & 0.3811 \\
UL2 M & 0.62 & 1.45 & 0.15 & 90 & 4.06 & 0.0001 \\
UL2 D & 0.72 & 1.30 & 0.14 & 90 & 5.28 & $<0.0001$ \\
UL3 M & -0.07 & 1.32 & 0.14 & 90 & -0.51 & 0.6111 \\
UL3 D & -0.14 & 1.33 & 0.14 & 90 & -1.02 & 0.3096 \\
UL4 M & 0.99 & 1.50 & 0.24 & 40 & 4.18 & 0.0002 \\
UL4 D & 1.60 & 1.51 & 0.24 & 40 & 6.72 & $<0.0001$ \\
UL5 M & -1.77 & 1.50 & 0.21 & 50 & -8.37 & $<0.0001$ \\
UL5 D & -1.38 & 1.23 & 0.17 & 50 & -7.91 & $<0.0001$ \\
UL6 M & -1.44 & 1.27 & 0.13 & 90 & -10.83 & $<0.0001$ \\
UL6 D & -0.69 & 1.20 & 0.13 & 90 & -5.44 & $<0.0001$ \\
UL7 M & -0.82 & 1.25 & 0.14 & 84 & -6.06 & $<0.0001$ \\
UL7 D & -0.06 & 1.56 & 0.17 & 84 & -0.38 & 0.7076 \\
\hline
\end{tabular}




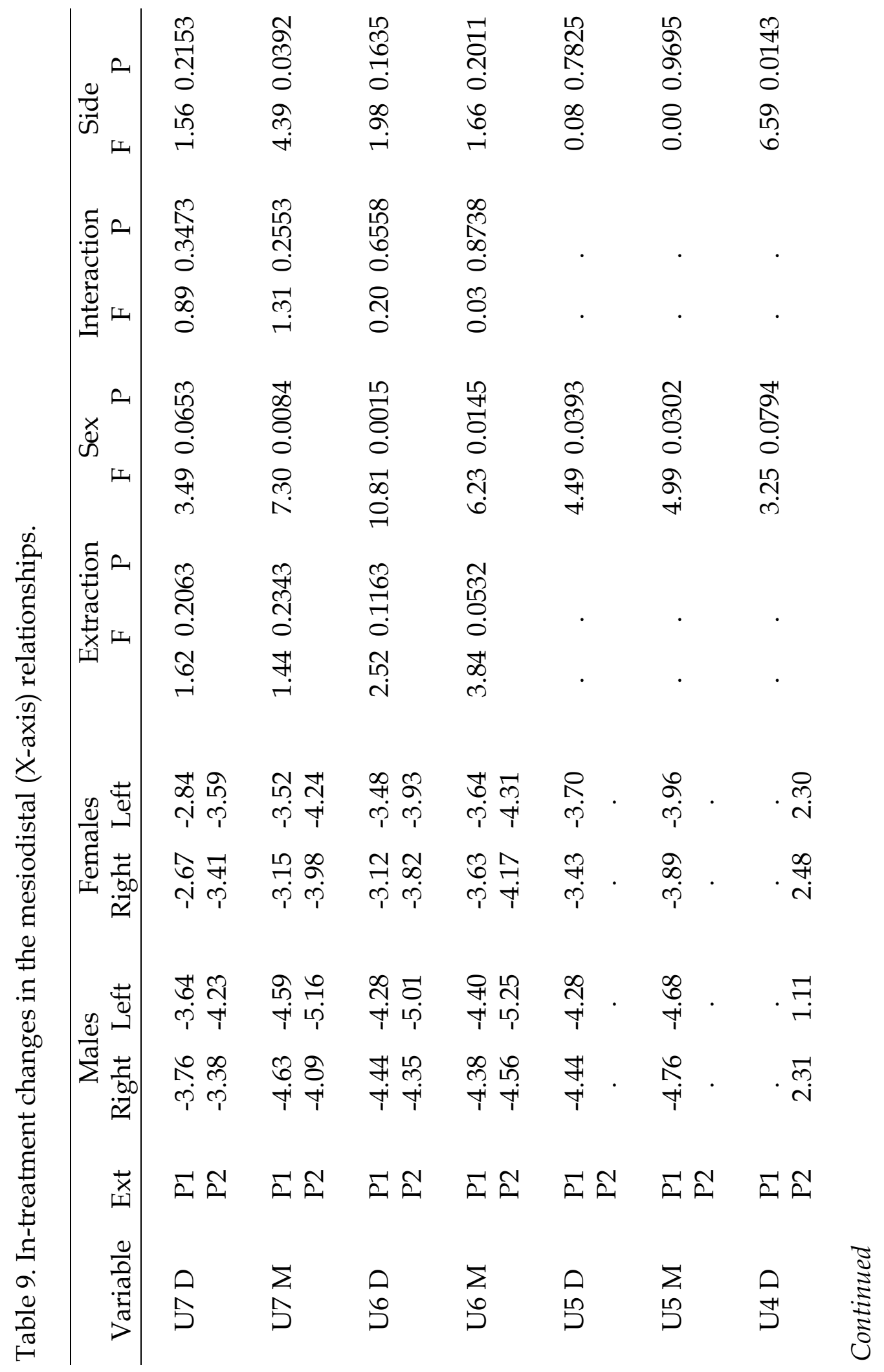




\begin{tabular}{|c|c|c|c|c|c|c|c|}
\hline $0^{f}$ & $\begin{array}{l}\text { సે } \\
\text { है } \\
0\end{array}$ & $\begin{array}{l}\sigma \\
8 \\
8 \\
0\end{array}$ & $\begin{array}{l}\infty \\
\infty \\
\infty \\
\infty \\
0\end{array}$ & 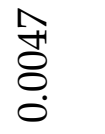 & $\begin{array}{l}\text { సै } \\
\text { హ్ }\end{array}$ & $\begin{array}{l}\stackrel{\circ}{\circ} \\
8 \\
0\end{array}$ & 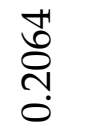 \\
\hline & बे & ڤึ & $\stackrel{H}{0}$ & $\underset{\infty}{\not}$ & ठ். & అై & $\underset{\sim}{\widetilde{\sigma}}$ \\
\hline . & . & $\begin{array}{l}\text { ळे } \\
\text { ڤై } \\
0\end{array}$ & $\begin{array}{l}0 \\
\frac{0}{0} \\
0 \\
0\end{array}$ & \begin{tabular}{l}
$\infty$ \\
\multirow{+}{0}{} \\
0
\end{tabular} & $\begin{array}{l}\infty \\
\stackrel{\infty}{\not} \\
\stackrel{+}{0}\end{array}$ & $\begin{array}{l}0 \\
\text { مी } \\
0\end{array}$ & $\begin{array}{l}\stackrel{9}{+} \\
\text { ठ․ }\end{array}$ \\
\hline 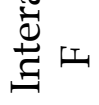 & 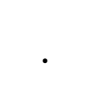 & ণิ & $\stackrel{8}{\circ}$ & $\begin{array}{l}\circ \\
\text { ஸे }\end{array}$ & $\begin{array}{l}\sigma \\
ٌ\end{array}$ & $\begin{array}{l}\infty \\
\infty \\
\infty\end{array}$ & $\begin{array}{l}\infty \\
\infty \\
\sim\end{array}$ \\
\hline$\stackrel{\leftrightarrow}{\infty}^{\sim}$ & $\begin{array}{c}\text { Y } \\
\text { On } \\
\stackrel{0}{0}\end{array}$ & 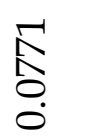 & $\begin{array}{l}N \\
\infty \\
0 \\
\swarrow \\
0 \\
0\end{array}$ & $\begin{array}{l}\text { ڤิ } \\
\text { กิ } \\
0\end{array}$ & \begin{tabular}{l} 
ڤ్ \\
\multirow{6}{0}{} \\
0
\end{tabular} & $\begin{array}{l}0 \\
10 \\
\text { กิ } \\
0\end{array}$ & 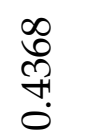 \\
\hline 工 & 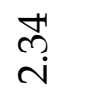 & $\begin{array}{l}\text { ते } \\
\text { n் }\end{array}$ & $\stackrel{m}{0}$ & $\stackrel{0}{\stackrel{0}{0}}$ & $\begin{array}{l}\text { ஸे } \\
\text { on }\end{array}$ & 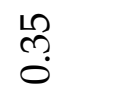 & రై \\
\hline 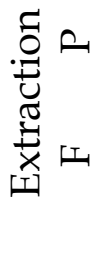 & . & 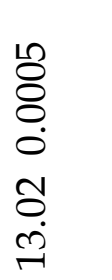 & $\begin{array}{l}\infty \\
\infty \\
5 \\
0 \\
0 \\
\infty \\
\infty \\
10\end{array}$ & 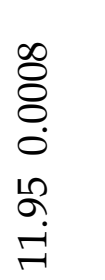 & 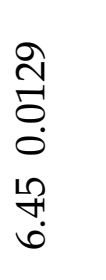 & 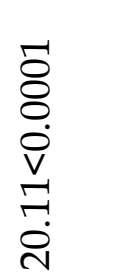 & 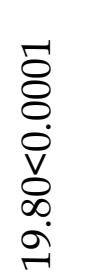 \\
\hline 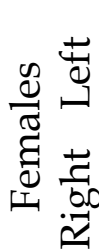 & 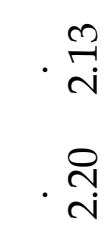 & 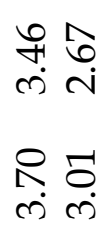 & 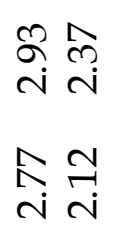 & $\begin{array}{l}\infty \\
\infty \\
\infty \\
\sim\end{array}$ & 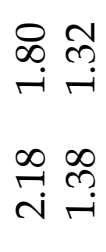 & 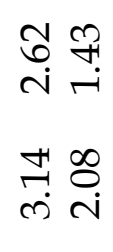 & 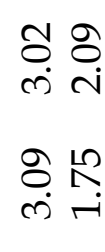 \\
\hline 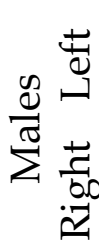 & $\cdot \stackrel{\rightleftarrows}{\rightleftarrows}$ & 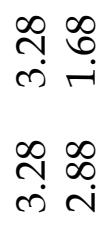 & 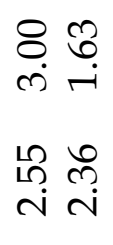 & 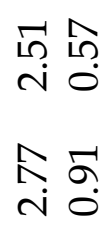 & 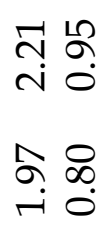 & 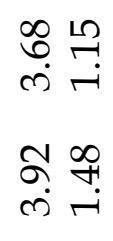 & 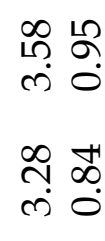 \\
\hline 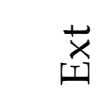 & $\Sigma \cong$ & $\Xi \cong$ & $\tilde{\epsilon} \cong$ & $\Phi \cong$ & $\Sigma \cong$ & $\Sigma \cong$ & $\bar{\sigma} \cong$ \\
\hline 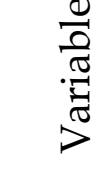 & $\begin{array}{l}\sum^{\prime} \\
\$\end{array}$ & $\stackrel{\oplus}{\oplus}$ & 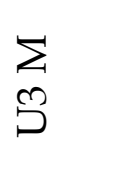 & $\stackrel{D}{\stackrel{D}{D}}$ & $\begin{array}{l}\sum \\
\stackrel{\Sigma}{D}\end{array}$ & $\begin{array}{l}\text { 口 } \\
\text { 与 }\end{array}$ & $\begin{array}{l}\Sigma \\
\vdots\end{array}$ \\
\hline
\end{tabular}


Table 9 also discloses several cases of sexual dimorphism, where the males changed during treatment, probably due to growth, more than the females. These instances are localized to the buccal segments, probably because of the combined effects of greater growth in the back of the palate and because the greater orthodontic correction of the anterior teeth (which is indifferent to the patient's sex).

In addition, five of the 14 ANOVA tests disclose a systematic side difference in the amounts of transverse change. Average transverse changes tend to be larger on the right side.

\section{Mesiodistal Changes}

Scanning the ANOVA results in Table 10 shows that there are few significant differences between the two extraction patterns or between the sexes. This does not mean that the landmarks did not move; instead, it shows the magnitudes of change are comparable in the two samples.

The left-right side differences are interesting. In the buccal segments, the significant side differences are due to greater mesiodistal changes that are greater on the patient's left side. But, in the anterior segment, teeth on the right side moved more (right $>$ left). 


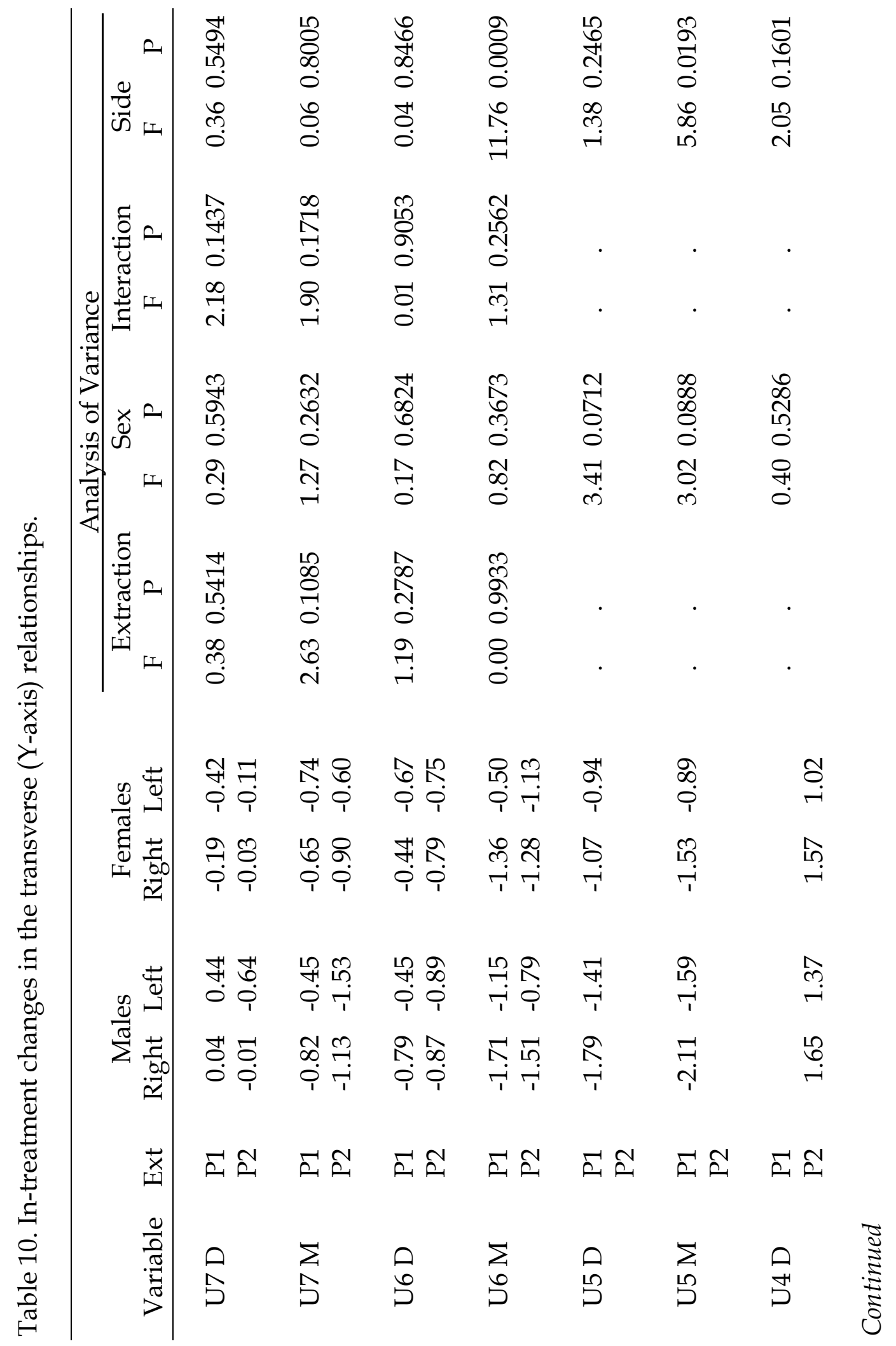




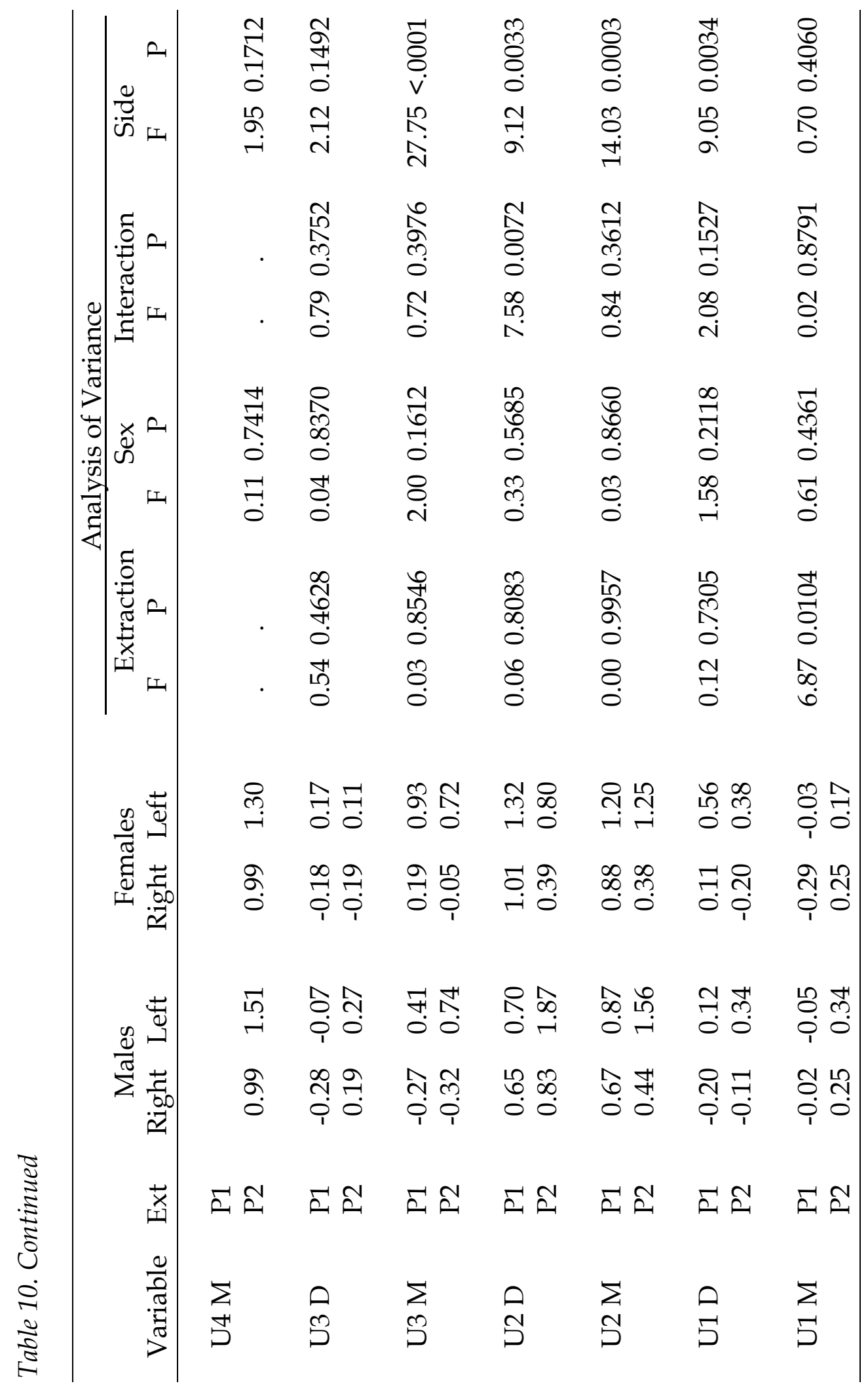




\section{$\underline{\text { Proxy Tooth Sizes }}$}

Conventionally, maximum mesiodistal crown diameters are obtained at the heights of contour (e.g., Moorrees 1957), but these points cannot be assessed on most teeth with the instrument used here. Instead, the occlusal landmarks closest to the anatomic contacts were used. This method provided crown diameters closely approximating those obtained by measuring at the height of contour. The straight-line distances between the mesial and distal coordinate of each tooth were calculated with the Pythagorean equation using the $X, Y$, and $Z$ axes. In most instances, our mesial and distal limits of each tooth type probably are a bit smaller than would be obtained on the casts with calipers. Still, the differences probably are small, and regardless, consistent criteria were used for size determination, so it is informative to assess the distributions of these "proxy" tooth diameters.

Based on a mixed-model ANOVA, crown sizes were tested for differences by extraction pattern, sex, pattern-by-sex interaction, and arcade (i.e., a repeated-measure test between homologous left and right dimensions). In actuality, the full mixed model for these tests was performed, but the ancillary interaction effects are omitted here to conserve space. (The three within-subject interaction effects omitted here are side- $x$-extraction, side- $x-$ sex, and side- $x$-extraction-x-sex.) 
Table 2 lists the results, and it is noteworthy that all of the mean sizes are larger in the first-premolar extraction sample than the second-premolar extraction sample. Most of the seven tests for first-premolar versus secondpremolar differences are significant statistically. Only the central incisor has a large P-value, and some of the F-ratios are fairly large (i.e., first-premolar, second-premolar, and first-molar). These statistical size differences seem to confirm orthodontists' tendency to extract the larger premolar in these Class I cases. Alternatively, these data support research by Adams (1982), Doris et al. (1981), and others that found that tooth size is in itself a risk factor for developing dentoalveolar malocclusions. The finding here that first-premolar extraction cases have bigger overjets is consistent with this supposition.

Analysis (Table 2) also discloses the characteristic tooth size sexual dimorphism seen in humans (Garn et al. 1967). Five of the seven tests show that males, on average, have significantly larger mesiodistal crown dimensions than females.

This inferential statistical model also discloses four cases where there is systematic directional asymmetry, where the left tooth dimension is larger than the homologue on the right (central incisor, later incisor, canine, and firstpremolar). Such examples of sidedness have never been reported from the human dentition (e.g., Ballard 1994; Doyle and Johnston 1977), though in fact, no one has previously looked at occlusal dimensions in this manner. Still, it is 
most parsimonious to attribute these small but systematic differences to operator errors (biases) in visualizing and locating landmarks on the corresponding left and right teeth. These differences are very small--on the order of 0.1 to $0.2 \mathrm{~mm}$ - which does not affect the other analyses in this study, especially since we control for side differences statistically.

We also combined some adjacent tooth dimensions (Table 2, bottom) to assess the effects of tooth segments. Four combinations were calculated, (1) the midarch segment (canine + first-premolar + second-premolar), (2) premolar segment (first-premolar + second-premolar), (3) molars (first-molar + second-molar), and (4) the buccal segment (first-premolar + secondpremolar + first-molar + second-molar). Of note, these "distances" were calculated as the distances from the mesial contact of the mesial tooth to the distal contact of the distal tooth in each segment, so the distances include the interproximal areas and, thus, are larger than if the individual tooth dimensions (top portion of Table 2) were summed.

All four arch segments are significantly larger in the first-premolar extraction sample and, as well, significantly larger in boys than girls. These results emphasize that orthodontists seem to be attuned to tooth sizes when treatment-planning a case in terms of which premolars to extract. More probably, the orthodontist evaluates the extent of crowding, incisor overjet, 
and canine relationships - and that promotes the decision of which pair of premolars to extract.

These data suggest that the sequence of events is (1) larger tooth sizes enhance malocclusions in the anterior segment and (2) the decision is made to alleviate the anterior problems by extracting first-premolars, which provide extraction spaces close to the sites of the problems (Graber 1972; Proffit and Fields 2000).

Predicting Tooth Movements from the Malocclusion

\section{Complete Sample}

This section addresses the central issue of how orthodontic tooth movement can be predicted in the statistical sense from evaluation of the pretreatment occlusion. Specifically, we looked at the mesiodistal changes of the dental landmarks, and evaluated which pretreatment occlusal conditions were significantly correlated with the amount of tooth movement. There were 17 occlusal measures to be used as predictor variables, along with the patient's sex and which left-right pair of premolars was extracted for treatment (Table 11). Multiple linear regression was used in the stepwise fashion to evaluate which subset of variables was predictive of the tooth's movement (Freund and Littell 1991). 
Table 11. Listing of the occlusal and demographic variables available for selection into the stepwise multiple linear regression model.

\begin{tabular}{l}
\hline \multicolumn{1}{c}{ Variable } \\
\hline Incisor Overjet ${ }^{1}$ \\
Incisor Overbite \\
Maxillary Incisor Irregularity \\
Maxillary Incisor Spacing \\
Maxillary 3-3 Width \\
Maxillary 6-6 Width \\
Mandibular 3-3 Width \\
Mandibular 6-6 Width \\
Mx-Md 3-3 Difference \\
Mx-Md 6-6 Difference \\
Maxillary 1-3 Chord* \\
Maxillary 1-6 Chord* \\
Mandibular 1-3 Chord* \\
Mandibular 1-6 Chord* \\
Midline Discrepancy \\
Buccal Segment Relation* \\
Canine Relation \\
\\
Extraction Pattern (P1 or P2) \\
Subject's Sex (Male or Female) \\
Ext-x-Sex Interaction \\
\hline
\end{tabular}

${ }^{1}$ The average of the left and right quadrants was used in the statistical analysis. 
The dental arch is basically symmetric around the midsagittal plane, so the homologous left and right dental landmarks should respond very similarly. The two sides were treated independently in the following paragraph in order to assess the consistency of the left and right changes. The changes are described from front to back in the dental arch. One generality to be seen is that more occlusal variables are significantly associated with the changes towards the front of the mouth. In other words, the distal tooth movements are predicted less well by the occlusal status of the patient.

Multiple linear regression was used in the stepwise mode, where the mesiodistal change in a dental landmark is the dependant variable and the 17 occlusal measurements (Table 11) are the predictor variables. Subject's sex also was included to account for sexual dimorphism in size, and extraction pattern (P1 or P2) was included for most of the models. "Extraction" is invariant for the four premolar landmarks because one tooth or the other was removed, so "extraction" is necessarily deleted from the model in these instances.

In brief, the stepwise procedure begins with calculating the univariate dependency between each predictor variable and the outcome variable. These are expressed as F-ratios. Step one in the model-building process is to select that variable with the largest F-ratio. Then the computer program recalculates the F-ratio for all of the variables accounting for the statistical variance 
explained by the selected variable. Step two selects the variable with the largest F-ratio (assuming one or more variables has a F-ratio with a P-value less than 0.05). These steps are repeated until none of the remaining variables has a significant F-ratio, so none contributes significantly more to accounting for the variance in the outcome variable. The procedure was used with the options for forward and backward stepping. Full details of the modelbuilding procedure are provided in Freund and Littell (1991).

Importantly, results discussed in the following sections are based on the total sample (first- and second-premolar extraction cases). Subsequent sections then describe analysis of each of the two extraction patterns individually.

\section{$\underline{\text { Incisors }}$}

Tables 12 and 13 list the results for the mesial contact landmarks of the left and right central incisor, respectively. Four variables entered the model for the left incisor and six variables for the right. Of these two analyses, there are three variables in common, namely maxillary 1-3 chord, maxillary 3-3 width, and incisor spacing. The maxillary 1-3 chord distance is predictive in that the longer the chord at the pretreatment examination, the greater the intreatment reduction (Fig. 34). This is anticipated since the 1-3 chord distance is sensitive to labioversion and proclination of the maxillary incisors 
Table 12. Results of stepwise multiple regression analysis with the intreatment change in the maxillary left central incisor (mesial) as the dependent variable. ${ }^{1}$

\begin{tabular}{lrrr}
\hline \multicolumn{1}{c}{ Variable } & F Ratio & P Value & Model \\
\hline Incisor Overjet & 3.14 & 0.0802 & \\
Incisor Overbite & 0.05 & 0.8247 & \\
Incisor Irregularity & 0.82 & 0.3678 \\
Incisor Spacing & 4.05 & 0.0474 \\
Max 3-3 Width & 16.16 & 0.0001 \\
Max 6-6 Width & 0.18 & 0.6759 \\
Mand 3-3 Width & 0.10 & 0.7517 \\
Mand 6-6 Width & 0.13 & 0.7249 \\
Mx-Md 3-3 Difference & 0.10 & 0.7517 \\
Mx-Md 6-6 Difference & 0.03 & 0.8746 \\
Mx 1-3 Chord & 22.39 & 0.0000 \\
Mx 1-6 Chord & 0.12 & 0.7334 \\
Md 1-3 Chord & 0.81 & 0.3722 \\
Md 1-6 Chord & 1.13 & 0.2918 \\
Midline Discrepancy & 3.66 & 0.0592 \\
Buccal Segment Relation & 0.32 & 0.5706 \\
Canine Relation & 4.78 & 0.0317 \\
Extraction Pattern & 4.23 & 0.0430 \\
Subject's Sex & 4.09 & 0.0465 \\
Ext-x-Sex Interaction & 2.72 & 0.0503 &
\end{tabular}

1Step history:

$\begin{array}{clc}\text { Step } & \text { Parameter } & \text { R-Squared } \\ 1 & \text { Mx 1-3 Chord } & 0.1636 \\ 2 & \text { Max 3-3 Width } & 0.2734 \\ 3 & \text { Canine Relation } & 0.3175 \\ 4 & \text { Incisor Spacing } & 0.3497\end{array}$


Table 13. Results of stepwise multiple regression analysis with the intreatment change in the maxillary right central incisor (mesial) as the dependent variable. ${ }^{1}$

\begin{tabular}{lccc}
\hline \multicolumn{1}{c}{ Variable } & F Ratio & P Value & Model \\
\hline Incisor Overjet & 5.45 & 0.0221 & Entered \\
Incisor Overbite & 0.09 & 0.7678 & \\
Incisor Irregularity & 0.89 & 0.3489 & \\
Incisor Spacing & 16.63 & 0.0001 & Entered \\
Max 3-3 Width & 20.88 & 0.0000 & Entered \\
Max 6-6 Width & 0.01 & 0.9284 & \\
Mand 3-3 Width & 0.14 & 0.7139 & \\
Mand 6-6 Width & 0.04 & 0.8400 & \\
Mx-Md 3-3 Difference & 0.14 & 0.7139 & \\
Mx-Md 6-6 Difference & 0.01 & 0.9053 & \\
Mx 1-3 Chord & 8.35 & 0.0050 & Entered \\
Mx 1-6 Chord & 0.00 & 0.9484 & \\
Md 1-3 Chord & 1.33 & 0.2517 & \\
Md 1-6 Chord & 0.47 & 0.4970 & \\
Midline Discrepancy & 0.88 & 0.3504 & \\
Buccal Segment Relation & 1.07 & 0.3033 & \\
Canine Relation & 2.31 & 0.1327 & \\
Extraction Pattern & 7.90 & 0.0062 & Entered \\
Subject's Sex & 6.69 & 0.0115 & Entered \\
Ext-x-Sex Interaction & 0.26 & 0.6100 & \\
\hline
\end{tabular}

1Step history:

$\begin{array}{clc}\text { Step } & \text { Parameter } & \text { R-Squared } \\ 1 & \text { Extraction Pattern } & 0.1775 \\ 2 & \text { Incisor Overjet } & 0.2910 \\ 3 & \text { Incisor Spacing } & 0.4042 \\ 4 & \text { Max 3-3 Width } & 0.4798 \\ 5 & \text { Mx 1-3 Chord } & 0.5079 \\ 6 & \text { Subject's Sex } & 0.5458\end{array}$




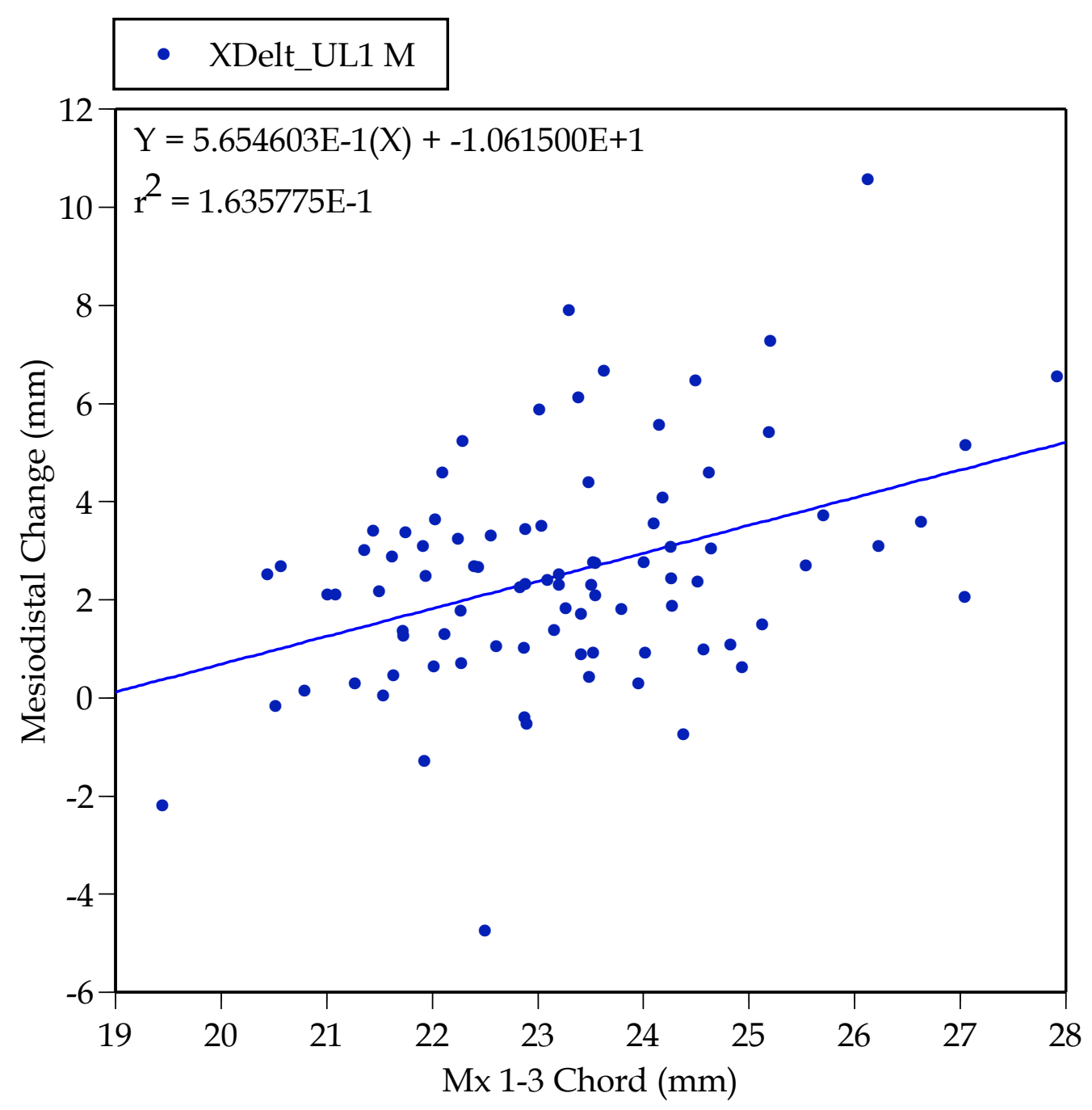

Fig. 34. Scatterplot showing the positive association between pretreatment arch chord (1-3) and the amount of retraction of the maxillary left central incisor (mesial). 
and, thus, indirectly of the amount of overjet. Fig. A-25 in the Appendix shows an extreme example of a long 1-3 chord distance treated with appreciable incisor retraction.

Maxillary 3-3 width entered the equation (Tables 12, 13) because those cases with large widths exhibited bucally "blocked-out" canines (buccoversion) treated with greater retraction of the anterior segment. The univariate association is, however, weak.

The influence of canine relationship is intuitive: the greater the Class II discrepancy of the canine, the greater the in-treatment retraction of the central incisors (Fig. 35).

Only a few of the cases exhibited inter-dental spacing of the incisors (Fig. 36), but these experienced greater amounts of incisor retraction, evidently to resolve the spacing issue.

The predictive models for the distal landmarks of the central incisors (Tables 14,15$)$ are primarily influenced by two occlusal variables, maxillary 16 chord and the extraction pattern. These two predictors account for about $30 \%$ of the variance in the mesiodistal tooth movement of the distal landmark. The relationship with arch chord (Fig. 37) is that cases with longer chords undergo more incisor retraction during treatment. As for the extraction pattern, those with first-premolars removed experienced greater incisor retraction $(\bar{x}=1.3 \mathrm{~mm})$ than in the sample in whom the second-premolars had 


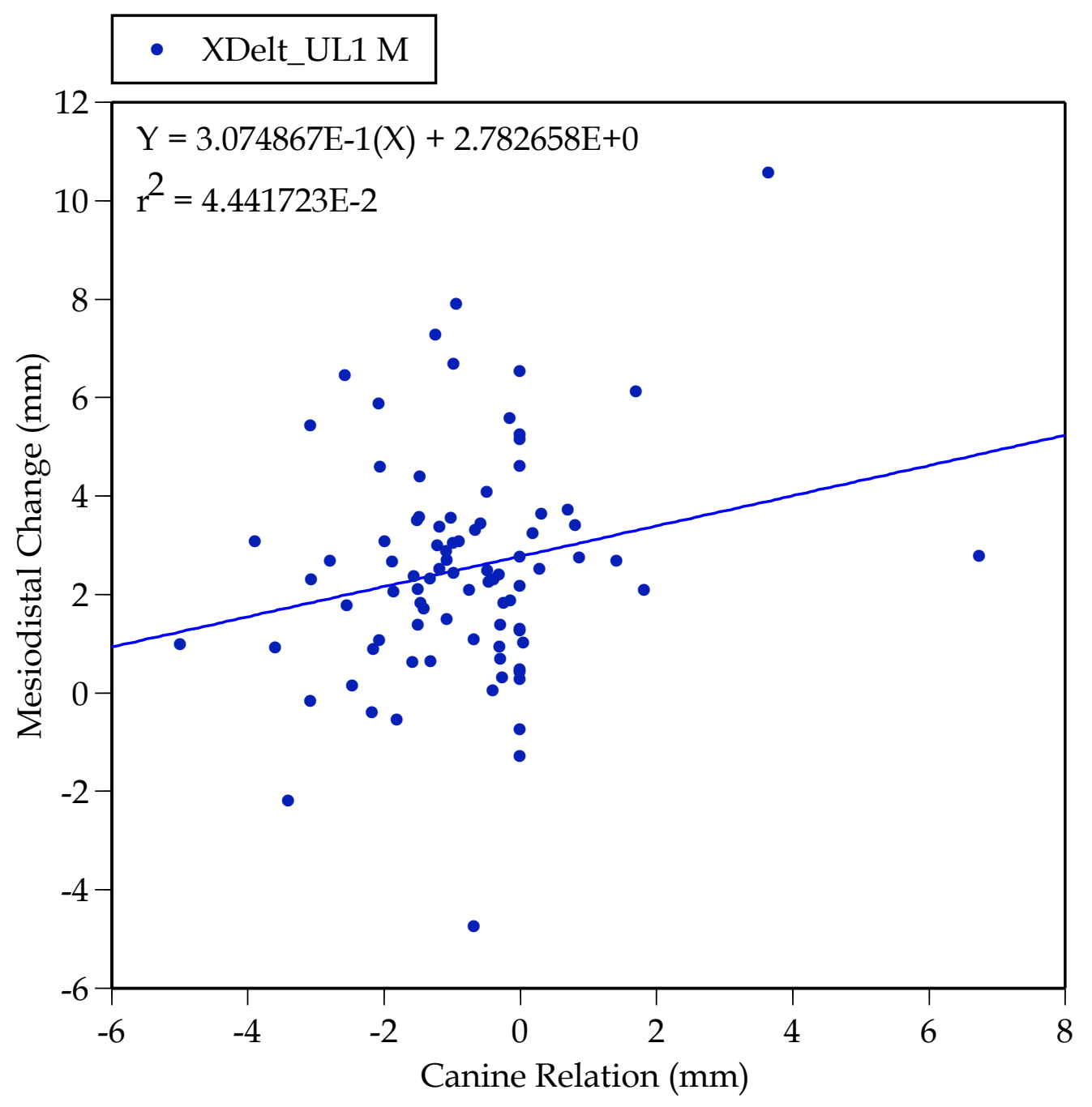

Fig. 35. Scatterplot showing the positive association between pretreatment canine relationship and the amount of retraction of the maxillary left central incisor (mesial). 


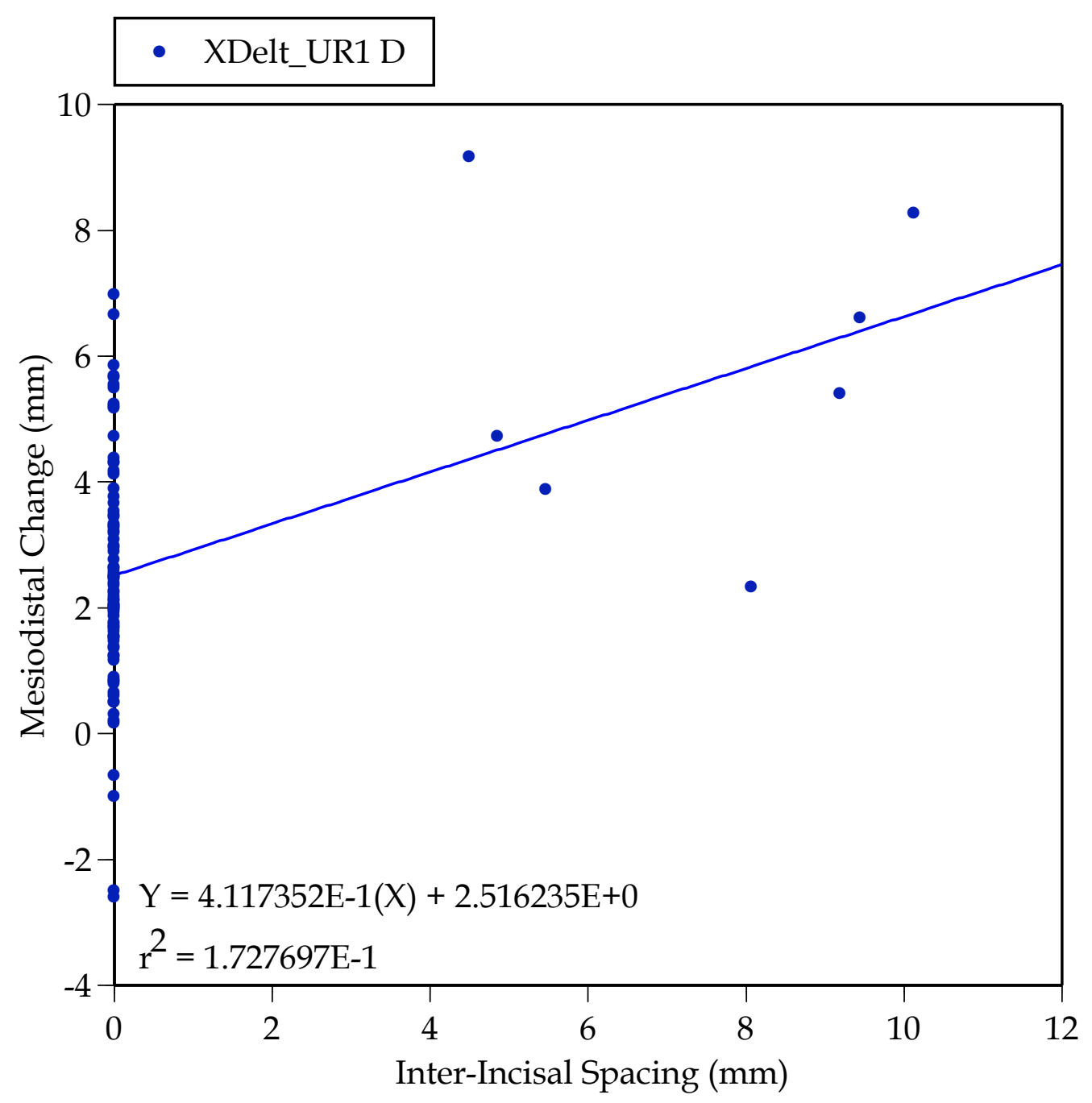

Fig. 36. Scatterplot showing the positive association between pretreatment interdental spacing and the amount of retraction of the maxillary left central incisor (mesial). 
Table 14. Results of stepwise multiple regression analysis with the intreatment change in the maxillary left central incisor (distal) as the dependent variable. ${ }^{1}$

\begin{tabular}{lccc}
\hline \multicolumn{1}{c}{ Variable } & F Ratio & P Value & Model \\
\hline Incisor Overjet & 0.01 & 0.9416 & \\
Incisor Overbite & 0.22 & 0.6433 \\
Incisor Irregularity & 5.78 & 0.0185 \\
Incisor Spacing & 1.96 & 0.1657 \\
Max 3-3 Width & 7.29 & 0.0084 \\
Max 6-6 Width & 0.73 & 0.3947 \\
Mand 3-3 Width & 0.07 & 0.7882 \\
Mand 6-6 Width & 0.03 & 0.8620 \\
Mx-Md 3-3 Difference & 0.07 & 0.7882 \\
Mx-Md 6-6 Difference & 0.79 & 0.3781 \\
Mx 1-3 Chord & 0.15 & 0.6981 \\
Mx 1-6 Chord & 12.71 & 0.0006 \\
Md 1-3 Chord & 0.94 & 0.3362 \\
Md 1-6 Chord & 1.98 & 0.1635 \\
Midline Discrepancy & 4.87 & 0.0301 \\
Buccal Segment Relation & 0.60 & 0.4426 \\
Canine Relation & 0.63 & 0.4295 \\
Extraction Pattern & 8.31 & 0.0050 \\
Subject's Sex & 0.24 & 0.6279 \\
Ext-x-Sex Interaction & 0.27 & 0.7662 & \\
\hline
\end{tabular}

1Step history:

$\begin{array}{clc}\text { Step } & \text { Parameter } & \text { R-Squared } \\ 1 & \text { Mx 1-6 Chord } & 0.2219 \\ 2 & \text { Extraction Pattern } & 0.3041 \\ 3 & \text { Max 3-3 Width } & 0.3493 \\ 4 & \text { Incisor Irregularity } & 0.3952 \\ 5 & \text { Midline Discrepancy } & 0.4296\end{array}$


Table 15. Results of stepwise multiple regression analysis with the intreatment change in the maxillary right central incisor (distal) as the dependent variable. ${ }^{1}$

\begin{tabular}{lrrl}
\hline \multicolumn{1}{c}{ Variable } & F Ratio & P Value & Model \\
\hline Incisor Overjet & 0.75 & 0.3894 & \\
Incisor Overbite & 0.71 & 0.4010 & \\
Incisor Irregularity & 0.63 & 0.4282 & \\
Incisor Spacing & 10.97 & 0.0014 & Entered \\
Max 3-3 Width & 0.57 & 0.4528 & \\
Max 6-6 Width & 0.43 & 0.5117 & \\
Mand 3-3 Width & 4.04 & 0.0478 & Entered \\
Mand 6-6 Width & 0.00 & 0.9561 & \\
Mx-Md 3-3 Difference & 0.57 & 0.4528 & \\
Mx-Md 6-6 Difference & 0.81 & 0.3719 & \\
Mx 1-3 Chord & 1.17 & 0.2836 & \\
Mx 1-6 Chord & 10.02 & 0.0022 & Entered \\
Md 1-3 Chord & 2.66 & 0.1071 & \\
Md 1-6 Chord & 0.37 & 0.5474 & \\
Midline Discrepancy & 1.22 & 0.2733 & \\
Buccal Segment Relation & 0.33 & 0.5659 & \\
Canine Relation & 2.62 & 0.1094 & \\
Extraction Pattern & 10.53 & 0.0017 & Entered \\
Subject's Sex & 1.85 & 0.1772 & \\
Ext-x-Sex Interaction & 1.11 & 0.3335 & \\
\hline
\end{tabular}

1Step history:

\begin{tabular}{clc} 
Step & \multicolumn{1}{c}{ Parameter } & R-Squared \\
1 & Mx 1-6 Chord & 0.2003 \\
2 & Extraction Pattern & 0.2824 \\
3 & Incisor Spacing & 0.3433 \\
4 & Mand 3-3 Width & 0.3741
\end{tabular}




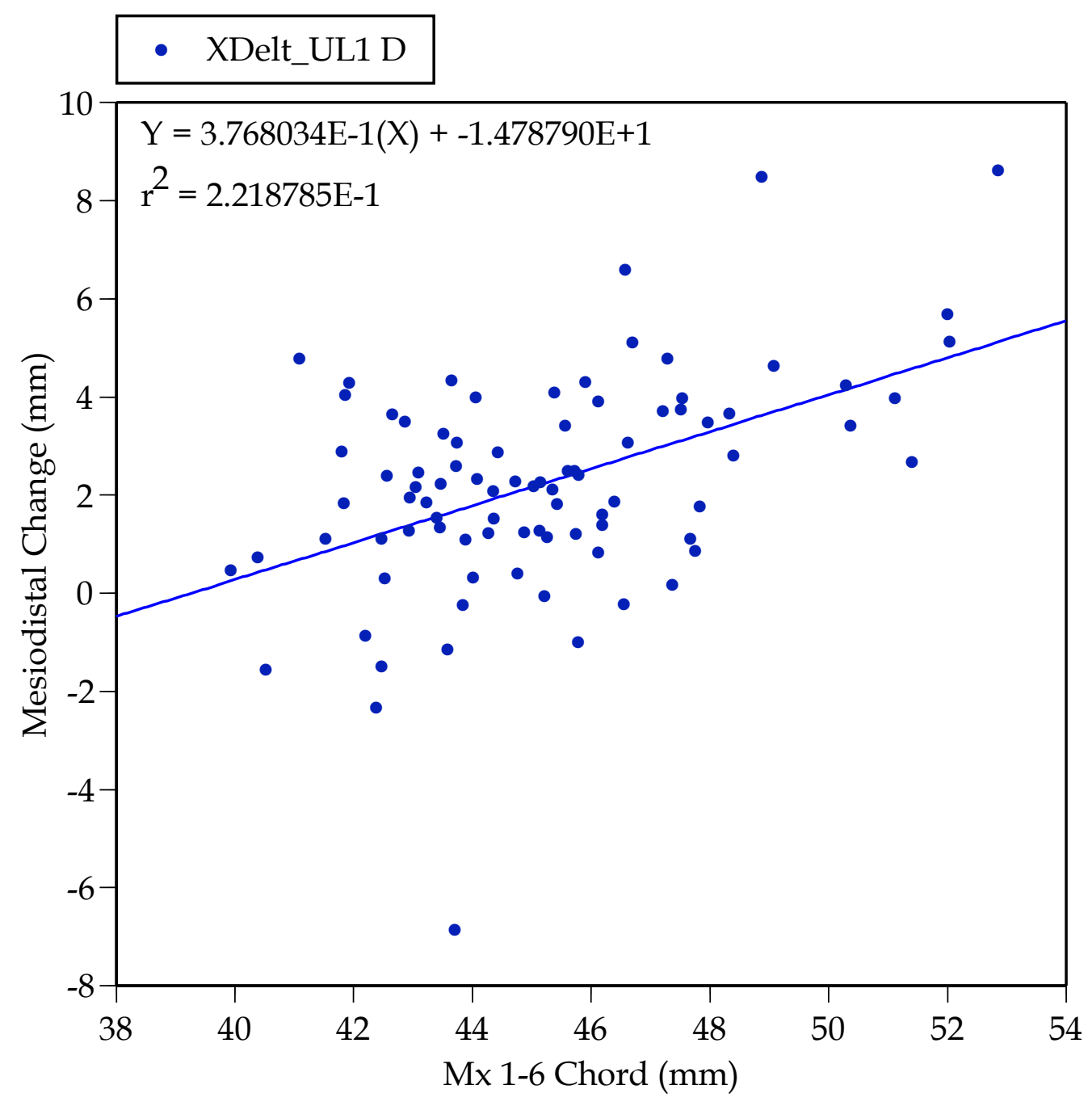

Fig. 37. Scatterplot showing the positive association between pretreatment maxillary arch chord (1-6) and the amount of retraction of the maxillary left central incisor (distal). 
been removed. By F-ratio, this univariate difference is highly significant statistically $(\mathrm{F}=16.4 ; \mathrm{P}<0.0001)$. Movement of the mesial contact of the maxillary lateral incisor (Tables 16,17 ) is best predicted by the amount of maxillary incisor irregularity (Fig. 38). With low levels of irregularity the trend was for this lateral incisor landmark to be retracted back into the arch form. With severe irregularity, the lateral incisors tended to be moved mesially (labially), often because the lateral incisors were well lingual of the central incisors in such cases. The plot in Fig. A-33 is an example of this latter situation.

Overbite also has a significant influence in combination with other variables (Table 17), but the effect is only slight when viewed univariately (Fig. 39). It appears that cases with deeper bites experience retraction of the lateral incisors, while those with shallow bites undergo labial incisor repositioning.

This association with overbite is more evident for the distal contact of the lateral incisor (Tables 18, 19), where it accounts for $17 \%$ of the variance for the lateral incisor on the left (though just $8 \%$ on the right side). The association is positive (Fig. 40): Cases with deep bites experience more incisor retraction than those with shallow bites. For example, the case in Fig. A-36 had a very deep bite $(10.3 \mathrm{~mm})$, and this lateral incisor landmark was retracted about $5 \mathrm{~mm}$. At the other extreme, Fig. A-20 shows a case with an anterior 
Table 16. Results of stepwise multiple regression analysis with the intreatment change in the maxillary left lateral incisor (mesial) as the dependent variable. ${ }^{1}$

\begin{tabular}{lrrr}
\hline \multicolumn{1}{c}{ Variable } & F Ratio & P Value & Model \\
\hline Incisor Overjet & 0.68 & 0.4121 \\
Incisor Overbite & 2.63 & 0.1086 \\
Incisor Irregularity & 14.49 & 0.0003 \\
Incisor Spacing & 0.00 & 0.9610 \\
Max 3-3 Width & 2.36 & 0.1285 \\
Max 6-6 Width & 0.56 & 0.4567 \\
Mand 3-3 Width & 1.04 & 0.3108 \\
Mand 6-6 Width & 0.03 & 0.8722 \\
Mx-Md 3-3 Difference & 0.89 & 0.3471 \\
Mx-Md 6-6 Difference & 1.10 & 0.2977 \\
Mx 1-3 Chord & 0.06 & 0.8006 \\
Mx 1-6 Chord & 1.66 & 0.2013 \\
Md 1-3 Chord & 0.06 & 0.8057 \\
Md 1-6 Chord & 0.32 & 0.5743 \\
Midline Discrepancy & 7.92 & 0.0061 \\
Buccal Segment Relation & 0.00 & 0.9583 \\
Canine Relation & 3.20 & 0.0771 \\
Extraction Pattern & 1.23 & 0.2712 \\
Subject's Sex & 0.07 & 0.7955 \\
Ext-x-Sex Interaction & 0.42 & 0.7390 \\
\hline
\end{tabular}

1Step history:

\begin{tabular}{clc} 
Step & \multicolumn{1}{c}{ Parameter } & R-Squared \\
1 & Incisor Irregularity & 0.1672 \\
2 & Midline Discrepancy & 0.2390
\end{tabular}


Table 17. Results of stepwise multiple regression analysis with the intreatment change in the maxillary right lateral incisor (mesial) as the dependent variable. ${ }^{1}$

\begin{tabular}{lccc}
\hline \multicolumn{1}{c}{ Variable } & F Ratio & P Value & Model \\
\hline Incisor Overjet & 0.025 & 0.8744 & \\
Incisor Overbite & 18.78 & 0.0000 & Entered \\
Incisor Irregularity & 19.407 & 0.0000 & Entered \\
Incisor Spacing & 3.451 & 0.0669 & \\
Max 3-3 Width & 0.035 & 0.8527 & \\
Max 6-6 Width & 1.365 & 0.2462 & \\
Mand 3-3 Width & 0.87 & 0.3537 & \\
Mand 6-6 Width & 1.272 & 0.2627 & \\
Mx-Md 3-3 Difference & 1.263 & 0.2645 & \\
Mx-Md 6-6 Difference & 0.166 & 0.6847 & \\
Mx 1-3 Chord & 0.145 & 0.7040 & \\
Mx 1-6 Chord & 0.905 & 0.3444 & \\
Md 1-3 Chord & 1.287 & 0.2599 & \\
Md 1-6 Chord & 1.218 & 0.2731 & \\
Midline Discrepancy & 0.01 & 0.9200 & \\
Buccal Segment Relation & 0.12 & 0.7252 & \\
Canine Relation & 1.13 & 0.2905 & \\
Extraction Pattern & 7.69 & 0.0069 & Entered \\
Subject's Sex & 5.52 & 0.0212 & Entered \\
Ext-x-Sex Interaction & 0.92 & 0.3394 & \\
\hline
\end{tabular}

1Step history:

$\begin{array}{clc}\text { Step } & \text { Parameter } & \text { R-Squared } \\ 1 & \text { Incisor Irregularity } & 0.1563 \\ 2 & \text { Incisor Overbite } & 0.2627 \\ 3 & \text { Sex } & 0.3216\end{array}$




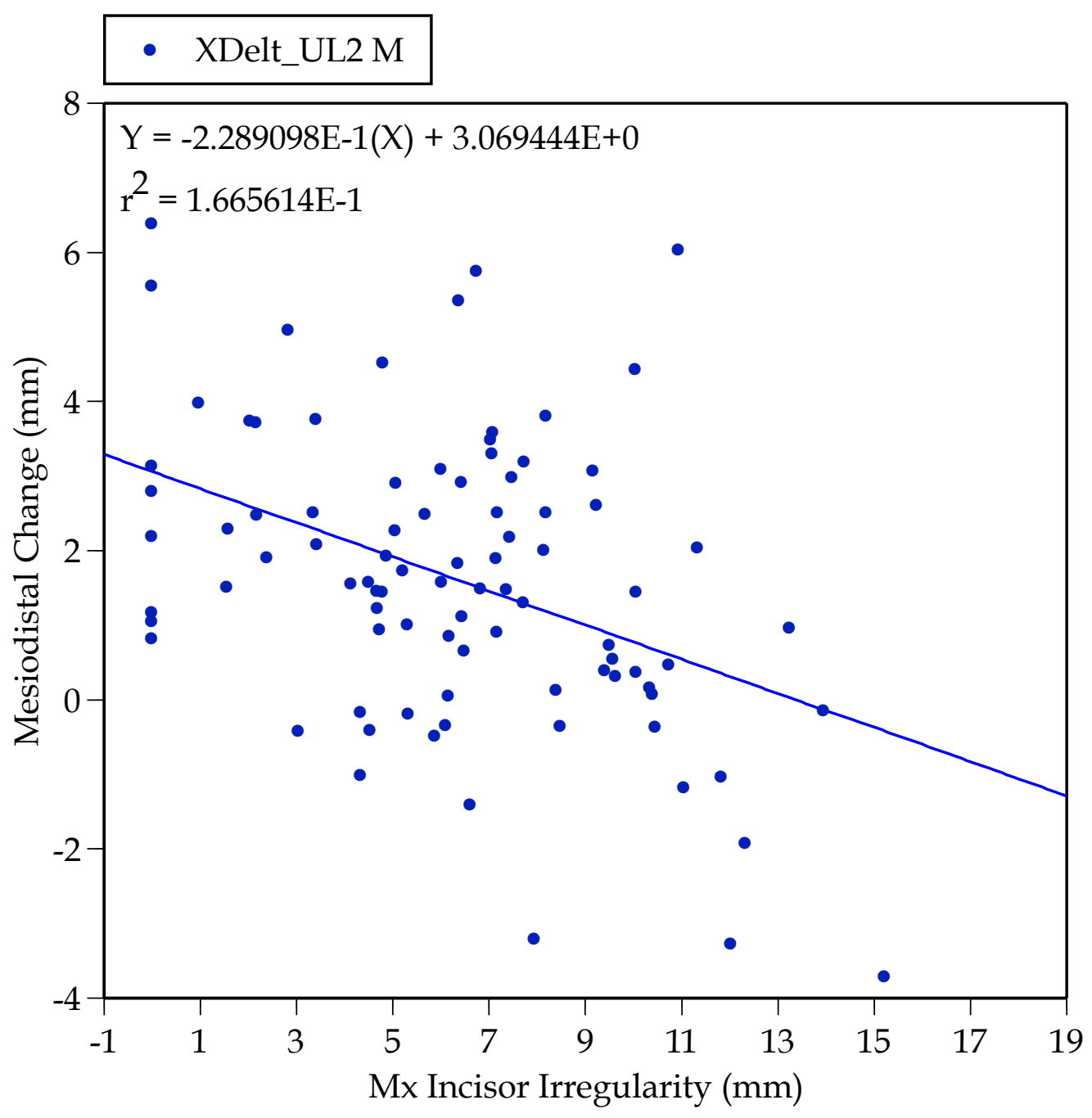

Fig. 38. Scatterplot showing the negative association between pretreatment incisor irregularity and the amount of retraction of the maxillary left lateral incisor (mesial). 


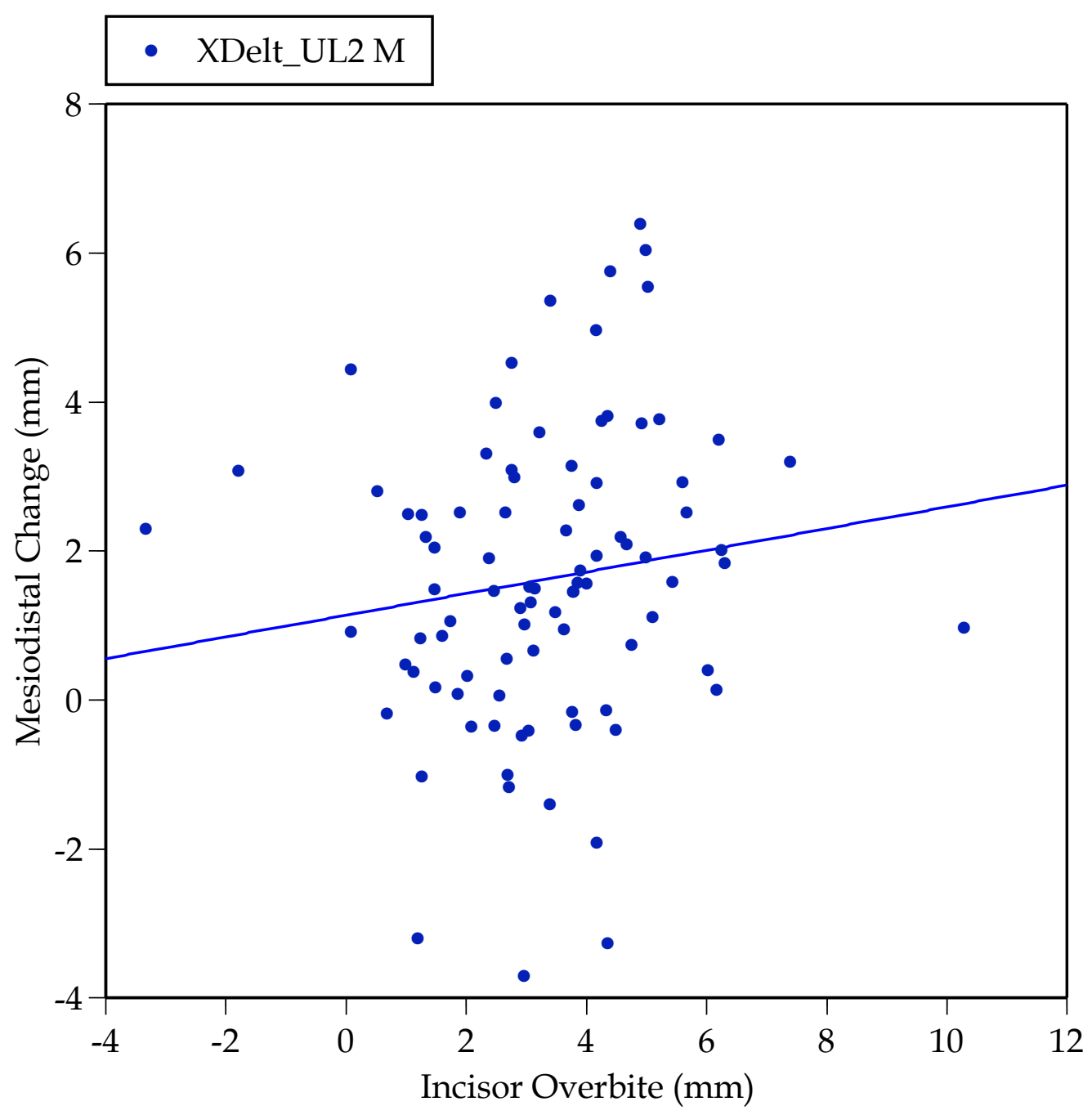

Fig. 39. Scatterplot showing the positive association between pretreatment overbite and mesiodistal change of the maxillary left lateral incisor (mesial). 
Table 18. Results of stepwise multiple regression analysis with the intreatment change in the maxillary left lateral incisor (distal) as the dependent variable. ${ }^{1}$

\begin{tabular}{lccc}
\hline \multicolumn{1}{c}{ Variable } & F Ratio & P Value & Model \\
\hline Incisor Overjet & 0.00 & 0.9747 \\
Incisor Overbite & 8.65 & 0.0043 \\
Incisor Irregularity & 1.83 & 0.1795 \\
Incisor Spacing & 0.00 & 0.9816 \\
Max 3-3 Width & 1.37 & 0.2449 \\
Max 6-6 Width & 0.13 & 0.7178 \\
Mand 3-3 Width & 0.01 & 0.9329 \\
Mand 6-6 Width & 0.26 & 0.6131 \\
Mx-Md 3-3 Difference & 1.66 & 0.2008 \\
Mx-Md 6-6 Difference & 0.03 & 0.8673 \\
Mx 1-3 Chord & 0.54 & 0.4667 \\
Mx 1-6 Chord & 8.99 & 0.0036 \\
Md 1-3 Chord & 0.52 & 0.4711 \\
Md 1-6 Chord & 0.19 & 0.6623 \\
Midline Discrepancy & 4.80 & 0.0314 \\
Buccal Segment Relation & 0.00 & 0.9634 \\
Canine Relation & 5.86 & 0.0177 \\
Extraction Pattern & 8.05 & 0.0057 \\
Subject's Sex & 1.27 & 0.2624 \\
Ext-x-Sex Interaction & 0.76 & 0.4701 &
\end{tabular}

1Step history:

$\begin{array}{clc}\text { Step } & \text { Parameter } & \text { R-Squared } \\ 1 & \text { Mx 1-6 Chord } & 0.1270 \\ 2 & \text { Extraction Pattern } & 0.1851 \\ 3 & \text { Incisor Overbite } & 0.2488 \\ 4 & \text { Canine Relation } & 0.2989\end{array}$


Table 19. Results of stepwise multiple regression analysis with the intreatment change in the maxillary right lateral incisor (distal) as the dependent variable. ${ }^{1}$

\begin{tabular}{lrrl}
\hline \multicolumn{1}{c}{ Variable } & F Ratio & P Value & Model \\
\hline Incisor Overjet & 0.25 & 0.6184 & \\
Incisor Overbite & 33.90 & 0.0000 & Entered \\
Incisor Irregularity & 6.60 & 0.0120 & Entered \\
Incisor Spacing & 1.29 & 0.2590 & \\
Max 3-3 Width & 0.15 & 0.7034 & \\
Max 6-6 Width & 1.37 & 0.2461 & \\
Mand 3-3 Width & 1.44 & 0.2343 & \\
Mand 6-6 Width & 0.55 & 0.4618 & \\
Mx-Md 3-3 Difference & 0.38 & 0.5386 & \\
Mx-Md 6-6 Difference & 0.93 & 0.3368 & \\
Mx 1-3 Chord & 0.61 & 0.4382 & \\
Mx 1-6 Chord & 1.79 & 0.1846 & \\
Md 1-3 Chord & 1.06 & 0.3066 & \\
Md 1-6 Chord & 2.27 & 0.1359 & \\
Midline Discrepancy & 0.44 & 0.5090 & \\
Buccal Segment Relation & 0.14 & 0.7135 & \\
Canine Relation & 6.05 & 0.0160 & Entered \\
Extraction Pattern & 9.43 & 0.0029 & Entered \\
Subject's Sex & 4.08 & 0.0467 & Entered \\
Ext-x-Sex Interaction & 0.32 & 0.5719 & \\
\hline
\end{tabular}

1Step history:

$\begin{array}{clc}\text { Step } & \text { Parameter } & \text { R-Squared } \\ 1 & \text { Incisor Overbite } & 0.1740 \\ 2 & \text { Extraction Pattern } & 0.2690 \\ 3 & \text { Canine Relation } & 0.3440 \\ 4 & \text { Incisor Irregularity } & 0.3813 \\ 5 & \text { Subject's Sex } & 0.4110\end{array}$




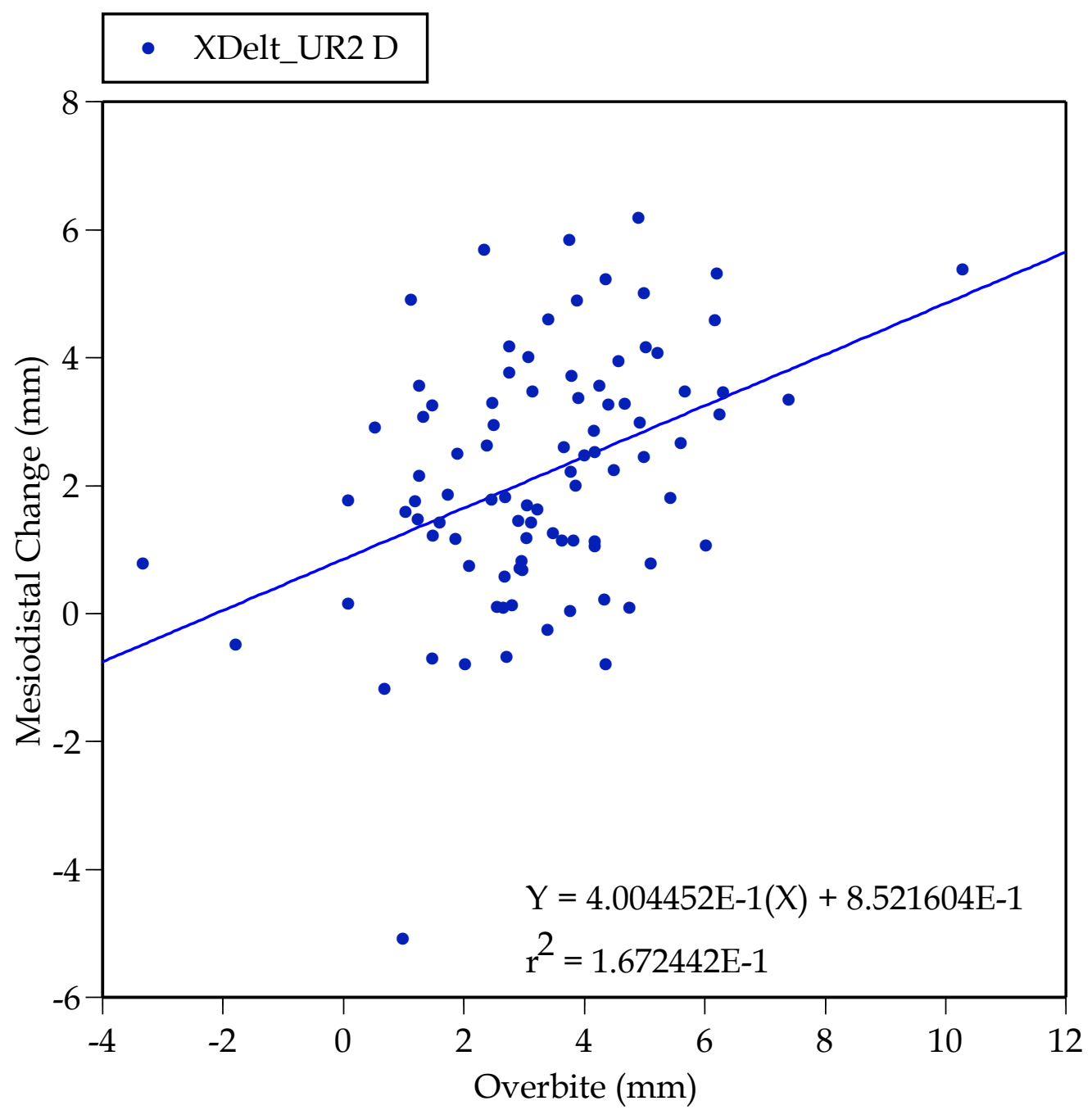

Fig. 40. Scatterplot showing the positive association between pretreatment overbite and the amount of retraction of the maxillary left lateral incisor (distal). 
openbite $(-3.3 \mathrm{~mm})$, and the lateral incisors were moved almost not at all during treatment.

Extraction pattern also significantly influenced the mesiodistal movement of the mesial aspect of the lateral incisor (Tables 18, 19). The variance accounted for by the right tooth is higher, and this landmark was retracted a mean of $2.1 \mathrm{~mm}$ in the first-premolar group, but moved labially an average of $1.2 \mathrm{~mm}$ in the second-premolar group, a difference that is significant by univariate $\mathrm{F}-$ ratio $(\mathrm{F}=6.3, \mathrm{P}=0.0137)$.

\section{$\underline{\text { Canine }}$}

The four anatomic contacts on the canine are dealt with together (Tables 20 through 23) because there is little consistency in the occlusal variables entered in each model and none of the variables has a particularly high associated $\mathrm{r}^{2}$. Two variables do recur among the four statistical analyses, namely extraction pattern and incisor irregularity. Extraction pattern gained the largest variance for the distal contact of the left tooth (Fig. 41) where mean retraction was $3.4 \mathrm{~mm}$ in the first-premolar sample, which is significantly greater than the $2.3 \mathrm{~mm}$ observed in the second-premolar sample $(\mathrm{F}=14.2 ; \mathrm{P}$ $=0.0003)$.

Fig. 42 shows the relationship between incisor irregularity and the amount of mesiodistal change in canine position. The greater the 
Table 20. Results of stepwise multiple regression analysis with the intreatment change in the maxillary left canine (mesial) as the dependent variable. ${ }^{1}$

\begin{tabular}{lccc}
\hline \multicolumn{1}{c}{ Variable } & F Ratio & P Value & Model \\
\hline Incisor Overjet & 0.10 & 0.7529 & \\
Incisor Overbite & 8.03 & 0.0058 \\
Incisor Irregularity & 1.07 & 0.3045 & \\
Incisor Spacing & 0.03 & 0.8724 \\
Max 3-3 Width & 0.01 & 0.9138 \\
Max 6-6 Width & 3.82 & 0.0540 \\
Mand 3-3 Width & 1.70 & 0.1964 \\
Mand 6-6 Width & 3.21 & 0.0767 \\
Mx-Md 3-3 Difference & 1.25 & 0.2674 \\
Mx-Md 6-6 Difference & 0.82 & 0.3676 \\
Mx 1-3 Chord & 0.35 & 0.5576 \\
Mx 1-6 Chord & 0.04 & 0.8358 \\
Md 1-3 Chord & 1.94 & 0.1678 \\
Md 1-6 Chord & 1.15 & 0.2861 \\
Midline Discrepancy & 0.26 & 0.6097 \\
Buccal Segment Relation & 0.03 & 0.8728 \\
Canine Relation & 2.95 & 0.0898 \\
Extraction Pattern & 10.99 & 0.0014 \\
Subject's Sex & 1.94 & 0.1672 \\
Ext-x-Sex Interaction & 1.18 & 0.3140 \\
\hline
\end{tabular}

1Step history:

$\begin{array}{clc}\text { Step } & \text { Parameter } & \text { R-Squared } \\ 1 & \text { Extraction Pattern } & 0.0862 \\ 2 & \text { Incisor Overbite } & 0.1659\end{array}$


Table 21. Results of stepwise multiple regression analysis with the intreatment change in the maxillary right canine (mesial) as the dependent variable. ${ }^{1}$

\begin{tabular}{lccc}
\hline \multicolumn{1}{c}{ Variable } & F Ratio & P Value & Model \\
\hline Incisor Overjet & 2.94 & 0.0904 & \\
Incisor Overbite & 6.87 & 0.0104 & Entered \\
Incisor Irregularity & 9.57 & 0.0027 & Entered \\
Incisor Spacing & 0.01 & 0.9236 & \\
Max 3-3 Width & 0.05 & 0.8189 & \\
Max 6-6 Width & 0.00 & 0.9753 & \\
Mand 3-3 Width & 0.03 & 0.8601 & \\
Mand 6-6 Width & 0.26 & 0.6093 & \\
Mx-Md 3-3 Difference & 0.02 & 0.8785 & \\
Mx-Md 6-6 Difference & 0.41 & 0.5252 & \\
Mx 1-3 Chord & 2.54 & 0.1150 & \\
Mx 1-6 Chord & 2.00 & 0.1606 & \\
Md 1-3 Chord & 9.38 & 0.0030 & Entered \\
Md 1-6 Chord & 0.00 & 0.9808 & \\
Midline Discrepancy & 2.34 & 0.1299 & \\
Buccal Segment Relation & 0.00 & 0.9703 & \\
Canine Relation & 2.79 & 0.0986 & \\
Extraction Pattern & 3.62 & 0.0606 & \\
Subject's Sex & 0.37 & 0.5423 & \\
Ext-x-Sex Interaction & 1.47 & 0.2293 & \\
\hline
\end{tabular}

1Step history:

\begin{tabular}{clc} 
Step & \multicolumn{1}{c}{ Parameter } & R-Squared \\
1 & Incisor Irregularity & 0.0459 \\
2 & Md 1-3 Chord & 0.1052 \\
3 & Incisor Overbite & 0.1736
\end{tabular}


Table 22. Results of stepwise multiple regression analysis with the intreatment change in the maxillary left canine (distal) as the dependent variable. ${ }^{1}$

\begin{tabular}{lccc}
\hline \multicolumn{1}{c}{ Variable } & F Ratio & P Value & Model \\
\hline Incisor Overjet & 0.73 & 0.3941 \\
Incisor Overbite & 1.24 & 0.2695 \\
Incisor Irregularity & 3.44 & 0.0671 \\
Incisor Spacing & 0.00 & 0.9629 \\
Max 3-3 Width & 0.43 & 0.5152 \\
Max 6-6 Width & 1.16 & 0.2839 \\
Mand 3-3 Width & 2.06 & 0.1549 \\
Mand 6-6 Width & 1.33 & 0.2528 \\
Mx-Md 3-3 Difference & 0.32 & 0.5748 \\
Mx-Md 6-6 Difference & 0.08 & 0.7814 \\
Mx 1-3 Chord & 0.03 & 0.8547 \\
Mx 1-6 Chord & 0.04 & 0.8522 \\
Md 1-3 Chord & 1.03 & 0.3130 \\
Md 1-6 Chord & 0.71 & 0.4011 \\
Midline Discrepancy & 0.19 & 0.6651 \\
Buccal Segment Relation & 0.24 & 0.6278 \\
Canine Relation & 1.77 & 0.1867 \\
Extraction Pattern & 14.12 & 0.0003 \\
Subject's Sex & 3.34 & 0.0713 \\
Ext-x-Sex Interaction & 2.50 & 0.0883 \\
\hline
\end{tabular}

1Step history:

$\begin{array}{ccc}\text { Step } & \text { Parameter } & \text { R-Squared } \\ 1 & \text { Extraction Pattern } & 0.1425\end{array}$


Table 23. Results of stepwise multiple regression analysis with the intreatment change in the maxillary right canine (distal) as the dependent variable. ${ }^{1}$

\begin{tabular}{lrrl}
\hline \multicolumn{1}{c}{ Variable } & F Ratio & P Value & Model \\
\hline Incisor Overjet & 5.73 & 0.0190 & Entered \\
Incisor Overbite & 2.27 & 0.1356 & \\
Incisor Irregularity & 15.77 & 0.0002 & Entered \\
Incisor Spacing & 0.00 & 0.9572 & \\
Max 3-3 Width & 0.39 & 0.5335 & \\
Max 6-6 Width & 0.05 & 0.8297 & \\
Mand 3-3 Width & 0.39 & 0.5335 & \\
Mand 6-6 Width & 0.04 & 0.8414 & \\
Mx-Md 3-3 Difference & 5.78 & 0.0185 & Entered \\
Mx-Md 6-6 Difference & 0.01 & 0.9314 & \\
Mx 1-3 Chord & 0.00 & 0.9549 & \\
Mx 1-6 Chord & 0.01 & 0.9275 & \\
Md 1-3 Chord & 0.50 & 0.4827 & \\
Md 1-6 Chord & 0.44 & 0.5077 & \\
Midline Discrepancy & 8.13 & 0.0055 & Entered \\
Buccal Segment Relation & 1.35 & 0.2486 & \\
Canine Relation & 0.65 & 0.4232 & \\
Extraction Pattern & 8.74 & 0.0041 & Entered \\
Subject's Sex & 0.19 & 0.6660 & \\
Ext-x-Sex Interaction & 0.10 & 0.9094 & \\
\hline
\end{tabular}

1Step history:

$\begin{array}{clc}\text { Step } & \text { Parameter } & \text { R-Squared } \\ 1 & \text { Incisor Irregularity } & 0.0981 \\ 2 & \text { Midline Discrepancy } & 0.1728 \\ 3 & \text { Extraction Pattern } & 0.2197 \\ 4 & \text { Mx-Md 3-3 Difference } & 0.2611 \\ 5 & \text { Incisor Overjet } & 0.3099\end{array}$




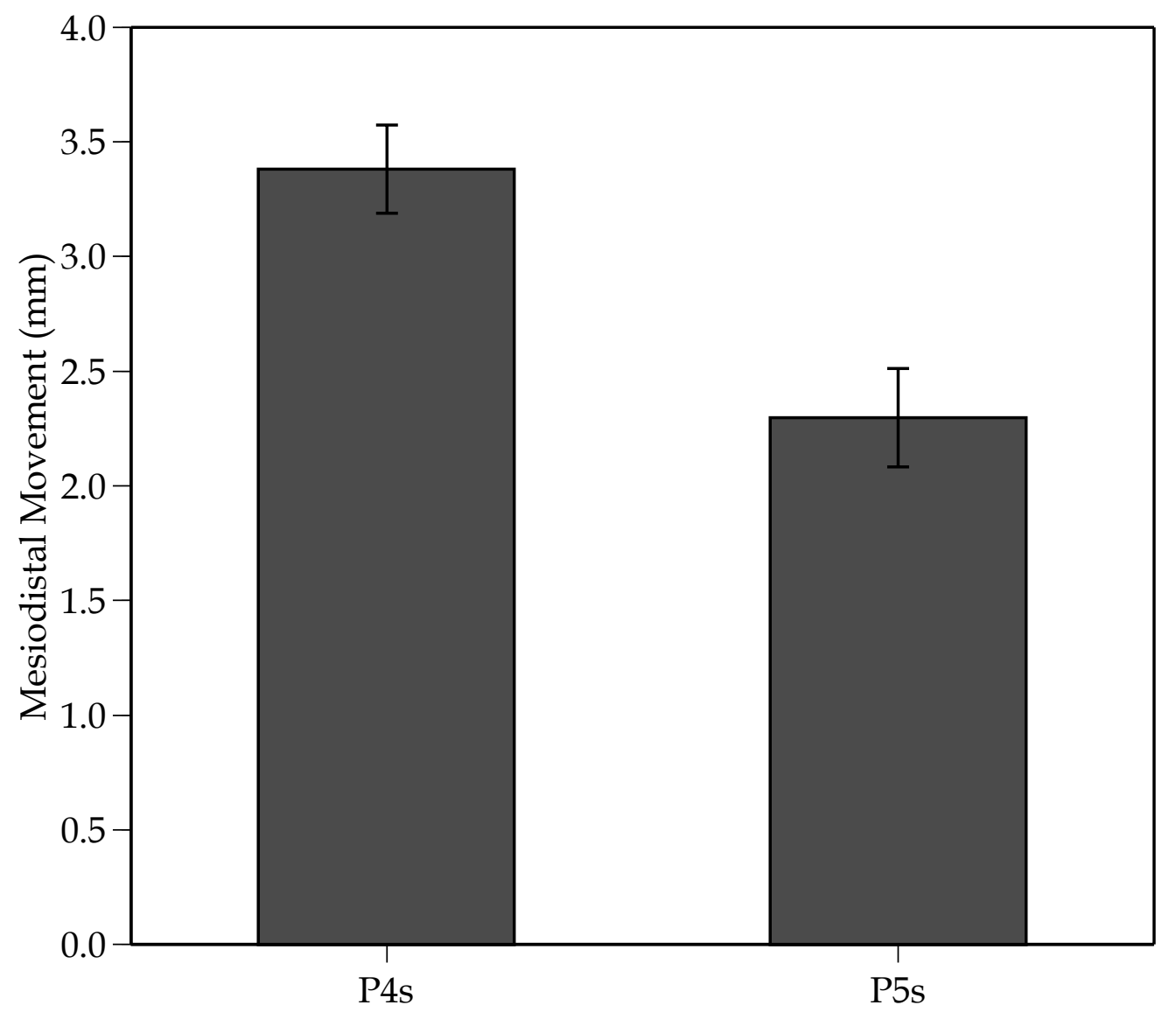

Fig. 41. Bar chart showing the greater retraction of the canine (left, distal contact) in the first-premolar sample (P4s) versus the sample treated with second-premolar extractions (P5s). Error bars are standard errors of the mean. 


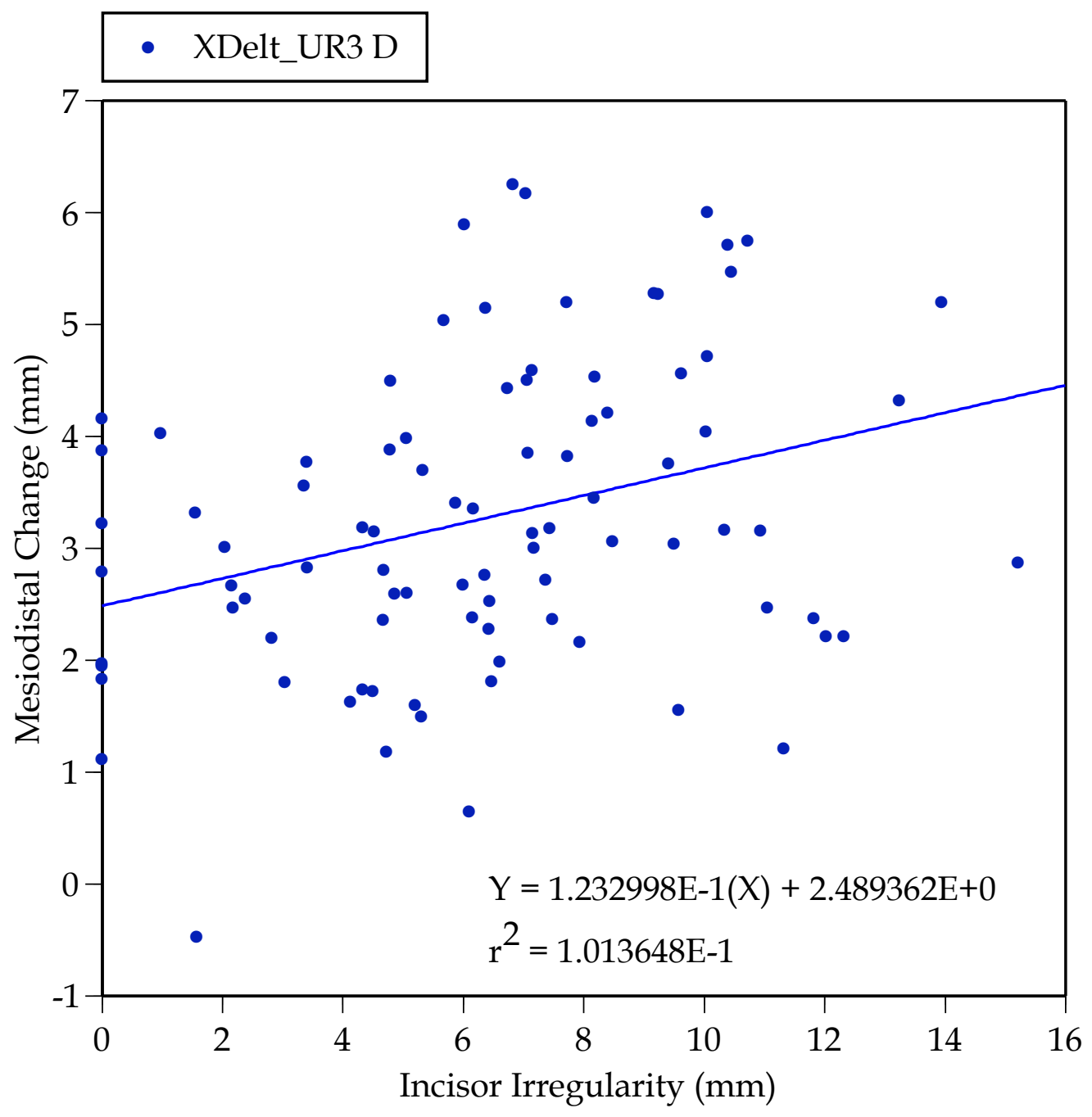

Fig. 42. Scatterplot showing the positive association between pretreatment incisor irregularity and the amount of retraction of the maxillary right central canine (distal). 
pretreatment irregularity, the greater the typical retraction of the canine. Looking at the regression line, canine retraction in the absence of incisor irregularity (where the change in the $\mathrm{X}$ axis is 0 ) is about $2.5 \mathrm{~mm}$.

\section{$\underline{\text { First Premolar }}$}

These four analyses (Tables 24, 25) are based just on the subset of the sample in which the second premolar had been extracted. There is a considerable difference for the left and right contact of the P1; none of the variance was accounted for on the left (Table 24), but half (49\%) was accounted for by three variables for the right landmark. For this right mesial landmark (Table 25), overjet was the predominant predictor of how far the first premolar was moved mesiodistally $\left(\mathrm{r}^{2}=22 \%\right)$, and the relationship is that the greater the overjet, the less the first premolar was moved (Fig. 43) in the 40 P2-extraction cases. One might suppose that the cases with greater overjet would experience incisor retraction, but this is not case (Fig. 43). Instead, cases in the upper-left quadrant-who underwent the most P1 extractioncharacteristically exhibited little overjet at the start of treatment. Inspection of these cases (e.g., Figs. A-6, A-11, A-36, A-52) shows that the second premolars were extracted and the first premolars moved back into those spaces to accommodate buccolingually displaced (ectopic) lateral incisors or canines not to resolve any issue of excessive overjet. 
Table 24. Results of stepwise multiple regression analysis with the intreatment change in the maxillary left first premolar (mesial) as the dependent variable. ${ }^{1}$

\begin{tabular}{lccl}
\hline \multicolumn{1}{c}{ Variable } & F Ratio & P Value & Model \\
\hline Incisor Overjet & 2.74 & 0.1067 & \\
Incisor Overbite & 0.81 & 0.3748 & \\
Incisor Irregularity & 0.19 & 0.6677 & \\
Incisor Spacing & 0.08 & 0.7789 & \\
Max 3-3 Width & 0.76 & 0.3901 \\
Max 6-6 Width & 1.36 & 0.2505 \\
Mand 3-3 Width & 1.39 & 0.2468 \\
Mand 6-6 Width & 1.03 & 0.3164 \\
Mx-Md 3-3 Difference & 0.02 & 0.8796 & \\
Mx-Md 6-6 Difference & 0.22 & 0.6451 & \\
Mx 1-3 Chord & 0.06 & 0.8098 \\
Mx 1-6 Chord & 0.14 & 0.7130 \\
Md 1-3 Chord & 0.71 & 0.4044 \\
Md 1-6 Chord & 0.18 & 0.6704 \\
Midline Discrepancy & 0.11 & 0.7401 & \\
Buccal Segment Relation & 0.00 & 0.9825 \\
Canine Relation & 2.90 & 0.0972 \\
Sex & N/A & \\
Subject's Sex & 3.69 & 0.0629 \\
Ext-x-Sex Interaction & N/A & & \\
\hline
\end{tabular}

1Step history:

Step Parameter

R-Squared

None 
Table 25. Results of stepwise multiple regression analysis with the intreatment change in the maxillary right first premolar (mesial) as the dependent variable. ${ }^{1}$

\begin{tabular}{lrrl}
\hline \multicolumn{1}{c}{ Variable } & F Ratio & P Value & Model \\
\hline Incisor Overjet & 18.43 & 0.0001 & Entered \\
Incisor Overbite & 0.00 & 0.9926 & \\
Incisor Irregularity & 16.81 & 0.0002 & Entered \\
Incisor Spacing & 2.67 & 0.1119 & \\
Max 3-3 Width & 1.22 & 0.2784 & \\
Max 6-6 Width & 1.86 & 0.1814 & \\
Mand 3-3 Width & 0.21 & 0.6509 & \\
Mand 6-6 Width & 0.45 & 0.5090 & \\
Mx-Md 3-3 Difference & 0.79 & 0.3808 & \\
Mx-Md 6-6 Difference & 1.22 & 0.2780 & \\
Mx 1-3 Chord & 7.51 & 0.0097 & Entered \\
Mx 1-6 Chord & 0.78 & 0.3825 & \\
Md 1-3 Chord & 0.39 & 0.5366 & \\
Md 1-6 Chord & 0.73 & 0.3996 & \\
Midline Discrepancy & 0.75 & 0.3920 & \\
Buccal Segment Relation & 1.15 & 0.2922 & \\
Canine Relation & 0.70 & 0.4097 & \\
Sex & $\mathrm{N} / \mathrm{A}$ & & \\
Subject's Sex & 0.77 & 0.3874 & \\
Ext-x-Sex Interaction & $\mathrm{N} / \mathrm{A}$ & & \\
\hline
\end{tabular}

1Step history:

$\begin{array}{clc}\text { Step } & \text { Parameter } & \text { R-Squared } \\ 1 & \text { Incisor Overjet } & 0.2209 \\ 2 & \text { Incisor Irregularity } & 0.3828 \\ 3 & \text { Mx 1-3 Chord } & 0.4945\end{array}$




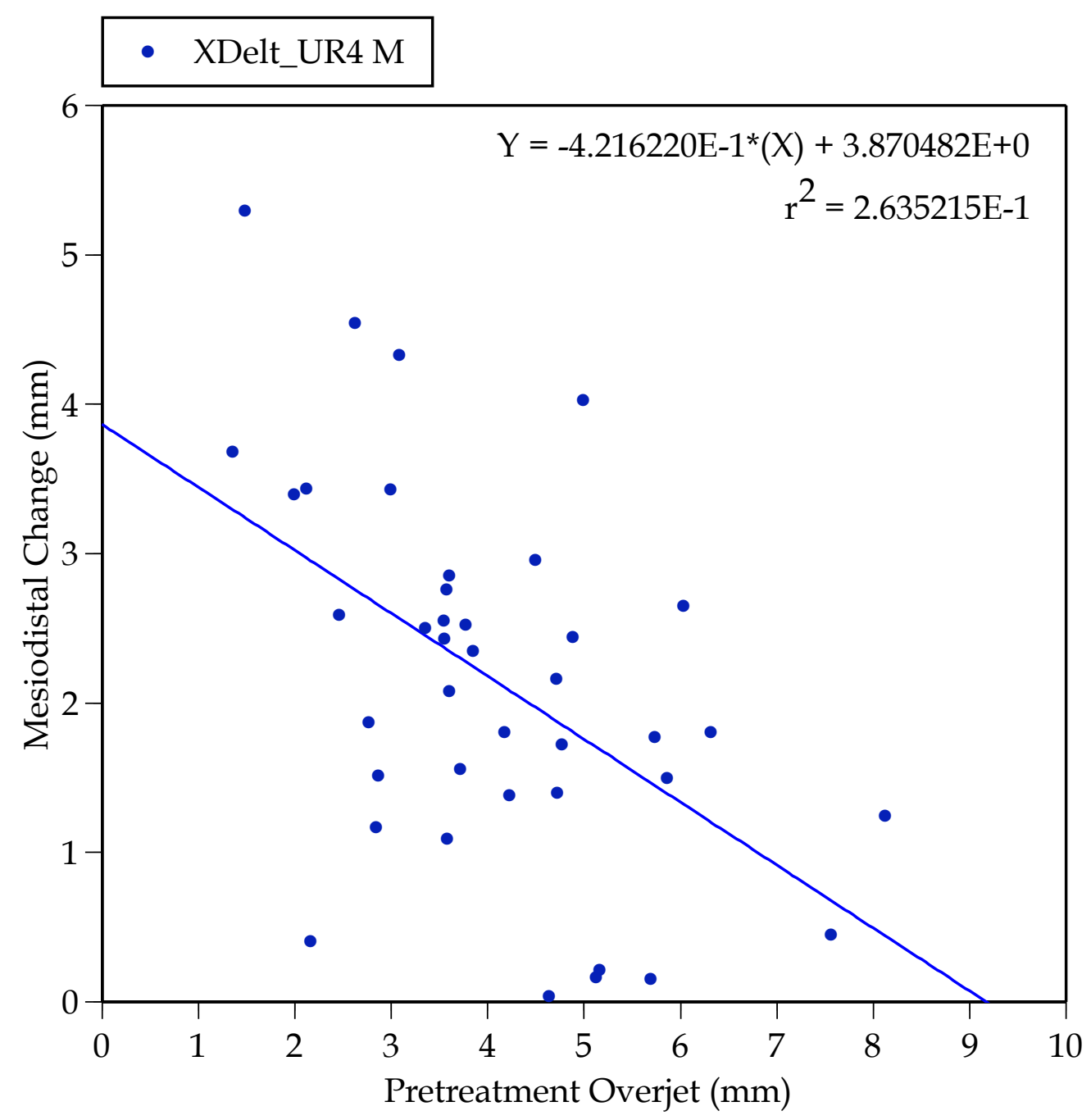

Fig. 43. Scatterplot showing the negative association between pretreatment overjet and the amount of retraction of the maxillary right first premolar (mesial). 
Results for analyses of the distal landmarks of the first premolars are listed in Tables 26 and 27. The significant predictors are wholly different for the left and right landmarks. On the left (Table 26), canine relationship is predictive $\left(\mathrm{r}^{2}=16 \%\right)$, and the situation (Fig. 44$)$ is that when the canine is close to its correct position (e.g., Figs. A-14, A-16), the first premolar is retracted a lot into the P2-extraction space, but so is the mandibular P1, so interdigitation between the two arches is preserved. In contrast, when the canines are in overt mesiversion because of the maxillary anterior teeth are procumbent (e.g., Figs. A-27, A-61), then most of the P2 extraction space is used to adjust the buccal segment relationship rather than anterior retraction.

\section{$\underline{\text { Second Premolar }}$}

Mesiodistal changes of the second premolars are, of course, limited to the cases in whom first premolars were extracted. Results for the mesial landmarks in the two quadrants (Tables 28, 29) are not concordant. Incisor irregularity is predictive on the left tooth $\left(\mathrm{r}^{2}=36 \%\right)$, and the relationship is that the greater the incisor irregularity, the less P2 moved mesially during treatment (Fig. 45). This is reasonable since one supposes that cases with considerable irregularity (e.g., Fig. A-33) will use up most of the extraction space to resolve the crowding in the anterior segments. On the other hand, 
Table 26. Results of stepwise multiple regression analysis with the intreatment change in the maxillary left first premolar (distal) as the dependent variable. ${ }^{1}$

\begin{tabular}{lccc}
\hline \multicolumn{1}{c}{ Variable } & F Ratio & P Value & Model \\
\hline Incisor Overjet & 1.93 & 0.1737 & \\
Incisor Overbite & 2.79 & 0.1039 & \\
Incisor Irregularity & 2.24 & 0.1438 & \\
Incisor Spacing & 0.04 & 0.8387 & \\
Max 3-3 Width & 1.24 & 0.2737 & \\
Max 6-6 Width & 0.93 & 0.3407 & \\
Mand 3-3 Width & 2.54 & 0.1197 & \\
Mand 6-6 Width & 1.85 & 0.1831 & \\
Mx-Md 3-3 Difference & 0.07 & 0.7927 & \\
Mx-Md 6-6 Difference & 0.08 & 0.7766 & \\
Mx 1-3 Chord & 0.18 & 0.6742 & \\
Mx 1-6 Chord & 0.33 & 0.5675 & \\
Md 1-3 Chord & 1.31 & 0.2601 & \\
Md 1-6 Chord & 0.46 & 0.5032 & \\
Midline Discrepancy & 0.18 & 0.6777 & \\
Buccal Segment Relation & 2.34 & 0.1354 & \\
Canine Relation & 7.01 & 0.0120 & Entered \\
Sex & N/A & & \\
Subject's Sex & 3.70 & 0.0625 & \\
Ext-x-Sex Interaction & N/A & & \\
\hline
\end{tabular}

1Step history:

$\begin{array}{ccc}\text { Step } & \text { Parameter } & \text { R-Squared } \\ 1 & \text { Canine Relation } & 0.1629\end{array}$


Table 27. Results of stepwise multiple regression analysis with the intreatment change in the maxillary right first premolar (distal) as the dependent variable. ${ }^{1}$

\begin{tabular}{lrrl}
\hline \multicolumn{1}{c}{ Variable } & F Ratio & P Value & Model \\
\hline Incisor Overjet & 17.36 & 0.0002 & Entered \\
Incisor Overbite & 0.02 & 0.8831 & \\
Incisor Irregularity & 17.28 & 0.0002 & Entered \\
Incisor Spacing & 0.48 & 0.4955 & \\
Max 3-3 Width & 0.01 & 0.9344 & \\
Max 6-6 Width & 1.80 & 0.1888 & \\
Mand 3-3 Width & 0.01 & 0.9417 & \\
Mand 6-6 Width & 0.87 & 0.3590 & \\
Mx-Md 3-3 Difference & 0.04 & 0.8457 & \\
Mx-Md 6-6 Difference & 0.51 & 0.4790 & \\
Mx 1-3 Chord & 9.37 & 0.0043 & Entered \\
Mx 1-6 Chord & 0.92 & 0.3455 & \\
Md 1-3 Chord & 0.74 & 0.3945 & \\
Md 1-6 Chord & 0.25 & 0.6240 & \\
Midline Discrepancy & 3.32 & 0.0777 & \\
Buccal Segment Relation & 0.87 & 0.3592 & \\
Canine Relation & 0.00 & 0.9491 & \\
Sex & $\mathrm{N} / \mathrm{A}$ & & \\
Subject's Sex & 0.96 & 0.3339 & \\
Ext-x-Sex Interaction & $\mathrm{N} / \mathrm{A}$ & & \\
\hline
\end{tabular}

1Step history:

$\begin{array}{clc}\text { Step } & \text { Parameter } & \text { R-Squared } \\ 1 & \text { Incisor Irregularity } & 0.2090 \\ 2 & \text { Incisor Overjet } & 0.3404 \\ 3 & \text { Mx 1-3 Chord } & 0.4829\end{array}$




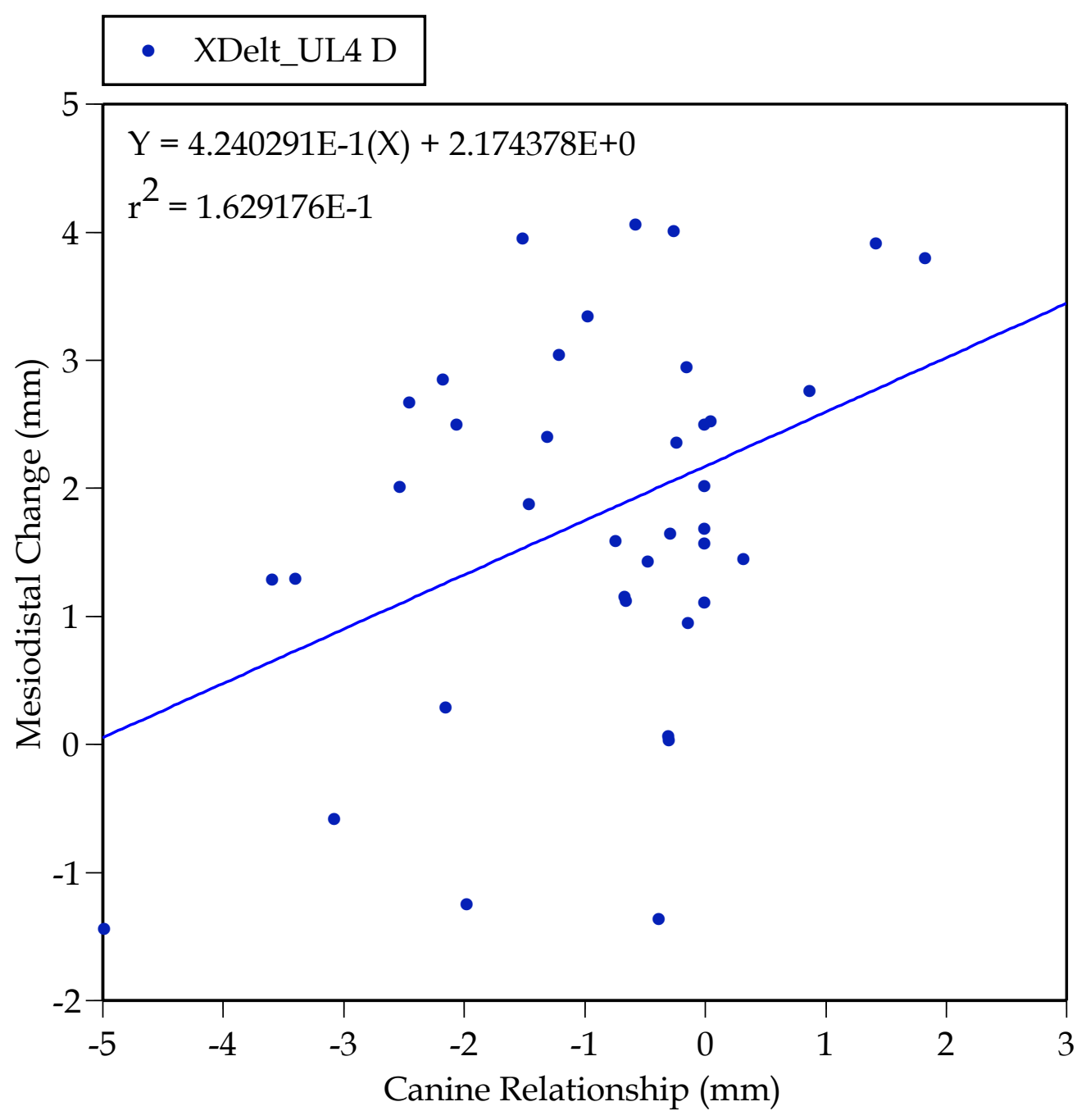

Fig. 44. Scatterplot showing the positive association between pretreatment canine relationship and the amount of retraction of the maxillary right first premolar (distal). 
Table 28. Results of stepwise multiple regression analysis with the intreatment change in the maxillary left second premolar (mesial) as the dependent variable. ${ }^{1}$

\begin{tabular}{lrrl}
\hline \multicolumn{1}{c}{ Variable } & F Ratio & P Value & Model \\
\hline Incisor Overjet & 0.05 & 0.8249 & \\
Incisor Overbite & 10.79 & 0.0020 & Entered \\
Incisor Irregularity & 35.66 & 0.0000 & Entered \\
Incisor Spacing & 0.13 & 0.7161 & \\
Max 3-3 Width & 2.28 & 0.1384 & \\
Max 6-6 Width & 0.02 & 0.8879 & \\
Mand 3-3 Width & 0.33 & 0.5699 & \\
Mand 6-6 Width & 0.08 & 0.7733 & \\
Mx-Md 3-3 Difference & 6.17 & 0.0168 & Entered \\
Mx-Md 6-6 Difference & 0.03 & 0.8667 & \\
Mx 1-3 Chord & 1.13 & 0.2940 & \\
Mx 1-6 Chord & 0.32 & 0.5732 & \\
Md 1-3 Chord & 0.04 & 0.8341 & \\
Md 1-6 Chord & 0.29 & 0.5927 & \\
Midline Discrepancy & 0.03 & 0.8681 & \\
Buccal Segment Relation & 1.42 & 0.2392 & \\
Canine Relation & 0.21 & 0.6493 & \\
Sex & $\mathrm{N} / \mathrm{A}$ & & \\
Subject's Sex & 3.72 & 0.0601 & \\
Ext-x-Sex Interaction & $\mathrm{N} / \mathrm{A}$ & & \\
\hline
\end{tabular}

1Step history:

$\begin{array}{clc}\text { Step } & \text { Parameter } & \text { R-Squared } \\ 1 & \text { Incisor Irregularity } & 0.3601 \\ 2 & \text { Incisor Overbite } & 0.4816\end{array}$


Table 29. Results of stepwise multiple regression analysis with the intreatment change in the maxillary right second premolar (mesial) as the dependent variable. ${ }^{1}$

\begin{tabular}{lccc}
\hline \multicolumn{1}{c}{ Variable } & F Ratio & P Value & Model \\
\hline Incisor Overjet & 0.57 & 0.4537 & \\
Incisor Overbite & 2.23 & 0.1424 & \\
Incisor Irregularity & 1.81 & 0.1850 & \\
Incisor Spacing & 0.04 & 0.8531 & \\
Max 3-3 Width & 1.93 & 0.1711 & \\
Max 6-6 Width & 3.62 & 0.0636 & \\
Mand 3-3 Width & 1.58 & 0.2149 & \\
Mand 6-6 Width & 1.69 & 0.2000 & \\
Mx-Md 3-3 Difference & 0.36 & 0.5531 & \\
Mx-Md 6-6 Difference & 0.77 & 0.3843 & \\
Mx 1-3 Chord & 0.05 & 0.8233 & \\
Mx 1-6 Chord & 16.66 & 0.0002 & Entered \\
Md 1-3 Chord & 0.44 & 0.5102 & \\
Md 1-6 Chord & 1.64 & 0.2072 & \\
Midline Discrepancy & 5.20 & 0.0273 & Entered \\
Buccal Segment Relation & 0.53 & 0.4719 & \\
Canine Relation & 1.02 & 0.3180 & \\
Sex & $\mathrm{N} / \mathrm{A}$ & & \\
Subject's Sex & 0.18 & 0.6775 & \\
Ext-x-Sex Interaction & $\mathrm{N} / \mathrm{A}$ & & \\
\hline
\end{tabular}

1Step history:

$\begin{array}{clc}\text { Step } & \text { Parameter } & \text { R-Squared } \\ 1 & \text { Mx 1-6 Chord } & 0.2023 \\ 2 & \text { Midline Discrepancy } & 0.2833\end{array}$




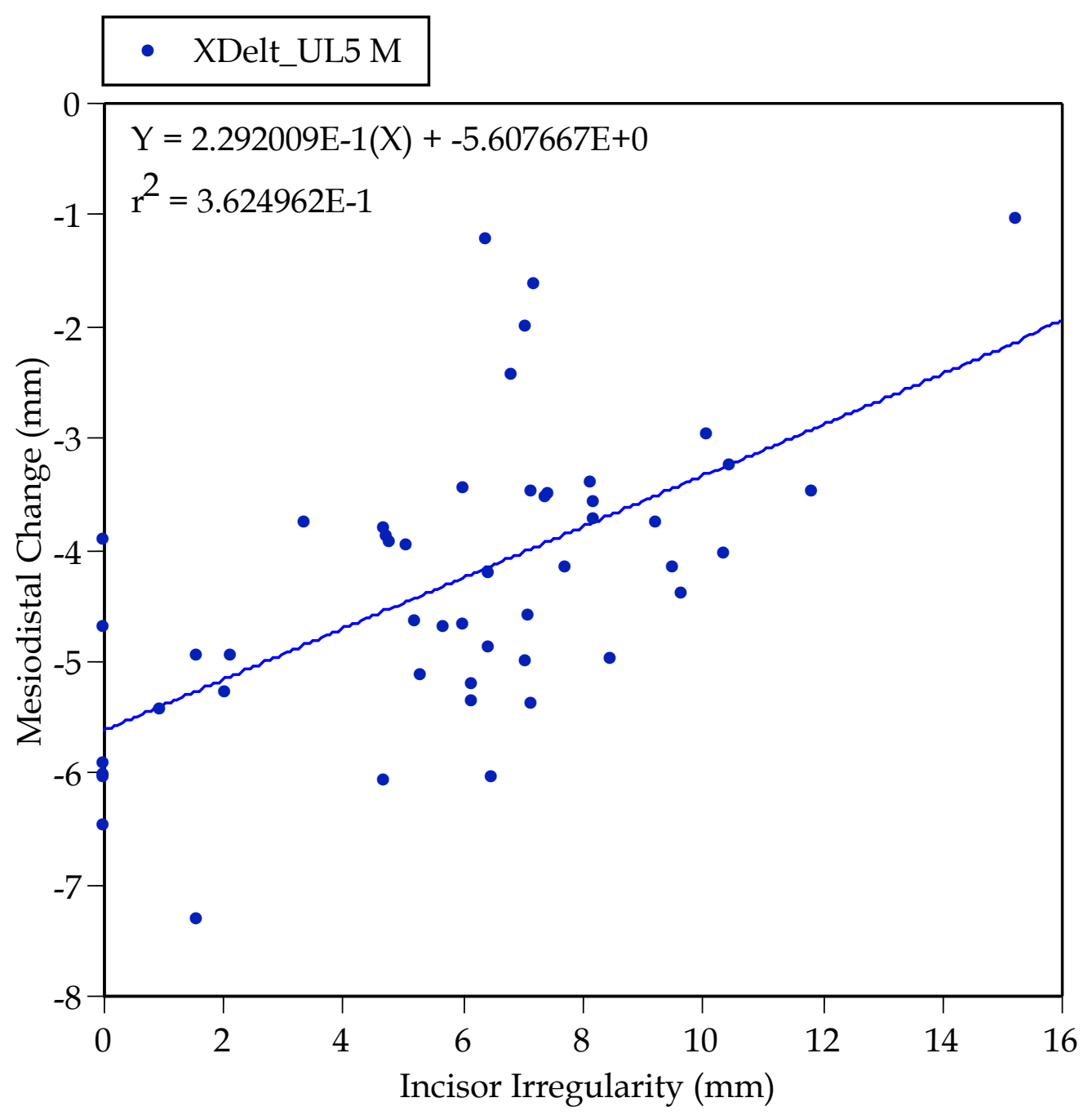

Fig. 45. Scatterplot showing the positive association between incisor irregularity and the amount of retraction of the maxillary left second premolar (mesial). 
cases presenting with little incisor irregularity (e.g., Figs. A-20, A-89) use most of the P1 extraction space to resolve other issues.

On the right, the paramount predictor for the mesial landmark is the maxillary 1-6 chord length $\left(r^{2}=20 \%\right.$; Table 29). The longer the arch chord (which probably reflects incisor procumbency), the more the second premolar was moved mesially (Fig. 46). Fig. A-59 is an example of a malocclusion with a short-broad arch form, where P2 was moved mesially very little. In contrast, Fig. A-25 shows a longer arch form, where P2 was moved mesially about 4 $\mathrm{mm}$.

Tables 30 and 31 show the statistical results for the distal landmark for the left and right P2, respectively, and the major predictor for each tooth is the same as for the mesial landmarks. That is, incisor irregularity is predictive for the left tooth $(\mathrm{r}=32 \%)$ and maxillary 1-6 arch chord is predictive for the right tooth $\left(\mathrm{r}^{2}=25 \%\right)$.

\section{$\underline{\text { First Molar }}$}

Incisor irregularity is the preeminent predictor of how much the first molar (mesial) contact was moved mesiodistally during treatment (Tables 32, 33). The greater the irregularity at the start of treatment, the less the molar was moved mesially (Fig. 47). This, of course, is intuitive since in those cases with considerable irregularity the premolar extraction space is used 


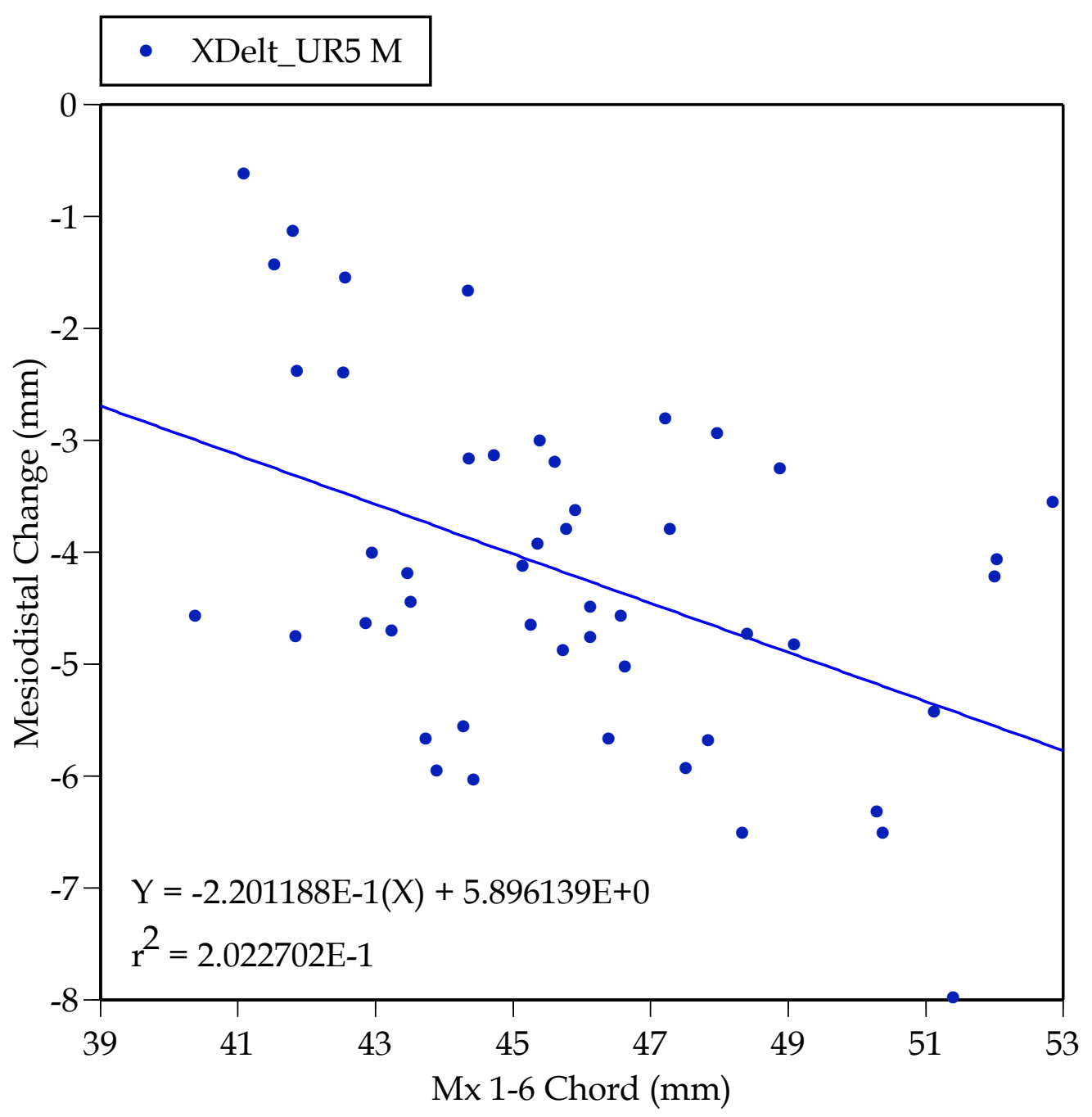

Fig. 46. Scatterplot showing the negative association between pretreatment arch chord (1-6) and the amount of mesial movement of the maxillary right second premolar (mesial). 
Table 30. Results of stepwise multiple regression analysis with the intreatment change in the maxillary left second premolar (distal) as the dependent variable. ${ }^{1}$

\begin{tabular}{lrrl}
\hline \multicolumn{1}{c}{ Variable } & F Ratio & P Value & Model \\
\hline Incisor Overjet & 0.00 & 0.9584 & \\
Incisor Overbite & 12.43 & 0.0010 & Entered \\
Incisor Irregularity & 31.21 & 0.0000 & Entered \\
Incisor Spacing & 0.01 & 0.9325 & \\
Max 3-3 Width & 0.22 & 0.6390 & \\
Max 6-6 Width & 1.01 & 0.3209 & \\
Mand 3-3 Width & 1.25 & 0.2694 & \\
Mand 6-6 Width & 0.94 & 0.3374 & \\
Mx-Md 3-3 Difference & 2.47 & 0.1228 & \\
Mx-Md 6-6 Difference & 0.13 & 0.7223 & \\
Mx 1-3 Chord & 0.17 & 0.6824 & \\
Mx 1-6 Chord & 0.07 & 0.7912 & \\
Md 1-3 Chord & 0.23 & 0.6309 & \\
Md 1-6 Chord & 0.31 & 0.5789 & \\
Midline Discrepancy & 0.01 & 0.9091 & \\
Buccal Segment Relation & 0.28 & 0.6029 & \\
Canine Relation & 0.76 & 0.3873 & \\
Sex & $\mathrm{N} / \mathrm{A}$ & & \\
Subject's Sex & 1.32 & 0.2564 & \\
Ext-x-Sex Interaction & $\mathrm{N} / \mathrm{A}$ & & \\
\hline
\end{tabular}

1Step history:

$\begin{array}{clc}\text { Step } & \text { Parameter } & \text { R-Squared } \\ 1 & \text { Incisor Irregularity } & 0.3197 \\ 2 & \text { Incisor Overbite } & 0.4644\end{array}$


Table 31. Results of stepwise multiple regression analysis with the intreatment change in the maxillary right second premolar (distal) as the dependent variable. ${ }^{1}$

\begin{tabular}{lccc}
\hline \multicolumn{1}{c}{ Variable } & F Ratio & P Value & Model \\
\hline Incisor Overjet & 0.03 & 0.8720 & \\
Incisor Overbite & 0.86 & 0.3600 & \\
Incisor Irregularity & 2.15 & 0.1493 & \\
Incisor Spacing & 0.00 & 0.9554 & \\
Max 3-3 Width & 0.55 & 0.4615 & \\
Max 6-6 Width & 4.24 & 0.0453 & Entered \\
Mand 3-3 Width & 0.31 & 0.5782 & \\
Mand 6-6 Width & 0.03 & 0.8632 & \\
Mx-Md 3-3 Difference & 0.15 & 0.7052 & \\
Mx-Md 6-6 Difference & 0.03 & 0.8632 & \\
Mx 1-3 Chord & 0.02 & 0.8805 & \\
Mx 1-6 Chord & 22.12 & 0.0000 & Entered \\
Md 1-3 Chord & 0.08 & 0.7740 & \\
Md 1-6 Chord & 0.46 & 0.4992 & \\
Midline Discrepancy & 6.31 & 0.0157 & Entered \\
Buccal Segment Relation & 0.26 & 0.6135 & \\
Canine Relation & 1.47 & 0.2314 & \\
Sex & $\mathrm{N} / \mathrm{A}$ & & \\
Subject's Sex & 0.60 & 0.4436 & \\
Ext-x-Sex Interaction & $\mathrm{N} / \mathrm{A}$ & & \\
\hline
\end{tabular}

1Step history:

$\begin{array}{clc}\text { Step } & \text { Parameter } & \text { R-Squared } \\ 1 & \text { Mx 1-6 Chord } & 0.2505 \\ 2 & \text { Midline Discrepancy } & 0.3358 \\ 3 & \text { Max 6-6 Width } & 0.3930\end{array}$


Table 32. Results of stepwise multiple regression analysis with the intreatment change in the maxillary left first molar (mesial) as the dependent variable. ${ }^{1}$

\begin{tabular}{lrrl}
\hline \multicolumn{1}{c}{ Variable } & F Ratio & P Value & Model \\
\hline Incisor Overjet & 1.34 & 0.2508 & \\
Incisor Overbite & 7.38 & 0.0080 & Entered \\
Incisor Irregularity & 17.54 & 0.0001 & Entered \\
Incisor Spacing & 0.18 & 0.6742 & \\
Max 3-3 Width & 0.19 & 0.6664 & \\
Max 6-6 Width & 0.03 & 0.8750 & \\
Mand 3-3 Width & 0.26 & 0.6116 & \\
Mand 6-6 Width & 0.20 & 0.6593 & \\
Mx-Md 3-3 Difference & 0.00 & 0.9579 & \\
Mx-Md 6-6 Difference & 0.15 & 0.6992 & \\
Mx 1-3 Chord & 1.47 & 0.2291 & \\
Mx 1-6 Chord & 3.17 & 0.0789 & \\
Md 1-3 Chord & 0.88 & 0.3499 & \\
Md 1-6 Chord & 0.38 & 0.5397 & \\
Midline Discrepancy & 0.00 & 0.9986 & \\
Buccal Segment Relation & 2.40 & 0.1253 & \\
Canine Relation & 7.50 & 0.0075 & Entered \\
Extraction Pattern & 9.43 & 0.0029 & Entered \\
Subject's Sex & 3.36 & 0.0703 & \\
Ext-x-Sex Interaction & 1.80 & 0.1725 & \\
\hline
\end{tabular}

1Step history:

$\begin{array}{clc}\text { Step } & \text { Parameter } & \text { R-Squared } \\ 1 & \text { Incisor Irregularity } & 0.0920 \\ 2 & \text { Extraction Pattern } & 0.1809 \\ 3 & \text { Canine Relation } & 0.2488\end{array}$


Table 33. Results of stepwise multiple regression analysis with the intreatment change in the maxillary right first molar (mesial) as the dependent variable. ${ }^{1}$

\begin{tabular}{lrrl}
\hline \multicolumn{1}{c}{ Variable } & F Ratio & P Value & Model \\
\hline Incisor Overjet & 5.94 & 0.0170 & \\
Incisor Overbite & 8.71 & 0.0041 & Entered \\
Incisor Irregularity & 24.43 & 0.0000 & Entered \\
Incisor Spacing & 0.05 & 0.8232 & \\
Max 3-3 Width & 1.50 & 0.2244 & \\
Max 6-6 Width & 0.06 & 0.8153 & \\
Mand 3-3 Width & 0.13 & 0.7231 & \\
Mand 6-6 Width & 0.02 & 0.8897 & \\
Mx-Md 3-3 Difference & 1.65 & 0.2032 & \\
Mx-Md 6-6 Difference & 0.05 & 0.8298 & \\
Mx 1-3 Chord & 0.07 & 0.7907 & \\
Mx 1-6 Chord & 0.97 & 0.3280 & \\
Md 1-3 Chord & 0.03 & 0.8739 & \\
Md 1-6 Chord & 0.03 & 0.8601 & \\
Midline Discrepancy & 8.03 & 0.0058 & Entered \\
Buccal Segment Relation & 0.97 & 0.3286 & \\
Canine Relation & 10.25 & 0.0020 & Entered \\
Extraction Pattern & 6.54 & 0.0125 & Entered \\
Subject's Sex & 1.95 & 0.1667 & \\
Ext-x-Sex Interaction & 1.18 & 0.3123 & \\
\hline
\end{tabular}

1Step history:

$\begin{array}{clc}\text { Step } & \text { Parameter } & \text { R-Squared } \\ 1 & \text { Incisor Irregularity } & 0.1326 \\ 2 & \text { Canine Relation } & 0.2149 \\ 3 & \text { Midline Discrepancy } & 0.2896 \\ 4 & \text { Incisor Overbite } & 0.3314 \\ 5 & \text { Extraction Pattern } & 0.3644 \\ 6 & \text { Incisor Overjet } & 0.4083\end{array}$




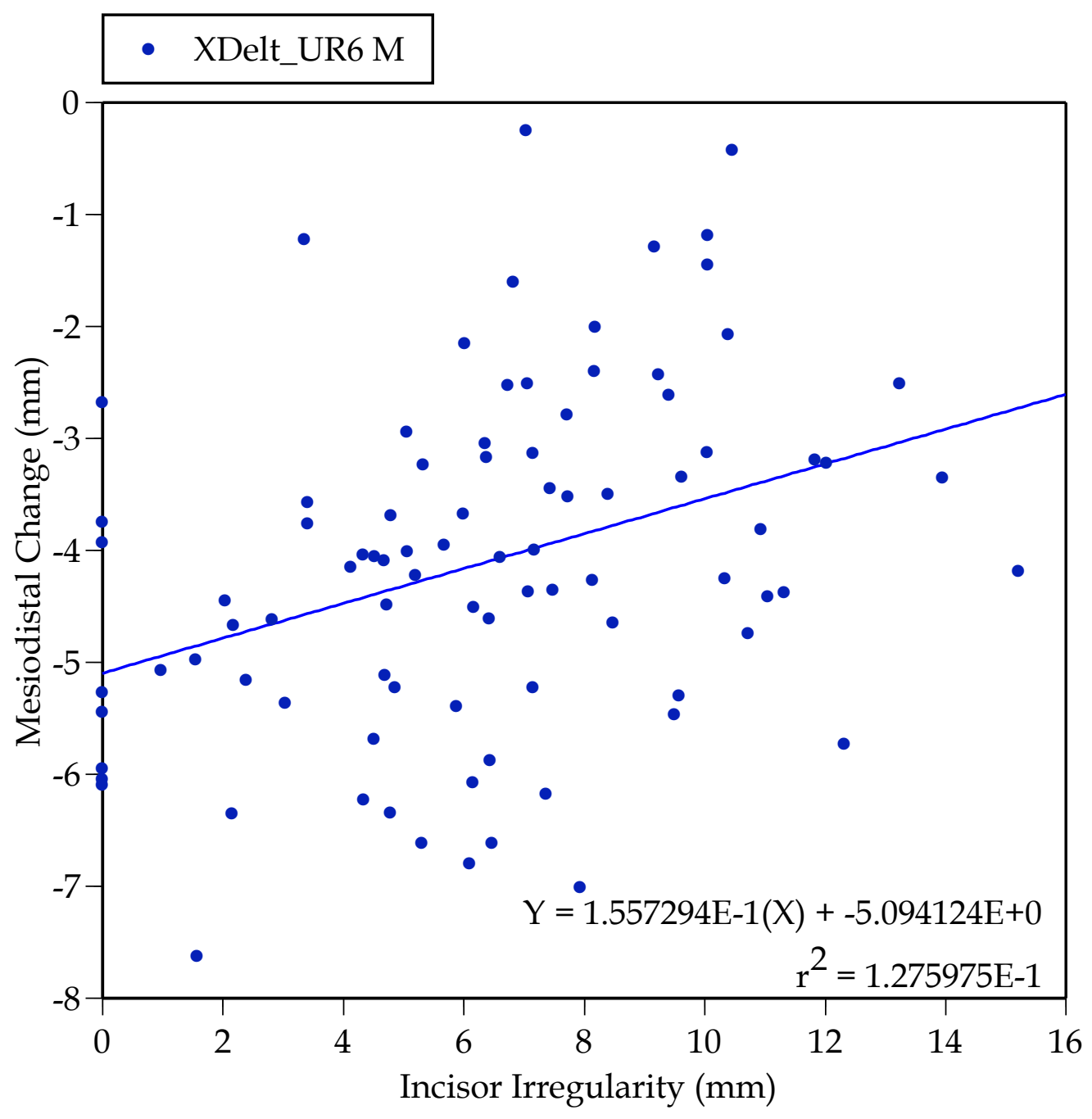

Fig. 47. Scatterplot showing the positive association between pretreatment incisor irregularity and the amount of mesial movement of the maxillary right first molar (mesial). 
predominantly to provide space for aligning the anterior teeth. Conversely, there is considerable mesial molar movement when irregularity is slight and changes need to be made in the buccal segment. Figs. A-3 and A-51 are examples of this latter situation where there was considerable mesial movement of the maxillary first molars (mesial). In contrast, Figs. A-36 and A39 exemplify the other extreme, where there was considerable incisor irregularity that was resolved by using the extraction space to align the anterior teeth (with little molar movement).

Extraction pattern entered the models for the right and left sides because, predictably, first premolars were extracted to alleviate anterior crowding, so the first molars were moved forward less than in secondpremolar extraction cases. Statistically, by ANOVA, the univariate difference is significant. For the right side, the first molar (mesial contact) moved mesially an average of $4.0 \mathrm{~mm}$ in the first-premolar sample $(\mathrm{sd}=0.22)$ whereas the molar moved forward $4.7 \mathrm{~mm}(\mathrm{sd}=0.25)$ in the second-premolar sample $(\mathrm{F}=4.45 ; \mathrm{P}=0.0375)$

The third predictive variable in common between sides (Tables 32, 33) is canine relationship. The association here parallels that just discussed for incisor irregularity (Fig. 48). The greater the canine discrepancy, the less the first molar was moved forward. Figs. A-33, A-37, and A-73 are representative of cases treated with very little molar movement (so the canine position could 


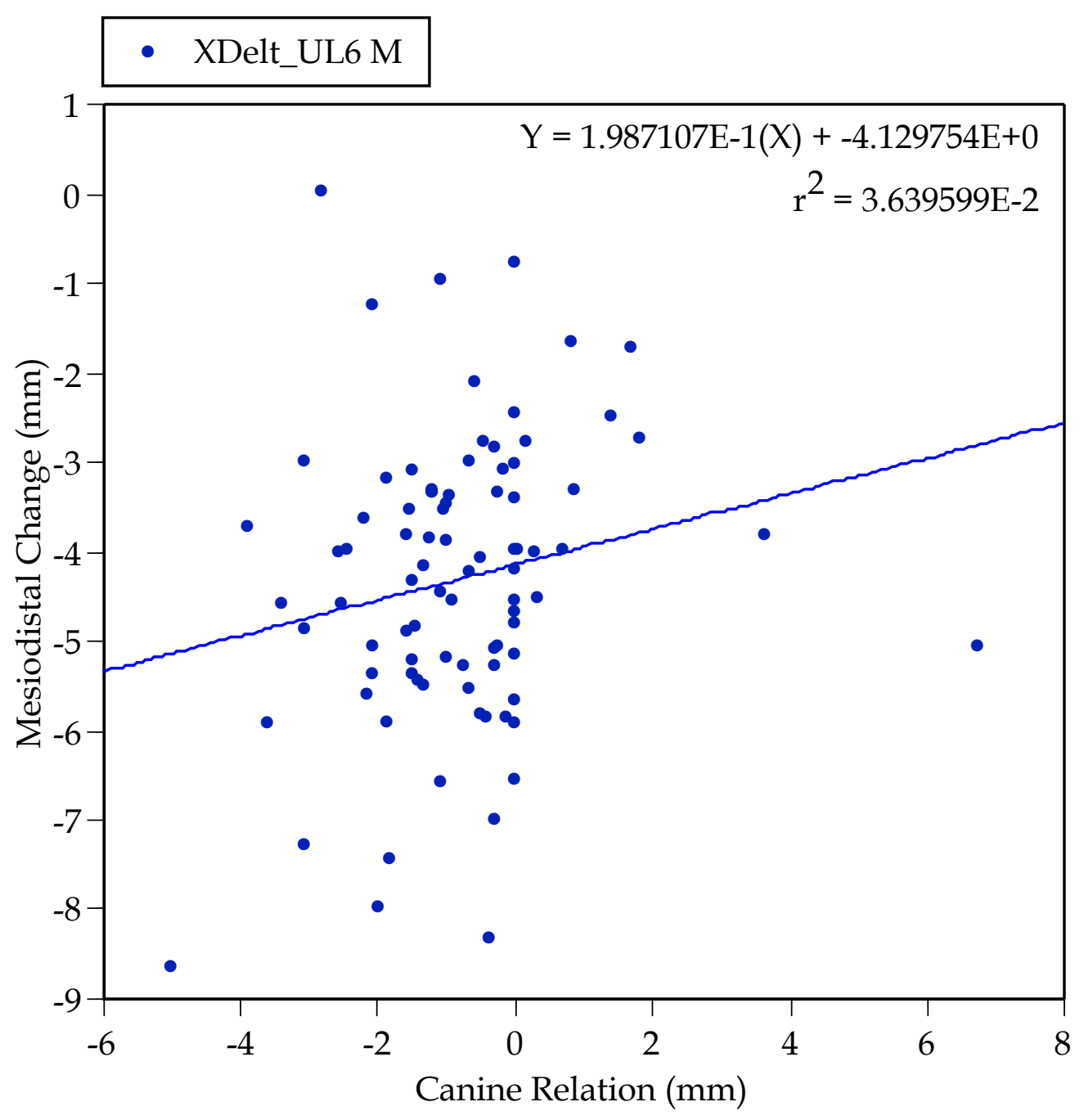

Fig. 48. Scatterplot showing the positive association between pretreatment canine relationship and the amount of mesial movement of the maxillary left first molar (mesial). 
be corrected). At the other extreme, Figs. A-27, A-45, and A-61 show cases in which each experienced a lot of mesial first molar movement (and, conversely, little positional change in canine position mesiodistally).

Examining changes in the distal contact of the first molar (Tables 34, 35), several predictors entered each model, but just two variables occur in both, namely incisor irregularity and overbite. The relationship with irregularity is the same as for the mesial landmark (Fig. 49): Greater incisor irregularity is associated with less mesial molar movement.

The relationship with pretreatment overbite is graphed in Fig. 50. Cases with deep bites underwent less mesial molar movement than those with a shallow bite or, even, an anterior openbite.

\section{$\underline{\text { Second Molar }}$}

The predictive models for the left and right second molars (mesial contact) share no variable in common (Tables 36,37$)$. The major predictor for the landmark on the left is the patients' $\operatorname{sex}\left(\mathrm{r}^{2}=7 \%\right)$. This may be a finding relevant just to this data set, because "sex" is not predictive of any of the other tooth movements investigated in this section. In this sample (left second molar), the sample of females experienced less second molar movement than males. Mean mesial movement was $3.8 \mathrm{~mm}(\mathrm{sd}=1.7 \mathrm{~mm})$ in girls and $4.8 \mathrm{~mm}$ 
Table 34. Results of stepwise multiple regression analysis with the intreatment change in the maxillary left first molar (distal) as the dependent variable. ${ }^{1}$

\begin{tabular}{lrrl}
\hline \multicolumn{1}{c}{ Variable } & F Ratio & P Value & Model \\
\hline Incisor Overjet & 1.92 & 0.1693 & \\
Incisor Overbite & 7.77 & 0.0066 & Entered \\
Incisor Irregularity & 16.39 & 0.0001 & Entered \\
Incisor Spacing & 0.16 & 0.6888 & \\
Max 3-3 Width & 0.44 & 0.5096 & \\
Max 6-6 Width & 0.17 & 0.6803 & \\
Mand 3-3 Width & 0.01 & 0.9391 & \\
Mand 6-6 Width & 0.29 & 0.5935 & \\
Mx-Md 3-3 Difference & 0.89 & 0.3483 & \\
Mx-Md 6-6 Difference & 0.00 & 0.9494 & \\
Mx 1-3 Chord & 0.45 & 0.5037 & \\
Mx 1-6 Chord & 1.00 & 0.3210 & \\
Md 1-3 Chord & 0.10 & 0.7486 & \\
Md 1-6 Chord & 0.07 & 0.7895 & \\
Midline Discrepancy & 0.02 & 0.8880 & \\
Buccal Segment Relation & 0.22 & 0.6440 & \\
Canine Relation & 9.34 & 0.0030 & Entered \\
Extraction Pattern & 8.72 & 0.0041 & Entered \\
Subject's Sex & 6.54 & 0.0124 & \\
Ext-x-Sex Interaction & 0.08 & 0.7752 & \\
\hline
\end{tabular}

1Step history:

$\begin{array}{clc}\text { Step } & \text { Parameter } & \text { R-Squared } \\ 1 & \text { Incisor Irregularity } & 0.1014 \\ 2 & \text { Canine Relation } & 0.1803 \\ 3 & \text { Incisor Overbite } & 0.3396 \\ 4 & \text { Subject's Sex } & 0.3396\end{array}$


Table 35. Results of stepwise multiple regression analysis with the intreatment change in the maxillary right first molar (distal) as the dependent variable. ${ }^{1}$

\begin{tabular}{lccc}
\hline \multicolumn{1}{c}{ Variable } & F Ratio & P Value & Model \\
\hline Incisor Overjet & 1.77 & 0.1868 & \\
Incisor Overbite & 5.24 & 0.0247 & Entered \\
Incisor Irregularity & 7.35 & 0.0082 & Entered \\
Incisor Spacing & 0.57 & 0.4533 & \\
Max 3-3 Width & 1.96 & 0.1651 & \\
Max 6-6 Width & 1.45 & 0.2327 & \\
Mand 3-3 Width & 0.73 & 0.3971 & \\
Mand 6-6 Width & 0.03 & 0.8718 & \\
Mx-Md 3-3 Difference & 0.94 & 0.3349 & \\
Mx-Md 6-6 Difference & 3.52 & 0.0642 & \\
Mx 1-3 Chord & 2.48 & 0.1195 & \\
Mx 1-6 Chord & 13.17 & 0.0005 & Entered \\
Md 1-3 Chord & 1.00 & 0.3200 & \\
Md 1-6 Chord & 1.60 & 0.2093 & \\
Midline Discrepancy & 8.85 & 0.0039 & Entered \\
Buccal Segment Relation & 0.64 & 0.4280 & \\
Canine Relation & 4.09 & 0.0464 & Entered \\
Extraction Pattern & 8.31 & 0.0050 & Entered \\
Subject's Sex & 2.74 & 0.1015 & \\
Ext-x-Sex Interaction & 1.82 & 0.1680 & \\
\hline
\end{tabular}

1Step history:

$\begin{array}{clc}\text { Step } & \text { Parameter } & \text { R-Squared } \\ 1 & \text { Mx 1-6 Chord } & 0.1542 \\ 2 & \text { Midline Discrepancy } & 0.2316 \\ 3 & \text { Incisor Irregularity } & 0.2833 \\ 4 & \text { Extraction Pattern } & 0.3361 \\ 5 & \text { Incisor Overbite } & 0.3764\end{array}$




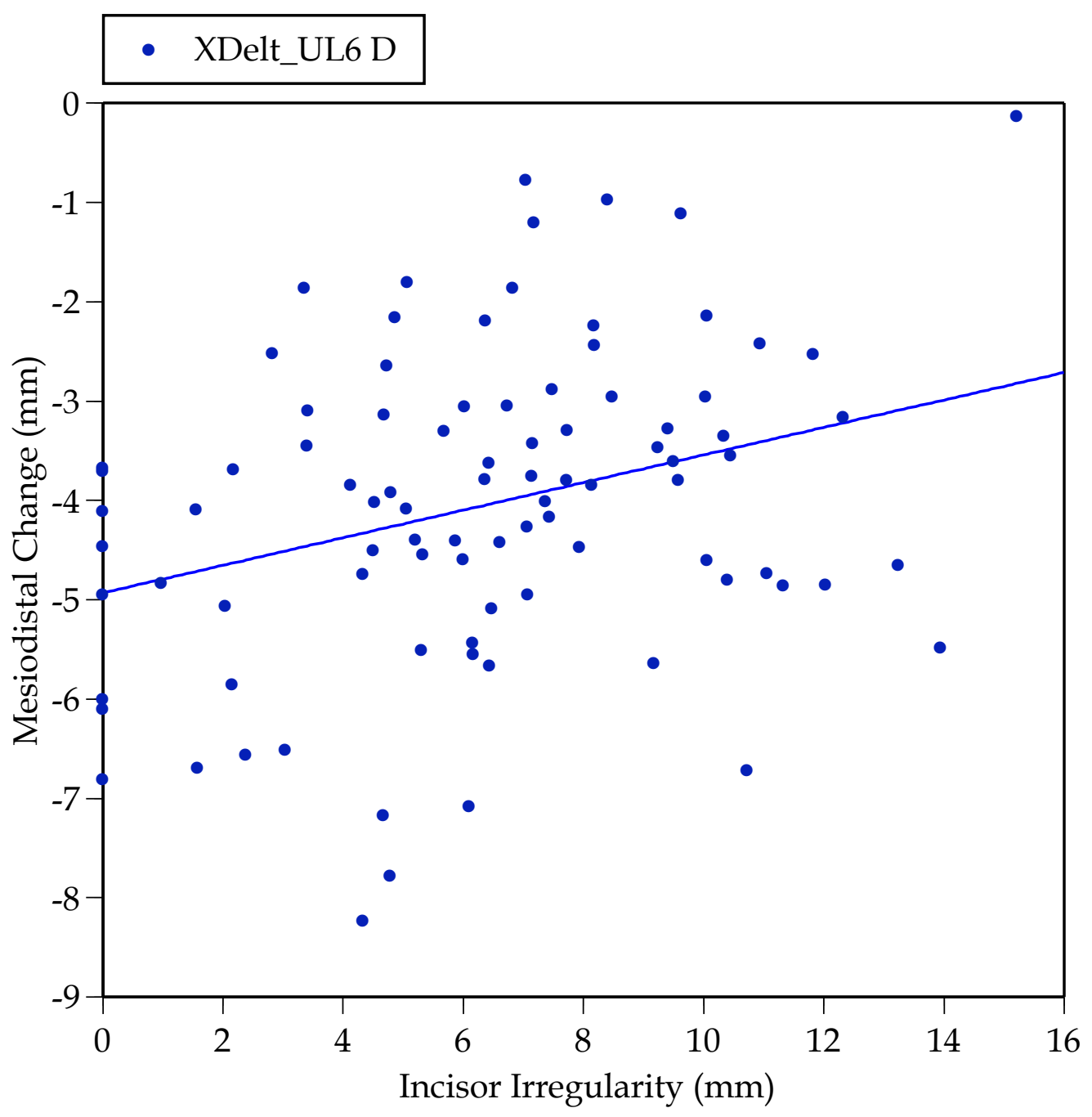

Fig. 49. Scatterplot showing the positive association between pretreatment incisor irregularity and the amount of mesial movement of the maxillary left first molar (distal). 


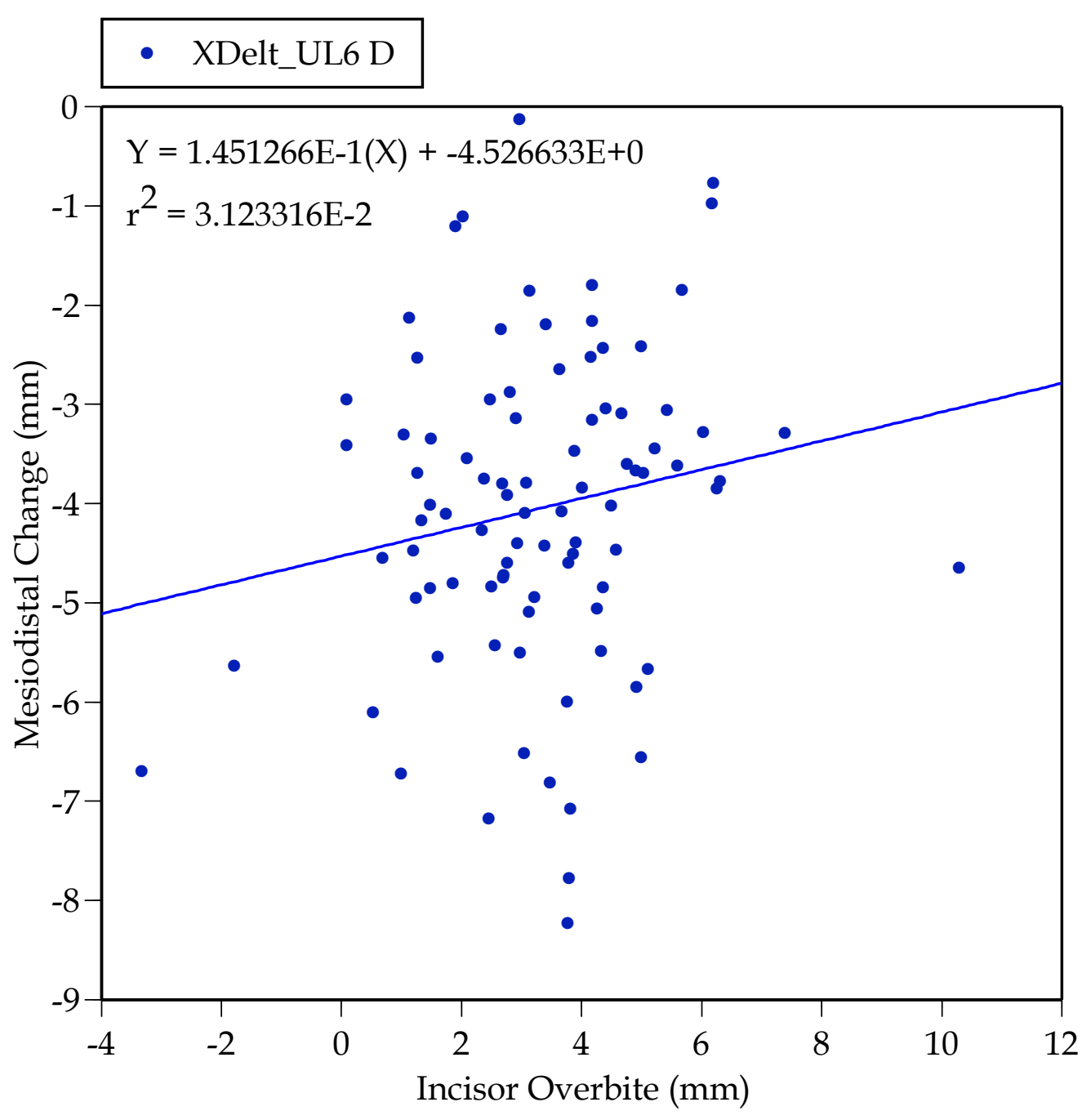

Fig. 50. Scatterplot showing the positive association between pretreatment overbite and the amount of mesial movement of the maxillary left first molar (distal). 
Table 36. Results of stepwise multiple regression analysis with the intreatment change in the maxillary left second molar (mesial) as the dependent variable. ${ }^{1}$

\begin{tabular}{lccc}
\hline \multicolumn{1}{c}{ Variable } & F Ratio & P Value & Model \\
\hline Incisor Overjet & 0.21 & 0.6485 & \\
Incisor Overbite & 6.52 & 0.0127 & Entered \\
Incisor Irregularity & 5.68 & 0.0197 & Entered \\
Incisor Spacing & 1.75 & 0.1894 & \\
Max 3-3 Width & 0.57 & 0.4529 & \\
Max 6-6 Width & 1.21 & 0.2756 & \\
Mand 3-3 Width & 0.01 & 0.9316 & \\
Mand 6-6 Width & 0.41 & 0.5231 & \\
Mx-Md 3-3 Difference & 1.16 & 0.2854 & \\
Mx-Md 6-6 Difference & 0.90 & 0.3453 & \\
Mx 1-3 Chord & 0.60 & 0.4418 & \\
Mx 1-6 Chord & 0.20 & 0.6545 & \\
Md 1-3 Chord & 0.01 & 0.9241 & \\
Md 1-6 Chord & 0.15 & 0.6960 & \\
Midline Discrepancy & 0.00 & 0.9921 & \\
Buccal Segment Relation & 0.17 & 0.6857 & \\
Canine Relation & 3.92 & 0.0513 & \\
Extraction Pattern & 8.98 & 0.0037 & Entered \\
Subject's Sex & 7.54 & 0.0075 & Entered \\
Ext-x-Sex Interaction & 0.11 & 0.7363 & \\
\hline
\end{tabular}

1Step history:

$\begin{array}{clc}\text { Step } & \text { Parameter } & \text { R-Squared } \\ 1 & \text { Subject's Sex } & 0.0712 \\ 2 & \text { Incisor Overbite } & 0.1209 \\ 3 & \text { Extraction Pattern } & 0.1839 \\ 4 & \text { Incisor Irregularity } & 0.2407\end{array}$


Table 37. Results of stepwise multiple regression analysis with the intreatment change in the maxillary right second molar (mesial) as the dependent variable. ${ }^{1}$

\begin{tabular}{lccc}
\hline \multicolumn{1}{c}{ Variable } & F Ratio & P Value & Model \\
\hline Incisor Overjet & 0.65 & 0.4238 & \\
Incisor Overbite & 1.87 & 0.1758 & \\
Incisor Irregularity & 2.42 & 0.1237 & \\
Incisor Spacing & 0.00 & 0.9884 & \\
Max 3-3 Width & 0.41 & 0.5242 & \\
Max 6-6 Width & 1.84 & 0.1794 & \\
Mand 3-3 Width & 0.11 & 0.7363 & \\
Mand 6-6 Width & 0.00 & 0.9939 & \\
Mx-Md 3-3 Difference & 0.20 & 0.6537 & \\
Mx-Md 6-6 Difference & 3.79 & 0.0552 & \\
Mx 1-3 Chord & 0.72 & 0.4000 & \\
Mx 1-6 Chord & 15.11 & 0.0002 & Entered \\
Md 1-3 Chord & 0.34 & 0.5598 & \\
Md 1-6 Chord & 1.03 & 0.3139 & \\
Midline Discrepancy & 5.48 & 0.0218 & Entered \\
Buccal Segment Relation & 0.19 & 0.6620 & \\
Canine Relation & 2.20 & 0.1419 & \\
Extraction Pattern & 4.61 & 0.0350 & \\
Subject's Sex & 0.95 & 0.3324 & \\
Ext-x-Sex Interaction & 2.58 & 0.0598 & \\
\hline
\end{tabular}

1Step history:

\begin{tabular}{clc} 
Step & \multicolumn{1}{c}{ Parameter } & R-Squared \\
1 & Mx 1-6 Chord & 0.1254 \\
2 & Midline Discrepancy & 0.1828
\end{tabular}


$(\mathrm{sd}=1.6 \mathrm{~mm})$ in boys, which is a significant difference by ANOVA $(\mathrm{F}=6.5 ; \mathrm{P}$

$=0.0130)$.

For the right landmark (Table 37), maxillary 1-6 chord accounted for $12 \%$ of the variance in mesiodistal molar movement in the multivariate model. The relationship (Fig. 51) is that cases with long 1-6 arch chord underwent less second molar movement. This seems to occur because a long arch chord reflects incisor proclination and procumbency (and a long-tapered arch form) and most of the premolar space is used to correct problems in the anterior segment rather than "burning anchorage."

Predictive models for the distal landmarks of the second molars (Tables $38,39)$ both disclose overbite as the prime correlate of mesiodistal movement, though the explained variance is low $\left(\mathrm{r}^{2}=5 \%\right)$. As graphed in Fig. 52, cases with deep bites underwent less second molar movements; cases with shallow (or negative) overbites experienced more mesial movement of the second molar (distal).

\section{First Premolar Extractions}

The prior section described the analysis of the overall sample, both the cases treated with first- and with second-premolars. "Extraction" was included in the statistical models to account for differences in the two groups. Still, the patterns and/or intensities of the relationships may not be the same 


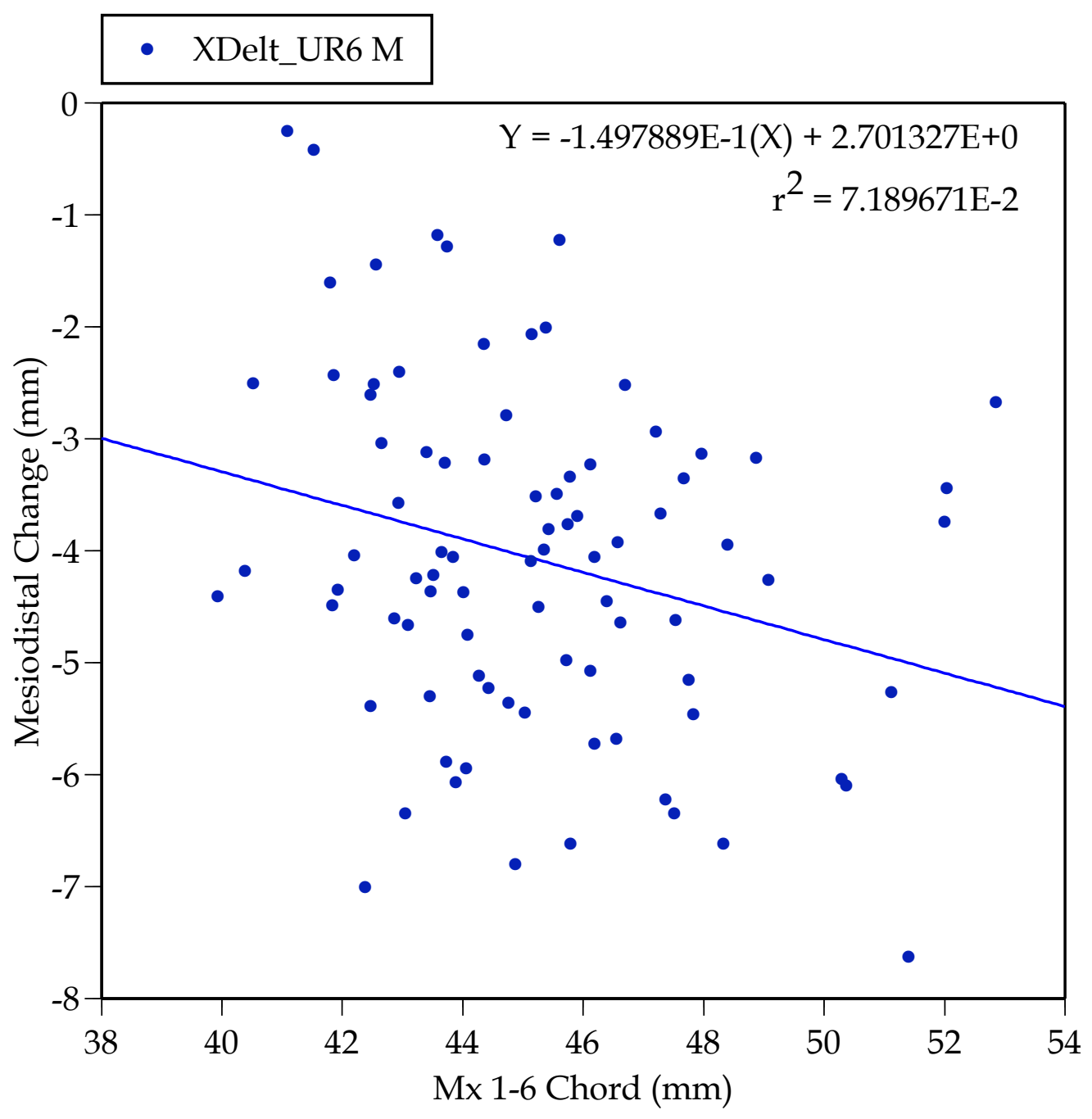

Fig. 51. Scatterplot showing the positive association between pretreatment arch chord (1-6) and the amount of mesiodistal movement of the maxillary left second molar (mesial). 
Table 38. Results of stepwise multiple regression analysis with the intreatment change in the maxillary left second molar (distal) as the dependent variable. ${ }^{1}$

\begin{tabular}{lccc}
\hline \multicolumn{1}{c}{ Variable } & F Ratio & P Value & Model \\
\hline Incisor Overjet & 0.03 & 0.8700 & \\
Incisor Overbite & 7.20 & 0.0089 & Entered \\
Incisor Irregularity & 4.47 & 0.0377 & \\
Incisor Spacing & 0.49 & 0.4874 & \\
Max 3-3 Width & 1.22 & 0.2728 & \\
Max 6-6 Width & 0.05 & 0.8268 & \\
Mand 3-3 Width & 0.01 & 0.9293 & \\
Mand 6-6 Width & 0.07 & 0.7968 & \\
Mx-Md 3-3 Difference & 1.88 & 0.1748 & \\
Mx-Md 6-6 Difference & 0.00 & 0.9995 & \\
Mx 1-3 Chord & 0.00 & 0.9850 & \\
Mx 1-6 Chord & 0.04 & 0.8387 & \\
Md 1-3 Chord & 0.21 & 0.6469 & \\
Md 1-6 Chord & 0.03 & 0.8736 & \\
Midline Discrepancy & 0.65 & 0.4224 & \\
Buccal Segment Relation & 0.31 & 0.5767 & \\
Canine Relation & 2.62 & 0.1097 & \\
Extraction Pattern & 7.63 & 0.0072 & Entered \\
Subject's Sex & 3.85 & 0.0533 & \\
Ext-x-Sex Interaction & 0.09 & 0.7709 & \\
\hline
\end{tabular}

1Step history:

$\begin{array}{clc}\text { Step } & \text { Parameter } & \text { R-Squared } \\ 1 & \text { Incisor Overbite } & 0.0483 \\ 2 & \text { Extraction Pattern } & 0.0969 \\ 3 & \text { Subject's Sex } & 0.1525 \\ 4 & \text { Incisor Irregularity } & 0.1996\end{array}$


Table 39. Results of stepwise multiple regression analysis with the intreatment change in the maxillary right second molar (distal) as the dependent variable. ${ }^{1}$

\begin{tabular}{lccc}
\hline \multicolumn{1}{c}{ Variable } & F Ratio & P Value & Model \\
\hline Incisor Overjet & 1.68 & 0.1986 & \\
Incisor Overbite & 4.34 & 0.0405 & Entered \\
Incisor Irregularity & 4.12 & 0.0458 & \\
Incisor Spacing & 3.07 & 0.0838 & \\
Max 3-3 Width & 0.05 & 0.8319 & \\
Max 6-6 Width & 0.71 & 0.4008 & \\
Mand 3-3 Width & 1.11 & 0.2964 & \\
Mand 6-6 Width & 1.78 & 0.1860 & \\
Mx-Md 3-3 Difference & 0.57 & 0.4521 & \\
Mx-Md 6-6 Difference & 0.15 & 0.7013 & \\
Mx 1-3 Chord & 1.51 & 0.2234 & \\
Mx 1-6 Chord & 3.48 & 0.0660 & \\
Md 1-3 Chord & 1.90 & 0.1721 & \\
Md 1-6 Chord & 1.21 & 0.2757 & \\
Midline Discrepancy & 1.12 & 0.2942 & \\
Buccal Segment Relation & 0.65 & 0.4233 & \\
Canine Relation & 1.33 & 0.2524 & \\
Extraction Pattern & 1.46 & 0.2299 & \\
Subject's Sex & 3.17 & 0.0787 & \\
Ext-x-Sex Interaction & 2.68 & 0.0528 & \\
\hline
\end{tabular}

1Step history:

$\begin{array}{ccc}\text { Step } & \text { Parameter } & \text { R-Squared } \\ 1 & \text { Incisor Overbite } & 0.0521\end{array}$




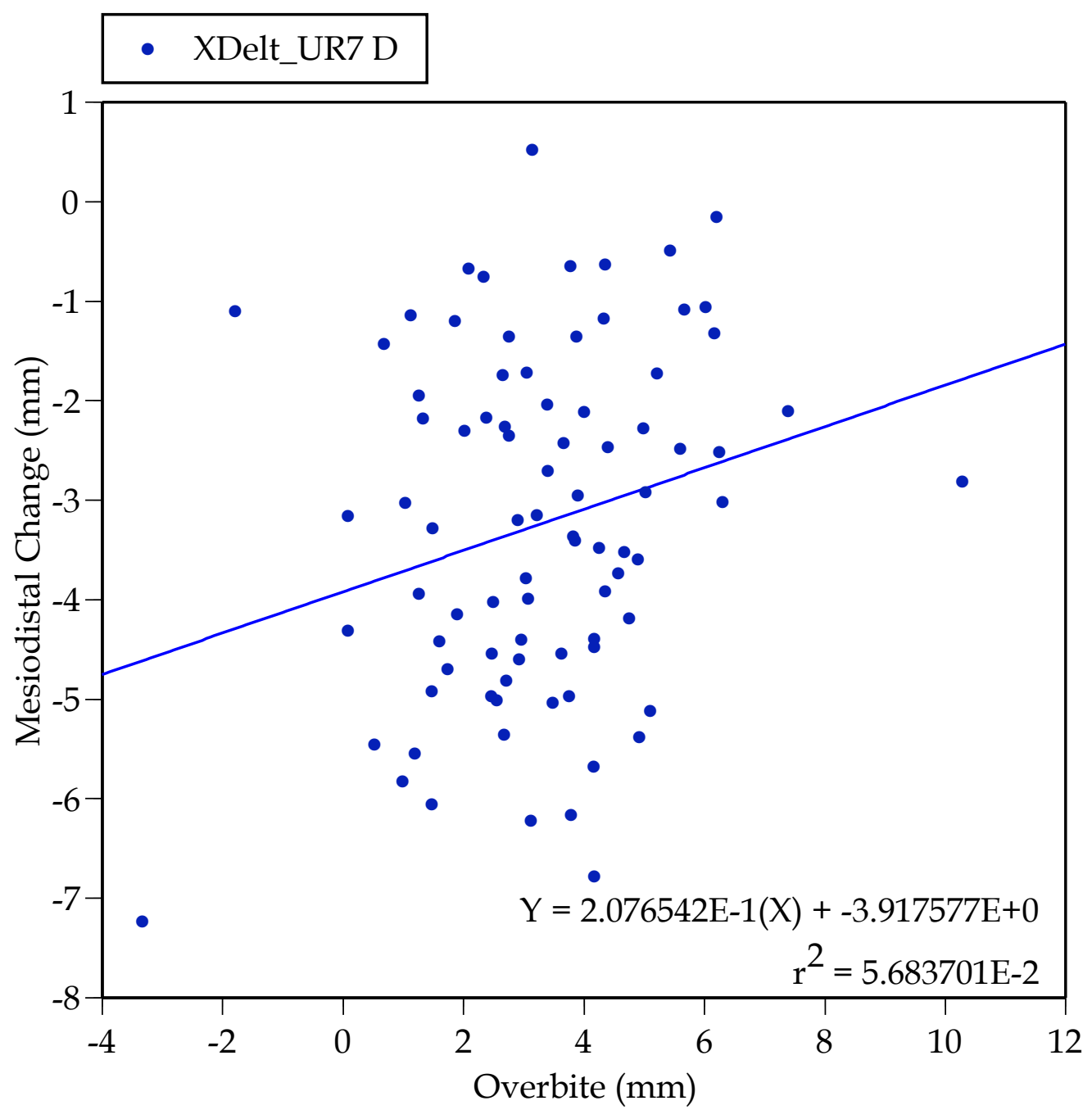

Fig. 52. Scatterplot showing the positive association between pretreatment incisor overbite and the amount of mesial movement of the maxillary right second molar (distal). 
in the two extraction patterns. The present section repeats the prior analysis, but just on the subsample of first-premolar extraction cases. Again, the stepwise linear regression models begin with the assessment of 17 occlusal variables measured from the pretreatment dental casts (Table 11) plus the patient's sex.

\section{Central Incisor}

Results for mesiodistal movement of the mesial landmarks on the central incisors are listed in Tables 40 (left quadrant) and 41 (right). Statistical results are concordant for these antimeric landmarks: the same three occlusal variables entered both models, namely overjet, spacing, and maxillary intercanine (3-3) width. Of these, interdental spacing among the incisors had the highest $\mathrm{r}^{2}(c a .20 \%)$ with the amounts of mesiodistal change in this landmark.

To recall, we measured Little's incisor irregularity index, but this approach does not distinguish between distances between the anatomic contacts due to crowding (often with torsiversion) and those due to spacing. We accounted for this by separately recording interdental spacing. Figs. A-3 and A-5 are examples of patients with spacing, and, while spacing is not common ( 7 cases out of 50), it is obvious from these examples that spacing is resolved by moving the incisors back into a smaller radius. The graph in 
Table 40. Results of stepwise linear regression predicting the amount of mesiodistal tooth movement from the pretreatment malocclusion: results for maxillary left central incisor (mesial) in the subset in whom first premolars were extracted.

\begin{tabular}{lrrrr}
\hline \multicolumn{1}{c}{ Variable } & $\begin{array}{c}\text { Sum of } \\
\text { Squares }\end{array}$ & F Ratio & P Value & $\mathrm{r}^{2}$ \\
\hline Incisor Overjet & 36.85 & 11.68 & $\underline{\mathbf{0 . 0 0 1 4}}$ & 0.1466 \\
Incisor Overbite & 0.16 & 0.05 & 0.8261 & \\
Irregularity Index & 3.39 & 1.08 & 0.3055 & \\
Incisor Spacing & 57.22 & 18.14 & $\underline{\mathbf{0 . 0 0 0 1}}$ & 0.1869 \\
Max 3-3 Width & 15.40 & 4.88 & $\underline{\mathbf{0 . 0 3 2 3}}$ & 0.0652 \\
Max 6-6 Width & 4.05 & 1.29 & 0.2616 & \\
Mand 3-3 Width & 1.77 & 0.56 & 0.4597 & \\
Mand 6-6 Width & 2.15 & 0.68 & 0.4154 & \\
UL 3-3 Width Difference & 1.77 & 0.56 & 0.4597 & \\
UL 6-6 Width Difference & 1.47 & 0.46 & 0.5013 & \\
Max 1-3 Chord & 5.77 & 1.86 & 0.1791 & \\
Max 1-6 Chord & 6.61 & 2.15 & 0.1497 & \\
Mand 1-3 Chord & 6.07 & 1.97 & 0.1679 & \\
Mand 1-6 Chord & 9.33 & 3.10 & 0.0854 & \\
Midline Deviation & 6.88 & 2.24 & 0.1416 & \\
Buccal Segment Relation & 6.52 & 2.12 & 0.1526 & \\
Canine Discrepancy & 5.19 & 1.67 & 0.2032 & \\
Patient's Sex & 0.02 & 0.01 & 0.9334 & \\
\hline
\end{tabular}


Table 41. Results of stepwise linear regression predicting the amount of mesiodistal tooth movement from the pretreatment malocclusion: results for maxillary right central incisor (mesial) in the subset in whom first premolars were extracted.

\begin{tabular}{lrrrr}
\hline \multicolumn{1}{c}{ Variable } & $\begin{array}{c}\text { Sum of } \\
\text { Squares }\end{array}$ & F Ratio & P Value & $\mathrm{r}^{2}$ \\
\hline Incisor Overjet & & & & \\
Incisor Overbite & 31.47 & 14.81 & $\underline{\mathbf{0 . 0 0 0 4}}$ & 0.1587 \\
Irregularity Index & 0.07 & 0.03 & 0.8598 & \\
Incisor Spacing & 1.46 & 0.68 & 0.4129 & \\
Max 3-3 Width & 67.42 & 31.73 & $\underline{\mathbf{0 . 0 0 0 0}}$ & 0.2089 \\
Max 6-6 Width & 32.82 & 15.44 & $\underline{\mathbf{0 . 0 0 0 3}}$ & 0.1501 \\
Mand 3-3 Width & 1.23 & 0.57 & 0.4536 & \\
Mand 6-6 Width & 0.55 & 0.26 & 0.6164 & \\
UL 3-3 Width Difference & 0.96 & 0.45 & 0.5066 & \\
UL 6-6 Width Difference & 0.55 & 0.26 & 0.6164 & \\
Max 1-3 Chord & 0.19 & 0.09 & 0.7711 & \\
Max 1-6 Chord & 0.89 & 0.41 & 0.5239 & \\
Mand 1-3 Chord & 0.71 & 0.33 & 0.5698 & \\
Mand 1-6 Chord & 2.92 & 1.39 & 0.2452 & \\
Midline Deviation & 0.94 & 0.44 & 0.5119 & \\
Buccal Segment Relation & 2.35 & 1.11 & 0.2984 & \\
Canine Discrepancy & 0.17 & 0.08 & 0.7799 & \\
Patient's Sex & 1.08 & 0.50 & 0.4823 & \\
\hline
\end{tabular}


Fig. 53 shows that the greater the spacing, the more these central incisor landmarks were repositioned distally.

Incisor overjet also was a significant predictor of central incisor retraction. As shown in Fig. 54, those with excessive overjets tended to experience the most central incisor retraction. Figs. A-17 and A-25 illustrate two such examples. At the other extreme, Fig. A-42 illustrates a case with only about $3 \mathrm{~mm}$ of overjet, and these incisors were effectively stationary during treatment.

The third significant predictor was maxillary intercanine width, which has a negative association with the amount of incisor retraction (Fig. 55). Looking at some of the extreme cases, Figs. A-66 and A-73 illustrate two cases with comparatively narrow intercanine widths, but with appreciable overjet and incisor crowding, so most of the first-premolar extraction space was used to retract the anterior segment, including the canines. Cases with broad 3-3 widths tend to have the canines blocked-out to the labial, and these tend to experience less incisor retraction (see, e.g., Figs. A-22 and A-76).

Mesiodistal changes in the lateral anatomic contact of the central incisors are shown in Tables 42 and 43. Three occlusal variables entered each model, but just two are held in common, namely overbite and maxillary 1-6 chord distance. 


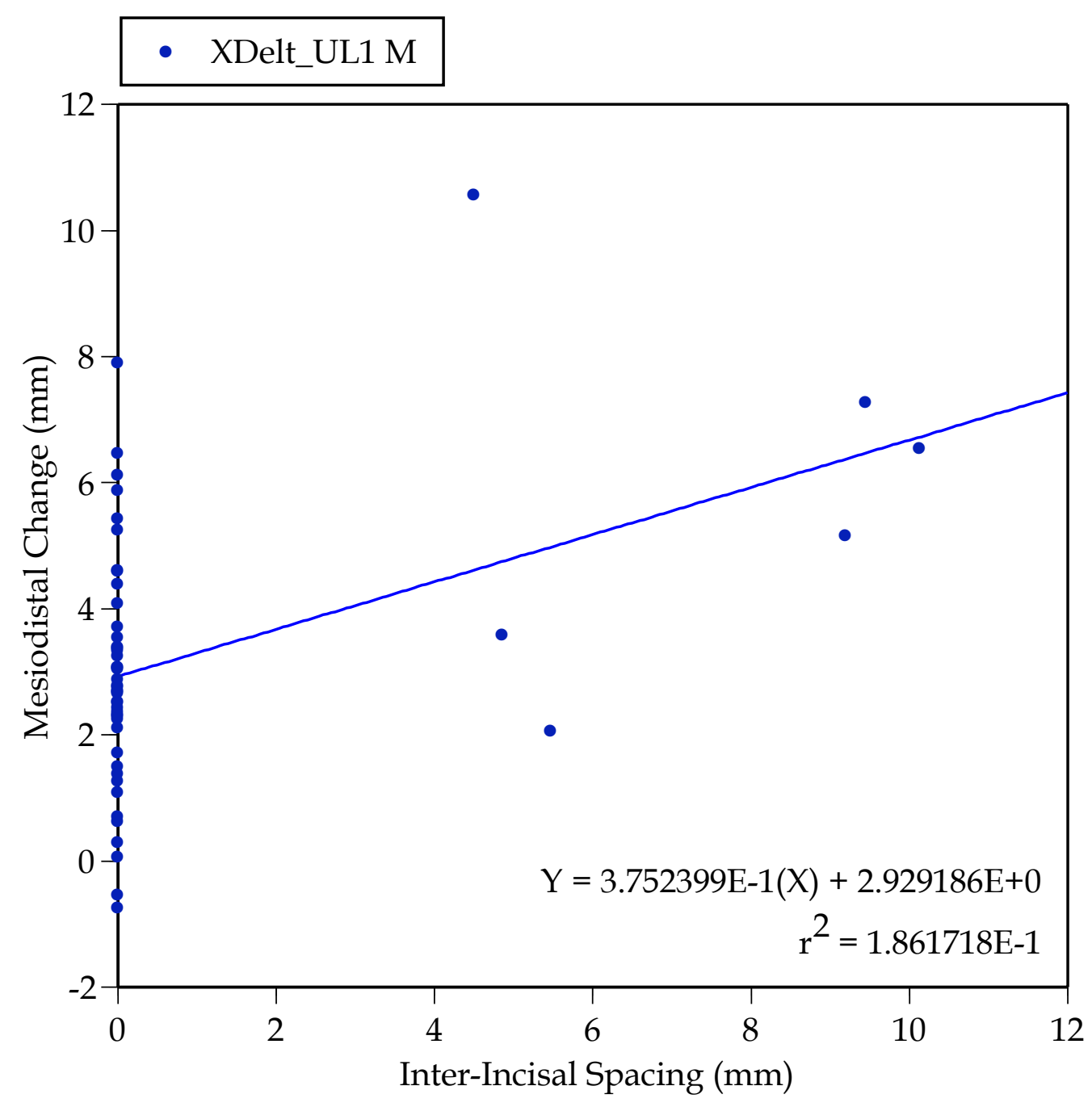

Fig. 53. Scatterplot showing the association between pretreatment spacing and the amount of mesiodistal change of the maxillary right central incisor (mesial) analyzed in just the first-premolar extraction sample. 


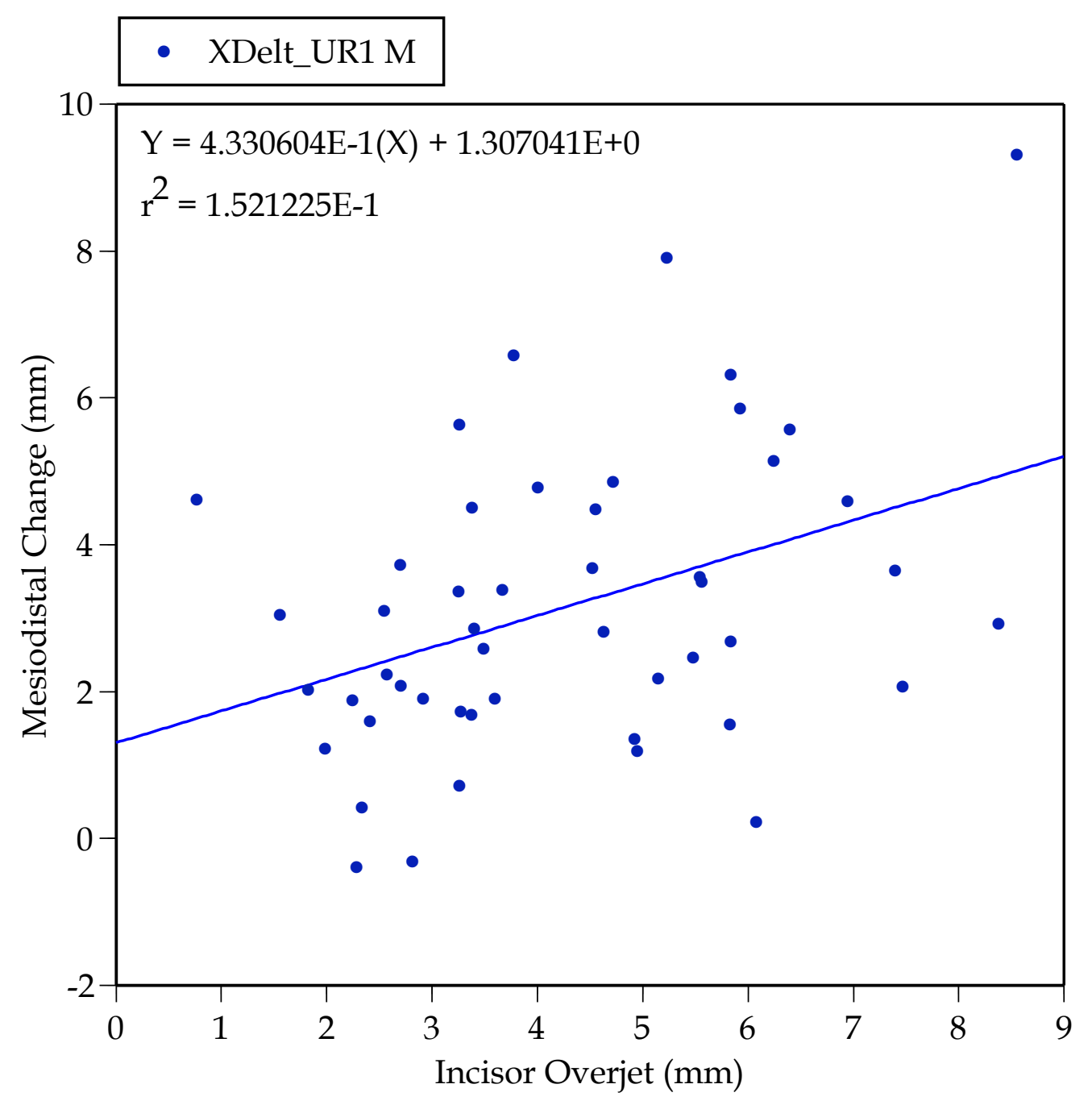

Fig. 54. Scatterplot showing the association between pretreatment overjet and the amount of mesiodistal change of the maxillary right central incisor (mesial) analyzed in just the first-premolar extraction sample. 


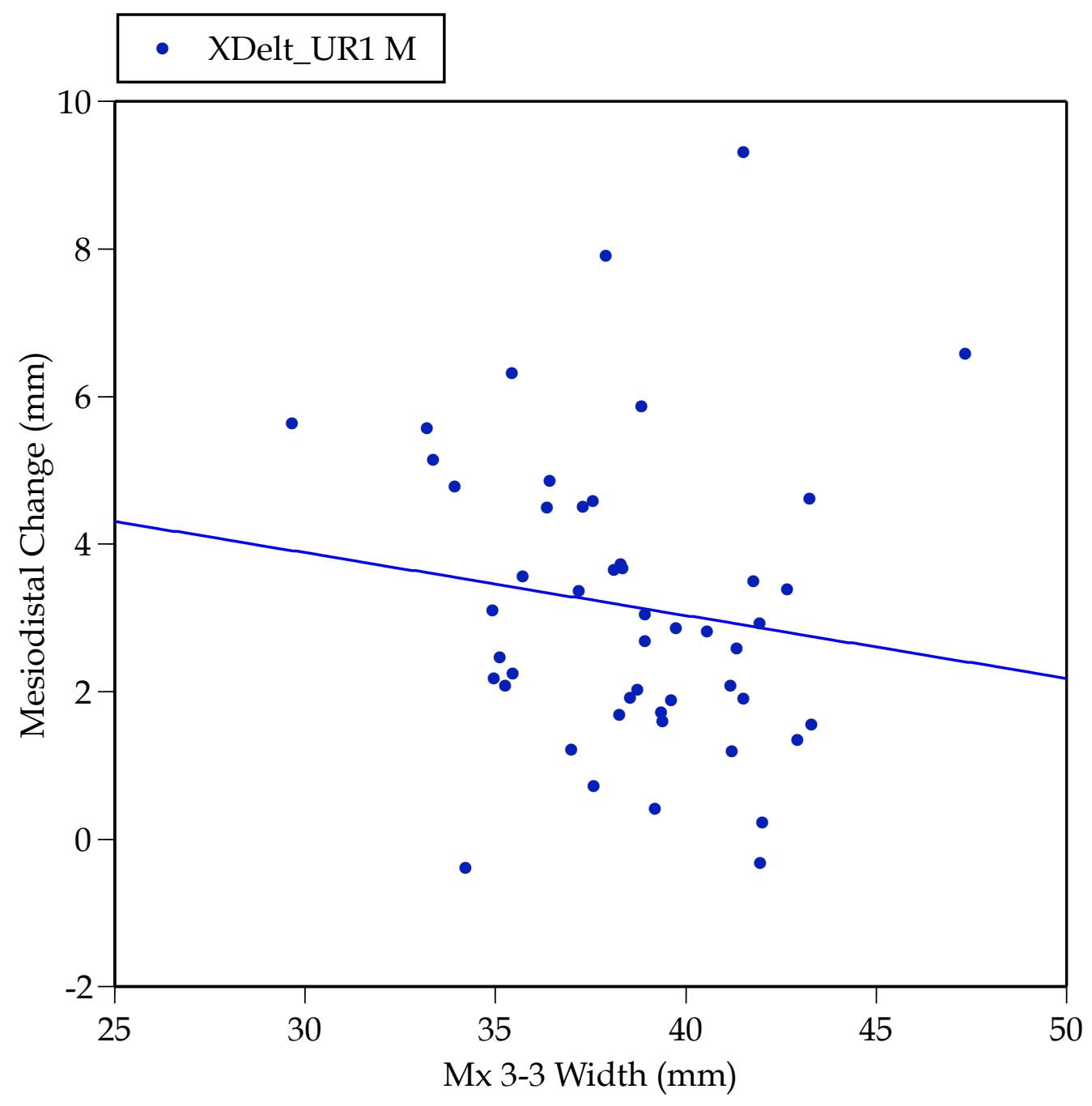

Fig. 55. Scatterplot showing the association between pretreatment intercanine width and the amount of mesiodistal change of the maxillary right central incisor (mesial) analyzed in just the first-premolar extraction sample. 
Table 42. Results of stepwise linear regression predicting the amount of mesiodistal tooth movement from the pretreatment malocclusion: results for maxillary left central incisor (distal) in the subset in whom first premolars were extracted.

\begin{tabular}{lrrrr}
\hline \multicolumn{1}{c}{ Variable } & $\begin{array}{c}\text { Sum of } \\
\text { Squares }\end{array}$ & F Ratio & P Value & $\mathrm{r}^{2}$ \\
\hline Incisor Overjet & & & & \\
Incisor Overbite & 1.89 & 1.16 & 0.2876 & \\
Irregularity Index & 30.39 & 18.54 & $\underline{\mathbf{0 . 0 0 0 1}}$ & 0.1422 \\
Incisor Spacing & 0.06 & 0.03 & 0.8544 & \\
Max 3-3 Width & 4.29 & 2.62 & 0.1130 & \\
Max 6-6 Width & 1.55 & 0.95 & 0.3360 & \\
Mand 3-3 Width & 1.04 & 0.63 & 0.4328 & \\
Mand 6-6 Width & 1.55 & 0.95 & 0.3360 & \\
UL 3-3 Width Difference & 0.39 & 0.23 & 0.6327 & \\
UL 6-6 Width Difference & 18.53 & 11.30 & $\underline{\mathbf{0 . 0 0 1 6}}$ & 0.1064 \\
Max 1-3 Chord & 4.82 & 3.08 & 0.0865 & \\
Max 1-6 Chord & 0.24 & 0.14 & 0.7084 & \\
Mand 1-3 Chord & 33.42 & 20.39 & $\underline{\mathbf{0 . 0 0 0 0}}$ & 0.2946 \\
Mand 1-6 Chord & 0.34 & 0.20 & 0.6558 & \\
Midline Deviation & 0.15 & 0.09 & 0.7649 & \\
Buccal Segment Relation & 0.51 & 0.30 & 0.5844 & \\
Canine Discrepancy & 0.10 & 0.06 & 0.8048 & \\
Patient's Sex & 1.21 & 0.73 & 0.3967 & \\
\hline
\end{tabular}


Table 43. Results of stepwise linear regression predicting the amount of mesiodistal tooth movement from the pretreatment malocclusion: results for maxillary right central incisor (distal) in the subset in whom first premolars were extracted.

\begin{tabular}{lrrrr}
\hline \multicolumn{1}{c}{ Variable } & $\begin{array}{c}\text { Sum of } \\
\text { Squares }\end{array}$ & F Ratio & P Value & $\mathrm{r}^{2}$ \\
\hline Incisor Overjet & & & & \\
Incisor Overbite & 31.39 & 1.68 & 0.2016 & \\
Irregularity Index & 1.29 & 12.05 & $\underline{\mathbf{0 . 0 0 1 2}}$ & 0.1207 \\
Incisor Spacing & 17.10 & 0.48 & 0.4922 & \\
Max 3-3 Width & 4.57 & 1.45 & $\underline{\mathbf{0 . 0 1 4 6}}$ & 0.2627 \\
Max 6-6 Width & 0.63 & 0.24 & 0.1927 & \\
Mand 3-3 Width & 0.11 & 0.04 & 0.8423 & \\
Mand 6-6 Width & 0.03 & 0.01 & 0.9186 & \\
UL 3-3 Width Difference & 4.25 & 1.62 & 0.2092 & \\
UL 6-6 Width Difference & 1.63 & 0.61 & 0.4399 & \\
Max 1-3 Chord & 10.15 & 4.09 & 0.0492 & \\
Max 1-6 Chord & 12.81 & 4.83 & $\underline{\mathbf{0 . 0 3 3 2}}$ & 0.0597 \\
Mand 1-3 Chord & 0.18 & 0.07 & 0.7975 & \\
Mand 1-6 Chord & 0.62 & 0.23 & 0.6350 & \\
Midline Deviation & 0.31 & 0.11 & 0.7369 & \\
Buccal Segment Relation & 0.97 & 0.36 & 0.5509 & \\
Canine Discrepancy & 0.95 & 0.35 & 0.5561 & \\
Patient's Sex & 6.97 & 2.73 & 0.1058 & \\
\hline
\end{tabular}


The relationship with overbite is shown in Fig. 56. Cases with deepbites tended to experience the greater amounts of incisor retraction, while those with shallow bites or frank openbites were treated with comparatively little change in central incisor position.

\section{Lateral Incisor}

Regression models for change in the mesiodistal axis of the medial contact of the lateral incisor are shown in Tables 44 and 45. Incisor irregularity is the single important predictor here. The greater the irregularity, the less the lateral incisors were moved mesiodistally. Inspection of the individual cases helps clarify this negative association. Where there is a lot of maxillary irregularity (e.g., Fig. A-33), the incisors commonly can be "unraveled" with torsiversions and comparatively minor tooth displacements. Conversely, when the incisors are already rather well aligned, they have to be retracted a fair amount to get proper coupling with the mandibular teeth. Figs. A-17 and A-25 are examples where irregularity was low but there was appreciable retraction of all four maxillary incisors during treatment. In passing, notice that overbite also was significantly predictive of incisor movement in the left quadrant (Table 44).

The two regression models for the distal landmark of UI2 are listed Tables 46 and 47, and the predominant predictor in both models is incisor 


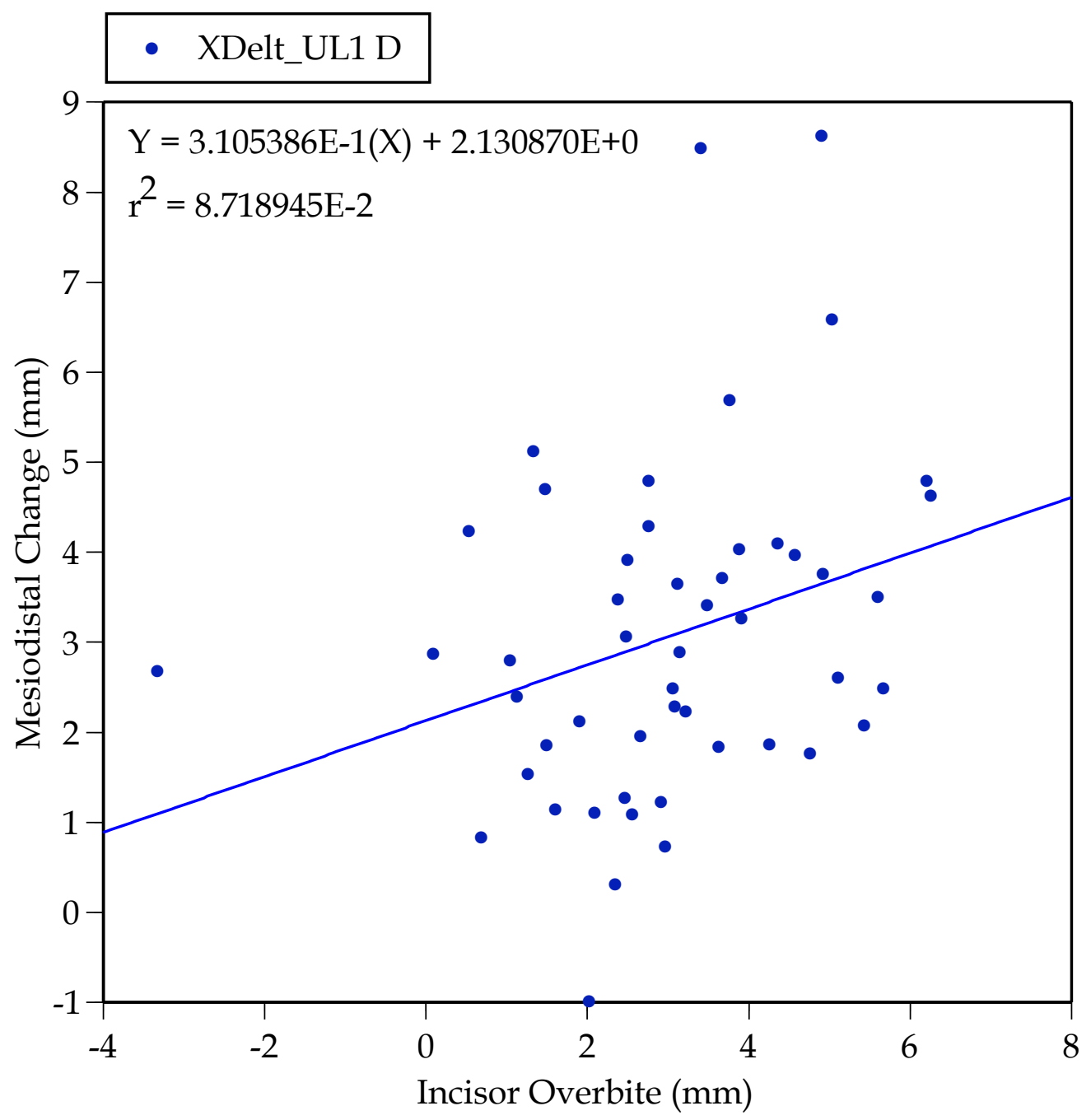

Fig. 56. Scatterplot showing the association between pretreatment overbite and the amount of mesiodistal change of the maxillary right central incisor (distal) analyzed in just the first-premolar extraction sample. 
Table 44. Results of stepwise linear regression predicting the amount of mesiodistal tooth movement from the pretreatment malocclusion: results for maxillary left lateral incisor (mesial) in the subset in whom first premolars were extracted.

\begin{tabular}{lrrrr}
\hline \multicolumn{1}{c}{ Variable } & $\begin{array}{c}\text { Sum of } \\
\text { Squares }\end{array}$ & F Ratio & P Value & $\mathrm{r}^{2}$ \\
\hline Incisor Overjet & 5.19 & 2.79 & 0.1017 & \\
Incisor Overbite & 9.58 & 4.95 & $\underline{\mathbf{0 . 0 3 1 0}}$ & 0.0616 \\
Irregularity Index & 51.50 & 26.64 & $\underline{\mathbf{0 . 0 0 0 0}}$ & 0.3666 \\
Incisor Spacing & 0.83 & 0.43 & 0.5171 & \\
Max 3-3 Width & 5.75 & 3.11 & 0.0845 & \\
Max 6-6 Width & 2.13 & 1.11 & 0.2985 & \\
Mand 3-3 Width & 0.60 & 0.31 & 0.5816 & \\
Mand 6-6 Width & 0.58 & 0.29 & 0.5910 & \\
UL 3-3 Width Difference & 5.47 & 2.95 & 0.0928 & \\
UL 6-6 Width Difference & 2.15 & 1.12 & 0.2962 & \\
Max 1-3 Chord & 0.27 & 0.14 & 0.7151 & \\
Max 1-6 Chord & 1.12 & 0.58 & 0.4523 & \\
Mand 1-3 Chord & 0.51 & 0.26 & 0.6119 & \\
Mand 1-6 Chord & 0.04 & 0.02 & 0.8844 & \\
Midline Deviation & 6.69 & 3.66 & 0.0621 & \\
Buccal Segment Relation & 1.05 & 0.54 & 0.4671 & \\
Canine Discrepancy & 4.94 & 2.65 & 0.1108 & \\
Patient's Sex & 3.19 & 1.67 & 0.2023 & \\
\hline
\end{tabular}


Table 45. Results of stepwise linear regression predicting the amount of mesiodistal tooth movement from the pretreatment malocclusion: results for maxillary right lateral incisor (mesial) in the subset in whom first premolars were extracted.

\begin{tabular}{lrrrr}
\hline \multicolumn{1}{c}{ Variable } & $\begin{array}{c}\text { Sum of } \\
\text { Squares }\end{array}$ & F Ratio & P Value & $\mathrm{r}^{2}$ \\
\hline Incisor Overjet & 0.47 & 0.18 & 0.6736 & \\
Incisor Overbite & 5.84 & 2.33 & 0.1337 & \\
Irregularity Index & 25.27 & 9.81 & $\underline{\mathbf{0 . 0 0 3 0}}$ & 0.1727 \\
Incisor Spacing & 5.28 & 2.10 & 0.1541 & \\
Max 3-3 Width & 0.97 & 0.37 & 0.5455 & \\
Max 6-6 Width & 0.28 & 0.11 & 0.7455 & \\
Mand 3-3 Width & 0.95 & 0.36 & 0.5498 & \\
Mand 6-6 Width & 0.39 & 0.15 & 0.7033 & \\
UL 3-3 Width Difference & 0.10 & 0.04 & 0.8493 & \\
UL 6-6 Width Difference & 0.00 & 0.00 & 0.9853 & \\
Max 1-3 Chord & 2.50 & 0.97 & 0.3295 & \\
Max 1-6 Chord & 0.98 & 0.38 & 0.5421 & \\
Mand 1-3 Chord & 0.27 & 0.10 & 0.7487 & \\
Mand 1-6 Chord & 0.67 & 0.26 & 0.6158 & \\
Midline Deviation & 0.06 & 0.02 & 0.8817 & \\
Buccal Segment Relation & 1.08 & 0.41 & 0.5232 & \\
Canine Discrepancy & 1.33 & 0.51 & 0.4775 & \\
Patient's Sex & 6.70 & 2.70 & 0.1074 & \\
\hline
\end{tabular}


Table 46. Results of stepwise linear regression predicting the amount of mesiodistal tooth movement from the pretreatment malocclusion: results for maxillary left lateral incisor (distal) in the subset in whom first premolars were extracted.

\begin{tabular}{lrrrr}
\hline \multicolumn{1}{c}{ Variable } & $\begin{array}{c}\text { Sum of } \\
\text { Squares }\end{array}$ & F Ratio & P Value & $\mathrm{r}^{2}$ \\
\hline Incisor Overjet & 0.19 & 0.12 & 0.7269 & \\
Incisor Overbite & 26.89 & 18.09 & $\underline{\mathbf{0 . 0 0 0 1}}$ & 0.2044 \\
Irregularity Index & 0.73 & 0.49 & 0.4884 & \\
Incisor Spacing & 0.00 & 0.00 & 0.9736 & \\
Max 3-3 Width & 2.33 & 1.59 & 0.2145 & \\
Max 6-6 Width & 0.16 & 0.10 & 0.7497 & \\
Mand 3-3 Width & 0.32 & 0.21 & 0.6464 & \\
Mand 6-6 Width & 0.16 & 0.10 & 0.7497 & \\
UL 3-3 Width Difference & 4.23 & 2.97 & 0.0917 & \\
UL 6-6 Width Difference & 6.99 & 4.71 & $\underline{\mathbf{0 . 0 3 5 4}}$ & 0.0584 \\
Max 1-3 Chord & 0.41 & 0.27 & 0.6044 & \\
Max 1-6 Chord & 7.66 & 5.15 & $\underline{\mathbf{0 . 0 2 8 1}}$ & 0.1790 \\
Mand 1-3 Chord & 0.02 & 0.01 & 0.9069 & \\
Mand 1-6 Chord & 0.04 & 0.03 & 0.8721 & \\
Midline Deviation & 2.09 & 1.42 & 0.2403 & \\
Buccal Segment Relation & 0.29 & 0.19 & 0.6630 & \\
Canine Discrepancy & 0.03 & 0.02 & 0.8805 & \\
Patient's Sex & 2.24 & 1.53 & 0.2234 & \\
\hline
\end{tabular}


Table 47. Results of stepwise linear regression predicting the amount of mesiodistal tooth movement from the pretreatment malocclusion: results for maxillary right lateral incisor (distal) in the subset in whom first premolars were extracted.

\begin{tabular}{lrccc}
\hline \multicolumn{1}{c}{ Variable } & $\begin{array}{c}\text { Sum of } \\
\text { Squares }\end{array}$ & F Ratio & P Value & $\mathrm{r}^{2}$ \\
\hline Incisor Overjet & 2.28 & 0.86 & 0.3575 & \\
Incisor Overbite & 24.67 & 9.37 & $\underline{\mathbf{0 . 0 0 3 7}}$ & 0.1395 \\
Irregularity Index & 1.45 & 0.55 & 0.4640 & \\
Incisor Spacing & 4.88 & 1.89 & 0.1763 & \\
Max 3-3 Width & 0.35 & 0.13 & 0.7187 & \\
Max 6-6 Width & 11.56 & 4.39 & $\mathbf{0 . 0 4 1 7}$ & 0.0750 \\
Mand 3-3 Width & 0.01 & 0.01 & 0.9457 & \\
Mand 6-6 Width & 1.00 & 0.37 & 0.5440 & \\
UL 3-3 Width Difference & 0.50 & 0.19 & 0.6685 & \\
UL 6-6 Width Difference & 1.00 & 0.37 & 0.5440 & \\
Max 1-3 Chord & 0.05 & 0.02 & 0.8966 & \\
Max 1-6 Chord & 0.03 & 0.01 & 0.9183 & \\
Mand 1-3 Chord & 0.05 & 0.02 & 0.8901 & \\
Mand 1-6 Chord & 0.03 & 0.01 & 0.9155 & \\
Midline Deviation & 2.13 & 0.81 & 0.3739 & \\
Buccal Segment Relation & 0.02 & 0.01 & 0.9288 & \\
Canine Discrepancy & 5.68 & 2.21 & 0.1439 & \\
Patient's Sex & 3.10 & 1.18 & 0.2830 & \\
\hline
\end{tabular}


overbite. Fig. 57 shows that cases with shallow overbites (e.g., Figs. A-9, A-72) experience little mesiodistal change in the lateral incisor, whereas those with deepbites (e.g., Figs. A-59, A-76) tended to undergo more incisor retraction.

\section{Canine}

Mesiodistal changes in the mesial and distal contact points of the canine can be discussed together (Tables 48-51) because the major determinant in each model is overbite. For completeness, all four bivariate associations are provided (Figs. 58-61); in each situation, cases with deep overbites underwent appreciable canine retraction, while those with shallow bites tended to undergo essentially no change along this parasagittal axis.

Examination of the two regression models for the distal contacts of the canines show that maxillary incisor irregularity also contributed significantly to explaining the variation in canine movement (Tables 50,51). The greater the irregularity, the larger the typical amount of canine retraction. This association tends to be intuitive, since the canines need to be moved back in the arch to create space for "unraveling" the incisor irregularity. 


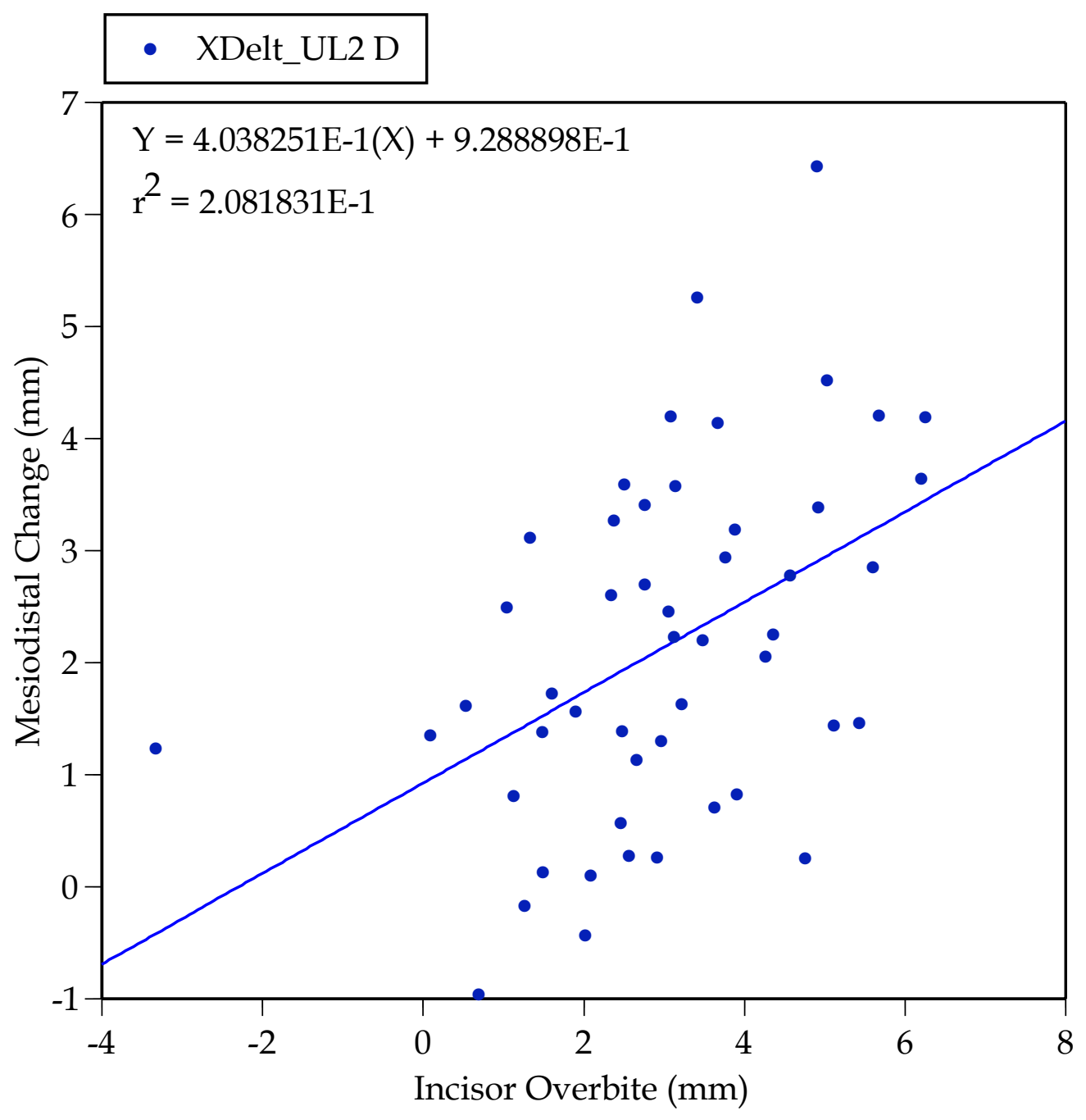

Fig. 57. Scatterplot showing the association between pretreatment overbite and the amount of mesiodistal change of the maxillary left lateral incisor (distall) analyzed in just the first-premolar extraction sample. 
Table 48. Results of stepwise linear regression predicting the amount of mesiodistal tooth movement from the pretreatment malocclusion: results for maxillary left canine (mesial) in the subset in whom first premolars were extracted.

\begin{tabular}{lrrrr}
\hline \multicolumn{1}{c}{ Variable } & $\begin{array}{c}\text { Sum of } \\
\text { Squares }\end{array}$ & F Ratio & P Value & $\mathrm{r}^{2}$ \\
\hline Incisor Overjet & 0.65 & 0.38 & 0.5390 & \\
Incisor Overbite & 23.66 & 14.18 & $\underline{\mathbf{0 . 0 0 0 5}}$ & 0.2318 \\
Irregularity Index & 5.14 & 3.23 & 0.0790 & \\
Incisor Spacing & 0.53 & 0.31 & 0.5794 & \\
Max 3-3 Width & 0.86 & 0.51 & 0.4787 & \\
Max 6-6 Width & 3.64 & 2.24 & 0.1411 & \\
Mand 3-3 Width & 1.15 & 0.68 & 0.4131 & \\
Mand 6-6 Width & 2.65 & 1.61 & 0.2111 & \\
UL 3-3 Width Difference & 5.04 & 3.16 & 0.0820 & \\
UL 6-6 Width Difference & 1.41 & 0.85 & 0.3627 & \\
Max 1-3 Chord & 0.87 & 0.52 & 0.4763 & \\
Max 1-6 Chord & 0.19 & 0.11 & 0.7382 & \\
Mand 1-3 Chord & 0.16 & 0.09 & 0.7625 & \\
Mand 1-6 Chord & 0.60 & 0.35 & 0.5549 & \\
Midline Deviation & 0.04 & 0.02 & 0.8860 & \\
Buccal Segment Relation & 0.37 & 0.22 & 0.6410 & \\
Canine Discrepancy & 1.58 & 0.94 & 0.3365 & \\
Patient's Sex & 2.16 & 1.30 & 0.2599 & \\
\hline
\end{tabular}


Table 49. Results of stepwise linear regression predicting the amount of mesiodistal tooth movement from the pretreatment malocclusion: results for maxillary right canine (mesial) in the subset in whom first premolars were extracted.

\begin{tabular}{lrrrr}
\hline \multicolumn{1}{c}{ Variable } & $\begin{array}{c}\text { Sum of } \\
\text { Squares }\end{array}$ & F Ratio & P Value & $\mathrm{r}^{2}$ \\
\hline Incisor Overjet & 3.85 & 1.64 & 0.2070 & \\
Incisor Overbite & 15.89 & 6.67 & $\underline{\mathbf{0 . 0 1 3 0}}$ & 0.1242 \\
Irregularity Index & 6.50 & 2.84 & 0.0990 & \\
Incisor Spacing & 0.19 & 0.08 & 0.7826 & \\
Max 3-3 Width & 0.06 & 0.02 & 0.8784 & \\
Max 6-6 Width & 0.30 & 0.12 & 0.7285 & \\
Mand 3-3 Width & 1.12 & 0.47 & 0.4982 & \\
Mand 6-6 Width & 0.33 & 0.14 & 0.7134 & \\
UL 3-3 Width Difference & 1.72 & 0.72 & 0.4011 & \\
UL 6-6 Width Difference & 0.03 & 0.01 & 0.9082 & \\
Max 1-3 Chord & 3.46 & 1.46 & 0.2324 & \\
Max 1-6 Chord & 0.94 & 0.39 & 0.5368 & \\
Mand 1-3 Chord & 0.58 & 0.24 & 0.6259 & \\
Mand 1-6 Chord & 0.03 & 0.01 & 0.9139 & \\
Midline Deviation & 1.75 & 0.73 & 0.3980 & \\
Buccal Segment Relation & 2.77 & 1.17 & 0.2854 & \\
Canine Discrepancy & 3.69 & 1.57 & 0.2171 & \\
Patient's Sex & 3.03 & 1.28 & 0.2642 & \\
\hline
\end{tabular}


Table 50. Results of stepwise linear regression predicting the amount of mesiodistal tooth movement from the pretreatment malocclusion: results for maxillary left canine (distal) in the subset in whom first premolars were extracted.

\begin{tabular}{lrrrr}
\hline \multicolumn{1}{c}{ Variable } & $\begin{array}{c}\text { Sum of } \\
\text { Squares }\end{array}$ & F Ratio & P Value & $\mathrm{r}^{2}$ \\
\hline Incisor Overjet & 0.61 & 0.45 & 0.5038 & \\
Incisor Overbite & 7.87 & 5.94 & $\underline{\mathbf{0 . 0 1 8 8}}$ & 0.0971 \\
Irregularity Index & 17.32 & 13.08 & $\underline{\mathbf{0 . 0 0 0 8}}$ & 0.0872 \\
Incisor Spacing & 0.00 & 0.00 & 0.9646 & \\
Max 3-3 Width & 0.00 & 0.00 & 0.9687 & \\
Max 6-6 Width & 0.00 & 0.00 & 0.9966 & \\
Mand 3-3 Width & 2.08 & 1.59 & 0.2138 & \\
Mand 6-6 Width & 0.25 & 0.19 & 0.6667 & \\
UL 3-3 Width Difference & 0.69 & 0.52 & 0.4757 & \\
UL 6-6 Width Difference & 0.27 & 0.20 & 0.6590 & \\
Max 1-3 Chord & 0.02 & 0.01 & 0.9078 & \\
Max 1-6 Chord & 0.67 & 0.50 & 0.4819 & \\
Mand 1-3 Chord & 0.60 & 0.44 & 0.5088 & \\
Mand 1-6 Chord & 10.68 & 8.07 & $\underline{\mathbf{0 . 0 0 6 8}}$ & 0.0799 \\
Midline Deviation & 0.00 & 0.00 & 0.9806 & \\
Buccal Segment Relation & 2.61 & 2.02 & 0.1627 & \\
Canine Discrepancy & 0.70 & 0.53 & 0.4724 & \\
Patient's Sex & 2.48 & 1.91 & 0.1739 & \\
\hline
\end{tabular}


Table 51. Results of stepwise linear regression predicting the amount of mesiodistal tooth movement from the pretreatment malocclusion: results for maxillary right canine (distal) in the subset in whom first premolars were extracted.

\begin{tabular}{lcccc}
\hline \multicolumn{1}{c}{ Variable } & $\begin{array}{c}\text { Sum of } \\
\text { Squares }\end{array}$ & F Ratio & P Value & $\mathrm{r}^{2}$ \\
\hline Incisor Overjet & 4.00 & 2.52 & 0.1196 & \\
Incisor Overbite & 8.18 & 4.98 & $\underline{\mathbf{0 . 0 3 0 5}}$ & 0.0891 \\
Irregularity Index & 9.85 & 6.00 & $\underline{\mathbf{0 . 0 1 8 2}}$ & 0.0884 \\
Incisor Spacing & 0.03 & 0.02 & 0.8985 & \\
Max 3-3 Width & 3.89 & 2.44 & 0.1253 & \\
Max 6-6 Width & 1.78 & 1.09 & 0.3025 & \\
Mand 3-3 Width & 2.62 & 1.62 & 0.2096 & \\
Mand 6-6 Width & 0.48 & 0.29 & 0.5928 & \\
UL 3-3 Width Difference & 1.18 & 0.71 & 0.4032 & \\
UL 6-6 Width Difference & 1.79 & 1.09 & 0.3015 & \\
Max 1-3 Chord & 0.01 & 0.01 & 0.9406 & \\
Max 1-6 Chord & 0.19 & 0.11 & 0.7392 & \\
Mand 1-3 Chord & 0.38 & 0.23 & 0.6354 & \\
Mand 1-6 Chord & 0.26 & 0.16 & 0.6958 & \\
Midline Deviation & 2.60 & 1.60 & 0.2122 & \\
Buccal Segment Relation & 2.93 & 1.81 & 0.1849 & \\
Canine Discrepancy & 4.64 & 2.94 & 0.0931 & \\
Patient's Sex & 1.50 & 0.91 & 0.3448 & \\
\hline
\end{tabular}




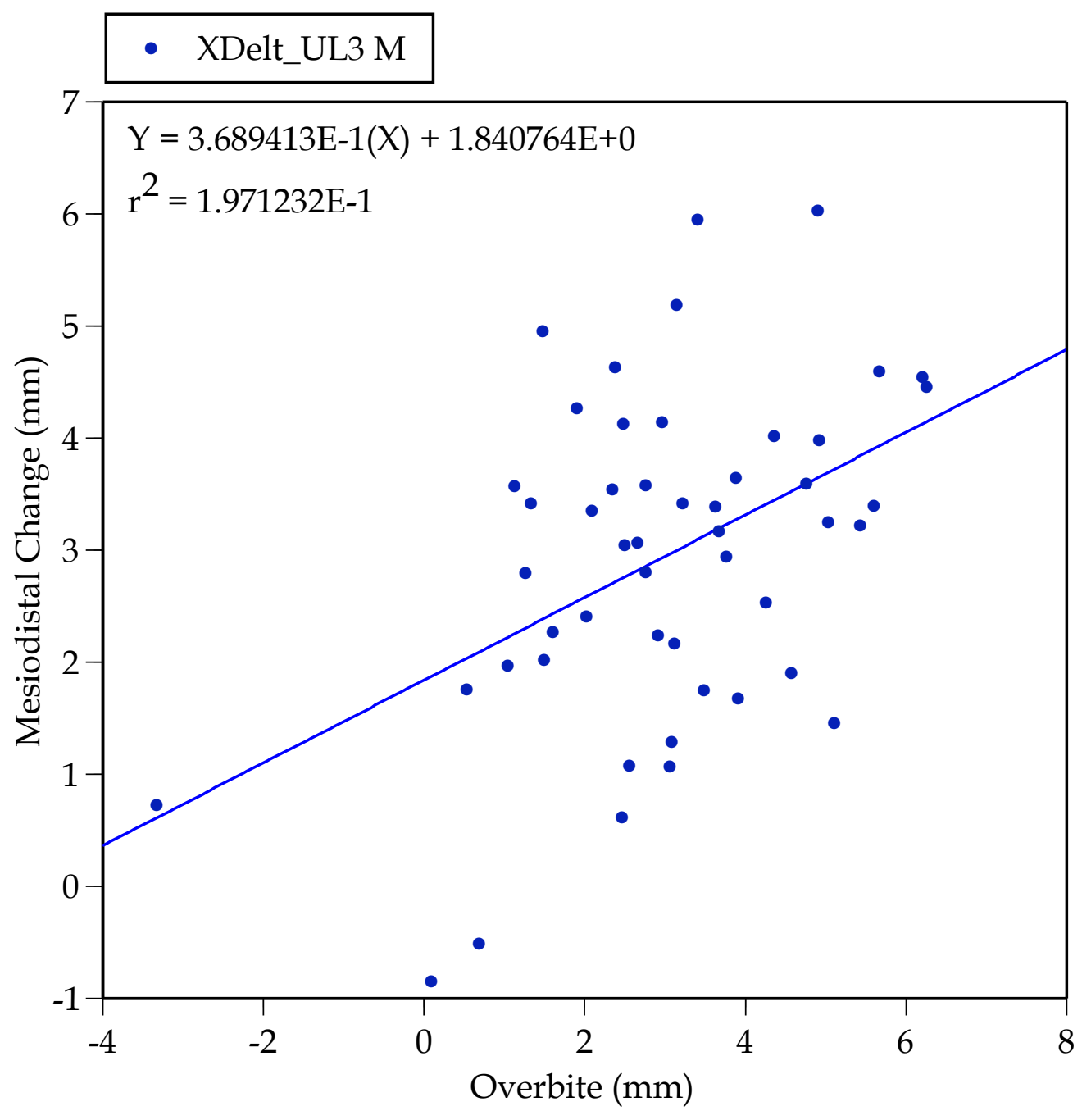

Fig. 58. Scatterplot showing the association between pretreatment spacing and the amount of mesiodistal change of the maxillary left canine (mesial) analyzed in just the firstpremolar extraction sample. 


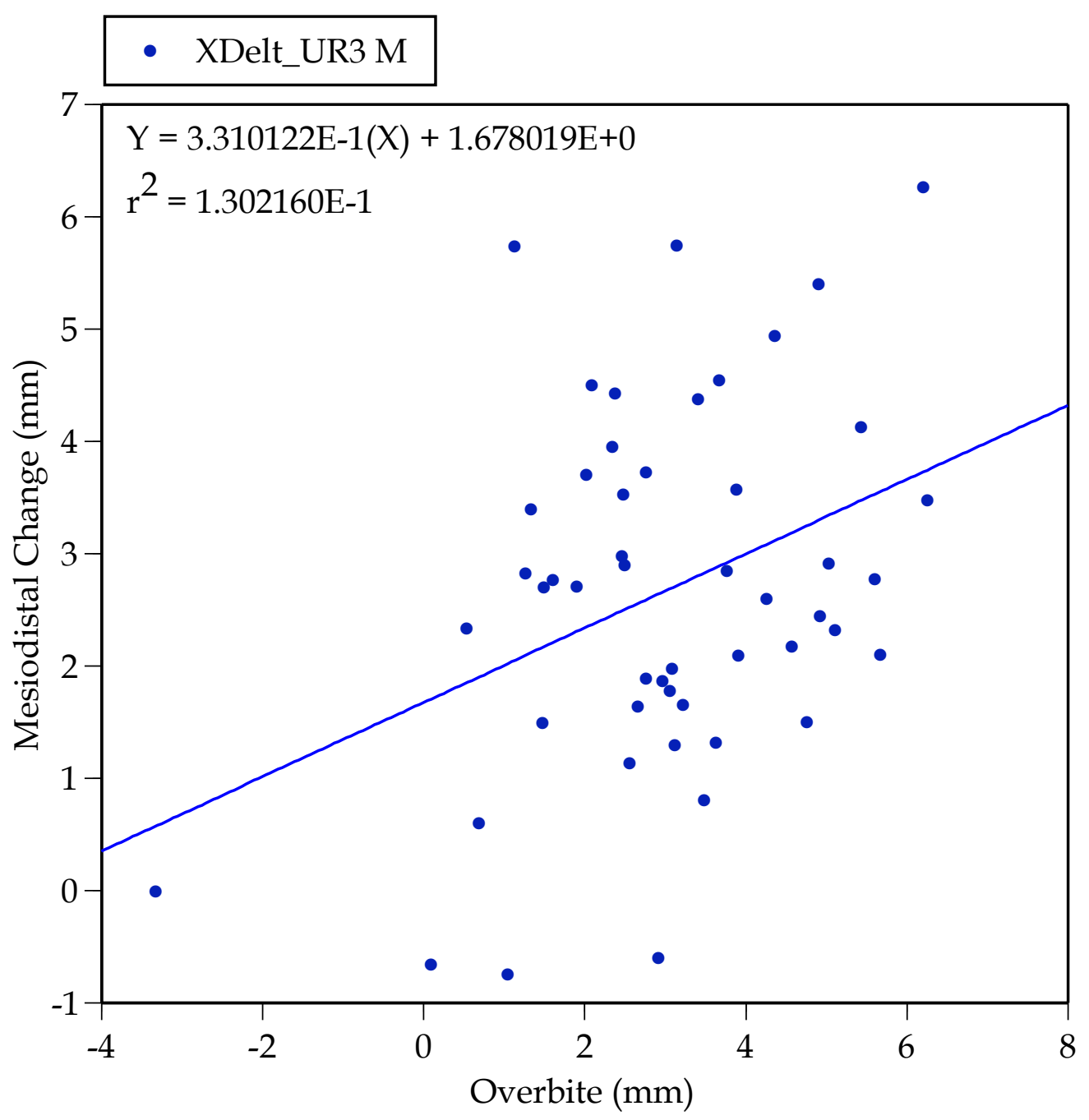

Fig. 59. Scatterplot showing the association between pretreatment spacing and the amount of mesiodistal change of the maxillary right canine (mesial) analyzed in just the firstpremolar extraction sample. 


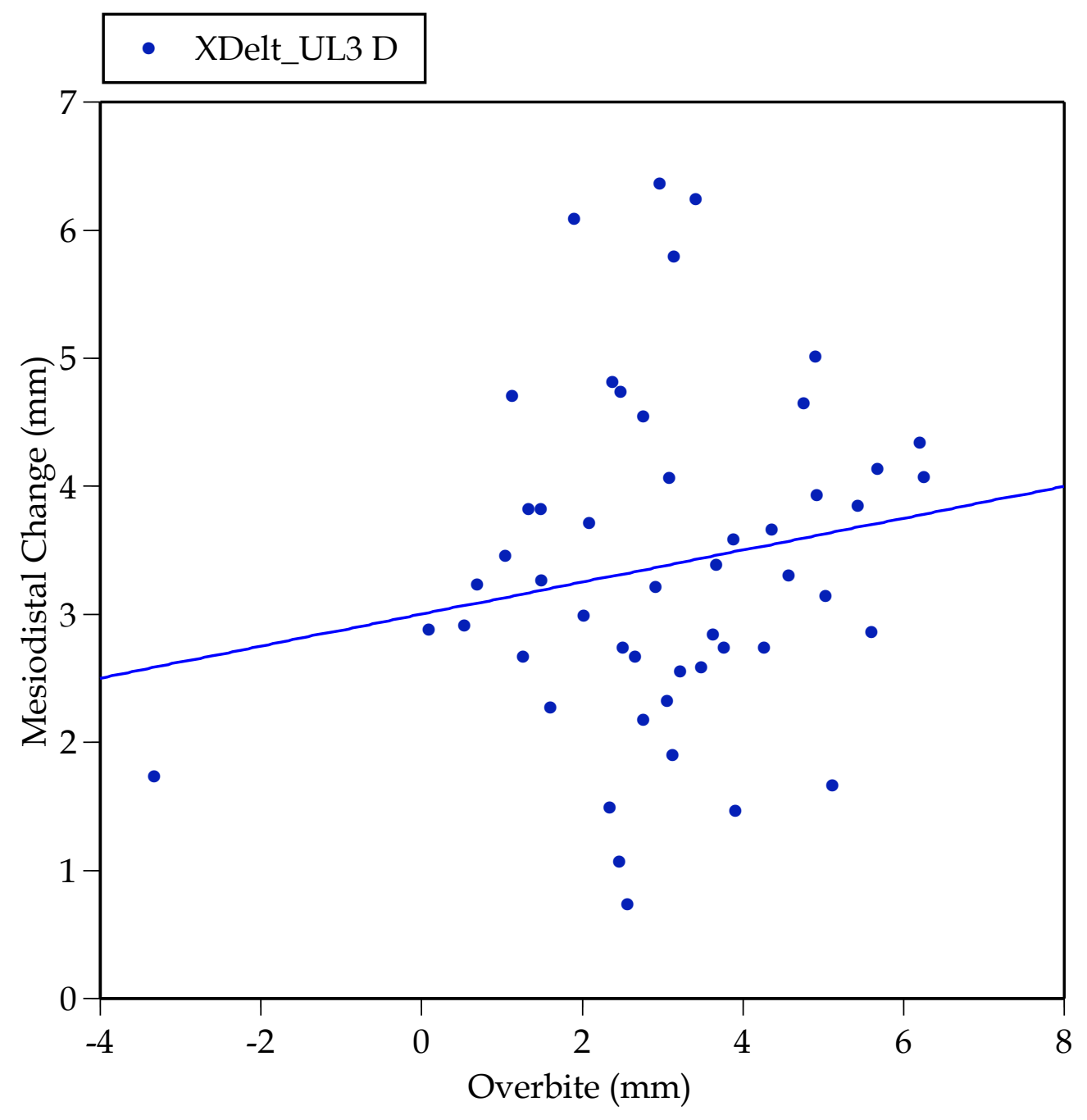

Fig. 60. Scatterplot showing the association between pretreatment spacing and the amount of mesiodistal change of the maxillary left canine (distal) analyzed in just the firstpremolar extraction sample. 


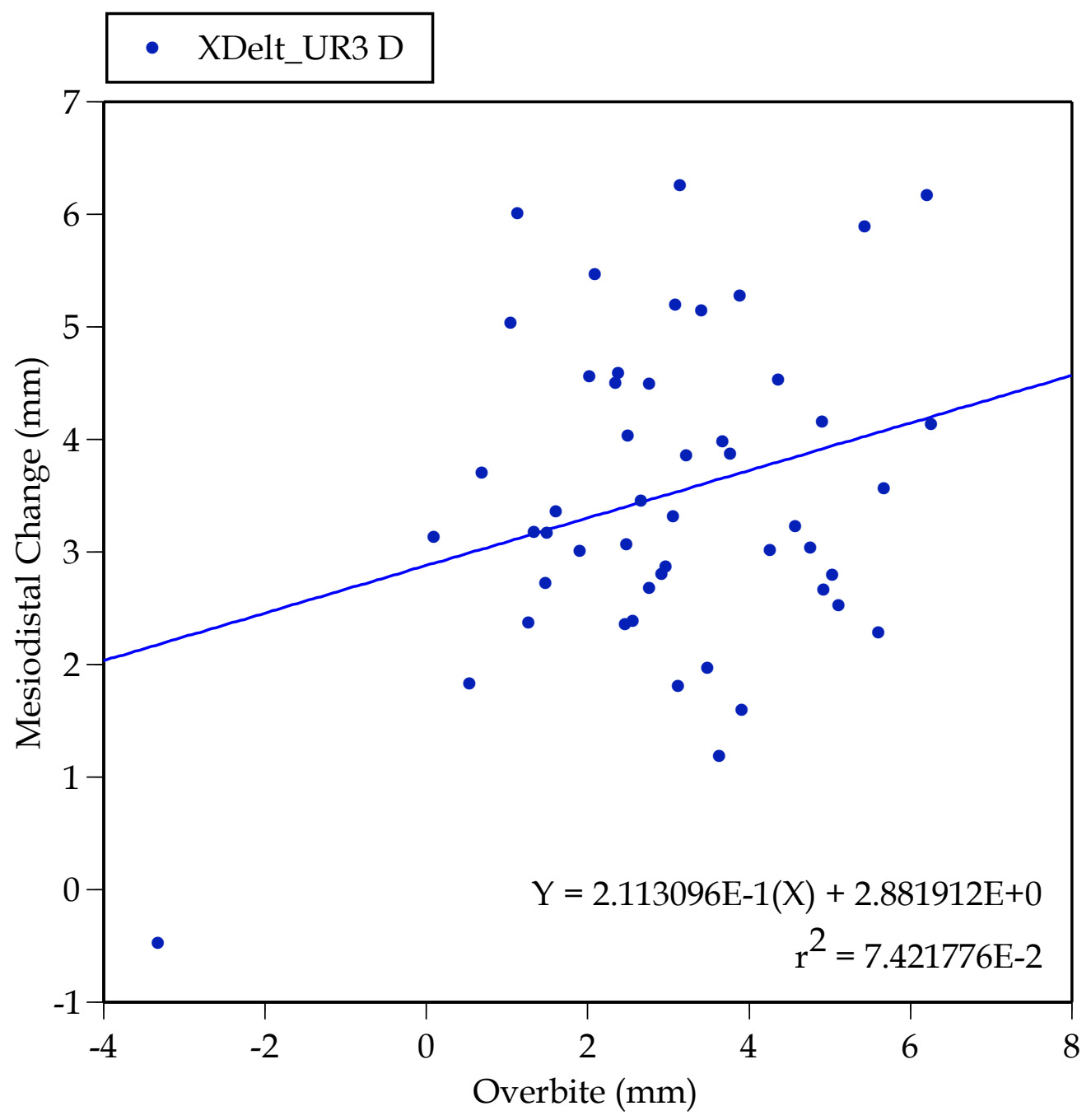

Fig. 61. Scatterplot showing the association between pretreatment spacing and the amount of mesiodistal change of the maxillary right canine (distal) analyzed in just the firstpremolar extraction sample. 


\section{$\underline{\text { Second Premolar }}$}

Results for predicting meisodistal change of the second premolar mesial from occlusal variables are interesting (Tables 52, 53) in that (1) both models account for 20 to $30 \%$ of the variation in premolar movement but (2) none of the significant predictors is common to the two models. Results are, however, readily interpretable for the two homologous landmarks and not dissimilar. Incisor irregularity is the preeminent predictor for the left second premolar (mesial contact), and, as shown in Fig. 55, the greater the irregularity the less the premolar was moved mesially into the extraction space, presumably because more of the extraction space is being used to align teeth in the anterior segment. In this situation, it is assumed that less mesial force was placed on the posterior segment during extraction space closure because a majority of the space was consumed by the incisor irregularity.

Similarly, for the right quadrant (Table 53), maxillary 1-6 chord length was the strongest predictor $\left(\mathrm{r}^{2}=20 \%\right)$. The relationship is shown in Fig. 62 . In cases with short 1-6 lengths, the canines tend to be labioverted and there is appreciable incisor irregularity, so much of the premolar extraction space is used to alleviate these anterior-segment issues, and the second molar is not moved much to close the remaining extraction space. Figs. A-37, A-39, and A59 are examples of these situations. At the other extreme, such as illustrated in Figs. A-3, A-20, and A-89, teeth in the anterior segment are already pretty well 
Table 52. Results of stepwise linear regression predicting the amount of mesiodistal tooth movement from the pretreatment malocclusion: results for maxillary left second premolar (mesial) in the subset in whom first premolars were extracted.

\begin{tabular}{lrrrr}
\hline \multicolumn{1}{c}{ Variable } & $\begin{array}{c}\text { Sum of } \\
\text { Squares }\end{array}$ & F Ratio & P Value & $\mathrm{r}^{2}$ \\
\hline Incisor Overjet & 0.03 & 0.03 & 0.8560 & \\
Incisor Overbite & 16.62 & 21.95 & $\underline{\mathbf{0 . 0 0 0 0}}$ & 0.1215 \\
Irregularity Index & 20.34 & 26.86 & $\underline{\mathbf{0 . 0 0 0 0}}$ & 0.3601 \\
Incisor Spacing & 0.40 & 0.52 & 0.4733 & \\
Max 3-3 Width & 0.79 & 1.05 & 0.3112 & \\
Max 6-6 Width & 1.36 & 1.83 & 0.1830 & \\
Mand 3-3 Width & 0.79 & 1.05 & 0.3112 & \\
Mand 6-6 Width & 0.58 & 0.77 & 0.3857 & \\
UL 3-3 Width Difference & 6.05 & 8.00 & $\underline{\mathbf{0 . 0 0 7 0}}$ & 0.0625 \\
UL 6-6 Width Difference & 0.81 & 1.08 & 0.3057 & \\
Max 1-3 Chord & 0.16 & 0.21 & 0.6517 & \\
Max 1-6 Chord & 0.16 & 0.21 & 0.6488 & \\
Mand 1-3 Chord & 0.26 & 0.34 & 0.5608 & \\
Mand 1-6 Chord & 1.45 & 1.96 & 0.1688 & \\
Midline Deviation & 0.08 & 0.10 & 0.7486 & \\
Buccal Segment Relation & 0.62 & 0.81 & 0.3726 & \\
Canine Discrepancy & 0.29 & 0.38 & 0.5434 & \\
Patient's Sex & 4.17 & 5.51 & $\underline{\mathbf{0 . 0 2 3 5}}$ & 0.0507 \\
\hline
\end{tabular}


Table 53. Results of stepwise linear regression predicting the amount of mesiodistal tooth movement from the pretreatment malocclusion: results for maxillary right second premolar (mesial) in the subset in whom first premolars were extracted.

\begin{tabular}{lcccc}
\hline \multicolumn{1}{c}{ Variable } & $\begin{array}{c}\text { Sum of } \\
\text { Squares }\end{array}$ & F Ratio & P Value & $\mathrm{r}^{2}$ \\
\hline Incisor Overjet & 1.01 & 0.57 & 0.4537 & \\
Incisor Overbite & 3.80 & 2.23 & 0.1424 & \\
Irregularity Index & 3.12 & 1.81 & 0.1850 & \\
Incisor Spacing & 0.06 & 0.04 & 0.8531 & \\
Max 3-3 Width & 3.32 & 1.93 & 0.1711 & \\
Max 6-6 Width & 6.00 & 3.62 & 0.0636 & \\
Mand 3-3 Width & 2.74 & 1.58 & 0.2149 & \\
Mand 6-6 Width & 2.92 & 1.69 & 0.2000 & \\
UL 3-3 Width Difference & 0.63 & 0.36 & 0.5531 & \\
UL 6-6 Width Difference & 1.36 & 0.77 & 0.3843 & \\
Max 1-3 Chord & 0.09 & 0.05 & 0.8233 & \\
Max 1-6 Chord & 29.18 & 16.66 & $\underline{\mathbf{0 . 0 0 0 2}}$ & 0.2023 \\
Mand 1-3 Chord & 0.78 & 0.44 & 0.5102 & \\
Mand 1-6 Chord & 2.83 & 1.64 & 0.2072 & \\
Midline Deviation & 9.11 & 5.20 & $\underline{\mathbf{0 . 0 2 7 3}}$ & 0.0810 \\
Buccal Segment Relation & 0.93 & 0.53 & 0.4719 & \\
Canine Discrepancy & 1.79 & 1.02 & 0.3180 & \\
Patient's Sex & 0.31 & 0.18 & 0.6775 & \\
\hline
\end{tabular}




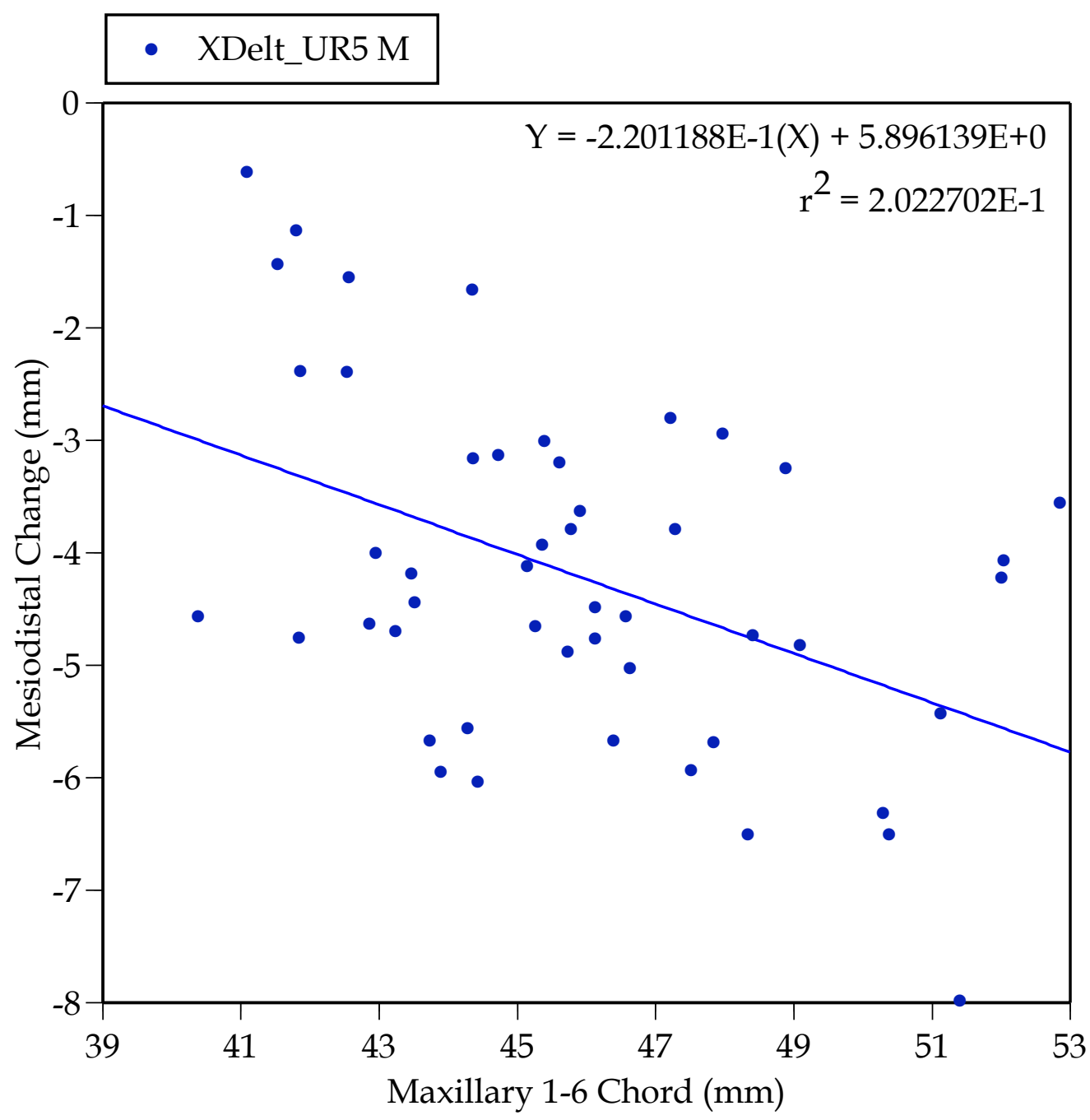

Fig. 62. Scatterplot showing the association between pretreatment maxillary 1-6 chord distance and the amount of mesiodistal change of the maxillary right second premolar (mesial) analyzed in just the first-premolar extraction sample. 
aligned, so much of the P1 extraction space is filled by mesial movement of the second-premolar.

As with the mesial contacts of P2, the distal contacts of P2 in the two quadrants both possess statistically significant occlusal predictors, but they are wholly different in the two models (Tables 54, 55). Incisor irregularity dominates the field for the left landmark $\left(r^{2}=32 \%\right)$, while maxillary 1-6 chord is the single important predictor on the right $\left(\mathrm{r}^{2}=25 \%\right)$. Obviously, these two variables are the same as disclosed for the mesial landmarks on P2 (Tables 52, 53), with the same kinds of associations

\section{$\underline{\text { First Molar }}$}

The statistical relationships just discussed for the second-premolar carry over to the first-molar that is butted against it. Indeed, except for tipping and rotational differences, these two teeth (P2, M1) should respond quite similarly. The one strong predictor of mesiodistal change in the left quadrant is incisor irregularity $\left(\mathrm{r}^{2}=33 \%\right)$; (Table 56), while on the right, the model is governed by maxillary $1-6$ chord distance $\left(r^{2}=16 \%\right.$; Table 57$)$.

The relationships with incisor irregularity (Table 56) is the same as discussed above for the second premolar (Fig. 63): Cases with a lot of incisor irregularity (e.g., Figs. A-10, A-22) expend most of the extraction space resolving those anterior-segment issues, and there is, consequently, little 
Table 54. Results of stepwise linear regression predicting the amount of mesiodistal tooth movement from the pretreatment malocclusion: results for maxillary left second premolar (distal) in the subset in whom first premolars were extracted.

\begin{tabular}{lrrrr}
\hline \multicolumn{1}{c}{ Variable } & $\begin{array}{c}\text { Sum of } \\
\text { Squares }\end{array}$ & F Ratio & P Value & $\mathrm{r}^{2}$ \\
\hline Incisor Overjet & 0.00 & 0.00 & 0.9584 & \\
Incisor Overbite & 14.61 & 12.43 & $\underline{\mathbf{0 . 0 0 1 0}}$ & 0.1447 \\
Irregularity Index & 36.67 & 31.21 & $\underline{\mathbf{0 . 0 0 0 0}}$ & 0.3197 \\
Incisor Spacing & 0.01 & 0.01 & 0.9325 & \\
Max 3-3 Width & 0.27 & 0.22 & 0.6390 & \\
Max 6-6 Width & 1.18 & 1.01 & 0.3209 & \\
Mand 3-3 Width & 1.46 & 1.25 & 0.2694 & \\
Mand 6-6 Width & 1.11 & 0.94 & 0.3374 & \\
UL 3-3 Width Difference & 2.82 & 2.47 & 0.1228 & \\
UL 6-6 Width Difference & 0.15 & 0.13 & 0.7223 & \\
Max 1-3 Chord & 0.20 & 0.17 & 0.6824 & \\
Max 1-6 Chord & 0.09 & 0.07 & 0.7912 & \\
Mand 1-3 Chord & 0.28 & 0.23 & 0.6309 & \\
Mand 1-6 Chord & 0.37 & 0.31 & 0.5789 & \\
Midline Deviation & 0.02 & 0.01 & 0.9091 & \\
Buccal Segment Relation & 0.33 & 0.28 & 0.6029 & \\
Canine Discrepancy & 0.90 & 0.76 & 0.3873 & \\
Patient's Sex & 1.54 & 1.32 & 0.2564 & \\
\hline
\end{tabular}


Table 55. Results of stepwise linear regression predicting the amount of mesiodistal tooth movement from the pretreatment malocclusion: results for maxillary right second premolar (distal) in the subset in whom first premolars were extracted.

\begin{tabular}{lrrrr}
\hline \multicolumn{1}{c}{ Variable } & $\begin{array}{c}\text { Sum of } \\
\text { Squares }\end{array}$ & F Ratio & P Value & $\mathrm{r}^{2}$ \\
\hline Incisor Overjet & 0.69 & 0.39 & 0.5381 & \\
Incisor Overbite & 1.21 & 0.69 & 0.4121 & \\
Irregularity Index & 3.82 & 2.23 & 0.1424 & \\
Incisor Spacing & 0.01 & 0.00 & 0.9489 & \\
Max 3-3 Width & 3.06 & 1.77 & 0.1898 & \\
Max 6-6 Width & 6.96 & 4.24 & 0.0453 & \\
Mand 3-3 Width & 2.47 & 1.42 & 0.2404 & \\
Mand 6-6 Width & 2.84 & 1.64 & 0.2075 & \\
UL 3-3 Width Difference & 0.60 & 0.34 & 0.5633 & \\
UL 6-6 Width Difference & 2.10 & 1.20 & 0.2791 & \\
Max 1-3 Chord & 0.29 & 0.17 & 0.6869 & \\
Max 1-6 Chord & 38.23 & 21.75 & $\underline{\mathbf{0 . 0 0 0 0}}$ & 0.2505 \\
Mand 1-3 Chord & 0.67 & 0.37 & 0.5439 & \\
Mand 1-6 Chord & 2.88 & 1.66 & 0.2042 & \\
Midline Deviation & 10.39 & 5.91 & $\underline{\mathbf{0 . 0 1 9 0}}$ & 0.0853 \\
Buccal Segment Relation & 0.44 & 0.25 & 0.6230 & \\
Canine Discrepancy & 5.66 & 3.38 & 0.0724 & \\
Patient's Sex & 0.74 & 0.41 & 0.5230 & \\
\hline
\end{tabular}


Table 56. Results of stepwise linear regression predicting the amount of mesiodistal tooth movement from the pretreatment malocclusion: results for maxillary left first molar (mesial) in the subset in whom first premolars were extracted.

\begin{tabular}{lrrrr}
\hline \multicolumn{1}{c}{ Variable } & $\begin{array}{c}\text { Sum of } \\
\text { Squares }\end{array}$ & F Ratio & P Value & $\mathrm{r}^{2}$ \\
\hline Incisor Overjet & 0.38 & 0.25 & 0.6208 & \\
Incisor Overbite & 11.45 & 7.54 & $\underline{\mathbf{0 . 0 0 8 6}}$ & 0.0941 \\
Irregularity Index & 44.73 & 29.47 & $\underline{\mathbf{0 . 0 0 0 0}}$ & 0.3325 \\
Incisor Spacing & 0.24 & 0.16 & 0.6938 & \\
Max 3-3 Width & 0.00 & 0.00 & 0.9597 & \\
Max 6-6 Width & 1.58 & 1.04 & 0.3126 & \\
Mand 3-3 Width & 1.10 & 0.72 & 0.3997 & \\
Mand 6-6 Width & 0.98 & 0.64 & 0.4286 & \\
UL 3-3 Width Difference & 0.95 & 0.62 & 0.4343 & \\
UL 6-6 Width Difference & 0.61 & 0.40 & 0.5321 & \\
Max 1-3 Chord & 0.26 & 0.17 & 0.6835 & \\
Max 1-6 Chord & 0.17 & 0.11 & 0.7418 & \\
Mand 1-3 Chord & 0.21 & 0.13 & 0.7168 & \\
Mand 1-6 Chord & 0.41 & 0.26 & 0.6105 & \\
Midline Deviation & 0.05 & 0.03 & 0.8561 & \\
Buccal Segment Relation & 0.63 & 0.41 & 0.5256 & \\
Canine Discrepancy & 1.21 & 0.80 & 0.3768 & \\
Patient's Sex & 2.28 & 1.52 & 0.2238 & \\
\hline
\end{tabular}


Table 57. Results of stepwise linear regression predicting the amount of mesiodistal tooth movement from the pretreatment malocclusion: results for maxillary right first molar (mesial) in the subset in whom first premolars were extracted.

\begin{tabular}{lcccc}
\hline \multicolumn{1}{c}{ Variable } & $\begin{array}{c}\text { Sum of } \\
\text { Squares }\end{array}$ & F Ratio & P Value & $\mathrm{r}^{2}$ \\
\hline Incisor Overjet & 0.78 & 0.39 & 0.5347 & \\
Incisor Overbite & 3.29 & 1.70 & 0.1984 & \\
Irregularity Index & 4.04 & 2.11 & 0.1534 & \\
Incisor Spacing & 0.25 & 0.12 & 0.7276 & \\
Max 3-3 Width & 5.46 & 2.90 & 0.0958 & \\
Max 6-6 Width & 7.53 & 4.09 & 0.0491 & \\
Mand 3-3 Width & 3.22 & 1.67 & 0.2035 & \\
Mand 6-6 Width & 1.50 & 0.76 & 0.3886 & \\
UL 3-3 Width Difference & 1.53 & 0.77 & 0.3835 & \\
UL 6-6 Width Difference & 4.65 & 2.45 & 0.1249 & \\
Max 1-3 Chord & 0.17 & 0.09 & 0.7723 & \\
Max 1-6 Chord & 26.55 & 13.52 & $\underline{\mathbf{0 . 0 0 0 6}}$ & 0.1586 \\
Mand 1-3 Chord & 1.20 & 0.60 & 0.4412 & \\
Mand 1-6 Chord & 3.24 & 1.68 & 0.2020 & \\
Midline Deviation & 11.92 & 6.07 & $\underline{\mathbf{0 . 0 1 7 5}}$ & 0.0981 \\
Buccal Segment Relation & 0.12 & 0.06 & 0.8102 & \\
Canine Discrepancy & 7.68 & 4.18 & 0.0467 & \\
Patient's Sex & 0.14 & 0.07 & 0.7954 & \\
\hline
\end{tabular}




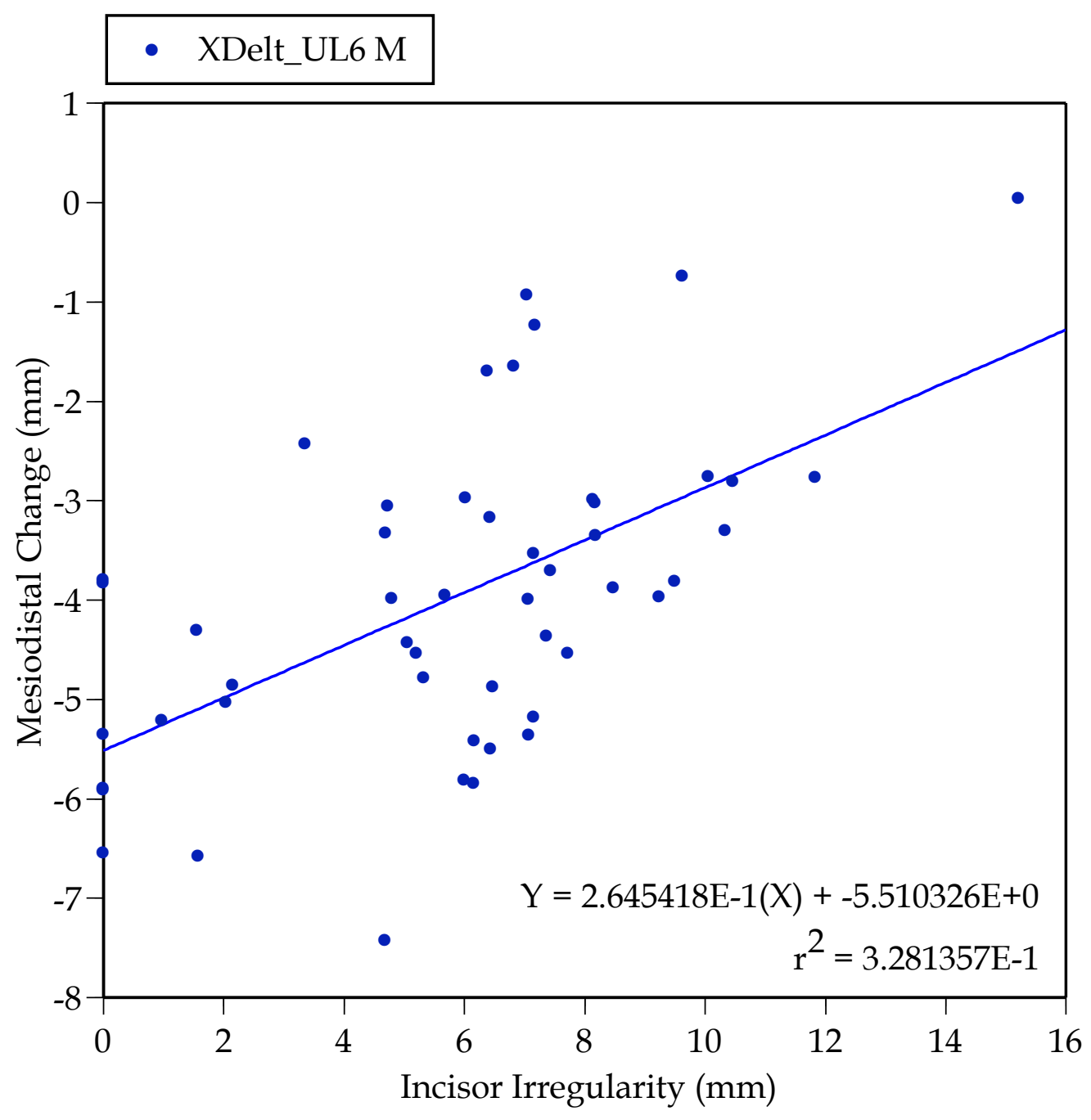

Fig. 63. Scatterplot showing the association between pretreatment incisor irregularity and the amount of mesiodistal change of the maxillary left first molar (mesial) analyzed in just the first-premolar extraction sample. 
mesial molar movement. At the other extreme (e.g., Figs. A-3, A-20), the anterior segment already exhibits overall alignment, and most of the extraction space was "burned" with mesial movement of the second premolar and molars in each quadrant.

The relationship for maxillary 1-6 arch chord (Table 57) is graphed in Fig. 64: The greater the 1-6 arch chord the greater, on average, the mesial molar movement. Cases with little molar movement during treatment (e.g., Figs. A-39, A-59) tended to merit incisor retraction (and/or the canines were in labioversion), so most of the extraction space was used to resolve issues in the anterior portion of the dental arch. Where the anterior teeth were fairly well aligned and well positioned, but first-premolars were extracted, such as in Figs. A-3, and A-89, most of the premolar extraction space was used to move the first molars mesially.

The two regression models for the first molar (distal contact) are listed in Tables 58 and 59. Once again the results are asymmetric: Incisor irregularity is the prime predictor of molar movement in the left quadrant $\left(\mathrm{r}^{2}\right.$ $=35 \%$, while maxillary 1-6 chord distance is, by far, the strongest predictor on the right $\left(\mathrm{r}^{2}=31 \%\right)$. These results are identical to the outcomes for the mesial landmarks of this tooth, which is predictable since the two landmarks on a tooth are invariant and movements will be the same along the parasagittal axis aside from any rotations. 


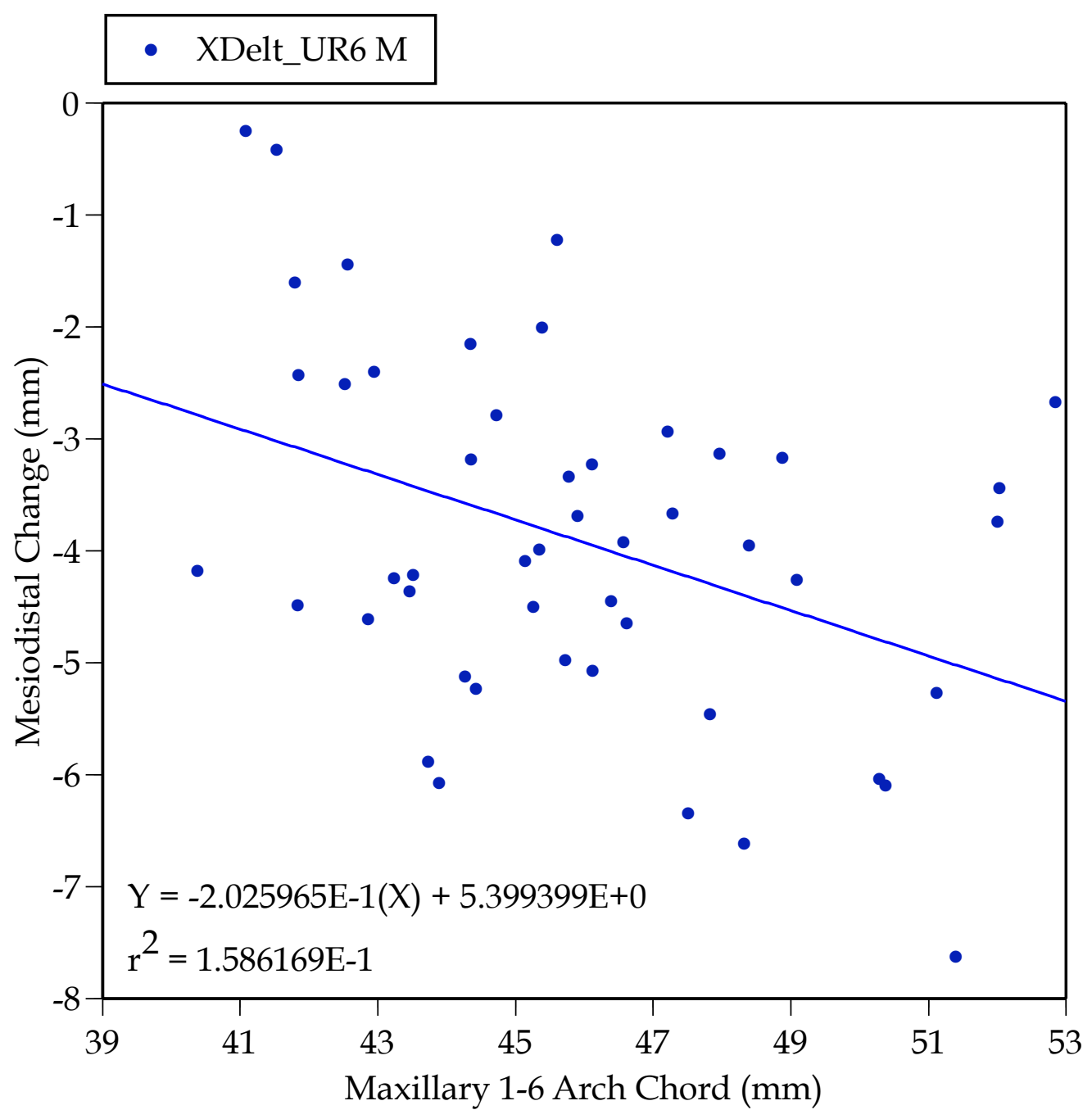

Fig. 64. Scatterplot showing the association between maxillary 1-6 arch chord and the amount of mesiodistal change of the maxillary right first molar (mesial) analyzed in just the first-premolar extraction sample. 
Table 58. Results of stepwise linear regression predicting the amount of mesiodistal tooth movement from the pretreatment malocclusion: results for maxillary left first molar (distal) in the subset in whom first premolars were extracted.

\begin{tabular}{lrrrr}
\hline \multicolumn{1}{c}{ Variable } & $\begin{array}{c}\text { Sum of } \\
\text { Squares }\end{array}$ & F Ratio & P Value & $\mathrm{r}^{2}$ \\
\hline Incisor Overjet & 1.02 & 0.65 & 0.4231 & \\
Incisor Overbite & 7.14 & 4.61 & $\underline{\mathbf{0 . 0 3 7 0}}$ & 0.0596 \\
Irregularity Index & 44.75 & 28.91 & $\underline{\mathbf{0 . 0 0 0 0}}$ & 0.3462 \\
Incisor Spacing & 0.20 & 0.13 & 0.7253 & \\
Max 3-3 Width & 0.03 & 0.02 & 0.8847 & \\
Max 6-6 Width & 0.26 & 0.17 & 0.6838 & \\
Mand 3-3 Width & 0.10 & 0.06 & 0.8013 & \\
Mand 6-6 Width & 0.03 & 0.02 & 0.8961 & \\
UL 3-3 Width Difference & 0.25 & 0.16 & 0.6917 & \\
UL 6-6 Width Difference & 0.44 & 0.28 & 0.5990 & \\
Max 1-3 Chord & 0.57 & 0.36 & 0.5492 & \\
Max 1-6 Chord & 1.23 & 0.79 & 0.3777 & \\
Mand 1-3 Chord & 0.01 & 0.01 & 0.9242 & \\
Mand 1-6 Chord & 0.00 & 0.00 & 0.9815 & \\
Midline Deviation & 0.00 & 0.00 & 0.9939 & \\
Buccal Segment Relation & 0.28 & 0.18 & 0.6774 & \\
Canine Discrepancy & 2.26 & 1.48 & 0.2308 & \\
Patient's Sex & 2.14 & 1.39 & 0.2439 & \\
\hline
\end{tabular}


Table 59. Results of stepwise linear regression predicting the amount of mesiodistal tooth movement from the pretreatment malocclusion: results for maxillary right first molar (distal) in the subset in whom first premolars were extracted.

\begin{tabular}{lrrrr}
\hline \multicolumn{1}{c}{ Variable } & $\begin{array}{c}\text { Sum of } \\
\text { Squares }\end{array}$ & F Ratio & P Value & $\mathrm{r}^{2}$ \\
\hline Incisor Overjet & 0.16 & 0.09 & 0.7633 & \\
Incisor Overbite & 2.02 & 1.18 & 0.2841 & \\
Irregularity Index & 1.39 & 0.81 & 0.3745 & \\
Incisor Spacing & 0.58 & 0.33 & 0.5680 & \\
Max 3-3 Width & 3.06 & 1.80 & 0.1861 & \\
Max 6-6 Width & 2.16 & 1.26 & 0.2673 & \\
Mand 3-3 Width & 1.28 & 0.74 & 0.3945 & \\
Mand 6-6 Width & 0.12 & 0.07 & 0.7993 & \\
UL 3-3 Width Difference & 1.13 & 0.65 & 0.4239 & \\
UL 6-6 Width Difference & 2.39 & 1.40 & 0.2432 & \\
Max 1-3 Chord & 0.13 & 0.08 & 0.7853 & \\
Max 1-6 Chord & 56.82 & 32.92 & $\underline{\mathbf{0 . 0 0 0 0}}$ & 0.3108 \\
Mand 1-3 Chord & 1.20 & 0.69 & 0.4094 & \\
Mand 1-6 Chord & 2.44 & 1.43 & 0.2388 & \\
Midline Deviation & 18.30 & 10.60 & $\underline{\mathbf{0 . 0 0 2 1}}$ & 0.1291 \\
Buccal Segment Relation & 0.48 & 0.27 & 0.6031 & \\
Canine Discrepancy & 1.71 & 0.99 & 0.3246 & \\
Patient's Sex & 2.13 & 1.24 & 0.2718 & \\
\hline
\end{tabular}


$\underline{\text { Second Molar }}$

Statistically, the maxillary arch chord dimensions are driving the mesiodistal changes in the second molar (mesial), but the 1-3 chord is more important on the left (Table 60) while the 1-6 chord is more important on the right (Table 61).

The relationship on the left (Fig. 65) is that malocclusions with long 1-3 chords experience more mesial molar movement, as in the cases graphed in Figs. A-5, A-64, and A-89. Maxillary 1-3 chord length often reflects the procumbency of the central incisors (but not irregularity), and its seems that those cases flagged here as having long 1-3 chords were treated by moving the central incisors lingually, so much of the premolar extraction space was consumed by mesial molar movement.

The relationship in the right quadrant (Table 61) is comparable, but depends on the maxillary 1-6 rather than the 1-3 chord distance (Fig. 66): The relationship seems to be that when the 1-6 chord is short, there tends to be considerable crowding in the anterior segment (e.g., Figs. A-7, A-33) that uses up much of the first premolar extraction space, and, thus, there is little mesial movement of the second molar. Conversely, when the 1-6 chord is much larger (e.g., Figs. A-64, A-89) there tends to be little TSALD in the anterior 
Table 60. Results of stepwise linear regression predicting the amount of mesiodistal tooth movement from the pretreatment malocclusion: results for maxillary left second molar (mesial) in the subset in whom first premolars were extracted.

\begin{tabular}{lcccc}
\hline \multicolumn{1}{c}{ Variable } & $\begin{array}{c}\text { Sum of } \\
\text { Squares }\end{array}$ & F Ratio & P Value & $\mathrm{r}^{2}$ \\
\hline Incisor Overjet & 2.16 & 0.85 & 0.3608 & \\
Incisor Overbite & 6.52 & 2.67 & 0.1091 & \\
Irregularity Index & 6.86 & 2.82 & 0.1001 & \\
Incisor Spacing & 1.15 & 0.45 & 0.5068 & \\
Max 3-3 Width & 0.00 & 0.00 & 0.9704 & \\
Max 6-6 Width & 0.03 & 0.01 & 0.9110 & \\
Mand 3-3 Width & 0.04 & 0.01 & 0.9078 & \\
Mand 6-6 Width & 0.06 & 0.02 & 0.8826 & \\
UL 3-3 Width Difference & 0.01 & 0.00 & 0.9632 & \\
UL 6-6 Width Difference & 0.35 & 0.14 & 0.7149 & \\
Max 1-3 Chord & 29.58 & 11.70 & $\underline{0.0013}$ & 0.1993 \\
Max 1-6 Chord & 0.60 & 0.24 & 0.6301 & \\
Mand 1-3 Chord & 0.01 & 0.00 & 0.9482 & \\
Mand 1-6 Chord & 0.06 & 0.02 & 0.8816 & \\
Midline Deviation & 0.03 & 0.01 & 0.9186 & \\
Buccal Segment Relation & 1.01 & 0.40 & 0.5322 & \\
Canine Discrepancy & 0.07 & 0.03 & 0.8744 & \\
Patient's Sex & 3.20 & 1.27 & 0.2653 & \\
\hline
\end{tabular}


Table 61. Results of stepwise linear regression predicting the amount of mesiodistal tooth movement from the pretreatment malocclusion: results for maxillary right second molar (mesial) in the subset in whom first premolars were extracted.

\begin{tabular}{lrrrr}
\hline \multicolumn{1}{c}{ Variable } & $\begin{array}{c}\text { Sum of } \\
\text { Squares }\end{array}$ & F Ratio & P Value & $\mathrm{r}^{2}$ \\
\hline Incisor Overjet & 0.00 & 0.00 & 0.9864 & \\
Incisor Overbite & 2.27 & 1.10 & 0.2989 & \\
Irregularity Index & 1.92 & 0.93 & 0.3401 & \\
Incisor Spacing & 0.07 & 0.04 & 0.8526 & \\
Max 3-3 Width & 1.16 & 0.56 & 0.4589 & \\
Max 6-6 Width & 4.90 & 2.45 & 0.1243 & \\
Mand 3-3 Width & 0.47 & 0.23 & 0.6366 & \\
Mand 6-6 Width & 0.37 & 0.18 & 0.6747 & \\
UL 3-3 Width Difference & 0.44 & 0.21 & 0.6498 & \\
UL 6-6 Width Difference & 4.85 & 2.43 & 0.1263 & \\
Max 1-3 Chord & 0.08 & 0.04 & 0.8483 & \\
Max 1-6 Chord & 53.75 & 26.09 & $\underline{\mathbf{0 . 0 0 0 0}}$ & 0.2696 \\
Mand 1-3 Chord & 0.42 & 0.20 & 0.6571 & \\
Mand 1-6 Chord & 1.05 & 0.50 & 0.4823 & \\
Midline Deviation & 17.67 & 8.58 & $\underline{\mathbf{0 . 0 0 5 3}}$ & 0.1149 \\
Buccal Segment Relation & 0.88 & 0.42 & 0.5195 & \\
Canine Discrepancy & 5.27 & 2.65 & 0.1103 & \\
Patient's Sex & 4.62 & 2.31 & 0.1359 & \\
\hline
\end{tabular}




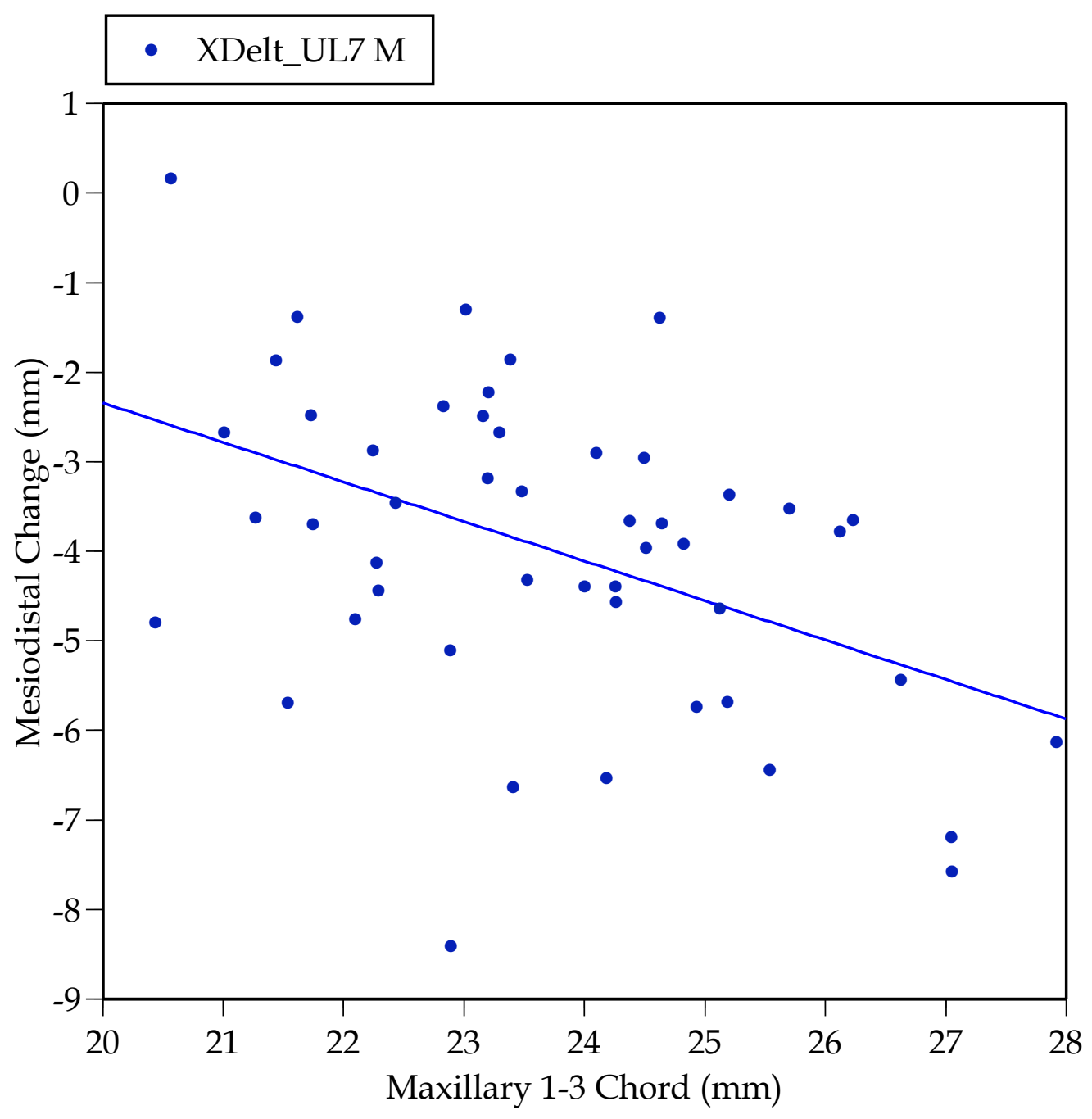

Fig. 65. Scatterplot showing the association between maxillary 1-3 chord distance and the amount of mesiodistal change of the maxillary left second molar (mesial) analyzed in just the first-premolar extraction sample. 


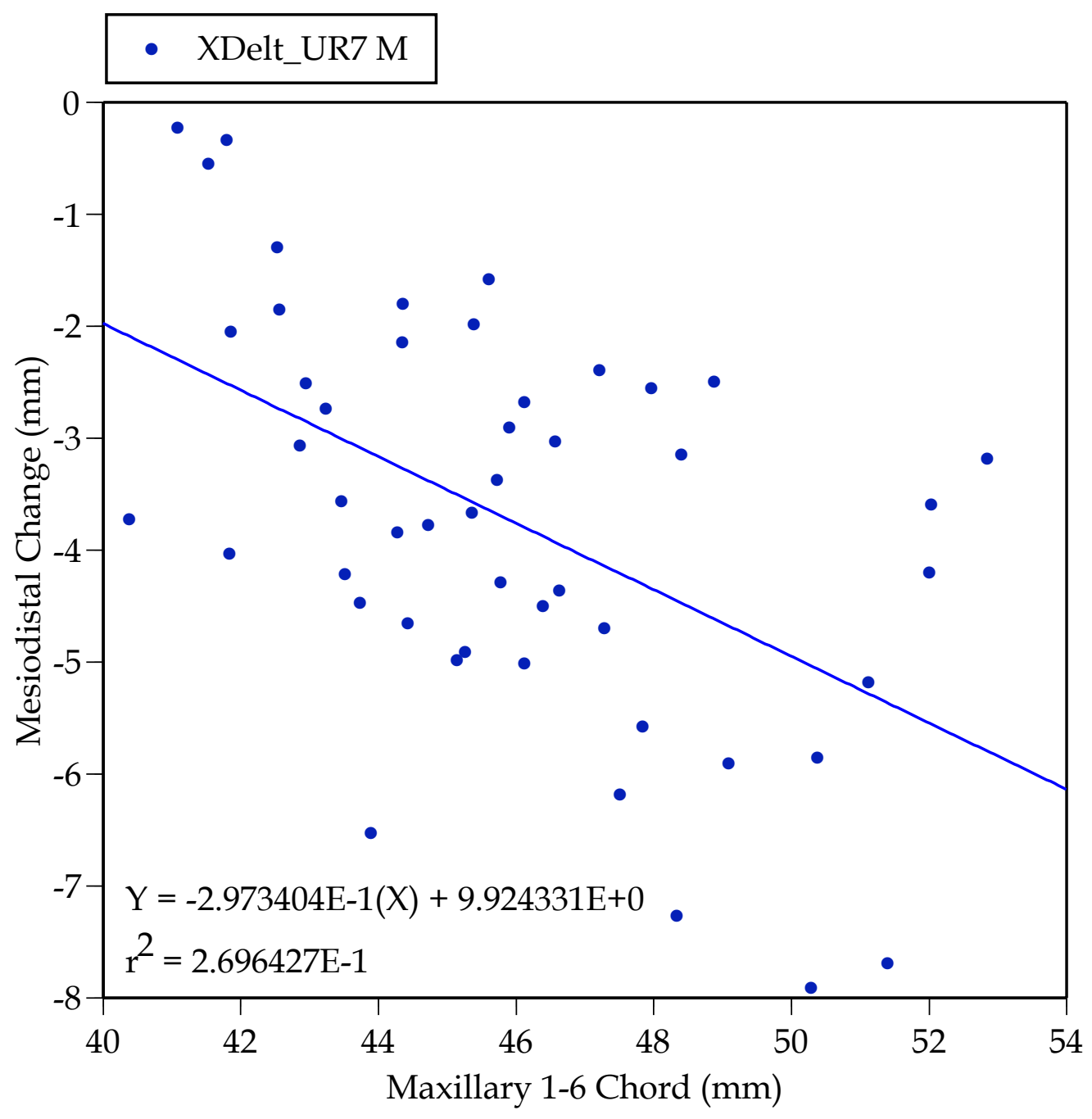

Fig. 66. Scatterplot showing the association between maxillary 1-6 chord and the amount of mesiodistal change of the maxillary right second molar (mesial) analyzed in just the first-premolar extraction sample. 
segments, and much of the extraction space is "burned" by mesial movement of the molars.

Concerning the distal landmarks on the second molar, incisor irregularity is the single significant predictor in the left quadrant $\left(\mathrm{r}^{2}=19 \%\right.$; Table 62), while maxillary 1-6 chord distance is the most important predictor in the right quadrant $\left(\mathrm{r}^{2}=17 \%\right.$; Table 63$)$.

The relationships with incisor irregularity have been described several times in this section, and, again here, in cases with considerable irregularity most of the first-premolar extraction space is used to resolve the anterior issues and rather little of the space is used by mesial molar movement (Fig. 67). Indeed, this same relationship has been noted in this section for the whole "chain" of six dental landmarks distal to the first-premolar extraction space (P2, M1, and M2); the more of the extraction space used to align teeth in the anterior segment, the less the need to "burn" anchorage to close-up the little remaining extraction space.

The association for the maxillary 1-6 chord in the right quadrant (Table 63 ) is identical to that described above for the mesial landmark on this tooth. Simply, the more extraction space used to resolve anterior problems, the less space remaining to be closed by mesial molar movement. 
Table 62. Results of stepwise linear regression predicting the amount of mesiodistal tooth movement from the pretreatment malocclusion: results for maxillary left second molar (distal) in the subset in whom first premolars were extracted.

\begin{tabular}{lrrrr}
\hline \multicolumn{1}{c}{ Variable } & $\begin{array}{c}\text { Sum of } \\
\text { Squares }\end{array}$ & F Ratio & P Value & $\mathrm{r}^{2}$ \\
\hline Incisor Overjet & 0.02 & 0.01 & 0.9383 & \\
Incisor Overbite & 6.83 & 2.63 & 0.1119 & \\
Irregularity Index & 29.01 & 10.79 & $\underline{\mathbf{0 . 0 0 1 9}}$ & 0.1867 \\
Incisor Spacing & 0.05 & 0.02 & 0.8889 & \\
Max 3-3 Width & 1.38 & 0.51 & 0.4792 & \\
Max 6-6 Width & 0.00 & 0.00 & 0.9930 & \\
Mand 3-3 Width & 0.09 & 0.03 & 0.8536 & \\
Mand 6-6 Width & 0.18 & 0.07 & 0.7966 & \\
UL 3-3 Width Difference & 1.31 & 0.48 & 0.4917 & \\
UL 6-6 Width Difference & 0.43 & 0.16 & 0.6935 & \\
Max 1-3 Chord & 1.08 & 0.40 & 0.5329 & \\
Max 1-6 Chord & 1.74 & 0.64 & 0.4274 & \\
Mand 1-3 Chord & 1.41 & 0.52 & 0.4744 & \\
Mand 1-6 Chord & 0.35 & 0.13 & 0.7241 & \\
Midline Deviation & 0.02 & 0.01 & 0.9379 & \\
Buccal Segment Relation & 4.09 & 1.54 & 0.2209 & \\
Canine Discrepancy & 0.05 & 0.02 & 0.8958 & \\
Patient's Sex & 1.61 & 0.59 & 0.4453 & \\
\hline
\end{tabular}


Table 63. Results of stepwise linear regression predicting the amount of mesiodistal tooth movement from the pretreatment malocclusion: results for maxillary right second molar (distal) in the subset in whom first premolars were extracted.

\begin{tabular}{lcccc}
\hline \multicolumn{1}{c}{ Variable } & $\begin{array}{c}\text { Sum of } \\
\text { Squares }\end{array}$ & F Ratio & P Value & $\mathrm{r}^{2}$ \\
\hline Incisor Overjet & 0.00 & 0.00 & 0.9854 & \\
Incisor Overbite & 4.74 & 2.14 & 0.1501 & \\
Irregularity Index & 2.12 & 0.94 & 0.3387 & \\
Incisor Spacing & 0.61 & 0.27 & 0.6092 & \\
Max 3-3 Width & 3.00 & 1.33 & 0.2543 & \\
Max 6-6 Width & 5.34 & 2.43 & 0.1258 & \\
Mand 3-3 Width & 0.05 & 0.02 & 0.8805 & \\
Mand 6-6 Width & 0.93 & 0.41 & 0.5275 & \\
UL 3-3 Width Difference & 3.78 & 1.69 & 0.1996 & \\
UL 6-6 Width Difference & 3.61 & 1.61 & 0.2105 & \\
Max 1-3 Chord & 0.32 & 0.14 & 0.7103 & \\
Max 1-6 Chord & 33.24 & 14.68 & $\mathbf{0 . 0 0 0 4}$ & 0.1745 \\
Mand 1-3 Chord & 3.57 & 1.60 & 0.2131 & \\
Mand 1-6 Chord & 0.05 & 0.02 & 0.8871 & \\
Midline Deviation & 13.11 & 5.79 & $\mathbf{0 . 0 2 0 2}$ & 0.0923 \\
Buccal Segment Relation & 4.20 & 1.89 & 0.1757 & \\
Canine Discrepancy & 4.42 & 1.99 & 0.1648 & \\
Patient's Sex & 1.60 & 0.70 & 0.4065 & \\
\hline
\end{tabular}




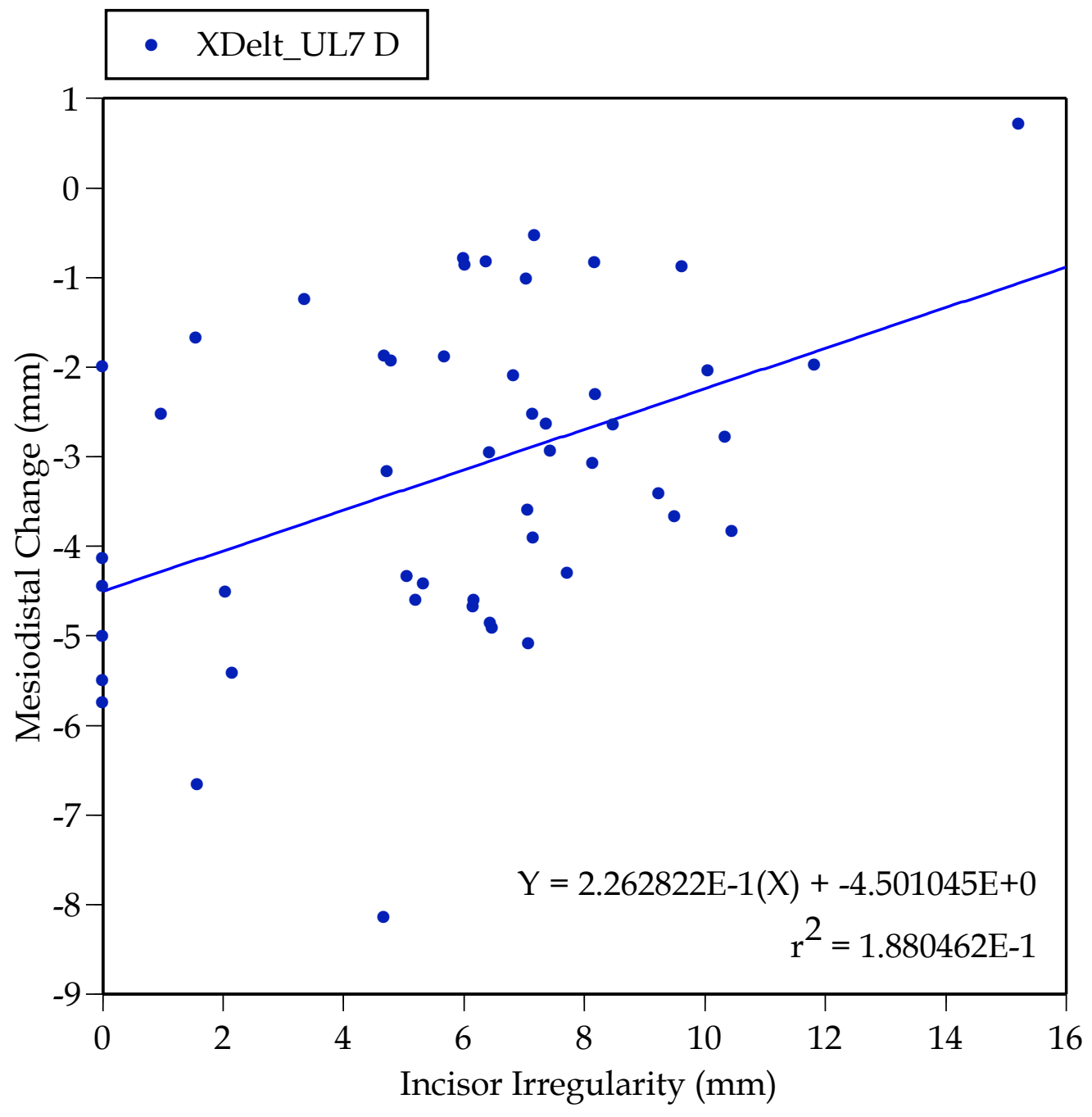

Fig. 67. Scatterplot showing the association between incisor irregularity and the amount of mesiodistal change of the maxillary left second molar (distall) analyzed in just the firstpremolar extraction sample. 


\section{Second Premolar Extraction}

This section complements the prior section; here we describe the statistical dependencies between the amounts of mesiodistal tooth movement in the sample of the cases where second premolars were extracted.

\section{Central Incisor}

Three variables were significantly predictors of the mesiodistal change of the central incisor (mesial contact) in the right arcade (Table 64), but just one in the left arcade (Table 65), and none of these variables is shared in common between sides. Overall, the predominant predictor $\left(\mathrm{r}^{2}=22 \%\right)$ is maxillary 1-3 arch chord (Table 64). The nature of the association here (Fig. 68 ) is that when the anterior arch form is long and tapered (e.g., Figs. A-8, A78), the maxillary 1-3 arch chord is large and is treated by retracting the central incisors (mesial contact) distally to create a broader, more rounded arch form. In this situation the incisors are being brought into a broader section of the arch. When, in contrast, the maxillary central incisors are linguoverted at the start of treatment (e.g., Figs. A-61, A-68), there tends to be little mesiodistal movement of the central incisors themselves, and retraction of the other anterior teeth is used to develop the arch form.

Predictive models for the central incisor (distal) are listed in Tables 66 and 67 , and the major association in each model is with maxillary incisor 
Table 64. Results of stepwise linear regression predicting the amount of mesiodistal tooth movement from the pretreatment malocclusion: results for maxillary right central incisor (mesial) in the subset in whom second premolars were extracted.

\begin{tabular}{lrrrr}
\hline \multicolumn{1}{c}{ Variable } & $\begin{array}{c}\text { Sum of } \\
\text { Squares }\end{array}$ & F Ratio & P Value & $\mathrm{r}^{2}$ \\
\hline Incisor Overjet & 0.14 & 0.08 & 0.7826 & \\
Incisor Overbite & 1.61 & 0.94 & 0.3396 & \\
Irregularity Index & 0.63 & 0.36 & 0.5522 & \\
Incisor Spacing & 0.50 & 0.29 & 0.5971 & \\
Max 3-3 Width & 3.67 & 2.22 & 0.1454 & \\
Max 6-6 Width & 12.33 & 7.21 & $\underline{\mathbf{0 . 0 1 1 2}}$ & 0.1330 \\
Mand 3-3 Width & 0.04 & 0.02 & 0.8835 & \\
Mand 6-6 Width & 0.27 & 0.15 & 0.6997 & \\
UL 3-3 Width Difference & 4.39 & 2.69 & 0.1105 & \\
UL 6-6 Width Difference & 0.27 & 0.15 & 0.6997 & \\
Max 1-3 Chord & 34.55 & 20.19 & $\underline{\mathbf{0 . 0 0 0 1}}$ & 0.2218 \\
Max 1-6 Chord & 6.89 & 4.43 & 0.0430 & \\
Mand 1-3 Chord & 2.81 & 1.67 & 0.2049 & \\
Mand 1-6 Chord & 1.82 & 1.07 & 0.3089 & \\
Midline Deviation & 1.02 & 0.59 & 0.4493 & \\
Buccal Segment Relation & 1.91 & 1.12 & 0.2980 & \\
Canine Discrepancy & 2.09 & 1.23 & 0.2753 & \\
Patient's Sex & 9.77 & 5.71 & $\underline{\mathbf{0 . 0 2 2 6}}$ & 0.0930 \\
\hline
\end{tabular}


Table 65. Results of stepwise linear regression predicting the amount of mesiodistal tooth movement from the pretreatment malocclusion: results for maxillary left central incisor (mesial) in the subset in whom second premolars were extracted.

\begin{tabular}{lrlll}
\hline \multicolumn{1}{c}{ Variable } & $\begin{array}{c}\text { Sum of } \\
\text { Squares }\end{array}$ & F Ratio & P Value & $\mathrm{r}^{2}$ \\
\hline Incisor Overjet & 1.96 & 0.55 & 0.4654 & \\
Incisor Overbite & 2.08 & 0.58 & 0.4516 & \\
Irregularity Index & 17.44 & 4.91 & $\underline{0.0331}$ & 0.1200 \\
Incisor Spacing & 1.34 & 0.37 & 0.5466 & \\
Max 3-3 Width & 0.80 & 0.22 & 0.6410 & \\
Max 6-6 Width & 1.02 & 0.28 & 0.5993 & \\
Mand 3-3 Width & 0.80 & 0.22 & 0.6427 & \\
Mand 6-6 Width & 3.50 & 0.99 & 0.3279 & \\
UL 3-3 Width Difference & 0.05 & 0.01 & 0.9100 & \\
UL 6-6 Width Difference & 0.74 & 0.20 & 0.6545 & \\
Max 1-3 Chord & 5.17 & 1.47 & 0.2329 & \\
Max 1-6 Chord & 7.30 & 2.12 & 0.1544 & \\
Mand 1-3 Chord & 2.24 & 0.62 & 0.4348 & \\
Mand 1-6 Chord & 5.66 & 1.62 & 0.2113 & \\
Midline Deviation & 0.31 & 0.08 & 0.7733 & \\
Buccal Segment Relation & 10.40 & 3.10 & 0.0870 & \\
Canine Discrepancy & 3.91 & 1.11 & 0.3003 & \\
Patient's Sex & 8.68 & 2.55 & 0.1194 & \\
\hline
\end{tabular}




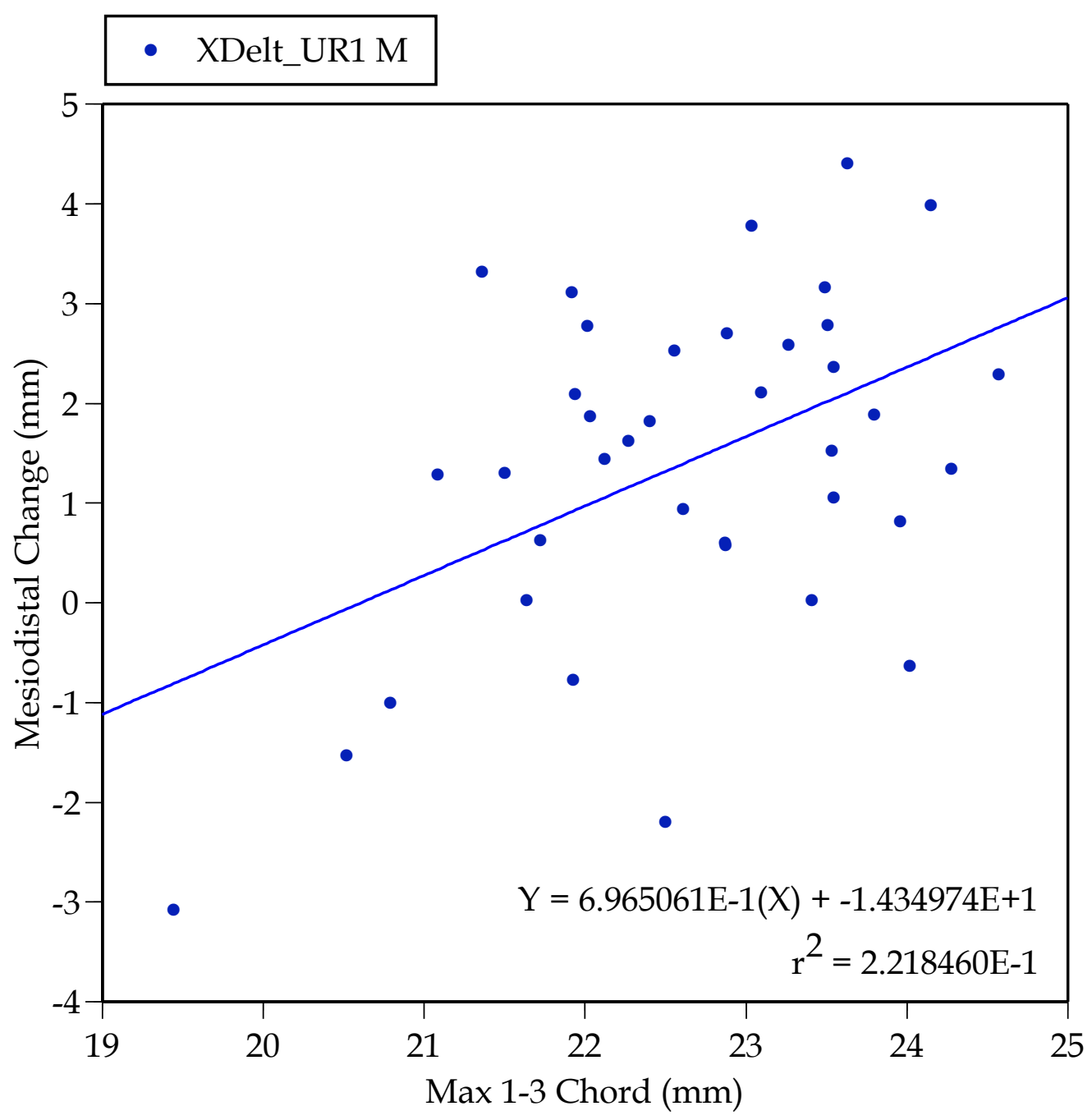

Fig. 68. Scatterplot showing the association between pretreatment maxillary 1-3 chord distance and the amount of mesiodistal change of the maxillary right central incisor (mesial) analyzed in just the second-premolar extraction sample. 
Table 66. Results of stepwise linear regression predicting the amount of mesiodistal tooth movement from the pretreatment malocclusion: results for maxillary left central incisor (distal) in the subset in whom second premolars were extracted.

\begin{tabular}{lrlll}
\hline \multicolumn{1}{c}{ Variable } & $\begin{array}{c}\text { Sum of } \\
\text { Squares }\end{array}$ & F Ratio & P Value & $\mathrm{r}^{2}$ \\
\hline Incisor Overjet & 0.01 & 0.00 & 0.9556 & \\
Incisor Overbite & 4.50 & 0.99 & 0.3264 & \\
Irregularity Index & 19.56 & 4.30 & $\underline{\mathbf{0 . 0 4 5 3}}$ & 0.1068 \\
Incisor Spacing & 0.23 & 0.05 & 0.8251 & \\
Max 3-3 Width & 5.16 & 1.14 & 0.2933 & \\
Max 6-6 Width & 0.16 & 0.03 & 0.8548 & \\
Mand 3-3 Width & 0.04 & 0.01 & 0.9274 & \\
Mand 6-6 Width & 0.09 & 0.02 & 0.8917 & \\
UL 3-3 Width Difference & 9.56 & 2.17 & 0.1495 & \\
UL 6-6 Width Difference & 0.98 & 0.21 & 0.6486 & \\
Max 1-3 Chord & 0.14 & 0.03 & 0.8622 & \\
Max 1-6 Chord & 4.94 & 1.09 & 0.3037 & \\
Mand 1-3 Chord & 3.14 & 0.68 & 0.4139 & \\
Mand 1-6 Chord & 4.71 & 1.04 & 0.3157 & \\
Midline Deviation & 16.15 & 3.83 & 0.0583 & \\
Buccal Segment Relation & 5.44 & 1.20 & 0.2799 & \\
Canine Discrepancy & 0.03 & 0.01 & 0.9322 & \\
Patient's Sex & 0.02 & 0.01 & 0.9457 & \\
\hline
\end{tabular}


Table 67. Results of stepwise linear regression predicting the amount of mesiodistal tooth movement from the pretreatment malocclusion: results for maxillary right central incisor (distal) in the subset in whom second premolars were extracted.

\begin{tabular}{lrlll}
\hline \multicolumn{1}{c}{ Variable } & $\begin{array}{c}\text { Sum of } \\
\text { Squares }\end{array}$ & F Ratio & P Value & $\mathrm{r}^{2}$ \\
\hline Incisor Overjet & 0.79 & 0.41 & 0.5245 & \\
Incisor Overbite & 0.16 & 0.08 & 0.7775 & \\
Irregularity Index & 10.31 & 5.52 & $\underline{\mathbf{0 . 0 2 4 8}}$ & 0.1305 \\
Incisor Spacing & 2.69 & 1.46 & 0.2361 & \\
Max 3-3 Width & 5.85 & 3.35 & 0.0763 & \\
Max 6-6 Width & 1.59 & 0.85 & 0.3646 & \\
Mand 3-3 Width & 5.12 & 2.89 & 0.0984 & \\
Mand 6-6 Width & 1.19 & 0.63 & 0.4331 & \\
UL 3-3 Width Difference & 1.40 & 0.74 & 0.3954 & \\
UL 6-6 Width Difference & 0.36 & 0.19 & 0.6677 & \\
Max 1-3 Chord & 0.26 & 0.14 & 0.7149 & \\
Max 1-6 Chord & 0.70 & 0.37 & 0.5485 & \\
Mand 1-3 Chord & 8.05 & 4.31 & $\underline{\mathbf{0 . 0 4 5 6}}$ & 0.0852 \\
Mand 1-6 Chord & 0.04 & 0.02 & 0.8918 & \\
Midline Deviation & 2.33 & 1.26 & 0.2702 & \\
Buccal Segment Relation & 15.49 & 8.29 & $\underline{\mathbf{0 . 0 0 6 8}}$ & 0.1114 \\
Canine Discrepancy & 0.09 & 0.05 & 0.8311 & \\
Patient's Sex & 0.66 & 0.35 & 0.5612 & \\
\hline
\end{tabular}


irregularity $\left(\mathrm{r}^{2}=10 \%\right)$. In cases with high incisor irregularity (e.g., Figs. A-36, A-80), the tendency is for the central incisors to be rotated into alignment without a great deal of labiolingual displacement (Fig. 69). Conversely, greater mesiodistal displacement tends to occur where there is less irregularity. One example is illustrated in Fig. A-57, where there is winging of the central incisors, so the distal aspects need to be rotated lingually. A different situation is shown in Fig. A-78, where, again, there is little incisor irregularity, but there was appreciable incisor retraction to round-out the anterior segment and diminish overjet.

\section{$\underline{\text { Lateral Incisor }}$}

There was no significant occlusal predictor for the lateral incisor (mesial) in the left quadrant, and the stronger of the two predictors in the right quadrant was overbite $($ Tables 68,69$)$. The association with overbite $\left(\mathrm{r}^{2}=\right.$ $24 \%$ ) is that people with shallow bites (e.g., Figs. A-48, A-61) tend to undergo incisor labioversion, assumedly to deepen the bite. People with deep bites (e.g., Figs. A-36, A-46) undergo linguoversion, partly to resolve the irregularity but to also open the bite (Fig. 70).

There are, likewise, few predictors for change in the distal aspects of the lateral incisors (Tables 70, 71); no occlusal predictor is significant in the left quadrant, and incisor overbite is the most strongly associated of the two 


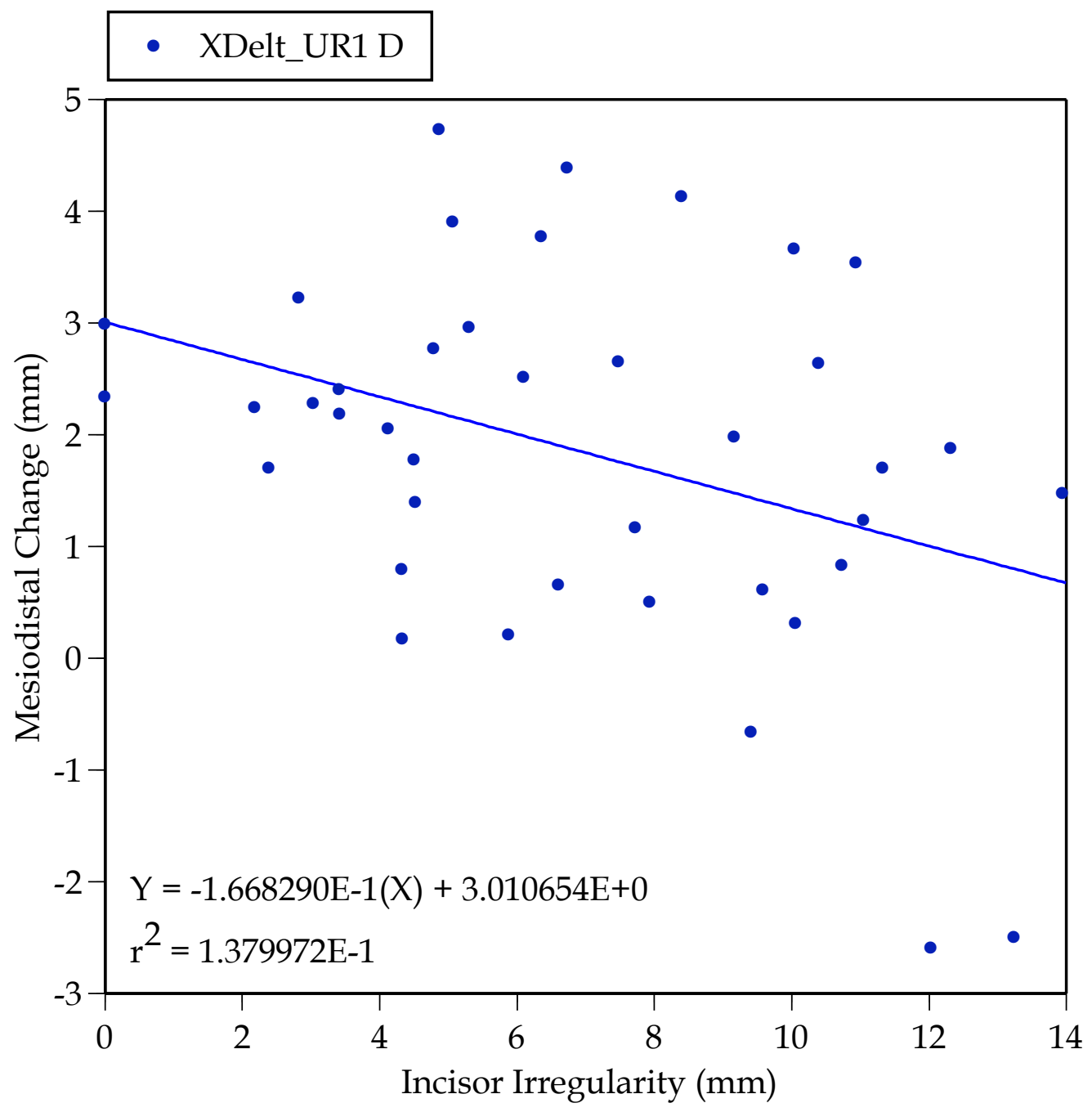

Fig. 69. Scatterplot showing the association between pretreatment incisor irregularity and the amount of mesiodistal change of the maxillary right central incisor (distal) analyzed in just the second-premolar extraction sample. 
Table 68. Results of stepwise linear regression predicting the amount of mesiodistal tooth movement from the pretreatment malocclusion: results for maxillary left lateral incisor (mesial) in the subset in whom second premolars were extracted.

\begin{tabular}{lccll}
\hline \multicolumn{1}{c}{ Variable } & $\begin{array}{c}\text { Sum of } \\
\text { Squares }\end{array}$ & F Ratio & P Value & $\mathrm{r}^{2}$ \\
\hline Incisor Overjet & & & & \\
Incisor Overbite & 1.88 & 0.39 & 0.5390 & \\
Irregularity Index & 0.58 & 0.12 & 0.7324 & \\
Incisor Spacing & 0.27 & 1.10 & 0.3015 & \\
Max 3-3 Width & 2.53 & 0.02 & 0.8833 & \\
Max 6-6 Width & 0.22 & 0.52 & 0.4757 & \\
Mand 3-3 Width & 0.16 & 0.04 & 0.8351 \\
Mand 6-6 Width & 0.00 & 0.00 & 0.8580 \\
UL 3-3 Width Difference & 3.40 & 0.70 & 0.9818 & \\
UL 6-6 Width Difference & 0.41 & 0.08 & 0.7734 \\
Max 1-3 Chord & 0.72 & 0.15 & 0.7048 \\
Max 1-6 Chord & 5.74 & 1.20 & 0.2805 \\
Mand 1-3 Chord & 7.34 & 1.55 & 0.2210 \\
Mand 1-6 Chord & 7.05 & 1.49 & 0.2307 \\
Midline Deviation & 12.24 & 2.66 & 0.1114 \\
Buccal Segment Relation & 0.14 & 0.03 & 0.8651 \\
Canine Discrepancy & 7.35 & 1.55 & 0.2208 \\
Patient's Sex & 1.01 & 0.21 & 0.6532 & \\
\hline
\end{tabular}


Table 69. Results of stepwise linear regression predicting the amount of mesiodistal tooth movement from the pretreatment malocclusion: results for maxillary right lateral incisor (mesial) in the subset in whom second premolars were extracted.

\begin{tabular}{lrrrr}
\hline \multicolumn{1}{c}{ Variable } & $\begin{array}{c}\text { Sum of } \\
\text { Squares }\end{array}$ & F Ratio & P Value & $\mathrm{r}^{2}$ \\
\hline Incisor Overjet & 0.32 & 0.15 & 0.7020 & \\
Incisor Overbite & 35.29 & 16.62 & $\underline{\mathbf{0 . 0 0 0 3}}$ & 0.2426 \\
Irregularity Index & 17.84 & 8.40 & $\underline{\mathbf{0 . 0 0 6 4}}$ & 0.1466 \\
Incisor Spacing & 2.36 & 1.11 & 0.2987 & \\
Max 3-3 Width & 0.01 & 0.01 & 0.9428 & \\
Max 6-6 Width & 0.02 & 0.01 & 0.9182 & \\
Mand 3-3 Width & 0.54 & 0.25 & 0.6213 & \\
Mand 6-6 Width & 0.02 & 0.01 & 0.9300 & \\
UL 3-3 Width Difference & 0.51 & 0.23 & 0.6321 & \\
UL 6-6 Width Difference & 0.00 & 0.00 & 0.9630 & \\
Max 1-3 Chord & 0.24 & 0.11 & 0.7429 & \\
Max 1-6 Chord & 0.00 & 0.00 & 0.9824 & \\
Mand 1-3 Chord & 1.27 & 0.59 & 0.4466 & \\
Mand 1-6 Chord & 0.06 & 0.03 & 0.8674 & \\
Midline Deviation & 0.01 & 0.01 & 0.9432 & \\
Buccal Segment Relation & 0.00 & 0.00 & 0.9854 & \\
Canine Discrepancy & 4.19 & 2.03 & 0.1634 & \\
Patient's Sex & 1.73 & 0.81 & 0.3738 & \\
\hline
\end{tabular}




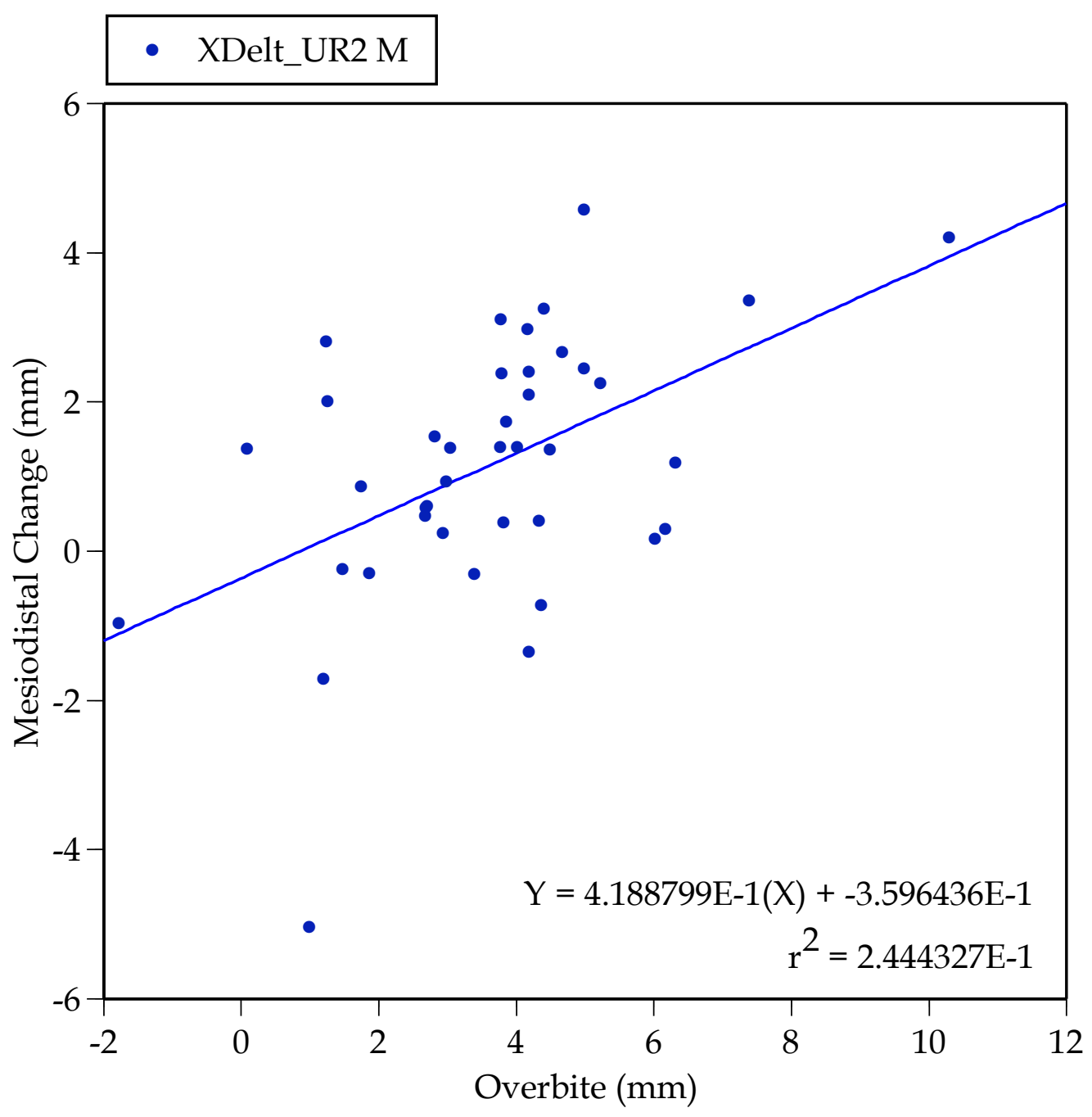

Fig. 70. Scatterplot showing the association between pretreatment incisor overbite and the amount of mesiodistal change of the maxillary right lateral incisor (mesial) analyzed in just the second-premolar extraction sample. 
Table 70. Results of stepwise linear regression predicting the amount of mesiodistal tooth movement from the pretreatment malocclusion: results for maxillary left lateral incisor (distal) in the subset in whom second premolars were extracted.

\begin{tabular}{lccll}
\hline \multicolumn{1}{c}{ Variable } & $\begin{array}{c}\text { Sum of } \\
\text { Squares }\end{array}$ & F Ratio & P Value & $\mathrm{r}^{2}$ \\
\hline Incisor Overjet & 0.04 & 0.01 & 0.9214 & \\
Incisor Overbite & 1.21 & 0.28 & 0.6014 & \\
Irregularity Index & 7.23 & 1.73 & 0.1971 & \\
Incisor Spacing & 0.03 & 0.01 & 0.9302 & \\
Max 3-3 Width & 2.02 & 0.47 & 0.4987 & \\
Max 6-6 Width & 0.48 & 0.11 & 0.7410 \\
Mand 3-3 Width & 0.03 & 0.01 & 0.9339 \\
Mand 6-6 Width & 0.04 & 0.01 & 0.9242 & \\
UL 3-3 Width Difference & 3.51 & 0.82 & 0.3717 \\
UL 6-6 Width Difference & 0.65 & 0.15 & 0.7028 \\
Max 1-3 Chord & 2.91 & 0.68 & 0.4168 \\
Max 1-6 Chord & 7.25 & 1.73 & 0.1964 \\
Mand 1-3 Chord & 4.00 & 0.94 & 0.3398 \\
Mand 1-6 Chord & 3.88 & 0.91 & 0.3470 \\
Midline Deviation & 10.18 & 2.48 & 0.1239 \\
Buccal Segment Relation & 2.43 & 0.56 & 0.4576 \\
Canine Discrepancy & 14.37 & 3.61 & 0.0656 \\
Patient's Sex & 2.77 & 0.64 & 0.4282 & \\
\hline
\end{tabular}


Table 71. Results of stepwise linear regression predicting the amount of mesiodistal tooth movement from the pretreatment malocclusion: results for maxillary right lateral incisor (distal) in the subset in whom second premolars were extracted.

\begin{tabular}{lrrrr}
\hline \multicolumn{1}{c}{ Variable } & $\begin{array}{c}\text { Sum of } \\
\text { Squares }\end{array}$ & F Ratio & P Value & $\mathrm{r}^{2}$ \\
\hline Incisor Overjet & 0.40 & 0.17 & 0.6840 & \\
Incisor Overbite & 58.12 & 25.14 & $\mathbf{0 . 0 0 0 0}$ & 0.3212 \\
Irregularity Index & 8.76 & 4.13 & 0.0500 & \\
Incisor Spacing & 0.93 & 0.39 & 0.5347 & \\
Max 3-3 Width & 0.14 & 0.06 & 0.8077 & \\
Max 6-6 Width & 0.53 & 0.22 & 0.6389 & \\
Mand 3-3 Width & 0.23 & 0.10 & 0.7595 & \\
Mand 6-6 Width & 0.74 & 0.31 & 0.5798 & \\
UL 3-3 Width Difference & 0.00 & 0.00 & 0.9903 & \\
UL 6-6 Width Difference & 0.00 & 0.00 & 0.9798 & \\
Max 1-3 Chord & 0.44 & 0.18 & 0.6705 & \\
Max 1-6 Chord & 0.01 & 0.00 & 0.9567 & \\
Mand 1-3 Chord & 0.68 & 0.29 & 0.5951 & \\
Mand 1-6 Chord & 0.48 & 0.20 & 0.6554 & \\
Midline Deviation & 0.06 & 0.03 & 0.8736 & \\
Buccal Segment Relation & 1.99 & 0.86 & 0.3610 & \\
Canine Discrepancy & 16.91 & 7.32 & $\mathbf{0 . 0 1 0 5}$ & 0.1174 \\
Patient's Sex & 6.06 & 2.75 & 0.1063 & \\
\hline
\end{tabular}


significant variables on the right $\left(\mathrm{r}^{2}=32 \%\right)$. Just as seen for the mesial aspect of this tooth (Table 69), case with shallow bites tend to experience incisor labioversion, while cases with deep bites undergo linguoversion during treatment, which helps open the bite.

\section{Canine}

Assessment of the canine movements in these second-premolar extraction cases carries along prior findings for the incisors that there are few significant occlusal predictors - probably precisely because the second premolars were removed rather than the first.

Regression models for the canine changes (mesial) are listed in Tables 72 and 73. Again, nothing is significant for the left quadrant. In the right quadrant, three predictors achieve significance, namely overjet, overbite, and mandibular 1-6 chord. Of these, the strongest association is with overjet $\left(\mathrm{r}^{2}=\right.$ $17 \%)$, but it is difficult from inspection of the maxillary dental changes alone to understand this association. Indeed, at face value it seems counter-intuitive (Fig. 71) that case with the greatest overjet should tend to be associated with the least maxillary canine movement. Inspection of the dental casts obviously helps understand the situation.

Cases at the upper-left of the graph (Fig. 71) have little overjet at the start of treatment, and they also tend to have maxillary canines that are 
Table 72. Results of stepwise linear regression predicting the amount of mesiodistal tooth movement from the pretreatment malocclusion: results for maxillary left canine (mesial) in the subset in whom second premolars were extracted.

\begin{tabular}{lccll}
\hline \multicolumn{1}{c}{ Variable } & $\begin{array}{c}\text { Sum of } \\
\text { Squares }\end{array}$ & F Ratio & P Value & $\mathrm{r}^{2}$ \\
\hline Incisor Overjet & 3.91 & 1.95 & 0.1714 & \\
Incisor Overbite & 0.69 & 0.33 & 0.5686 & \\
Irregularity Index & 0.10 & 0.05 & 0.8288 & \\
Incisor Spacing & 0.00 & 0.00 & 0.9658 \\
Max 3-3 Width & 3.75 & 1.86 & 0.1807 & \\
Max 6-6 Width & 3.59 & 1.78 & 0.1902 \\
Mand 3-3 Width & 4.47 & 2.25 & 0.1427 \\
Mand 6-6 Width & 2.88 & 1.42 & 0.2416 & \\
UL 3-3 Width Difference & 0.06 & 0.03 & 0.8647 \\
UL 6-6 Width Difference & 0.51 & 0.24 & 0.6263 \\
Max 1-3 Chord & 0.11 & 0.05 & 0.8211 \\
Max 1-6 Chord & 0.11 & 0.05 & 0.8175 \\
Mand 1-3 Chord & 7.12 & 3.72 & 0.0618 \\
Mand 1-6 Chord & 4.55 & 2.29 & 0.1389 \\
Midline Deviation & 0.22 & 0.11 & 0.7477 \\
Buccal Segment Relation & 0.03 & 0.02 & 0.9022 \\
Canine Discrepancy & 2.09 & 1.02 & 0.3198 \\
Patient's Sex & 3.95 & 1.97 & 0.1688 & \\
\hline
\end{tabular}


Table 73. Results of stepwise linear regression predicting the amount of mesiodistal tooth movement from the pretreatment malocclusion: results for maxillary right canine (mesial) in the subset in whom second premolars were extracted.

\begin{tabular}{lcccc}
\hline \multicolumn{1}{c}{ Variable } & $\begin{array}{c}\text { Sum of } \\
\text { Squares }\end{array}$ & F Ratio & P Value & $\mathrm{r}^{2}$ \\
\hline Incisor Overjet & 8.84 & 7.73 & $\underline{\mathbf{0 . 0 0 8 8}}$ & 0.1754 \\
Incisor Overbite & 0.88 & 0.77 & 0.3874 & \\
Irregularity Index & 9.23 & 8.07 & $\underline{\mathbf{0 . 0 0 7 5}}$ & 0.1331 \\
Incisor Spacing & 0.10 & 0.09 & 0.7693 & \\
Max 3-3 Width & 0.27 & 0.23 & 0.6370 & \\
Max 6-6 Width & 0.28 & 0.24 & 0.6253 & \\
Mand 3-3 Width & 0.00 & 0.00 & 0.9564 & \\
Mand 6-6 Width & 2.83 & 2.59 & 0.1173 & \\
UL 3-3 Width Difference & 0.34 & 0.29 & 0.5932 & \\
UL 6-6 Width Difference & 1.07 & 0.94 & 0.3398 & \\
Max 1-3 Chord & 2.55 & 2.31 & 0.1378 & \\
Max 1-6 Chord & 0.25 & 0.21 & 0.6489 & \\
Mand 1-3 Chord & 0.03 & 0.03 & 0.8719 & \\
Mand 1-6 Chord & 12.85 & 11.23 & $\underline{\mathbf{0 . 0 0 2 0}}$ & 0.1311 \\
Midline Deviation & 1.01 & 0.88 & 0.3553 & \\
Buccal Segment Relation & 0.28 & 0.24 & 0.6273 & \\
Canine Discrepancy & 0.11 & 0.09 & 0.7668 & \\
Patient's Sex & 0.12 & 0.10 & 0.7503 & \\
\hline
\end{tabular}




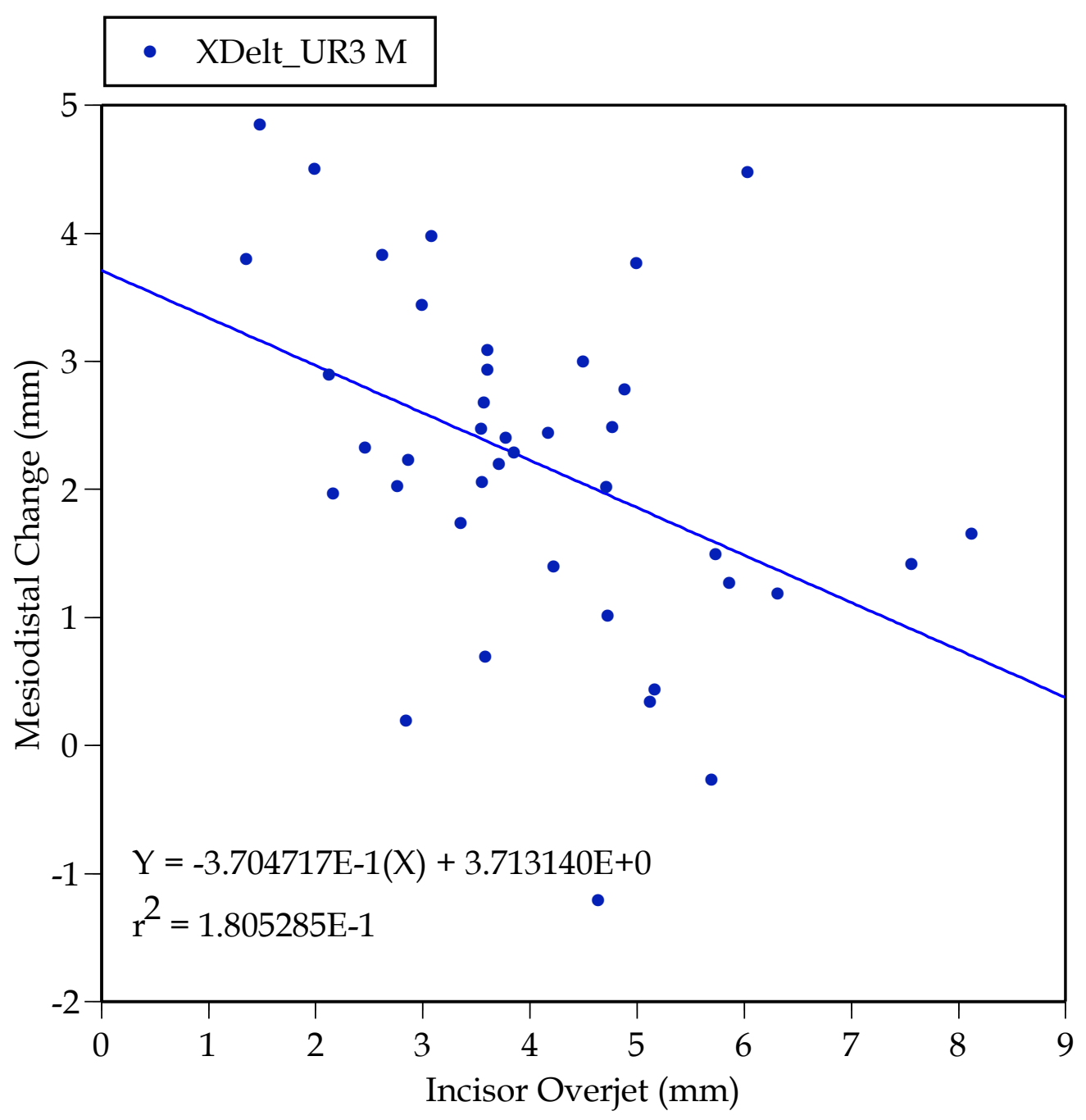

Fig. 71. Scatterplot showing the association between pretreatment overjet and the amount of mesiodistal change of the maxillary right canine (mesial) analyzed in just the second-premolar extraction sample. 
ectopic (labioverted), so the canines needed to be retracted to align them in the arch form. Figs. A-11 and A-61 are examples.

At the other extreme, cases such as illustrated in Figs. A-23 and A-85 have large overjets with linguoverted, overly-upright lower incisors (but good interdigitation of the canines). What is not discernible from study of the maxillary casts alone, is that these sorts of cases were corrected with little mesiodistal canine change but a good deal of change in the mandibular incisors (which alleviated the large overjets).

The two regression models for the canine (distal contact) are provided in Tables 74 and 75, and, again, there are considerable differences between the statistical results for the two quadrants. This may have to do with side differences in the frequency and extent of dental malocclusions (e.g., Kula et al. 1998; Harris and Bodford, 2007), but it also reflects the basic difference seen throughout the present study that orthodontist changed tooth positions in the anterior segment less in P2-extraction cases, so there is less, patterned variation to be assessed statistically. In other words, one can readily see that more-predictive models were developed for the P1-extraction sample (above), simply because there was more, patterned change in the anterior teeth in that sample.

Just one marginally-significant occlusal predictor was found for the left quadrant (Table 74), but there were several variables for the right quadrant 
Table 74. Results of stepwise linear regression predicting the amount of mesiodistal tooth movement from the pretreatment malocclusion: results for maxillary left canine (distal) in the subset in whom second premolars were extracted.

\begin{tabular}{lcccc}
\hline \multicolumn{1}{c}{ Variable } & $\begin{array}{c}\text { Sum of } \\
\text { Squares }\end{array}$ & F Ratio & P Value & $\mathrm{r}^{2}$ \\
\hline Incisor Overjet & 5.97 & 3.33 & 0.0764 & \\
Incisor Overbite & 0.28 & 0.14 & 0.7096 & \\
Irregularity Index & 1.00 & 0.52 & 0.4775 & \\
Incisor Spacing & 0.51 & 0.26 & 0.6105 & \\
Max 3-3 Width & 1.59 & 0.83 & 0.3685 & \\
Max 6-6 Width & 4.20 & 2.28 & 0.1401 & \\
Mand 3-3 Width & 2.92 & 1.56 & 0.2206 & \\
Mand 6-6 Width & 4.13 & 2.24 & 0.1436 & \\
UL 3-3 Width Difference & 0.06 & 0.03 & 0.8616 & \\
UL 6-6 Width Difference & 0.27 & 0.14 & 0.7116 & \\
Max 1-3 Chord & 0.04 & 0.02 & 0.8916 & \\
Max 1-6 Chord & 0.25 & 0.13 & 0.7207 & \\
Mand 1-3 Chord & 4.41 & 2.40 & 0.1303 & \\
Mand 1-6 Chord & 2.91 & 1.55 & 0.2217 & \\
Midline Deviation & 0.09 & 0.05 & 0.8290 & \\
Buccal Segment Relation & 0.00 & 0.00 & 0.9723 & \\
Canine Discrepancy & 4.87 & 2.67 & 0.1112 & \\
Patient's Sex & 8.46 & 4.44 & $\underline{\mathbf{0 . 0 4 2 2}}$ & 0.1097 \\
\hline
\end{tabular}


Table 75. Results of stepwise linear regression predicting the amount of mesiodistal tooth movement from the pretreatment malocclusion: results for maxillary right canine (distal) in the subset in whom second premolars were extracted.

\begin{tabular}{lrrrr}
\hline \multicolumn{1}{c}{ Variable } & $\begin{array}{c}\text { Sum of } \\
\text { Squares }\end{array}$ & F Ratio & P Value & $\mathrm{r}^{2}$ \\
\hline Incisor Overjet & 3.41 & 4.32 & 0.0460 & \\
Incisor Overbite & 0.13 & 0.14 & 0.7088 & \\
Irregularity Index & 21.46 & 24.66 & $\underline{\mathbf{0 . 0 0 0 0}}$ & 0.1902 \\
Incisor Spacing & 1.42 & 1.66 & 0.2067 & \\
Max 3-3 Width & 0.82 & 0.95 & 0.3386 & \\
Max 6-6 Width & 0.83 & 0.96 & 0.3356 & \\
Mand 3-3 Width & 0.82 & 0.95 & 0.3386 & \\
Mand 6-6 Width & 5.06 & 5.82 & $\underline{\mathbf{0 . 0 2 1 8}}$ & 0.0784 \\
UL 3-3 Width Difference & 5.77 & 6.63 & $\underline{\mathbf{0 . 0 1 4 8}}$ & 0.0721 \\
UL 6-6 Width Difference & 0.83 & 0.96 & 0.3356 & \\
Max 1-3 Chord & 3.54 & 4.52 & 0.0416 & \\
Max 1-6 Chord & 4.45 & 5.90 & $\underline{\mathbf{0 . 0 2 1 1}}$ & 0.1300 \\
Mand 1-3 Chord & 2.42 & 2.95 & 0.0959 & \\
Mand 1-6 Chord & 11.24 & 12.91 & $\mathbf{0 . 0 0 1 1}$ & 0.1326 \\
Midline Deviation & 11.62 & 13.35 & $\underline{\mathbf{0 . 0 0 0 9}}$ & 0.0953 \\
Buccal Segment Relation & 0.24 & 0.27 & 0.6106 & \\
Canine Discrepancy & 0.00 & 0.00 & 0.9628 & \\
Patient's Sex & 1.39 & 1.63 & 0.2117 & \\
\hline
\end{tabular}


(summary $\mathrm{r}^{2}=69.9 \%$ ). The strongest single predictor was incisor irregularity $\left(r^{2}=19 \%\right)$. The association here (Fig. 72$)$ is straightforward: Cases with considerable incisor irregularity required more canine retraction to align the anterior teeth. Conversely, cases with better alignment of the incisors required less canine retraction. It is worth recalling here that Little's measure of incisor irregularity includes any positional discrepancies at the left or right canine-lateral incisor contacts, so this association between the incisors and the canine both depend on canine positions.

\section{$\underline{\text { First Premolar }}$}

There was no occlusal predictor of the mesiodistal change of P1 in the left quadrant (Table 76). For the right quadrant (Table 77), incisor overjet was the major determinant in the model $\left(\mathrm{r}^{2}=22 \%\right)$. The relationship (Fig. 73) is essentially identical to that between overjet and canine movement (Fig. 71) discussed above: Cases with severe overjet (e.g., Figs. A-23, A-27, A-85) had a mandibular problem (such as linguoverted and retroclined lower incisors) rather than labioverted maxillary incisors. Cases in Fig. 73 with minor overjet (e.g., Figs. A-14, A-36) tended to exhibit labially displaced canines that warranted distal retraction (along with distal movement of the first premolars) to move the canines into the desired arch form. 


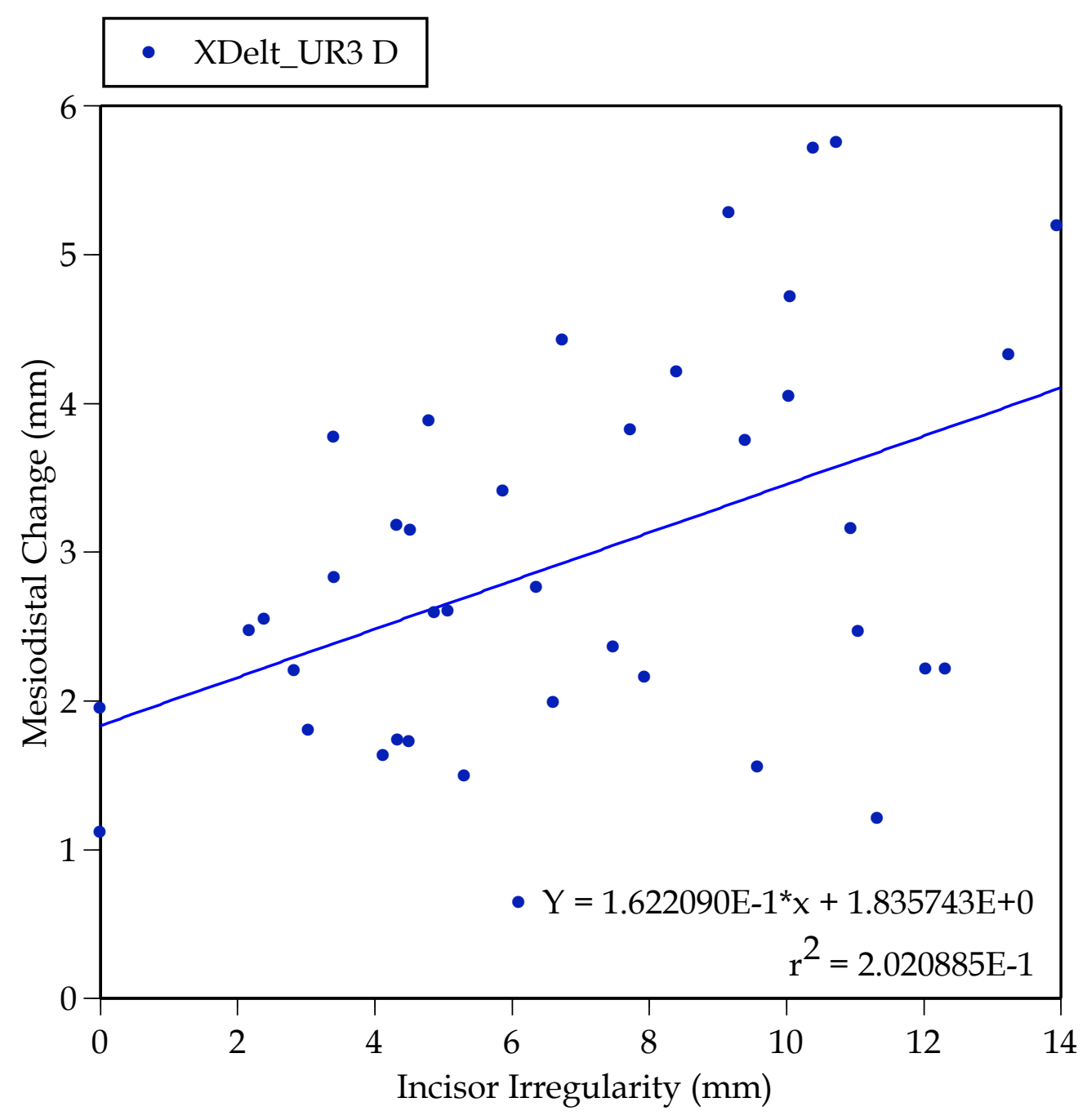

Fig. 72. Scatterplot showing the association between pretreatment maxillary incisor irregularity and the amount of mesiodistal change of the maxillary right caninie (distal) analyzed in just the second-premolar extraction sample. 
Table 76. Results of stepwise linear regression predicting the amount of mesiodistal tooth movement from the pretreatment malocclusion: results for maxillary left first premolar (mesial) in the subset in whom second premolars were extracted.

\begin{tabular}{lccll}
\hline \multicolumn{1}{c}{ Variable } & $\begin{array}{c}\text { Sum of } \\
\text { Squares }\end{array}$ & F Ratio & P Value & $\mathrm{r}^{2}$ \\
\hline Incisor Overjet & 5.60 & 2.74 & 0.1067 & \\
Incisor Overbite & 1.74 & 0.81 & 0.3748 & \\
Irregularity Index & 0.41 & 0.19 & 0.6677 & \\
Incisor Spacing & 0.18 & 0.08 & 0.7789 & \\
Max 3-3 Width & 1.63 & 0.76 & 0.3901 \\
Max 6-6 Width & 2.89 & 1.36 & 0.2505 \\
Mand 3-3 Width & 2.94 & 1.39 & 0.2468 \\
Mand 6-6 Width & 2.21 & 1.03 & 0.3164 \\
UL 3-3 Width Difference & 0.05 & 0.02 & 0.8796 \\
UL 6-6 Width Difference & 0.47 & 0.22 & 0.6451 \\
Max 1-3 Chord & 0.13 & 0.06 & 0.8098 \\
Max 1-6 Chord & 0.30 & 0.14 & 0.7130 \\
Mand 1-3 Chord & 1.54 & 0.71 & 0.4044 \\
Mand 1-6 Chord & 0.40 & 0.18 & 0.6704 \\
Midline Deviation & 0.25 & 0.11 & 0.7401 \\
Buccal Segment Relation & 0.00 & 0.00 & 0.9825 \\
Canine Discrepancy & 5.91 & 2.90 & 0.0972 \\
Patient's Sex & 7.36 & 3.69 & 0.0629 & \\
\hline
\end{tabular}


Table 77. Results of stepwise linear regression predicting the amount of mesiodistal tooth movement from the pretreatment malocclusion: results for maxillary right first premolar (mesial) in the subset in whom second premolars were extracted.

\begin{tabular}{lrrrr}
\hline \multicolumn{1}{c}{ Variable } & $\begin{array}{c}\text { Sum of } \\
\text { Squares }\end{array}$ & F Ratio & P Value & $\mathrm{r}^{2}$ \\
\hline Incisor Overjet & 16.78 & 18.43 & $\underline{\mathbf{0 . 0 0 0 1}}$ & 0.2209 \\
Incisor Overbite & 0.00 & 0.00 & 0.9926 & \\
Irregularity Index & 15.31 & 16.81 & $\underline{\mathbf{0 . 0 0 0 2}}$ & 0.1619 \\
Incisor Spacing & 2.31 & 2.67 & 0.1119 & \\
Max 3-3 Width & 1.10 & 1.22 & 0.2784 & \\
Max 6-6 Width & 1.66 & 1.86 & 0.1814 & \\
Mand 3-3 Width & 0.19 & 0.21 & 0.6509 & \\
Mand 6-6 Width & 0.41 & 0.45 & 0.5090 & \\
UL 3-3 Width Difference & 0.72 & 0.79 & 0.3808 & \\
UL 6-6 Width Difference & 1.10 & 1.22 & 0.2780 & \\
Max 1-3 Chord & 6.84 & 7.51 & $\underline{\mathbf{0 . 0 0 9 7}}$ & 0.1117 \\
Max 1-6 Chord & 0.72 & 0.78 & 0.3825 & \\
Mand 1-3 Chord & 0.36 & 0.39 & 0.5366 & \\
Mand 1-6 Chord & 0.67 & 0.73 & 0.3996 & \\
Midline Deviation & 0.69 & 0.75 & 0.3920 & \\
Buccal Segment Relation & 1.04 & 1.15 & 0.2922 & \\
Canine Discrepancy & 0.64 & 0.70 & 0.4097 & \\
Patient's Sex & 0.70 & 0.77 & 0.3874 & \\
\hline
\end{tabular}




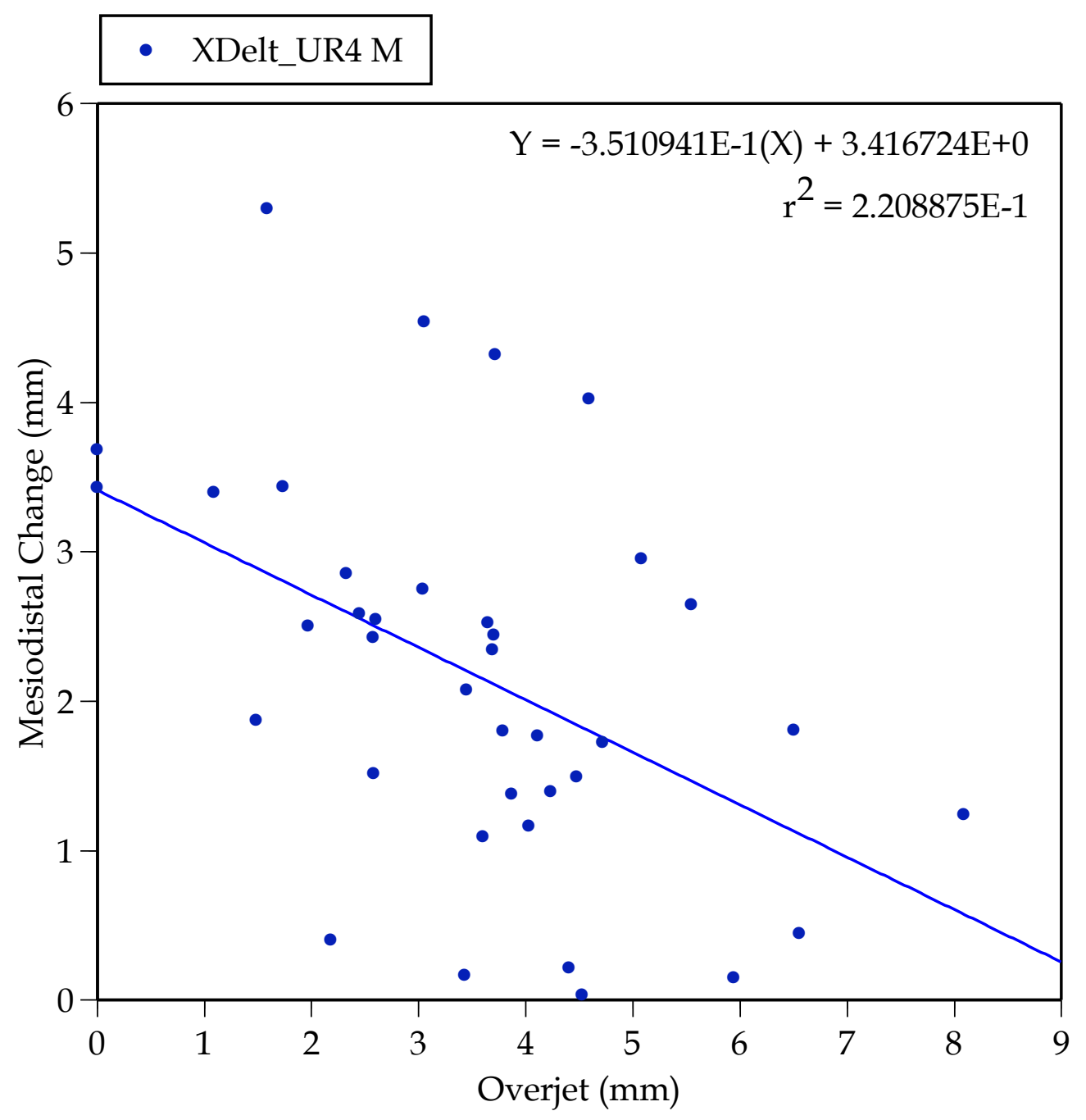

Fig. 73. Scatterplot showing the association between pretreatment overjet and the amount of mesiodistal change of the maxillary right first premolar (mesial) analyzed in just the second-premolar extraction sample. 
Mesiodistal movement of the first premolar (distal) are modeled in Tables 78 and 79. Canine discrepancy is the single occlusal predictor for the left quadrant $\left(\mathrm{r}^{2}=16 \%\right)$. Recalling that a "Class II" canine relationship was assigned a negative value, cases with a large negative value at pretreatment (e.g., Figs. A-27, A-61) were corrected primarily by mesial movement of the mandibular canine and premolar, with rather little mesiodistal change in the upper midarch. Such cases are in the lower-right of the plot in Fig. 74. At the other extreme (upper right quadrant, Fig. 74), cases (e.g., Figs. A-14, A-16) exhibit greater incisor irregularity and labioversion of the canines, requiring greater premolar retraction to develop the desired arch form.

\section{$\underline{\text { First Molar }}$}

Four occlusal variables are significantly associated with first molar movement in the left quadrant (Table 80). Of these, canine discrepancy is the major predictor $\left(\mathrm{r}^{2}=22 \%\right)$. Here (Fig. 75$)$, the greater the "Class II" canine discrepancy-the farther mesial the upper canine is of the lower caninepremolar embrasure-the more the first molar (mesial) is moved mesially. Extreme cases of this are illustrated in Figs. A-27 and A-61. In these cases, most of the P2 extraction space was used to move the maxillary first molar forward. Alternatively, where there was little canine discrepancy (upper-right 
Table 78. Results of stepwise linear regression predicting the amount of mesiodistal tooth movement from the pretreatment malocclusion: results for maxillary left first premolar (distal) in the subset in whom second premolars were extracted.

\begin{tabular}{lcccc}
\hline \multicolumn{1}{c}{ Variable } & $\begin{array}{c}\text { Sum of } \\
\text { Squares }\end{array}$ & F Ratio & P Value & $\mathrm{r}^{2}$ \\
\hline Incisor Overjet & 3.51 & 1.93 & 0.1737 & \\
Incisor Overbite & 4.95 & 2.79 & 0.1039 & \\
Irregularity Index & 4.03 & 2.24 & 0.1438 & \\
Incisor Spacing & 0.08 & 0.04 & 0.8387 & \\
Max 3-3 Width & 2.29 & 1.24 & 0.2737 & \\
Max 6-6 Width & 1.74 & 0.93 & 0.3407 & \\
Mand 3-3 Width & 4.55 & 2.54 & 0.1197 & \\
Mand 6-6 Width & 3.36 & 1.85 & 0.1831 & \\
UL 3-3 Width Difference & 0.13 & 0.07 & 0.7927 & \\
UL 6-6 Width Difference & 0.16 & 0.08 & 0.7766 & \\
Max 1-3 Chord & 0.34 & 0.18 & 0.6742 & \\
Max 1-6 Chord & 0.63 & 0.33 & 0.5675 & \\
Mand 1-3 Chord & 2.42 & 1.31 & 0.2601 & \\
Mand 1-6 Chord & 0.87 & 0.46 & 0.5032 & \\
Midline Deviation & 0.34 & 0.18 & 0.6777 & \\
Buccal Segment Relation & 4.20 & 2.34 & 0.1354 & \\
Canine Discrepancy & 13.07 & 7.01 & $\underline{0.0120}$ & 0.1629 \\
Patient's Sex & 6.42 & 3.70 & 0.0625 & \\
\hline
\end{tabular}


Table 79. Results of stepwise linear regression predicting the amount of mesiodistal tooth movement from the pretreatment malocclusion: results for maxillary right first premolar (distal) in the subset in whom second premolars were extracted.

\begin{tabular}{lrrrr}
\hline \multicolumn{1}{c}{ Variable } & $\begin{array}{c}\text { Sum of } \\
\text { Squares }\end{array}$ & F Ratio & P Value & $\mathrm{r}^{2}$ \\
\hline Incisor Overjet & & & & \\
Incisor Overbite & 20.71 & 17.36 & $\underline{\mathbf{0 . 0 0 0 2}}$ & 0.1314 \\
Irregularity Index & 0.03 & 0.02 & 0.8831 & \\
Incisor Spacing & 20.62 & 17.28 & $\underline{\mathbf{0 . 0 0 0 2}}$ & 0.2090 \\
Max 3-3 Width & 0.58 & 0.48 & 0.4955 & \\
Max 6-6 Width & 0.01 & 0.01 & 0.9344 & \\
Mand 3-3 Width & 2.10 & 1.80 & 0.1888 & \\
Mand 6-6 Width & 0.01 & 0.01 & 0.9417 & \\
UL 3-3 Width Difference & 1.04 & 0.87 & 0.3590 & \\
UL 6-6 Width Difference & 0.05 & 0.04 & 0.8457 & \\
Max 1-3 Chord & 0.62 & 0.51 & 0.4790 & \\
Max 1-6 Chord & 11.18 & 9.37 & $\mathbf{0 . 0 0 4 3}$ & 0.1425 \\
Mand 1-3 Chord & 1.10 & 0.92 & 0.3455 & \\
Mand 1-6 Chord & 0.89 & 0.74 & 0.3945 & \\
Midline Deviation & 0.30 & 0.25 & 0.6240 & \\
Buccal Segment Relation & 3.70 & 3.32 & 0.0777 & \\
Canine Discrepancy & 1.04 & 0.87 & 0.3592 & \\
Patient's Sex & 0.01 & 0.00 & 0.9491 & \\
\hline
\end{tabular}




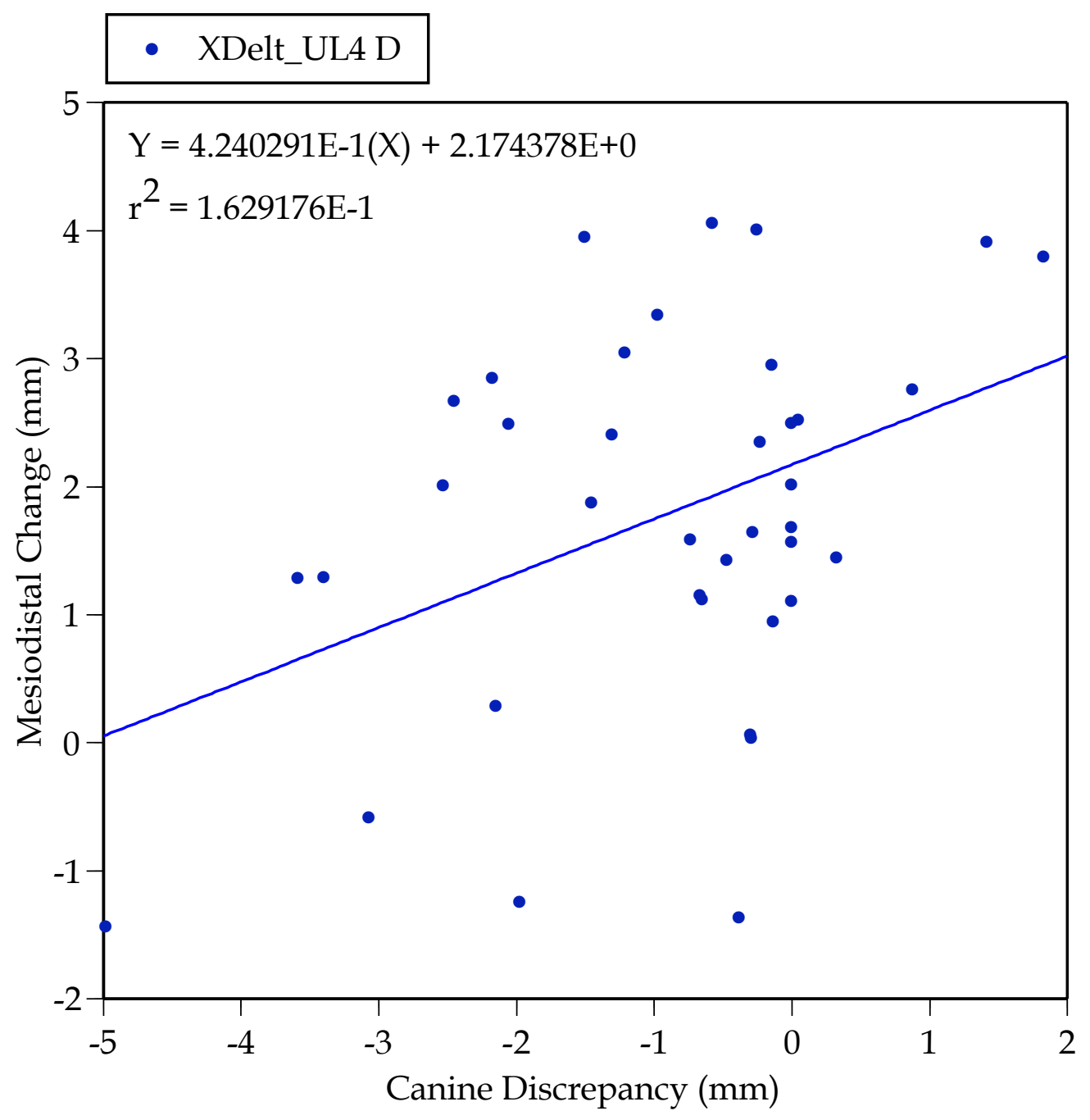

Fig. 74. Scatterplot showing the association between pretreatment canine discrepancy and the amount of mesiodistal change of the maxillary left first premolar (distal) analyzed in just the second-premolar extraction sample. 
Table 80. Results of stepwise linear regression predicting the amount of mesiodistal tooth movement from the pretreatment malocclusion: results for maxillary left first molar (mesial) in the subset in whom second premolars were extracted.

\begin{tabular}{lrrrr}
\hline \multicolumn{1}{c}{ Variable } & $\begin{array}{c}\text { Sum of } \\
\text { Squares }\end{array}$ & F Ratio & P Value & $\mathrm{r}^{2}$ \\
\hline Incisor Overjet & 0.39 & 0.32 & 0.5785 & \\
Incisor Overbite & 20.17 & 16.48 & $\underline{\mathbf{0 . 0 0 0 3}}$ & 0.1290 \\
Irregularity Index & 1.85 & 1.53 & 0.2248 & \\
Incisor Spacing & 0.23 & 0.18 & 0.6745 & \\
Max 3-3 Width & 0.12 & 0.09 & 0.7625 & \\
Max 6-6 Width & 0.02 & 0.02 & 0.8928 & \\
Mand 3-3 Width & 0.78 & 0.63 & 0.4335 & \\
Mand 6-6 Width & 16.41 & 13.41 & $\underline{\mathbf{0 . 0 0 0 9}}$ & 0.1320 \\
UL 3-3 Width Difference & 1.56 & 1.28 & 0.2655 & \\
UL 6-6 Width Difference & 0.02 & 0.02 & 0.8928 & \\
Max 1-3 Chord & 0.00 & 0.00 & 0.9703 & \\
Max 1-6 Chord & 6.01 & 4.91 & $\underline{\mathbf{0 . 0 3 3 6}}$ & 0.0670 \\
Mand 1-3 Chord & 2.29 & 1.92 & 0.1752 & \\
Mand 1-6 Chord & 0.98 & 0.80 & 0.3793 & \\
Midline Deviation & 0.04 & 0.03 & 0.8554 & \\
Buccal Segment Relation & 0.99 & 0.81 & 0.3760 & \\
Canine Discrepancy & 33.22 & 27.15 & $\underline{\mathbf{0 . 0 0 0 0}}$ & 0.2212 \\
Patient's Sex & 0.49 & 0.39 & 0.5353 & \\
\hline
\end{tabular}




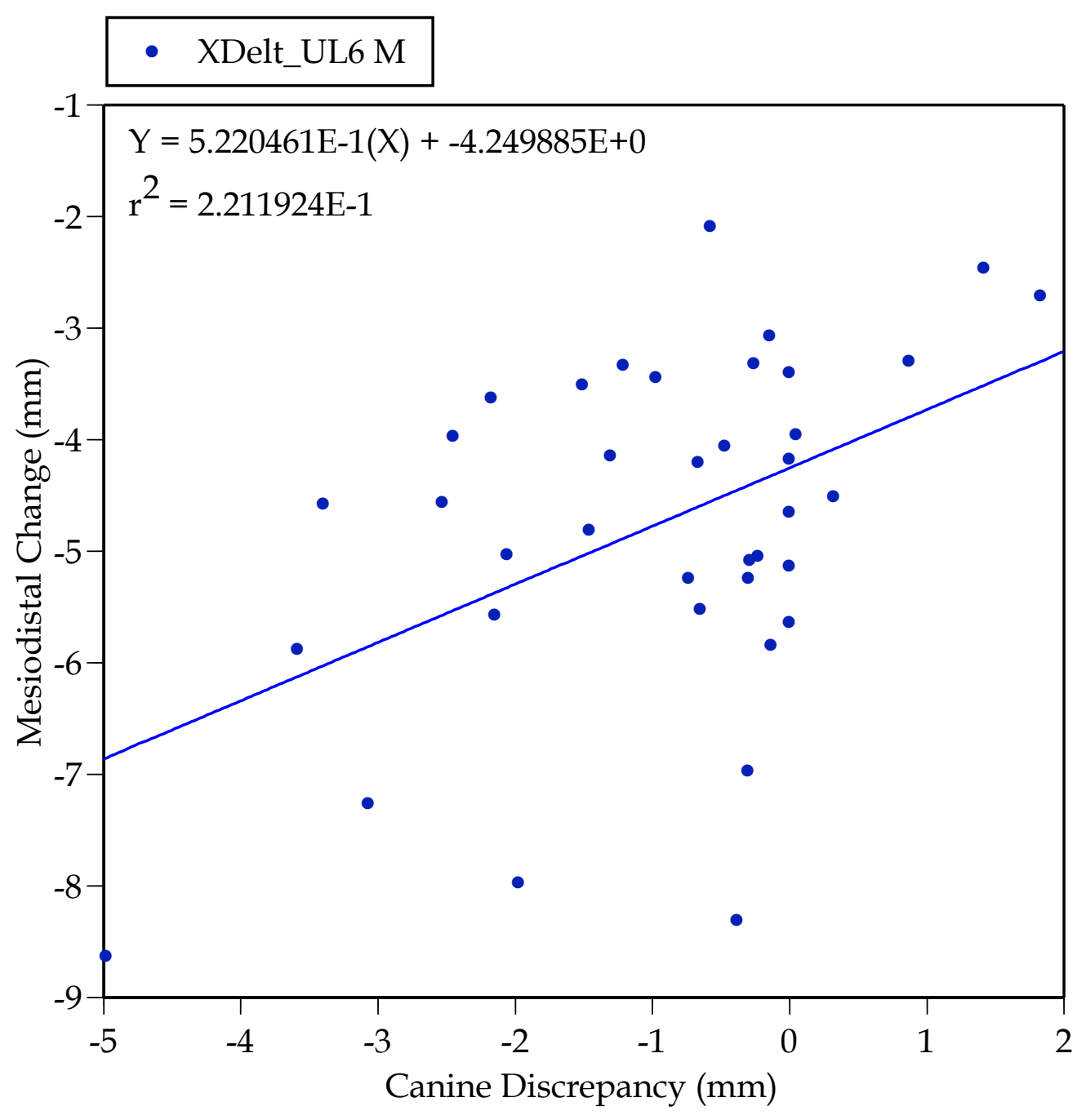

Fig. 75. Scatterplot showing the association between pretreatment canine discrepancy and the amount of mesiodistal change of the maxillary left first molar (mesial) analyzed in just the second-premolar extraction sample. 
of Fig. 75), there also was little molar movement. The plots in Fig. A-14 and A-16 illustrate this latter situation.

Two, wholly different predictive variables were significant for the right quadrant (Table 81), with incisor irregularity being the more important single predictor. The relationship here (Fig. 76) is easy to understand: The greater the irregularity the less the first molar (mesial) is moved mesially, which we suppose is because more of the extraction space is used to resolve the incisor irregularity.

There is no commonality of the occlusal predictors for the left and right first molar changes (distal contact) as shown in Tables 82 and 83. Canine discrepancy was the more powerful predictor on the left $\left(\mathrm{r}^{2}=19 \%\right)$. This relationship (Fig. 77) is that the greater the Class II canine discrepancy, the more the first molar is moved mesially into the P2 extraction space. Not surprisingly, this is the same relationship seen (above) for the first molar's mesial contact.

Comparably, for the right quadrant (Table 83), incisor irregularity is the stronger of the two occlusal predictors $\left(\mathrm{r}^{2}=17 \%\right)$. The greater the irregularity (Fig. 78), the less the first molar was moved mesially, evidently because the extraction space was used to align the anterior teeth. 
Table 81. Results of stepwise linear regression predicting the amount of mesiodistal tooth movement from the pretreatment malocclusion: results for maxillary right first molar (mesial) in the subset in whom second premolars were extracted.

\begin{tabular}{lrlll}
\hline \multicolumn{1}{c}{ Variable } & $\begin{array}{c}\text { Sum of } \\
\text { Squares }\end{array}$ & F Ratio & P Value & $\mathrm{r}^{2}$ \\
\hline Incisor Overjet & 10.17 & 6.51 & $\underline{\mathbf{0 . 0 1 5 3}}$ & 0.1273 \\
Incisor Overbite & 1.95 & 1.26 & 0.2701 & \\
Irregularity Index & 11.28 & 7.22 & $\underline{\mathbf{0 . 0 1 1 0}}$ & 0.1881 \\
Incisor Spacing & 0.29 & 0.18 & 0.6746 & \\
Max 3-3 Width & 3.28 & 2.17 & 0.1499 & \\
Max 6-6 Width & 0.72 & 0.46 & 0.5042 & \\
Mand 3-3 Width & 0.19 & 0.12 & 0.7341 & \\
Mand 6-6 Width & 0.31 & 0.19 & 0.6648 & \\
UL 3-3 Width Difference & 4.59 & 3.11 & 0.0866 & \\
UL 6-6 Width Difference & 0.35 & 0.22 & 0.6451 & \\
Max 1-3 Chord & 8.33 & 6.11 & 0.0186 & \\
Max 1-6 Chord & 1.32 & 0.84 & 0.3658 & \\
Mand 1-3 Chord & 0.44 & 0.27 & 0.6042 & \\
Mand 1-6 Chord & 2.20 & 1.43 & 0.2405 & \\
Midline Deviation & 1.08 & 0.69 & 0.4128 & \\
Buccal Segment Relation & 0.10 & 0.07 & 0.8009 & \\
Canine Discrepancy & 2.32 & 1.51 & 0.2278 & \\
Patient's Sex & 1.95 & 1.26 & 0.2704 & \\
\hline
\end{tabular}




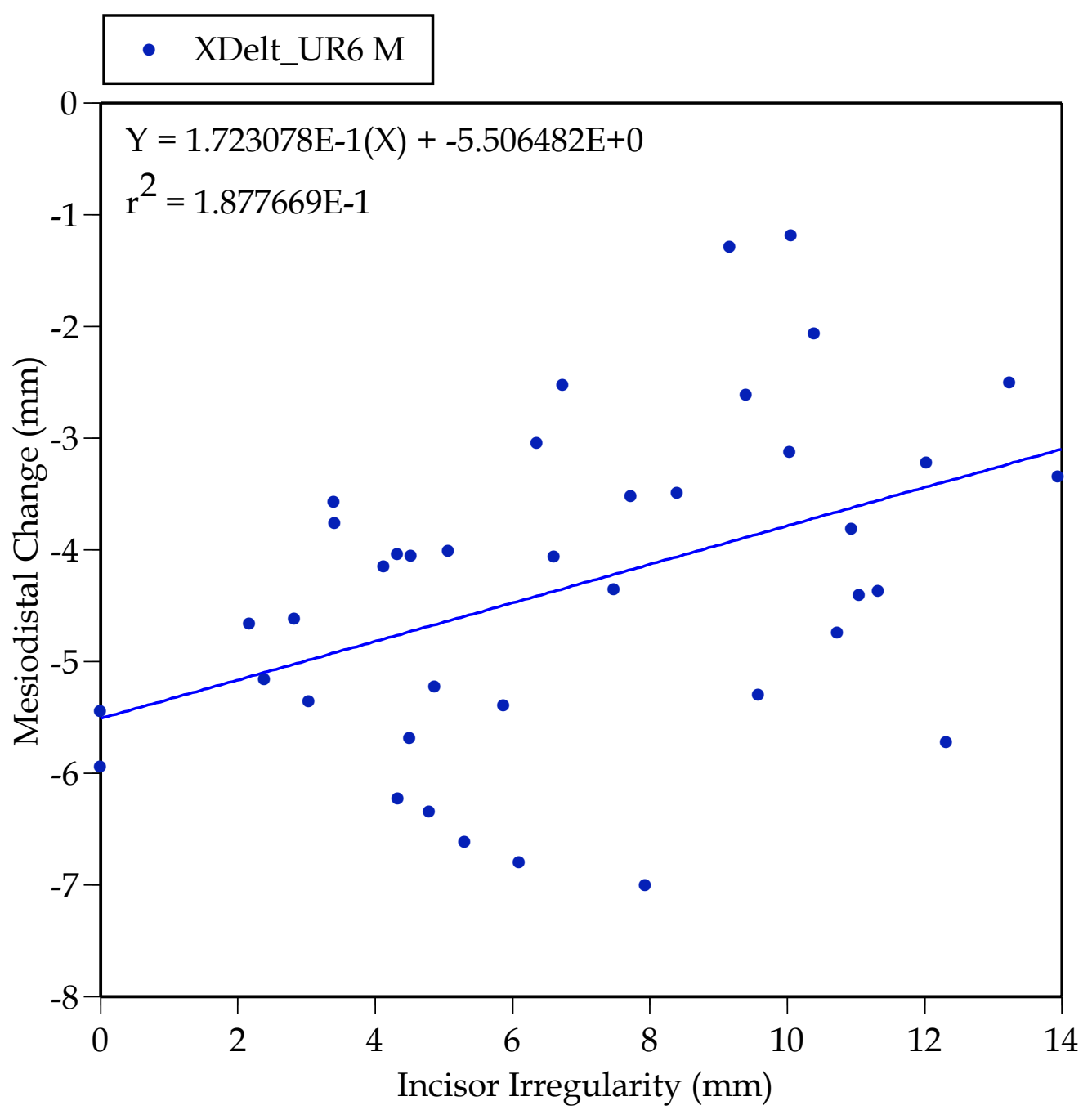

Fig. 76. Scatterplot showing the association between pretreatment maxillary incisor irregularity and the amount of mesiodistal change of the maxillary right first molar (mesial) analyzed in just the second-premolar extraction sample. 
Table 82. Results of stepwise linear regression predicting the amount of mesiodistal tooth movement from the pretreatment malocclusion: results for maxillary left first molar (distal) in the subset in whom second premolars were extracted.

\begin{tabular}{lrrrr}
\hline \multicolumn{1}{c}{ Variable } & $\begin{array}{c}\text { Sum of } \\
\text { Squares }\end{array}$ & F Ratio & P Value & $\mathrm{r}^{2}$ \\
\hline Incisor Overjet & & & & \\
Incisor Overbite & 11.07 & 1.15 & 0.2920 & \\
Irregularity Index & 3.85 & 6.49 & $\underline{\mathbf{0 . 0 1 5 4}}$ & 0.1260 \\
Incisor Spacing & 0.11 & 2.19 & 0.1479 & \\
Max 3-3 Width & 0.00 & 0.00 & 0.8107 & \\
Max 6-6 Width & 2.18 & 1.21 & 0.9720 & \\
Mand 3-3 Width & 2.55 & 1.42 & 0.2790 & \\
Mand 6-6 Width & 4.19 & 2.40 & 0.1306 & \\
UL 3-3 Width Difference & 3.74 & 2.13 & 0.1537 & \\
UL 6-6 Width Difference & 0.12 & 0.06 & 0.8043 & \\
Max 1-3 Chord & 1.95 & 1.08 & 0.3066 & \\
Max 1-6 Chord & 0.56 & 0.31 & 0.5846 & \\
Mand 1-3 Chord & 2.12 & 1.17 & 0.2863 & \\
Mand 1-6 Chord & 0.49 & 0.27 & 0.6102 & \\
Midline Deviation & 1.87 & 1.03 & 0.3172 & \\
Buccal Segment Relation & 1.84 & 1.02 & 0.3205 & \\
Canine Discrepancy & 24.70 & 13.61 & $\underline{\mathbf{0 . 0 0 0 8}}$ & 0.1926 \\
Patient's Sex & 3.06 & 1.72 & 0.1986 & \\
\hline
\end{tabular}


Table 83. Results of stepwise linear regression predicting the amount of mesiodistal tooth movement from the pretreatment malocclusion: results for maxillary right first molar (distal) in the subset in whom second premolars were extracted.

\begin{tabular}{lrlll}
\hline \multicolumn{1}{c}{ Variable } & $\begin{array}{c}\text { Sum of } \\
\text { Squares }\end{array}$ & F Ratio & P Value & $\mathrm{r}^{2}$ \\
\hline Incisor Overjet & 6.69 & 4.13 & $\underline{\mathbf{0 . 0 4 9 8}}$ & 0.00873 \\
Incisor Overbite & 3.93 & 2.53 & 0.1210 & \\
Irregularity Index & 10.26 & 6.33 & $\underline{\mathbf{0 . 0 1 6 6}}$ & 0.1720 \\
Incisor Spacing & 0.22 & 0.13 & 0.7210 & \\
Max 3-3 Width & 0.28 & 0.17 & 0.6866 & \\
Max 6-6 Width & 0.05 & 0.03 & 0.8603 & \\
Mand 3-3 Width & 0.08 & 0.05 & 0.8300 & \\
Mand 6-6 Width & 0.90 & 0.55 & 0.4642 & \\
UL 3-3 Width Difference & 1.24 & 0.76 & 0.3887 & \\
UL 6-6 Width Difference & 2.49 & 1.56 & 0.2205 & \\
Max 1-3 Chord & 6.52 & 4.41 & 0.0432 & \\
Max 1-6 Chord & 0.14 & 0.08 & 0.7773 & \\
Mand 1-3 Chord & 0.24 & 0.14 & 0.7076 & \\
Mand 1-6 Chord & 0.00 & 0.00 & 0.9891 & \\
Midline Deviation & 0.59 & 0.36 & 0.5547 & \\
Buccal Segment Relation & 0.89 & 0.54 & 0.4680 & \\
Canine Discrepancy & 1.32 & 0.81 & 0.3753 & \\
Patient's Sex & 2.65 & 1.66 & 0.2057 & \\
\hline
\end{tabular}




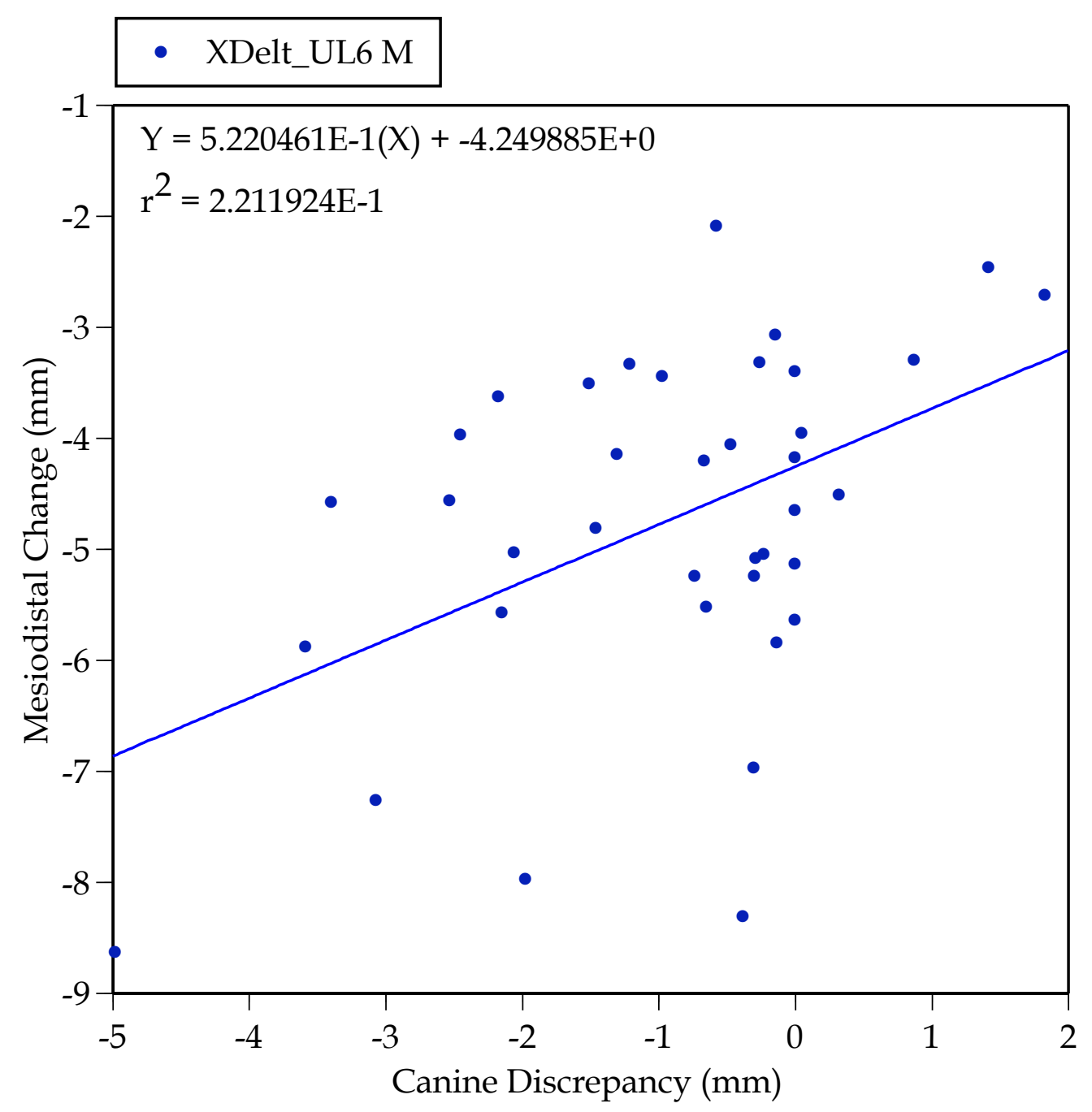

Fig. 77. Scatterplot showing the association between pretreatment canine discrepany and the amount of mesiodistal change of the maxillary left first molar (distal) analyzed in just the second-premolar extraction sample. 


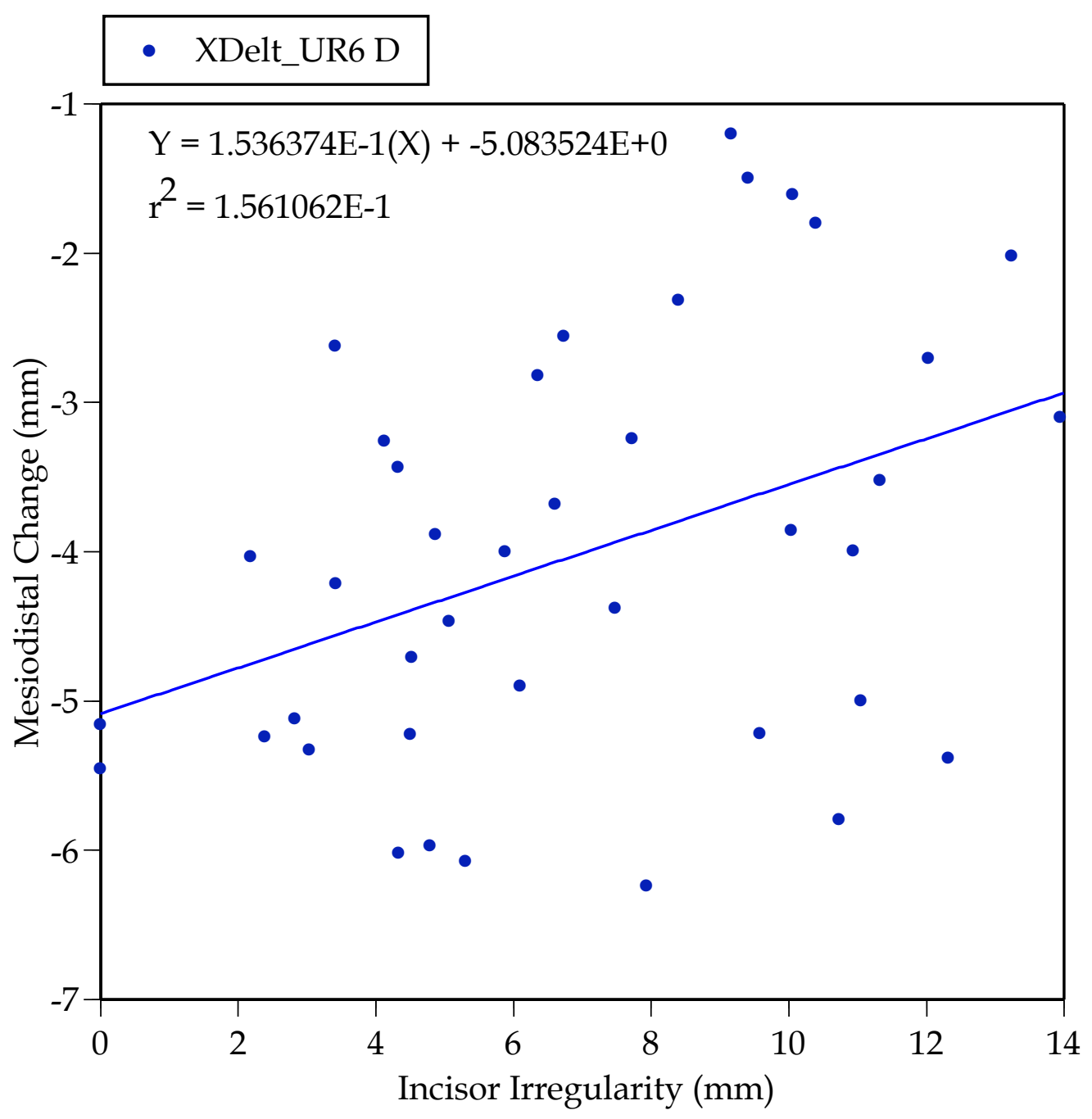

Fig. 78. Scatterplot showing the association between pretreatment incisor irregularity and the amount of mesiodistal change of the maxillary right first molar (distal) analyzed in just the second-premolar extraction sample. 


\section{Second Molar}

There was one significant occlusal predictor for the left and right second molars (mesial) as shown in Tables 84 and 85, respectively. In the left quadrant, maxillary 6-6 width is a significantly predictor of how much the second molar (mesial) was moved (Fig. 79). The narrower the 6-6 width, the more the second molar was moved mesially. Examples with narrow 6-6 widths are shown in Figs. A-45 and A-85; examples with broad width are in Figs. A-23 and A-78.

For the right quadrant, overjet is predictive of second molar (mesial) movement $\left(r^{2}=20 \%\right)$. As shown in Fig. 80 , the greater the overjet, the more the second molar was moved forward. This may seem counter-intuitive, but similar examples are noted above. Where there is severe overjet (e.g., Figs. A$23, \mathrm{~A}-45, \mathrm{~A}-85$ ) much of the overjet correction is achieved by changes in the mandibular incisors and most of the extraction space actually is used to correct buccal segment issues. At the other extreme, examples of where there was little overjet and little mesiodistal changes of the second molar (mesial) are shown in Figs. A-11, A-14, and A-36.

The final, most distal dental landmark (distal aspect of the second molars) is modeled in Table 86 (left quadrant) and Table 87 (right). Two variables are predictive for the left quadrant, namely incisor overbite $\left(\mathrm{r}^{2}=\right.$ 
Table 84. Results of stepwise linear regression predicting the amount of mesiodistal tooth movement from the pretreatment malocclusion: results for maxillary left second molar (mesial) in the subset in whom second premolars were extracted.

\begin{tabular}{lcccc}
\hline \multicolumn{1}{c}{ Variable } & $\begin{array}{c}\text { Sum of } \\
\text { Squares }\end{array}$ & F Ratio & P Value & $\mathrm{r}^{2}$ \\
\hline Incisor Overjet & 0.92 & 0.40 & 0.5312 & \\
Incisor Overbite & 6.46 & 3.08 & 0.0899 & \\
Irregularity Index & 0.01 & 0.00 & 0.9499 & \\
Incisor Spacing & 0.00 & 0.00 & 1.0000 & \\
Max 3-3 Width & 0.06 & 0.03 & 0.8733 & \\
Max 6-6 Width & 9.72 & 4.33 & $\underline{\mathbf{0 . 0 4 6 0}}$ & 0.1262 \\
Mand 3-3 Width & 0.26 & 0.11 & 0.7419 & \\
Mand 6-6 Width & 0.10 & 0.05 & 0.8335 & \\
UL 3-3 Width Difference & 0.07 & 0.03 & 0.8589 & \\
UL 6-6 Width Difference & 0.10 & 0.05 & 0.8335 & \\
Max 1-3 Chord & 0.50 & 0.22 & 0.6438 & \\
Max 1-6 Chord & 0.00 & 0.00 & 0.9759 & \\
Mand 1-3 Chord & 0.88 & 0.38 & 0.5410 & \\
Mand 1-6 Chord & 1.40 & 0.62 & 0.4381 & \\
Midline Deviation & 0.02 & 0.01 & 0.9272 & \\
Buccal Segment Relation & 2.37 & 1.06 & 0.3115 & \\
Canine Discrepancy & 5.98 & 2.83 & 0.1034 & \\
Patient's Sex & 8.14 & 3.99 & 0.0552 & \\
\hline
\end{tabular}


Table 85. Results of stepwise linear regression predicting the amount of mesiodistal tooth movement from the pretreatment malocclusion: results for maxillary right second molar (mesial) in the subset in whom second premolars were extracted.

\begin{tabular}{lrlll}
\hline \multicolumn{1}{c}{ Variable } & $\begin{array}{c}\text { Sum of } \\
\text { Squares }\end{array}$ & F Ratio & P Value & $\mathrm{r}^{2}$ \\
\hline Incisor Overjet & 17.13 & 7.64 & $\underline{\mathbf{0 . 0 0 9 7}}$ & 0.2029 \\
Incisor Overbite & 4.03 & 1.85 & 0.1848 & \\
Irregularity Index & 2.03 & 0.90 & 0.3502 & \\
Incisor Spacing & 0.00 & 0.00 & 1.0000 & \\
Max 3-3 Width & 1.76 & 0.78 & 0.3850 & \\
Max 6-6 Width & 1.04 & 0.45 & 0.5063 & \\
Mand 3-3 Width & 0.23 & 0.10 & 0.7565 & \\
Mand 6-6 Width & 0.03 & 0.01 & 0.9175 & \\
UL 3-3 Width Difference & 2.51 & 1.13 & 0.2975 & \\
UL 6-6 Width Difference & 3.90 & 1.78 & 0.1923 & \\
Max 1-3 Chord & 8.02 & 3.92 & 0.0572 & \\
Max 1-6 Chord & 1.38 & 0.61 & 0.4421 & \\
Mand 1-3 Chord & 0.06 & 0.03 & 0.8683 & \\
Mand 1-6 Chord & 0.77 & 0.34 & 0.5664 & \\
Midline Deviation & 0.01 & 0.00 & 0.9482 & \\
Buccal Segment Relation & 0.01 & 0.01 & 0.9468 & \\
Canine Discrepancy & 0.02 & 0.01 & 0.9348 & \\
Patient's Sex & 0.19 & 0.08 & 0.7752 & \\
\hline
\end{tabular}




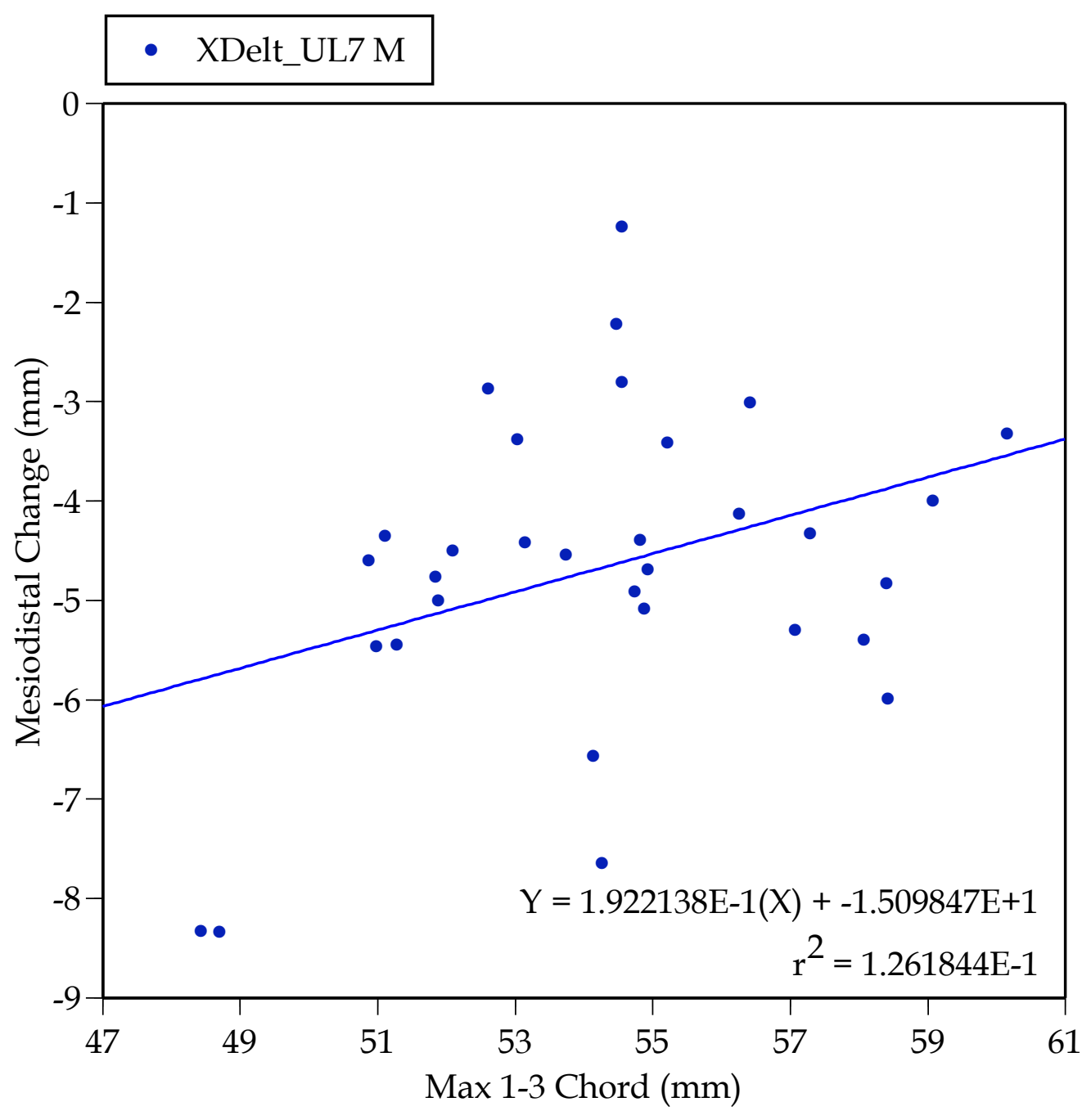

Fig. 79. Scatterplot showing the association between pretreatment maxillary 6-6 width and the amount of mesiodistal change of the maxillary left second molar (mesial) analyzed in just the second-premolar extraction sample. 


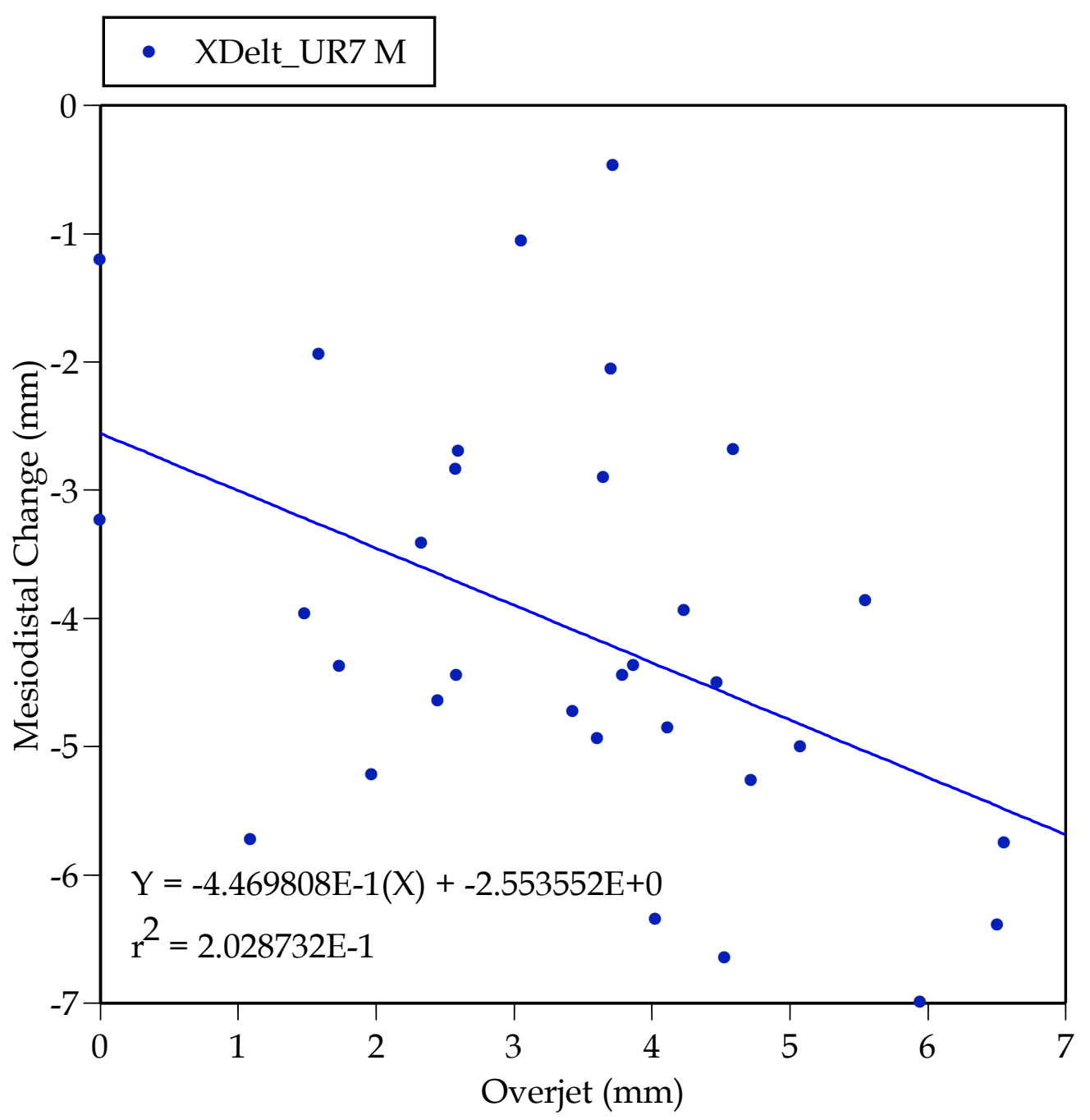

Fig. 80. Scatterplot showing the association between pretreatment overjet and the amount of mesiodistal change of the maxillary right second molar (mesial) analyzed in just the second-premolar extraction sample. 
Table 86. Results of stepwise linear regression predicting the amount of mesiodistal tooth movement from the pretreatment malocclusion: results for maxillary left second molar (distal) in the subset in whom second premolars were extracted.

\begin{tabular}{lrrrr}
\hline \multicolumn{1}{c}{ Variable } & $\begin{array}{r}\text { Sum of } \\
\text { Squares }\end{array}$ & F Ratio & P Value & $\mathrm{r}^{2}$ \\
\hline Incisor Overjet & 0.55 & 0.25 & 0.6187 & \\
Incisor Overbite & 24.58 & 11.68 & $\underline{\mathbf{0 . 0 0 1 9}}$ & 0.1377 \\
Irregularity Index & 2.04 & 0.97 & 0.3330 & \\
Incisor Spacing & 0.00 & 0.00 & 1.0000 & \\
Max 3-3 Width & 0.00 & 0.00 & 0.9636 & \\
Max 6-6 Width & 0.07 & 0.03 & 0.8564 & \\
Mand 3-3 Width & 0.71 & 0.33 & 0.5714 & \\
Mand 6-6 Width & 0.39 & 0.18 & 0.6755 & \\
UL 3-3 Width Difference & 1.52 & 0.71 & 0.4056 & \\
UL 6-6 Width Difference & 0.25 & 0.12 & 0.7374 & \\
Max 1-3 Chord & 0.23 & 0.11 & 0.7448 & \\
Max 1-6 Chord & 0.39 & 0.18 & 0.6729 & \\
Mand 1-3 Chord & 1.79 & 0.84 & 0.3660 & \\
Mand 1-6 Chord & 1.42 & 0.67 & 0.4216 & \\
Midline Deviation & 0.17 & 0.08 & 0.7831 & \\
Buccal Segment Relation & 2.74 & 1.31 & 0.2613 & \\
Canine Discrepancy & 22.74 & 10.81 & $\underline{\mathbf{0 . 0 0 2 7}}$ & 0.2340 \\
Patient's Sex & 0.38 & 0.18 & 0.6777 & \\
\hline
\end{tabular}


Table 87. Results of stepwise linear regression predicting the amount of mesiodistal tooth movement from the pretreatment malocclusion: results for maxillary right second molar (distal) in the subset in whom second premolars were extracted.

\begin{tabular}{lccll}
\hline \multicolumn{1}{c}{ Variable } & $\begin{array}{c}\text { Sum of } \\
\text { Squares }\end{array}$ & F Ratio & P Value & $\mathrm{r}^{2}$ \\
\hline Incisor Overjet & 2.78 & 0.95 & 0.3373 & \\
Incisor Overbite & 4.62 & 1.61 & 0.2138 & \\
Irregularity Index & 2.01 & 0.68 & 0.4150 \\
Incisor Spacing & 0.00 & 0.00 & 1.0000 & \\
Max 3-3 Width & 2.55 & 0.87 & 0.3580 \\
Max 6-6 Width & 0.05 & 0.02 & 0.9008 \\
Mand 3-3 Width & 0.02 & 0.01 & 0.9396 \\
Mand 6-6 Width & 2.81 & 0.96 & 0.3350 \\
UL 3-3 Width Difference & 6.28 & 2.24 & 0.1452 \\
UL 6-6 Width Difference & 5.00 & 1.76 & 0.1952 \\
Max 1-3 Chord & 7.59 & 2.75 & 0.1078 \\
Max 1-6 Chord & 1.01 & 0.34 & 0.5656 \\
Mand 1-3 Chord & 2.36 & 0.80 & 0.3770 \\
Mand 1-6 Chord & 5.19 & 1.83 & 0.1868 \\
Midline Deviation & 0.00 & 0.00 & 0.9766 \\
Buccal Segment Relation & 0.02 & 0.01 & 0.9328 \\
Canine Discrepancy & 0.04 & 0.01 & 0.9107 \\
Patient's Sex & 0.01 & 0.00 & 0.9618 & \\
\hline
\end{tabular}


$14 \%)$ and canine discrepancy $\left(\mathrm{r}^{2}=23 \%\right)$. In contrast, no predictor was statistically significant for this landmark's movement in the right quadrant.

The relationship between canine discrepancy and second molar (distal) movement in the left quadrant is shown in Fig. 81. Examples with large Class II canine discrepancies (and appreciable M2 mesial movement) are illustrated in Figs. A2 and A36. Conversely, cases with a slight Class III canine relationship (and little M2 movement) are shown in Figs. A-14 and A-16. 


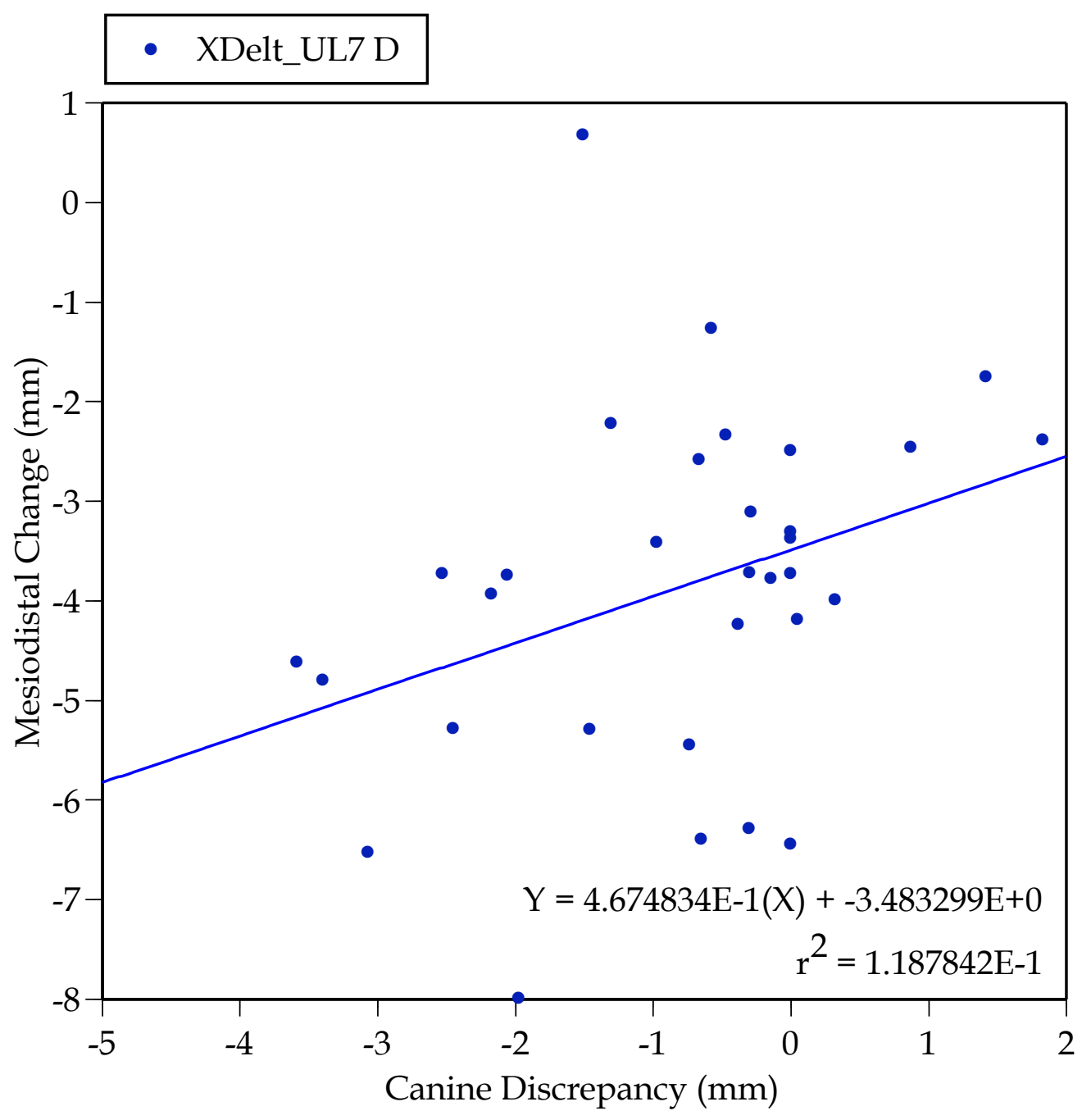

Fig. 81. Scatterplot showing the association between pretreatment canine discrepancy distance and the amount of mesiodistal change of the maxillary right second molar (distal) analyzed in just the second-premolar extraction sample. 


\section{CHAPTER V}

\section{DISCUSSION}

The historical controversy surrounding the use of extractions for orthodontic treatment is further convoluted by the fact that, in the current literature that compares extraction to nonextraction treatment, neither has been found to be superior (Weintraub et al. 1996). Even with the controversy and debate over efficacy of extraction treatment, a previous study reported that $95 \%$ of orthodontists extract teeth (Keim et al. 2002).

Previous surveys confirm that premolars are the most common teeth removed for orthodontic treatment (Proffit 1994). Conveniently located between the anterior and posterior segments, premolars would appear to be the obvious choice for correcting crowding and anteroposterior discrepancies.

Several clinicians have published recommendations as to which premolars (first or second premolars) to extract given particular malocclusions. Generally, the first-premolars are removed to correct problems such as anterior crowding, excessive overjet, and protrusion (Schoppe 1964; Graber 1972; Dewel 1973; Moyers 1973; Proffit 2000). Second-premolar extractions often are utilized in situations that present with mild anterior crowding, posterior crowding or when the molar anchorage needs to be intentionally lost (Dewel 1973; Myers 1973; de Castro 1974; Creekmore 1997). 
The extent of tooth movements surrounding first- and second-premolar extraction sites was evaluated in the present study to determine how the space gained by the extraction pattern was used to resolve the patients' malocclusions. Orthodontists choose to have teeth extracted and select their extraction patterns based on patients' individual dental, skeletal, and facial characteristics. The literature highlights several variables that should be considered when diagnosing and choosing an extraction pattern (e.g., Schoppe 1964; Moyers 1973; Baumrind et al. 1996; Proffit 2000). First-premolar extractions are typically recommended for more severe TSALDs and protrusion, while second premolar extraction patterns are often suggested for mild or posterior crowding, anterior openbites, and cases without incisor protrusion. Premolar extractions can also be used to correct for dental and skeletal anteroposterior discrepancies. The recurring theme in the literature is that different extraction patterns are used to treat different malocclusions by providing space at alternative locations in the dental arch.

The present study also examined how orthodontic tooth movement can be predicted statistically from pretreatment occlusial characteristics. More specifically, we wanted to provide an informative guide as to what occlusal movements the clinician can expect during treatment based on pretreatment occlusal variables. It has been documented that, with extraction treatment, the dental arches undergo changes in arch width and depth (Bishara et al. 1994, 
1997; Vaden et al. 1997; O’Higgins and Lee 2000; Gianelly 2003). Incisor irregularity and TSALD have also been shown to influence how extraction space is used during treatment (Ong and Woods 2001). One of the purposes of the present study was to determine how these and other variables influence tooth movement.

\section{Complete Sample}

This section combines results based on both the first- and secondpremolar extraction samples combined to assess relationships between the amount and nature of the pretreatment malocclusion and the amount of anteroposterior tooth movement.

Occlusal Characteristics

\section{$\underline{\text { Pretreatment Status }}$}

Comparing the pretreatment casts reveals that, prior to treatment, the first-premolar extraction sample possessed longer and broader arch forms compared to the second-premolar extraction sample. For the first-premolar extraction sample, we assumed that this extraction pattern was chosen to correct for maxillary protrusion and greater overjet as described by others, 
such as Schoppe (1964), Graber (1972), Dewel (1973), Moyers (1973), Proffit (2000).

It is noteworthy that all of the mean mesiodistal crown dimensions tooth sizes are larger in the first-premolar extraction sample than the secondpremolar extraction sample. A majority of the seven tests for differences between the samples were significant statistically. This occurrence has yet to be documented in the literature, but can possibly be explained by the fact that first-premolars are often extracted in cases that possess more crowding and the cases that were more crowded had excess tooth structure.

\section{$\underline{\text { Posttreatment Status }}$}

At posttreatment, the first premolar extraction sample continued to have longer arch forms. Therefore, the longer arch lengths in the firstpremolar group at the start of treatment, which we attributed primarily to protrusion of the maxillary incisors and, thus, greater overjet, was not completely resolved during treatment. This supposition is substantiated by the significantly greater overjet in the first-premolar sample at the end of treatment. The first-premolar extraction sample also maintained their mediolaterally broader arch forms compared to the second-premolar extraction sample. 


\section{$\underline{\text { Sexual Dimorphism }}$}

For the pretreatment cases, sexual dimorphism (males larger than females was apparent for the first-premolar sample. An unexpected feature of the second-premolar extraction sample was the lack of sexual dimorphism for the arch dimensions, where the male and female coordinates were commonly superimposed. Sexual dimorphism, for both samples is anticipated since several researchers have concluded that males possess larger arches and palatal dimensions (Moorrees 1959; Knott 1961; Burris and Harris 1998).

At posttreatment, the first-premolar extraction sample continued to exhibit sexual dimorphism, with the males having longer and broader arches. This difference, however, between males and females following treatment was less than at the start of treatment, presumable because treatment creates more uniform arch sizes and forms, especially with premolar extractions, which diminishes the male-female size differences. The second-premolar extraction sample, which failed to express sexual dimorphism at the start of treatment, continued to lack any extent of sexual dimorphism at posttreatment. For both groups, the decreased extent of sexual dimorphism can be expected as a more consistent arch from was developed through the use of arch wires during treatment. 


\section{$\underline{\text { In-Treatment Changes }}$}

There were two issues of particular interest when reviewing the intreatment changes in tooth position: (1) whether a dental landmark moved systematically during treatment and (2) whether the average changes differed significantly between the two extraction patterns.

For the first question, we look for significant trends in the direction of dental landmark changes. Given the nature of the changes, the three posterior teeth (P2, M1, M2) exhibited arch constriction whereas the four mesial tooth types characteristically experienced arch expansion.

Testing for differences in the amounts of change by extraction pattern, none of the four molar landmarks changed differently. In contrast, the six canine and incisor landmarks expanded in both groups and significantly more in the first-premolar extraction sample. The greater transverse correction in the first-premolar group might imply that they have narrower, more tapered anterior segments that merit greater expansion to move the teeth into alignment. For both groups, however, the general expansion seen in the anterior segment would merit support for the "expansion theory" of the arches that purports that canines are retracted into a broader arch diameter where the premolars had been located (Bishara et al. 1994; Vaden et al. 1997). The greater expansion observed in the first-premolar extraction sample could then be explained by the greater canine retraction observed (discussed below). 


\section{Predicting Tooth Movements}

This section addresses how orthodontic tooth movement can be predicted in the statistical sense from evaluations of the pretreatment occlusion.

\section{$\underline{\text { Incisors }}$}

Of the predictive variables we examined, probably the most discussed in the literature is extraction pattern. Several have previously looked at firstversus second-premolar extraction patterns with regard to incisor retraction, and have concluded that first-premolar extraction patterns yield more incisor retraction (Steyn et al. 1997; Ong and Woods 2001). The results of the present study were in agreement with this generality. Our first-premolar extraction sample experienced a mean $1.3 \mathrm{~mm}$ more incisor retraction than the secondpremolar extraction sample.

Longer maxillary 1-3 chord and 1-6 chord distances were found to be associated with greater incisor retraction. This seems somewhat intuitive in that, the more protrusive the incisors, the more they would be retracted to correct for the protrusion and achieve alignment.

Maxillary 3-3 width had a positive association with incisor retraction, in that, the wider the 3-3 distance, the more the incisors were retracted. Examination of the casts reveals that the cases with the widest 3-3 width 
presented with "blocked out" or buccally displaced canines. Once the premolars were removed, the canines could be moved into the extraction space without taxing the molar anchorage and, in turn, provide space for incisor retraction.

While it has not been examined in this way before, it seems somewhat obvious that cases with a Class II discrepancy of the canine would experience greater incisor retraction because one of the goals of treatment in these situations is greater retraction of the anterior segment to obtain a Class I sagittal molar relationship.

Inter-dental spacing and low levels of incisor irregularity also were positively associated with greater incisor retraction. This concurs with the results of Ong and Woods (2001). In these situations, little or none of the premolar extraction space was consumed by resolving anterior crowding, allowing all of the space to be used for retraction. When examining the firstand second-premolar extraction samples separately, incisor irregularity was still a predictive variable for incisor retraction. The few cases in the present study that had protrusive incisors with spacing showed even greater retraction because both the inter-dental spacing and the premolar extraction site were closed.

Overbite or in this situation, deeper bites, were found to be predictive of greater incisor retraction. This association is not commonly addressed in 
the literature. The association can be explained by these individuals having

greater inter-digitation of the buccal segments and stronger biting forces that provided an internal form of posterior anchorage when the incisors were being retracted.

\section{Canine}

Analysis of anteroposterior movements of the canine during treatment disclosed two significant predictive variables. Extraction pattern is the most significant, where mean canine retraction was $3.4 \mathrm{~mm}$ in the first-premolar extraction sample compared to the $2.3 \mathrm{~mm}$ observed in the second-premolar extraction sample. This phenomenon is hard to compare with previous studies, because most studies have focused on incisor retraction and/or molar protraction. There is essentially no comparative data. One could assume, however, since the canine is directly in contact with the first premolar, that when the first premolar is removed, the canine can more readily drift or be retracted into the extraction site. For the second premolar extraction cases, both the first premolar and the canine must be retracted, which could explain the greater anchorage loss and reduced amounts of retraction.

The pretreatment incisor irregularity also is predictive for this tooth. The association here is the greater the incisor irregularity, the greater the typical retraction of the canine. This is different than what is seen for the 
incisors, but is intuitive in that the canine must be retracted into the extraction space to gain anterior arch space to alleviate crowding.

\section{$\underline{\text { First Molar }}$}

The orthodontic literature is inundated with articles concerning maxillary first molar movement. With its known ease of -but often unwantedmesial movement, it is frequently the tooth of concern if a Class I molar is to be attained or maintained during treatment. There a plethora of articles concerning first molar movement and how it is controlled, either by extraction pattern or orthodontic techniques.

Extraction pattern was itself predictive of the amount of mesial molar movement. The first-premolar extraction sample's first molar moved forward an average of $4.0 \mathrm{~mm}$, whereas the first molar moved forward an average of $4.7 \mathrm{~mm}$ in the second-premolar extraction sample. Ong and Woods (2001) previously described this; however they recorded a larger difference between the first- and second-premolar extraction samples with mesial molar movement being $3.7 \mathrm{~mm}$ and $4.5 \mathrm{~mm}$, respectfully.

Assuming that the average mesiodistal diameter of a premolar is 7.5 $\mathrm{mm}$, over half of the extraction space for both groups was consumed by the buccal segments moving mesially. Others have written only approximately $33 \%$ of the extraction space in first-premolar extraction cases is consumed by 
the buccal segments moving forward (e.g., Williams and Hosila 1976; Creekmore 1977). Techniques to measure molar movement, sample characteristics and the mechanics employed could explain this difference.

Incisor irregularity is the preeminent predictor for how much the first molar moved mesiodistally during treatment. The greater the incisor irregularity at the start of treatment, the less the molar was moved mesially. This relationship is intuitive in that more of the extraction space was used to "unravel" the anterior irregularity and achieve alignment rather than moving the buccal segment mesially. This association between incisor irregularity and mesial molar movement is in agreement with what Ong and Woods (2001) found in their study looking at molar movement on a lateral cephalogram.

Another predictive variable for the first molar is canine discrepancy. The greater the canine discrepancy, the less the first molar moved forward. In these situations, it is assumed that more of the extraction space was consumed by the canine being retracted.

Overbite also was predictive of the first molar movement. Cases with deep bites underwent less mesial molar movement than those with shallow bites, or even, anterior openbites. The same association is assumed here as was with incisor retraction; cases with deeper bites had greater interdigitation of the posterior segments and stronger biting forces, which served as internal anchorage. In malocclusions with shallow or open bites, the lack of 
intercuspation between the buccal segments allowed for greater posterior anchorage loss.

\section{$\underline{\text { Second Molar }}$}

One would assume that the second molar, located directly distal to the first molar, would have the same set of predictive variables as the first molar. However, the present study found a few significant differences. Sex is predictive in that females experienced less second-molar movement than males. This seems to be a finding relevant just to this particular data set, because "sex" is not predictive of any of the other tooth movements investigated in this section.

Maxillary 1-6 chord distance also is predictive of second molar movement. The association is that cases with long 1-6 arch chords underwent less second molar movement. This apparently occurs because more of the extraction space was used correct the incisor proclination and procumbency rather than "burning anchorage."

Statistical differences between first and second molars can be explained by the way the study examined movement. The study looked only at parasagittal movement, disregarding any findings related to rotations or movements in other planes of space. This explains why the second molar, immediately distal to the first, had different predictive variables. 


\section{$\underline{\text { First Premolar Sample }}$}

\section{Predicting Tooth Movements}

$\underline{\text { Incisors }}$

Mesiodistal incisor movement plays an important role as extraction spaces are closed and overjet is corrected. The most significant predictor for mesiodistal movement of the central incisors in this sample is incisor spacing. In these cases the extra available space was removed by incisor retraction. Using this as a diagnostic indicator, one should expect a greater reduction in protrusion when there is incisor spacing.

Overjet also is positively associated with incisor retraction in this sample. Used together or individually, the clinician can expect greater incisor retraction in first-premolar extraction cases when there is increased overjet and/or incisor spacing.

When searching for predictive variables for movement of the lateral incisor, incisor irregularity was the single important predictor. The greater the incisor irregularity, the less retraction the lateral incisor experienced. This association was also found for the central incisors in the second-premolar extraction sample. Ong and Woods (2001) found this same association when looking at all four incisors and irregularity. In this circumstance, a majority of 
the available extraction space was used to properly align the incisors. This finding could prove beneficial to clinicians when treatment planning cases with increased crowding and overjet. Realizing that more of the extraction space will be consumed to resolve crowding, the clinician would then seek alternative methods to correct for the overjet.

\section{Canine}

The major determinant for canine movement is overbite. Cases with deep bites underwent appreciable canine retraction, while those with shallow bites tended to undergo essentially no change along the parasagittal axis. The same association was found in the second-premolar extraction series. The literature fails to mention the relation between overbite and mesiodistal canine movement. The current authors feel that much of this association can be attributed to cases with deep bites having better interdigitation of the buccal segments and stronger biting forces that served as a form of posterior anchorage.

Incisor irregularity has a positive association with canine movement, but this affiliation is not evident in the second-premolar extraction sample. Cases with greater irregularity experienced greater canine retraction. This association is intuitive, since the canines need to be moved back in the arch to accommodate resolution of the incisor irregularity. This indicates that the 
clinician can be more confident in retracting the canines into the extraction space when increased crowding is present.

\section{$\underline{\text { Molar }}$}

Movement of the first molar commonly is of great to concern during treatment because unpredicted movement can cause the loss of ideal buccal segment relationship. The first molar along with the second premolar and second molar butted against it, all responded very similarly during treatment.

Incisor irregularity was a strong predictor of mesiodistal change of the first molar in both the first-premolar and second-premolar extraction samples when assessed individually.

Cases with increased incisor irregularity expend most of the extraction space resolving those anterior-segment issues, so there is little mesial molar movement. A clinician could expect little molar movement, and thus less anchorage requirements, when extracting premolars in cases with greater crowding. This finding is again in agreement with what Ong and Woods found (2001).

For 1-6 chord distance, the greater the measurement, the greater the mesial molar movement. For a good number of the cases with large 1-6 chord distances, a narrow tapered arch form with increased overjet was present. As 
the clinician was correcting the arch form and overjet, little mesial molar movement occurred.

\section{$\underline{\text { Second Premolar Sample }}$}

\section{Predicting Tooth Movements}

$\underline{\text { Incisors }}$

In this sample for the central incisor the predominant predictor is maxillary 1-3 arch chord.

The nature of the association here is that when the anterior arch form is long and tapered, the maxillary 1-3 arch chord is large and is treated by retracting the central incisors distally to create a broader, more rounded arch form.

Overbite is the significant predictor for the lateral incisor in this sample. The same association presents here as did for canines in both samples. It appears evident that a clinician can expect greater retraction of both the incisor and the canines when the patient has a deep bite, and less retraction when the patient has a shallow bite. 


\section{$\underline{\text { Canine }}$}

In the second-premolar extraction sample, overjet was the sole unique predictive variable for canine movement. The association is the greater the pretreatment overjet, the less the canine retraction. At face value, this appears to be antagonistic to what a clinician would want and expect. Examining this samples casts however, reveals several cases with increased overjets, overly upright lower incisors and good interdigitation of the canines. The treatment goals of these cases were obviously to reduce overjet by proclining the lower incisors, while maintaining the canine relationship. Second-premolar extractions would then prove beneficial when this circumstance presents clinically.

\section{$\underline{\text { Molar }}$}

Canine relationship, or in this case the tendency for a "Class II" canine, is predictive for molar movement in this sample. The greater the "Class II" the more the molar moved mesial. This can be interpreted as a malapropos action of the molar in this situation. In cases that are Class II, or have a Class II tendency, little or no molar movement is the usual intention. However, in a second-premolar extraction case, the molar is at a disadvantage being "pitted" against the more anterior incisors, canines, and first-premolars. Several have actually recommended this extraction pattern for moving the molar mesial 
(Logan 1973; de Castro 1974). The present research indicates that when greater molar anchorage is needed, other treatment plans rather than secondpremolar extractions should be explored. 


\section{CHAPTER VI \\ SUMMARY AND CONCLUSIONS}

Malocclusions can involve arch-size tooth-size discrepancies and, potentially, several other skeletodental disharmonies that have to be resolved by premolar extractions. The present study compared the amounts of intreatment tooth movement in two common extraction patterns (4/4 and 5/5). The goal was to better understand the kinds and amounts of tooth movement afforded by each extraction pattern - and how the dental malocclusion was resolved.

The first part of the project was to develop a system for quantifying the in-treatment changes in tooth position using a MicroScribe GX2 threedimension digitizer (Immersion Corporation, San Jose, CA) interfaced with Rhino software (Robert McNeel \& Associates, Seattle, WA). Highlights of this proof of concept phase were:

1. Registration of the casts was on the medial terminals of the left and right distal palatal rugae, which are the most-stable, least-affected parts of the rugae patterns during orthodontic tooth movement.

2. A battery of mesial and distal occlusal dental landmarks was developed so the anteroposterior, transverse, and angular changes of each tooth could be quantified as viewed in the occlusal plane. 
3. Repeatability accuracy was less than $1 \mathrm{~mm}$ for each landmark.

4. It was informative to have collected data on both the mesial and distal contact points of a tooth, since teeth undergo appreciable rotational changes during treatment (and a minimum of two landmarks is required to quantify angular changes). For example, the lateral incisors characteristically were rotated during treatment such that the lateral margins were moved distally.

5. Data collected from Rhino were collated in a spreadsheet program and then analyzed statistically with JMP statistical software (SAS Institute Inc; Cary, NC). Mean landmarks were plotted using graphing software for visual inspection.

6. The method disclosed significant left-right asymmetry in the dental arches, with the teeth on the left side of the arch systematically positioned more lateral-and-distal than those on the right side. This comports with the few prior studies that collected data where asymmetry can be tested.

7. In our opinion, there are no landmarks on a dental cast that can be used to monitor orthodontic changes in the vertical (craniocaudal) axis. It would be necessary to do this on the skull, using immobile bony fiducial landmarks. 
The second, analytic part of the study compared first-premolar (P1) with second-premolar (P2) orthodontic extraction cases. Results are summarized as follows:

8. Cases treated with P1 extractions exhibited (1) greater incisor irregularity, (2) greater overjet, (3) more frequent interdental spacing, (4) longer arch lengths (1-3 and 1-6), (5) greater overbite, and (6) broader arch widths (3-3 and 6-6) at the start of treatment.

9. Anterior teeth (both incisors and the canine) were moved distally significantly more in the P1 than the P2 samples. The central incisors were retracted an average of about $3 \mathrm{~mm}$ in the P1 cases, versus about 1.3 $\mathrm{mm}$ in the P2 cases, though these group means obscure considerable interindividual variation.

10. Posterior teeth (first and second molars) were moved mesially significantly more in the P2 than the P1 cases.

11. Second premolars (P2) were moved mesially significantly more in the P1 extraction cases than the first premolars (P1) were moved distally in the P2 extraction cases.

12. On the order of $4 \mathrm{~mm}$ of mesial movement of the first molar occurred in both the P1 and P2 samples. Given an average premolar dimension of 7.5 $\mathrm{mm}$, only about half the extraction space was used to resolve orthodontic 
problems on the average. One might consider judicious reproximation in cases where little extraction space is required.

13. Interpretations from this cast analysis are limited to changes in the anteroposterior and transverse axes (i.e., the occlusal plane). Additional diagnostic information from other skeletal and integumental variables should be considered as well. 
LIST OF REFERENCES 
Almeida MA, Phillips C, Kula K, Tulloch C. Stability of the palatal rugae as landmarks for analysis of dental casts. Angle Orthod 1995;65:43-8.

Ashmore JL, Kurland BF, King GJ, Wheeler TT, Ghafari J, Ramsay DS. A 3dimensional analysis of molar movement during headgear treatment. Am J Orthod Dentofacial Orthop 2002:121:18-30.

Bailey LJ, Esmailnejad A, Almeida MA. Stability of the palatal rugae as landmarks for analysis of dental casts in extraction and nonextraction cases. Angle Orthod 1996;66:73-8.

Baume LJ, Horowitz HS, Summers CJ, Dirks OB, Brown WAB, Carlos JP, Cohen LK, Freer TJ, Harvold EP, Moorrees CFA, Salzmann JA, Schmuth G, Solow B, Taatz J. A method for measuring occlusal traits. Int Dent J 1973;23:530-7.

Baumrind S, Korn EL, Boyd RL, Maxwell R. The decision to extract: part II. Analysis of clinicians' stated reasons for extraction. Am J Orthod Dentofacial Orthop1996;109:393-402.

Bishara SE, Bayati P, Zaher AR, Jakobsen JR. Comparisons of the dental arch changes in patients with Class II, division 1 malocclusions: extraction vs nonextraction treatments. Angle Orthod 1994;64:351-8.

Bishara SE, Cummins DM, Jakobsen JR, Zaher AR. Dentofacial and soft tissue changes in Class II, division 1 cases treated with and without extractions. Am J Orthod Dentofacial Orthop 1995;107:28-37.

Bishara SE, Cummins DM, Zaher AR. Treatment and posttreatment changes in patients with Class II, division 1 malocclusion after extraction and nonextraction treatment. Am J Orthod Dentofacial Orthop 1997;111:1827.

Bodford K, Harris EF. A study of left-right asymmetries in the dental arches of orthodontic patients. Paper presented at the 85 th Annual Meeting of the International Association for Dental Research, New Orleans, 2007.

Bolton WA. Disharmony in tooth size and its relation to the analysis and treatment of malocclusion. Angle Orthod 1958;28:113-30.

Brandt S, Safirstein GR. Different extractions for different malocclusions. Am J Orthod 1975;68:15-41. 
Brodie AG. Cephalometric appraisal of orthodontic results. Angle Orthod 1938;8:261-351.

Camporesi M, Franchi L, Baccetti T, Antonini A. Thin-plate spline analysis of arch form in a South European population with an ideal natural occlusion. Eur J Orthod 2006;28:135-40.

Carter NE. First premolar extractions and fixed appliances in the Class II division 1 malocclusion. J Orthod 1988;15:1-10.

Cole HJ. Certain results of extraction in treatment of malocclusion. Angle Orhod 1948;18:103-13.

Creekmore TD. Where teeth should be positioned in the face and jaws and how to get them there. J Clin Orthod 1997;31:586-608.

Cusimano C, McLaughlin RP, Zernik JH. Effects of first bicuspid extractions on facial height in high-angle cases. J Clin Orthod 1993;27:594-8.

Darendeliler N, Taner-Sarisoy L. The influence of orthodontic extraction treatment on dental structures: a two-factor evaluation. Eur J Orthod 2001;23:295-303.

De Castro N. Second premolar extraction in clinical practice. Am J Orthod 1974;65:115-37.

Dewel, BF. Second premolar extraction in orthodontics: principles, procedures, and case analysis. Am J Orthod 1955;41:107-20.

Dewel, BF. Extraction in orthodontics: premises and prerequisites. Angle Orthod 1973;43:65-87.

Doyle WJ, Johnston O. On the meaning of increased fluctuating dental asymmetry: a cross population study. Am J Phys Anthropol 1977;46:127-34.

English WR, Robison SF, Summitt JB, Oesterle LJ, Brannon RB, Morlang WM. Individuality of human palatal rugae. J Forensic Sci 1988;33:718-26. 
Erdine AE, Nanda RS, Isiksal E. Relapse of anterior crowding in patients treated with extraction and nonextraction of premolars. Am J Orthod Dentofacial Orthop 2006;129:775-84.

Freitas KMS, Freitas MR, Henriques JFC, Pinzan A, Janson G. Postretention relapse of mandibular anterior crowding in patients treated without mandibular premolar extraction. Am J Orthod Dentofacial Orthop 2004;125:480-7.

Freund RJ, Littel RC. SAS ${ }^{\circledR}$ system for regression, 2nd ed. Cary, NC: SAS Institute Inc., 1991.

Gianelly AA. Arch width after extraction and nonextraction treatment. Am J Orthod Dentofacial Orthop 2003;123:25-8.

Graber TM. Orthodontics: principles and practice, 3rd ed. Philadelphia: WB Saunders Company, 1972.

Harris EF. Tooth-coding systems in the clinical dental setting. Dental Anthropology 2005;18:43-9.

Harris EF, Bodford K. Bilateral asymmetry in the tooth relationships of orthodontic patients. Angle Orthod [In Press].

Hoggan BR, Sadowsky C. The use of palatal rugae for the assessment of anteroposterior tooth movements. Am J Orthod Dentofacial Orthop 2001;119:482-8.

Housley JA, Nanda RS, Currier GF, McCune DE. Stability of transverse expansion inthe mandibular arch. Am J Orthod Dentofacial Orthop 2003;124:288-93.

Jepsen A. Root surface measurement and a method for x-ray determination of root surface area. Acta Odontol Scand 1963;21:35-46.

Kahl-Nieke B, Fischbach H, Schwarze CW. Treatment and postretention changes in dental arch width dimensions - a long-term evaluation of influencing cofactors. Am J Orthod Dentofacial Orthop 1996;109:36878. 
Keim RG, Gottlieb EL, Nelson AH, Vogels DS. 2002 JCO study of orthodontic diagnosis and treatment procedures. Part 1. Results and trends. J Clin Orthod 2002;36:553-68.

Kocadereli I. The effect of first premolar extraction on vertical dimension. Am J Orthod Dentofacial Orthop 1999;116:41-5.

Kula K, Esmailnejad A, Hass A. Dental arch asymmetry in children with large 0overjets. Angle Orthod 1998;68:45-52.

Little RM. The irregularity index: a quantitative score of mandibular anterior alignment. Am J Orthod 1975;68:554-6.

Logan LR. Second premolar extraction in Class I and Class II. Am J Orthod 1973;63:115-47.

Luppanapornlarp S, Johnston LE. The effects of premolar-extraction: a longterm comparison of outcomes in "clear-cut" extraction and nonextraction Class II patients. Angle Orthod 1993;63:257-72.

Lysell L. Plicae palatinae transverse and papilla incisiva in man: a morphologic and genetic study. Acta Odont Scand 1955;13:106-33.

Moyers RE. Handbook of orthodontics, 4th ed. Chicago: Year Book Medical Publishers, Inc., 1973.

Nance HN. The limitations of orthodontic treatment, II. Am J Orthod 1947;33:253-301.

O'Higgins EA, Lee RT. How much space is created from expansion or premolar extraction? J Orthod 2000;27:11-3.

Ong HB, Woods MG. An occlusal and cephalometric analysis of maxillary first and second premolar extraction effects. Angle Orthod 2001;71:90102.

Peavy DC Jr, Dendrick GS. The effects of tooth movement on the palatine rugae. J Prosthet Dent 1967;18:536-42.

Peck S, Peck L. A time for change of tooth numbering systems. J Dent Ed 1993;57:643-47. 
Proffit W. Forty-year review of extraction frequencies at a university orthodontic clinic. Angle Orthod 1994;407-14.

Proffit W. Contemporary orthodontics, 3rd ed. St Louis: CV Mosby Company, 2000.

Saatci P, Yukay F. The effect of premolar extractions on tooth-size discrepancy. Am J Orthod Dentofacial Orthop 1997;111:428-34.

Sadowsky C, Schneider BJ, BeGole EA, Tahir E. Long-term stability after orthodontic treatment: nonextraction with prolinged retention. Am J Orthod Dentofacial Orthop 1994;106:243-9.

Schoppe RJ. An analysis of second premolar extraction procedures. Angle Orthod 1964;34:292-302.

Schwab DT. Extraction effects on the dental profile in borderline cases. Angle Orthod 1963;33:120-2.

Sheppe JH. Six arch changes in first bicuspid extraction therapy in normal arches. Int J Orthod 1969;7:166-70.

Smith RJ, Bailit HL. Variation in dental occlusion and arches among Melanesians of Bougainville Island, Papua New Guinea. I. Age changes, sex differences and population comparisons. Am J Phys Anthropol 1977;47:195-208.

Sokal RR, Rohlf FJ. Biometry: the principles and practice of statistics in biological research, 3rd ed. San Francisco: WH Freeman and Company. 1995.

Staggers JA. Vertical changes following first premolar extractions. Am J Orthod Dentofacial Orthop 1994;105:19-24.

Steyn CL, du Preez RJ, Harris AM. Differential premolar extractions. Am J Orthod Dentofacial Orthop 1997;112:480-6.

Thiruvenkatachari B, Pavithranand A, Rasjasigamani K, Kyung HM. Comparison and measurement of the amount of anchorage loss off the molars with and without the use of implant anchorage during canine retraction. Am J Orthod Dentofacial Orthop 2006;129:551-5. 
Tweed $\mathrm{CH}$. Indications for the extraction of teeth in orthodontic procedure. Am J Orthod Oral Surg 1944;30:405-28.

Vaden JL, Harris EF, Gardner RL. Relapse revisited. Am J Orthod Dentofacial Orthop 1997;111:543-53.

van der Linden FPGM. Changes in the position of posterior teeth in relation to ruga points. Am J Orthod 1978;74:142-61.

Weintraub JA, Vig PS, Brown C, Kowalski CJ. The prevalence of orthodontic extraction. Am J Orthod Dentofacial Orthop 1996;297-309.

Williams R, Hosila FJ. The effect of different extraction sites upon incisor retraction. Am J Orthod 1976;69:388-410.

Winberg M, Sadowsky C. Resolution of mandibular arch crowding in growing patients with Class I malocclusions treated nonextraction. Am J Orthod Dentofacial Orthop 1996;110:359-64.

Winer BJ, Brown DR, Michels KM. Statistical principles in experimental design, 3rd ed. New York: McGraw-Hill Book Company, 1991.

Yoa CC, Lee JJ, Chen HY, Chang ZC, Chang HF, Chen YJ. Molar intrusion with fixed appliances and mini-implant anchorage studied in three dimensions. Angle Orthod 2005;75:754-60. 


\begin{abstract}
APPENDIX
The following figures are scaled plots of the arch forms representing the dental casts used in this study. The pretreatment arch forms are superimposed over the posttreatment arch forms with repect to the posterior medial rugae. The figures are ordered with repect to the alphabetical order of the patient's name.
\end{abstract}




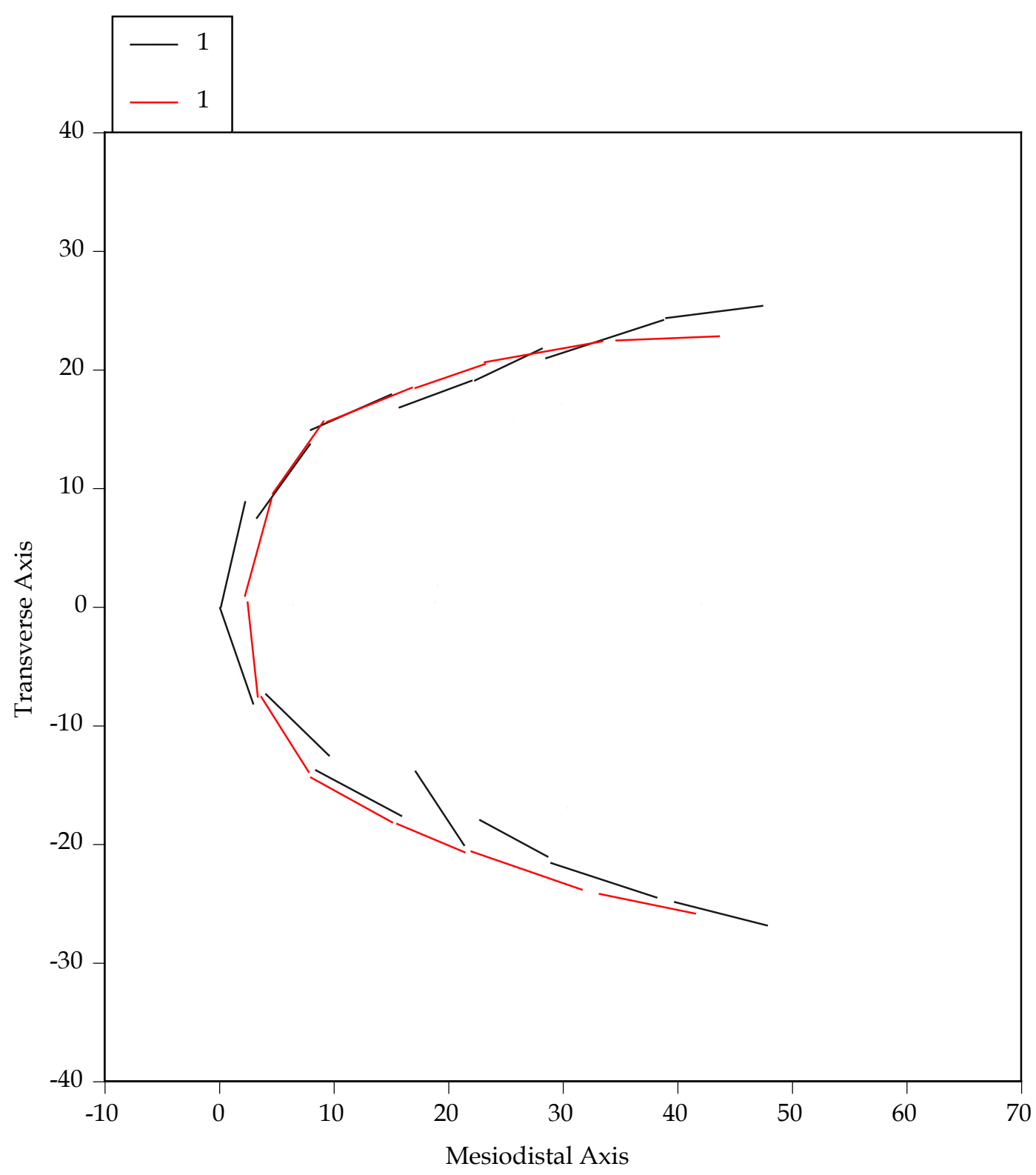

Fig. A-1. Plot of the maxillary dental arch relationships of case 1, a female in whom the maxillary second-premolars were extracted for treatment. Squares are the dental contacts at the start of treatment; circles are the contacts at the end of treatment. The $X$ and $Y$ axes are in millimeters. 


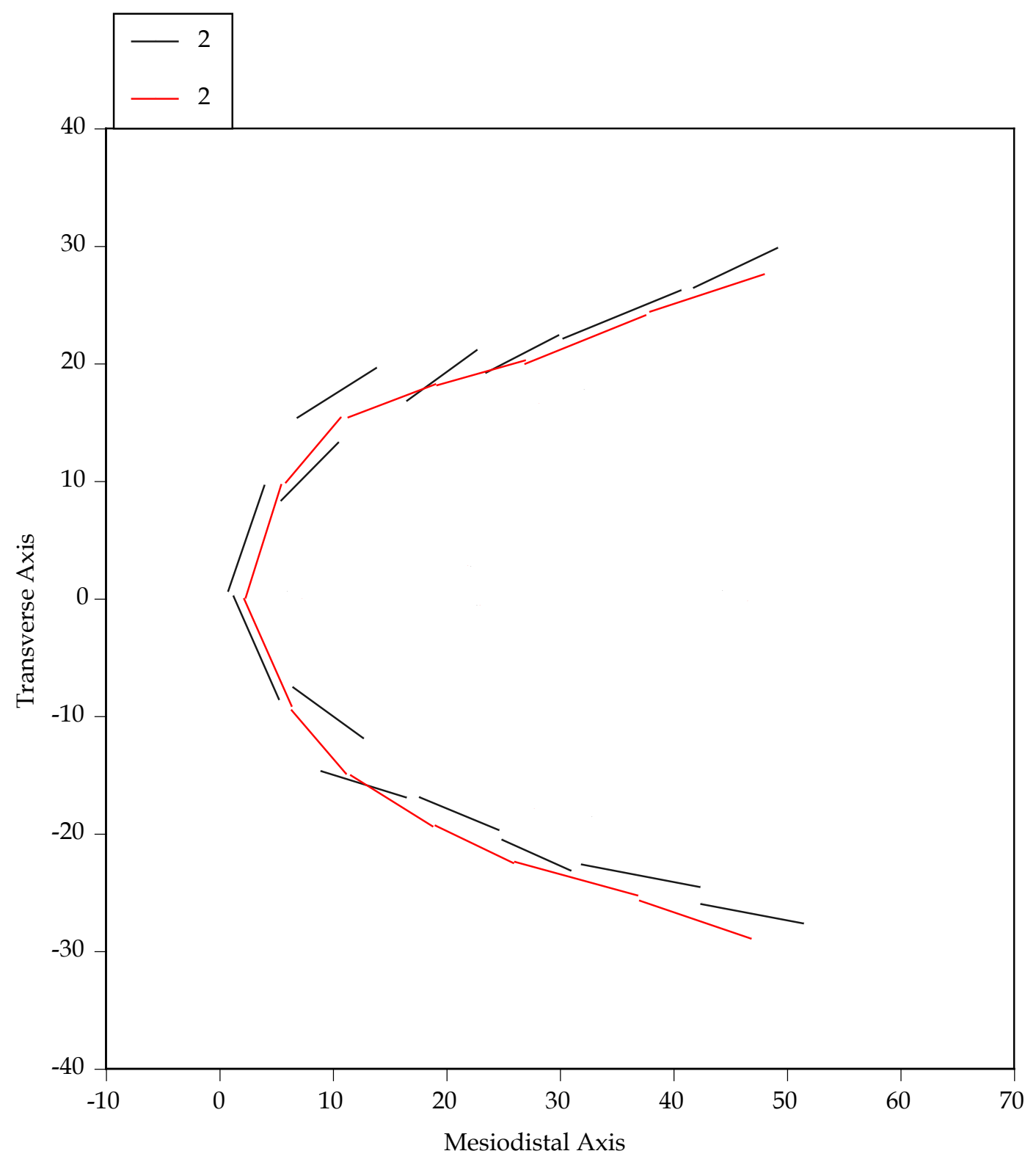

Fig. A-2. Plot of the maxillary dental arch relationships of case 2, a male in whom the maxillary second-premolars were extracted for treatment. Squares are the dental contacts at the start of treatment; circles are the contacts at the end of treatment. The $X$ and $\mathrm{Y}$ axes are in millimeters. 


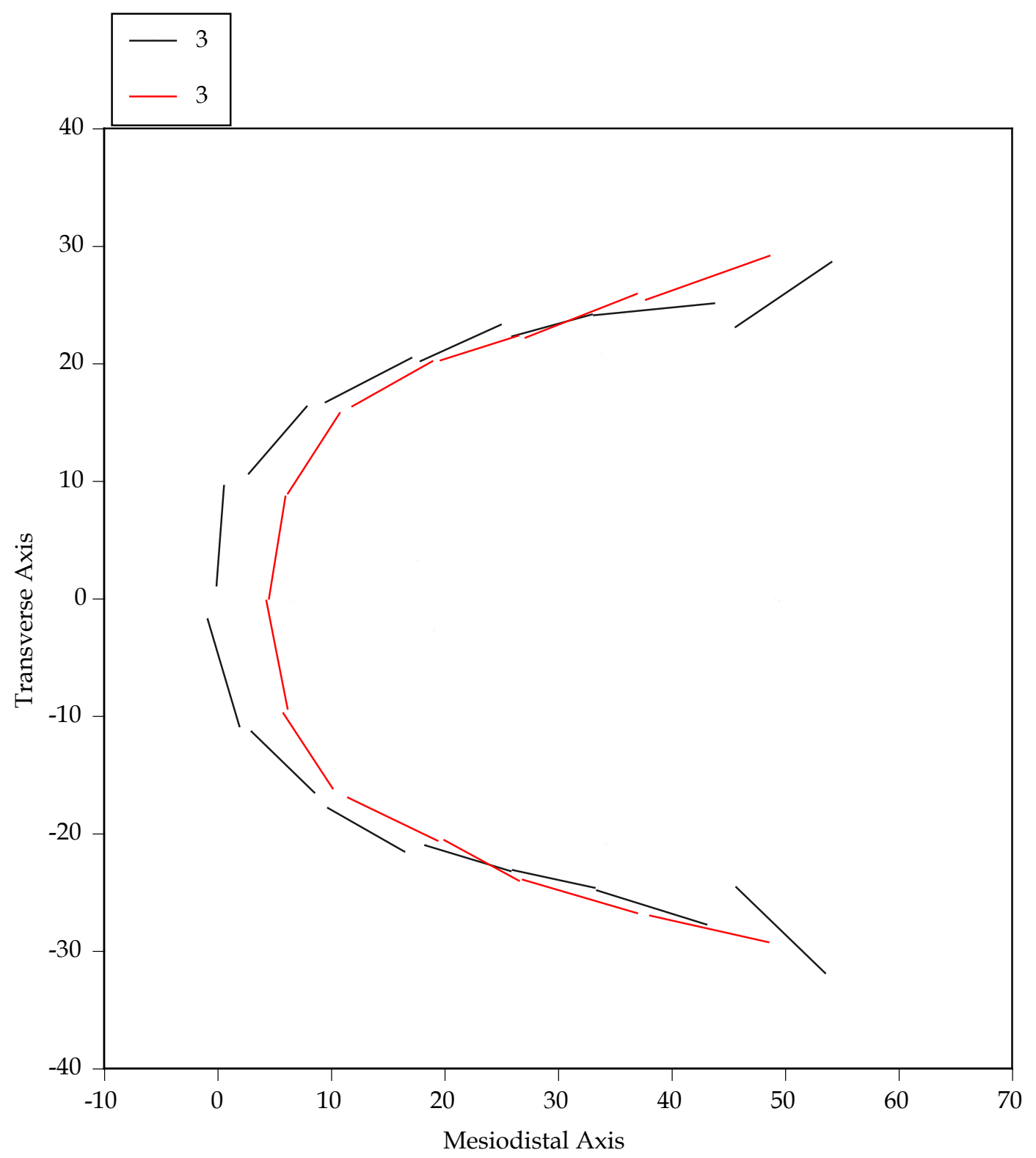

Fig. A-3. Plot of the maxillary dental arch relationships of case 3, a female in whom the maxillary first-premolars were extracted for treatment. Squares are the dental contacts at the start of treatment; circles are the contacts at the end of treatment. The $X$ and $\mathrm{Y}$ axes are in millimeters. 


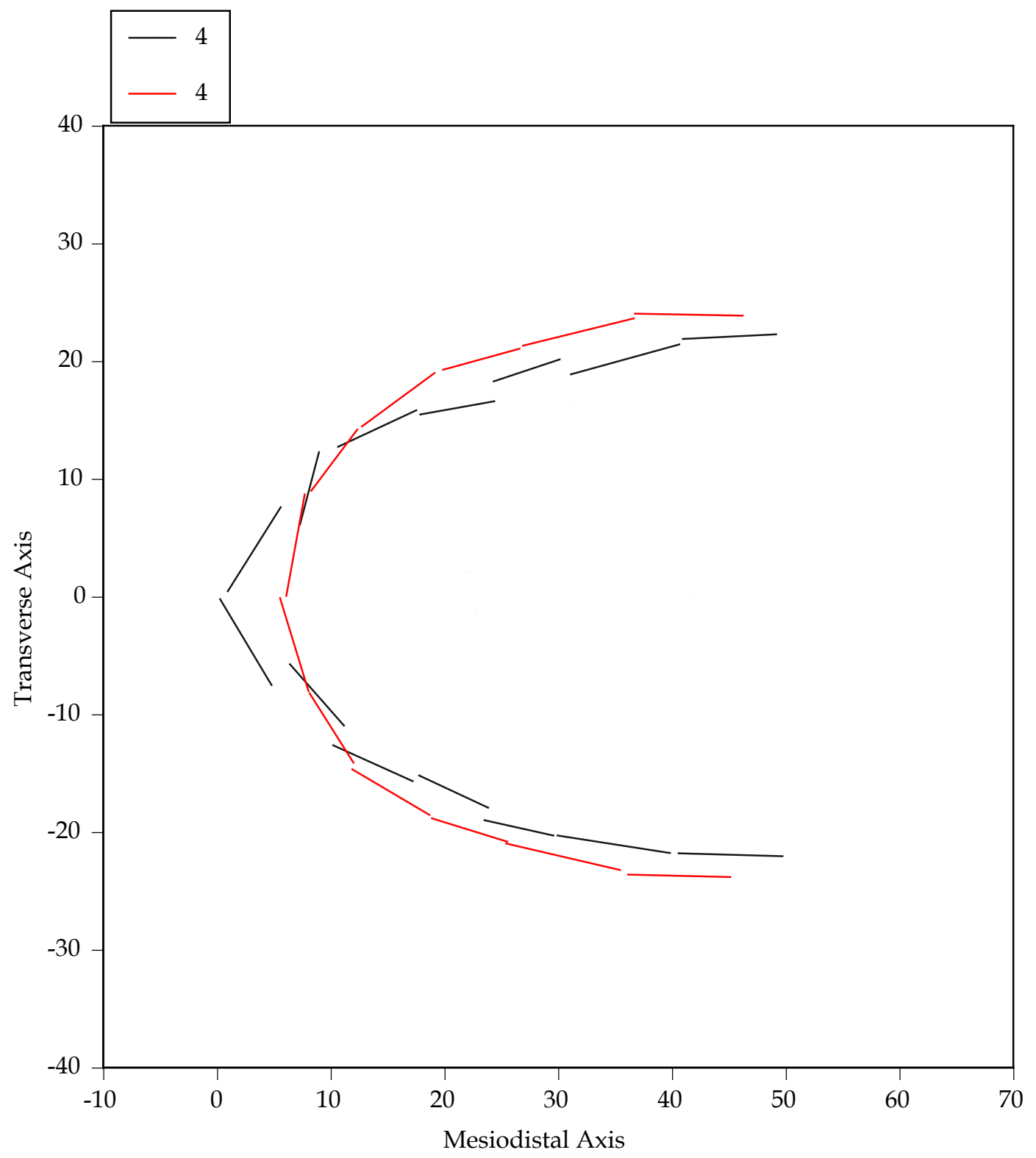

Fig. A-4. Plot of the maxillary dental arch relationships of case 4, a male in whom the maxillary first-premolars were extracted for treatment. Squares are the dental contacts at the start of treatment; circles are the contacts at the end of treatment. The $\mathrm{X}$ and $\mathrm{Y}$ axes are in millimeters. 


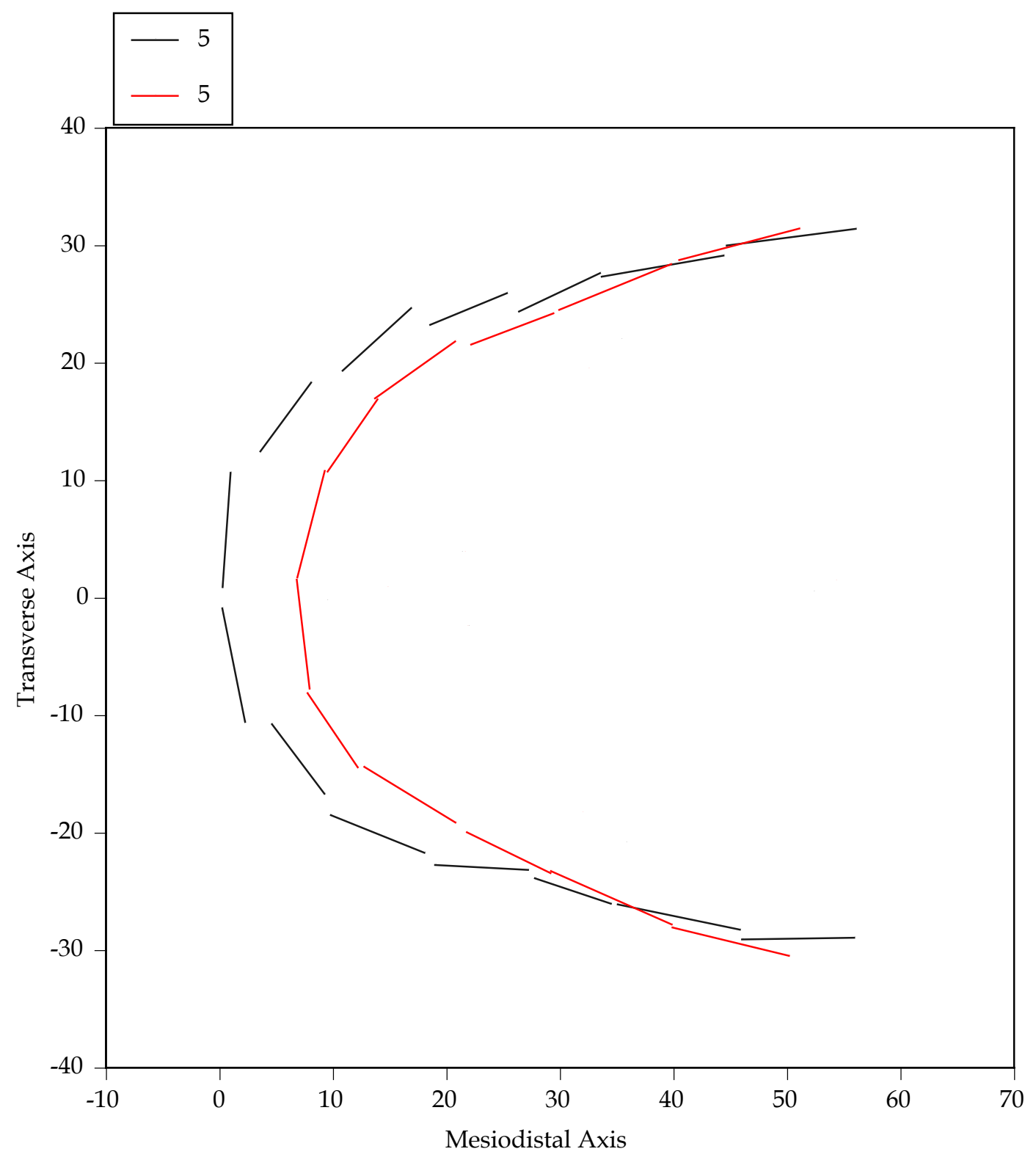

Fig. A-5. Plot of the maxillary dental arch relationships of case 5, a male in whom the maxillary first-premolars were extracted for treatment. Squares are the dental contacts at the start of treatment; circles are the contacts at the end of treatment. The $X$ and $\mathrm{Y}$ axes are in millimeters. 


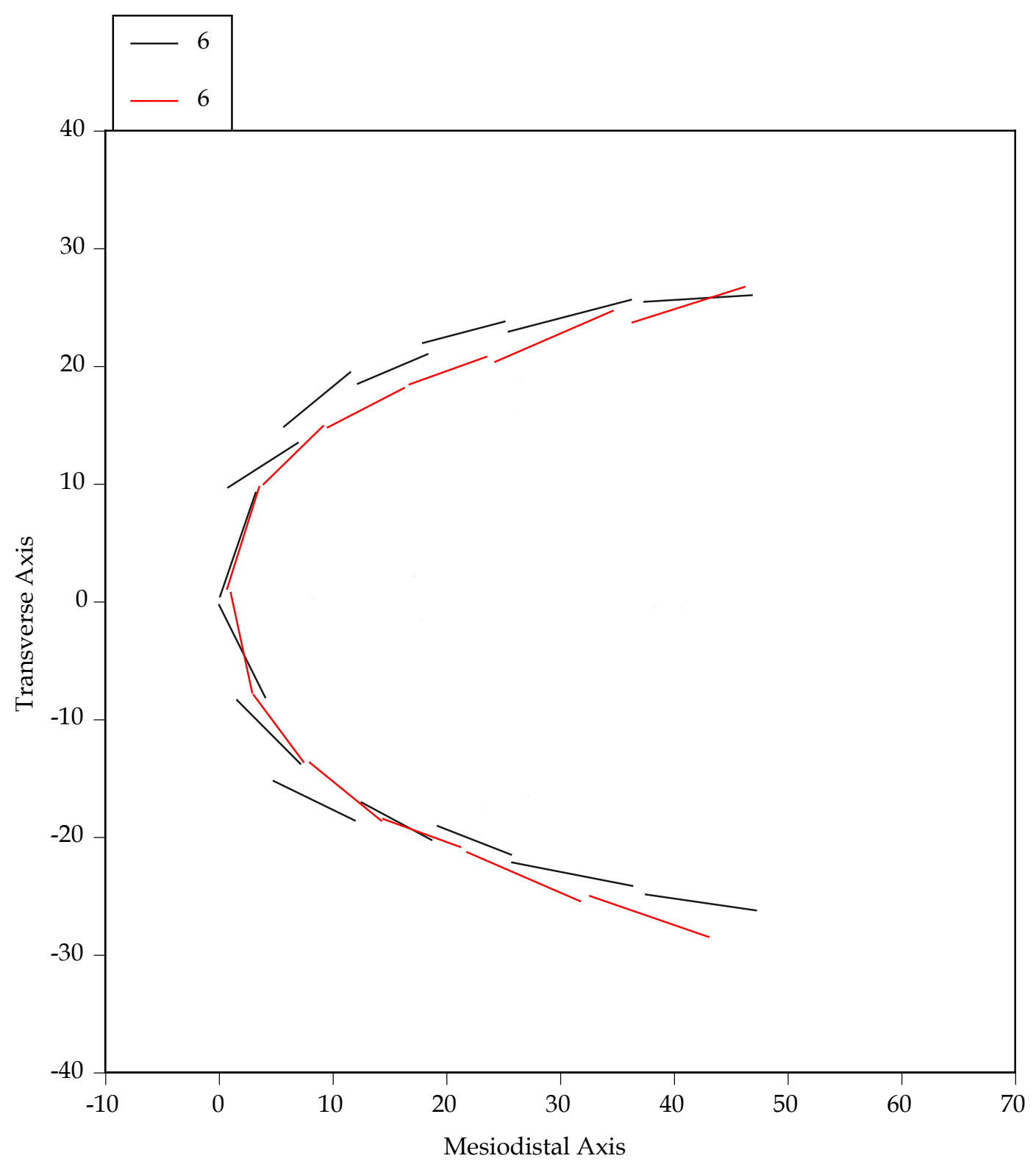

Fig. A-6. Plot of the maxillary dental arch relationships of case 6, a female in whom the maxillary second-premolars were extracted for treatment. Squares are the dental contacts at the start of treatment; circles are the contacts at the end of treatment. The $X$ and $\mathrm{Y}$ axes are in millimeters. 


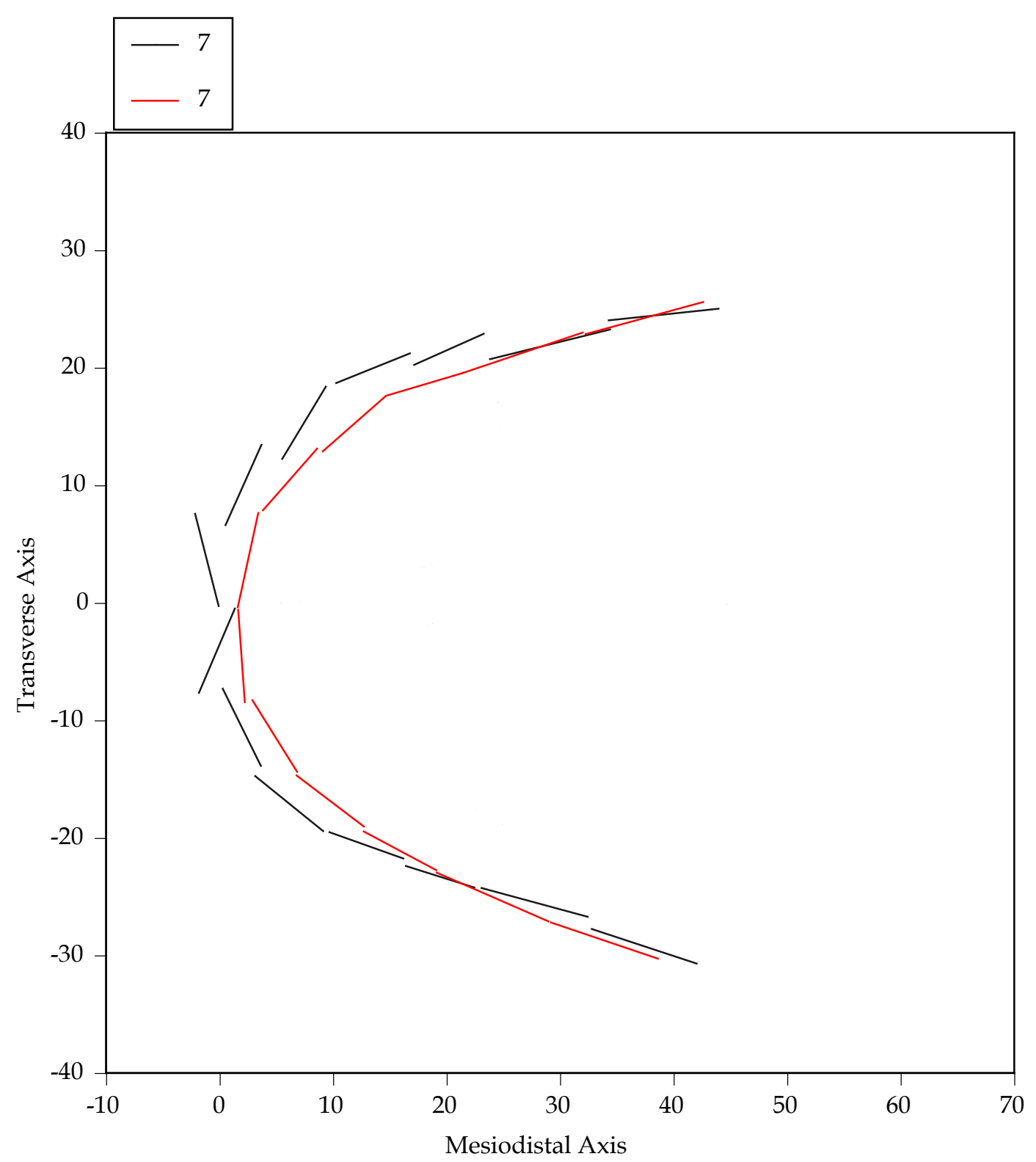

Fig. A-7. Plot of the maxillary dental arch relationships of case 7, a female in whom the maxillary first-premolars were extracted for treatment. Squares are the dental contacts at the start of treatment; circles are the contacts at the end of treatment. The $\mathrm{X}$ and $\mathrm{Y}$ axes 


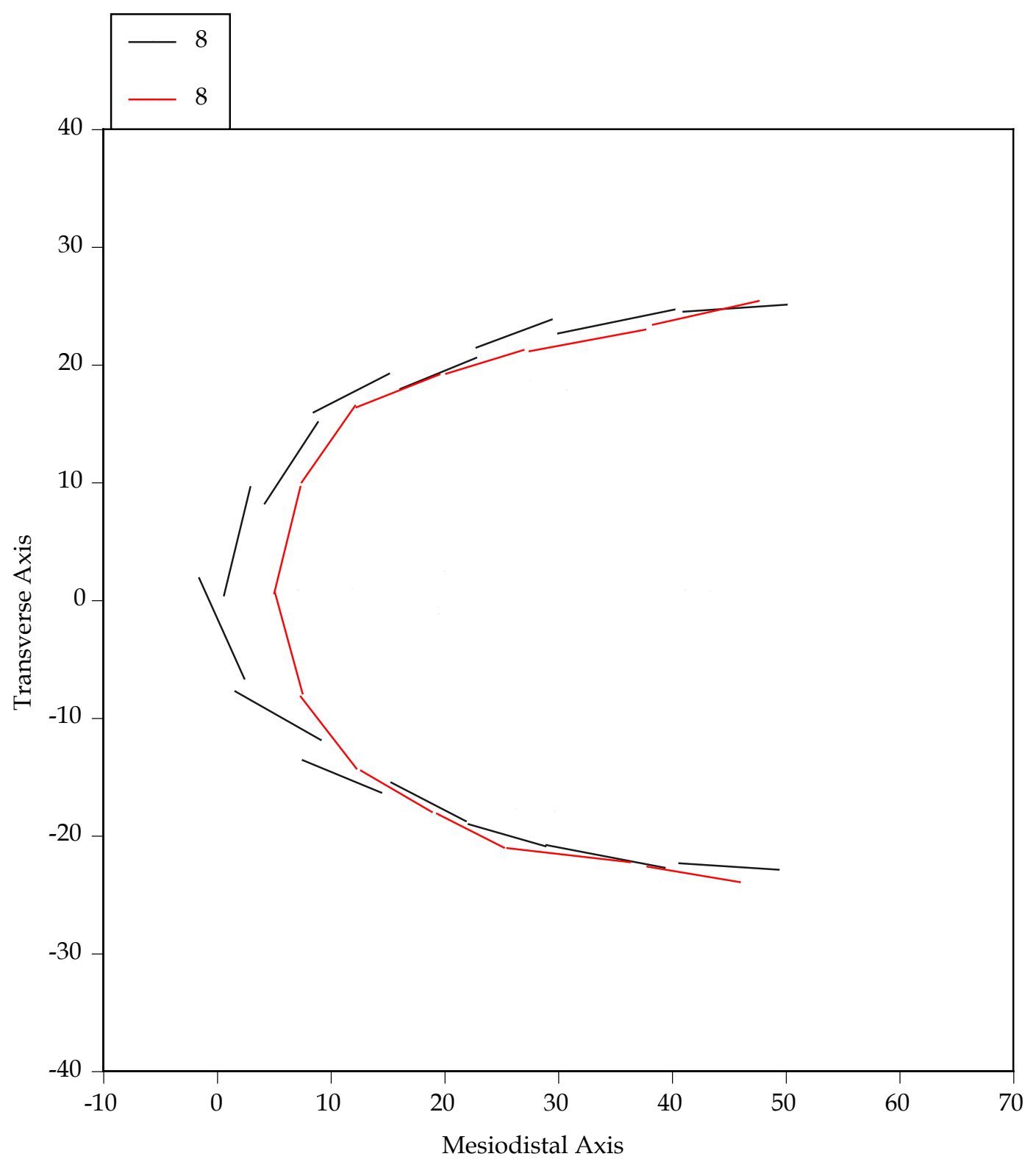

Fig. A-8. Plot of the maxillary dental arch relationships of case 8, a female in whom the maxillary second-premolars were extracted for treatment. Squares are the dental contacts at the start of treatment; circles are the contacts at the end of treatment. The $X$ and $\mathrm{Y}$ axes are in millimeters. 


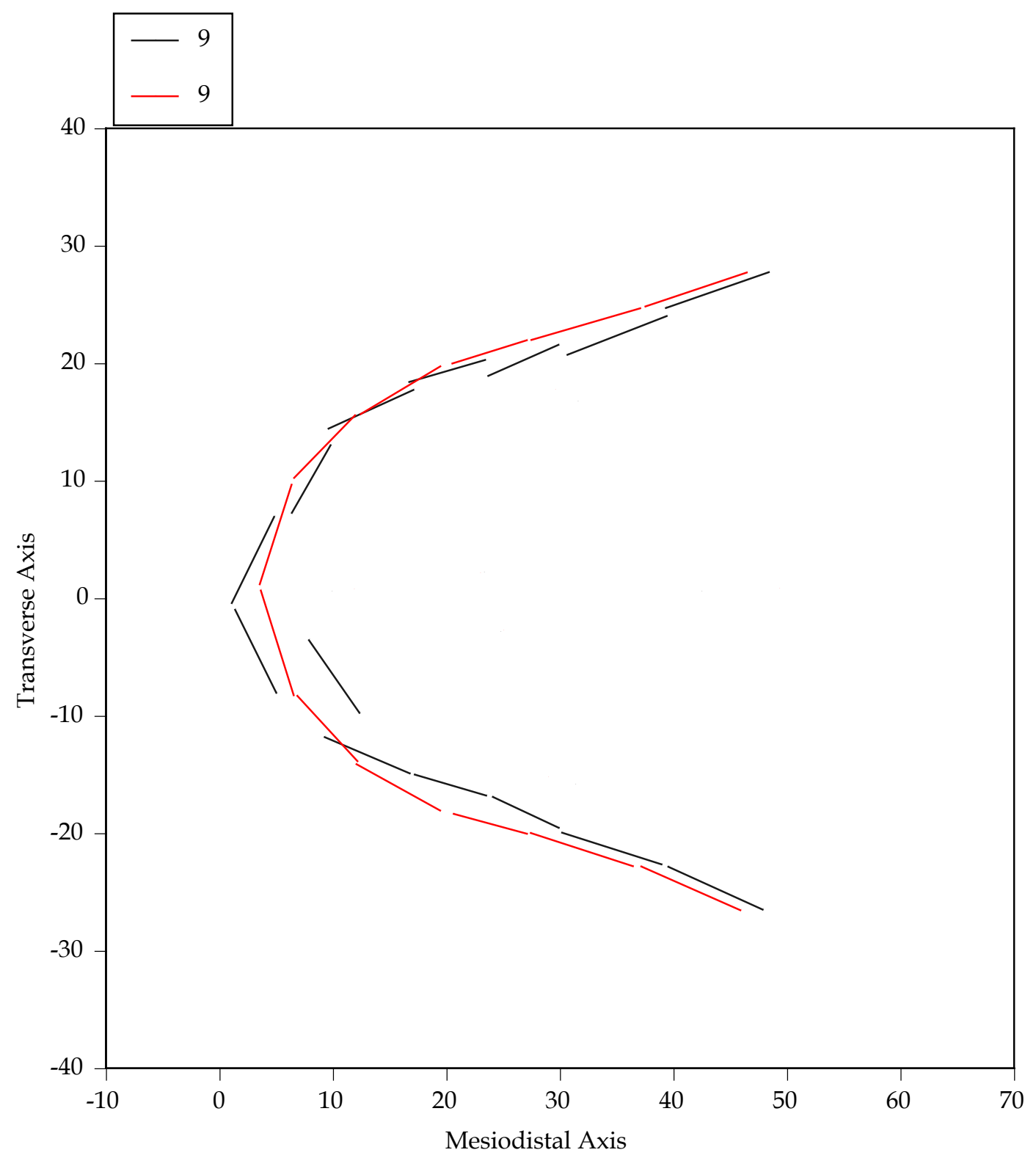

Fig. A-9. Plot of the maxillary dental arch relationships of case 9, a female in whom the maxillary first-premolars were extracted for treatment. Squares are the dental contacts at the start of treatment; circles are the contacts at the end of treatment. The $X$ and $\mathrm{Y}$ axes are in millimeters. 


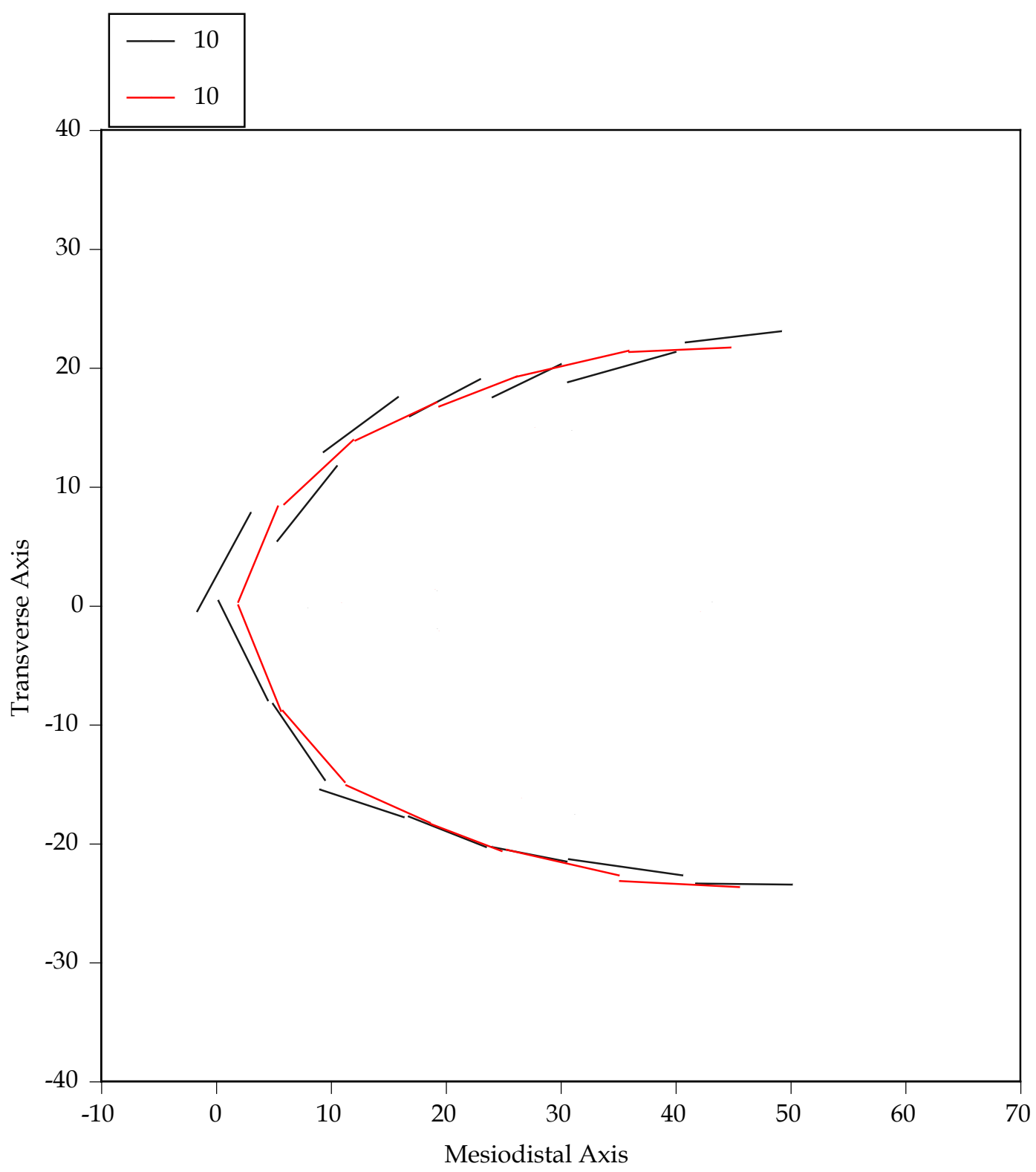

Fig. A-10. Plot of the maxillary dental arch relationships of case 10, a female in whom the maxillary first-premolars were extracted for treatment. Squares are the dental contacts at the start of treatment; circles are the contacts at the end of treatment. The $X$ and $\mathrm{Y}$ axes are in millimeters. 


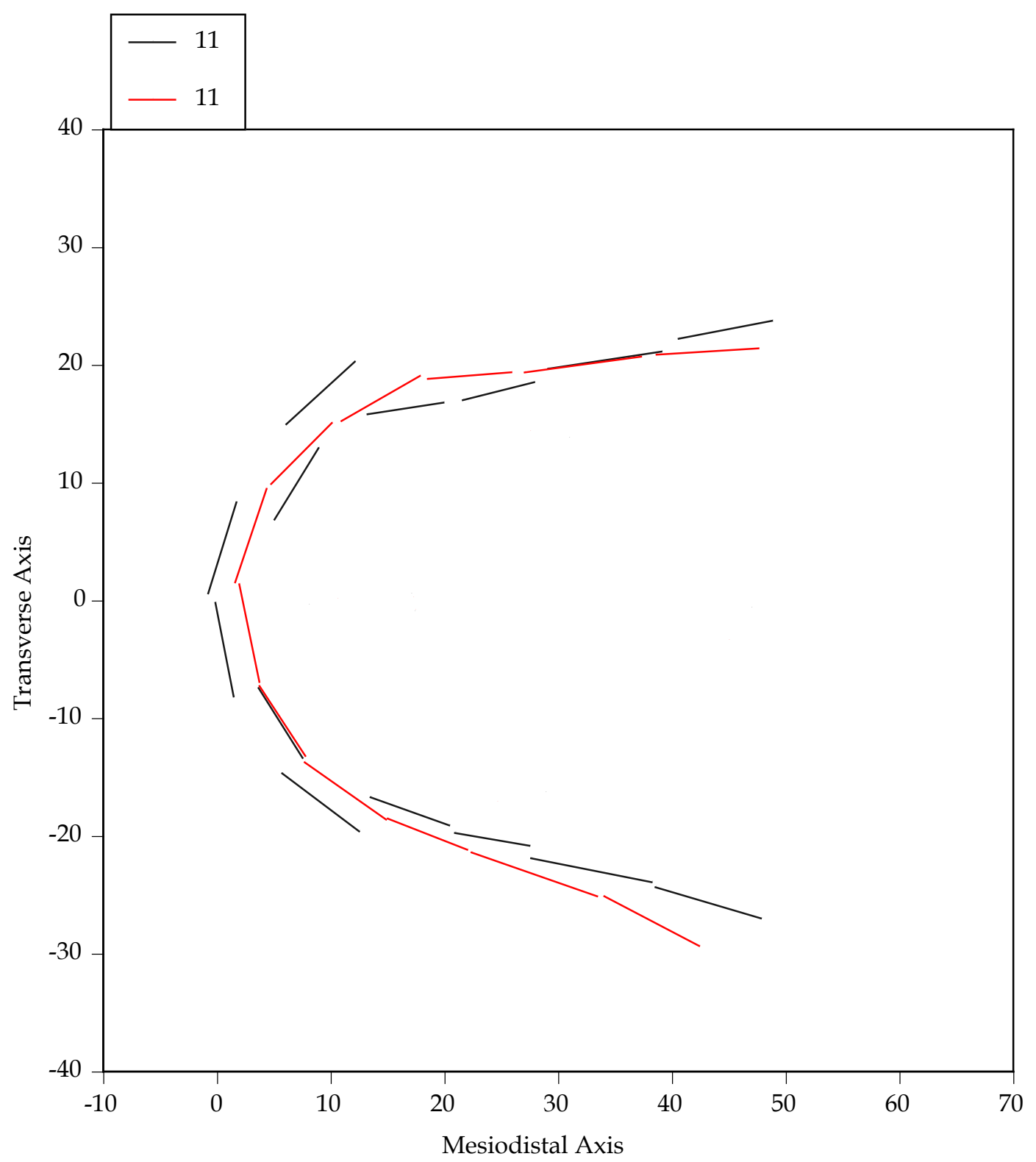

Fig. A-11. Plot of the maxillary dental arch relationships of case 11, a male in whom the maxillary second-premolars were extracted for treatment. Squares are the dental contacts at the start of treatment; circles are the contacts at the end of treatment. The $X$ and $\mathrm{Y}$ axes are in millimeters. 


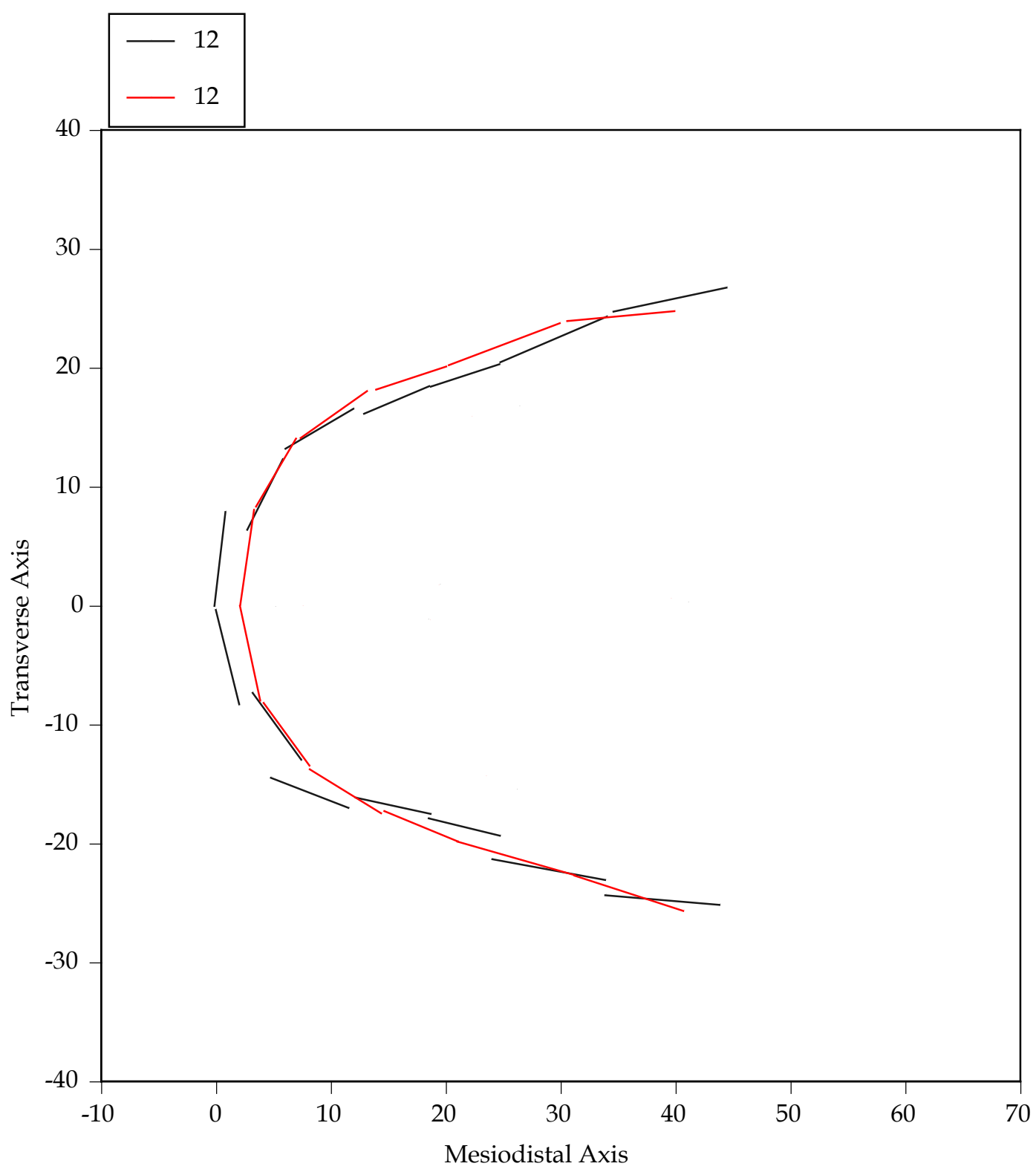

Fig. A-12. Plot of the maxillary dental arch relationships of case 12 , a female in whom the maxillary first-premolars were extracted for treatment. Squares are the dental contacts at the start of treatment; circles are the contacts at the end of treatment. The $X$ and $Y$ axes are in millimeters. 


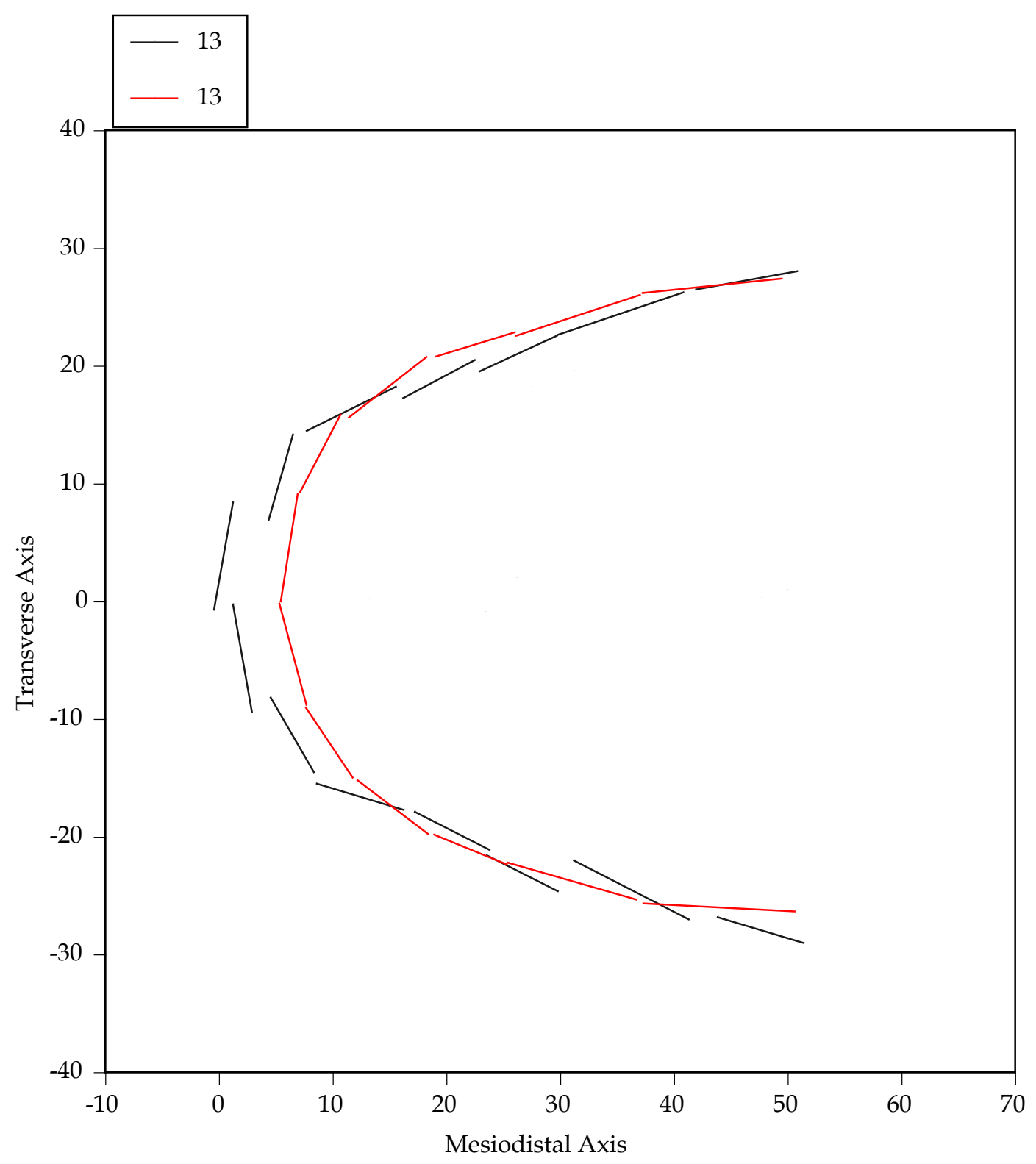

Fig. A-13. Plot of the maxillary dental arch relationships of case 13, a male in whom the maxillary first-premolars were extracted for treatment. Squares are the dental contacts at the start of treatment; circles are the contacts at the end of treatment. The $X$ and $\mathrm{Y}$ axes are in millimeters. 


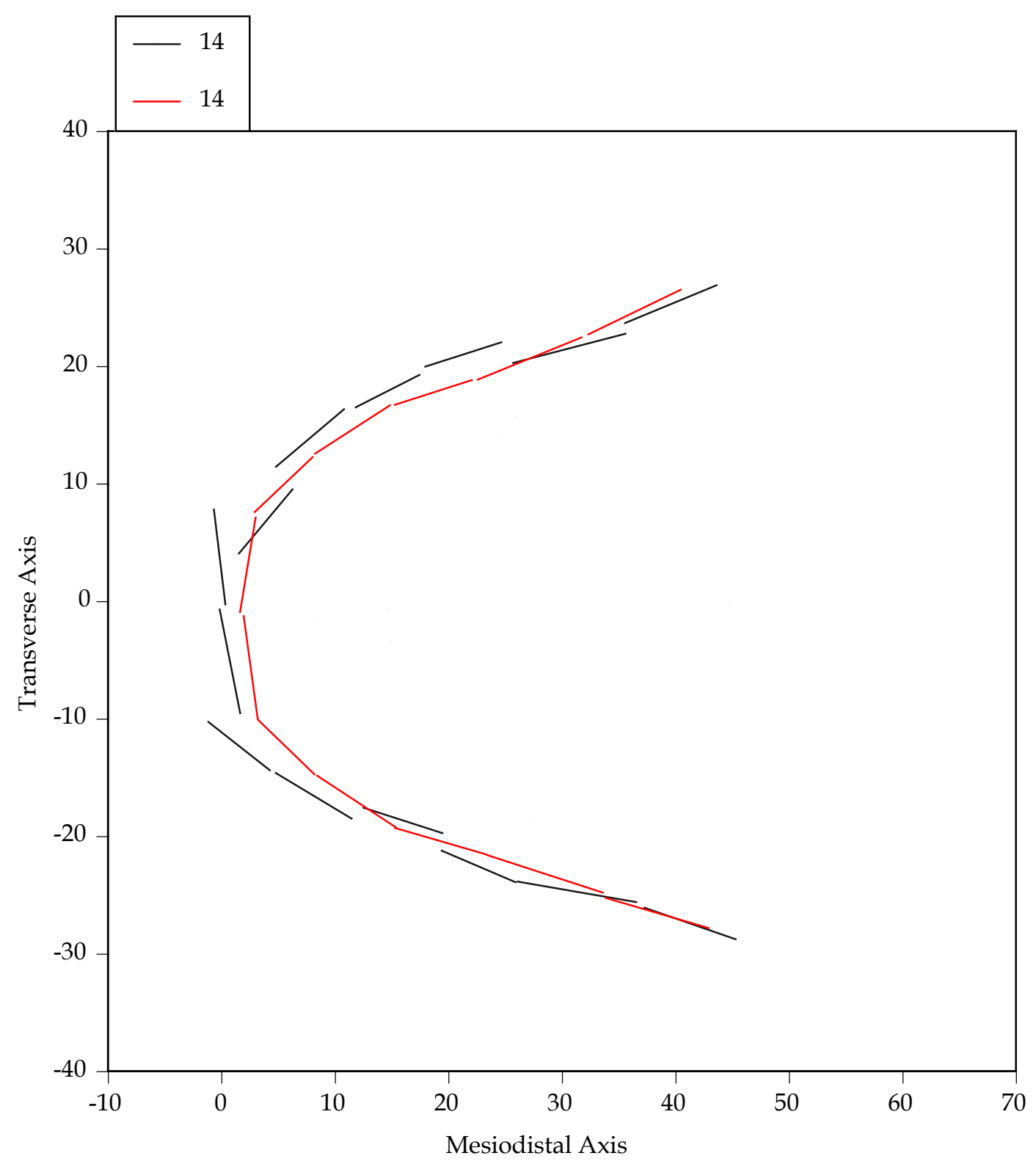

Fig. A-14. Plot of the maxillary dental arch relationships of case 14, a male in whom the maxillary second-premolars were extracted for treatment. Squares are the dental contacts at the start of treatment; circles are the contacts at the end of treatment. The $\mathrm{X}$ and $\mathrm{Y}$ axes are in millimeters. 


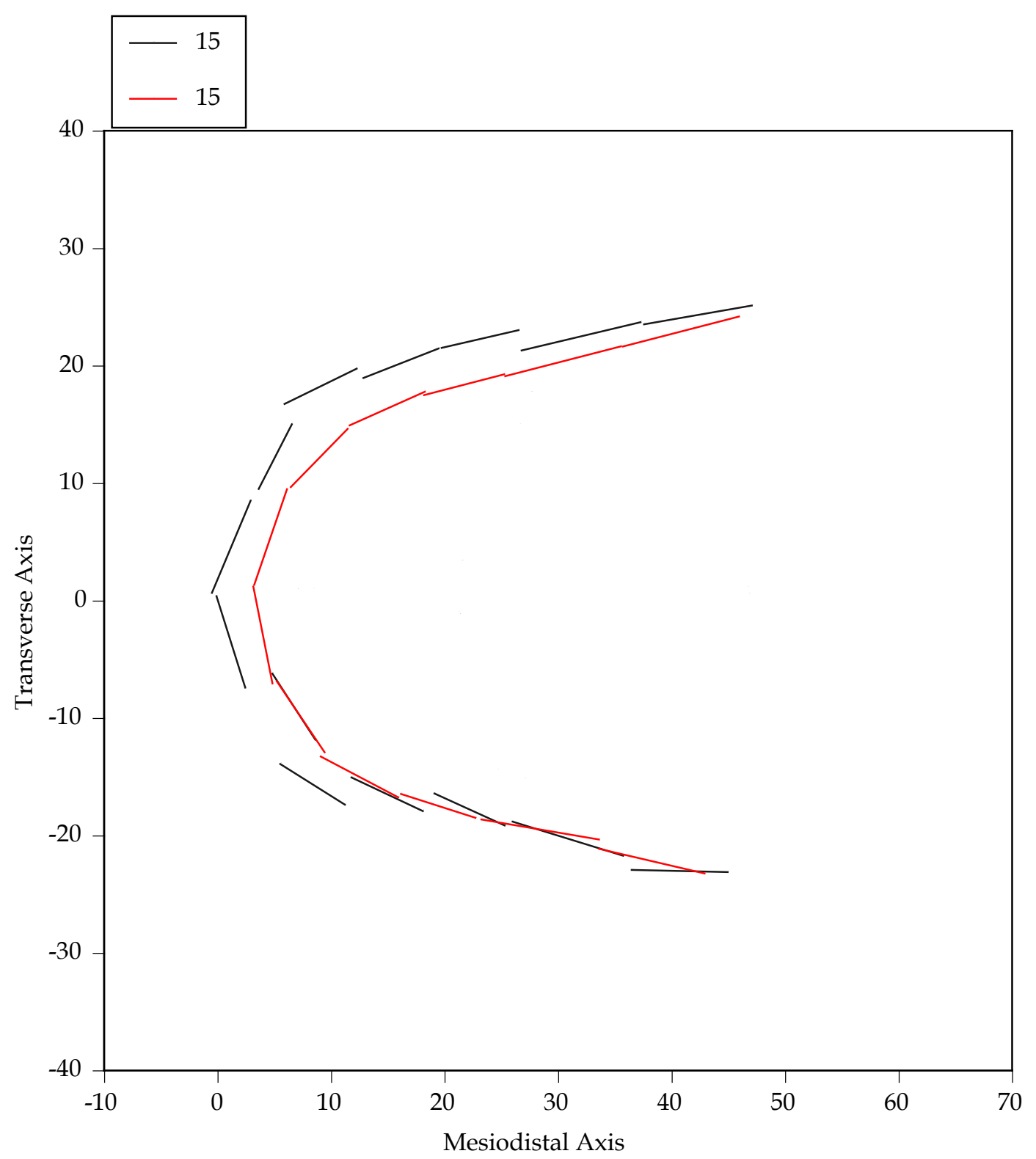

Fig. A-15. Plot of the maxillary dental arch relationships of case 15 , a female in whom the maxillary first-premolars were extracted for treatment. Squares are the dental contacts at the start of treatment; circles are the contacts at the end of treatment. The $X$ and $\mathrm{Y}$ axes are in millimeters. 


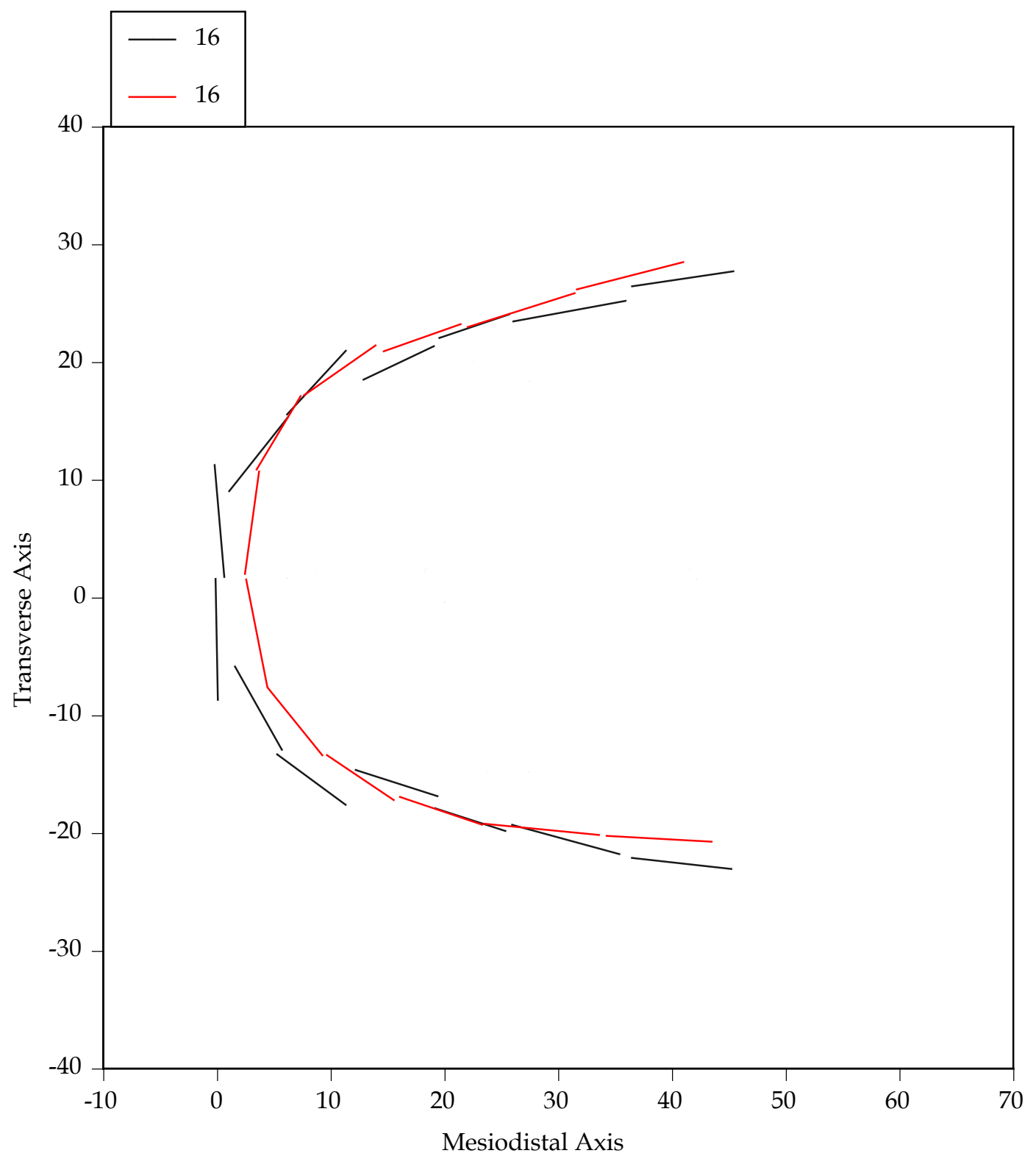

Fig. A-16. Plot of the maxillary dental arch relationships of case 16 , a female in whom the maxillary second-premolars were extracted for treatment. Squares are the dental contacts at the start of treatment; circles are the contacts at the end of treatment. The $\mathrm{X}$ and $\mathrm{Y}$ axes are in millimeters. 


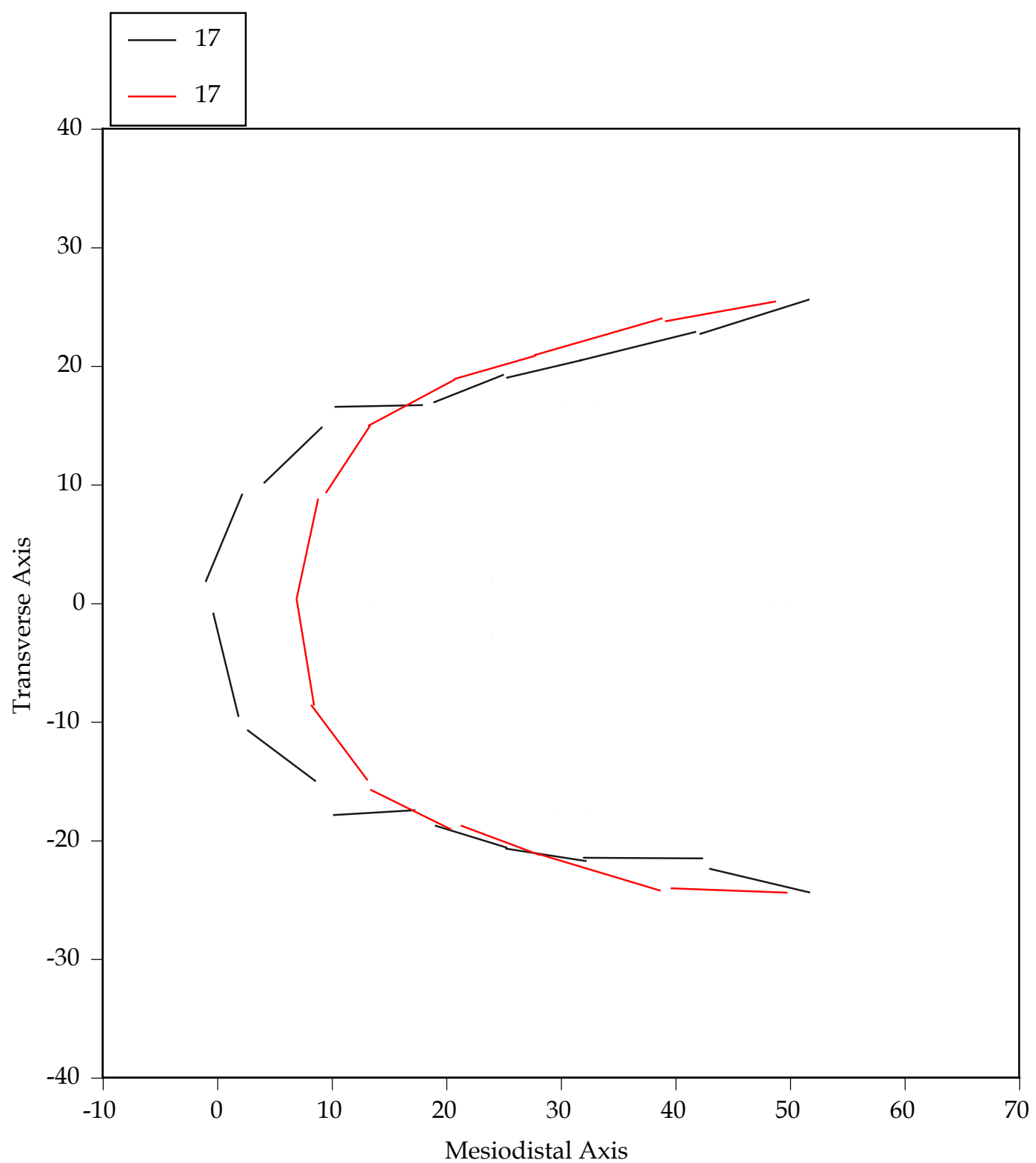

Fig. A-17. Plot of the maxillary dental arch relationships of case 17 , a male in whom the maxillary first-premolars were extracted for treatment. Squares are the dental contacts at the start of treatment; circles are the contacts at the end of treatment. The $\mathrm{X}$ and $\mathrm{Y}$ axes are in millimeters. 


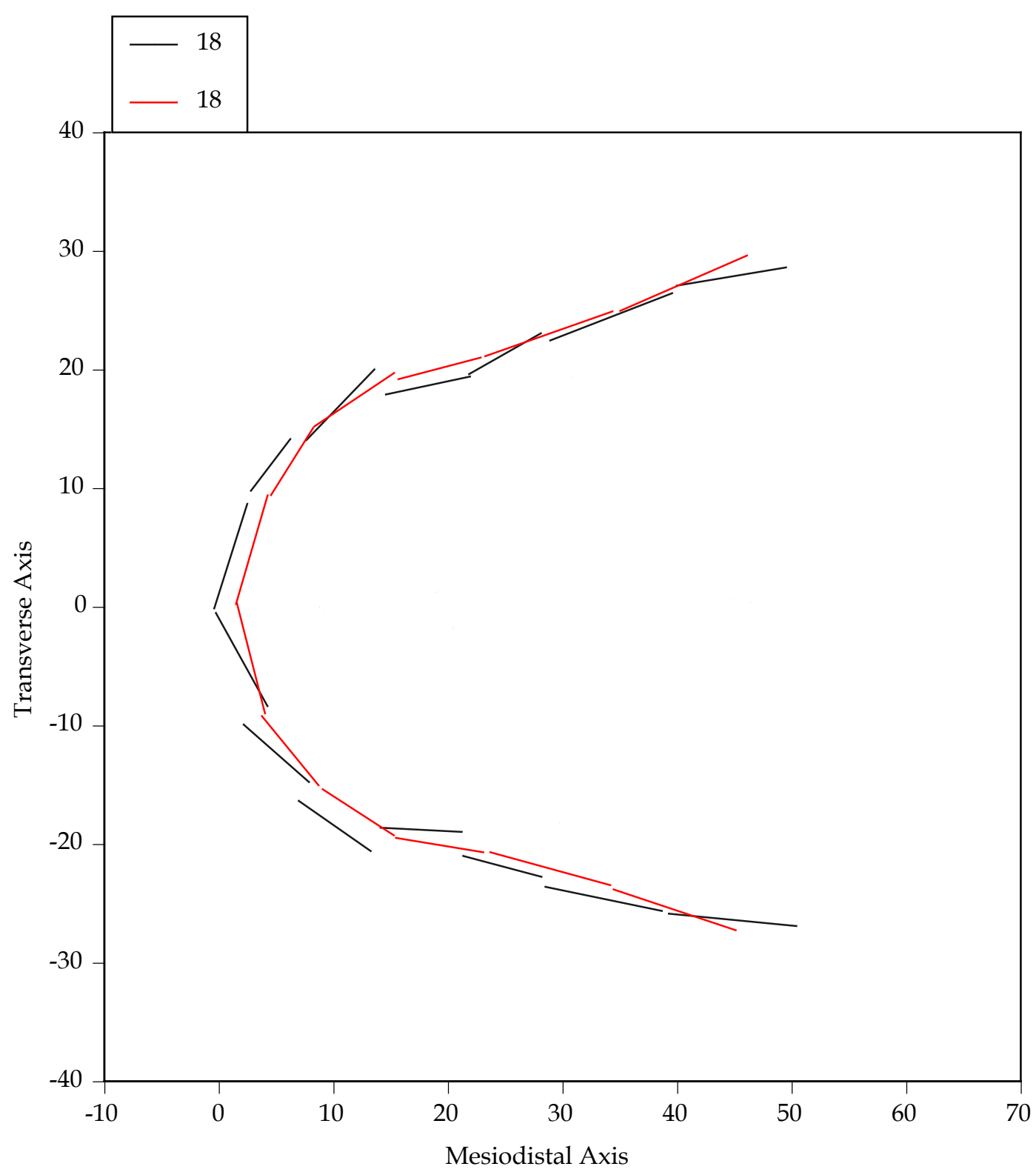

Fig. A-18. Plot of the maxillary dental arch relationships of case 18 , a male in whom the maxillary second-premolars were extracted for treatment. Squares are the dental contacts at the start of treatment; circles are the contacts at the end of treatment. The $X$ and $\mathrm{Y}$ axes are in millimeters. 


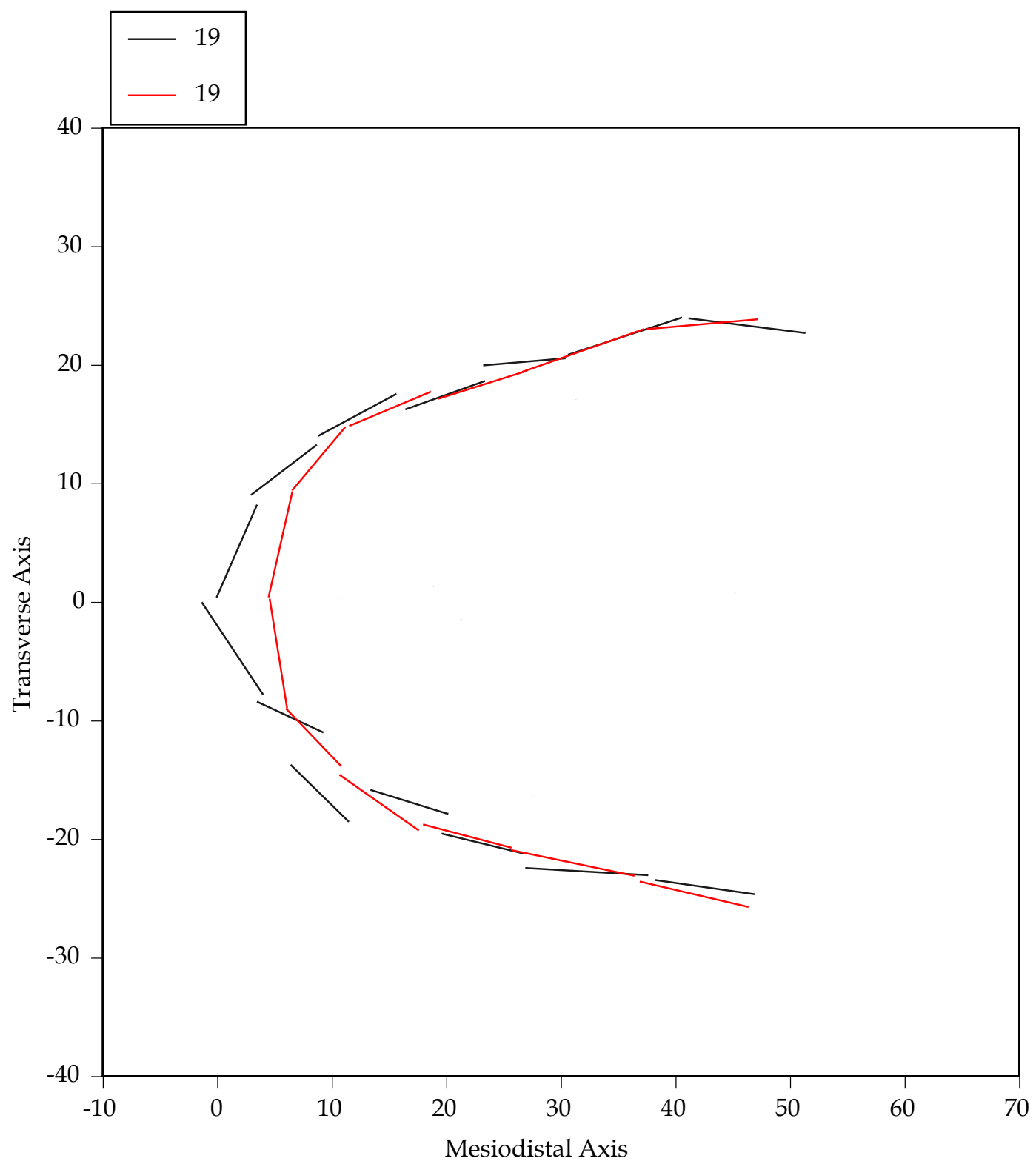

Fig. A-19. Plot of the maxillary dental arch relationships of case 19 , a female in whom the maxillary first-premolars were extracted for treatment. Squares are the dental contacts at the start of treatment; circles are the contacts at the end of treatment. The $X$ and $\mathrm{Y}$ axes are in millimeters. 


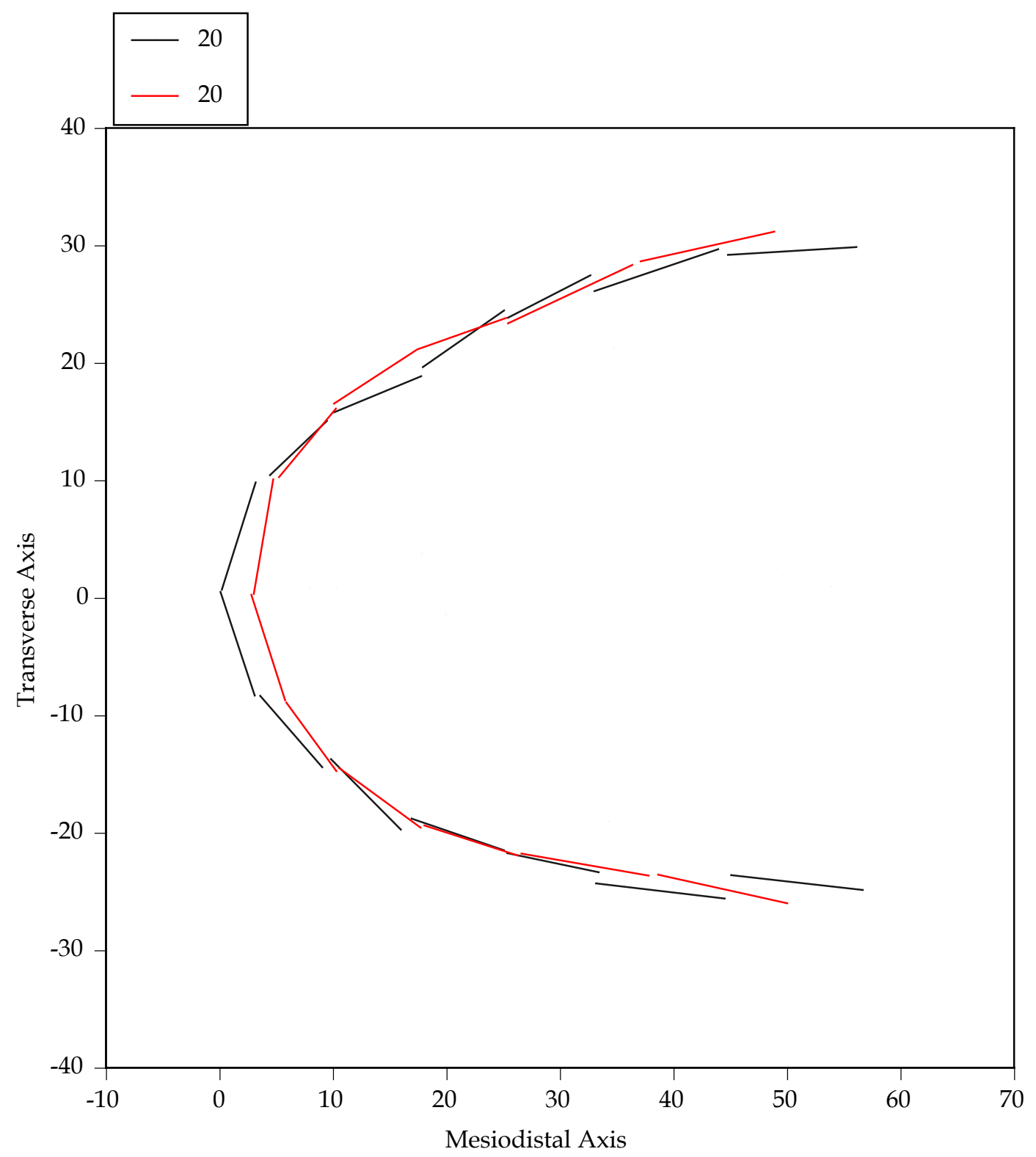

Fig. A-20. Plot of the maxillary dental arch relationships of case 20, a female in whom the maxillary first-premolars were extracted for treatment. Squares are the dental contacts at the start of treatment; circles are the contacts at the end of treatment. The $X$ and $\mathrm{Y}$ axes are in millimeters. 


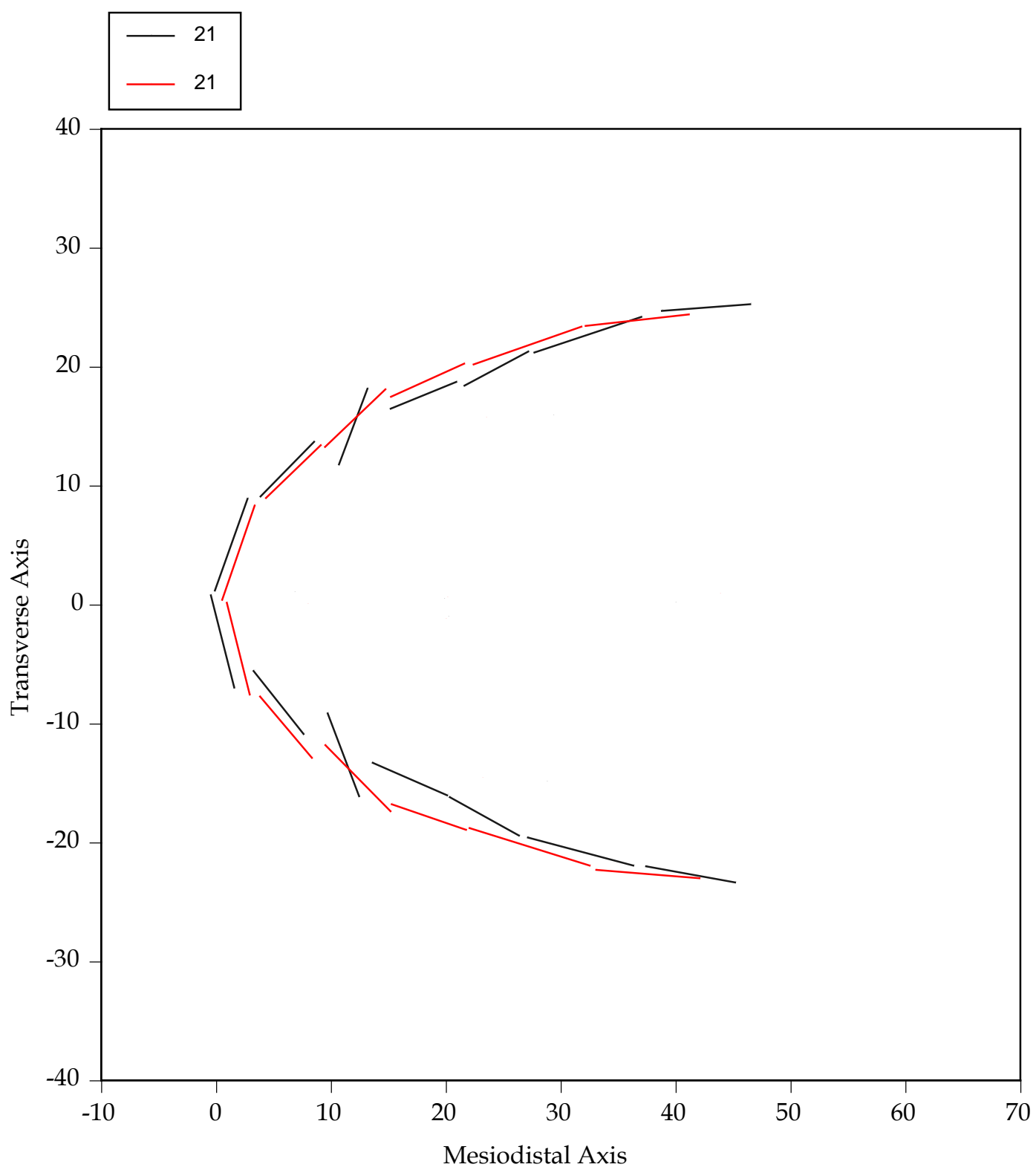

Fig. A-21. Plot of the maxillary dental arch relationships of case 21, a female in whom the maxillary second-premolars were extracted for treatment. Squares are the dental contacts at the start of treatment; circles are the contacts at the end of treatment. The $X$ and $\mathrm{Y}$ axes are in millimeters. 


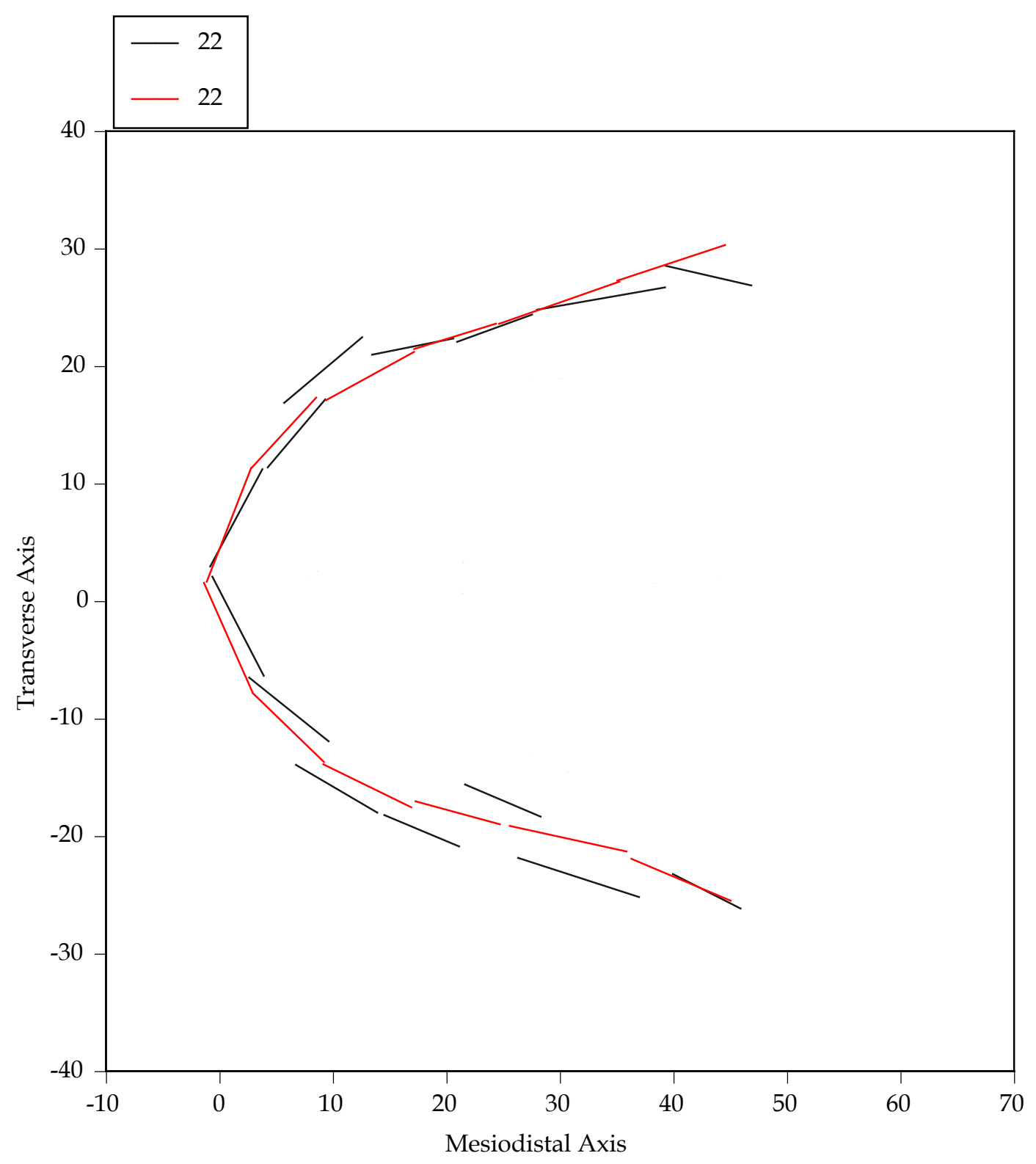

Fig. A-22. Plot of the maxillary dental arch relationships of case 22, a male in whom the maxillary first-premolars were extracted for treatment. Squares are the dental contacts at the start of treatment; circles are the contacts at the end of treatment. The $\mathrm{X}$ and $\mathrm{Y}$ axes are in millimeters. 


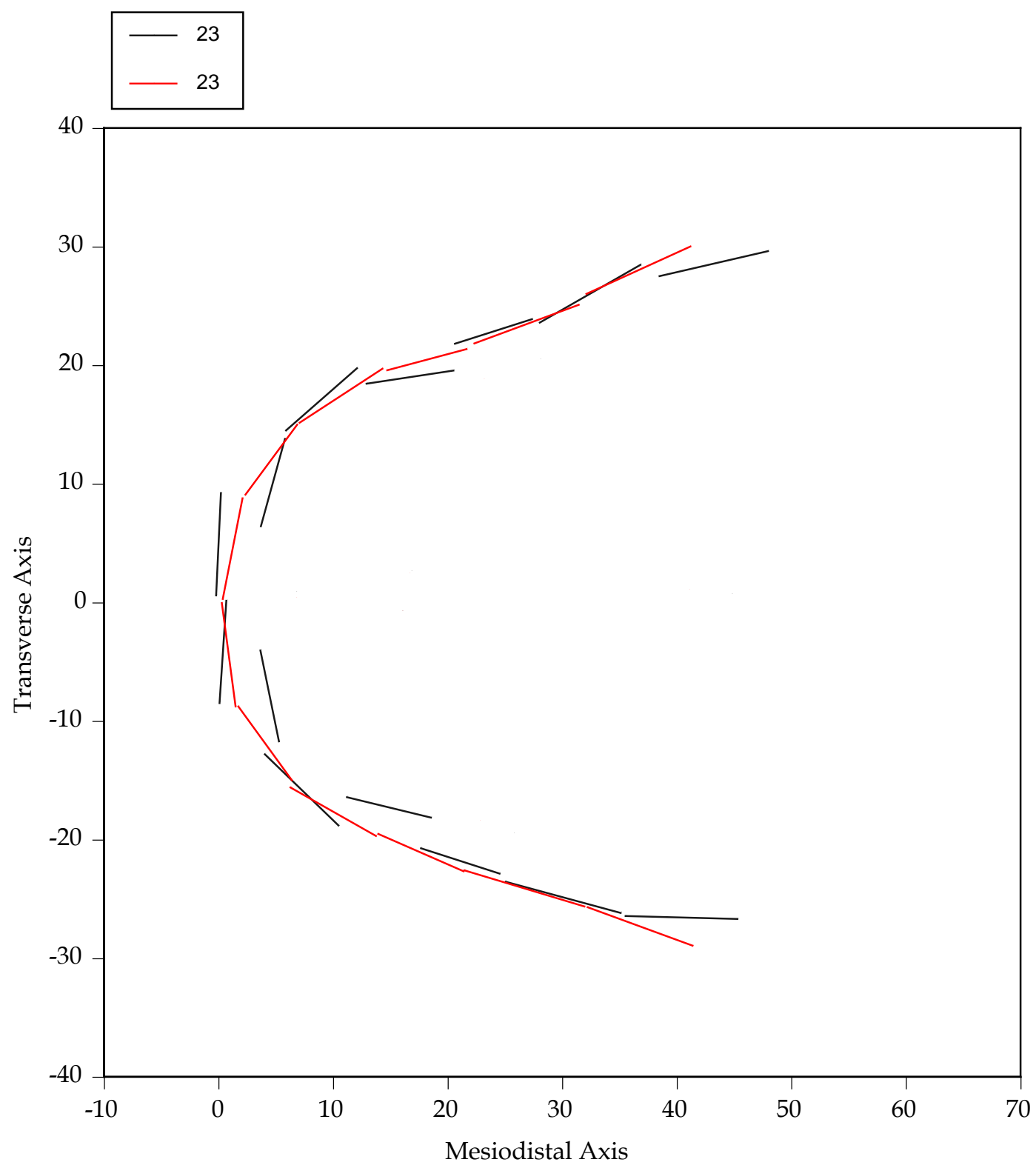

Fig. A-23. Plot of the maxillary dental arch relationships of case 23 , a female in whom the maxillary second-premolars were extracted for treatment. Squares are the dental contacts at the start of treatment; circles are the contacts at the end of treatment. The $X$ and $\mathrm{Y}$ axes are in millimeters. 


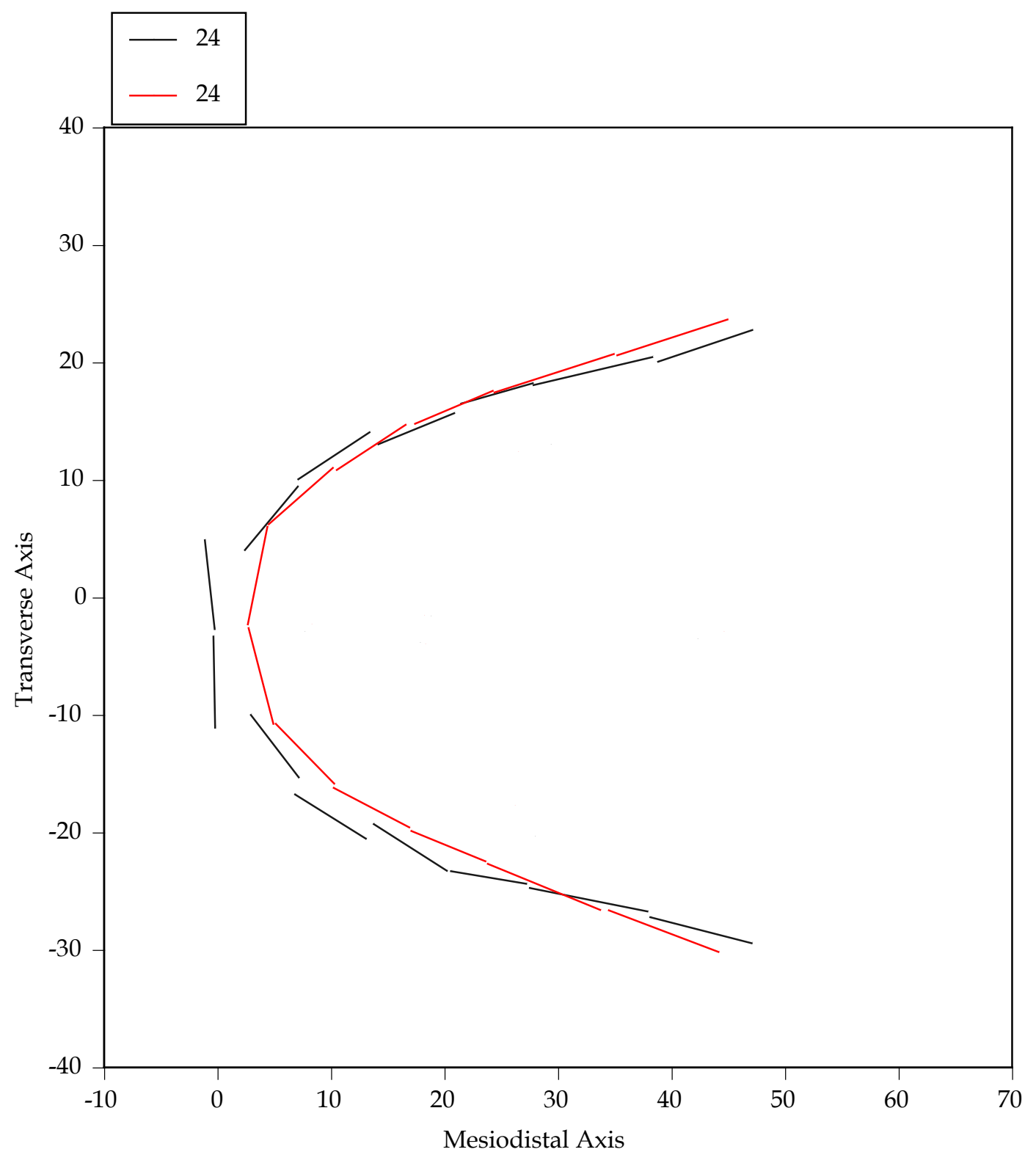

Fig. A-24. Plot of the maxillary dental arch relationships of case 24, a female in whom the maxillary first-premolars were extracted for treatment. Squares are the dental contacts at the start of treatment; circles are the contacts at the end of treatment. The $X$ and $\mathrm{Y}$ axes are in millimeters. 


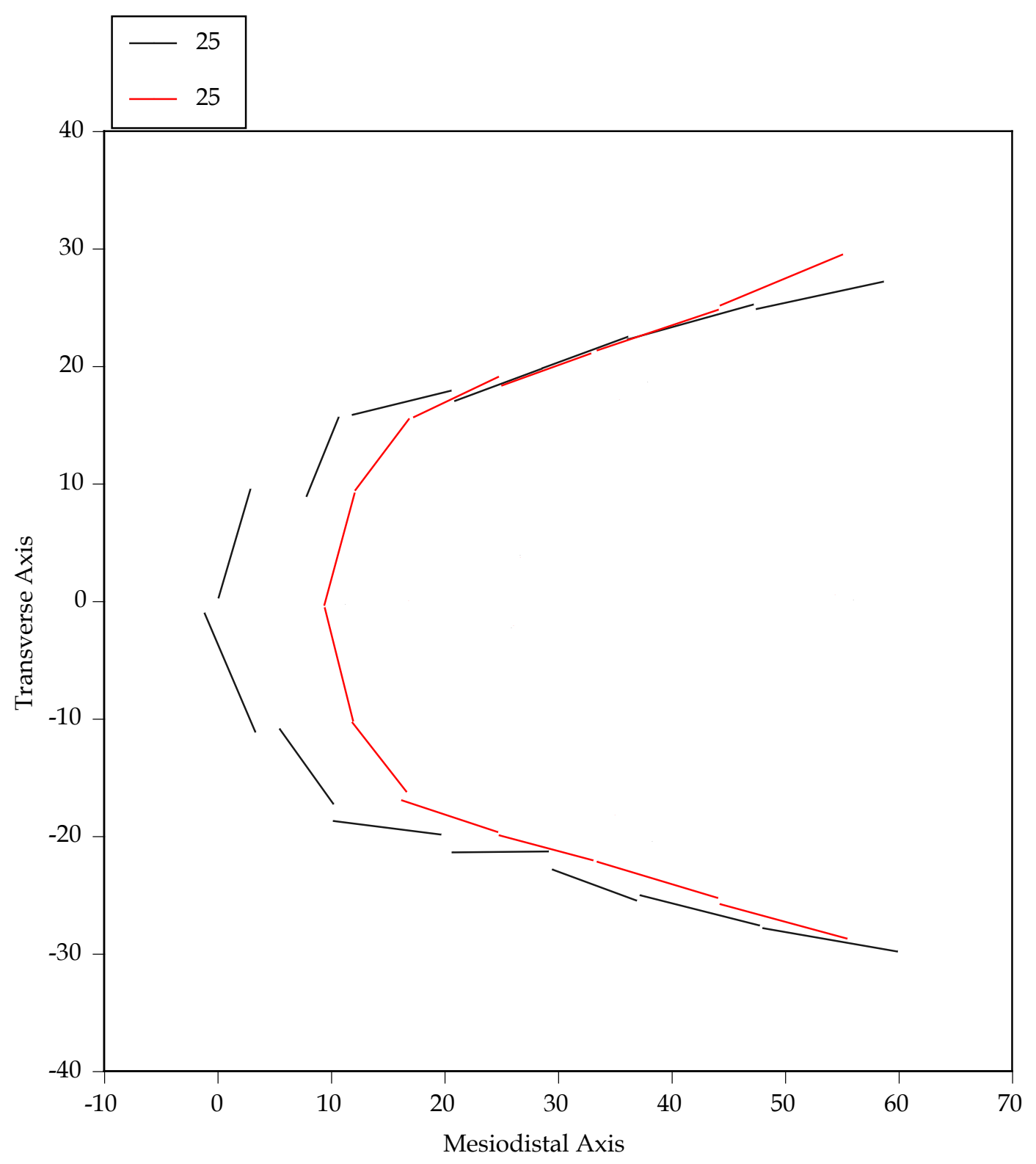

Fig. A-25. Plot of the maxillary dental arch relationships of case 25 , a male in whom the maxillary first-premolars were extracted for treatment. Squares are the dental contacts at the start of treatment; circles are the contacts at the end of treatment. The $X$ and $\mathrm{Y}$ axes are in millimeters. 


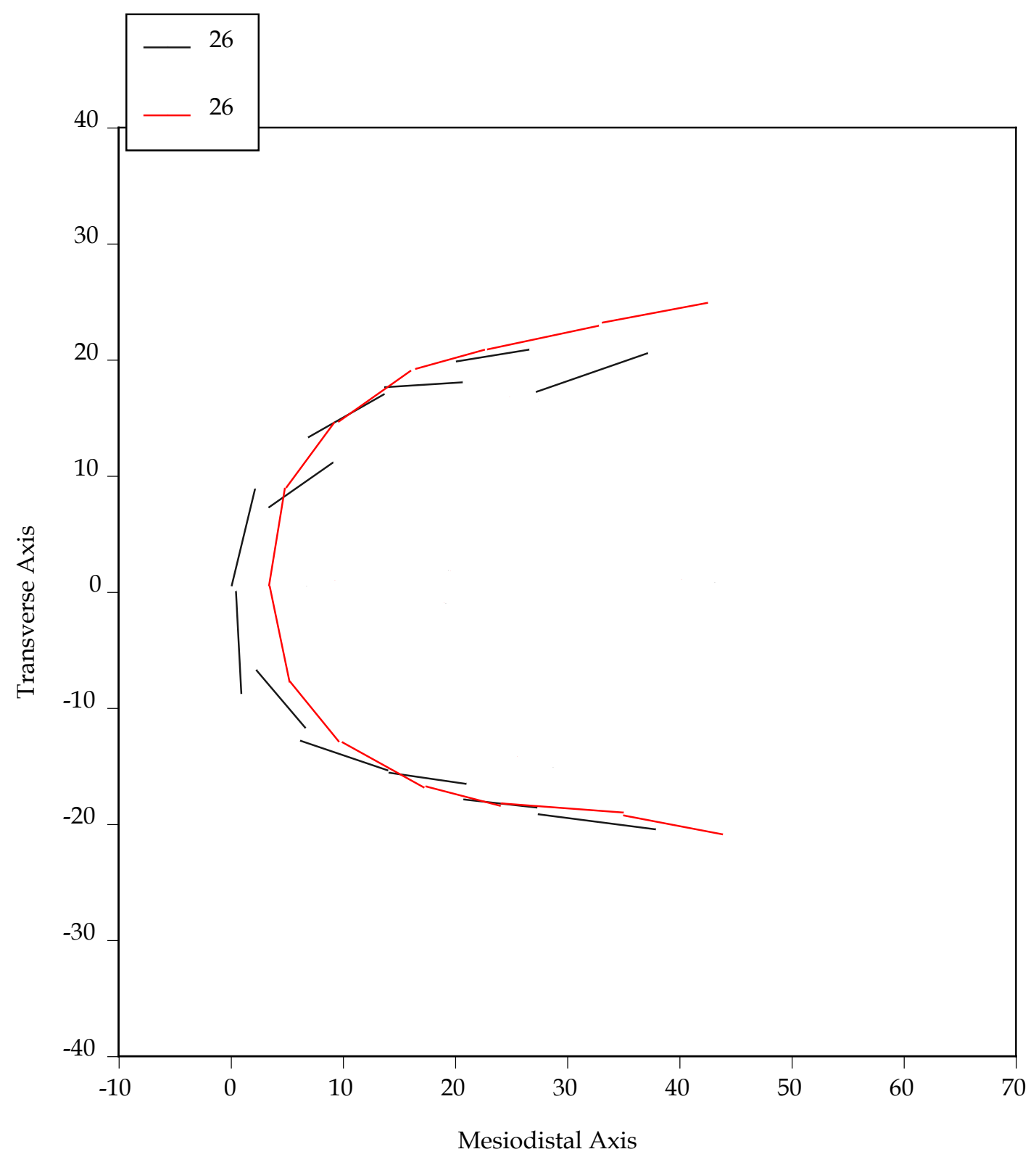

Fig. A-26. Plot of the maxillary dental arch relationships of case 26 , a female in whom the maxillary second-premolars were extracted for treatment. Squares are the dental contacts at the start of treatment; circles are the contacts at the end of treatment. The $\mathrm{X}$ and $\mathrm{Y}$ axes are in millimeters. 


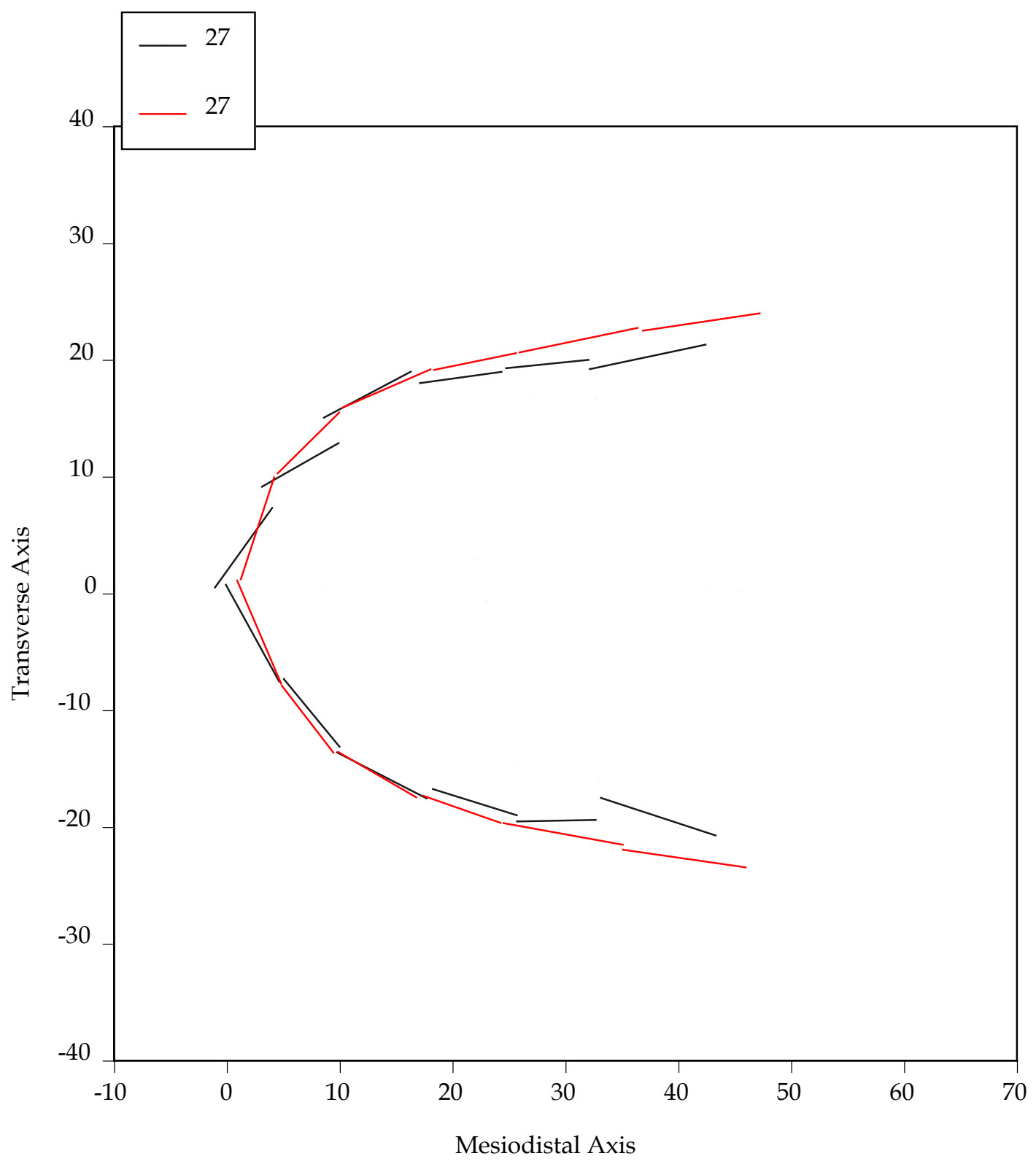

Fig. A-27. Plot of the maxillary dental arch relationships of case 27 , a male in whom the maxillary second-premolars were extracted for treatment. Squares are the dental contacts at the start of treatment; circles are the contacts at the end of treatment. The $\mathrm{X}$ and $\mathrm{Y}$ axes are in millimeters. 


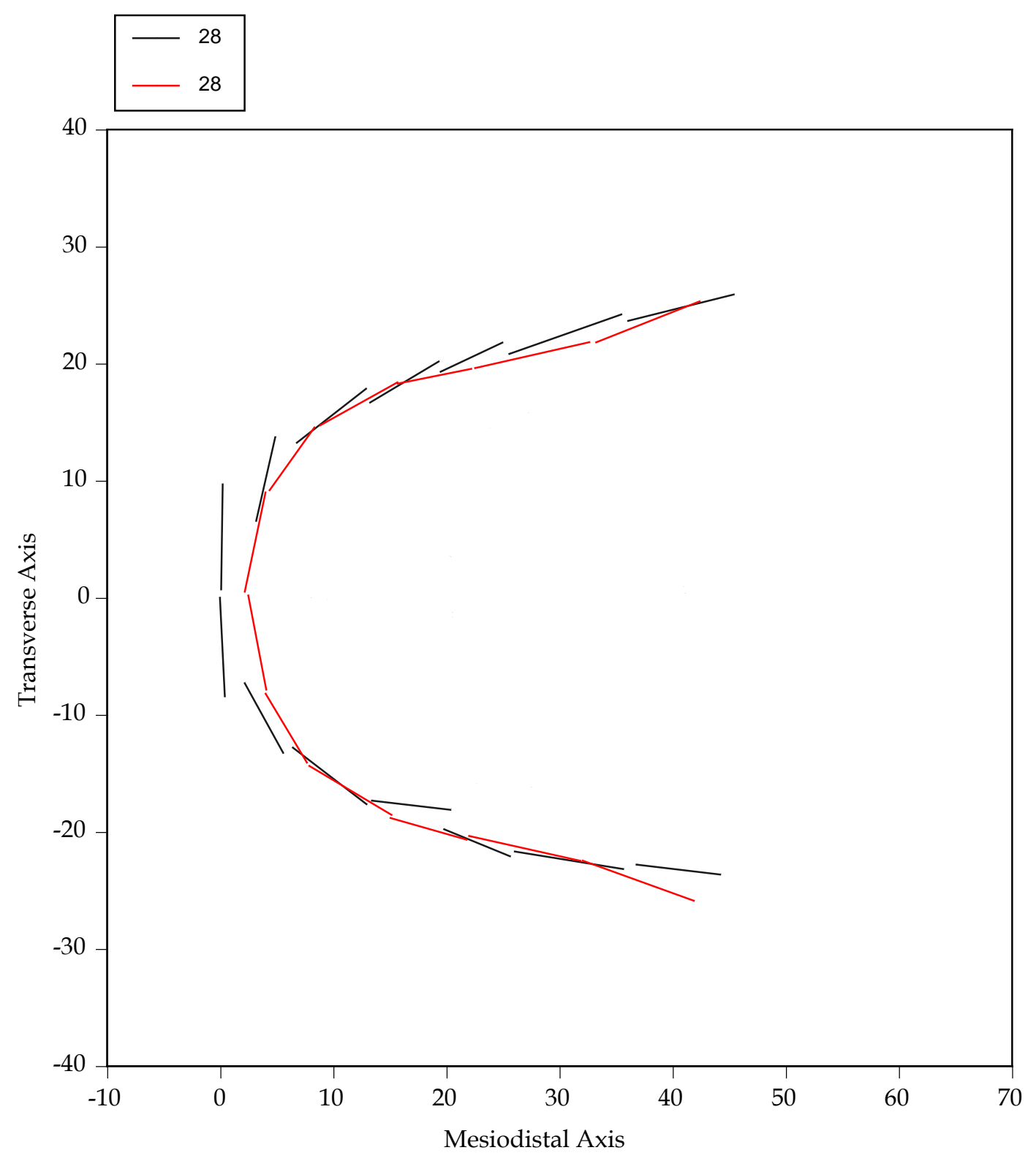

Fig. A-28. Plot of the maxillary dental arch relationships of case 28 , a female in whom the maxillary second-premolars were extracted for treatment. Squares are the dental contacts at the start of treatment; circles are the contacts at the end of treatment. The $\mathrm{X}$ and $\mathrm{Y}$ axes are in millimeters. 


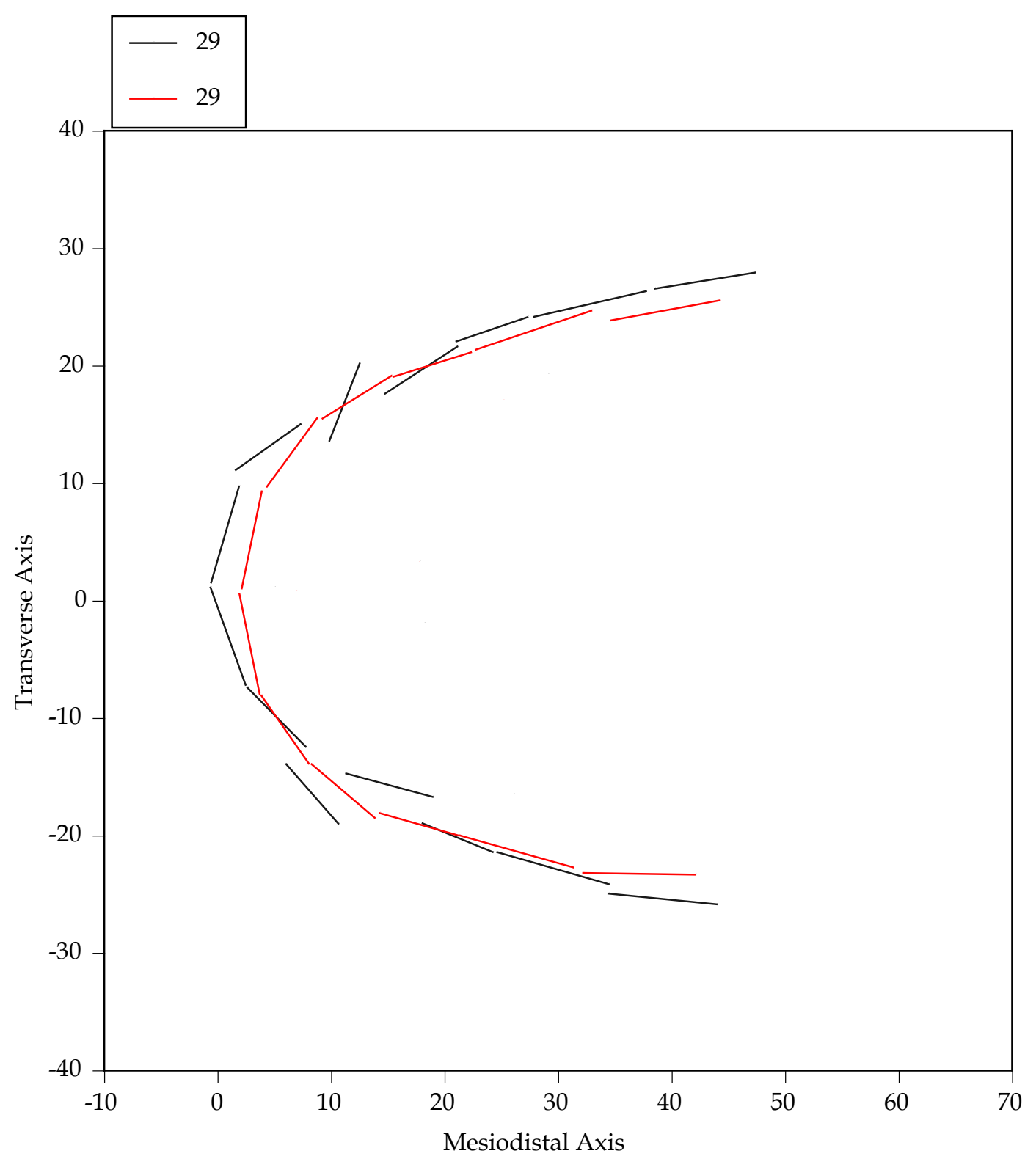

Fig. A-29. Plot of the maxillary dental arch relationships of case 29, a female in whom the maxillary first-premolars were extracted for treatment. Squares are the dental contacts at the start of treatment; circles are the contacts at the end of treatment. The $X$ and $\mathrm{Y}$ axes are in millimeters. 


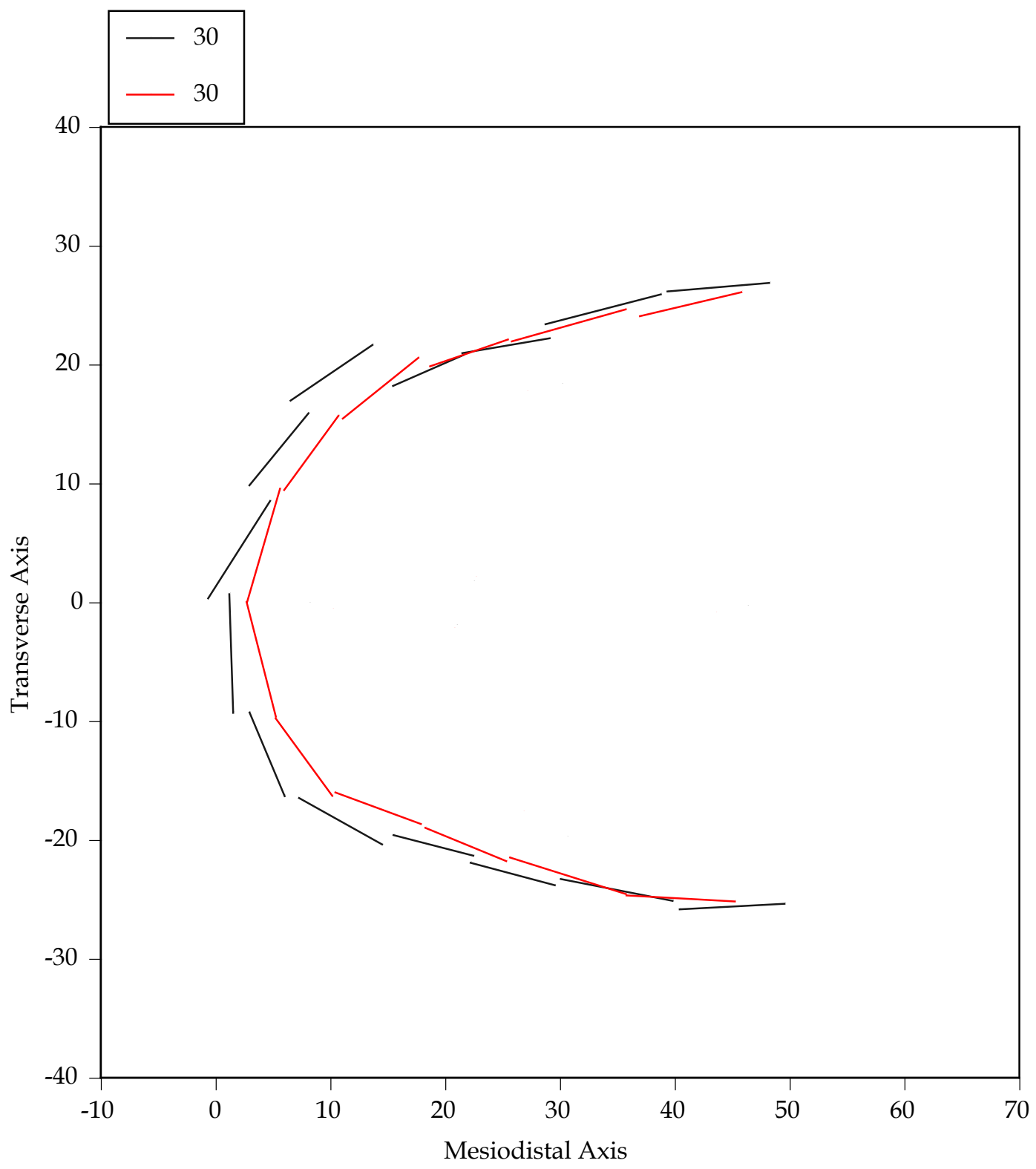

Fig. A-30. Plot of the maxillary dental arch relationships of case 30, a female in whom the maxillary first-premolars were extracted for treatment. Squares are the dental contacts at the start of treatment; circles are the contacts at the end of treatment. The $X$ and $\mathrm{Y}$ axes are in millimeters. 


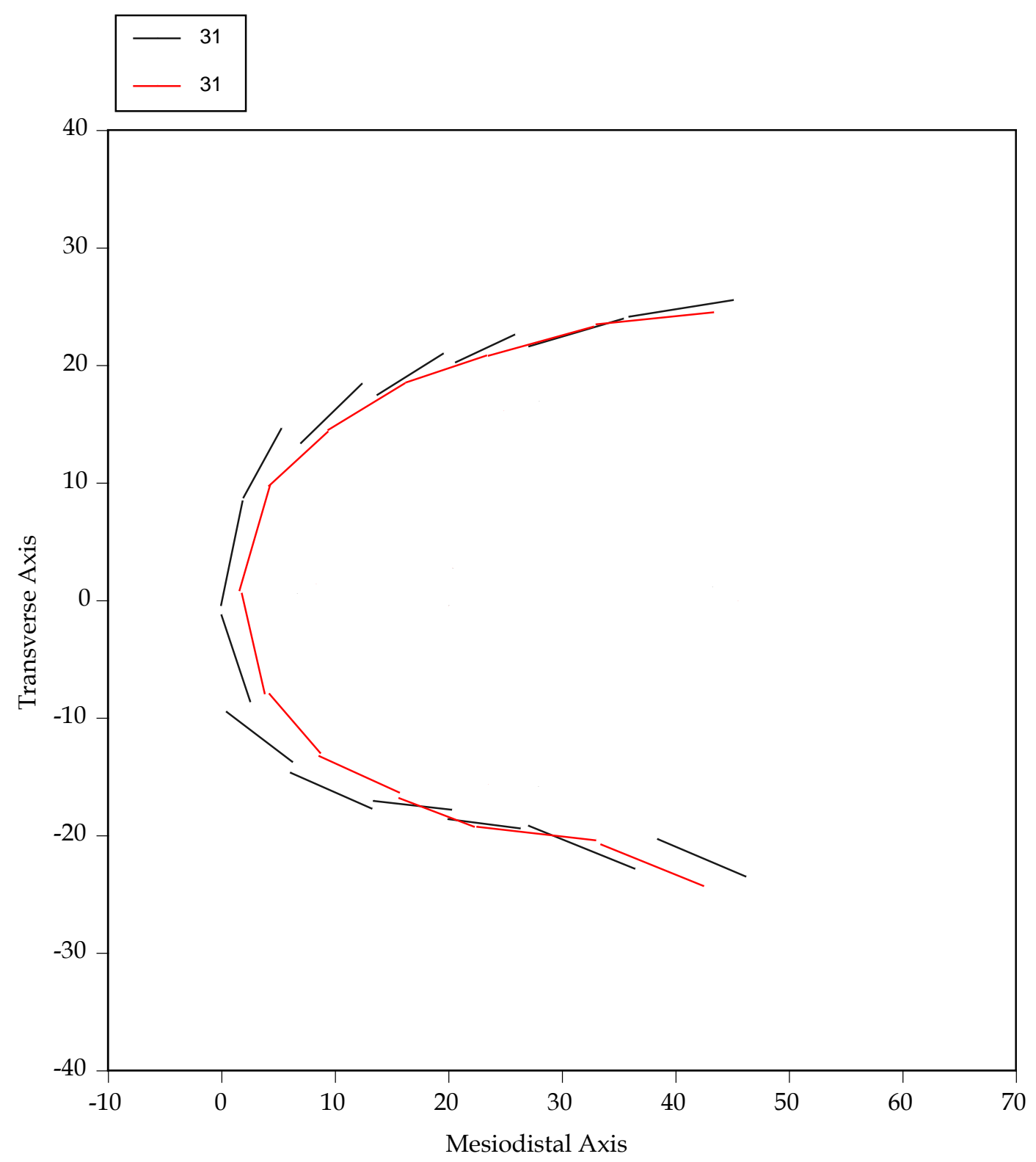

Fig. A-31. Plot of the maxillary dental arch relationships of case 31 , a female in whom the maxillary second-premolars were extracted for treatment. Squares are the dental contacts at the start of treatment; circles are the contacts at the end of treatment. The $X$ and $\mathrm{Y}$ axes are in millimeters. 


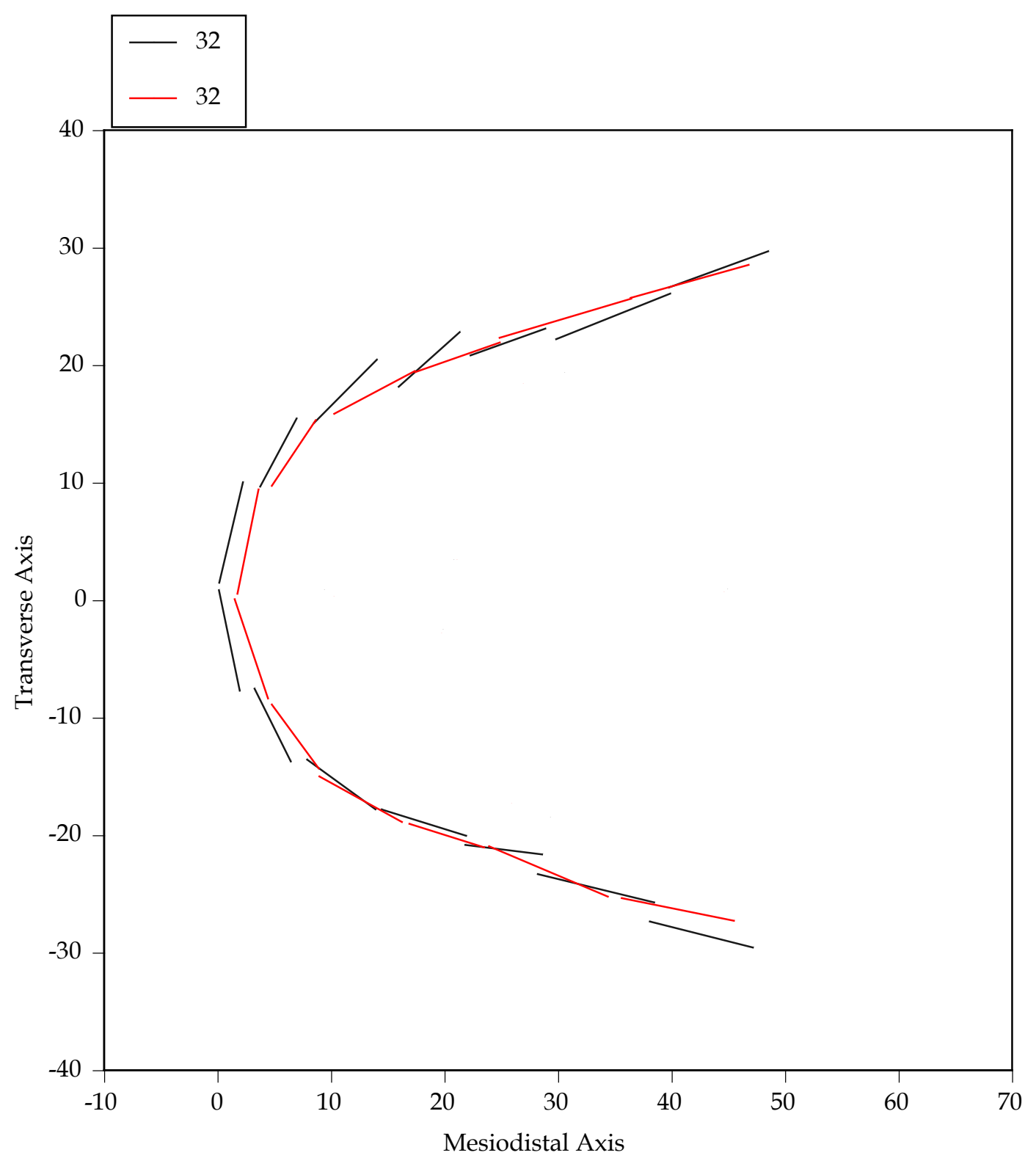

Fig. A-32. Plot of the maxillary dental arch relationships of case 32, a female in whom the maxillary first-premolars were extracted for treatment. Squares are the dental contacts at the start of treatment; circles are the contacts at the end of treatment. The $X$ and $\mathrm{Y}$ axes are in millimeters. 


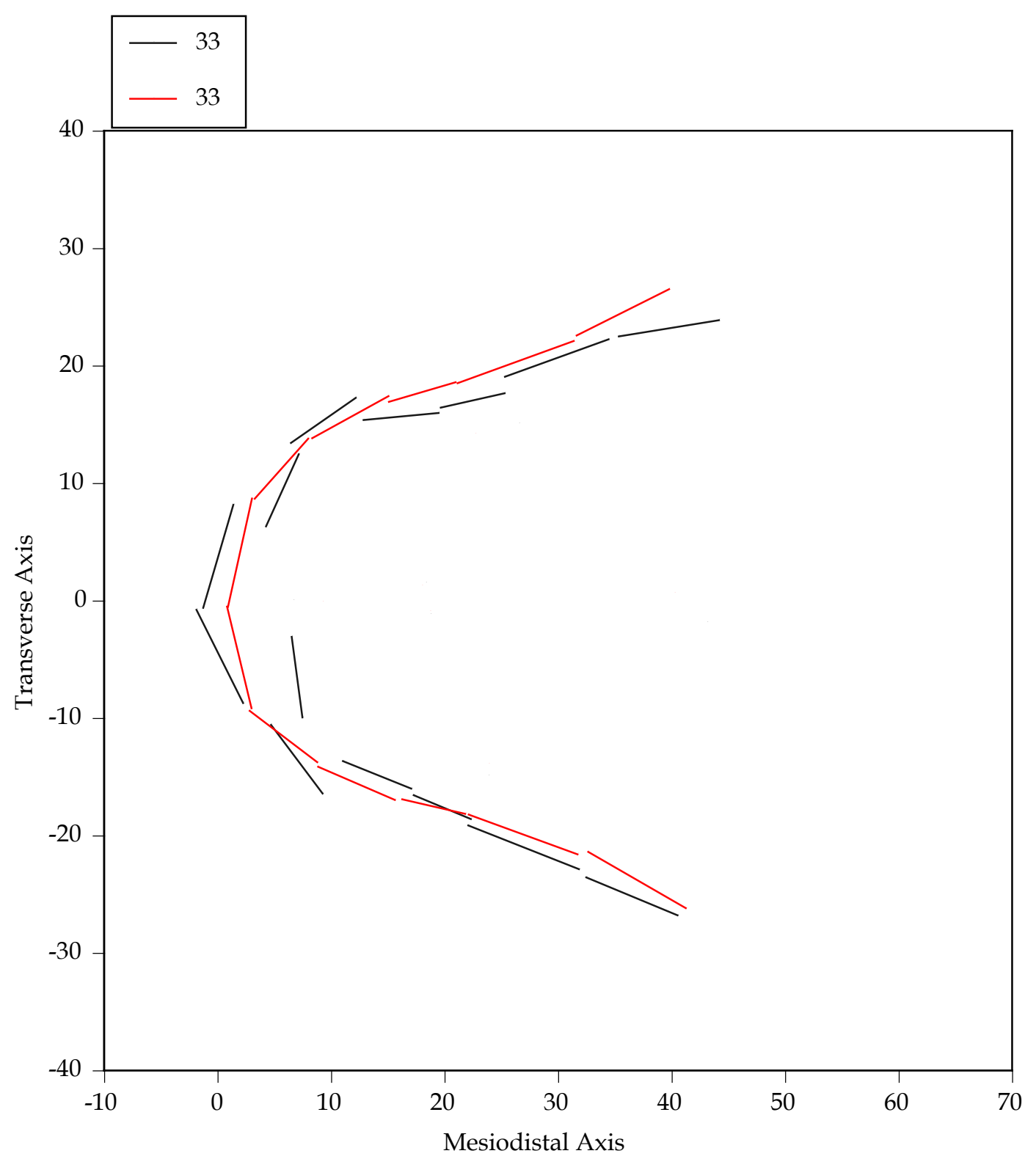

Fig. A-33. Plot of the maxillary dental arch relationships of case 33, a female in whom the maxillary first-premolars were extracted for treatment. Squares are the dental contacts at the start of treatment; circles are the contacts at the end of treatment. The $X$ and $Y$ axes are in millimeters. 


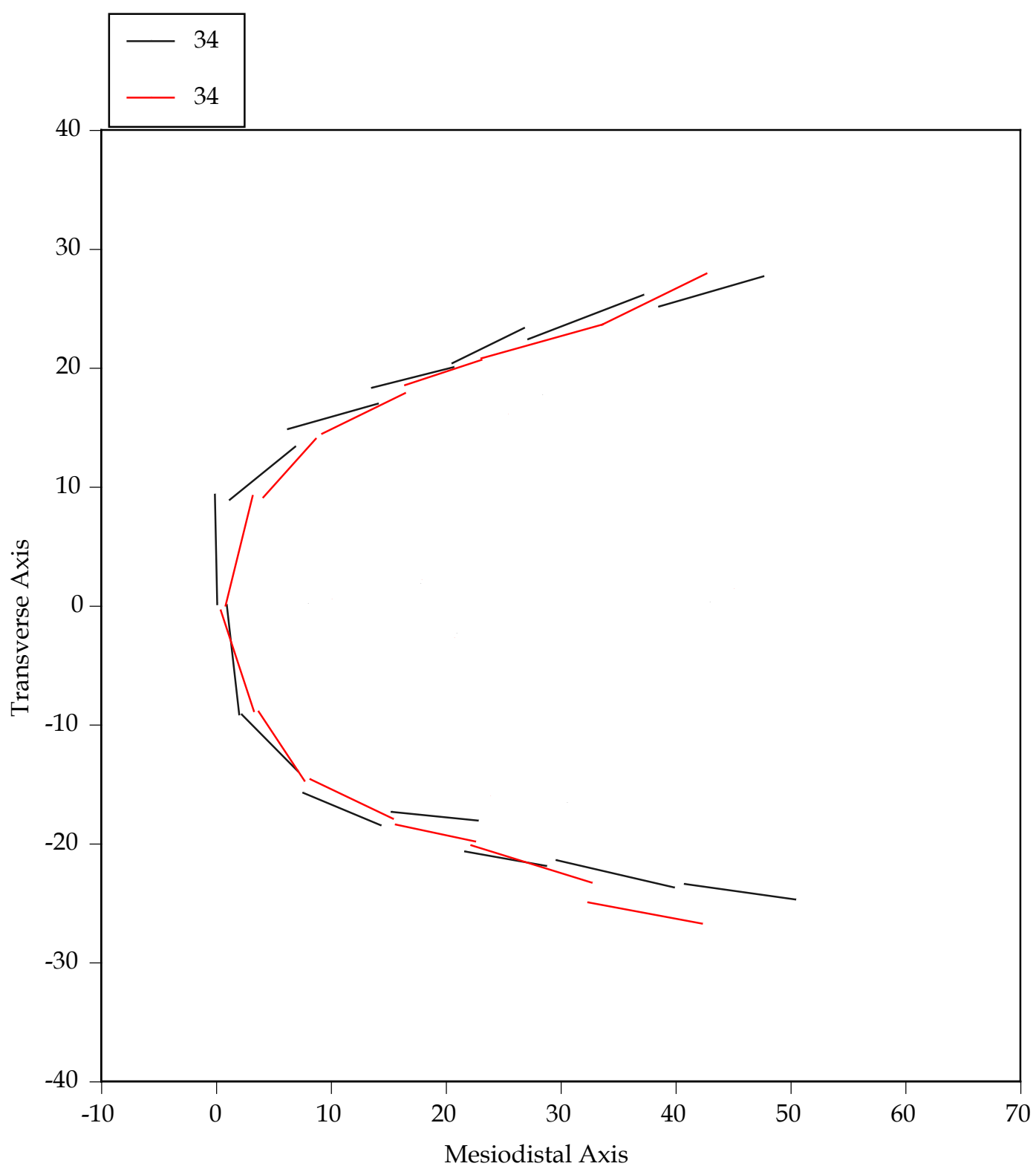

Fig. A-34. Plot of the maxillary dental arch relationships of case 34 , a female in whom the maxillary first-premolars were extracted for treatment. Squares are the dental contacts at the start of treatment; circles are the contacts at the end of treatment. The $X$ and $\mathrm{Y}$ axes are in millimeters. 


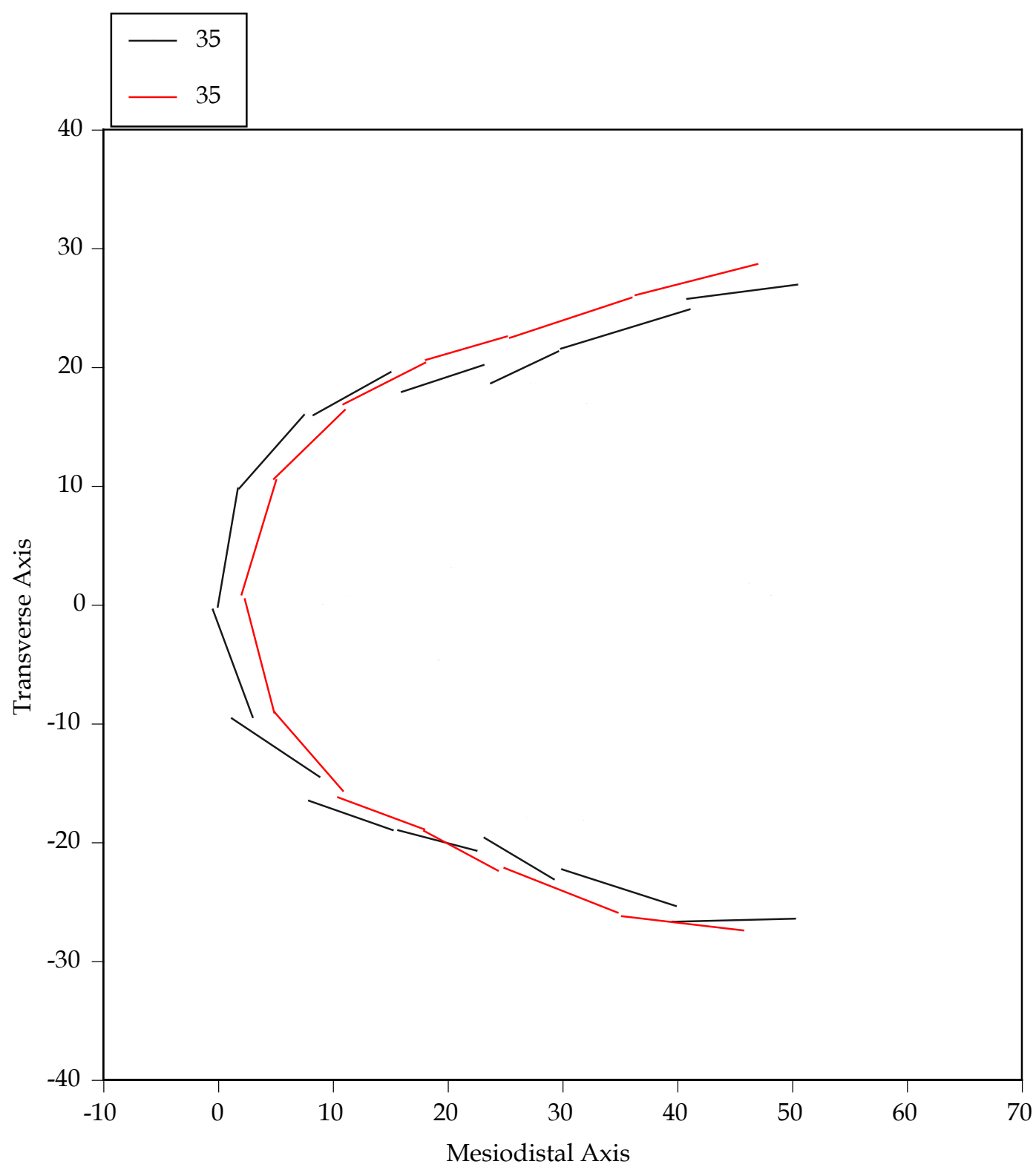

Fig. A-35. Plot of the maxillary dental arch relationships of case 35 , a female in whom the maxillary first-premolars were extracted for treatment. Squares are the dental contacts at the start of treatment; circles are the contacts at the end of treatment. The $\mathrm{X}$ and $\mathrm{Y}$ axes are in millimeters. 


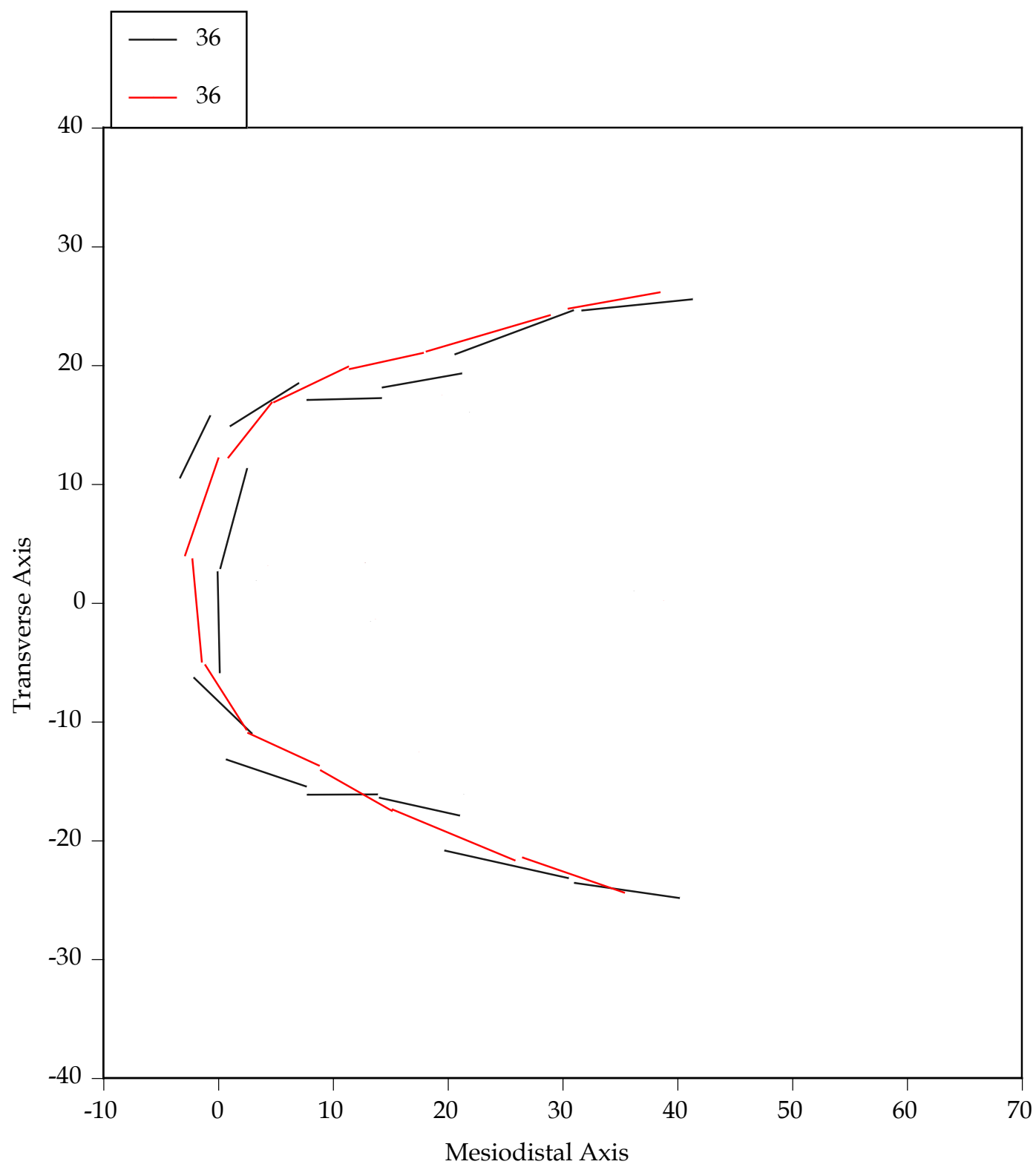

Fig. A-36. Plot of the maxillary dental arch relationships of case 36, a male in whom the maxillary second-premolars were extracted for treatment. Squares are the dental contacts at the start of treatment; circles are the contacts at the end of treatment. The $X$ and $\mathrm{Y}$ axes are in millimeters. 


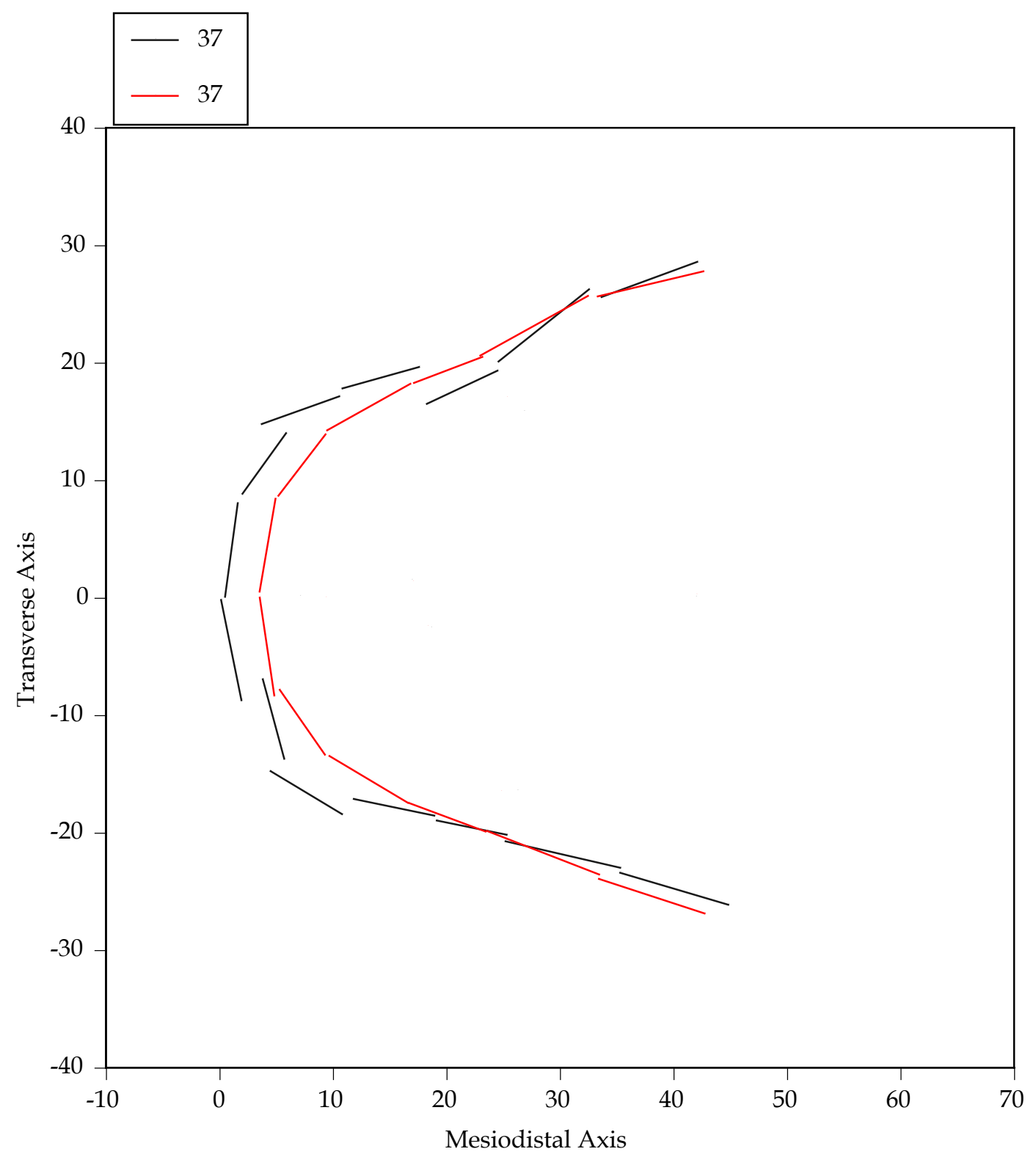

Fig. A-37. Plot of the maxillary dental arch relationships of case 37, a female in whom the maxillary first-premolars were extracted for treatment. Squares are the dental contacts at the start of treatment; circles are the contacts at the end of treatment. The $\mathrm{X}$ and $\mathrm{Y}$ axes are in millimeters. 


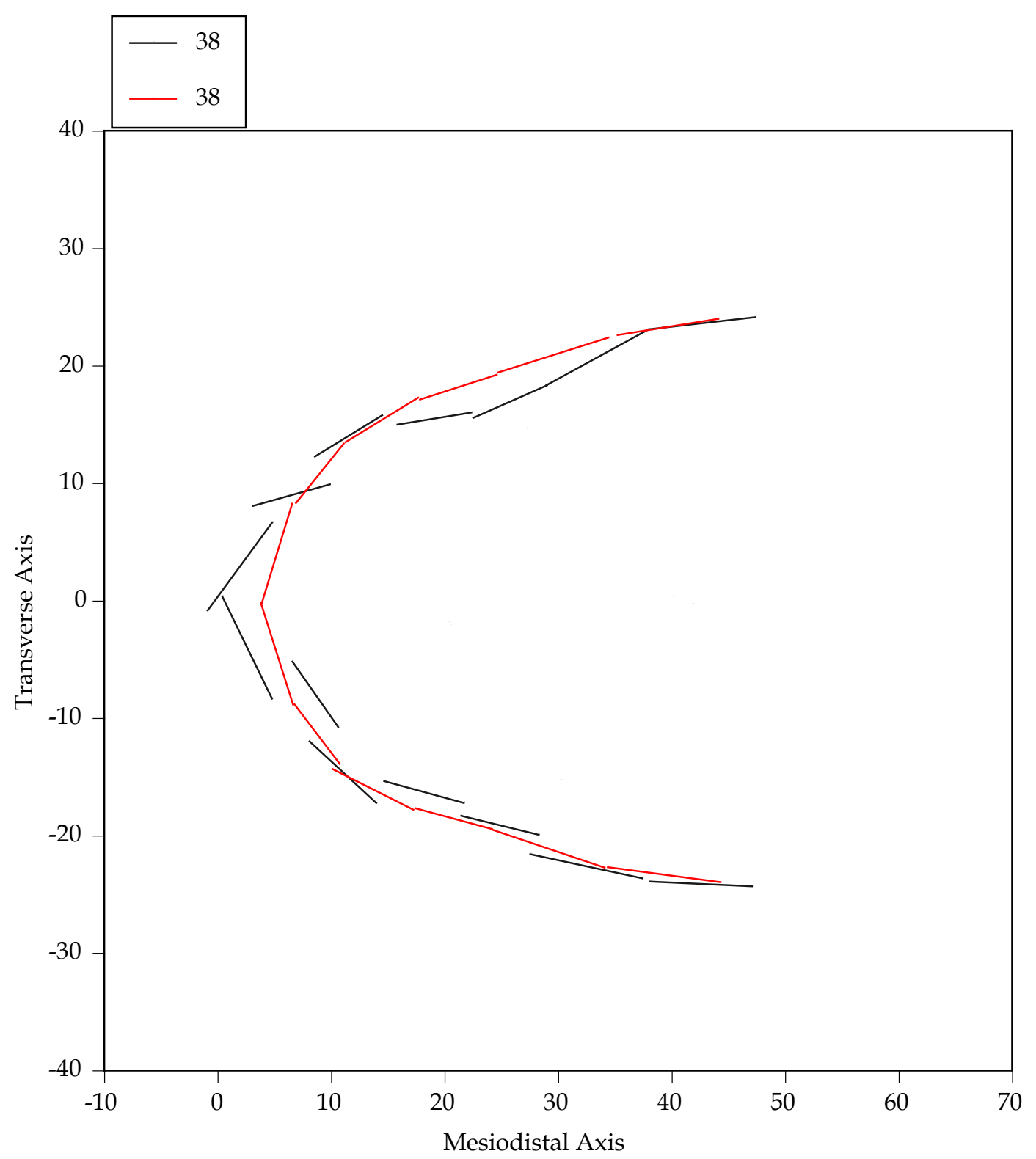

Fig. A-38. Plot of the maxillary dental arch relationships of case 38 , a female in whom the maxillary first-premolars were extracted for treatment. Squares are the dental contacts at the start of treatment; circles are the contacts at the end of treatment. The $X$ and $\mathrm{Y}$ axes are in millimeters. 


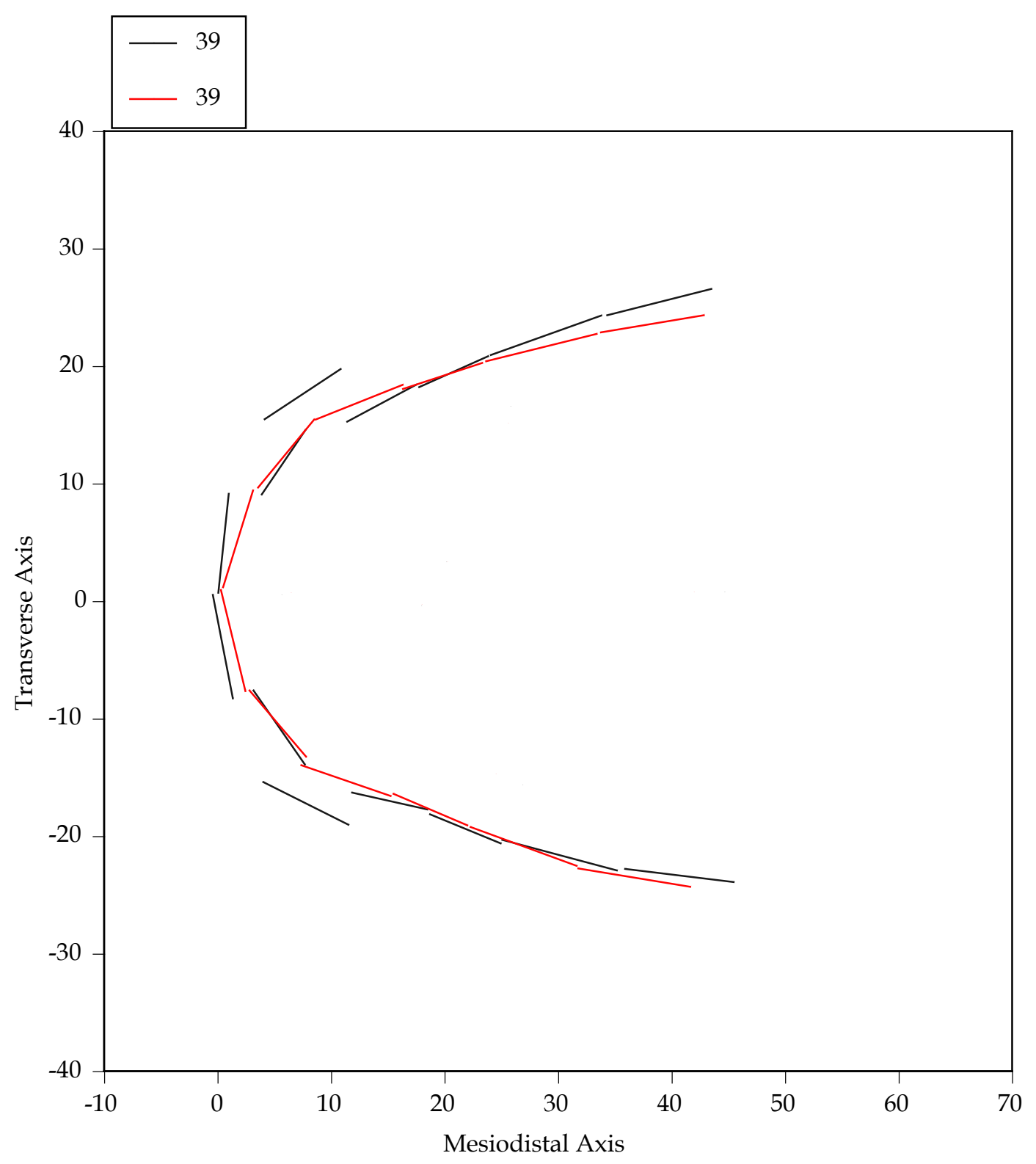

Fig. A-39. Plot of the maxillary dental arch relationships of case 39 , a female in whom the maxillary first-premolars were extracted for treatment. Squares are the dental contacts at the start of treatment; circles are the contacts at the end of treatment. The $\mathrm{X}$ and $\mathrm{Y}$ axes are in millimeters. 


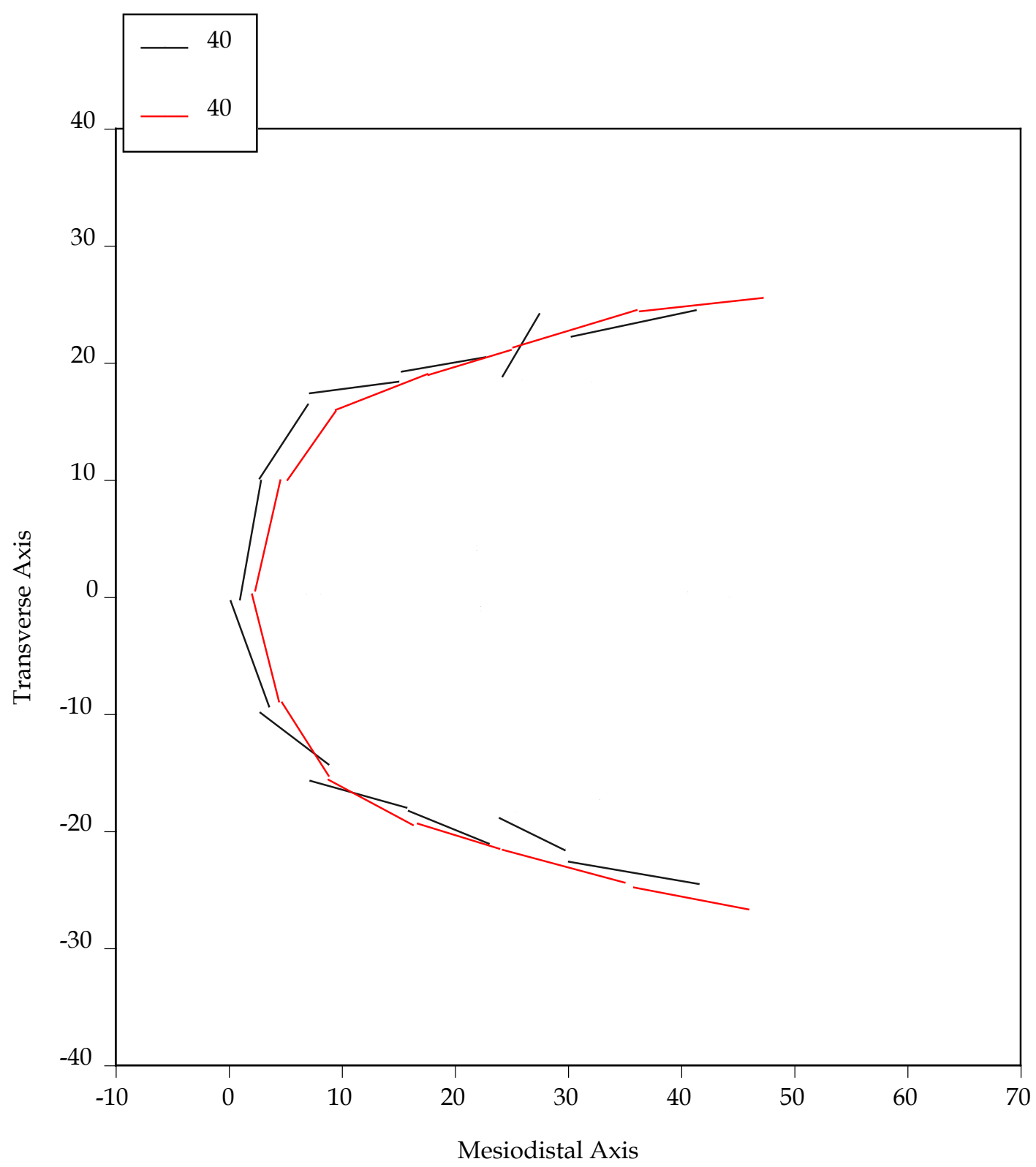

Fig. A-40. Plot of the maxillary dental arch relationships of case 40 , a male in whom the maxillary second-premolars were extracted for treatment. Squares are the dental contacts of at the start of treatment; circles are the contacts at the end of treatment. The $\mathrm{X}$ and $\mathrm{Y}$ axes are in millimeters. 


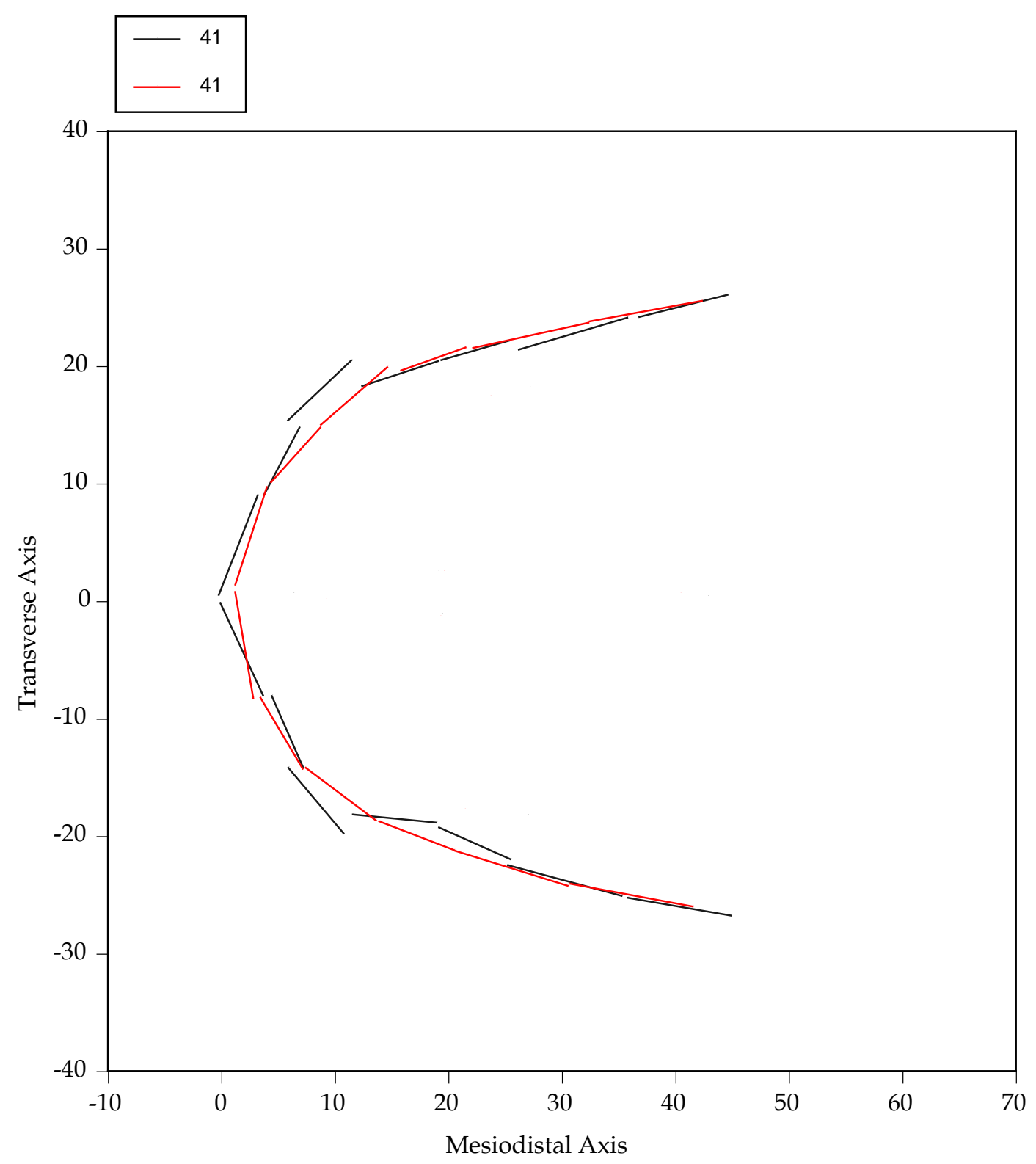

Fig. A-41. Plot of the maxillary dental arch relationships of case 41, a female in whom the maxillary second-premolars were extracted for treatment. Squares are the dental contacts at the start of treatment; circles are the contacts at the end of treatment. The $X$ and $\mathrm{Y}$ axes are in millimeters. 


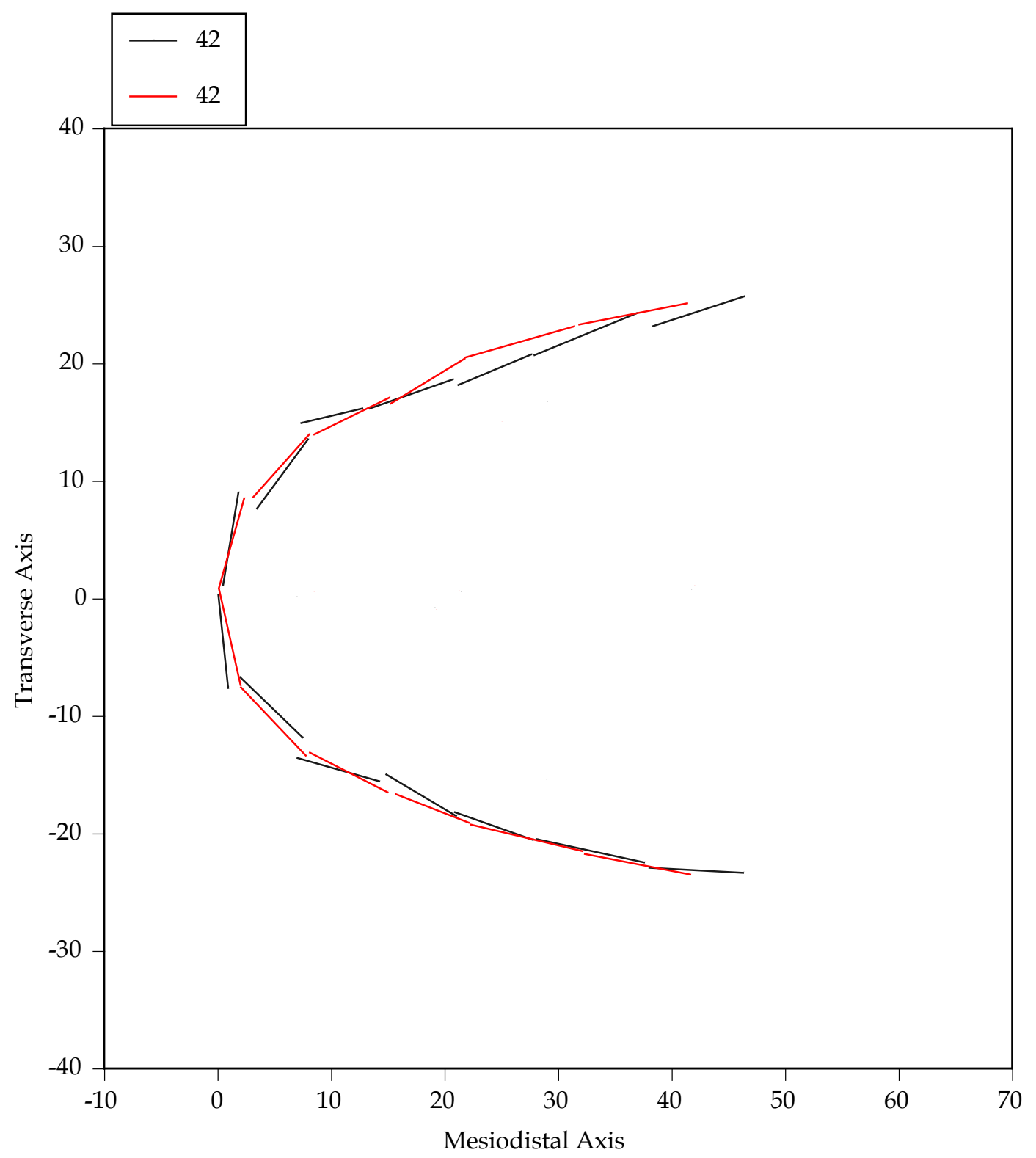

Fig. A-42. Plot of the maxillary dental arch relationships of case 42 , a male in whom the maxillary first-premolars were extracted for treatment. Squares are the dental contacts at the start of treatment; circles are the contacts at the end of treatment. The $X$ and $\mathrm{Y}$ axes are in millimeters. 


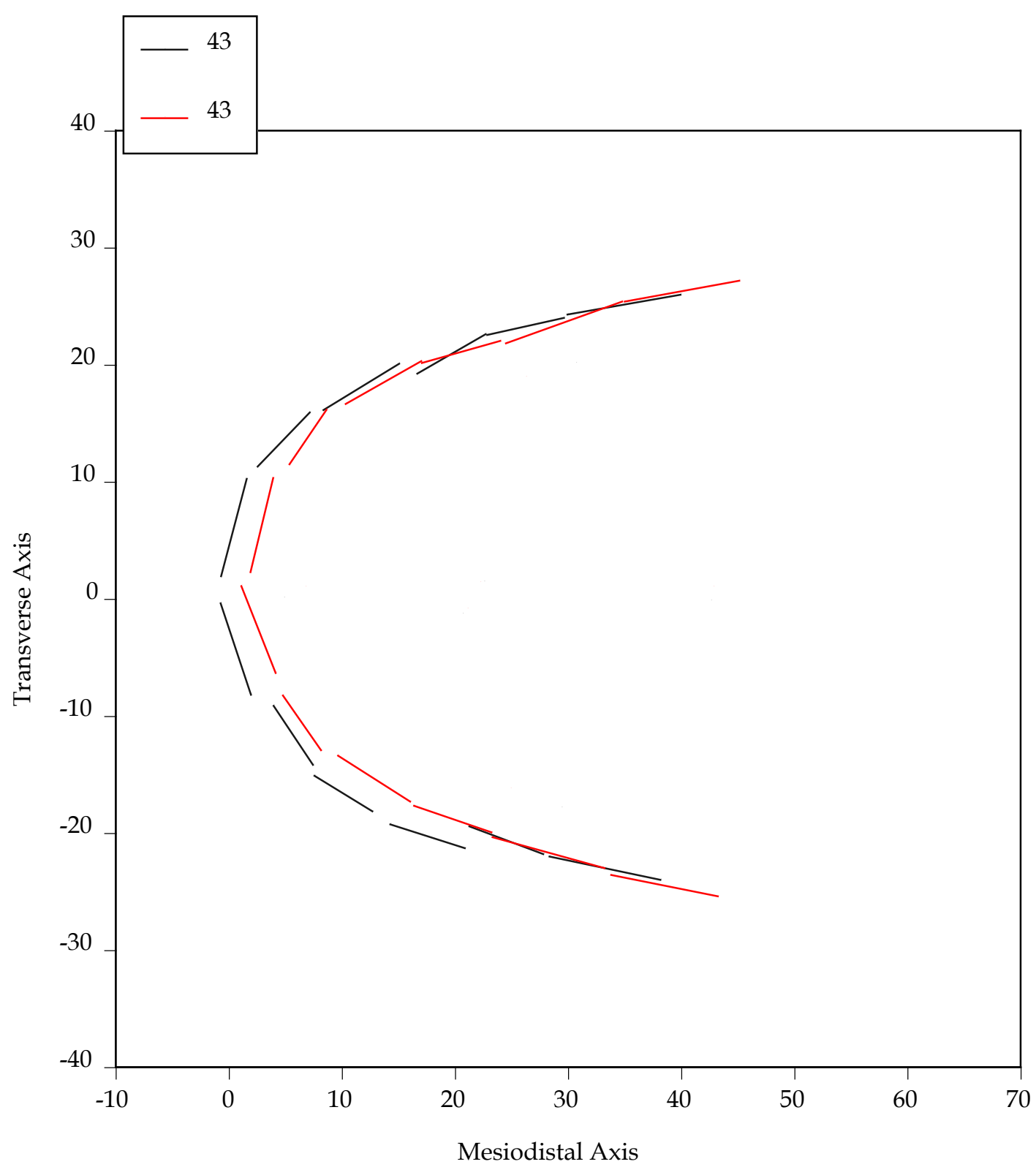

Fig. A-43. Plot of the maxillary dental arch relationships of case 43, a female in whom the maxillary second-premolars were extracted for treatment. Squares are the dental contacts at the start of treatment; circles are the contacts at the end of treatment. The $X$ and $Y$ axes are in millimeters. 


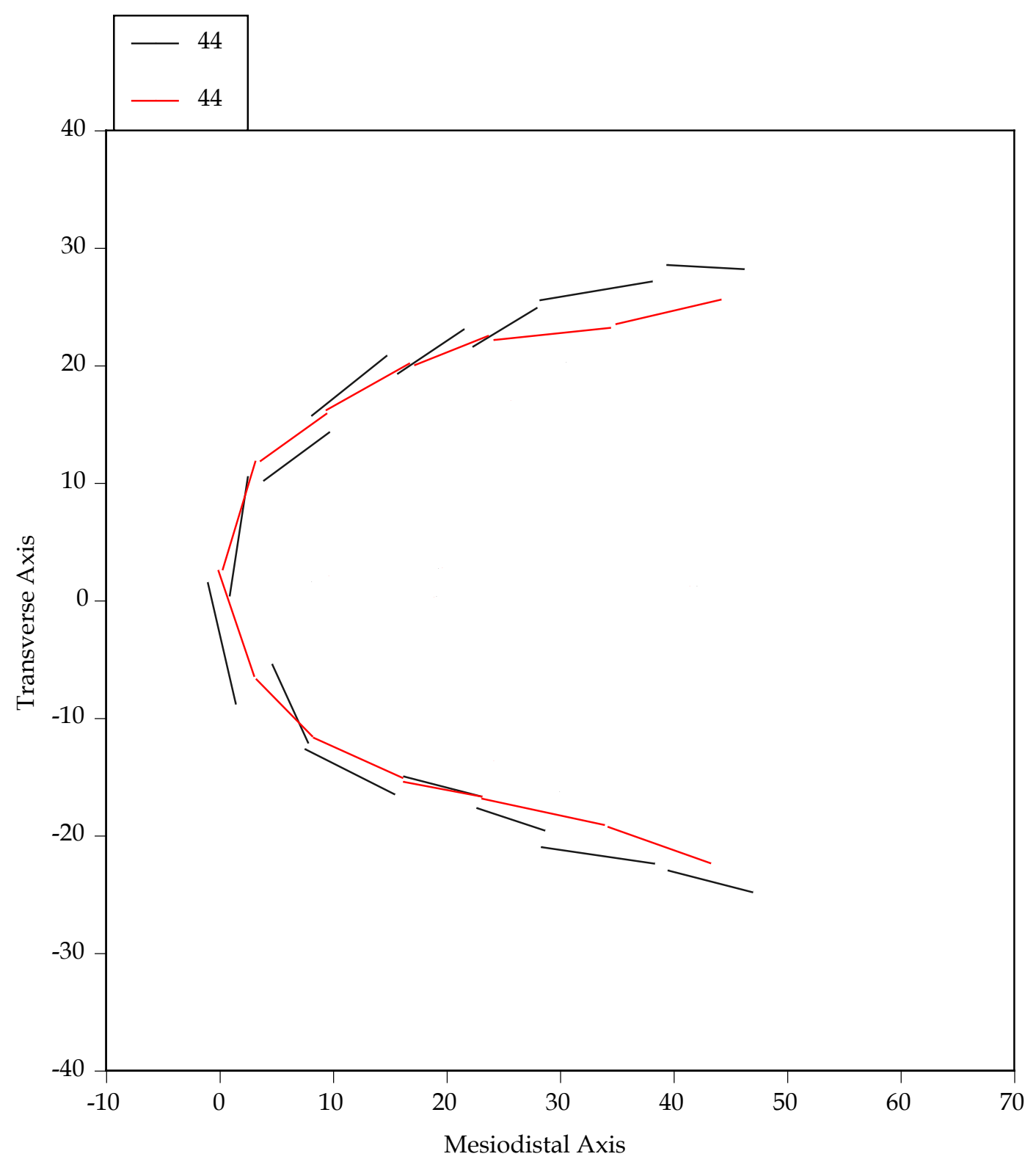

Fig. A-44. Plot of the maxillary dental arch relationships of case 44 , a male in whom the maxillary second-premolars were extracted for treatment. Squares are the dental contacts at the start of treatment; circles are the contacts at the end of treatment. The $\mathrm{X}$ and $\mathrm{Y}$ axes are in millimeters. 


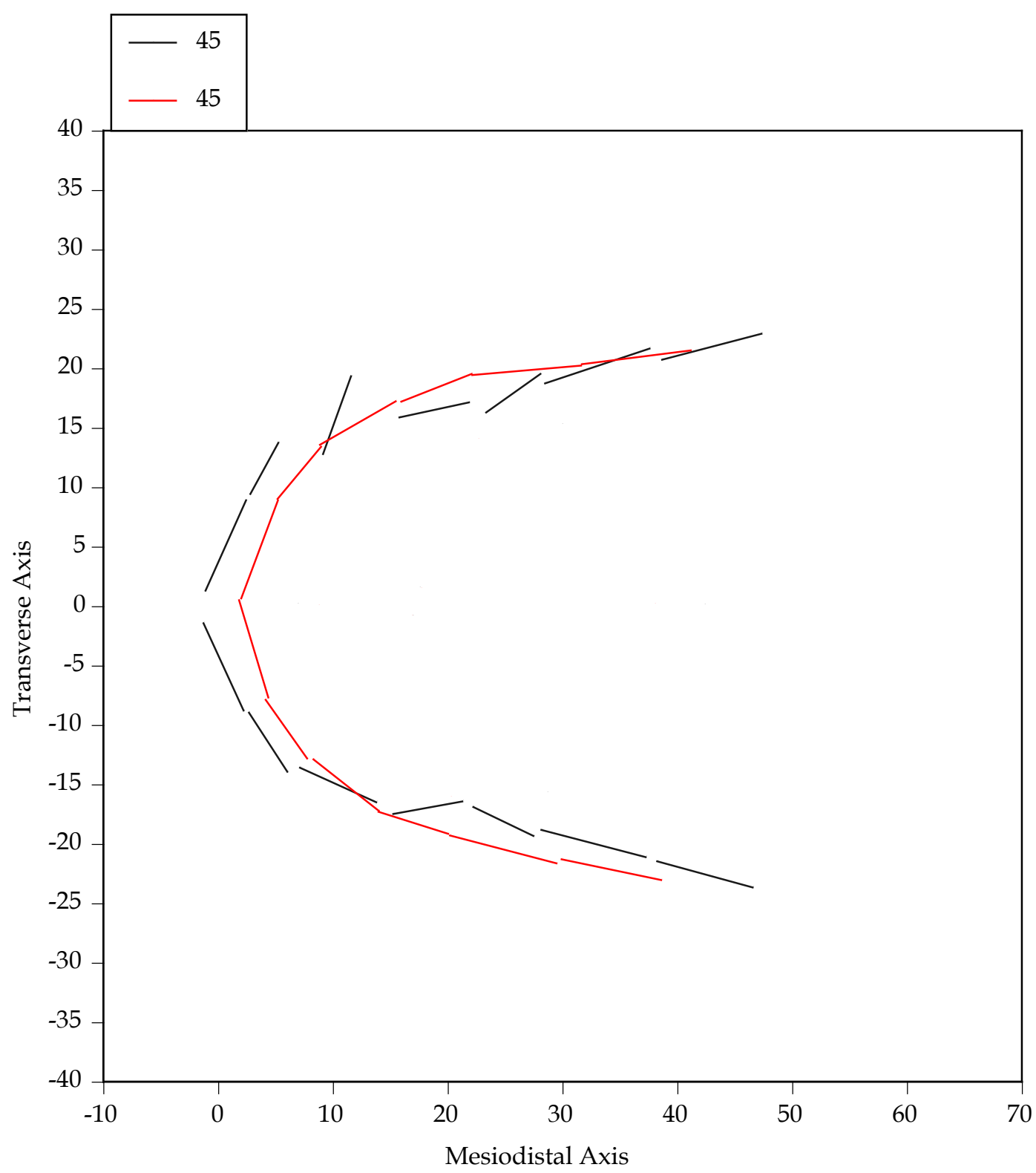

Fig. A-45. Plot of the maxillary dental arch relationships of case 45 , a female in whom the maxillary second-premolars were extracted for treatment. Squares are the dental contacts at the start of treatment; circles are the contacts at the end of treatment. The $X$ and $\mathrm{Y}$ axes are in millimeters. 


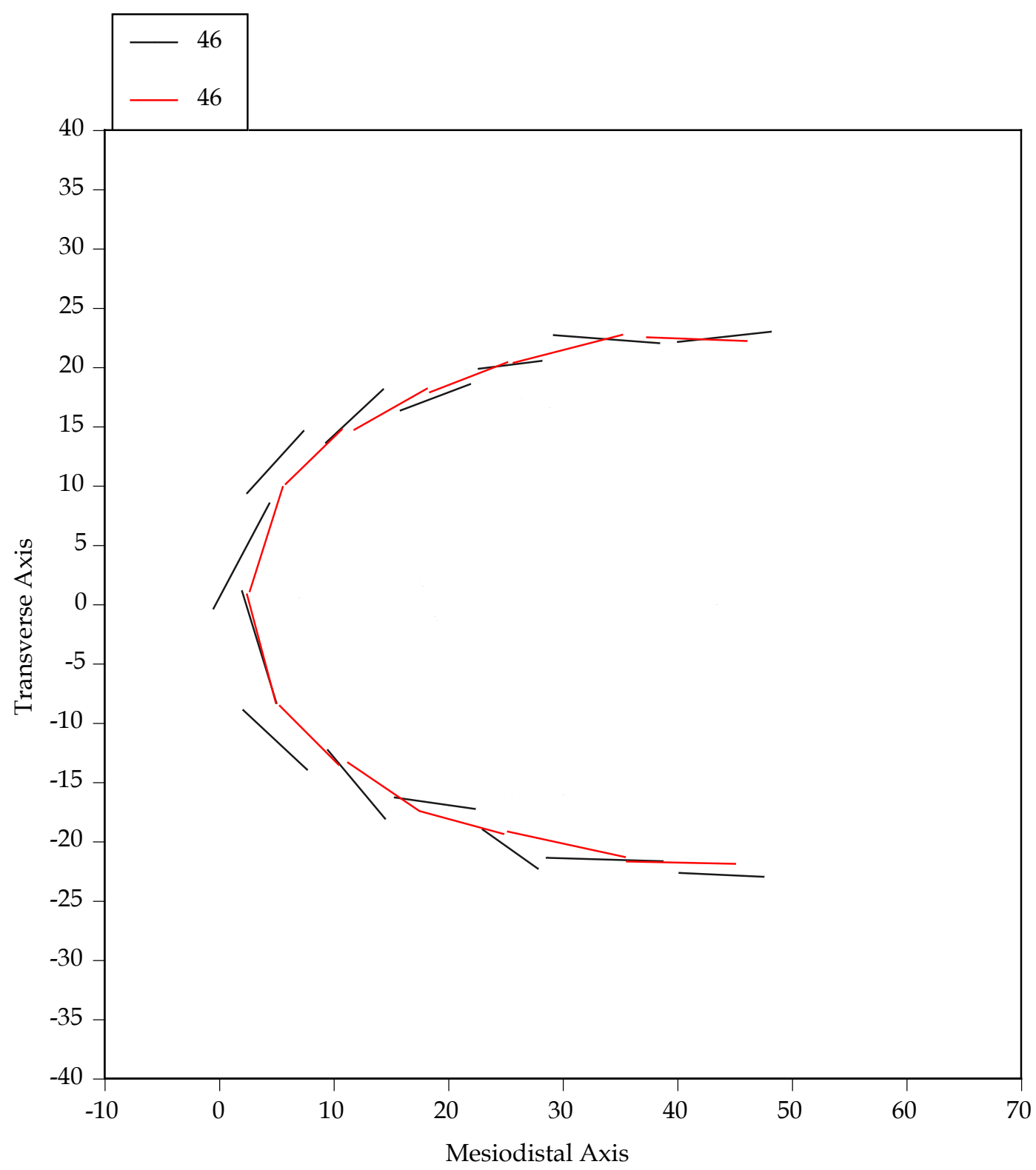

Fig. A-46. Plot of the maxillary dental arch relationships of case 46 , a female in whom the maxillary second-premolars were extracted for treatment. Squares are the dental contacts at the start of treatment; circles are the contacts at the end of treatment. The $X$ and $\mathrm{Y}$ axes are in millimeters. 


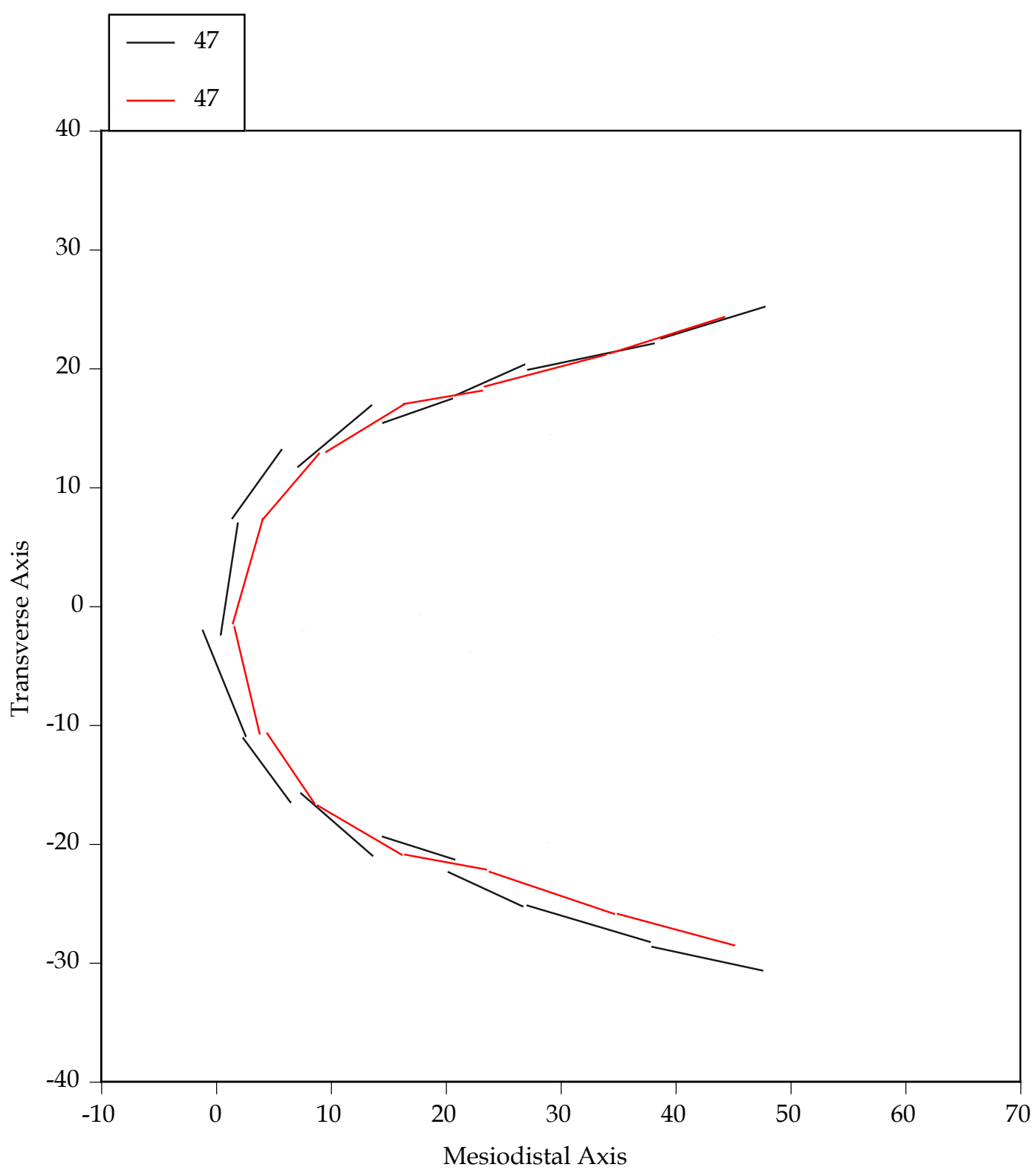

Fig. A-47. Plot of the maxillary dental arch relationships of case 47 , a female in whom the maxillary second-premolars were extracted for treatment. Squares are the dental contacts at the start of treatment; circles are the contacts at the end of treatment. The $\mathrm{X}$ and $\mathrm{Y}$ axes are in millimeters. 


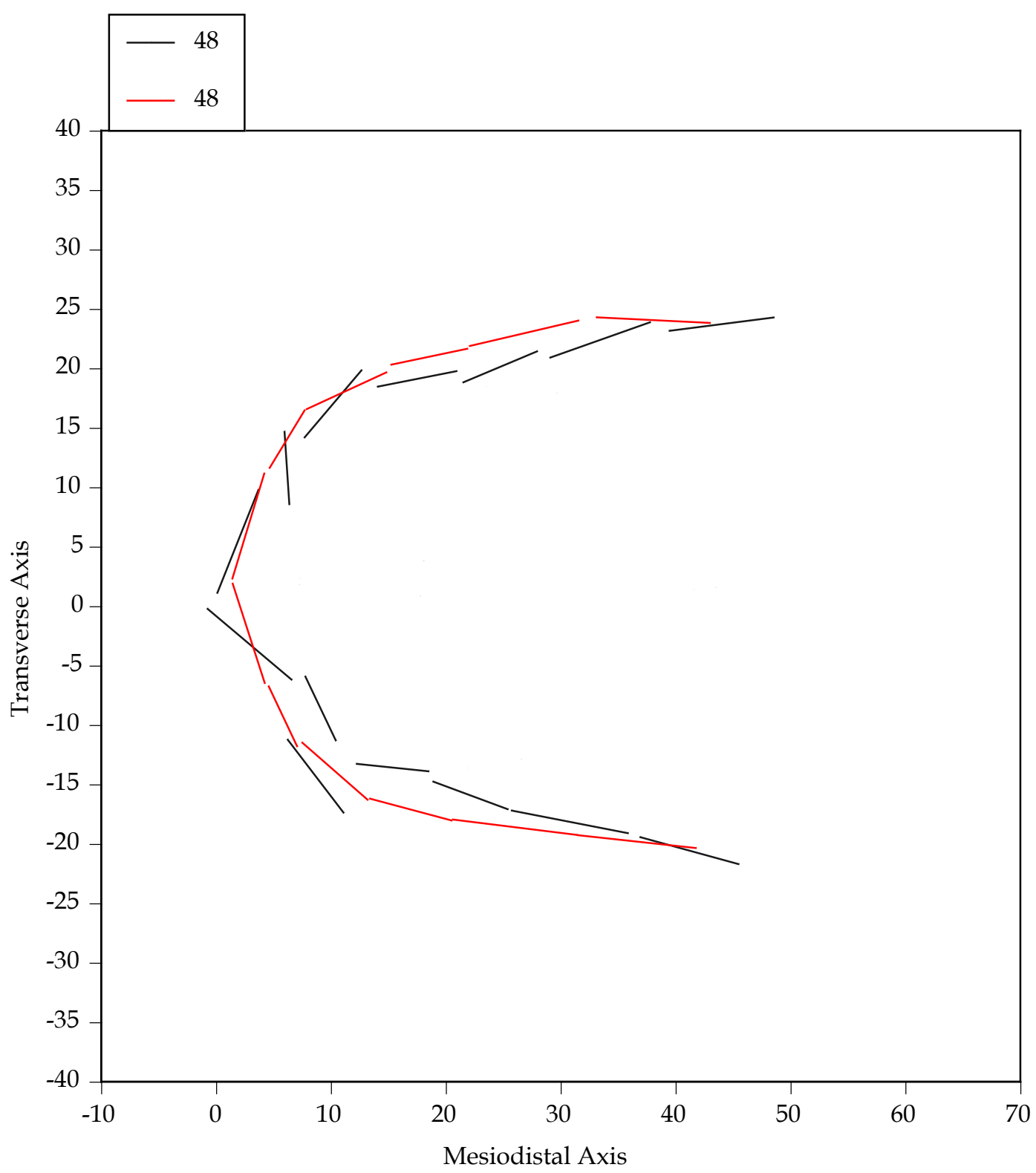

Fig. A-48. Plot of the maxillary dental arch relationships of case 48 , a female in whom the maxillary second-premolars were extracted for treatment. Squares are the dental contacts at the start of treatment; circles are the contacts at the end of treatment. The $X$ and $Y$ axes are in millimeters. 


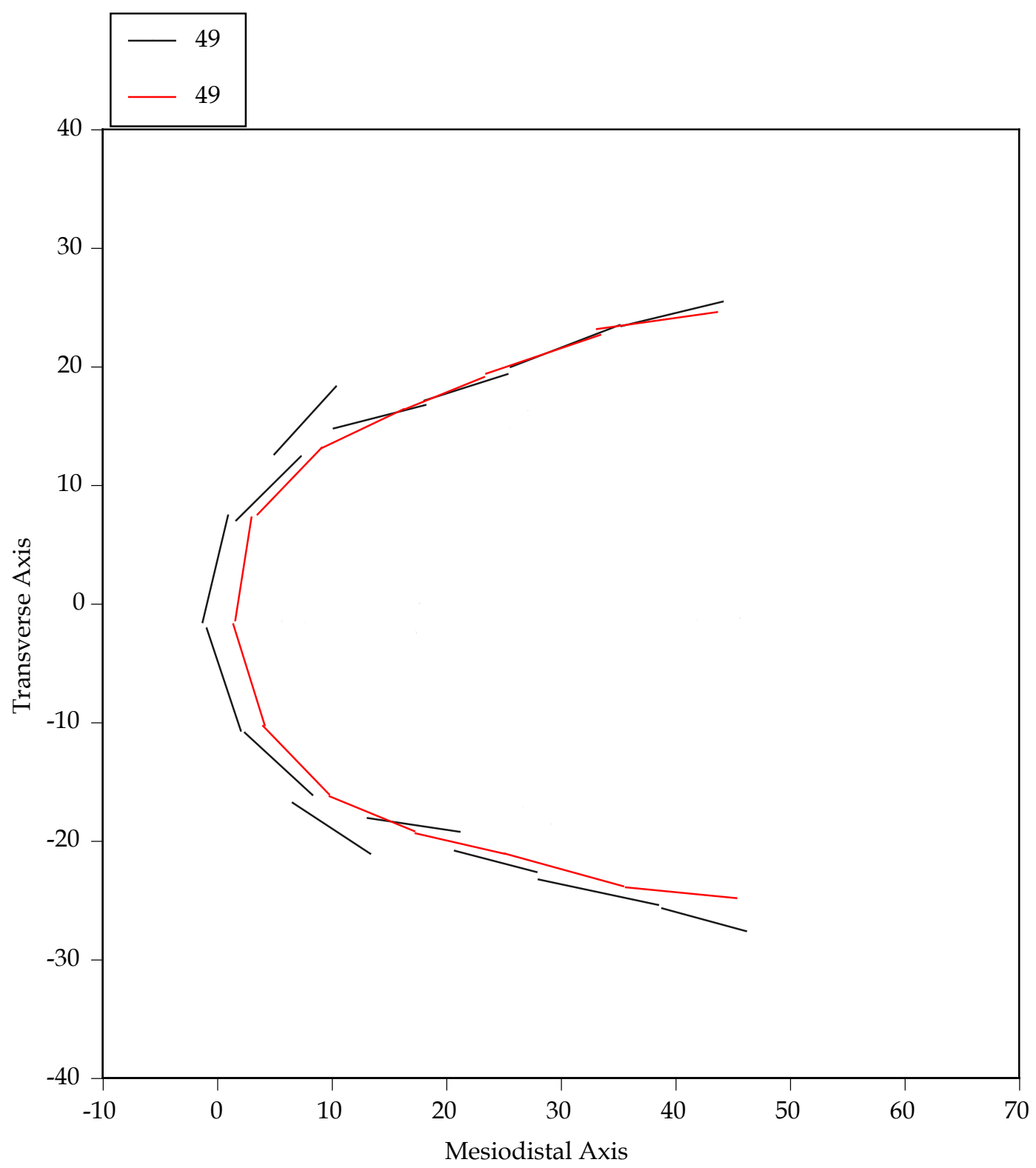

Fig. A-49. Plot of the maxillary dental arch relationships of case 49 , a female in whom the maxillary first-premolars were extracted for treatment. Squares are the dental contacts at the start of treatment; circles are the contacts at the end of treatment. The $X$ and $\mathrm{Y}$ axes are in millimeters. 


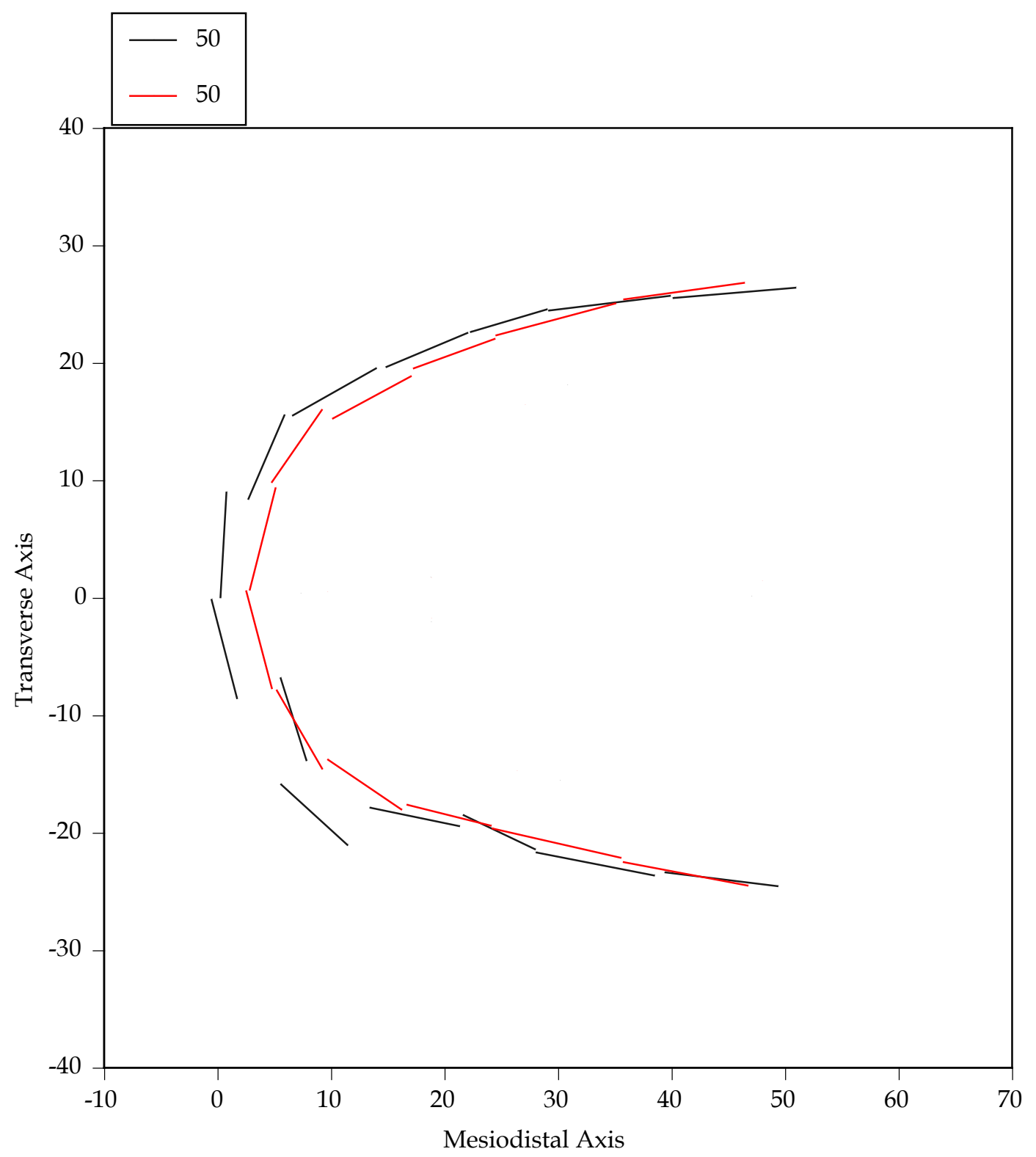

Fig. A-50. Plot of the maxillary dental arch relationships of case 50 , a male in whom the maxillary first-premolars were extracted for treatment. Squares are the dental contacts at the start of treatment; circles are the contacts at the end of treatment. The $X$ and $\mathrm{Y}$ axes are in millimeters. 


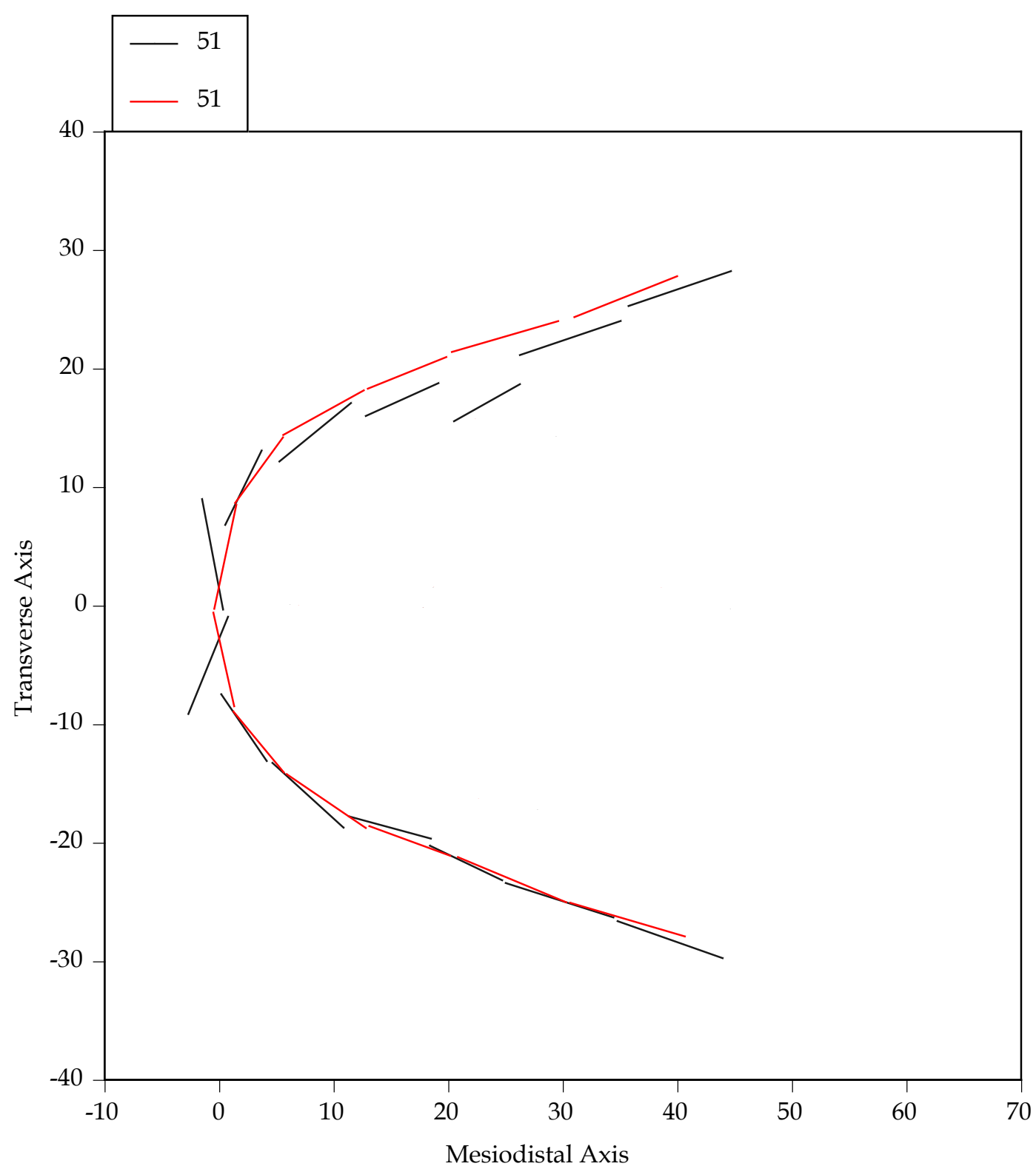

Fig. A-51. Plot of the maxillary dental arch relationships of case 51, a male in whom the maxillary second-premolars were extracted for treatment. Squares are the dental contacts at the start of treatment; circles are the contacts at the end of treatment. The $X$ and $\mathrm{Y}$ axes are in millimeters. 


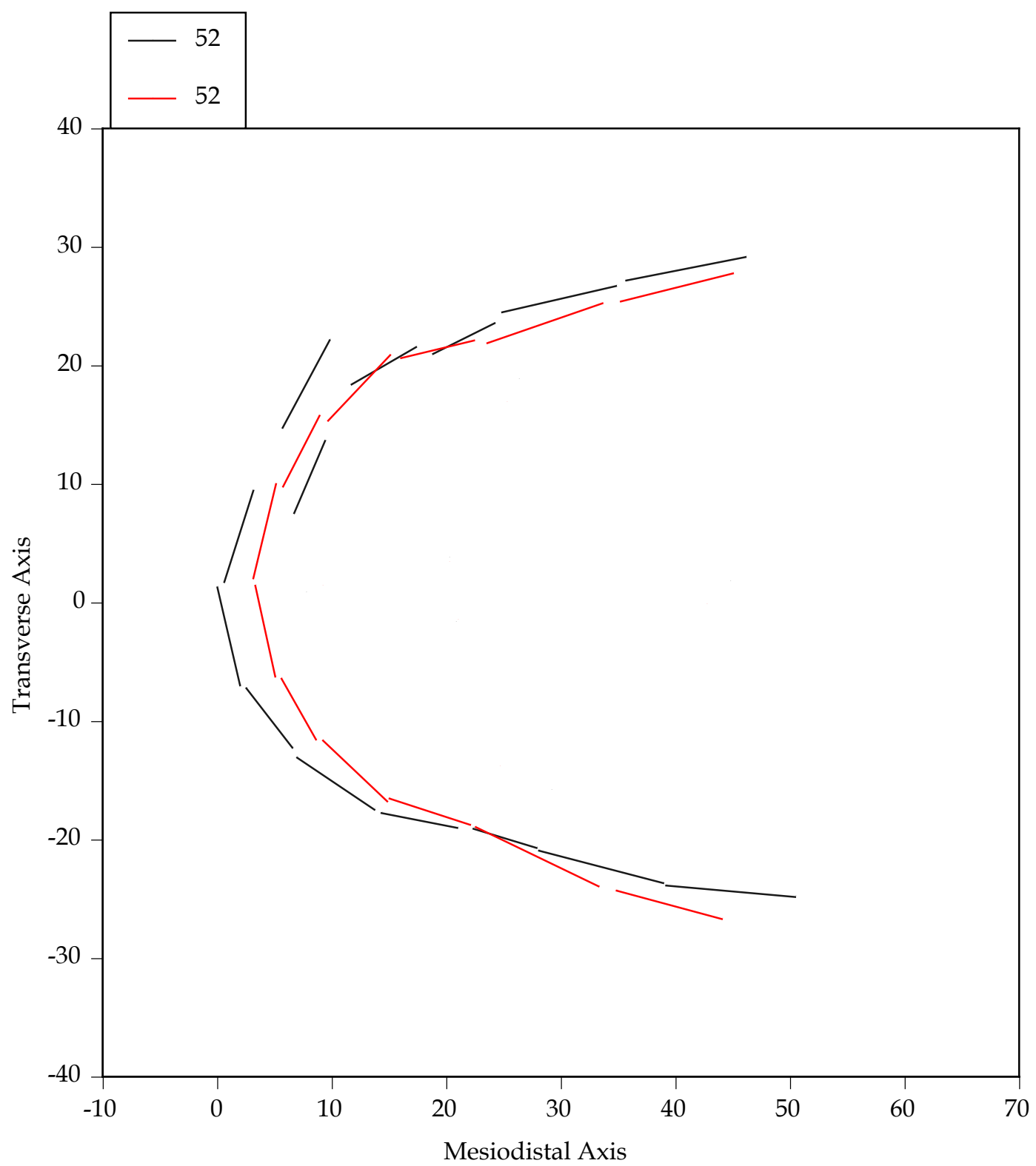

Fig. A-52. Plot of the maxillary dental arch relationships of case 52 , a male in whom the maxillary second-premolars were extracted for treatment. Squares are the dental contacts of at the start of treatment; circles are the contacts at the end of treatment. The $\mathrm{X}$ and $\mathrm{Y}$ axes are in millimeters. 


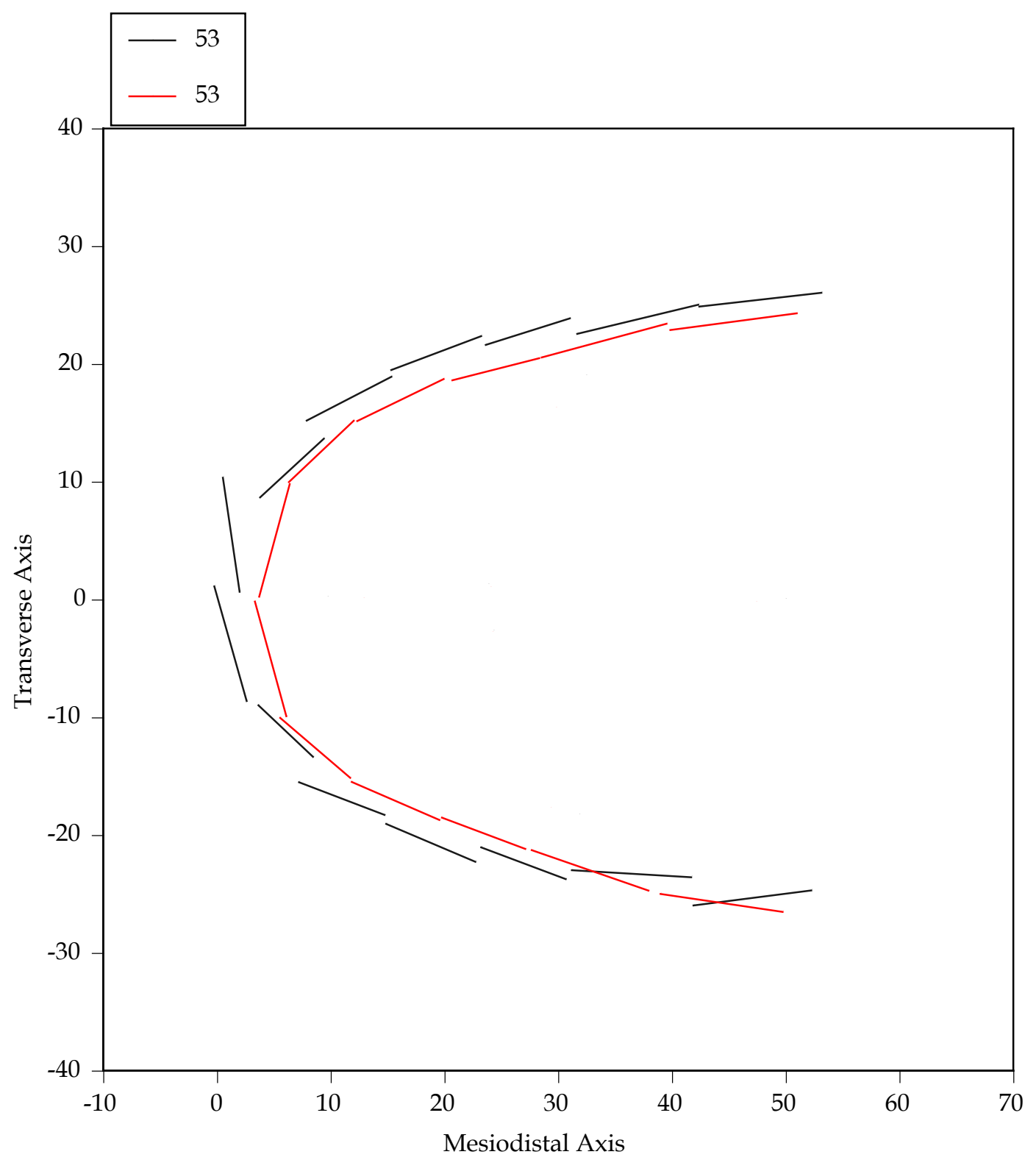

Fig. A-53. Plot of the maxillary dental arch relationships of case 53, a female in whom the maxillary first-premolars were extracted for treatment. Squares are the dental contacts at the start of treatment; circles are the contacts at the end of treatment. The $\mathrm{X}$ and $Y$ axes are in millimeters. 


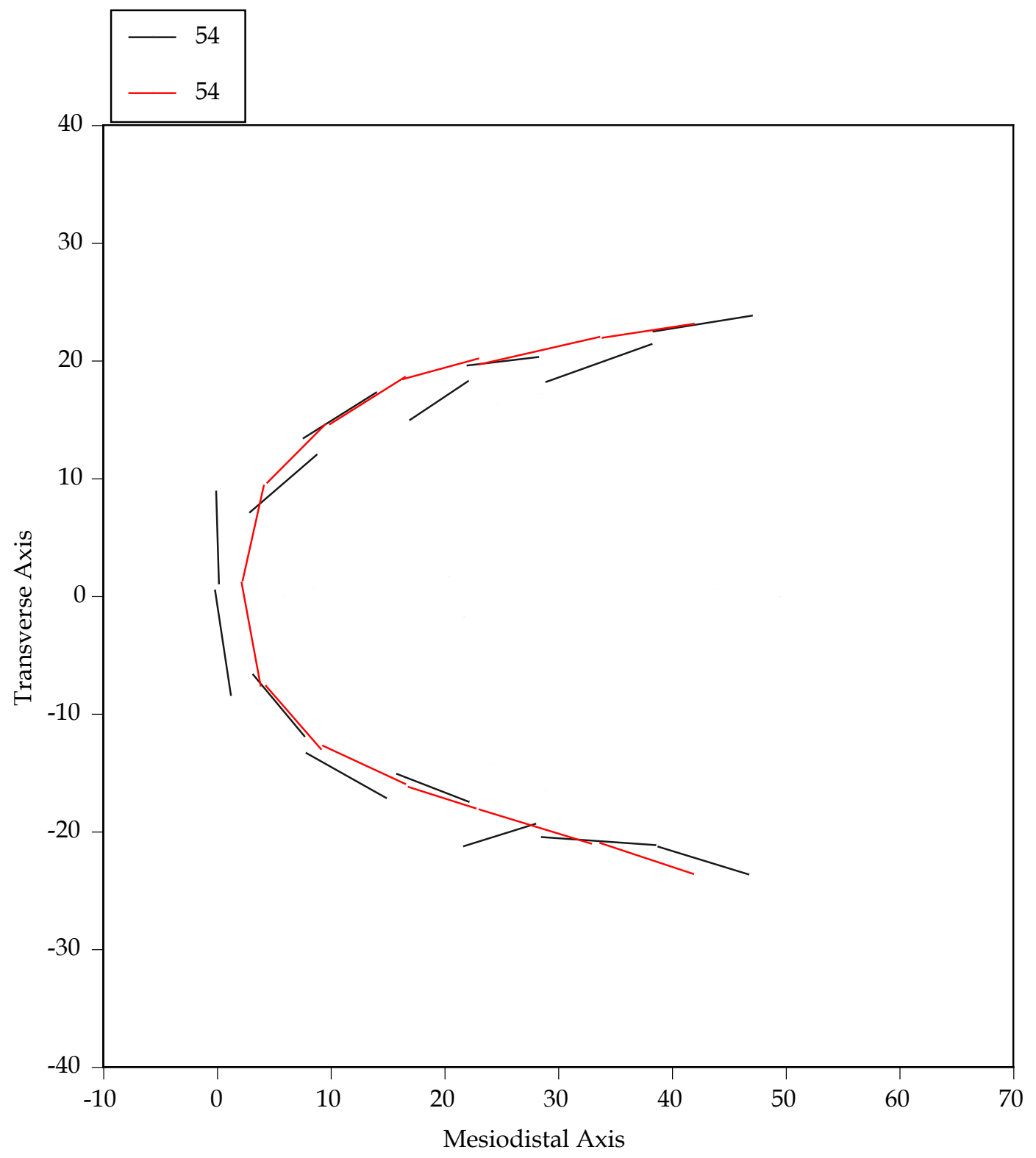

Fig. A-54. Plot of the maxillary dental arch relationships of case 54, a female in whom the maxillary first-premolars were extracted for treatment. Squares are the dental contacts at the start of treatment; circles are the contacts at the end of treatment. The $\mathrm{X}$ and $\mathrm{Y}$ axes are in millimeters. 


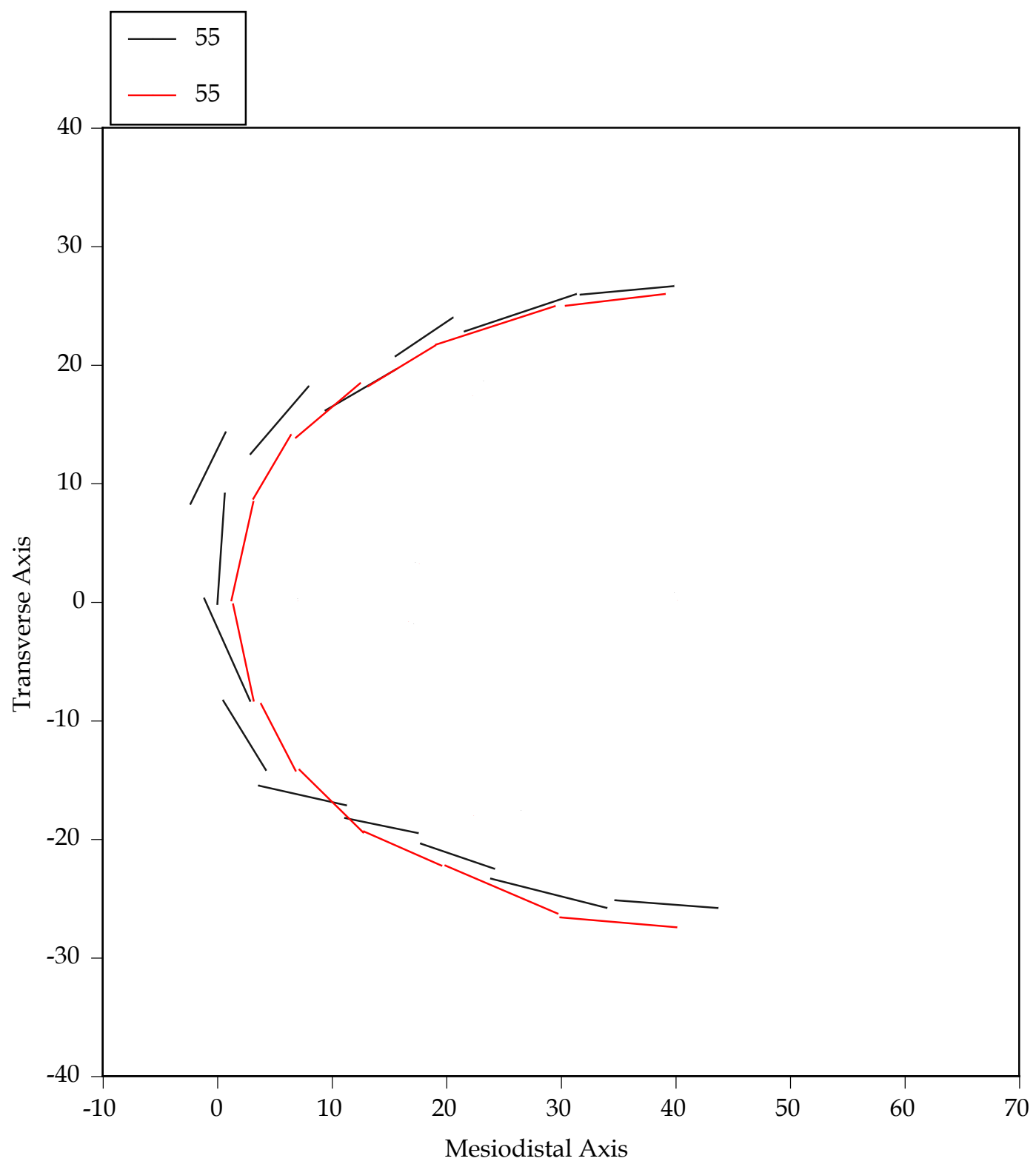

Fig. A-55. Plot of the maxillary dental arch relationships of case 55 , a male in whom the maxillary first-premolars were extracted for treatment. Squares are the dental contacts at the start of treatment; circles are the contacts at the end of treatment. The $X$ and $\mathrm{Y}$ axes are in millimeters. 


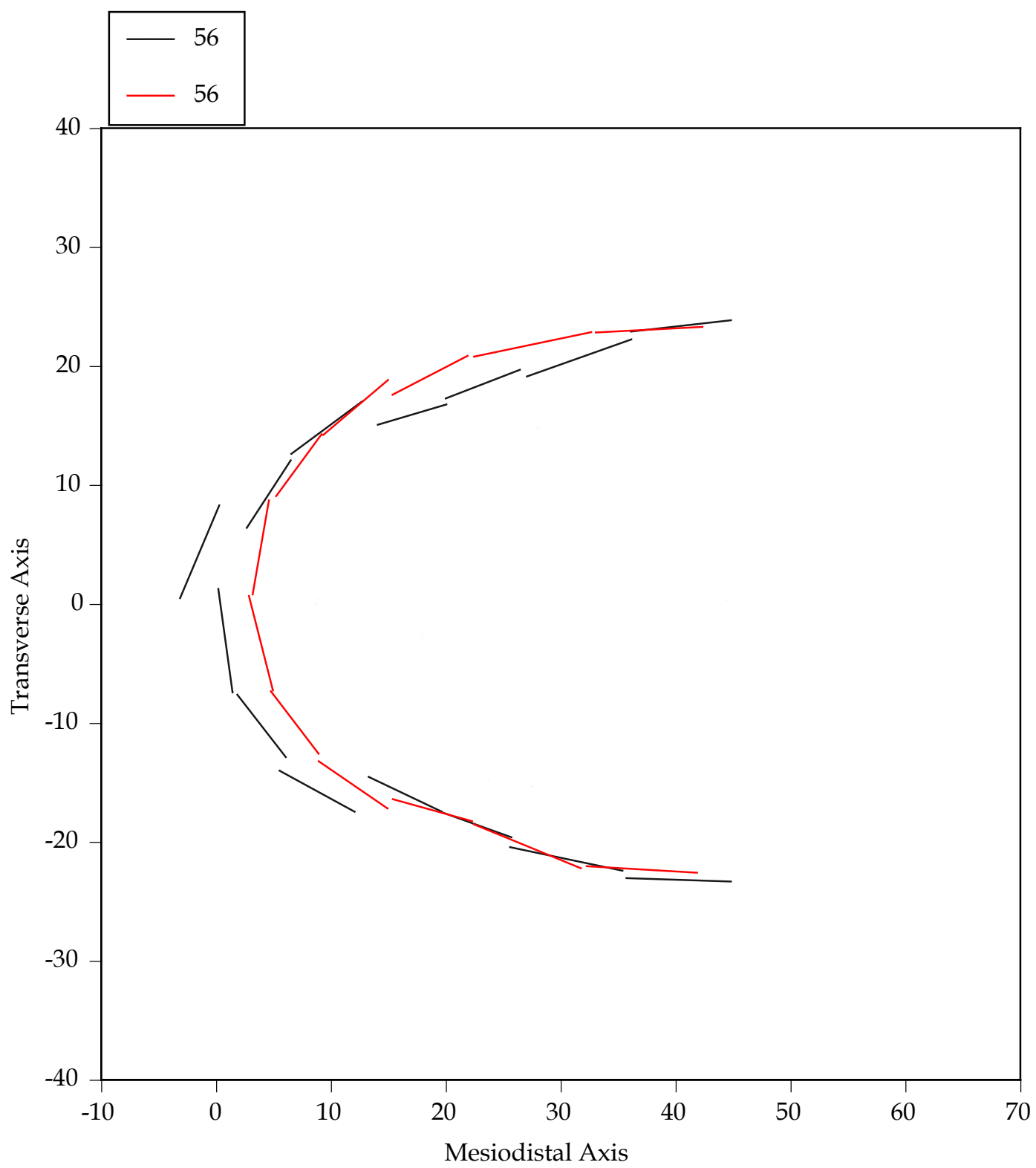

Fig. A-56. Plot of the maxillary dental arch relationships of case 56 , a female in whom the maxillary first-premolars were extracted for treatment. Squares are the dental contacts at the start of treatment; circles are the contacts at the end of treatment. The $X$ and $\mathrm{Y}$ axes are in millimeters. 


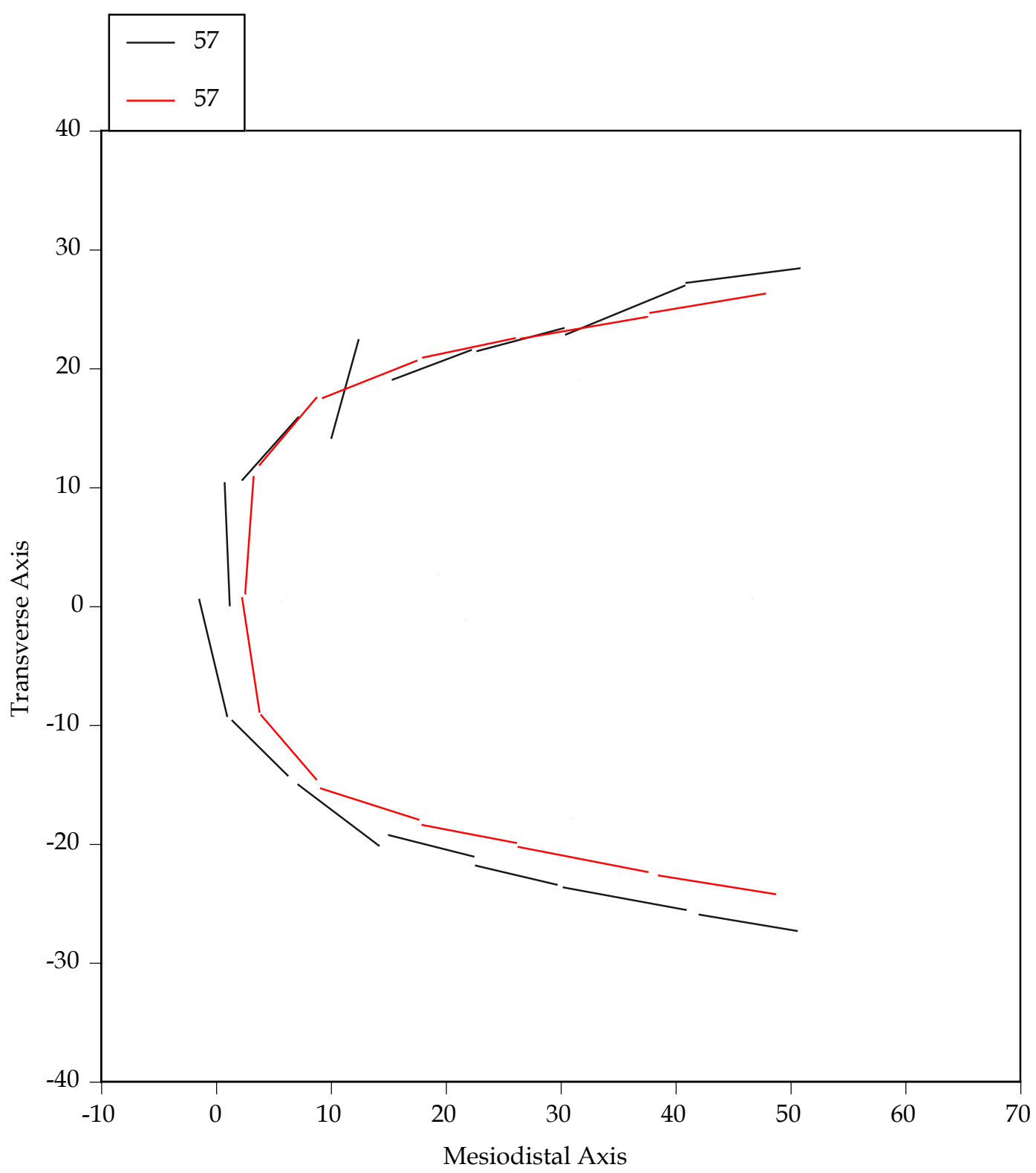

Fig. A-57. Plot of the maxillary dental arch relationships of case 57 , a female in whom the maxillary first-premolars were extracted for treatment. Squares are the dental contacts at the start of treatment; circles are the contacts at the end of treatment. The $\mathrm{X}$ and $\mathrm{Y}$ axes are in millimeters. 


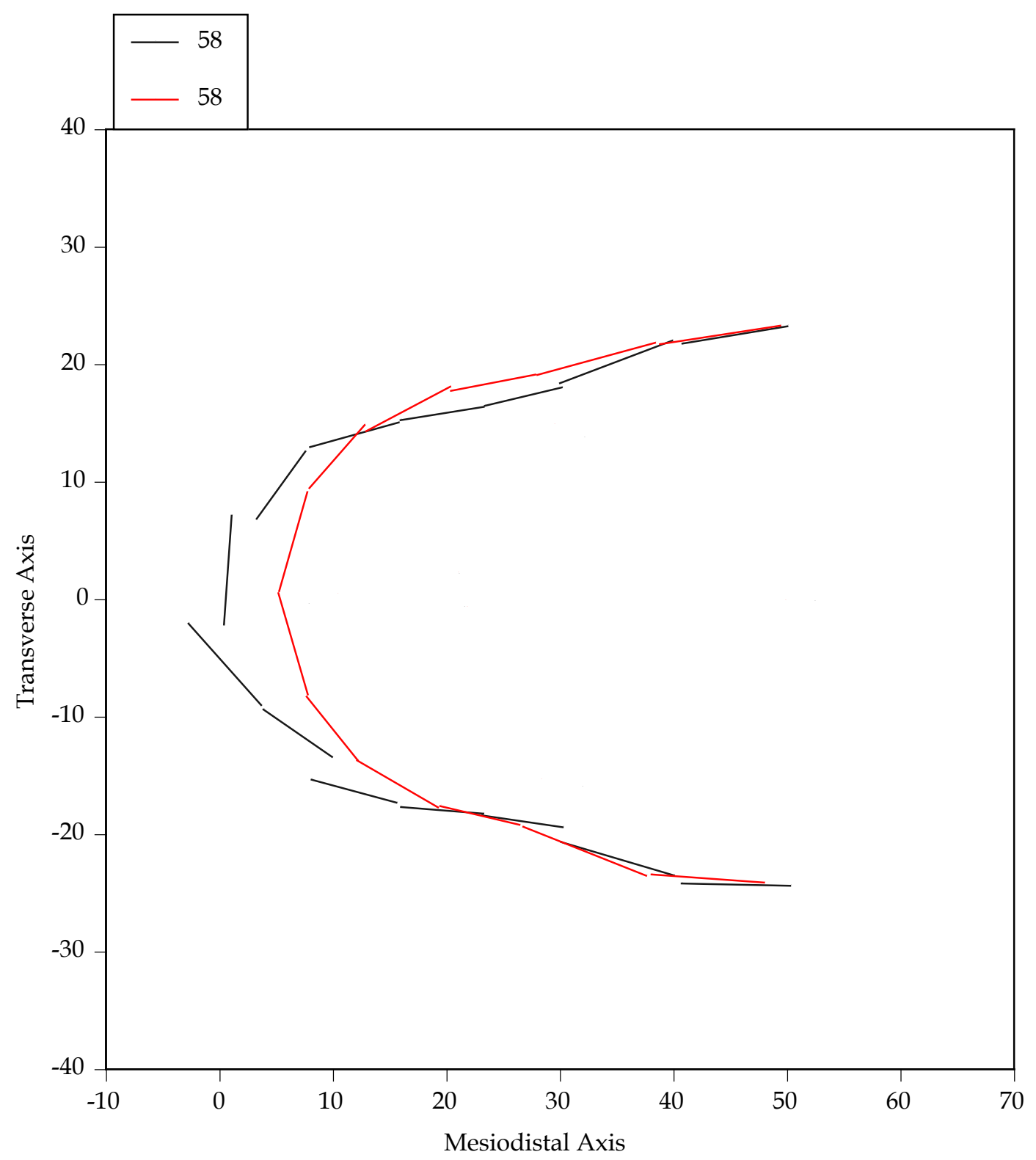

Fig. A-58. Plot of the maxillary dental arch relationships of case 58 , a female in whom the maxillary first-premolars were extracted for treatment. Squares are the dental contacts at the start of treatment; circles are the contacts at the end of treatment. The $\mathrm{X}$ and $\mathrm{Y}$ axes are in millimeters. 


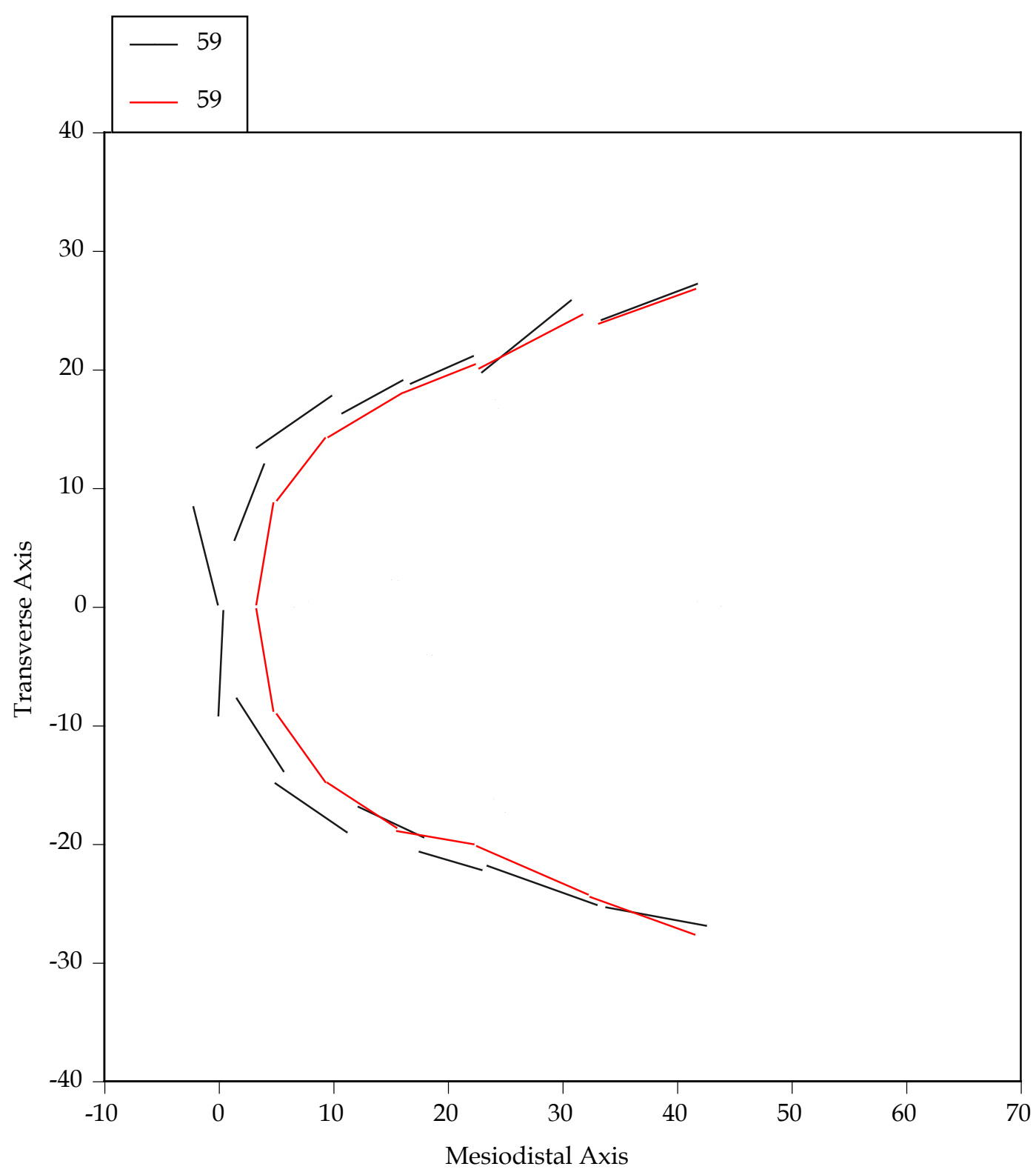

Fig. A-59. Plot of the maxillary dental arch relationships of case 59 , a female in whom the maxillary first-premolars were extracted for treatment. Squares are the dental contacts at the start of treatment; circles are the contacts at the end of treatment. The $\mathrm{X}$ and $\mathrm{Y}$ axes are in millimeters. 


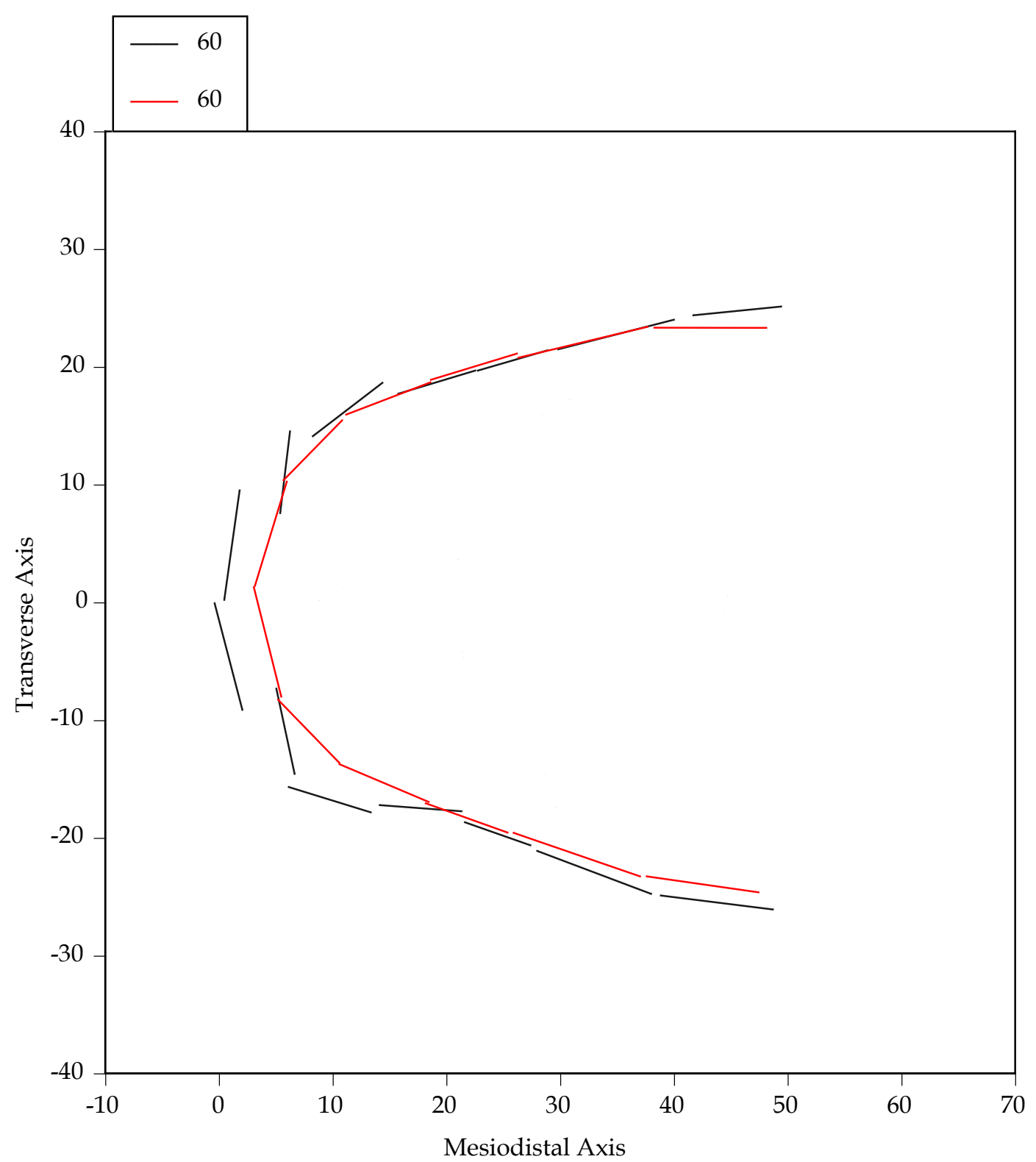

Fig. A-60. Plot of the maxillary dental arch relationships of case 60 , a female in whom the maxillary second-premolars were extracted for treatment. Squares are the dental contacts at the start of treatment; circles are the contacts at the end of treatment. The $\mathrm{X}$ and $\mathrm{Y}$ axes are in millimeters. 


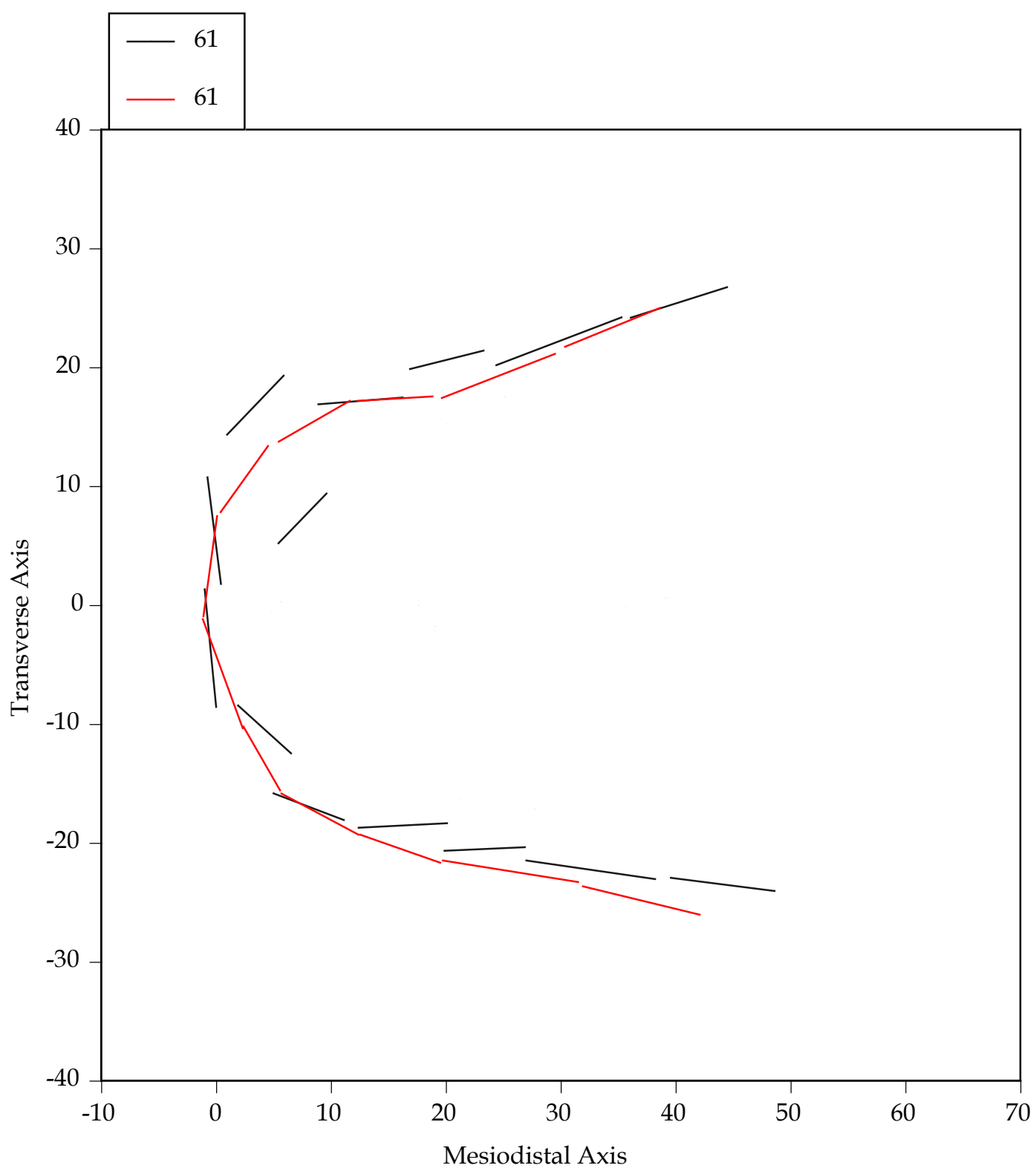

Fig. A-61. Plot of the maxillary dental arch relationships of case 61, a male in whom the maxillary second-premolars were extracted for treatment. Squares are the dental contacts at the start of treatment; circles are the contacts at the end of treatment. The $\mathrm{X}$ and $\mathrm{Y}$ axes are in millimeters. 


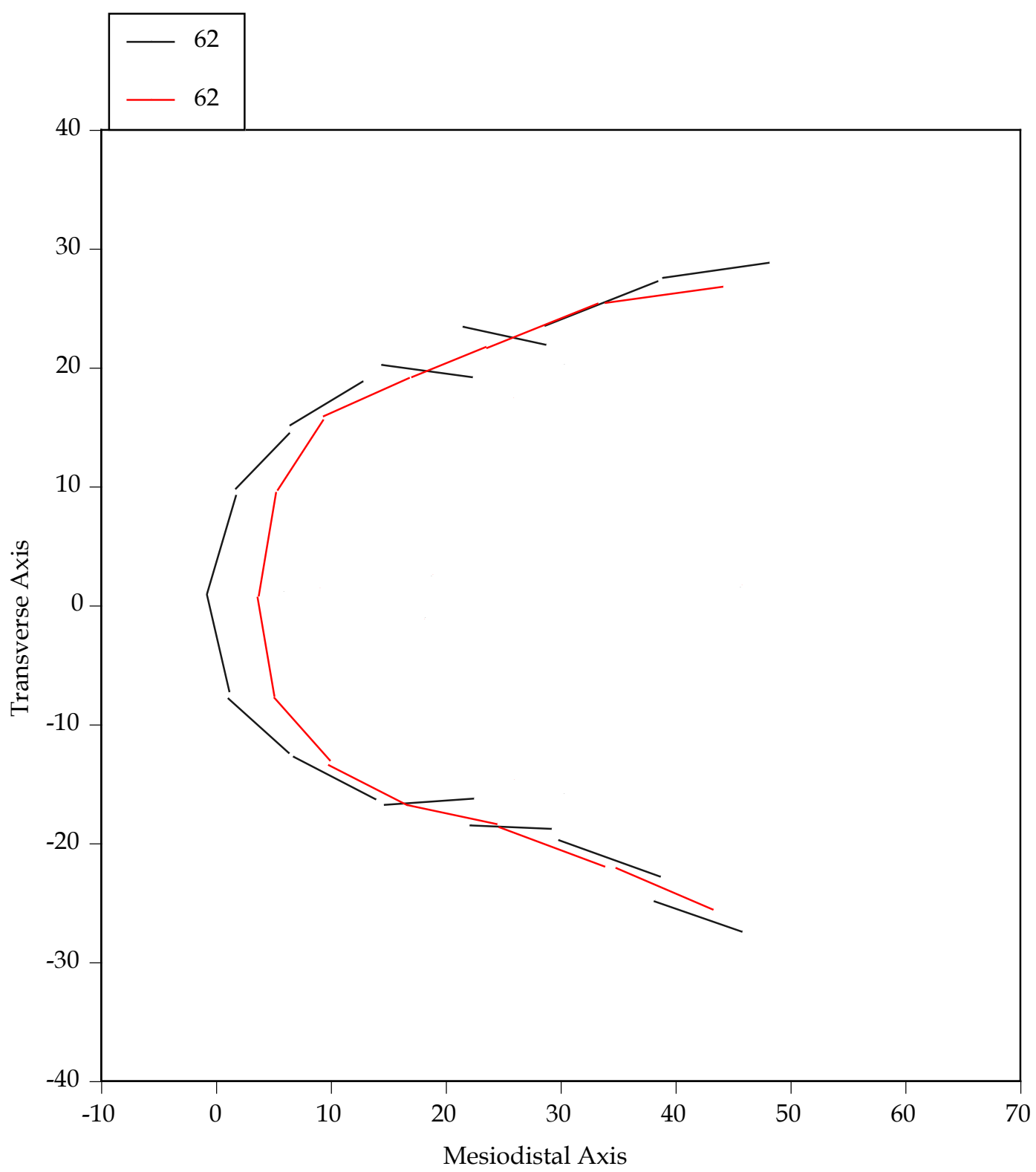

Fig. A-62. Plot of the maxillary dental arch relationships of case 62 , a male in whom the maxillary first-premolars were extracted for treatment. Squares are the dental contacts at the start of treatment; circles are the contacts at the end of treatment. The $X$ and $\mathrm{Y}$ axes are in millimeters. 


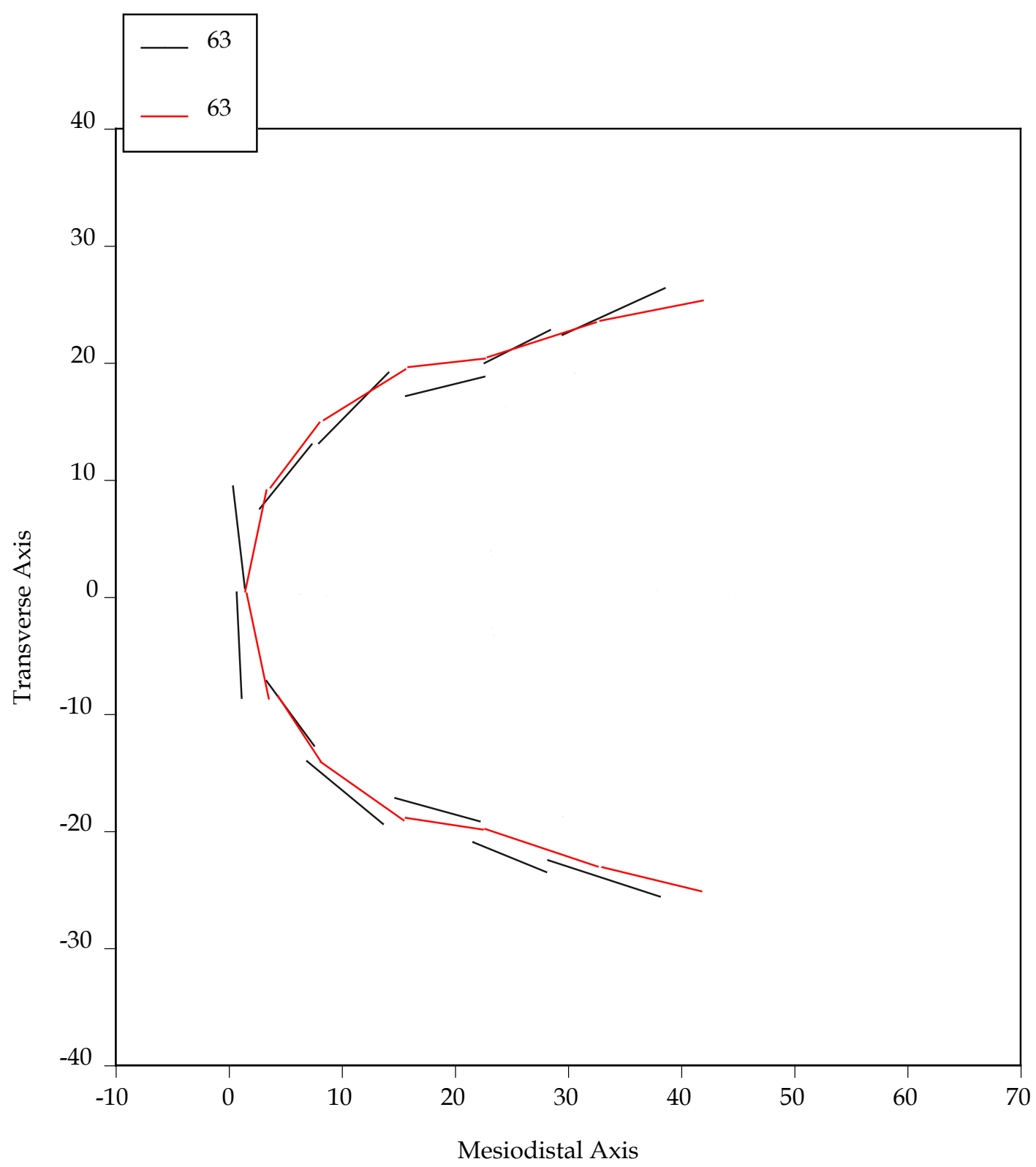

Fig. A-63. Plot of the maxillary dental arch relationships of case 63 , a male in whom the maxillary second-premolars were extracted for treatment. Squares are the dental contacts at the start of treatment; circles are the contacts at the end of treatment. The $X$ and $Y$ axes are in millimeters. 


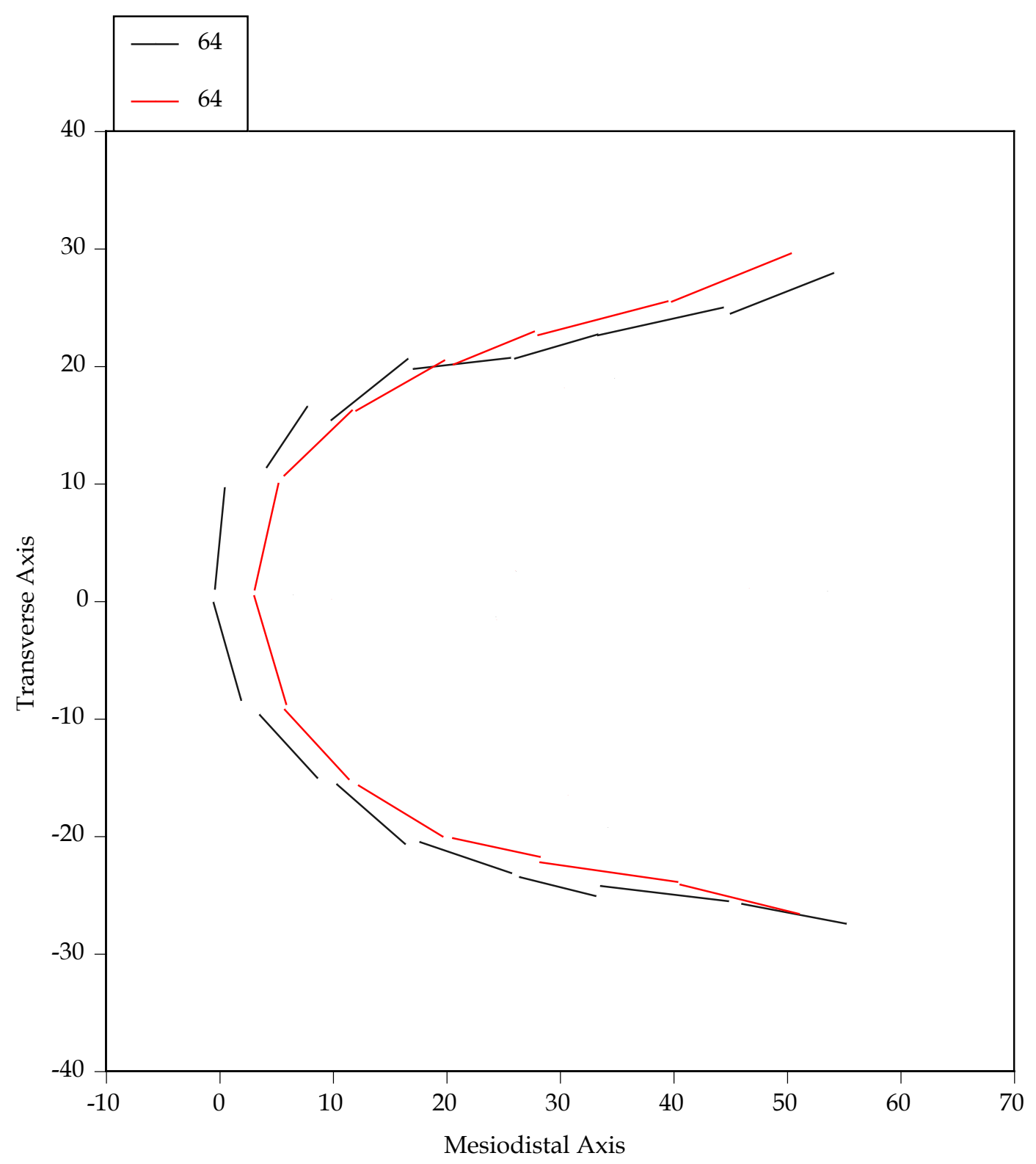

Fig. A-64. Plot of the maxillary dental arch relationships of case 64 , a male in whom the maxillary first-premolars were extracted for treatment. Squares are the dental contacts at the start of treatment; circles are the contacts at the end of treatment. The $X$ and $\mathrm{Y}$ axes are in millimeters. 


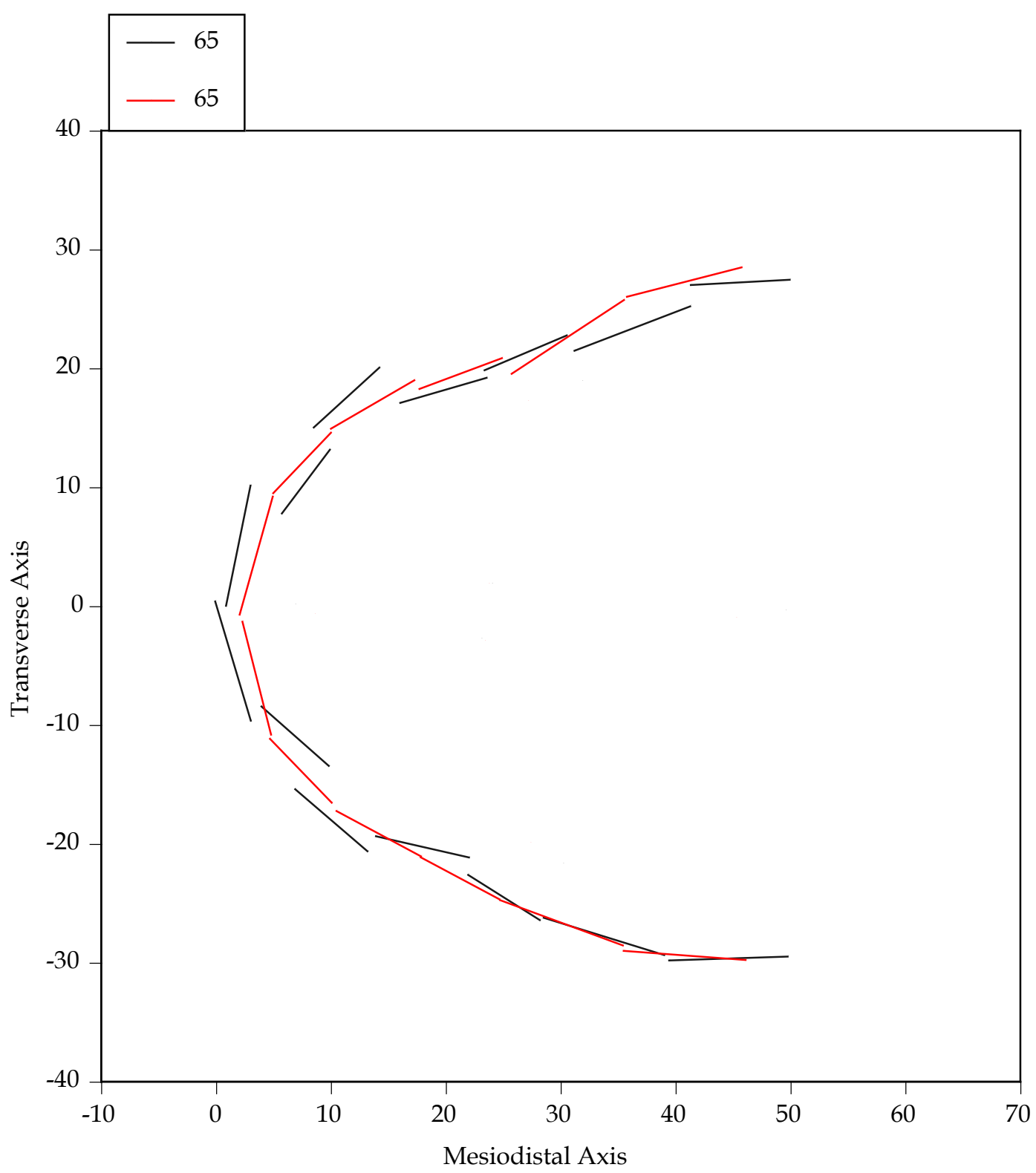

Fig. A-65. Plot of the maxillary dental arch relationships of case 65 , a male in whom the maxillary first-premolars were extracted for treatment. Squares are the dental contacts at the start of treatment; circles are the contacts at the end of treatment. The $X$ and $\mathrm{Y}$ axes are in millimeters. 


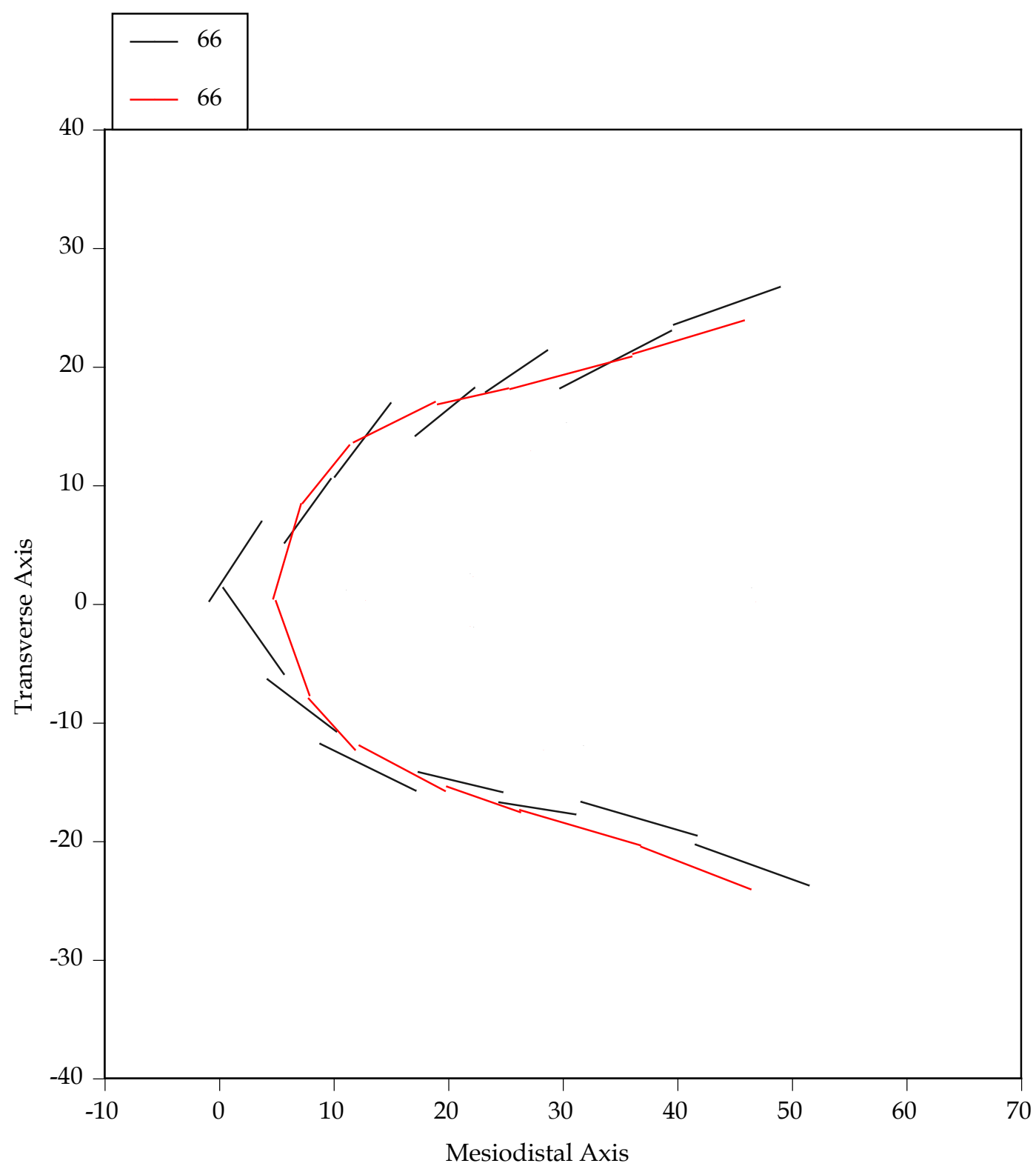

Fig. A-66. Plot of the maxillary dental arch relationships of case 66, a female in whom the maxillary first-premolars were extracted for treatment. Squares are the dental contacts at the start of treatment; circles are the contacts at the end of treatment. The $X$ and $\mathrm{Y}$ axes are in millimeters. 


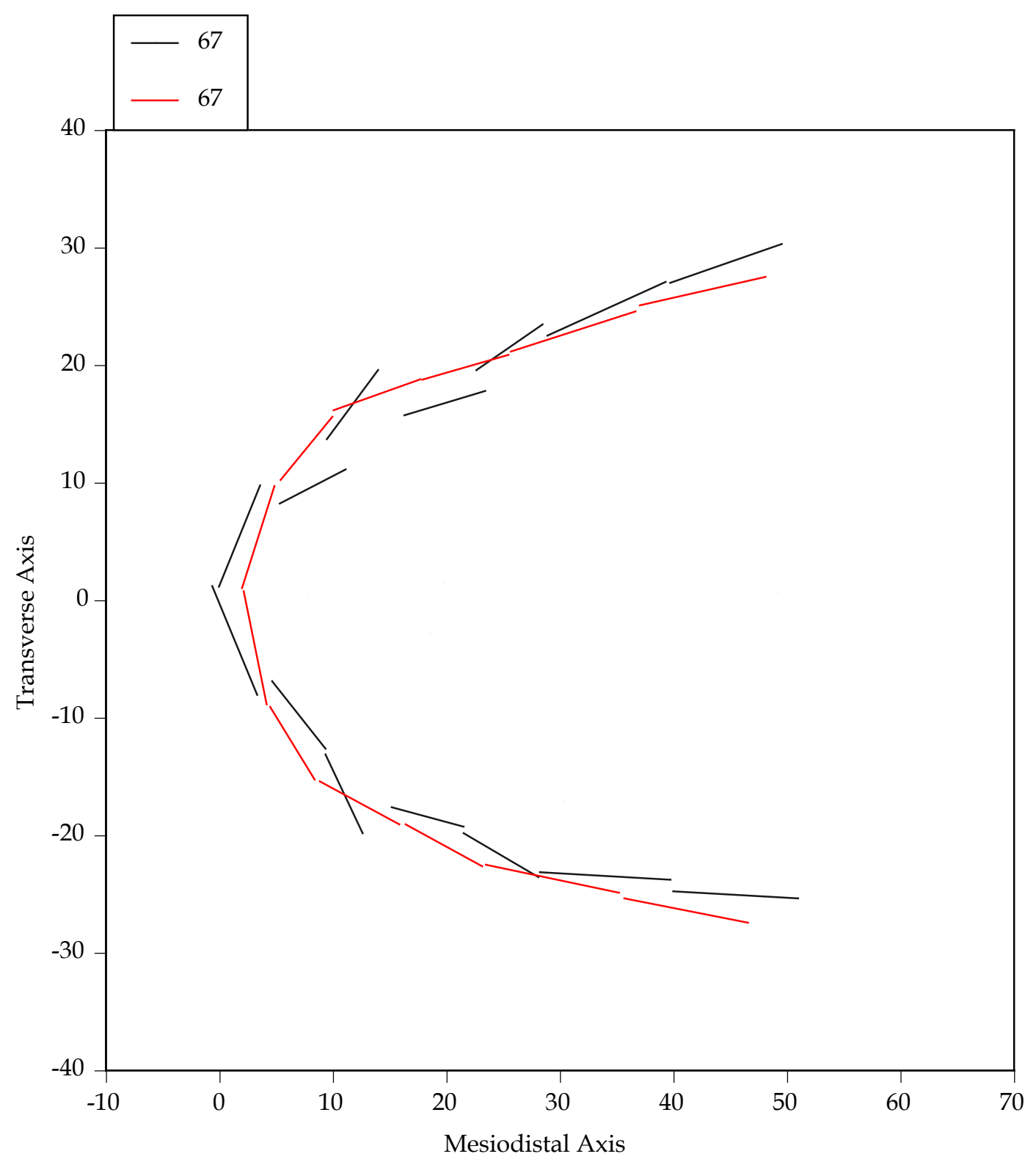

Fig. A-67. Plot of the maxillary dental arch relationships of case 67 , a male in whom the maxillary first-premolars were extracted for treatment. Squares are the dental contacts at the start of treatment; circles are the contacts at the end of treatment. The $\mathrm{X}$ and $Y$ axes are in millimeters. 


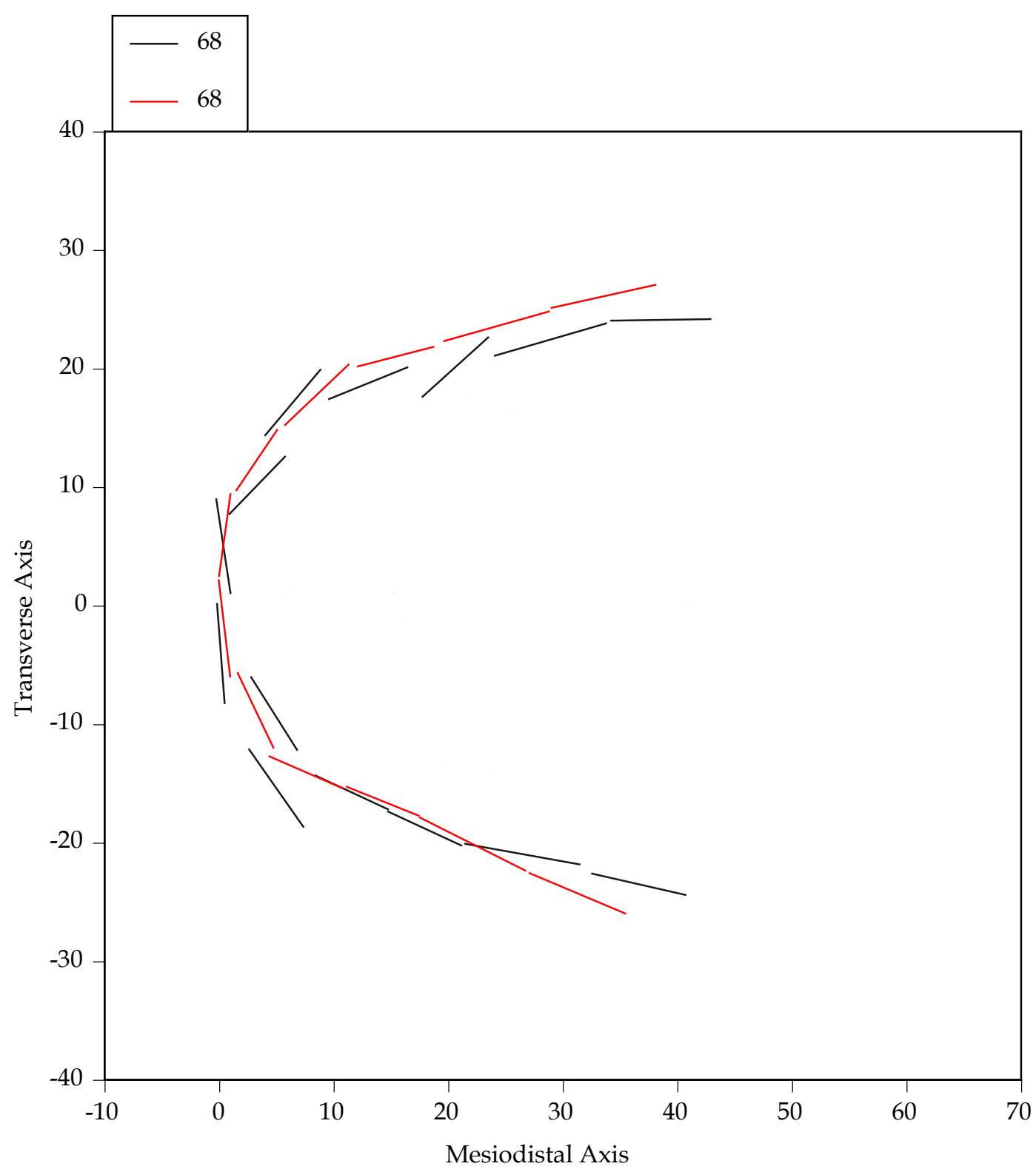

Fig. A-68. Plot of the maxillary dental arch relationships of case 68 , a female in whom the maxillary second-premolars were extracted for treatment. Squares are the dental contacts at the start of treatment; circles are the contacts at the end of treatment. The $X$ and $\mathrm{Y}$ axes are in millimeters. 


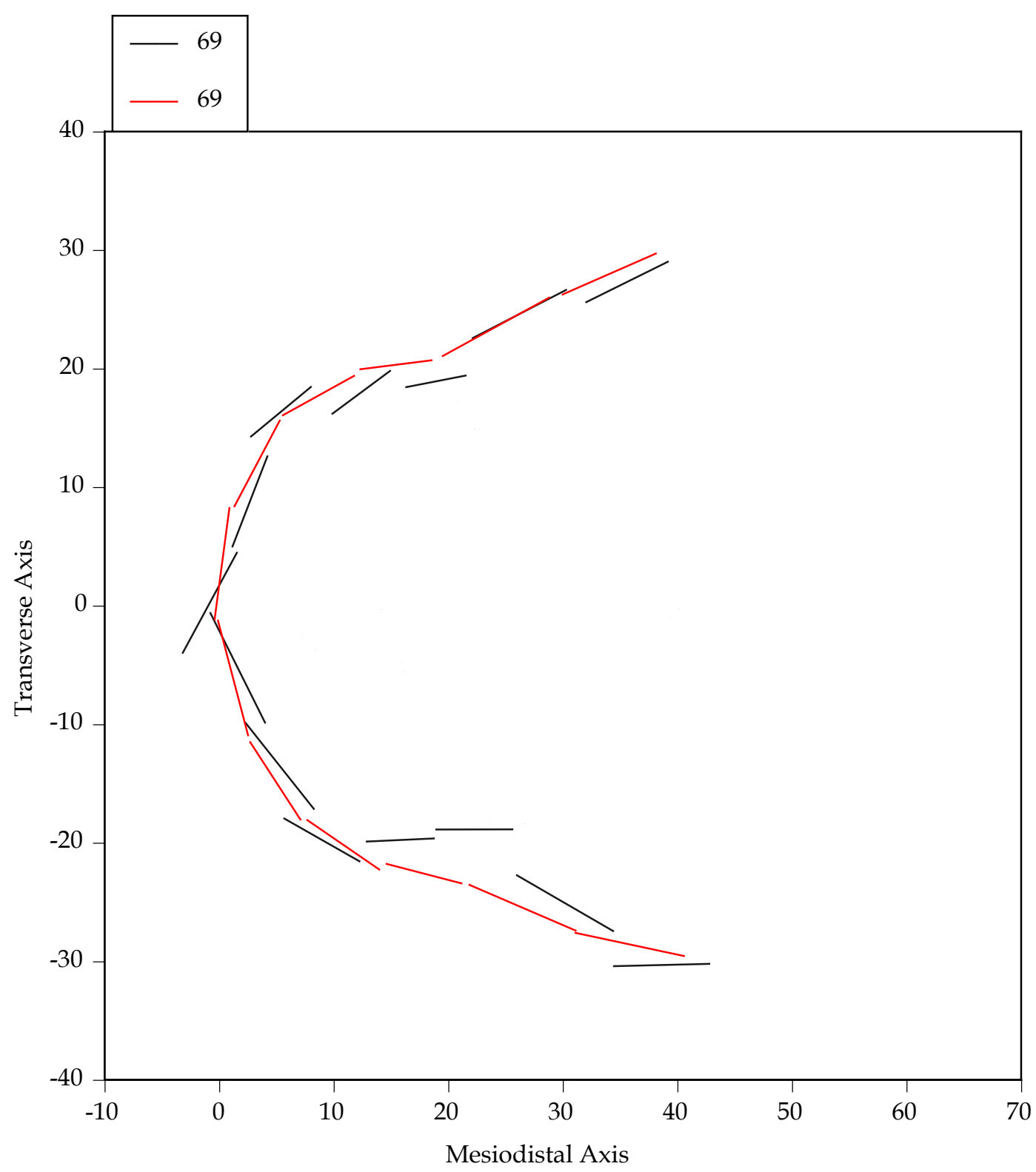

Fig. A-69. Plot of the maxillary dental arch relationships of case 69 , a female in whom the maxillary second-premolars were extracted for treatment. Squares are the dental contacts of at the start treatment; circles are the contacts at the end of treatment. The $X$ and $Y$ axes are in millimeters. 


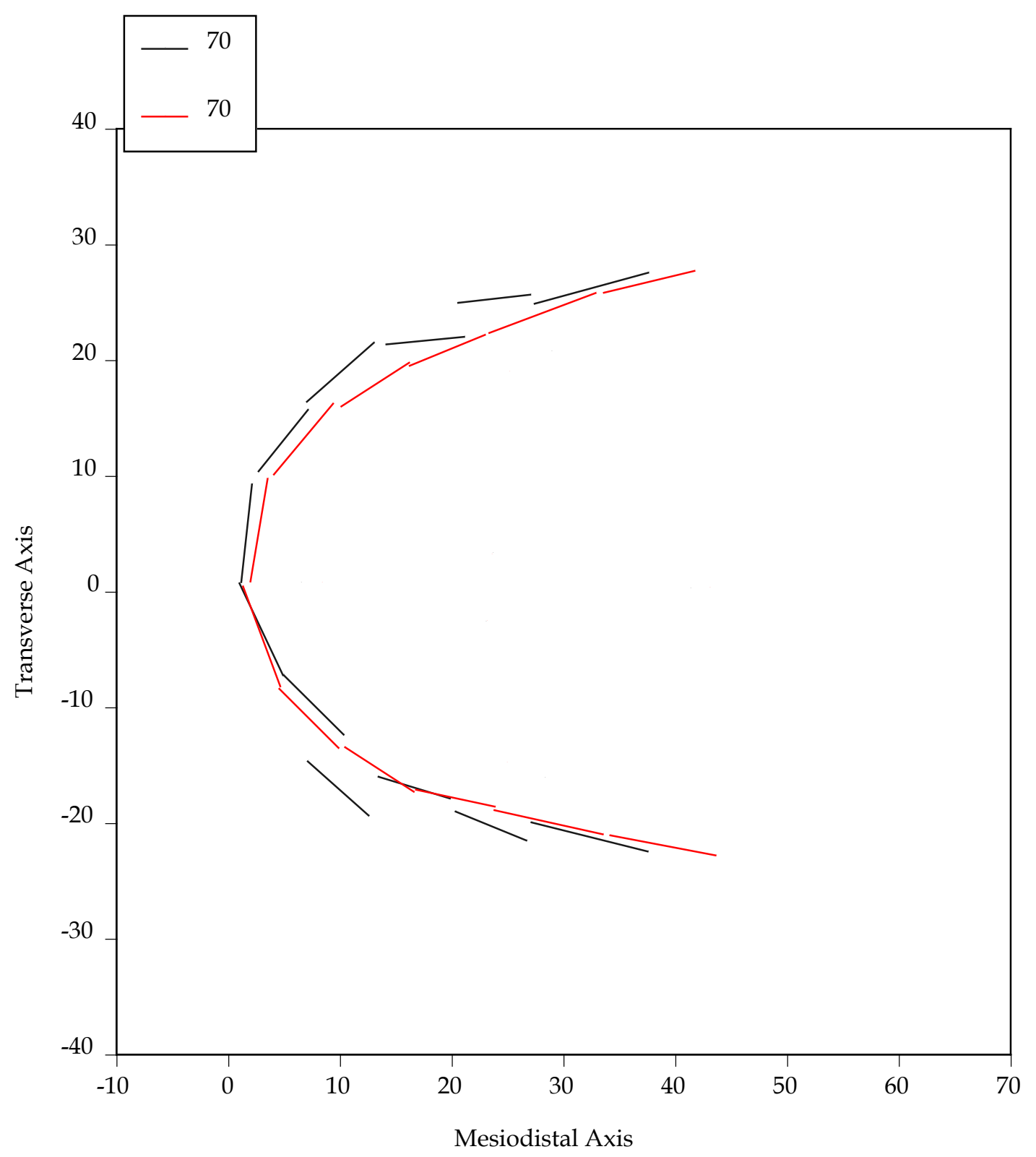

Fig. A-70. Plot of the maxillary dental arch relationships of case 70, a female in whom the maxillary second-premolars were extracted for treatment. Squares are the dental contacts at the start of treatment; circles are the contacts at the end of treatment. The $\mathrm{X}$ and $\mathrm{Y}$ axes are in millimeters. 


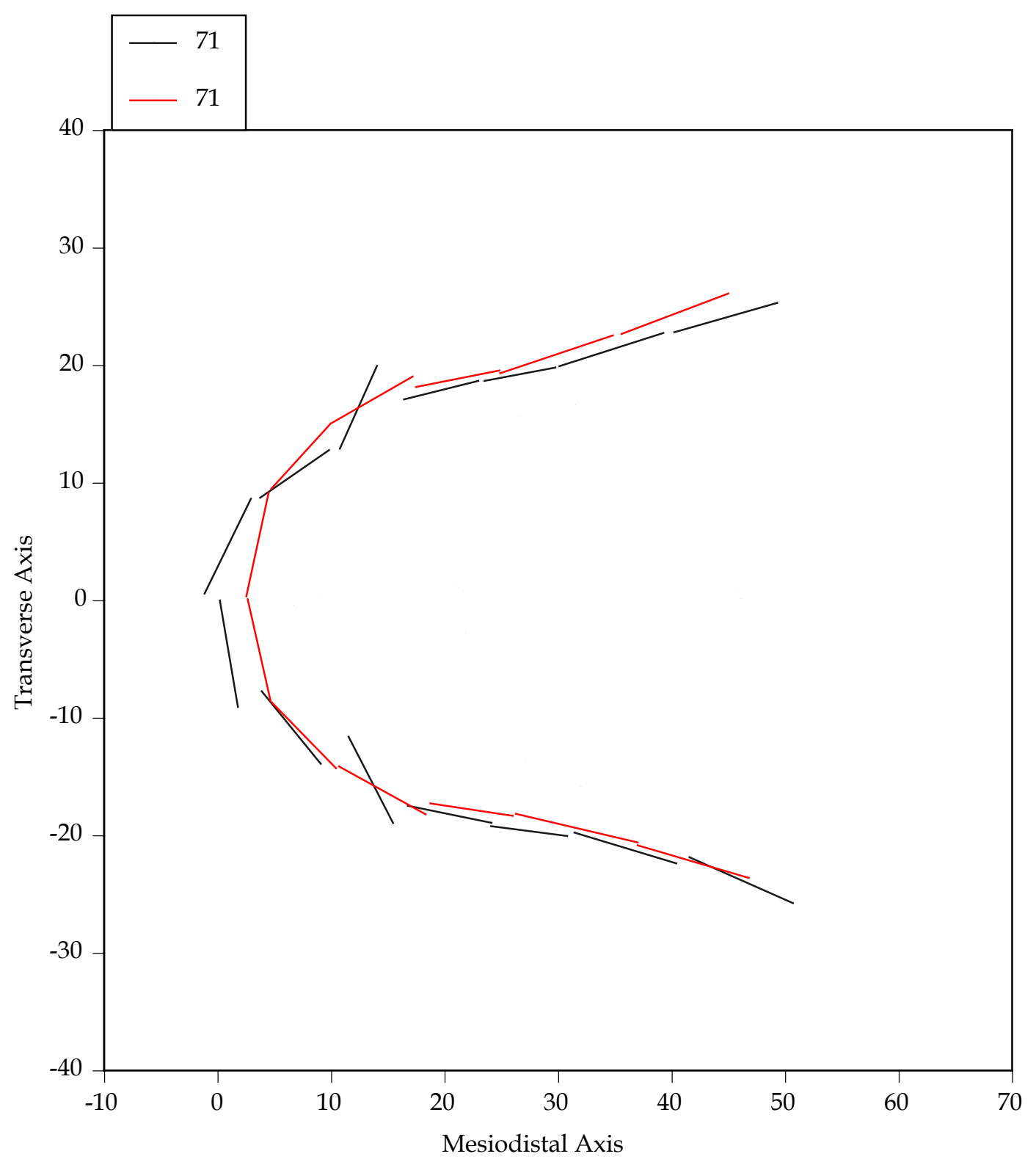

Fig. A-71. Plot of the maxillary dental arch relationships of case 71 , a female in whom the maxillary first-premolars were extracted for treatment. Squares are the dental contacts at the start of treatment; circles are the contacts at the end of treatment. The $\mathrm{X}$ and $\mathrm{Y}$ axes are in millimeters. 


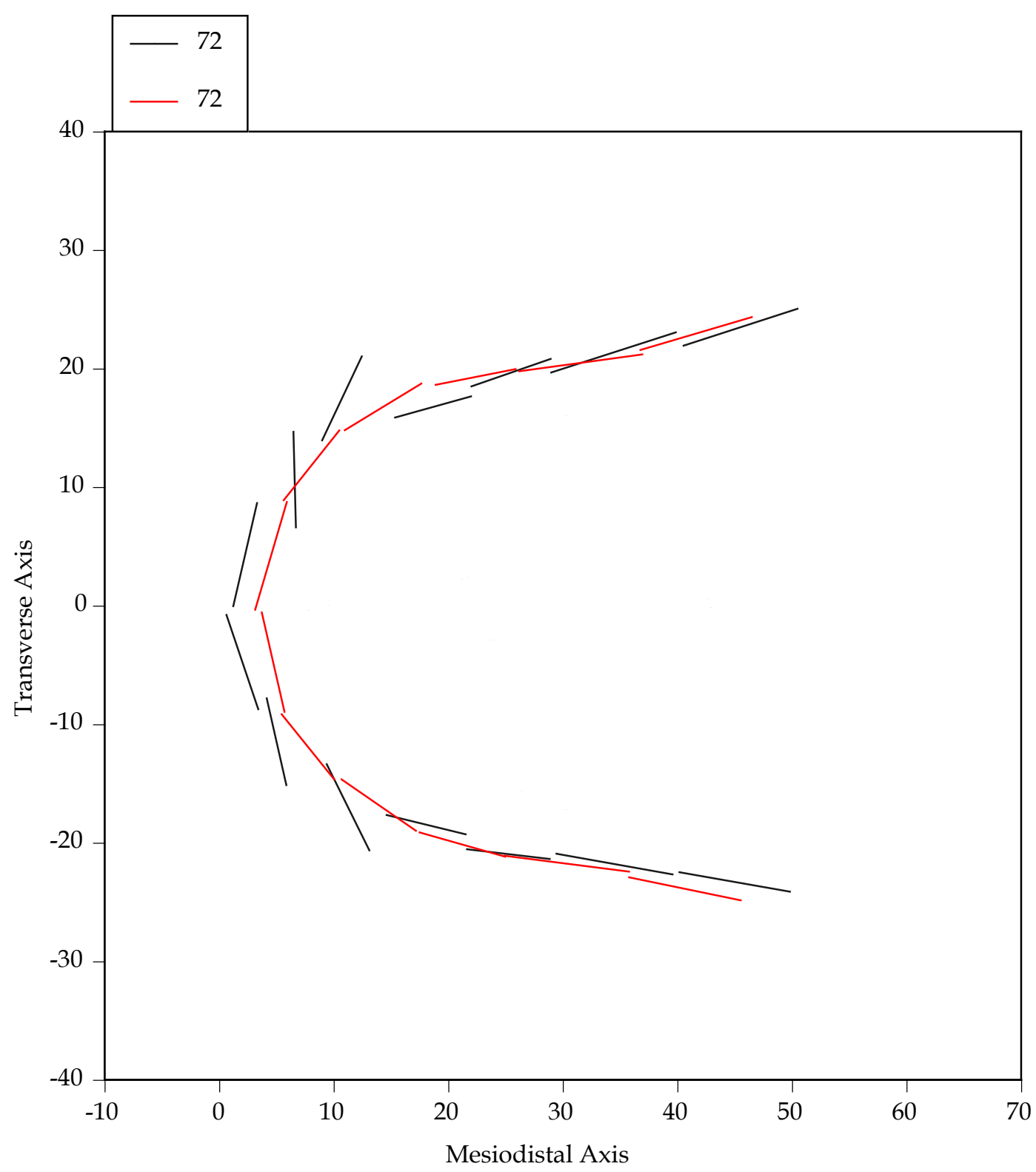

Fig. A-72. Plot of the maxillary dental arch relationships of case 72 , a male in whom the maxillary first-premolars were extracted for treatment. Squares are the dental contacts at the start of treatment; circles are the contacts at the end of treatment. The $X$ and $\mathrm{Y}$ axes are in millimeters. 


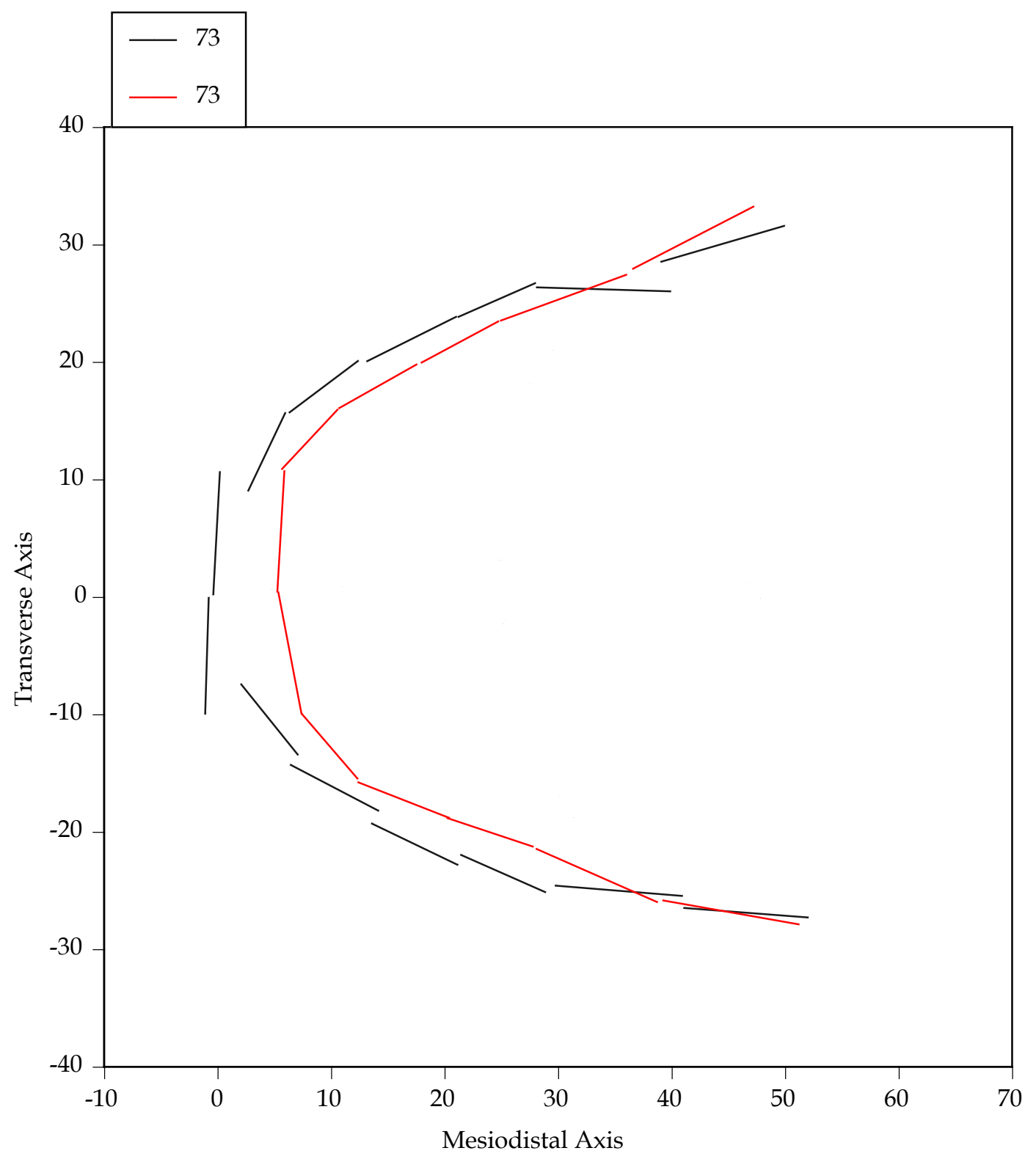

Fig. A-73. Plot of the maxillary dental arch relationships of case 73 , a male in whom the maxillary first-premolars were extracted for treatment. Squares are the dental contacts at the start of treatment; circles are the contacts at the end of treatment. The $X$ and $\mathrm{Y}$ axes are in millimeters. 


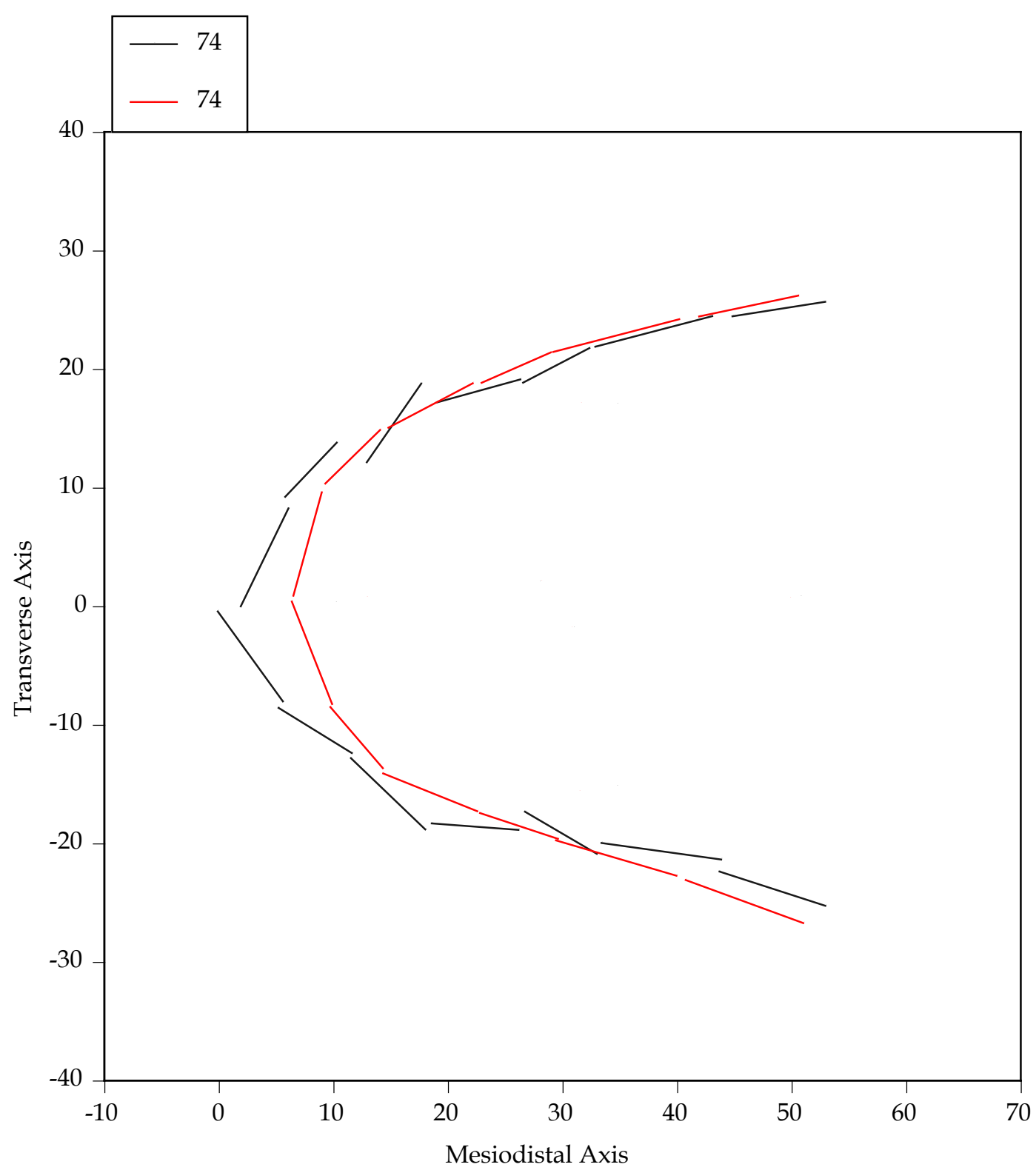

Fig. A-74. Plot of the maxillary dental arch relationships of case 74 , a female in whom the maxillary first-premolars were extracted for treatment. Squares are the dental contacts at the start of treatment; circles are the contacts at the end of treatment. The $X$ and $\mathrm{Y}$ axes are in millimeters. 


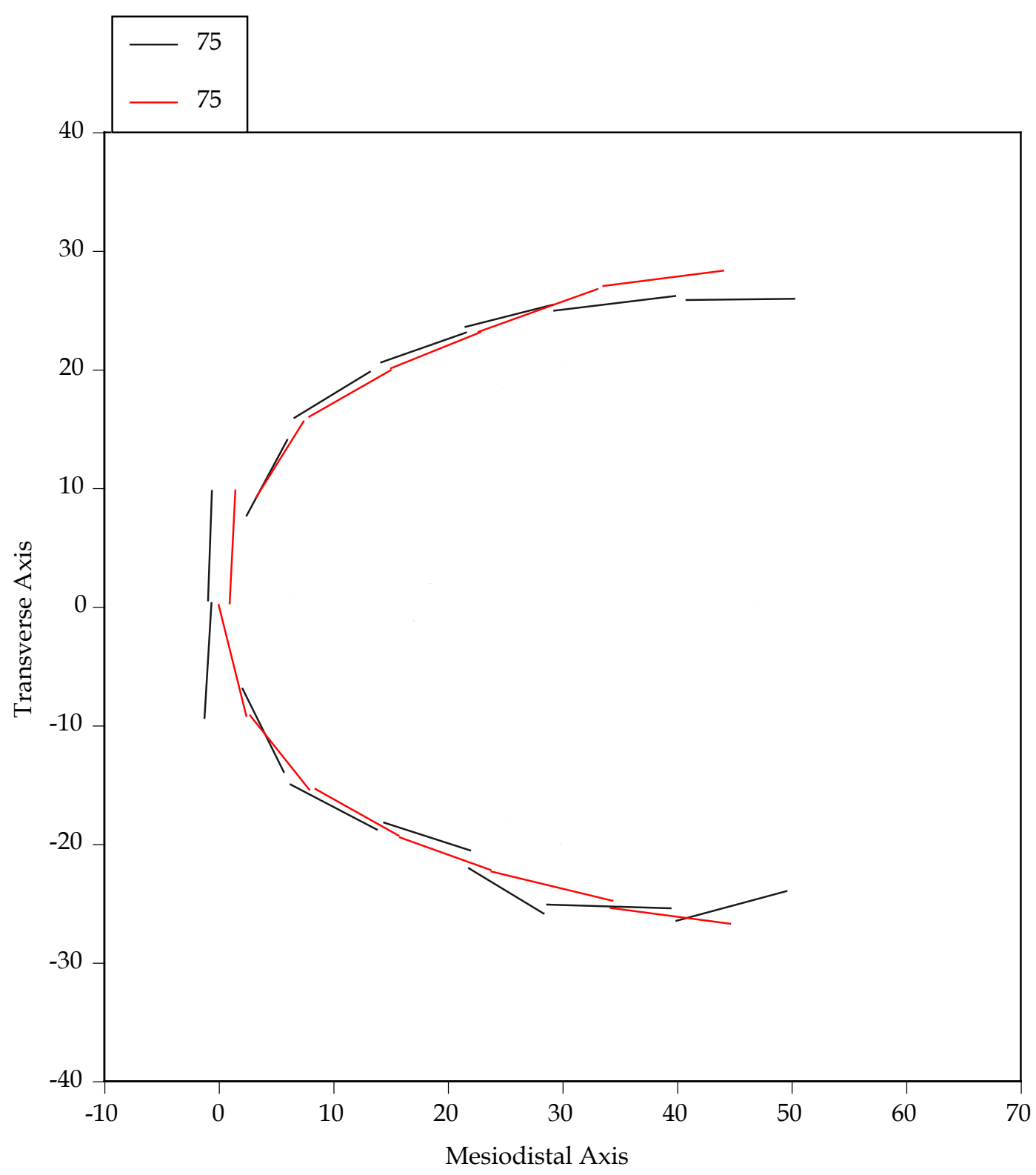

Fig.A-75. Plot of the maxillary dental arch relationships of case 75 , a male in whom the maxillary first-premolars were extracted for treatment. Squares are the dental contacts at the start of treatment; circles are the contacts at the end of treatment. The $X$ and $\mathrm{Y}$ axes are in millimeters. 


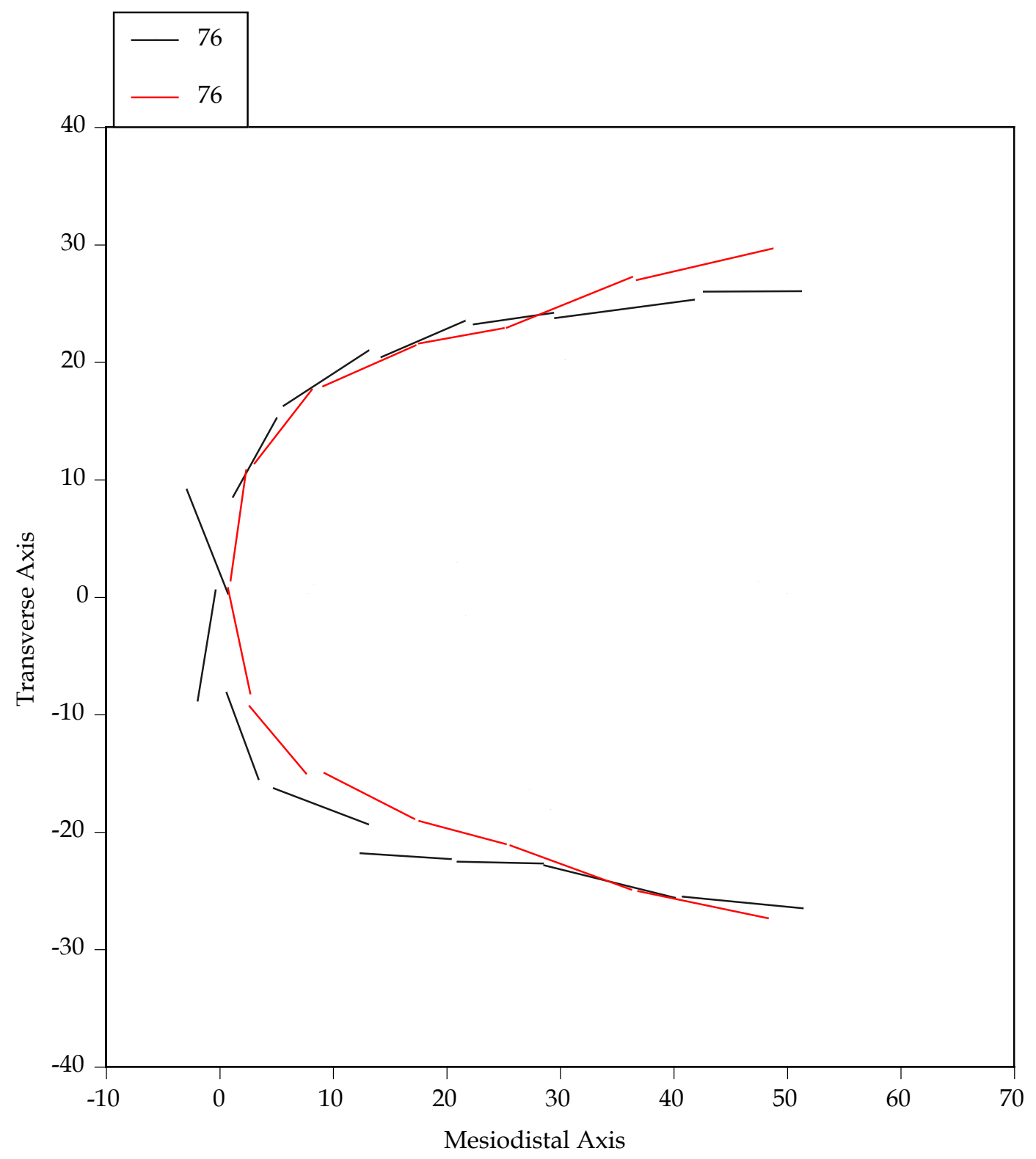

Fig. A-76. Plot of the maxillary dental arch relationships of case 76 , a male in whom the maxillary first-premolars were extracted for treatment. Squares are the dental contacts at the start of treatment; circles are the contacts at the end of treatment. The $X$ and $Y$ axes are in millimeters. 


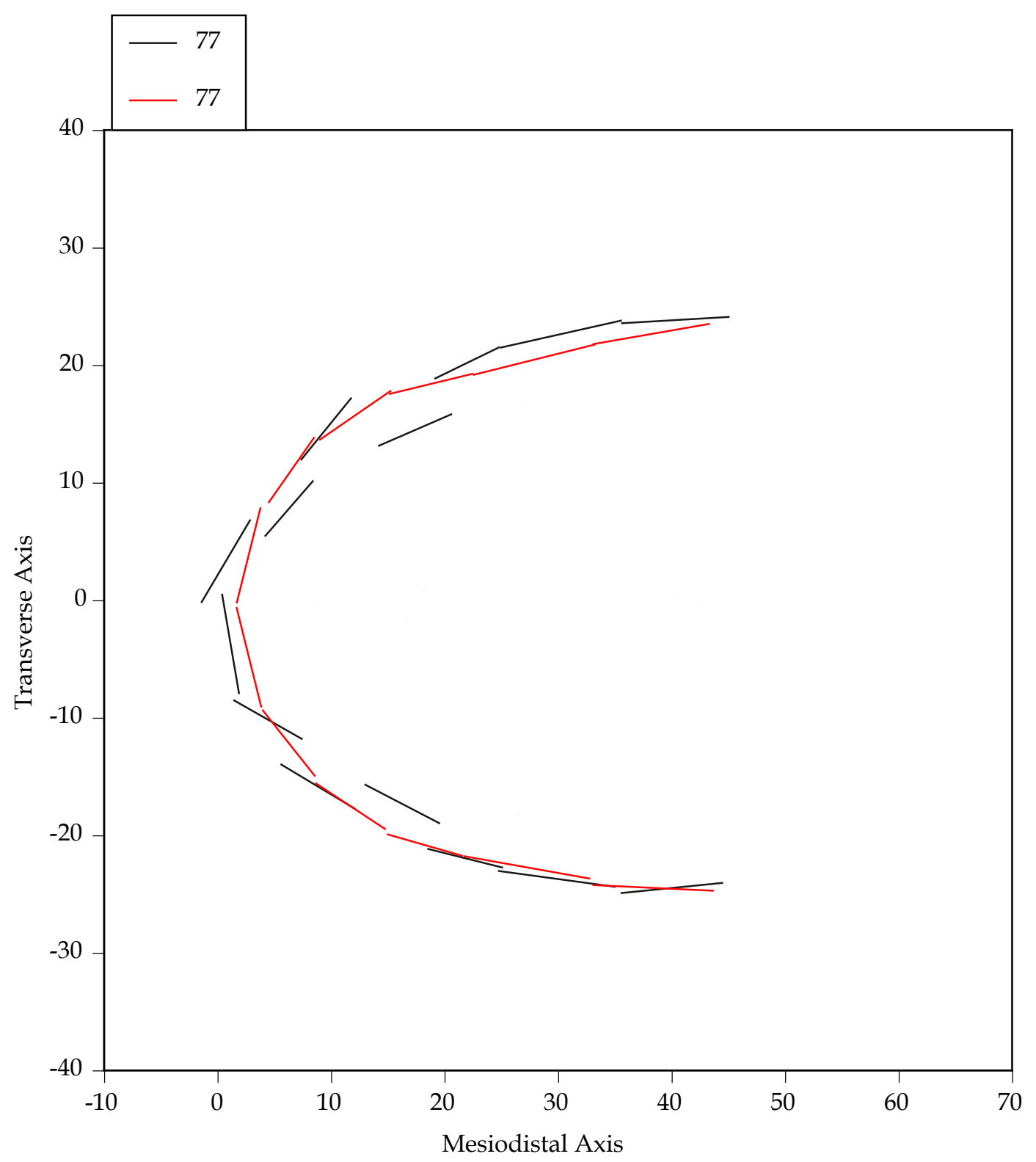

Fig. A-77. Plot of the maxillary dental arch relationships of case 77 , a female in whom the maxillary first-premolars were extracted for treatment. Squares are the dental contacts at the start of treatment; circles are the contacts at the end of treatment. The $\mathrm{X}$ and $\mathrm{Y}$ axes are in millimeters. 


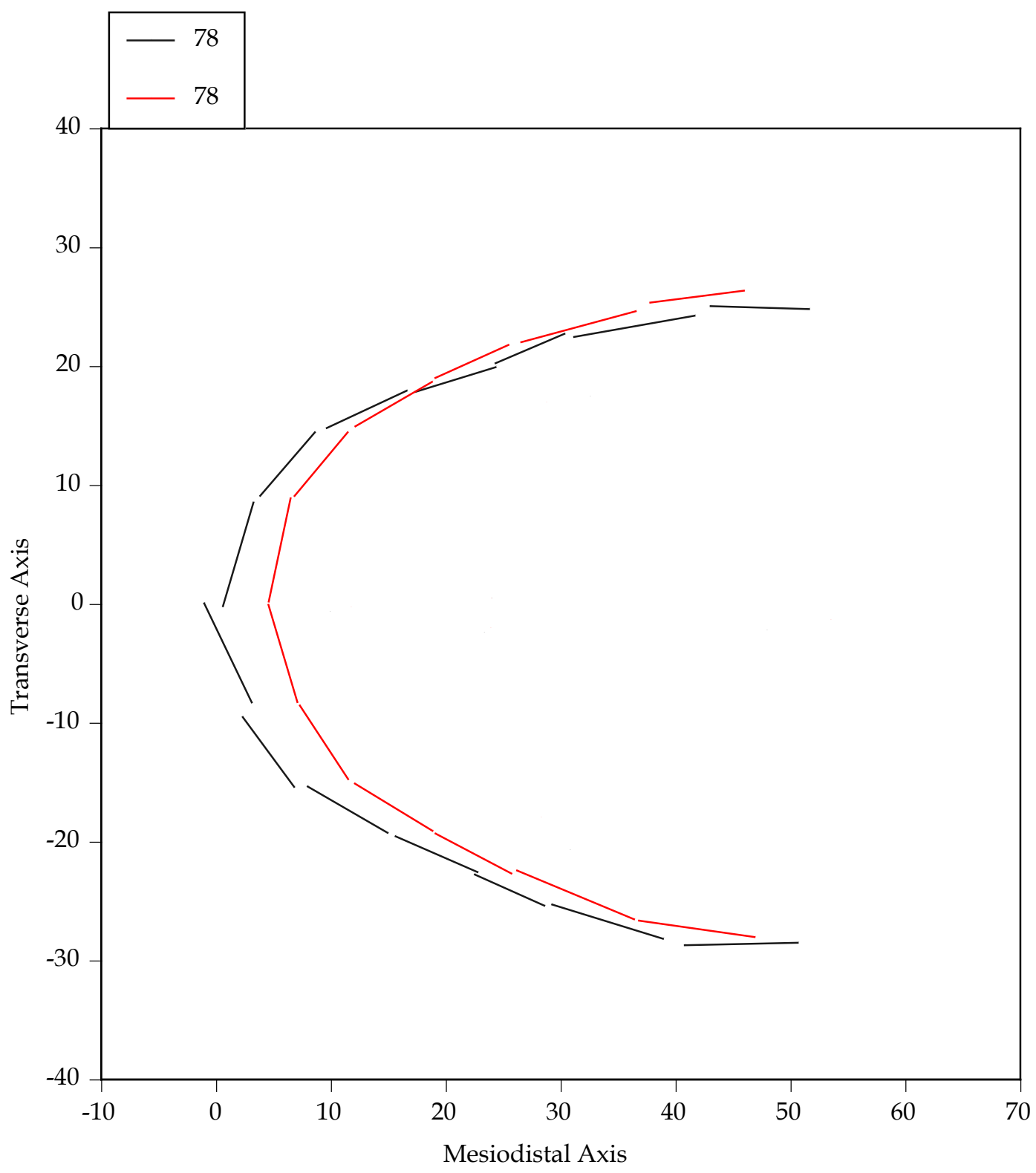

Fig. A-78. Plot of the maxillary dental arch relationships of case 78 , a male in whom the maxillary second-premolars were extracted for treatment. Squares are the dental contacts at the start of treatment; circles are the contacts at the end of treatment. The $\mathrm{X}$ and $\mathrm{Y}$ axes are in millimeters. 


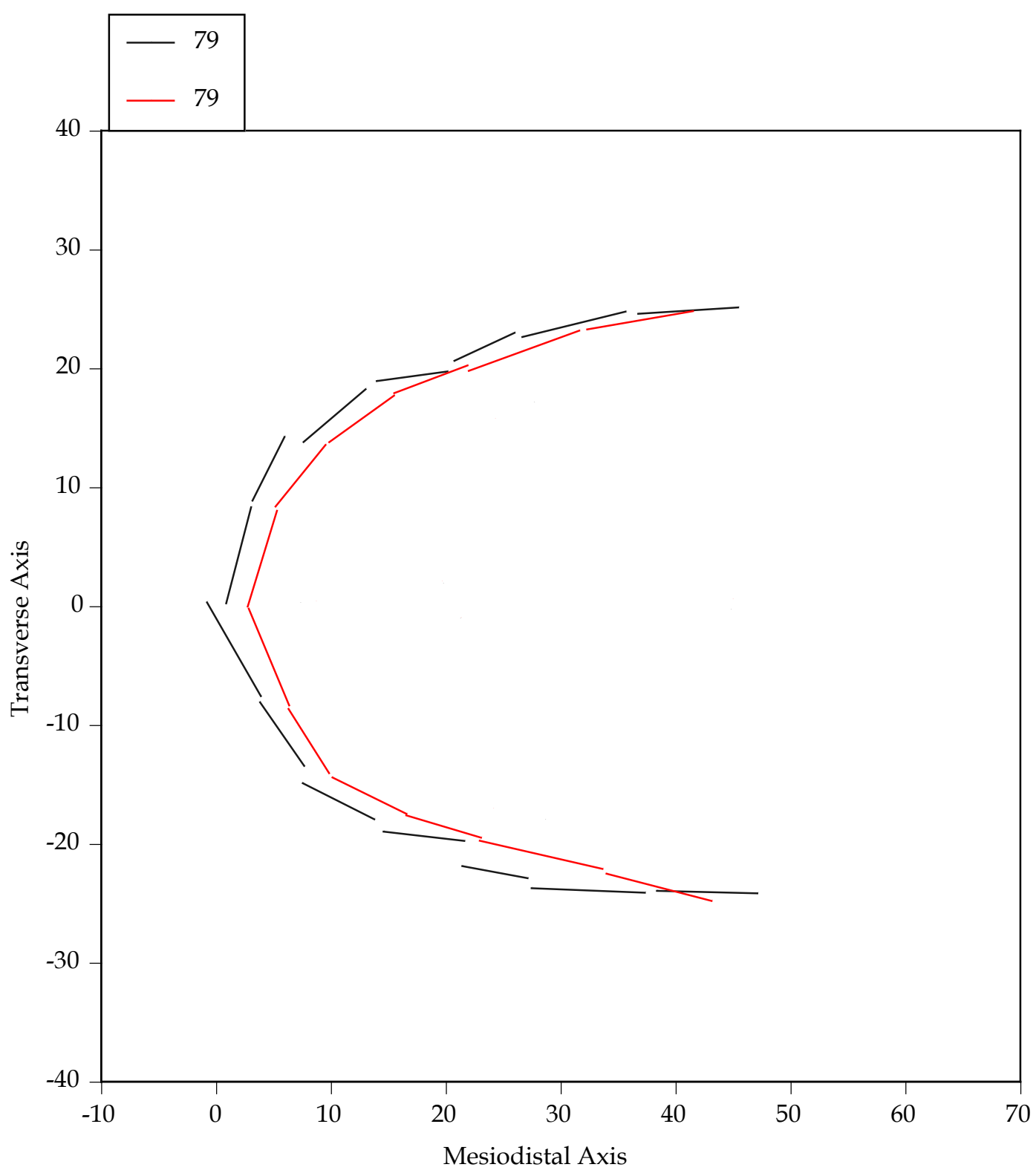

Fig. A-79. Plot of the maxillary dental arch relationships of case 79 , a female in whom the maxillary second-premolars were extracted for treatment. Squares are the dental contacts at the start of treatment; circles are the contacts at the end of treatment. The $X$ and $Y$ axes are in millimeters. 


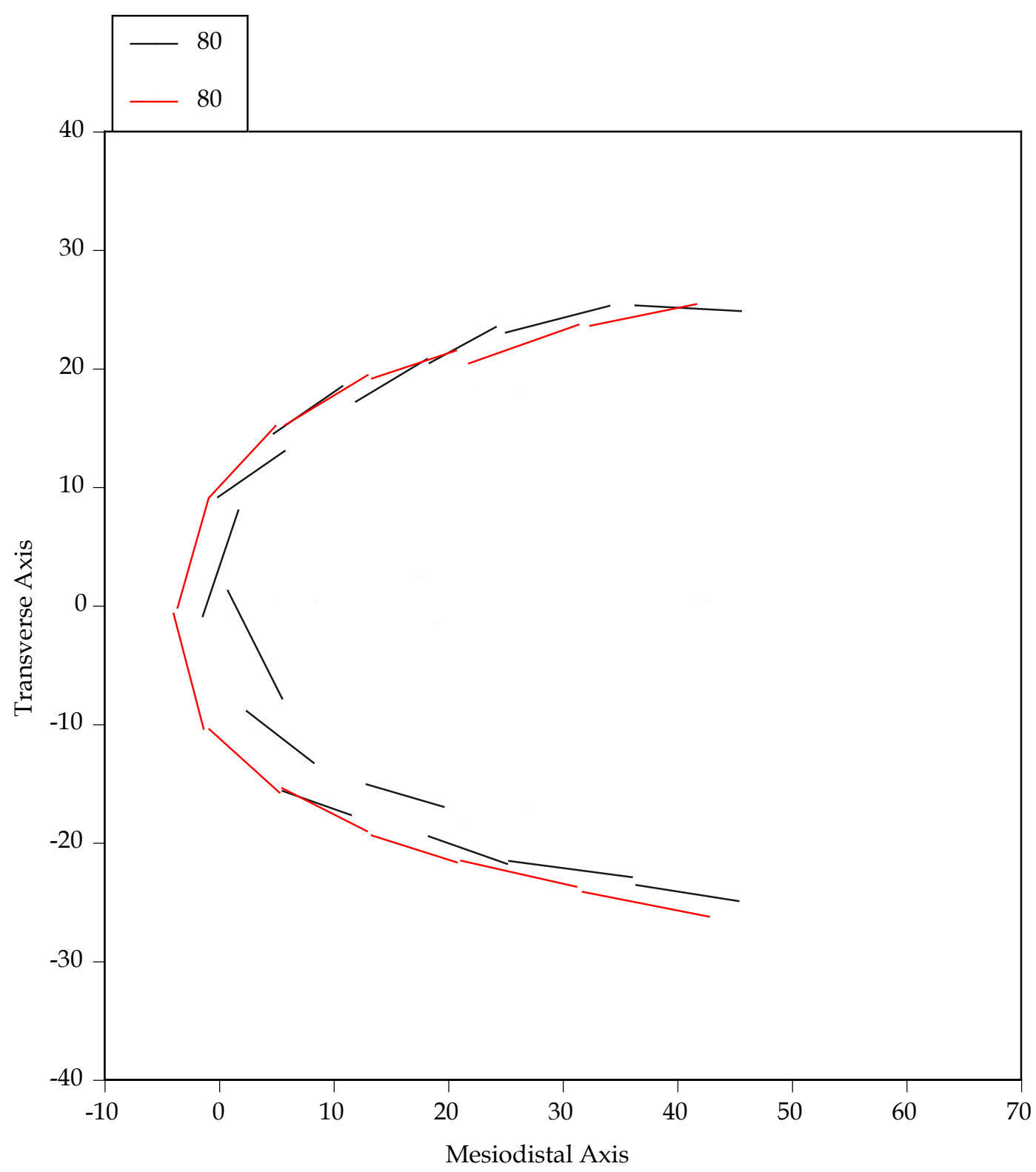

Fig. A-80. Plot of the maxillary dental arch relationships of case 80 , a male in whom the maxillary second-premolars were extracted for treatment. Squares are the dental contacts at the start of treatment; circles are the contacts at the end of treatment. The $\mathrm{X}$ and $\mathrm{Y}$ axes are in millimeters. 


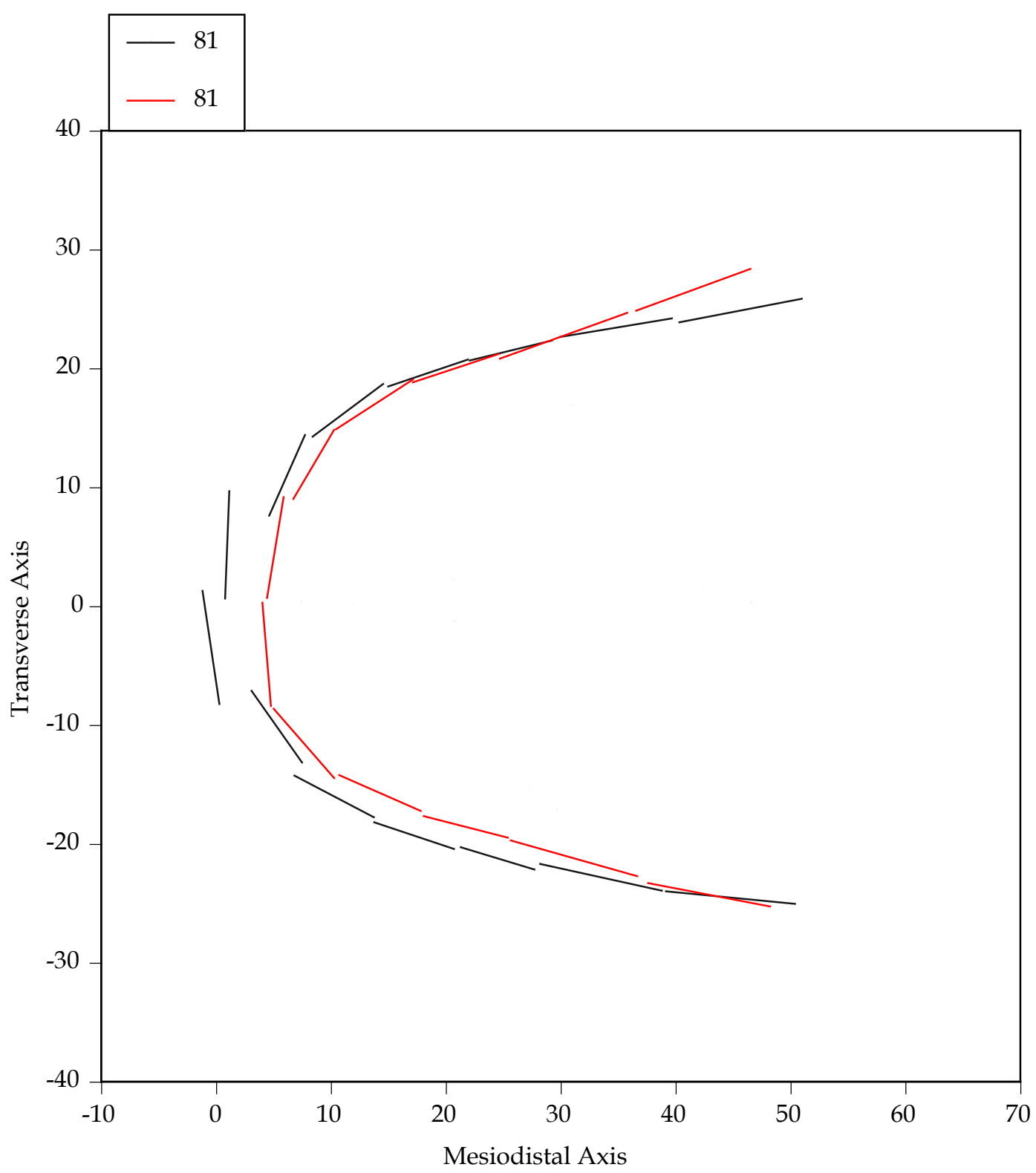

Fig. A-81. Plot of the maxillary dental arch relationships of case 81 , a female in whom the maxillary second-premolars were extracted for treatment. Squares are the dental contacts at the start of treatment; circles are the contacts at the end of treatment. The $\mathrm{X}$ and $\mathrm{Y}$ axes are in millimeters. 


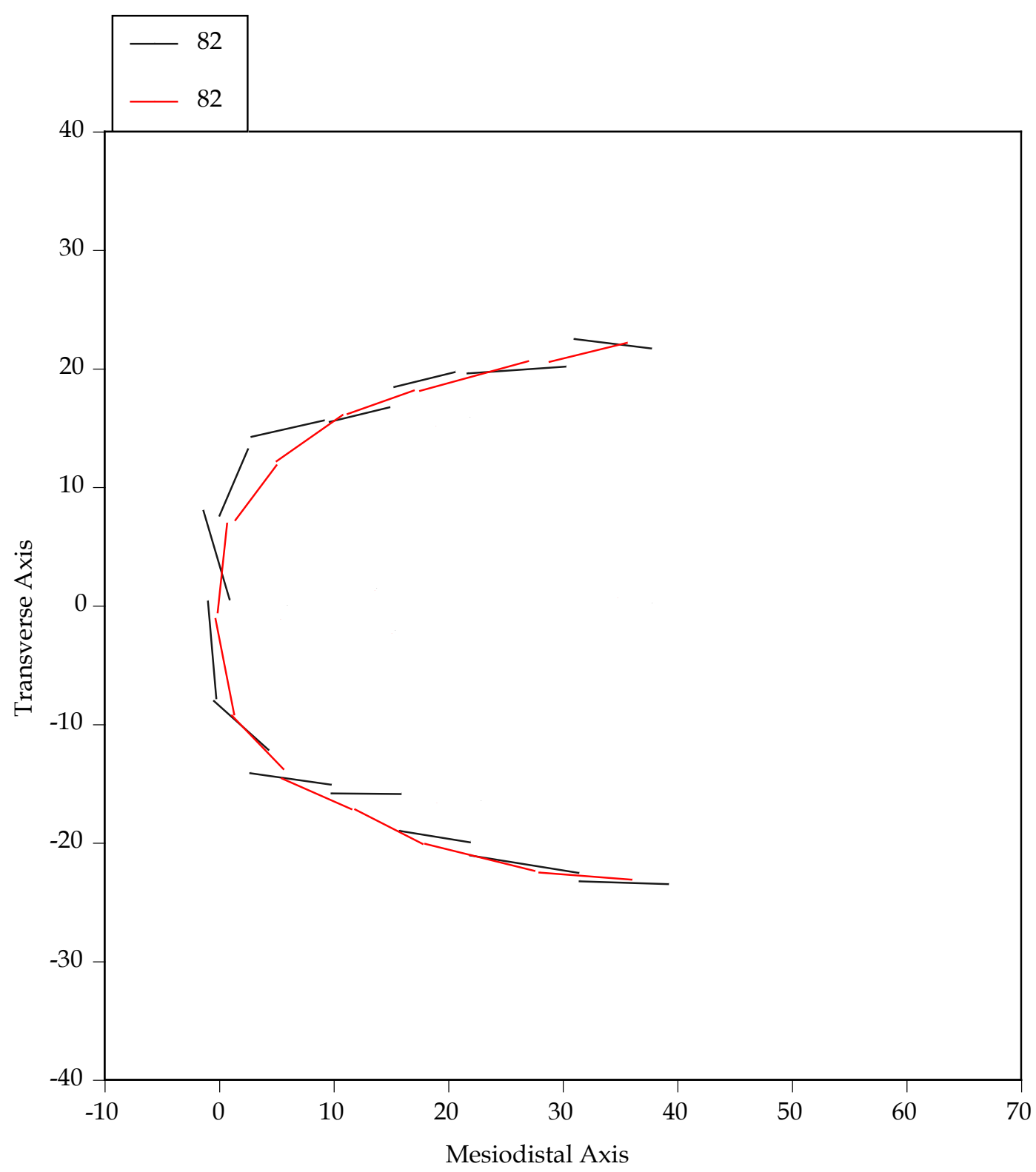

Fig. A-82. Plot of the maxillary dental arch relationships of case 82 , a female in whom the maxillary second-premolars were extracted for treatment. Squares are the dental contacts at the start of treatment; circles are the contacts at the end of treatment. The $X$ and $\mathrm{Y}$ axes are in millimeters. 


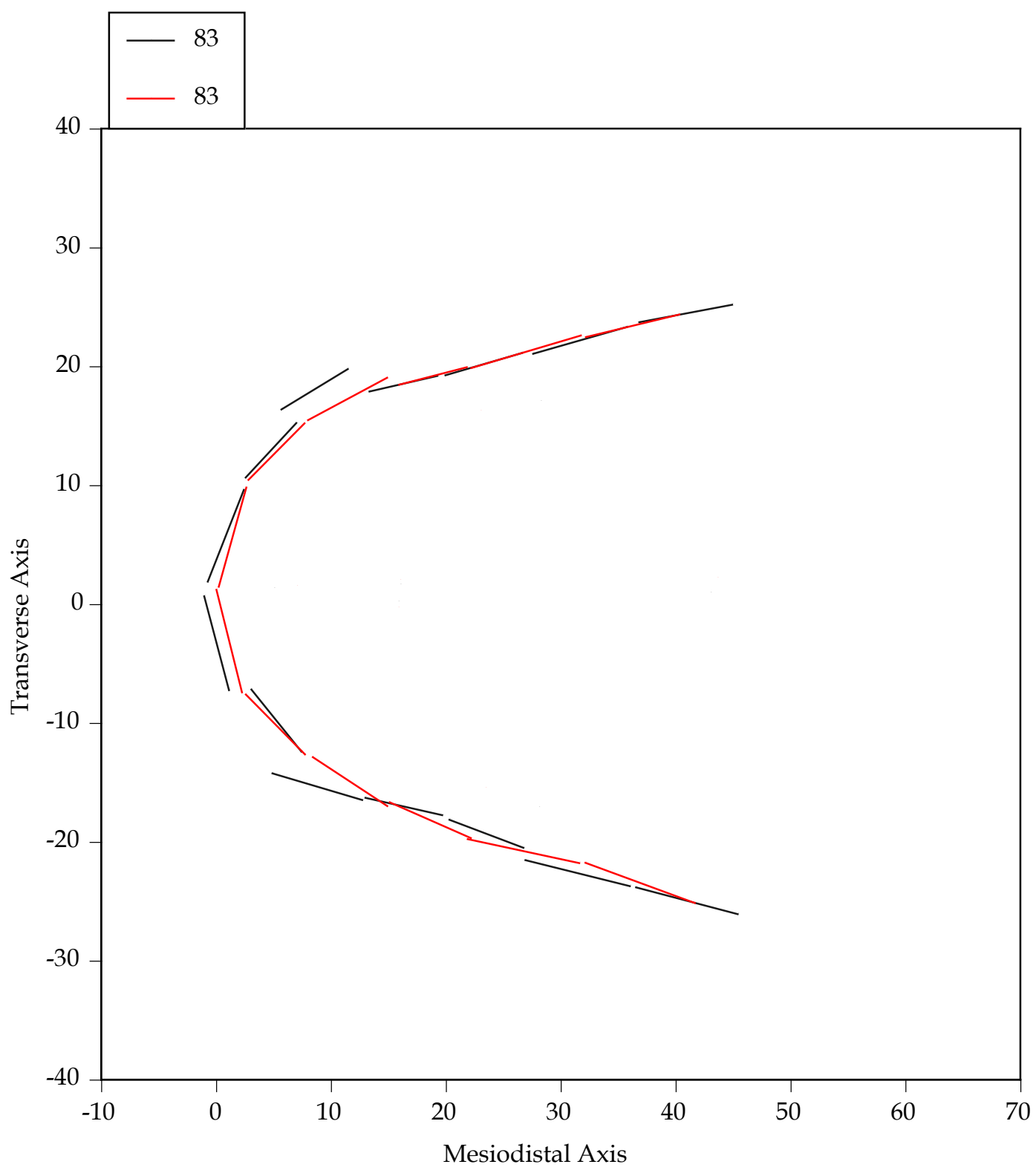

Fig. A-83. Plot of the maxillary dental arch relationships of case 83 , a female in whom the maxillary second-premolars were extracted for treatment. Squares are the dental contacts at the start of treatment; circles are the contacts at the end of treatment. The $X$ and $Y$ axes are in millimeters. 


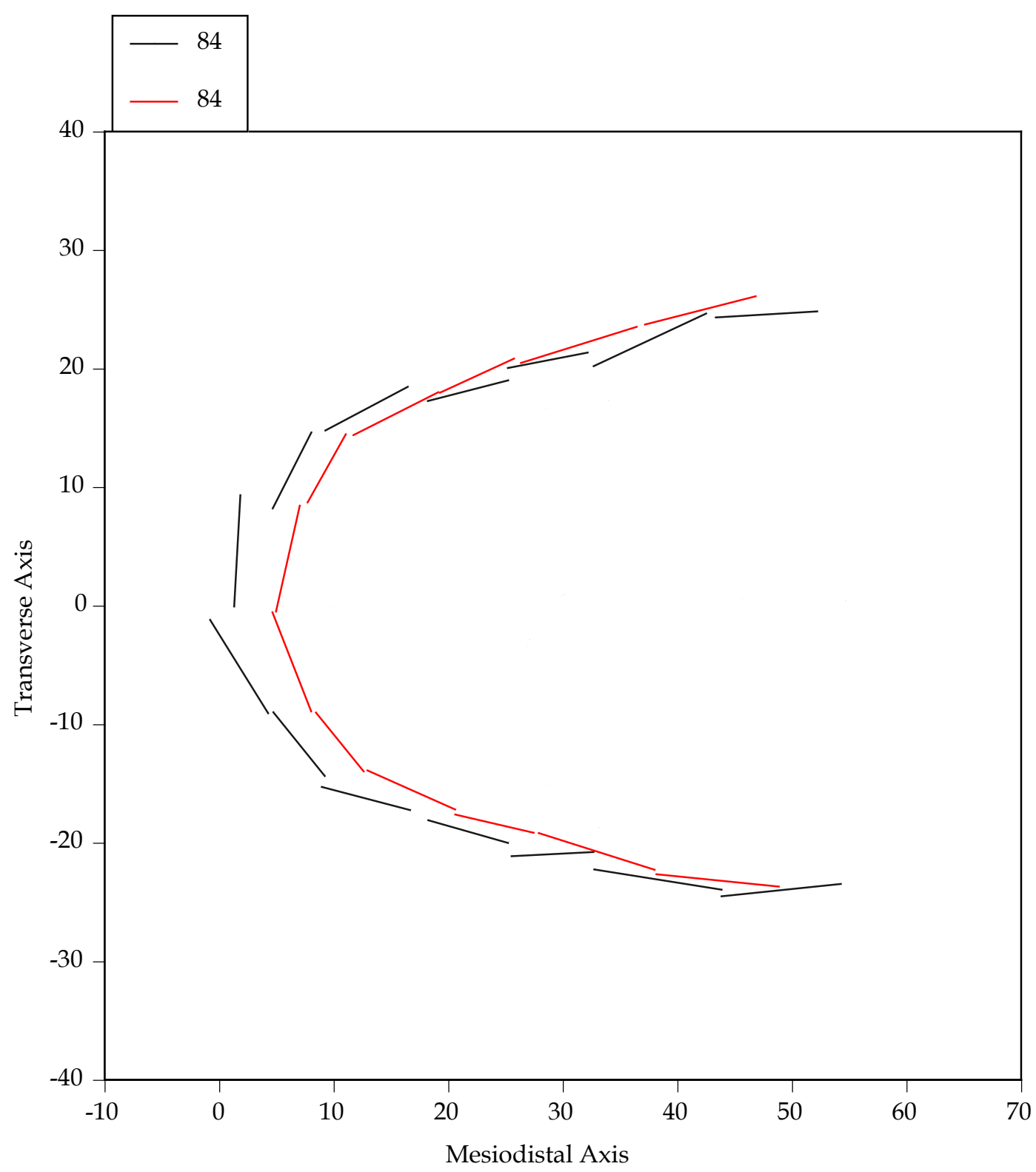

Fig. A-84. Plot of the maxillary dental arch relationships of case 84 , a male in whom the maxillary first-premolars were extracted for treatment. Squares are the dental contacts at the start of treatment; circles are the contacts at the end of treatment. The $X$ and $\mathrm{Y}$ axes are in millimeters. 


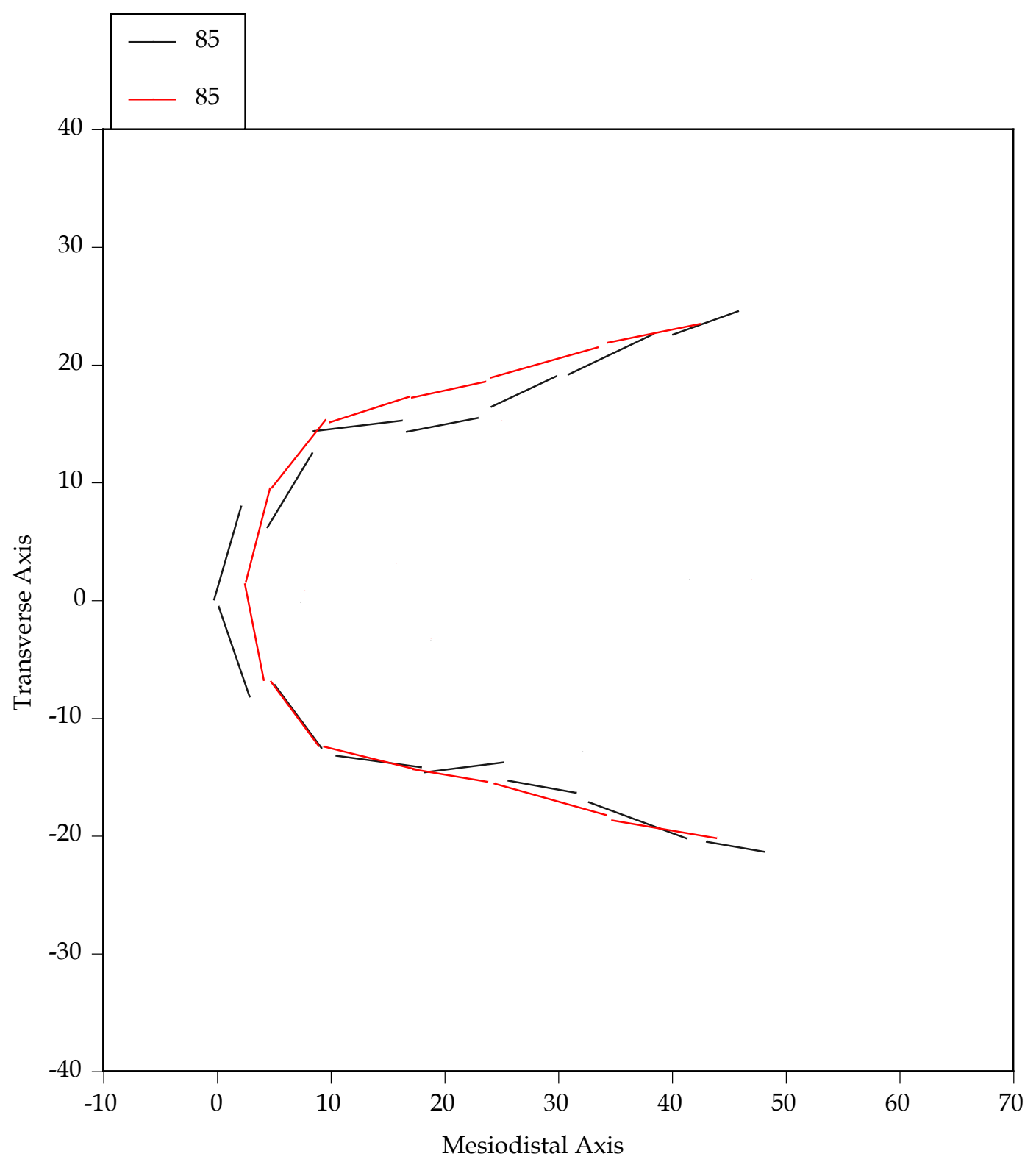

Fig. A-85. Plot of the maxillary dental arch relationships of case 85 , a male in whom the maxillary second-premolars were extracted for treatment. Squares are the dental contacts at the start of treatment; circles are the contacts at the end of treatment. The $\mathrm{X}$ and $\mathrm{Y}$ axes are in millimeters. 


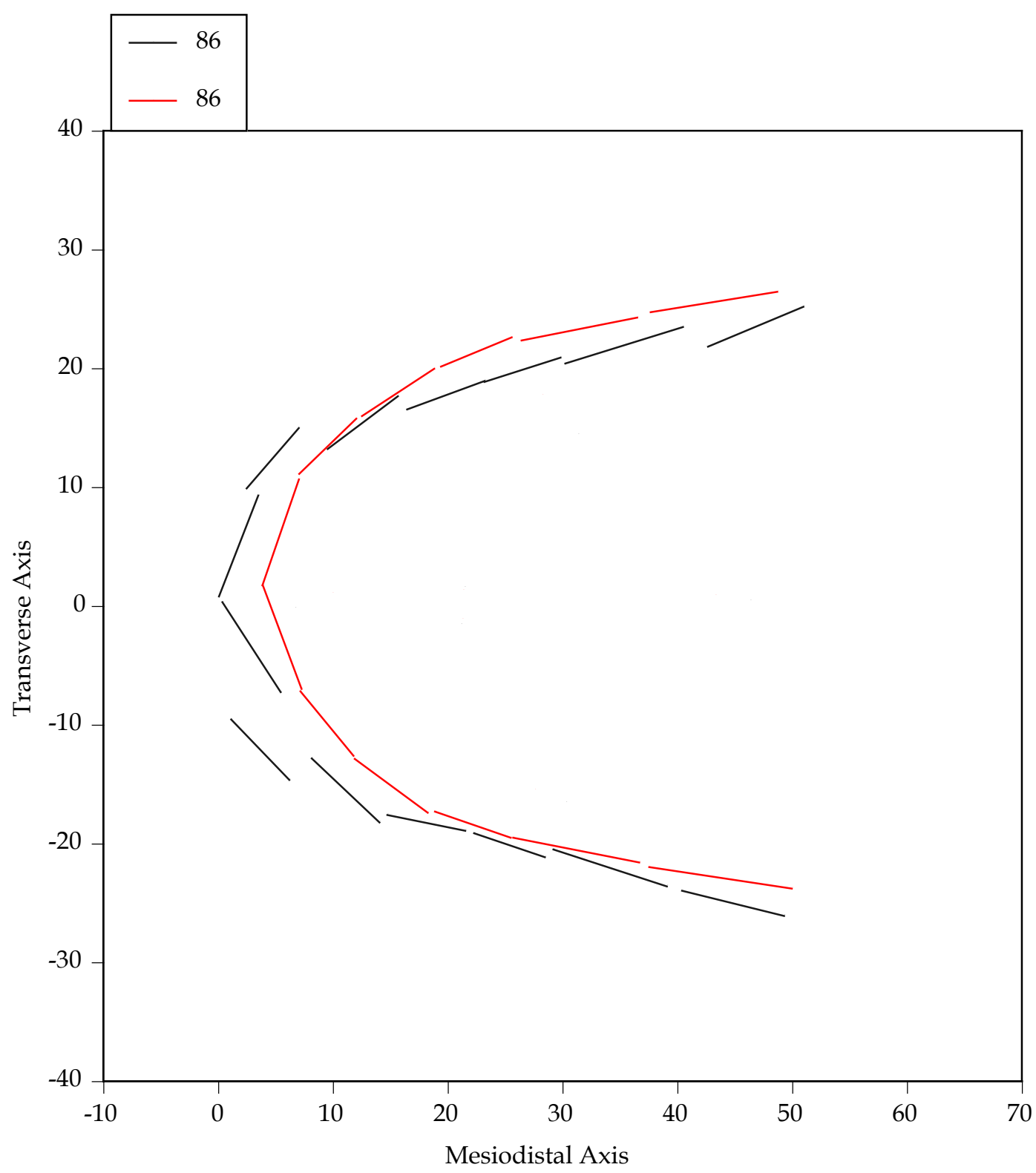

Fig. A-86. Plot of the maxillary dental arch relationships of case 86 , a female in whom the maxillary second-premolars were extracted for treatment. Squares are the dental contacts at the start of treatment; circles are the contacts at the end of treatment. The $\mathrm{X}$ and $\mathrm{Y}$ axes are in millimeters. 


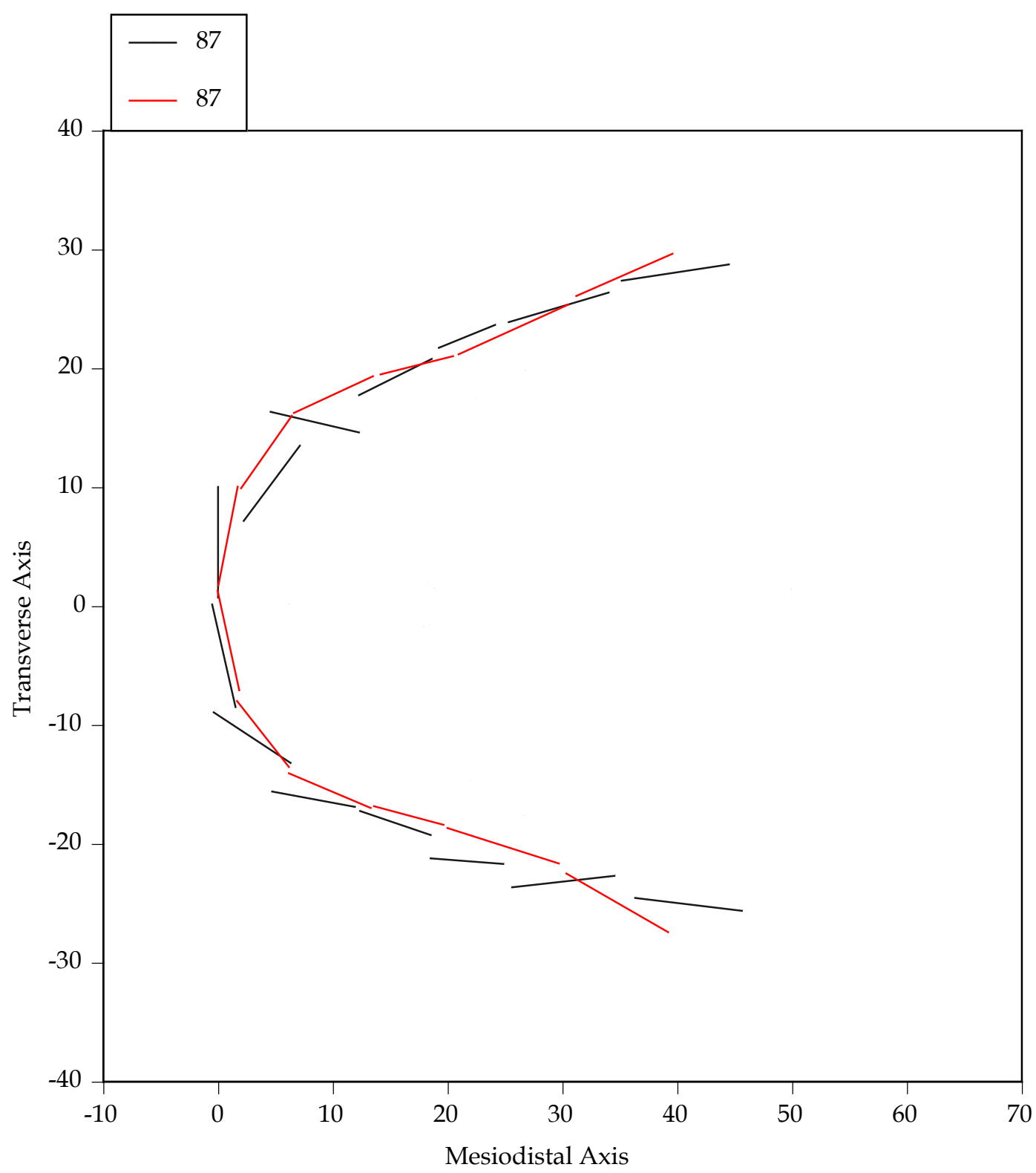

Fig. A-87. Plot of the maxillary dental arch relationships of case 87, a female in whom the maxillary second-premolars were extracted for treatment. Squares are the dental contacts at the start of treatment; circles are the contacts at the end of treatment. The $\mathrm{X}$ and $\mathrm{Y}$ axes are in millimeters. 


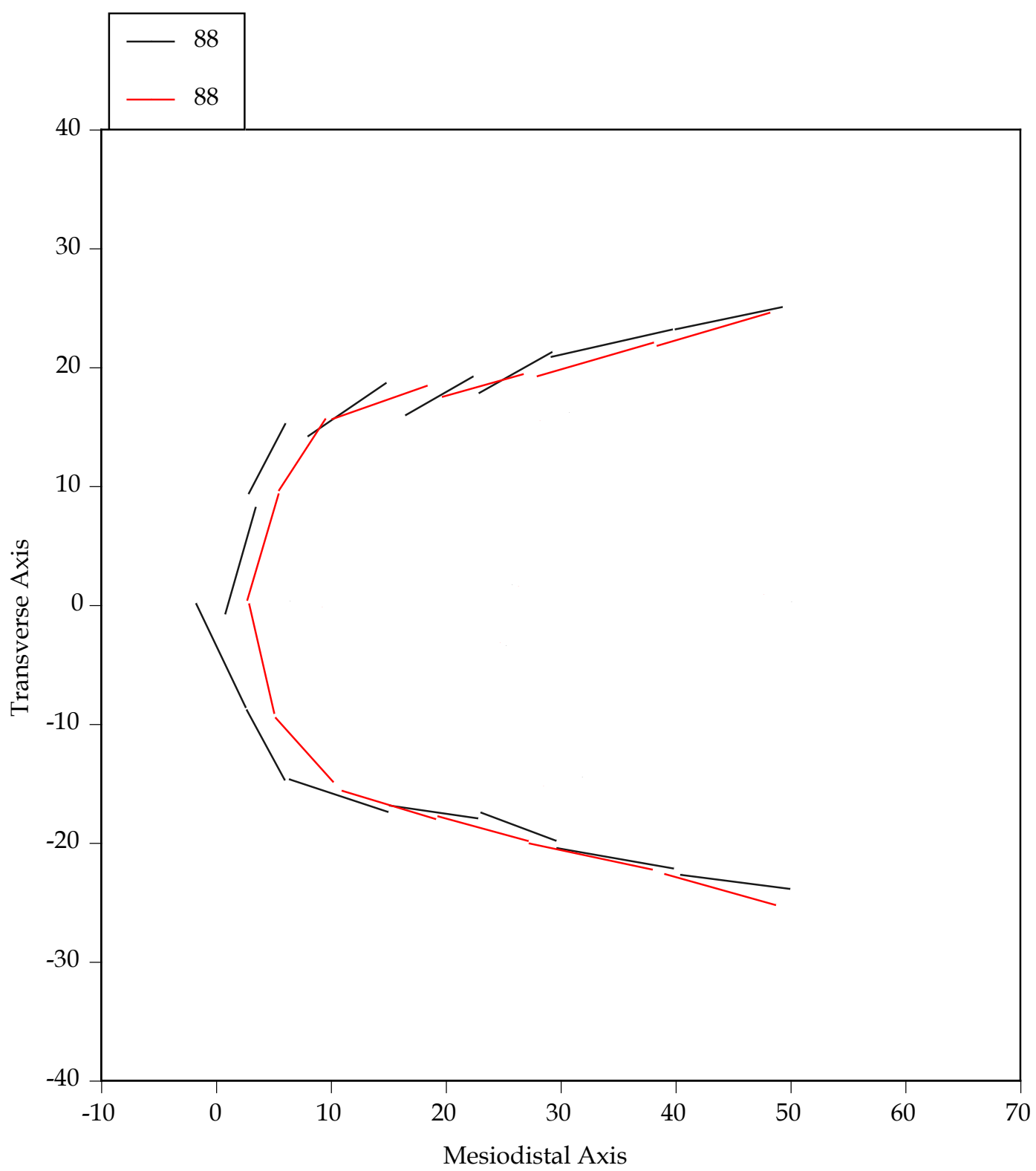

Fig. A-88. Plot of the maxillary dental arch relationships of case 88 , a male in whom the maxillary first-premolars were extracted for treatment. Squares are the dental contacts at the start of treatment; circles are the contacts at the end of treatment. The $X$ and $\mathrm{Y}$ axes are in millimeters. 


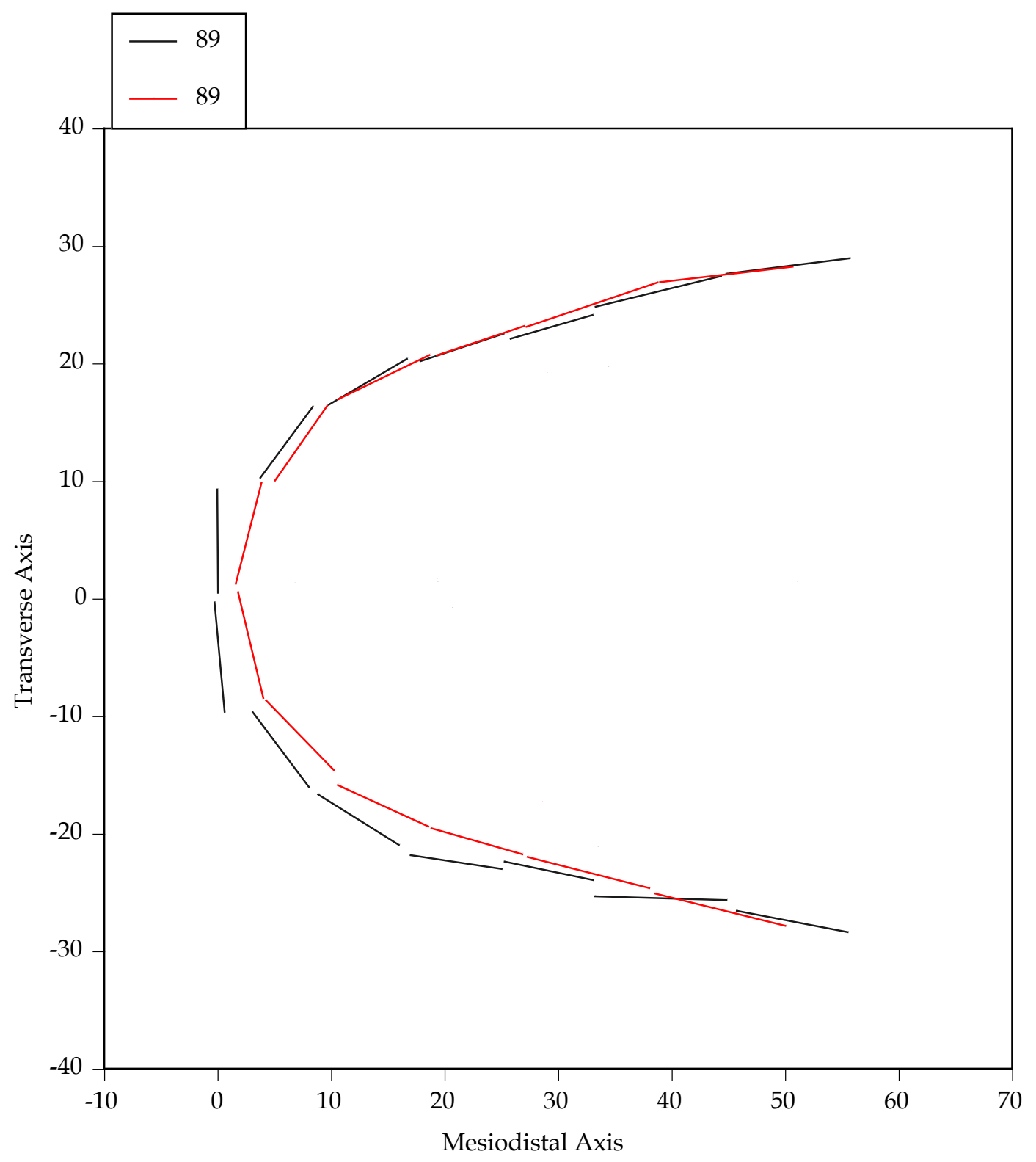

Fig. A-89. Plot of the maxillary dental arch relationships of case 89 , a male in whom the maxillary first-premolars were extracted for treatment. Squares are the dental contacts at the start of treatment; circles are the contacts at the end of treatment. The $X$ and $\mathrm{Y}$ axes are in millimeters. 


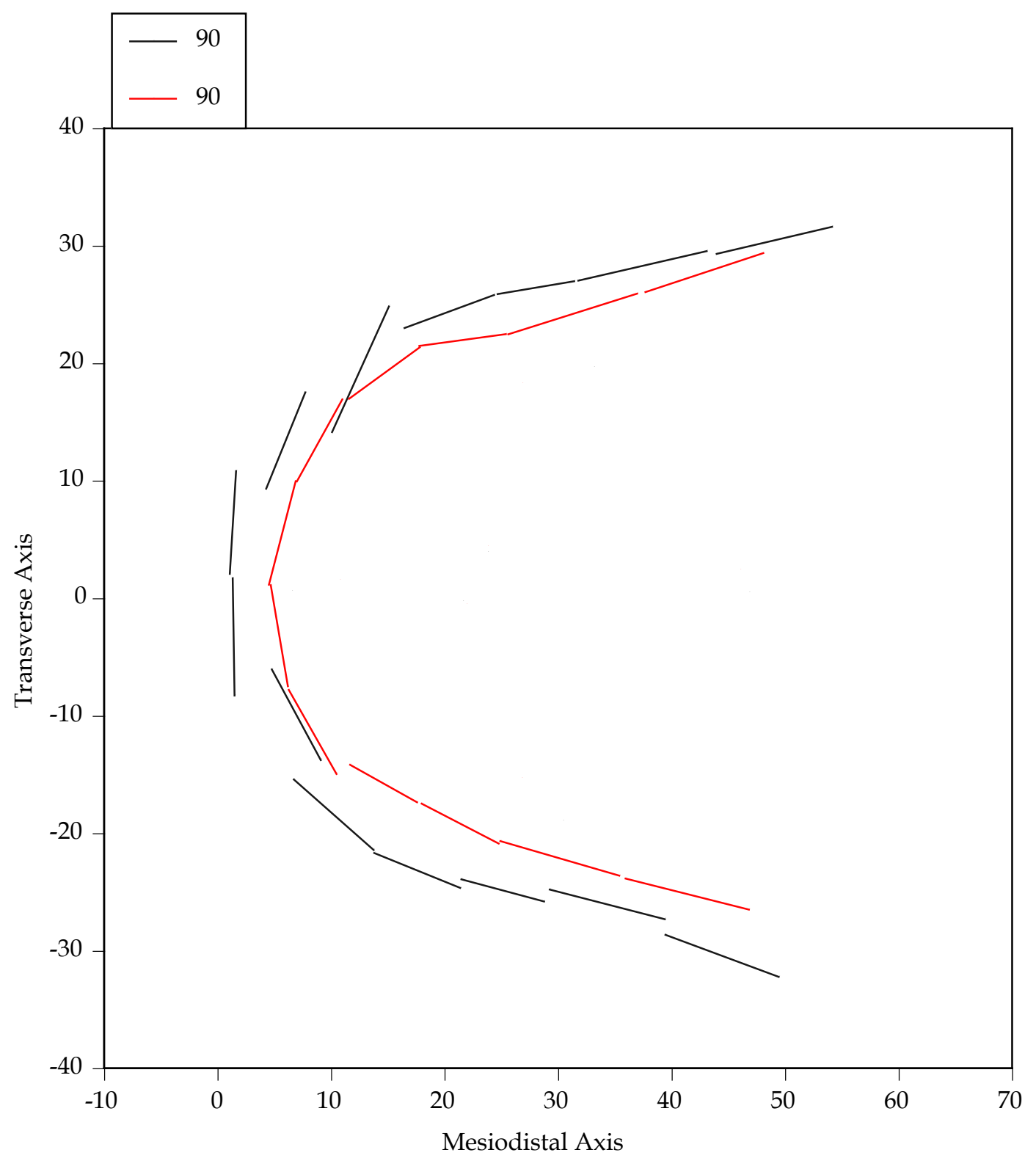

Fig. A-90. Plot of the maxillary dental arch relationships of case 90, a female in whom the maxillary first-premolars were extracted for treatment. Squares are the dental contacts at the start of treatment; circles are the contacts at the end of treatment. The $\mathrm{X}$ and $Y$ axes are in millimeters. 
Robert Andrew Turner was born in Knoxville, Tennessee, on November 7, 1977. He attended elementary school and graduated from Halls High School in May, 1996 in Knoxville, Tennessee. The following month he entered the University of Tennessee, Knoxville and in May, 2000 received the degree Bachelor of Sciences in Biology. He entered the University of Tennessee School of Dentistry in Memphis in August 2000 and in May, 2004 received the degree of Doctor of Dental Surgery. In August, 2004 he entered The Univeristy of Tennessee Health Science Center, Memphis, as a graduate student in Orthodontics and will receive his Master of Dental Science degree in May, 2007.

He plans to return to Knoxville, Tennessee, to enter private practice specializing in orthodontics. 